\title{
Universal Śaivism
}




\section{Gonda Indological Studies}

PUBLISHED UNDER THE AUSPICES OF THE J. GONDA FOUNDATION ROYAL NETHERLANDS ACADEMY OF ARTS AND SCIENCES

VOLUME 18

\section{Editor}

Peter C. Bisschop (Leiden)

\section{Editorial Board}

Hans T. Bakker (Groningen)

Dominic D.S. Goodall (Paris/Pondicherry)

Hans Harder (Heidelberg)

Stephanie Jamison (Los Angeles)

Ellen M. Raven (Leiden)

Jonathan A. Silk (Leiden)

The titles published in this series are listed at brill.com/gis 


\section{Universal Śaivism}

The Appeasement of All Gods and Powers in the Śāntyadhyāya of the Śivadharmaśāstra

Peter C. Bisschop

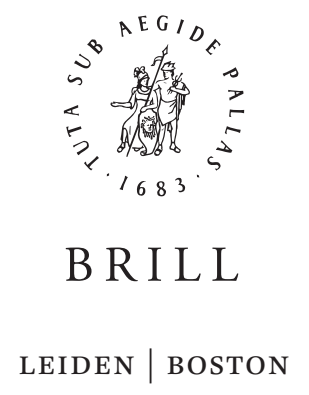


This is an open access title distributed under the terms of the prevailing CC-BY-NC License at the time of publication, which permits any non-commercial use, distribution, and reproduction in any medium, provided the original author(s) and source are credited.

This book has been realised thanks to the financial support of the European Research Council (ERC) and the Netherlands Organisation of Scientific Research (Nwo).

Cover illustration: Multi-faced Śiva-linga from Mt. Harșa (Rajasthan), Ajmer Government Museum. Photo by Peter C. Bisschop.

Library of Congress Control Number: 2018955596

Typeface for the Latin, Greek, and Cyrillic scripts: "Brill”. See and download: brill.com/brill-typeface.

\section{ISSN $1382-3442$}

ISBN 978-90-04-38246-6 (hardback)

ISBN 978-90-04-38436-1 (e-book)

Copyright 2018 by Peter C. Bisschop. Published by Koninklijke Brill Nv, Leiden, The Netherlands. Koninklijke Brill NV incorporates the imprints Brill, Brill Hes \& De Graaf, Brill Nijhoff, Brill Rodopi, Brill Sense, Hotei Publishing, mentis Verlag, Verlag Ferdinand Schöningh and Wilhelm Fink Verlag. Koninklijke Brill NV reserves the right to protect the publication against unauthorized use and to authorize dissemination by means of offprints, legitimate photocopies, microform editions, reprints, translations, and secondary information sources, such as abstracting and indexing services including databases. Requests for commercial re-use, use of parts of the publication, and/or translations must be addressed to Koninklijke Brill NV.

This book is printed on acid-free paper and produced in a sustainable manner. 


\section{Contents}

Preface vii

Introduction 1

The Śivadharma . . . . . . . . . . . . . . . . . . 4

The Teaching of the Śivadharmaśästra $\ldots \ldots \ldots$. . . . 6

The Date of the Śivadharmaśāstra . . . . . . . . . . . . . . . 9

The Provenance of the Śivadharmaśāstra . . . . . . . . . . . 25

The Śäntyadhyāya . . . . . . . . . . . . . . . . . . . . 27

Order and Organisation of the Mantra $\ldots . . . . . .27$

Iconographical Aspects . . . . . . . . . . . . . . . . . . . . 39

Universal Śaivism . . . . . . . . . . . . . . . . . . . . . . 41

Use of the Mantra . . . . . . . . . . . . . . . . . . . . 43

Introduction to the Edition . . . . . . . . . . . . . 49

Sources for the Edition . . . . . . . . . . . . . . $5^{2}$

The Transmission in Nepal . . . . . . . . . . . . . . . . . 54

The Transmission in Bengal . . . . . . . . . . . . . . 59

The Transmission in Kashmir . . . . . . . . . . . 6o

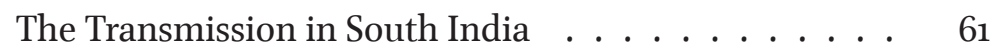

The Critical Apparatus . . . . . . . . . . . . . . 63

The Śāntyadhyāya: Edition $\quad 65$

The Chapter on Appeasement: Translation $\quad 147$

$\begin{array}{ll}\text { Appendix } & 189\end{array}$

Single-Text and Composite Manuscripts . . . . . . . . . . . 189

Śāntyadhyāya Single-Text Manuscripts . . . . . . . . . . 189

Śāntyadhyāya Composite Manuscripts . . . . . . . . 191 
A Commentary on the Śāntyadhyāya . . . . . . . . . . . . . . 195

Transcription . . . . . . . . . . . . . . . . 196

Translation . . . . . . . . . . . . . 197

$\begin{array}{ll}\text { Bibliography } & 201\end{array}$

$\begin{array}{ll}\text { Index } & 215\end{array}$ 


\section{Preface}

My first encounter with the Sivadharma goes back to the time of my PhD. In surveying the so-called Pañcāșțaka - five sets of eight Śaiva sacred sites (Bisschop 2006: 27-34) — I incorporated the readings of this list in the Śivadharmaśāstra on the basis of a Nepalese palm-leaf manuscript in the collection of the Cambridge University Library (MS Add. $1645=$ siglum $\mathrm{N}_{45}^{c}$ in the present work). In the years after I continued to be interested in the Sivadharma as an unexplored source on early, non-Tantric forms of Śiva worship. I started collecting images of manuscripts and gave a first presentation of my findings at the 'Third International Workshop on Early Tantra' at Hamburg University in the summer of 2010. As the title of my paper at the time shows ('The Sañntyadhyāya of the Sivadharma: Its Relevance for the Study of Early Śaivism and Tantra') my interest had focussed on one chapter in particular: the Śāntyadhyāya. I subsequently gave presentations on my work in progress at several workshops and conferences ('Empires of Faith: South Asia as a Field of Global Religious Interaction', Bochum, April 2013; 'Tantric Communities in Contexts: Sacred Secrets and Public Rituals', Vienna, February 2015; 'Asia Beyond Boundaries Seminar', London, October 2015; 'Books of Śaiva Knowledge', Hamburg, June 2016), and I got in touch with other scholars who had started working on different parts of the corpus. I also had the chance to read parts of my draft edition at the Leiden Summer School in Languages and Linguistics in 2014 and 2015. Finally, thanks to a grant of the European Research Council (ERC), I was able to organise 'The Śivadharma Workshop: Manuscripts, Editions, Perspectives' at Leiden University in September 2016.

As the above trajectory indicates, I have had occasion to discuss my work with many colleagues along the way, all of whom have contributed 
to it in one way or another. I should first of all like to thank the participants of the 2016 Leiden workshop, in particular Elizabeth Cecil, Csaba Dezső, Marco Franceschini, Dominic Goodall, Nirajan Kafle, Timothy Lubin, Florinda De Simini, Nina Mirnig, Judit Törzsök and Yuko Yokochi. Several of them I should mention once more: Elizabeth Cecil for challenging me to express my thoughts more clearly on a number of occasions; Dominic Goodall for pertinent remarks on Śaiva matters; Florinda De Simini and Nina Mirnig for providing me with images of several of the manuscripts that I have used for the edition; and Yuko Yokochi for reading through the entire work and providing me with critical feedback. Likewise, I thank Hans Bakker, Arlo Griffiths and Harunaga Isaacson for reading and commenting upon an earlier version of this book. Bill Mak enlightened me on several Jyotihśāstra peculiarities. Anil Kumar Acharya deserves thanks from all students of the Sivadharma for preparing an e-text. I would also like to thank Kristen de Joseph for proofreading and correcting my English.

I am indebted to the various libraries and institutions whose manuscripts I have used for my work: the Asha Archives, Kathmandu; the Asiatic Society, Kolkata; the Institut Française de Pondichéry; the National Archives of Kathmandu; the Nepal German Manuscript Preservation Project; the Oriental Research Institute and Manuscripts Library, Trivandrum; the Oriental Research Library, Srinagar; the University Library of Cambridge; and the University of Pennsylvania Libraries.

This project was made possible thanks to the support of the European Research Council (ERC) Synergy Project 6o9823, and the Netherlands Organisation for Scientific Research (NWO) Open Competition Project 36o-63-110. I am particularly grateful to the J. Gonda Fund Foundation for supporting the publication of this book in open access.

Leiden, May 2018 


\section{Introduction}

The traditions that have evolved around the worship of the god Siva show a remarkable tendency and capacity to absorb other cults, deities and principles. This point is well illustrated by the subject of the present study, a lengthy mantra, addressed to all gods and powers, for the pacification (śänti) of any malevolent influences. Running through the invocation is the underlying notion that all worldly and cosmic power is ultimately dependent upon and oriented towards Śiva. I refer to this ideology as 'Universal Śaivism'. At the time the mantra was composed, the worship of Śiva and his aniconic form, the linga, had been popular for several centuries, but in contrast to Brahmanism, Buddhism or Jainism, it had no recognisable canon, nor had Śaivism developed into an organised religion with well defined roles of ascetics, priests and laity. This period then sees an intensive production of texts, culminating in an overarching conception of Śaivism as the religion dedicated to and taught by Siva. The composers of these texts participated in and adapted already existing models to create their normative vision of Śaivism from what must actually have been a plurality of practices on the ground.

The mantra that forms the subject of this study is part of the Sivadharmaśāstra, a text belonging to the corpus of lay Śaiva literature generally referred to as Śivadharma 'Religion of Śiva'. It is taught in the Śivadharmaśāstra's sixth chapter, also going under the name of Śāntyadhyāya 'Chapter on Appeasement'. The Śivadharma had long been neglected by scholars, though it has arguably played a crucial role in the formation, development and institutionalisation of Śaivism, making it a key text for the study of the religion's social and ritual formations. ${ }^{1}$ The Sivadharmaśāstra is the first text that systematically targets and integrates

${ }^{1}$ For a long time the Śivadharma was known almost exclusively through Hazra's two summaries of the first two works of the corpus, the Sivadharmaśāstra and the Siva- 
the growing body of lay devotees of Śiva, offering them a distinctive social system and model of religious practice and ritual. ${ }^{2}$ While its main teaching centres around the linga cult, running through the text is an attempt to integrate and subsume all other religious traditions under the heading of 'Śaivism'.

The core of this book consists of a critical edition and annotated translation of the Śāntyadhyāya. The edition aims to provide the reader with an overview of some of the main variations in the transmission of the mantra, including manuscripts from regions as far apart as Nepal, Kashmir, Bengal and South India. The annotated translation draws attention to the rich data contained in the mantra and also includes translations of passages that do not occur in the main constituted text, being found only in certain lines of transmission. The large number of variant readings, including lengthy additional passages, are indicative of the mantra's intensive use over time and reveal much about the values and motives of the communities engaged in its transmission. These changes attest to the fact that the text has been a living tradition across the Indian subcontinent for many centuries.

In the remainder of this introduction I address several aspects con-

dharmottara (Hazra 1954, 1956). This situation has changed significantly in recent years. The full text of the corpus was first published by Naraharinātha in 1998, while over the past few years several scholars have started to work on individual parts of the corpus or referred to them in their studies. See, in particular, Acharya 20o9; Bisschop 2010, 2014; De Simini 2013, 2016a, 2016b, 2017; De Simini \& Mirnig 2017; Goodall 2011; Kafle 2013, 2015; Magnone 2005; Sanderson 2003/O4, 2012/13; Schwartz 2012. An edition of the Śivadharmaśástra alone, based on a single manuscript in the Adyar Library, has been published more recently as well (Jugnu \& Sharma 2014). The Śivopanișad, which also forms part of the Śivadharma corpus, was already published much earlier but was not recognised as such, being included in a collection of Upanișads (Kunhan Raja 1933).

${ }^{2}$ The last few decades have seen major advances in the study of Śaivism, culminating in Sanderson's influential study 'The Śaiva Age' (2009), in which he has shown, on the basis of extensive textual and epigraphic evidence, how Śaivism came to be integral to the religious landscape of early medieval South and Southeast Asia. Most of the groundbreaking scholarship in the field has been on the Tantric or initiatory forms of Śaivism. For two recent surveys, see Hatley 2010 and Goodall \& Isaacson 2011. The lay, non-initiatory forms of the religion, by contrast, have received considerably less attention, with the significant exception of the work done in the framework of the critical edition of the Skandapurāna. In addition to the volumes of the critical edition and various related studies, see Bakker 2014 for the historical context of the composition of the Skandapurāṇa. 
cerning the composition and transmission of the Śivadharmaśāstra, and its Sāntyadhyāya in particular, in order to position the text within the historical development of Śaivism.

The section 'The Sivadharma' briefly introduces the Śivadharma text corpus, with a specific focus on the Śivadharmaśāstra. A crucial step towards putting the text into context is to establish, as far as possible, its time of composition. The Śivadharmaśāstra's anonymous character makes this a difficult task. I have assembled the main body of available evidence that may help us locate the text in place and in time. As I will argue, the Śantyadhyāya in particular provides some important clues to its date of composition or redaction. I conclude that the Śantyadhyāya was most probably composed in North India towards the end of the sixth or the beginning of the seventh century at the latest. To what extent this date also applies to other parts of the Sivadharmaśāstra remains to be evaluated.

The section 'The Śāntyadhyāya' commences with an overview and discussion of the principles behind the order and organisation of the mantra. Here I also draw attention to the rich body of iconographical data contained in the mantra and take up the question of its ritual use. The available evidence indicates that the Śantyadhyāya's mantra was used for a variety of purposes in different regions of the Indian subcontinent over a long period of time, but always with a view to appeasing external forces, be it for oneself or for the benefit of the kingdom.

The section 'Introduction to the Edition' sets out the principles of the text edition. With at least 80 known surviving manuscripts from different parts of the Indian subcontinent, editing the Sivadharmaśāstra presents a major challenge. The edition of the Śāntyadhyāya does not aspire to reconstruct an urtext underlying all the variation, let alone strive for completeness. Its goals are more modest. I have made a careful and representative selection of manuscripts from different parts of the Indian subcontinent that should give the reader insight into the wide distribution of variations. As a methodological principle I have followed the readings of the oldest Nepalese palm-leaf manuscripts, mainly to provide an anchor against which the other readings can be read and compared. On the basis of regional distribution, four main groups of manuscripts may be distinguished: those from Nepal, Bengal, Kashmir and South In- 
dia. There is as yet no manuscript evidence that there was an independent recension of the text in Bengal, but there are clearly recognisable features that allow us to distinguish different recensions of the text in Nepal, Kashmir and South India. On the other hand, there is a lot of evidence for contamination, which makes drawing up of a stemma a hazardous task - if not an impossible one, at least for now. More future work on other parts of the Śivadharma may help us develop a more informed understanding of the individual lines of transmission of the text.

Two appendices conclude this book. The first gives an overview of several known single-text manuscripts of the Śantyadhyāya, along with composite manuscripts in which the chapter has been included in a bundle together with selected passages from other texts. The second appendix gives a transcript and translation of a Sanskrit commentary on the Sántyadhyāya. This is to be found in a unique Malayalam manuscript in the Trivandrum Manuscript Library, which contains the text of the Śivadharmaśāstra followed by a pratīka-style commentary (Śivadharmavivaraña) on the Śivadharmaśāstra and the Śivadharmottara.

The larger aims of this study are twofold. First, it intends to contribute to an understanding of the position of the Sivadharmaśāstra in the socio-historical formation of Śaivism as an institutionalised religion. While it seems doubtful that the lay devotees of Śiva who form the target audience of the Śivadharmaśāstra were organised in any systematic way, the authors of the text clearly intended to provide a blueprint of a complete and unified Śaiva society. ${ }^{3}$ Secondly, the edition aims to contribute to the development of a set of methodological principles for the critical edition of texts belonging to the Sivadharma corpus. The principles adopted for editing the Śantyadhyāya are set out below, not only with reference to the Śantyadhyāya but also with a view to possible future editions of other parts of the corpus.

\section{The Śivadharma}

The collective name 'Śivadharma' refers to a corpus of anonymous Śaiva texts of uncertain origins, written in Sanskrit. Texts belonging to the Śivadharma expound the rituals, activities and attitudes of devotion to be

${ }^{3}$ This becomes clear in particular from Śivadharmaśāstra 11 and 12. 
adopted by devotees of Śiva. It is prescriptive rather than descriptive. The corpus expanded over time with the addition of a number of new texts, which makes the Sivadharma into an ideal sourcebook for studying the historical development of Śaivism.

The most commonly encountered order of the texts found in Sivadharma manuscripts is the following: ${ }^{4}$

1. Śivadharmaśāstra 'Treatise on the Religion of Śiva'

2. Sivadharmottara 'Supplement on the Religion of Śiva'

3. Sivadharmasamgraha 'Compendium on the Religion of Śiva'

4. Umāmaheśvarasamvvāda 'Dialogue Between Umā and Maheśvara'

5. Uttarottaramahāsamvāada 'Great Dialogue of Questions and Answers'

6. Śivopanișad 'Mystery of Śiva'

7. Vrșasārasamgraha 'Compendium on the Essence of the Bull [of Dharma]'

\section{Dharmaputrikā 'Daughter of Dharma's}

About 18 such corpus manuscripts survive in the form of old palm-leaf manuscripts from Nepal, in addition to many later paper manuscripts. This tradition of eight Śivadharma texts is, however, unique to Nepal. From outside of Nepal only manuscripts of the Sivadharmaśästra and the Śivadharmottara survive, mostly transmitted as separate works.

There can be no doubt that the text heading the corpus, the Sivadharmaśāstra, is also historically the first of the Sivadharma texts. It is the most archaic, both in form and character, and provides the model for the Śivadharmottara that follows, which expands upon many of the

\footnotetext{
${ }^{4}$ For a thorough study of the corpus manuscripts and corpus organisation of the Sivadharma, see De Simini 2016a.

${ }^{5} \mathrm{~N}_{45}^{c}$, an eleventh-century Nepalese palmleaf manuscript in the Asiatic Society of Calcutta (G 4077), is unique in that it includes a longer text called Lalitavistara - not to be confused with the Buddhist text of the same name — instead of the Dharmaputrika as the last text of the corpus (De Simini and Mirnig 2017). For the list of sigla of the manuscripts of the Śivadharma used in this book, see p. $5^{2}$.
} 
topics introduced in the Śivadharmaśāstra. Each text consists of twelve chapters. ${ }^{6}$ After this, the relative chronology of the different texts that make up the corpus is less easy to determine. The third text in the corpus, the Sivadharmasamgraha, is the first to attest to the influence and impact of Tantric religion; it has been shown that it integrated large parts of the Niśvāsamukha. ${ }^{7}$ The Śivopanișad emphatically presents itself as the third text of the Śivadharma, after the Śivadharmaśāstra and the Śivadharmottara, but it did not come to occupy this position in the corpus manuscripts and may in fact have been a relatively late addition. ${ }^{8}$

The Śivadharmaśāstra is sometimes also referred to as Śivadharma, but to distinguish it from the Sivadharma corpus at large I refer to the individual text as Śivadharmaśāstra. This is the title given in the colophons of the majority of manuscripts. In origin it was an individual work and that is how it will be treated in this study.

\section{The Teaching of the Śivadharmaśāstra}

The Śivadharmaśāstra is introduced in the opening verses as a dialogue between the divine sage Sanatkumāra and Siva's foremost Gaṇa Nandikeśvara. At the request of Sanatkumāra, Nandikeśvara instructs Sanatkumāra and the sages dwelling on Mount Meru in the worship of Siva. ${ }^{9}$

\footnotetext{
${ }^{6}$ See also De Simini 2013: 34-35, 54-55.

${ }^{7}$ See Kafle 2015: 61-72. This was first noticed by Anil Kumar Acharya, who edited the first three chapters of the Śivadharmasamgraha for his PhD thesis at Pondicherry University (Acharya 2009). The Niśvāsatattvasaṃhitā consists of five books: Niśvāsamukha, Mūlasūtra, Uttarasūtra, Nayasūtra and Guhyasūtra. The Niśvāsamukha most probably belongs to the latest layer of the collection (Goodall et al. 2015: 22).

${ }^{8}$ See De Simini 2016a: 237-239.

${ }^{9}$ Sanatkumāra is well-known from the Mahābhārata and Purānic literature as the firstborn son of Brahmā. He also plays a prominent role in the Skandapurāna, for in that text it is Sanatkumāra who teaches Vyāsa about Śiva's mythology. Nandikeśvara is a distinct Śaiva figure, likewise known from the Skandapurāna. The Skandapurāna recounts the story of how Nandīśvara, also known as Nandin, became Śiva's favourite Gaṇa (SP 20-25). Moreover, the Skandapurāṇa contains a section of Śivadharma-like teachings that are put into his mouth (SP 27-28). These two chapters of the Skandapurāna are introduced by a question of Vyāsa concerning Śiva's teachings to Devī when she asked him about the benefits that his devotees receive from their devotional activities. Sanatkumāra tells Vyāsa that he once put the same question to Nandīśvara. He then reports to Vyāsa the dialogue between Śiva and Devī. This Skandapurāna episode matches the frame story of the Śivadharmaśāstra and indicates that Nandīśvara was
} 
Nandikeśvara tells him that the teaching that he is about to expound was originally revealed by Śiva himself to Pārvatī, Skanda (Șaṇmukha), Nandikeśvara and the other gods. ${ }^{10}$ In other words, the Śivadharma gives rules for the worship of Śiva that ultimately come from the mouth of the deity himself. This format of the teaching corresponds to Sanderson's definition of Śaivism in his seminal article 'Śaivism and the Tantric Traditions':

The term Śaivism here refers to a number of distinct but historically related systems comprising theology, ritual, observance and yoga, which have been propagated in India as the teachings of the Hindu deity Śiva. A Śaiva is one who practices such a system. (Sanderson 1988: 66o)

The frame story of the Sivadharmaśāstra matches the paradigm of Tantric teachings. Śaiva Tantras characteristically start with a section on the 'descent of the Tantra' (tantrāvatāra), which serves to explain how the esoteric teachings originally uttered by the deity have reached human ears." Significantly, the Niśvāsatattvasaṃhitā, the earliest surviving Śaiva Tantra, is likewise transmitted through the mouth of Nandikeśvara. In the opening verses of the Niśvāsamukha, Nandikeśvara initiates the sages and introduces them to the five streams of knowledge as they had been originally revealed by Siva to Devī (Niśvāsamukha 1.1-29). While the teachings of the Niśvāsatattvasamhitā and other Tantras are esoteric instructions requiring initiation, those of the Śivadharma are open to all Śiva's devotees. It is devotion (bhakti) that is key and Nandikeśvara represents the quintessential Śaiva devotee. ${ }^{12}$

In the first chapter Nandikeśvara introduces the nature and power of Rudra-Śiva devotion. A striking feature of his introduction is the notion that people who are exclusively devoted to Rudra are veritable Rudras on earth. His devotees are set apart from the rest of humankind:

held to be a teacher of the Śaiva religion at the time. However, although SP $27-28$ is very similar in content, there are no significant textual parallels between the two texts (SP IIA: 197).

${ }^{10}$ ŚiDhŚ 1.10-11 (draft edition Nina Mirnig): śrūyatām abhidhāsyāmi sukhopāyam mahatphalam | paramam sarvadharmānām śivadharmam śivātmakam || śivena kathitạ̣ pūrvam pārvatyāḥ șaṇmukhasya ca | gaṇānām devamukhyānām asmākam ca viśeșatah $\|$.

${ }^{11}$ See Oberhammer 1994 and TAK III, s.v. tantrāvatāra.

${ }^{12}$ On devotion and Śaivism, with some references to the Śivadharma, see Ganesan \& Sathyanarayanan 2010-11. 
ye 'rcayanti sadā rudram na te prakrtimānuṣāh | rudralokāt paribhrașțās te rudrā nātra saṃśayah $\|^{13}$

They who always worship Rudra, they are no ordinary men. They are Rudras who have come down from Rudraloka. There is no doubt about this.

This also finds expression in a much-cited idea that would come to have a long afterlife in Tantric traditions, but that seems to be voiced here for the first time:

nārudrạ̣ saṃsmared rudraṃ nārudro rudram arcayet $\mid$ nārudraḥ kīrtayed rudram nārudro rudram āpnuyāt $\|^{14}$

One who is not Rudra cannot call to mind Rudra. One who is not Rudra cannot worship Rudra. One who is not Rudra cannot praise Rudra. One who is not Rudra cannot attain Rudra.

The Śivadharmaśāstra consists of 12 chapters in total. The titles of the colophons, as given in the editio princeps of Naraharinātha, are as follows:

1. Sivabhakti 'Devotion to Śiva'

2. Śivadharmapraśnādhyāya 'Chapter of Questions on the Religion of Śiva'

3. Lingotpatti 'Origin of the Linga'

4. Āyatanādhyāya 'Chapter on the Temple'

5. Sivārcanadravyavidhi 'Rules on the Substances for the Honouring of Śiva'

6. Śāntyadhyāya 'Chapter on Appeasement'

7. Pūjādharma 'Practice of Worship'

8. Śivadānaphalādhyāya 'Chapter on the Fruits of Gifts to Siva'

${ }^{13}$ ŚiDhŚ 1.16 (draft edition Nina Mirnig).

${ }^{14}$ ŚiDhŚ 1.24 (draft edition Nina Mirnig). In the context of Tantric worship, variants of this verse (nādevo devam arcayet / nāsivah śivam arcayet) are cited to express that one has to ritually identify oneself with the deity before one may worship the deity. Cf. Goodall et al. 2005: 13. See also Davis 1991. The Śivadharma expresses a more general attitude of bhakti. 
9. Sivalingamahāvrata 'Great Observance of the Śivalinga'

10. Upavāsagopradānavidhi 'Rules on Fasting and the Gift of a Cow'

11. Śivāśrama 'Discipline of Śiva'

12. Śivabhaktyādyaśākhopaśākhādhyāya 'Chapter on the Primary and Secondary Branches of Devotion to Śiva'

The text addresses the concerns of lay devotees of Śiva and provides them with a range of activities to express their devotion, including gifting, undertaking observances and, most important of all, worshipping the linga. The bulk of the work may be characterised as a manual for linga worship. ${ }^{15}$ It is not surprising therefore that the only narrative part of the text should be concerned with the popular myth of the origin of the linga (chapter 3). In fact, as Kafle 2013 has argued, the Sivadharmaśāstra's version is quite likely one of the earliest versions of the myth. Significantly, the theme of the origin of the linga was also singled out for representation on the painted wooden covers of several of the surviving Nepalese palm-leaf manuscripts, which display the linga flanked by Brahmā and Viṣnu, as well as a range of other gods worshipping different lingas. ${ }^{16}$ This configuration closely follows the model of chapter 3 , which, after telling about Brahmā and Viṣnu's failed attempt to find the end of the linga and their subsequent worship of it, mentions that other gods acquired their respective position by worshipping lingas made of different materials. ${ }^{17}$ The final two chapters of the text set out the blueprint for an ideal Śaiva society.

\section{The Date of the Śivadharmaśāstra}

As with so much anonymous literature from premodern India, dating the Śivadharmaśāstra is a major challenge. Yet, in order to appreciate

${ }^{15}$ The chapters dealing with the linga cult form the subject of the FWF project 'The Sivalinga Cult on the Eve of the Tantric Age: A Study and Critical Edition of the Sivadharmaśāstra's Chapters $1-5$ and 7-9' by Nina Mirnig (Vienna).

${ }^{16}$ For an example, see Mookerjee 1946, referring to manuscript G 4077 in the Asiatic Society (our siglum $\mathrm{N}_{77}^{K o}$ ). The first cover shows the Lingodbhava and the second shows various gods worshiping different lingas.

${ }^{17}$ For details, see Bisschop forthc. a. 
the text's position and significance in the history of Śaivism and Indian religion at large, it is crucial to get a proper sense of the time, place and circumstances of its composition. As I will argue, the Sántyadhyāya in particular provides several important clues.

\section{Earlier Attempts}

The first author to have tackled the issue of the date of the Sivadharmaśāstra was R.C. Hazra (1954: 15). After listing the quotations of the Śivadharmaśāstra in medieval Dharmanibandha literature and observing that the text has to be earlier than the Sivadharmottara, ${ }^{18}$ Hazra states the following:

So, the Siva-dharma can by no means be dated later than 700 A.D. The fact that the Bhavisya-purāna mentions the Siva-dharma in one of its original chapters and that the Naksatras have been mentioned in the latter work in the order from Kṛttikā to Bharanī tend to indicate that the Śiva-dharma was composed earlier than 55 O A.D. From the mentioning of the Buddha and the Ārhata as worshipping Śiva-lingas and of the names of the zodiac signs (Rāsii) it appears that the Śiva-dharma was written later than the Yājñavalkya-smrti. So, this work is to be dated between 200 and 500 A.D.; and this early origin of the work explains why it is totally free from Tantric influence.

This argument is spurious. The listing of the Nakșatras in the 'old' order from Kṛttikā to Bharaṇī, rather than from Aśvinī to Revatī, does not allow us to date the text earlier than $55^{\circ} \mathrm{CE}$. While it may be true that the listing from Aśvinī to Revatī became the standard after Varāhamihira's Brrhatsamnitā, this does not mean that all subsequent texts necessarily followed this order. As a glance at the list of Nakșatras provided by Kirfel (1967: 138-141) on the basis of the Nakșatrakalpa, the Garudapurāna

${ }^{18}$ Hazra dates the Sivadharmottara to before 8oo CE. His arguments for the date of the Sivadharmottara are given in another article, dedicated to the Sivadharmottara (Hazra 1956: 46-49). There he argues that the Sivadharmottara must have been composed much later than the Sivadharmaśāstra because of its references to Tantra. He furthermore writes that it cannot be dated earlier than $700 \mathrm{CE}$ because of its mention of the use of the Nandināgarī script in chapter 2 of the Śivadharmottara. This particular issue will be addressed below. His argument for dating it before $800 \mathrm{CE}$ rests on a series of quotations in Dharmanibandha literature and the fact that the Vāyavīyasamhitā of the Śivapurāna, which he dates between 800 and 1000 CE, knows the text. 
and the Matsyapurāna illustrates, the 'old' order from Kṛttikā to Revatī remained in use in texts also after the composition of the Brhatsamphitā. Hazra's argument about the Yãjñavalkyasmriti is difficult to follow, but a date before $200 \mathrm{CE}$ is impossible at any rate.

Hazra furthermore argues that the opening verse of the Sivadharmaśāstra, which is shared with Bāna's Harșacarita, does not need to be taken into account, because it does not occur in all manuscripts of the text and 'might have been added to it sometime between 650 and 1000 A.D.' (Hazra 1954: 16). This is true. The opening verse of the Harșacarita is found only in Nepalese manuscripts of the Śivadharmaśāstra. ${ }^{19}$ Its presence may provide important evidence for a Nepalese recension of the text. ${ }^{20}$

As so often in Hazra's method of dating texts, the arguments rest on a series of suppositions about the dating of other works that requires reconsideration. Since Hazra, few scholars have attempted to date the text, although it is generally agreed that both the Sivadharmaśāstra and the Śivadharmottara reflect an early ascetic (Atimārga) milieu of Śaivism and most probably predate the development of Tantric Śaivism. ${ }^{21}$

The second work of the corpus, the Śivadharmottara, has received some more attention in recent years. Magnone (2005: 588-591) argues that it is a late South Indian work and even goes so far as to suggest that it would be a Vijayanagara-period text because of the prescription of the use of Nandināgara characters in chapter 2 of the text. This argument has rightly been refuted by Dominic Goodall, who has drawn attention to the existence of an early Nepalese palm-leaf manuscript (NAK 5-892, NGMPP A12/3) 'in a script that might be of the eighth or ninth century AD' (Goodall 2011: 232, n. 33). This fragmentary manuscript provides the text a terminus ante quem in the eighth century. Goodall furthermore adds the following observations on the date of the text:

${ }^{19}$ The only exception is a manuscript in the Cambridge University Library in Bengali script (Add. $1599=\mathrm{B}_{99}^{c}$ ), but this manuscript most probably derives from a Nepalese source (see below, p. 59). Cf. also De Simini 2016b: 193, n. 497.

${ }^{20}$ It is noteworthy that the Harșacarita verse was commonly cited also in early medieval inscriptions from the Kannada-speaking area of the Indian subcontinent (Griffiths \& Southworth 2007: $371-372$ ).

${ }^{21}$ This is the argument advanced by Sanderson (forthc.: 8): 'The Sivadharma and the Sivadharmottara were produced when initiatory Śaivism was restricted to ascetics or at least in the context of that form of Śaivism.' Bonazolli (1993) criticises Hazra's early dating, but he does not provide an alternative. 
Hazra, who has produced an article that characterises the Sivadharmottara, the second work of that corpus, argues that it was probably composed between 700 and $800 \mathrm{AD}[. .$.$] The first work of$ the Sivadharma-corpus that unmistakably shows evidence of Tantric influence is the Sivadharmasangraha [...] and that work seems both later than the Śivadharmottara and at the same time unlikely to be much later than eighth century $\mathrm{AD}$, since the evidence of the seventh-century Svāyambhuvasūtrasangraha suggests that the form of the Mantramārga that the Sivadharmasangraha reflects (that of the Guhyasütra of the Niśvassa) had by then been far superceded in doctrine, ritual and in terms of social religion. In short, the date of the Śivadharmottara is still unknown, but if I had to guess I would be inclined to place it at least a century earlier than does Hazra. (Goodall 2011: 232, n. 33-34)

Goodall thus proposes a seventh-century date for the Sivadharmottara. The time of composition of the Śivadharmaśâstra would necessarily have to precede it.

Before we continue, a few words should be added here about the Sivadharmottara's provision of the use of 'Nandināgara' characters:

He should have the Book of Śiva written with characters in the Nandināgara style, that are quadrangular, with even tops, not too thick nor too thin, their parts well filled, smooth, not too far separated [from each other] nor joined together, characterised by vowel signs, anusvāras, conjuncts, short and long signs, etc. ${ }^{22}$

As observed by Goodall, ${ }^{23}$ rather than to a Southern script, the description points to a Northern script, written with ink on the palm leaf instead of incised into the palm leaf and then subsequently inked as is the custom in South India. As for the meaning of the label 'Nandināgara', two options present themselves. The first one, proposed by De Simini (2016b: 113), would be to take it as a derivative of the town called Nandinagara. Nandinagara features in the Mahāmāyūrề's list of places where different deities reside: nandī va nandinagare 'And Nandin in Nandinagara. ${ }^{24}$ It

${ }^{22}$ Śivadharmottara 2.40-41 (edition De Simini 2013): caturasraih samaśírșair nātisthūlair na vā krśaịh (corr.; krșaih Ed.) | sampūrṇāvayavaih snigdhair nātivicchinnasaṃhataih || mātrānusvārasaṃyogahrasvadīrghādilakṣitaih | nandināgarakair varṇair lekhayec chivapustakam $\|$.

${ }^{23}$ In a message to the Indology list server in January 2010: http://list.indology.info/ pipermail/indology_list.indology.info/2010-January/o33994.html.

${ }^{24}$ Correcting the edition's $v a$ to $c a$, as suggested by De Simini 2013: 53, n. 185 . 
also features in several Buddhist donative inscriptions in Prakrit. ${ }^{25}$ Nandinagara has been identified with modern Nadner (Hoshangabad district, Madhya Pradesh), an ancient town in eastern Malwa on the banks of the Narmadā river. ${ }^{26}$ Nandināgara would then be the characters 'used in the Town of Nandi'. A second option, which does not necessarily exclude the first, would be to connect it to Nandin, also named Nandiśvara or Nandikeśvara, who is the teacher of the Sivadharma. A script with the name Nandināgara would certainly be fitting for writing down the words spoken by Nandin. ${ }^{27}$

Goodall's arguments for a seventh-century date of the Sivadharmottara find support from Sanderson (forthc.: 7, n. 11), who points out that the Sivadharmottara has been quoted by name by the Śvetāmbara Jain scholar Haribhadra in his Aștakaprakarana (4.2-3): $:^{28}$

dīkṣā mokṣārtham ākhyātā jñānadhyānaphalạ̣ ca sa|

śāstra ukto yatah sūtram śivadharmottare hy adah $\|$

püjayā vipulam rājyam agnikāryeña sampadah |

tapah pāpaviśuddhyartham jñānam dhyānam ca muktidam \|

Initiation is said to be for the sake of liberation, and that (liberation) is the fruit of knowledge and meditation. It is declared [to be such] in the treatise. For there is the following rule in the Śivadharmottara:

'By worship [one obtains] an extensive kingdom, by the fire sacrifice [one obtains] riches. Austerity is for the sake of purification of sin. Knowledge and meditation grant liberation.'

The verse quoted by Haribhadra can be identified with Sivadharmottara 3.60. ${ }^{29}$ Jain tradition knows of two authors named Haribhadra: Haribhadra Virahānka and Haribhadra Yākinīputra. The author of the Aștakaprakaraña can be attributed to Haribhadra Yākinīputra, who

\footnotetext{
${ }^{25}$ See Lüders's list, nos. 176, 472, 536, 563 and 852 (Epigraphia Indica 10, appendix).

${ }^{26}$ See Misra \& Sharma 2003: 138 for a summary of excavations carried out in 1986-87, 1987-88 and 1993 .

${ }^{27}$ This Nandin is a Gana close to Śiva and not to be confused with Śiva's Bull. See Bhattacharya 1977 .

${ }^{28}$ Translation mine.

${ }^{29}$ Numbering of the e-text based on the edition of Naraharinātha. The first line is identical, but the second line is phrased differently in the Sivadharmottara: japena $p \bar{a}-$ paśuddhị̣ syājjñānadhyānena mucyate.
} 
most probably lived in the eighth century. ${ }^{30}$ This would fit the dating of the Sivadharmottara proposed by Goodall.

\section{A Quotation in the Kāraṇdavyūhasūtra}

An important clue for the date of the Sivadharmaśāstra may be found in a Buddhist text, the Kärandaryūhasūtra. The context is an episode in the Kārandavyūhasūtra in which Avalokiteśvara produces several Brahmanical deities from different parts of his body. The most important deity and centre of attention is Śiva Maheśvara, to whom Avalokiteśvara delivers the following speech:

O Maheśvara, you will be there when the Kaliyuga arrives. Born as the primary god (ádideva) in the realm of wretched beings you will be called Creator and Agent [of the world]. All beings who hold the following discourse among the common people (prthagjana) will be deprived of the path to awakening (bodhimärga): 'Space they say is the linga, the earth its pedestal (pițhikâ). It is the dwelling (älaya) of all beings. Because of merging (līyanāt) into it, it is called "linga". ${ }^{11}$

The verse cited here by the Kāraṇdavyūhasūtra is Śivadharmaśāstra $3 \cdot 17:^{3^{2}}$

$3^{\circ}$ On the two Haribhadras, see Williams 1965 .

${ }^{31}$ Käraṇ davyūhasūtra 265.4-8, with corrections by Eltschinger (2014: 84): bhavișyasi tvam maheśvara kaliyuge pratipanne | kaștasattvadhātusamutpanna ādideva ākhyāyase sraștāam kartāram| te sarvasattvā bodhimārgeṇa viprahịnā bhavișyanti ya iddrśam prthagjaneșu sattveșu sāinkathyạ̣ kurvanti \| ākāsam lingam ity āhuḥ prithivī tasya pịthikā | àlayah sarvabhūtānām līyanāl lingam ucyate $\|$. Translation mine.

${ }^{32}$ Earlier authors (Regamey 1971 and Studholme 2002: 19-20, 28-29) tried in vain to trace this verse in the Skandapurāna, following a wrong lead by Alain Daniélou. Studholme in particular has a long argument about the influence of what he calls the Skandapurāna' on the Kärandaryūhasūtra on the basis of very slim evidence. He is not aware of the original Skandapurāna and its relation to later Khaṇdas, assembling his materials from different Khaṇuas and treating them all as one text. There can be no doubt that the Khaṇ̣as from which he is quoting are all post-Kāraṇdaryūhasūtra. Studholme was guided towards the Skandapurāna because Regamey had identified a verse on the linga in the Kāraṇdaryūhasūtra in Daniélou's anthology Polythéisme Hindou (Studholme 2002: 28). According to the information supplied by Daniélou, the verse came from the Skandapurāna, but he did not provide any reference for it. The verse as given by Daniélou has two variants: sarvadevānām and lāyanāl. According to Studholme, following Regamey, the Kārandaryūhasūtra 'changes lāyanāl to līyanāl, a unique Buddhist hybrid word derived, like the other, from the Sanskrit root lì-, "to 
ākāśam lingam ity āhuh prthivī tasya pịthikā | àlayah sarvabhütānām līyanāl lingam ucyate \|

While the verse is not explicitly attributed to the Sivadharmaśāstra, there is no particular reason to doubt that the Buddhist author would have cited it from our text. The main teaching of the Śivadharmaśāstra is linga worship and it is quite likely that the text was recognised as an important document of the burgeoning lay Śaivism by the author of the Kärandavyūhasūtra.

This naturally brings up the question of the date of the Kärandavyūhasütra. Eltschinger observes that, although the passage is not represented in the Gilgit manuscripts due to lack of folios, 'considering that the only known significant divergence between the Nepali and the Gilgit versions concerns a very neatly delineated section (Sarvanivaraṇavișkambhin's quest for the șadakșarì vidyā), I see no compelling reason to doubt the presence of this passage in the textual tradition reflected in the Gilgit manuscripts. ${ }^{33}$ This would yield an ante quem date of $630 \mathrm{CE}$ for this verse, as the two Gilgit manuscripts are dated to before $630 \mathrm{CE} .{ }^{34}$

\section{The Evidence of the Śāntyadhyāya}

The Śāntyadhyāya has several descriptions of deities that, taken together, provide important pointers for the time of composition of the text. In an earlier study I have argued that the invocation of two deities in particular, Vināyaka and Bhṛngin, includes information that seems hard to square with a date much before the beginning of the seventh century (Bisschop 2010: 244-246). Both Vināyaka and Bhṛngin are invoked in the Śāntyadhyāya as sons of Rudra. ${ }^{35}$ The figure of Bhringin does not make his appearance in literary sources before the Skandapurāna, dated to the sixth to seventh century, while Vināyaka (Ganeśa) appears to be adopted as a member of Śiva's family around the same time as well..$^{36}$ Other aspects

dissolve"' (Studholme 2002: 19-20). The form lìyanāl was in fact a conjecture for the edition's lïlaya by Regamey. In any case, there is no need to assume Buddhist hybridisation here, as the presence of the same form in the Śivadharmaśāstra shows.

33 Eltschinger 2014: 84, n. 198.

${ }^{34}$ Cf. Mette 1997: 7 .

35 Vināyaka: rudrasya tanayo (ŚiDhŚ 6.17a); Bhṛngin: rudrātmajo (ŚiDhŚ 6.25a).

${ }^{36}$ For details, see the notes to the translation at ŚiDhŚ 6.15-17 and 6.24-25. 
of the Śāntyadhyāya point in a similar direction. Most important in this respect is the lore of astronomy and astrology (Jyotihśāstra) included in the text.

For a start, the Śantyadhyāya has to be later than the fourth century, because in the invocation of the planets it follows the order of the seven days of the week - Sun, Moon, Mars, Mercury, Jupiter, Venus and Saturn (ŚiDhŚ 6.110-118) - that was introduced in India during the fourth century. ${ }^{37}$ Furthermore, the text includes descriptions of the appearance of the pseudoplanets Rāhu and Ketu (ŚiDhŚ 6.119-121). Material representations of all nine planets (Navagrahas), including Rāhu and Ketu, have only been documented from 6oo CE onwards. Varāhamihira (first half of the sixth century) is generally considered to be the first Jyotișa author to refer to the full group of nine planets. ${ }^{38}$ Recent work on the Gārgiyajyotisa, however, has shown that this text attributed to Garga was drawn upon by Varāhamihira, and Garga also describes the Navagrahas. ${ }^{39}$ On the other hand, the order of presentation of the planets in the Gärgìyajyotișa (Moon, Rāhu, Jupiter, Venus, Ketu, Saturn, Mars, Mercury and Sun) is different from the standard one ending in Rāhu and Ketu that we first find in Varāhamihira. ${ }^{40}$ The Śāntyadhyāya's detailed description of the nine planets in the standard order would suggest a sixth-century date for this part of the text at the earliest. ${ }^{41}$ There is a major caveat here, however, for the oldest of the manuscripts available for the Śāntyadhyā-

${ }^{37}$ Pingree 1965 and Yano 2004.

${ }^{38}$ Cf. Kochhar 2010: 'identification of Rāhu or Ketu with a planet in a text prior to Varāhamihira would be an exercise in anachronism' (Kochhar 2010: 296).

${ }^{39}$ See Geslani, Mak et al. 2017: 165-166 ('Garga's Treatment of the Planets').

${ }^{40}$ Bill Mak has argued that '[s]ince the seven weekdays in India, as in the Hellenistic tradition, do not include Rāhu and Ketu, the inclusion of the two pseudoplanets appears to be Varāhamihira's compromised attempt to bring the Garga tradition up to date' (Geslani, Mak et al. 2017: 165, n. 40).

${ }^{41}$ It is, however, not impossible that the notion of nine planets was first developed in Purānic circles before scholars like Varāhamihira started to adopt it in their astronomical system. In this connection it should also be noted that several Purānas have references to the Navagraha. These may provide important clues for dating Purānas as well. An example would be ViP 2.12.16-23, which refers to the chariots and horses of the nine planets. A passage like this in the Viṣnupurāna does not necessarily mean that the entire text has to be later than the sixth century, but that at least this part of the text may be later. For more details on the development of the Navagraha, see n. 70 on p. 166. 
$y a\left(\mathrm{~N}_{45}^{c}\right)$ precisely omits the invocation of Ketu $(120-121),{ }^{42}$ while the wide variety of readings found in the other manuscripts suggests that the invocation of Ketu may have been added independently at different moments in the transmission of the text. If so, this would rather point to an original time of composition when Ketu had not yet come to be included among the Navagrahas.

A late sixth or post-sixth century date is suggested by the invocation of the Dikpālas. The text includes the canonical list of eight Dikpālas and their cities - Indra in Amarāvatī, Agni in Tejovatī, Yama in Vaivasvatī, Nirṛti in Kṛṣnāâ, Varuṇa in Śuddhavatī, Vāyu in Gandhavatī, Kubera in Mahodayā and İsāana in Yaśovatī — and has detailed descriptions of each of them. The available textual and material evidence points towards the seventh century as the time when this set of eight became standardised. ${ }^{43}$

On the other hand, there are also several archaic elements in the mantra, such as Śiva's being four-headed (ŚiDhŚ 6.4c) and the listing of the Nakṣatras in the old order from Kṛttikā to Bharaṇi (see p. 10). These can, however, also survive in texts of a later date and do not therefore establish a terminus ante quem. In short, the above features suggest that the text may have been composed towards the end of the sixth century at the earliest. In the light of Goodall's dating of the Sivadharmottara to the seventh century, the Atimārga milieu of the text and the quotation in the Kärandaryūhasūtra, it will not have been much later than this. ${ }^{44}$ The terminus ante quem is the date of the incomplete Sivadharmottara manuscript, which was most probably written in the early ninth century.

\footnotetext{
$4^{2}$ See n. 82 on p. 168.

${ }^{43}$ For details, see n. $5^{2}$ on p. 161 .

44 The Atimārga milieu of the Śivadharmaśāstra is indicated by the descriptions of the activities of the śivayogins in chapter 12 of the text, which, for example, makes frequent reference to bathing in ashes. That the Sivadharmottara too was composed in an Atimārga environment is evinced by the references to the pāśupata and the mahāvratadhara as the highest recipients of gifts (12.203-207), as well as by the prescription of the installation of an icon of Lakuliśvara (ŚiDhUt 2.146-147). See De Simini 2016b: 51-53, for these references. Cf. also below, p. 20.
} 


\section{References in Inscriptions}

The oldest inscriptional records referring to the Sivadharma come from Southeast Asia. The recently published 'Stela from Khuê Trung' (C. 211), dated $899 \mathrm{CE},{ }^{45}$ suggests that the Śivadharma was known in the Campā kingdom. This inscription, belonging to the reign of the first of several kings named Śrī Jayasiṃhavarman in the history of Campā and dedicated to a foundation for Śiva in the form of Mahārudra, introduces a list of servants (sevaka) to the king who are said to be śivadharmmaparāyanāh 'devoted to Śiva's Law' (face B, stanza IV). The first of them, named Śrìkalpa, is an âcārya and is said to be śivadharmmabhāg 'an adherent of Śiva's Law' (face B, stanza V). While śivadharma could arguably just refer to the Śaiva religion in general, it seems plausible, given what we know about the extensive spread of the Sivadharma and the use of the term, that it refers more specifically to the teachings of the Sivadharma.

The first two texts of the Śivadharma corpus were also known in ancient Cambodia. A tenth-century inscription in Old Khmer from Pràsàt Roban Romãs (K. 150 $)^{46}$ cites a verse from the Śivadharmaśāstra on the sharing of merit, which is attributed explicitly to the 'Sacred Law of Siva' (vrah śivadharmma):

upadeștānumantā ca karttā kārayitā ca yaḥ | krtānupālakaś caiva pañca tulyaphalāḥ smrtāḥ $\|^{47}$

The one who teaches, the one who approves [of the teaching], the agent of the action and the instigator of the action, as well as the one who protects what has been done: the five are taught to share an equal fruit.

Furthermore, another Sanskrit inscription from Cambodia (K. 834), ${ }^{48}$ possibly datable to the reign of Süryavarman I (1002-1050 CE), attests to knowledge of the Sivadharmottara. As Sanderson (forthc.: 7, n. 11) points out, the sadangavidhi 'rite of six elements' said to be performed by the king in stanza 38 of the inscription must refer to the worship prescribed for the king in Sivadharmottara 1.80-90. He has also drawn attention

\footnotetext{
45 Griffiths et al. 2012: 263-270.

${ }^{46}$ Inscriptions du Cambodge 5: 191-194.

47 This is Śivadharmaśāstra 12.104 in De Simini's edition (2013, appendix 1). A second verse is cited after this one, which is, however, not from the Sivadharma.

${ }^{48}$ Inscriptions du Cambodge 5: 244-269.
} 
to parallels with passages from the Sivadharmottara in the Angkor Wat inscriptions identifying the sinners who go to different hells. ${ }^{49}$

The epigraphical evidence from the Indian subcontinent itself comes from South India from a somewhat later period, and concerns gifts to support the public recitation of the Sivadharma. Several inscriptions from the Cōla period in the Tamil-speaking South, beginning with an inscription dated to the reign of Rājendra Cōla (1012-44 CE), refer to public recitations of the text. ${ }^{50}$ However, most important for the present study is an inscription from Karnataka, dated to Śaka 1079 (1157 CE), on a stone close to the northwestern wall of the Praṇaveśvara temple at Tālagunda. ${ }^{1}$ It reports the donation of a village called Hiriya Tagulațti as an agrahāra. The epigraph attests to detailed knowledge of the Śivadharmaśāstra and the Śivadharmottara. ${ }^{5^{2}}$ The Tālagunda inscription refers specifically to the recitation of the Śivadharma's Śāntyadhyāya and thus provides important evidence on its use (see p. 48).

\section{Purāṇas and Other Testimonia}

A survey of the Sivadharmaśāstra's testimonia falls outside of the scope of the present study. Several Śaiva Purānas, including the Śivapurāna, appear to have made use of the text, but more systematic work is needed in this direction. Hazra (1954, Appendix I) has collected and identified a number of quotations attributed to the Sivadharma or the Sivadharmaśāstra by medieval Dharmanibandha authors. ${ }^{53}$ All these texts, however, postdate the earliest surviving manuscripts, and while they do evince

\footnotetext{
${ }^{49}$ For other evidence that the Sivadharmottara was known in ancient Cambodia, see Sanderson 2003/04: 422-423.

${ }^{50}$ See De Simini 2016b: 187, n. 483, for references. Furthermore, the second part of an inscription from Kưgōọ / Kurugōḍu (Bellary district, Karnataka) dated to Śaka 1103 (1181 $\mathrm{CE}$ ) reports, as part of a list of donations on the occasion of the grant of an agrahära by the Sinda ruler Rachamalla II, a donation for the public recitation of 'the Sivadharmapurāna' or 'the Śivadharma and the Purāna(s)' (EI 14, inscr. 19, 1.46; De Simini 2016b: 186-187).

${ }^{51}$ Epigraphia Carnatica 7 , Skt 185.

$5^{2}$ See the discussion in De Simini 2016b: 190-198.

53 The quotations identified by Hazra are from chapters 5, 7, 8, 9 and 10. The thirteenth-century author Hemādri, minister of the Yādava king of Devagiri, in particular cites extensively from the Sivadharmaśāstra. See also De Simini 2016b, on citations and adaptations of portions of text from the Sivadharmottara.
} 
the text's spread and influence, they do not help in narrowing down the time of composition of the text. It is also worth noting that the Sivadharma appears to have had a remarkable influence on the formation of Vīraśaivism, as attested in medieval works from South India. ${ }^{54}$

Aside from the earlier mentioned verse cited in the Kärandaryūhasūtra, a verse quoted by the Pāśupata author Kauṇịinya in his commentary on the Pāśupatasūtra is important for considering the date of composition of the text as well. In his lengthy commentary on Pāśupatasūtra 1.9, in the section where he discusses the Pāśupata concept of purity (śauca), Kauṇdinya cites a verse in praise of the merit of bathing in ashes:

yaḥ snānam àcaren nityam āgneyam samyatendriyaḥ |
kulaikaviṃśam uddhṛtya sa gacchet paramāṃ gatim $\mid{ }^{55}$

He who continually observes the Fiery Bath, with his senses in check, will rescue his lineage to the twenty-first generation and reach the highest state.

This verse corresponds almost verbatim with Śivadharmaśāstra 11.24, where it features in a long section praising the practice of bathing in ashes..$^{6}$ What is striking about the verse is that it addresses the concerns of a non-ascetic devotee of Śiva, in that it refers to the rescuing of one's ancestors. The ascetic has left behind his family duties and should be free of debts, and so should have no concern for this. This suggests

${ }^{54}$ Vīraśaivism displays several important shared concepts, including the characteristic distinction between the jangama and the sthâvara linga addressed in Śivadharmaśāstra 2 and 3. Moreover, several Vīraśaiva works, such as the Candrajñanāgama, the Kriyāsāra of Nīlakaṇṭhaśivācārya (between ca.1350 and 1530), the Siddhāntaśikhāmaṇi of Śivayogin (ca. 13th or early 14th c.), the Śrikarabhāşya of Śrīpati (ca. 140o) and the Lingadhāraṇacandrikā of Nandikeśvara, quote extensively from the Śivadharmaśāstra and the Sivadharmottara. For the dates of these authors, see Sanderson 2012/13: 84 . Similarly, Śaiva Siddhānta authors from earliest times have referred to the teachings of the Śivadharma (e.g. Rāmakaṇțha ad Kiraṇatantra 6.11d-12; Goodall 1998: 375-76) and there are indeed many similarities between the two traditions. For an example from a later period, see the many references to the Śivadharmaśāstra and the Sivadharmottara in the works of two South Indian Śaiva Siddhāntins, Nigamajñāna I and Nigamajñāna II (16th c.; Ganesan 2009).

${ }_{55}$ Pañcārthabhāṣya p. 30, ll. 1-2.

${ }^{5}$ Numbering according to the draft edition of Nirajan Kafle. Kauṇdinya reads uddhrtya instead of uttārya. 
that the verse belongs to the kind of non-initiatory Śaiva milieu of the Sivadharma. However, the question of whether Kaundinya really cites it from our text, or from an unknown earlier source, must remain open. Kaundinya may be dated to around the fourth or fifth century, which does not tally with the dates discussed above. The parallel may rather hint at the fact that parts of the Sivadharmaśāstra were composed on the basis of earlier, no longer surviving sources.

I have found three parallels for (parts of) the Śantyadhyāya in other texts. These may be considered testimonia for the text, if it can be shown that they have indeed been borrowed from the Śantyadhyāya. The first concerns three verses on the offering of bali to the Rudras, Mātris and Ganādhipas (53-55). These verses occur, with some variations, in Mrgendra, Kriyāpāda 7·33-35, and in Íśānaśivagurudevapaddhati (İśgP), Sāmānyapāda 14.178-18oab = Mantrapāda 28.96cd-98 = Krìyāpāda 15.4-16ab. The second passage concerns the lengthy invocation of the Ganas in the ten directions $\left(5^{6-75}\right)$. A very similar invocation occurs in İ́gP, Mantrapāda, 30.84, which gives a mantra meant to accompany the scattering of bali in the ten directions. While this is not a literal or direct quotation, the wording of the mantra in İsānassiva's text clearly goes back to that of the Śanntyadhyāya. That Ísaanaśiva had access to the Sivadharma becomes clear from his explicit citations from the 'Śivadharma' at Kriyāpāda 11.18-20 and at Kriyāpāda 13.115. The Mrgendra belongs to the early Śaiva Siddhānta scriptures, but represents a later stage among them (Goodall 1998: LVIII). The date of the Keralan İsāanaśiva, author of the Siddhāntasāra, more commonly known as Íśānaśivagurudevapaddhati, remains uncertain (Sanderson 2012/13: 23, n. 84). While these testimonia do not allow us to narrow down the time frame of the composition of the text, they do attest to the influence of the text on the Śaiva Siddhānta.

By far the most important and extensive parallel, however, is to be found in six chapters of the first parvan (Brähmaparvan) of the Bhavisyapurāna (BhavP 1.175-180). These chapters form part of a wholesale borrowing of the Śivadharmaśāstra, in which practically the entire work has been incorporated and revised in order to make it fit into a Saura context. The Bhavisyapurāna has long been known for its creative copying of earlier texts, including the Manusmrti and the Brihatsam hit $\bar{a},{ }^{57}$ but its adap-

${ }^{57}$ See, e.g., Hazra 1940: 167-173, László 1971 and Sternbach 1974. 
tation of the Sivadharmaśästra is in many respects the most extraordinary in terms of scope and wording. The part entitled 'Description of the Sauradharma' (sauradharmavarnana) starts at BhavP 1.151. Śatānīka tells Sumantu that he has heard many Dharmas, including the Vaiṣnava and Śaiva Dharmas, but now he wants to hear the Saura Dharma..$^{5}$ What follows - the moment Sumantu starts speaking — is taken verbatim from the Śivadharmaśāstra, but with all references to Śiva and his cult systematically replaced by Sürya and his cult. A verse from the first chapter of the Śivadharmaśāstra, cited earlier (ŚiDhŚ 1.16, p. 8), may serve as an example:

ye 'rcayanti sadā rudram na te prakrtimānuṣāḥ| rudralokāt paribhraștās te rudrā nātra saṃśayaḥ \|

They who always worship Rudra, they are no ordinary men. They are Rudras who have come down from Rudraloka. There is no doubt about this.

In BhavP 1.151.19 this has become:

stuvantiye sadā bhānuṃ na te prakrtimānușāḥ| svargalokāt paribhrașțās te jñeyā bhāskarā bhuvi \|

They who always praise Bhānu, they are no ordinary men. They should be known as Bhāskaras who have come down to earth from Svargaloka.

In this way, the entire Śivadharmaśāstra has been systematically incorporated and rewritten. Even the myth of the 'origin of the linga' (ŚiDhŚ 3) has been taken over, but turned into a Saura version that culminates in the gods' vision and worship of Sūrya (BhavP 1.153).

The Śantyadhyāya parallel itself starts with an introductory narrative that has no counterpart in the Śivadharmaśāstra. Garuḍa tells his brother Aruna, Sūrya's charioteer, that he is without wings due to the curse of a brahmavādini ${ }^{59}$ He wishes to know the means to regain his

${ }^{8}$ Cf. BhavP 1.151.8-9: śrutā me bahavo dharmāḥ śrutismrtyuditās tathā| vaiṣnavāḥ śaivadharmāś ca tathā paurāṇikāḥ śrutāḥ || śrotukāmo hy aham vipra sauram dharmam anaupamam | bhagavan sarvadhanyās te sauradharmaparāyaṇāh $\|$.

59 This must refer to the Sāṇḍilī episode (MBh 5.111.1-18), in which Garuḍa loses his wings, becoming like a ball of flesh, for despising a brahmin ascetic woman named Śāṇ̣ilī in a dream. 
wings. ${ }^{60}$ Aruna instructs him to worship the Sun and perform the fire rite (agnikärya), but Garudia answers that he cannot do this because of his mutilated state. He requests that his brother perform the fire rite called mahāśānti for his sake. ${ }^{61}$ Aruna agrees to do so, saying that the Sun had performed it for him before. ${ }^{62}$ After this there is a first brief parallel with the two opening verses of the Śantyadhyāya (BhavP 1.175.14-15 = ŚiDhŚ 6.1-2). ${ }^{63}$ Next Aruna gives ritual instructions on the lighting of the fire, the accompanying invocation, and the ceremony of a hundred thousand oblations (lakșahomavidhi). The rite, along with the śanti invocation, is to be performed for Sūrya by a Bhojaka priest. ${ }^{64}$ Following the lengthy mantra, divided over six sub-chapters, the narrative is resumed again at BhavP 1.180.40. Because of Aruna's recitation, Garuḍa retrieves his wings and becomes whole again thanks to the grace of the Sun. ${ }^{65}$ The chapter ends with an account of the transmission of the mantra: Sūrya $\rightarrow$ Aruna $\rightarrow$ Garuḍa $\rightarrow$ the Bhojakas of Sākadvīpa $\rightarrow$ Vyāsa. ${ }^{66}$

60 BhavP 1.175.5-7: sarvapatravihīnam me sarvarogavivarjitam| śāpena brahmavādinyāḥ paśyāngam dvijasattama || evam mattasya me tāta kiṃ kāryam avaśișyate | yenāhaṃ karmaṇā kalpo bhaveyaṃ patravān punaḥ || tan me brūhi khagaśreștha prapannasya khagādhipa | yat krtvā kalpatām prāpya pūjayāmi divākaram ||.

${ }^{61}$ BhavP 1.175.8-11: aruṇa uvāca| pūjayasva jagannātham bhāskaram timirāpaham | sūryāgnikāryaṃ satatam śuddhacittaḥ samācara || mahāśāntikaram khyātaṃ sarvopadravanāśanam | grahopaghātahantāram śubhakāryaṃkaram param || garuḍa uvāca $\mid$ nāham śakto 'smi vai kartum püjāṃ dinakarasya ca $\mid$ na cāgnikāryam śaknomi kartuṃ vikalatāị gatạ̣|| tasmān me kuru śāntyartham agnikāryam khagādhipa| mahāśāntir iti khyātam śāntaye mama suvrata $\|$.

${ }^{62}$ BhavP 1.175.12-13: evam eva yad āttha tvam vainateya khagādhipa| akalpas tvam na śaknoṣi mahāvyādhiprapīditaḥ || aham karomi te putra śāntaye pāvakārcanam | yat krtam mama cārkeṇa purā śāntidam ādarāt $\|$.

${ }_{3}^{6}$ All the parallels with the Bhavisyapurāna are given in the upper register of the apparatus of the critical edition.

${ }^{64}$ BhavP 1.175.35c-36ab: krtvaivam agnikāryam tu bhojako bhāskarāya vai \| śāntyartham sarvalokānām tatah śāntikam ācaret |. On the identity of the Zoroastrian Bhojakas, to be distinguished from the non-Zoroastrian Magas, see von Stietencron 1966: 236-247, 264-272. According to von Stietencron, the Bhojakas did not enter India before the second half of the sixth century and so this part of the Bhavisyapurāna must be dated at least after this. See also Bakker 2014: 67.

${ }_{5}$ BhavP 1.180.40-42: evam hi kurvatah śāntim aruṇasya mahīpate | śreyase vainateyasya garuḍasya mahātmanạ̣ || etasminn eva kāle tu suparnạh patravān abhüt | tejasā budhasaṃkāśo valena harịnā samaḥ \| sampūrṇāvayavo rājan yathāpūrvam tathābhavat | prasādād devadevasya bhāskarasya mahātmanah $\|$.

${ }^{66}$ BhavP 1.180.6o-62: sūryabhakte sadā deyam sūryeṇa kathitam purā $\mid$ aruṇasya 
Throughout the mantra, all references to devotion to Siva (rudrärcanapara, etc.) have been systematically replaced by expressions of devotion to Sürya (süryārcanarata, etc.). Here and there the order of verses has been revised and many of the invocations have been extensively rewritten or extended. Not surprisingly, the first deity to be invoked is not Siva but Sūrya, who heads the list of the Navagrahas (BhavP 1.175.36cd-50 = SiDhŚ 6.110-122). In the same vein, the invocation ends not with a jaya to Siva but with a jaya to Sūrya (BhavP 1.180.35-39 / ŚiDhŚ 236-242). The new narrative context has been taken into account too, and so there are occasional vocatives addressed to Garuda, for whose benefit Aruna recites the mantra, while the first person references of the original mantra have been changed into the second person. There are, however, a few slips where the original first person still remains, revealing the direction of change. ${ }^{67}$ The text has suffered from quite a few cases of accidental loss of verses. ${ }^{68}$

It is hard to put a date on the time of borrowing. The Brāhmaparvan is generally considered to be the oldest part of the Bhavisyapurāna. Indeed many of the verses quoted in the Dharmanibandha literature can be traced in this parvan (Hazra 1940: 167-173, 331-335), although Hazra also concludes that 'it is the result of a recast to which its prototype was subjected' (Hazra 1940: 171). Regarding the borrowing of the Śantyadhyāya, however, it is to be noted that the text transmitted in the extant version of the Bhavisyapurāna suggests a relatively early version of the text overall. None of the additional invocations found in some of the manuscripts appear in the Bhavisyapuranna's version, while its wording at the individual verse level often tends to agree with what appear to be the oldest

mahābāho garuḍasyāruṇena ca || garuḍena purā proktaṃ bhojakānām mahātmanām | sūryaśarmasukhādīnām śākadvīpe mahīpate || taiś cāpi kathitaṃ punyam muner vyāsasya dhìmatạ̣ | tenāpi kathitam punyam sarvapāpabhayāpaham $\|$.

${ }_{7}^{6}$ Strikingly, these only appear in chapter 178 : BhavP $1.178 .17 \mathrm{c}, 1.178 .19 \mathrm{~b}, 1.78 .27 \mathrm{c}$, $1.178 .40 \mathrm{Oc}, 1.178 .42 \mathrm{c}, 1.178 .47 \mathrm{~b}$.

${ }^{68}$ E.g. the invocations of the Ganas from the southwest to the north (ŚiDhŚ 6.62-69); Vāyu and Kubera among the Dikpālas (ŚiDhŚ 6.93-98); Śanaiścara and Rāhu among the Navagrahas (ŚiDhŚ 6.118-119); Vāsuki and Mahāpadma among the Nāgas (ŚiDhŚ 6.168169; 6.176-177); the second group of rivers (ŚiDhŚ 6.189-19o); Vibhāṇḍaka and Pūrṇabhadra among the Yakṣas (ŚiDhŚ 6.201-202; 6.205-206); the Yoginīs, Ḍākiṇis and Bhūtas in the last set of invocations (ŚiDhŚ 6.219-225). 
readings of the text in the Sivadharma manuscripts. ${ }^{69}$ Perhaps most significantly, it uniquely agrees with the oldest manuscript $\mathrm{N}_{77}^{K_{0}}$ in omitting the lengthy section on Viști, the Tithis and the Yogas (ŚiDhŚ 6.123-136). This may very well reflect an early stage of transmission of the text. ${ }^{70}$

\section{The Evidence of the Manuscripts}

The definite terminus ante quem is provided by the evidence of the surviving manuscripts. The earliest surviving Śivadharma manuscript is a fragmentary manuscript of the Śivadharmottara (NAK 5-892, NGMPP A12/3). Its script, which may be labeled 'Licchavi', is remarkably similar to the Nepalese palm-leaf manuscript of the Skandapurāna that is dated 810 CE (NAK 2-229, NGMPP B11/4). Unfortunately, the Śivadharmaśāstra is not included among the 42 surviving folios and it cannot be established with certainty that the two works were already copied together in a bundle (De Simini 2016a: 214). In any case, the Śivadharmaśāstra must have been composed before the Sivadharmottara. The earliest surviving manuscript that does contain the Sivadharmaśāstra is NAK 6/7, NGMPP A1o28/4. This may be dated to the end of the tenth century or early eleventh century on palaeographical grounds. Like other Nepalese palm-leaf manuscripts, this is a Sivadharma corpus manuscript, although it does not yet contain all texts belonging to the corpus (De Simini 2016a: 219-222). Unfortunately this manuscript is incomplete and lacks a large part of the beginning of the Sivadharmaśāstra, including the Śantyadhyāya. The earliest surviving manuscript that includes the Śāntyadhyāya is a Nepalese palm-leaf manuscript now at the Asiatic Society in Calcutta, G 4077 (siglum $\mathrm{N}_{77}^{K_{0}}$ in the present edition), dated [Nepāla] Saṃvat 156 (1035-36 CE).

\section{The Provenance of the Śivadharmaśāstra}

In his survey of the Śivadharmaśāstra, Hazra speculated about the possible provenance of the text:

The occurrence of the text of the Siva-dharma mostly in Newari mss. of Nepalese paper and the mention of the Devikā, a small river in Southern

\footnotetext{
${ }^{69}$ See the notes on the translation for further details.

${ }^{70}$ See below, p. 55 .
} 
Kashmir, and of the Candrabhāgā as a highly sacred river, seem to point to Southern Kashmir or the Northern Punjab as the place of origin of the Siva-dharma. (Hazra 1954: 16)

There are two elements to Hazra's argument: a text-external one and a text-internal one.

As for the text-external evidence, Hazra rightly points to the existence of the early manuscripts from Nepal. Approximately 18 palm-leaf manuscripts, written from the early ninth to the end of the fourteenth century, are known to survive (De Simini 2016a: 213-214). The number of subsequent paper manuscripts from Nepal easily doubles this figure. This is an exceptionally large number of manuscripts and it attests to the popularity of the Śivadharma in Nepal through the ages. It does not, however, tell us that the text was also composed there, for it is largely due to climatic conditions that such early palm-leaf manuscripts have survived there and not in other parts of the subcontinent. In fact, the two earliest texts of the corpus, the Śivadharmaśāstra and the Śivadharmottara, were distributed widely in the South as well. Some 20 palm-leaf and paper manuscripts from South India, written in Grantha and Telugu script, have been identified..$^{71}$ Furthermore, there are at least three manuscripts from Kashmir written in Sāradā script, two manuscripts written in Bengali script and several manuscripts written in Malayalam script (De Simini 2016a: 250-253). Copies of the first two texts of the corpus thus survive from across the Indian subcontinent, in particular from the border regions. The situation is different for the other texts belonging to the corpus, for these are only found in Nepal, suggesting that the corpus organisation of eight texts would have taken place in Nepal.

Referring to two rivers mentioned in the text, Hazra argues for an origin in southern Kashmir or the northern Punjab. The argument is based on the mention of two rivers, Devikā and Candrabhāgā, in a listing of rivers in the Śantyadhyāya (ŚiDhŚ 6.186-192). Although the list covers rivers from across the Indian subcontinent, also including, for example, a southern river like the Kāverī, there is indeed a noticeable presence of rivers from the northwestern part of the subcontinent. On the other hand, the mention of two minor rivers in the northeast, the Nairañja-

\footnotetext{
${ }^{71}$ Presentation by Marco Franceschini at the Śivadharma workshop held at Leiden
} University, 26-30 September 2016: 'The transmission of the Śivadharma in Tamil Nadu'. 
nā (near Bodhgayā) and the Varuṇā (near Vārāṇasī), also deserves to be taken into account.

Finally, mention may be made of a verse from chapter 11, which possibly indicates that Saiva religion was not yet the norm and that the text was targeted specifically at newly aspiring Śaiva rulers:

sudūram api gantavyam yatra māheśvaro janah | sa cayatnena draștaryas tatra sannihito harah \|\|$^{72}$

Even if one must search far and wide, where there are people devoted to Maheśvara, one must strive to see them, for there Hara is near.

\section{The Śāntyadhyāya}

The Śāntyadhyāya is the longest chapter of the Śivadharmaśāstra. Consisting essentially of an extensive mantra along with the fruits of reciting or hearing it, the Śāntyadhyāya has a markedly different character from the rest of the text. The mantra invokes a plethora of gods and cosmic powers for appeasement (śänti). Each deity is invoked in his or her own sphere, but always in relation to Śiva. It is from Śiva that they derive their power. The Śāntyadhyāya's invocation of all gods and powers does not stand on its own, but forms part of a long tradition of listing and invoking of cosmic powers. Sadovski, who has studied many of these lists and catalogues in Indo-Iranian traditions of oral poetry, observes the following:

By cataloguing the universe, worshippers try to find an underlying matrix system - but also to magically re-shape and re-create it over and over again. That is why the logic of arrangement of conceptual catalogues and their items in Indo-Iranian ritual poetry can shed light on the systems of motives, values and priorities of the worshippers and societies concerned. ${ }^{73}$

This observation holds true for the Sántyadhyāya as well. One way of looking at the mantra is by reading it as a catalogue of the cosmic powers recognised by the target community of the text. The question then arises: what are the principles of organisation of the invocation and what do they tell us about the values and priorities of its composer(s)?

\footnotetext{
$7^{2}$ ŚiDhŚ 11.51 (draft edition Nirajan Kafle).

${ }^{73}$ Sadovski 2013: 153, italics original.
} 


\section{Order and Organisation of the Mantra}

In order to establish the principles behind the selection and organisation of the mantra, we may first of all look at the general order of the deities and powers invoked. The structure of the mantra, as established in the main text of the edition, is as follows: ${ }^{74}$

- Maheśvara

- Umā

- The Inner Circle

- Kārttikeya

- Nandīśa

- Vināyaka

- Mahākāla

- Ambikā

- Mahāmahiṣamardanī

- Bhṛngiriți

- Caṇ̣eśvara

- Brahmā

- Viṣnu

- Mātṛs

- Brahmāṇī

- Rudrāṇī

- Kaumārī

- Vaiṣnavī

- Aindrī

74 There are numerous cases of additions and omissions in this list in the various manuscripts. These are discussed in the notes accompanying the translation. The list given here represents the main text of the edition. 
- Vārāhī

- Cāmuṇạā

- Lamboșțhì

- All Mātṛs

- Rudras, Mātṛs and Gaṇādhipas

- Gaṇas

- East

- Southeast

- South

- Southwest

- West

- Northwest

- North

- Northeast

- Below

- Above

- Dikpālas

- Indra in Amarāvatī (E)

- Agni in Tejovatī (SE)

- Yama in Vaivasvatì (S)

- Nirṛti in Kṛ̣̣nāā (SW)

- Varuṇa in Śuddhavatī (W)

- Vāyu in Gandhavatī (NW)

- Kubera in Mahodayā (N)

- İsāna in Yaśovatī (NE)

- Devas in the Seven Worlds 
- Devas in Caves, Forests etc.

- Four Goddesses

- Sarasvatī

- Śrī

- Jayā

- Aparājitā

- Navagrahas

- Sūrya

- Soma

- Añgāraka

- Budha

- Bṛhaspati

- Śukra

- Śanaiścara

- Rāhu

- Ketu

- Vișți

- Tithis

- Amāvāsī to Pūrṇamā

- Yogas

- Vișkambha, Prīti, Āyuṣmant, Saubhāgya, Śobhana, Atigaṇda, Sukarman, Dhṛti, Sūla, Gạ̣ḍa, Vṛddhi, Dhruva, Vyāghāta, Harșaṇa, Vajra, Siddhi, Vyatīpāta, Variyas, Parigha, Śiva, Siddhi, Sādhya, Śubha, Śukla, Brahman, Aindra, Vaidhṛti

- Nakṣatramātṛs 
- East: Kṛttikā, Rohiṇī, Mṛgaśirā, Ārdrā, Punarvasu, Puṣyā, Aśleșā

- South: Maghā, Pūrvaphālguṇī, Uttaraphālguṇī, Hastā, Citrā, Svātī, Višākhā

- West: Anurādhā, Jyeșṭhā, Mūlā, Pūrvāṣāạhā, Uttarāṣāạhā, Abhijit, Śravaṇā

- North: Dhaniṣṭhā, Śatabhiṣā, Pūrvabhādrapadā, Uttarabhadrā, Revatī, Aśvinī, Bharaṇī

- Rāśis

- East: Meșa (Aries), Siṃha (Leo), Dhanu (Sagittarius)

- South: Vṛ̣a (Taurus), Kanyā (Virgo), Makara (Capricorn)

- West: Mithuna (Gemini), Tulā (Libra), Kumbha (Aquarius)

- North: Karkața (Cancer), Vṛścika (Scorpio), Mīna (Pisces)

- Seven Sages (Ursa Major)

- Sages of Great Vows

- Kāśyapa, Gālava, Gārgya, Viśvāmitra, Manu, Dakṣa, Vasiștha, Mārkaṇḍa, Pulaha, Kratu, Nārada, Bhṛgu, Ātreya, Bharadvāja, Añgiras, Vālmīka, Kauśika, Kaṇva, Saakalya, Punarvasu, Sālaṅkāyana and others

- Wives, Daughters and Sons of the Sages

- Siddhas, Gandharvas, Apsarases, Vidyādharas, Garuḍas

- Daityas

- Namuci, Śan்kukarṇa, Mahānāda

- Mahājambha, Hayagrīva, Prahlāda, Anuhlādaka, Tāraka, Agnimukha, Kālanemi, Mahotkaṭa

- Virocana, Hiraṇyākṣa, Suparva, Sulomaka, Mucukunda, Sukunda, Revataka

- Wives, Daughters and Sons of the Daityas 
· Eight Nāgarājas

- Ananta

- Vāsuki

- Takșaka

- Karkoṭaka

- Padma

- Mahāpadma

- Śan்khapāla

- Kulika

- Other Nāgas

- Wives, Daughters and Sons of the Nāgas

- Rivers

- Gañgā, Yamunā, Narmadā, Gomatī, Kāverī, Varuṇā, Devikā

- Candrabhāgā, Godāvarī, Sarayū, Gaṇḍakī, Kauśikī, Sarasvatī

- Nairañjanā, Śoṇa, Mandākinī, Saṃnihitā

- and others

- Yakșas

- Mahāvaiśravaṇa

- Manibhadra

- Suviroma

- Pāñcika

- Vibhāṇḍaka

- Dhṛtarāṣṭra

- Pūrṇabhadra

- Virūpākṣa

- Other Yakșas 
- Wives, Sons and Daughters of the Yakșas

- Mountains

- Oceans

- Entourage of Bhairava

- Rākșasas

- Yoginīs

- Bhūtas

- Pretas

- Piśācas

- Grahas

\section{- All Deities}

After the announcement of the topic (1-3), the chapter opens with two verses addressed to the main object of devotion, referred to as Maheśvara 'Great Lord' $(4-5)$. The next four verses are addressed to his wife Umā (6-9).

The text continues with the members of Śiva's inner circle: Kārttikeya, Nandīśa, Vināyaka, Mahākāla, Ambikā, Mahāmahiṣamardanī, Bhṛngiriți and Caṇ̣eśvara (10-27). ${ }^{75}$ The distinction between Umā on the one hand and Ambikā on the other is not made explicit, but one distinctive feature of Ambikā is that she is addressed as 'Mother of the Troops', something not said of Umā. In her benevolent appearance Ambikā is the exact opposite of Mahāmahișamardanī, who is invoked after her. Several of the members of the inner circle - Nandiśa,

75 This group calls to mind the circle of eight Ganapatis in later Tantric sources. However, the standard list does not include Mahāmahiṣamardanī but has Vṛṣa instead. Cf. Goodall, s.v. ganapati, ganeśa, in TAK II: 'Variations in the order and form of the names occur; essentially they appear to be Śiva's Paurāṇika household: his wife (Umā), sons (Gajavaktra and Skanda), mount (Vṛsa), watchmen (Nandin and Mahākāla), and close devotees (Abala/Bhṛngin and Canda).' Three sources used for the edition $\left(\mathrm{E}^{N}\right.$, $\mathrm{P}_{32}^{T}$ and $\mathrm{P}_{72}^{T}$ ) add Vrṣa after the invocation of Caṇdeśvara. Several of the single-text manuscripts of the Śantyadhyāya that I have checked transmit the Vṛ̦a invocation as well; see p. $18 \mathrm{~g}$. There is a similar addition in $\hat{S}_{67}^{S}$, but there the additional verses refer to Kṛṣna and are preceded by two verses addressed to the Gaṇa Ghaṇțākarṇa. 
Mahākāla, Ambikā, Bhṛngiriṭi, and Caṇdeśvara — are characterised as 'three-eyed'. This iconographic feature reflects their closeness to Siva and sets them apart from the deities that follow.

After Śiva's inner circle, the two other main male gods of Brahmanical Hinduism are invoked: Brahmā and Viṣnu $(28-32) \cdot{ }^{76}$ No mention is made of Viṣnu's avatāra forms.

The mantra next continues with the Mātṛs $\left(33^{-55}\right)$. There is no unanimity about the number of these Mother goddesses. Several manuscripts have the standard seven Mothers (saptamātrkā), Brahmāṇī, Rudrāṇī, Kaumārī, Vaiṣnavīin, Aindrī, Vārāhī and Cāmuṇ̣̣ā, but most of the Nepalese manuscripts add an eighth one, the much less known Lamboșthì. Furthermore, compared to other lists of the Mothers and sets of surviving sculptures, the text reverses the common order of Aindrī and Vārāhī, invoking Aindrī before Vārāhī. ${ }^{77}$ While the addition of Lamboșthī as eighth Mother may well be an early addition in the Nepalese transmission of the text, it is possible that the group of eight is original because it is analogous to other groups of eight that we find throughout the mantra, such as eight Dikpālas, eight Nāgarājas and eight Yakșas. Thus it is also conceivable that Lamboșțī was removed by later scribes by the time the set of seven Mothers had become canonical. Iconographically, Lamboșthī appears as the fat counterpart of the emaciated Cāmuṇụa. The invocation of the Mātṛs concludes with the invocation of all Mothers collectively $\left(5^{\mathrm{O}}-5^{2}\right)$. Reference is made to the offering of bali to the Rudras, Mātṛs and Ganādhipas, a hint at the ritual background of the mantra (53-55).

The Mātṛs are followed by the Ganas $\left(5^{6-76}\right)$. They are invoked in separate groups, in accordance with the directions where they reside,

\footnotetext{
${ }^{76}$ Following these invocations there is a significant addition in $\mathrm{E}^{N}$ and $\hat{S}_{67}^{S}$, which includes Arhant, Buddha, Jayā / Vijayā, the Earth (Pṛthivī) and the Cows. $\mathrm{N}_{58}^{K}$ has a similar addition, invoking Arhant, Buddha, Vṛșabha and the Five Cows in Śivapura. The addition clearly reflects an attempt to expand the pantheon by incorporating the founders of Jainism and Buddhism, with some more deities added to the fold (Bisschop forthc. a). It should be noted, however, that Arhant and Buddha are not entirely foreign to the text, for they feature in another part of the Śivadharmaśāstra. In ŚiDhŚ 3.31-32 they appear in a list of deities who have received their status from worshipping Śiva's linga. The addition features in only two of the single-text Śantyadhyāya manuscripts that I have been able to check; see p. $189 \mathrm{f}$.

${ }^{77}$ Some later manuscripts have tried to repair this. See n. 36 on p. 155 .
} 
moving clockwise from east to northeast, followed by the lower and upper parts of the cosmos. In each case reference is made to the vertical division of the cosmos. The stereotypical phrasing of each set leads to a curious statement in the case of the Ganas residing in the lower and upper parts of the cosmos, for they are likewise said to be dwelling 'in heaven, in the sky, on earth and in the bottom of the nether region'. It is hard to imagine a lower or upper part of the universe again subdivided into heaven, sky, earth and nether region. The section on the Ganas is concluded with an instruction to provide bali offerings to the Ganas, which provides another clue to the ritual use of the mantra.

The text next returns to the theme of the directions, with invocations addressed to the eight Dikpālas 'Guardians of the directions' (77-101). These include fairly elaborate descriptions of their splendid cities of residence: Indra in Amarāvatī, Agni in Tejovatī, Yama in Vaivasvatī, Nirṛti in Kṛ̣ṇā, Varuṇa in Śuddhavatī, Vāyu in Gandhavatī, Kubera in Mahodayā and Îsāana in Yaśovatī. Like the previous section, it is arranged in a clockwise order from east to northeast.

After the invocations of the Guardians of the directions follow four verses addressed to the celestial gods in the seven worlds, Bhūloka, Bhuvarloka, Svarloka, Maharloka, Janarloka, Tapoloka and Satyaloka, as well as those dwelling in wild places on earth: on mountains, in caves, strongholds and forests $(102-105) \cdot{ }^{78}$

Four more verses follow, each addressed to a single goddess: Sarasvatī, Śrī, Jayā and Aparājitā (106-109). While the previously mentioned groups were deities associated with the wild, these four goddesses are protective deities of the civilised world. ${ }^{79}$

${ }^{78}$ Several sources $\left(\mathrm{N}_{58}^{K}, \hat{\mathrm{S}}_{67}^{S}, \mathrm{P}_{32}^{T}, \mathrm{P}_{72}^{T}, \mathrm{E}^{N}\right)$ include a few additional verses providing more information on the appearance of the gods in these seven worlds. The additions occur in different places in the text and are different in wording, suggesting that they have been added by scribes at different stages in the transmission of the text.

${ }^{79}$ Compare Arthaśāstra 2.4.17, which mentions shrines for Aparājita, Apratihata, Jayanta, Vaijayanta, Śrī etc. to be built in the centre of the city. It is noteworthy that the order of the deities in this part of the text partly resembles that seen in a section on the iconography of the gods in the Vișnudharmottarapurāna. From VDhP 3.5 o onwards we find the eight Dikpālas, followed by several gods not found in our text, continued by Sarasvatī (3.64), Śeșa (not in our text), Tumburu and his four sisters (3.66) and the Navagrahas (3.67-68). Manuscript $S_{67}^{S}$ adds Jayā and Aparājitā to Vijayā and Jayantī, thus completing the full group of four sisters of Tumburu (Goudriaan 1973). 
The mantra next includes a long section addressed to celestial powers (110-152). It is a veritable encyclopaedia of astronomical and astrological lore. There can be little doubt that it was composed by somebody with good training in Jyotihśāstra. It speaks of the Śaivas' attempts to engage with and take over the specialisms of rivalling ritualists. ${ }^{80}$ The section begins with an invocation of the Navagrahas: Sürya (Sun), Soma (Moon), Añgāraka (Mars), Budha (Mercury), Bṛhaspati (Jupiter), Śukra (Venus), Śanaiścara (Saturn), Rāhu and Ketu (110-121). ${ }^{81}$ The order of the seven planets follows the temporal order of the days of the week, which has been in use in India since the fourth century. A remarkably detailed depiction of the iconography of the Navagrahas is given. After a concluding verse (122), in which all the planets are requested to provide peace, Vișți is invoked in eight appearances (123-125). Viști is one of the 11 Karanas, artificial units of a half of a lunar day, and generally regarded as the most dangerous one. None of the other Karanas are mentioned, which makes this invocation stand out among the various lists that surround it. ${ }^{82}$ The 15 Tithis, the lunar phases of the moon's course, are up next, beginning with Amāvāsī, the night of the new moon, and ending with Pürnamāa, the night of the full moon (126-132). ${ }^{83}$ The Tithis are followed by the 27 Yogas, the lunisolar alignments, each one of them mentioned by name $\left(133^{-136)}\right.$. These are again followed by the 28 Nakșatramātṛs, the lunar mansions, usually simply called Nakṣatras. They are arranged from east to north, in the old order from Kṛttikā to Bharaṇi (137-147). The Nakṣatras are followed by the 12 Rāśis, the zodiac signs, again arranged from east to north, in accordance with the trigon (trikona) system $(148-151) .{ }^{84}$ The astronomical section ends with the Seven Sages

\footnotetext{
${ }^{80}$ See Sanderson 2004.

${ }^{81}$ The invocation of Ketu is absent in $\mathrm{N}_{45}^{C}$, while its wording shows remarkable variation in the different manuscripts. On the possible implications for the date of composition of the Śantyadhyāya and its transmission, see above, p. 17.

${ }^{82}$ Note that $\mathrm{N}_{77}^{K o}$ and the parallel in the Bhavisyapurạna omit this invocation of Viști, as well as those of the Tithis and the Yogas that follow (see below, p. 55). Two manuscripts show an attempt to link Viști to the other Karanas: $\mathrm{N}_{5^{8}}^{K}$ introduces a list of ten Karaṇas before Vișți, while $\mathrm{N}_{45}^{C}$ adds the other six movable Karaṇas after 136 .

${ }^{8} \mathrm{In}_{3^{2}}^{T}$ and $\mathrm{P}_{72}^{T}$ the verses dealing with the Tithis have been rewritten in such a way that the name of the deity presiding over each Tithi is included as well.

${ }^{84}$ There are some ambiguities in the transmission of this part of the text. For more on the trigon system, see n. 93 on p. $172 . S_{67}^{S}$ adds a passage that includes the Saṃkrāntis,
} 
(sapta rșayah), i.e. the constellation of Ursa Major, including Dhruva, the polar star (152).

The mention of the Seven Sages seems to have triggered the next section, which lists more sages $\left(153^{-155)}\right.$ ). Twenty-one sages (R̦sis) of great vows (mahāvrata) are invoked by name: Kāśyapa, Gālava, Gārgya, Viśvāmitra, Manu, Dakṣa, Vasișthha, Mārkaṇḍa, Pulaha, Kratu, Nārada, Bhṛgu, Ātreya, Bharadvāja, Añgiras, Vālmīka, Kauśika, Kaṇva, Saākalya, Punarvasu and Śālañkāyana. Many of them are famous Vedic sages, although this particular list is not found elsewhere. Their wives, sons and daughters are invoked as well $(156)$.

Next follow other classes of demigods: first, the Siddhas, Gandharvas, Apsarases and Garudias, all invoked as collective groups (157-158). These are all beings who have their natural abode in the sky. Next the text takes us to the underworld and from there it works its way up again to the level of the earth. First are listed the Daityas, who live in the nether region (Pātāla), as is indicated by the fact that they are said to be devoted to Hāțakeśvara, the name of Śiva in Pātāla. They are addressed by name and divided into three groups $(159-164):^{85}$

1. Namuci, Śaṅkukarṇa, Mahānāda

2. Mahājambha, Hayagrīva, Prahlāda, Tāraka, Agnimukha, Kālanemi, Mahotkața

3. Virocana, Hiraṇyākṣa, Suparva, Sulomaka, Mucukunda, Sukunda, Revataka

As in the case of the sages, the demons' wives, daughters and sons are invoked as well (165).

The next section takes us to the eight serpent lords (Nāgarāja): Ananta, Vāsuki, Takṣaka, Karkoțaka, Padma, Mahāpadma, Śan̉khapāla and Kulika (166-181). Each Nāgarāja is invoked in elaborate detail, with much attention paid to their individual iconography. Other Nāgas — in

the sun's transitions from one Rāśi to another, in accordance with the seven days of the week.

${ }^{85}$ These are not known as separate groups from other sources, but the order of names shows similarities to lists of demons dwelling in different layers of the Pātāla according to Purānic accounts. There these demons inhabit ever lower regions of the Pātāla. See n. 102 on p. 175 . 
the sky, in heaven, on earth, on mountains, in caves and forts and in the nether region - are also invoked, as well as the Nāgas' wives (182-184), daughters and sons. The Nāgas are addressed as being 'assembled here', another reminder of the ritual use of the mantra. Uniquely in the text, a śrutiphala concludes the section on the Nāgas (185).

Next we reach the earth, ground level so to say, with the invocation of the rivers. Seventeen rivers are mentioned by name, followed by an invocation of all rivers, flowing on earth, in heaven or in the sky. As in the case of the Daityas, the rivers are divided into three groups (186-192):

1. Gañgā, Yamunā, Narmadā, Gomatī, Kāverī, Varuṇā, Devikā

2. Candrabhāgā, Godāvarī, Sarayū, Gaṇḍakī, Kauśikī, Sarasvatī

3. Nairañjanā, Śoṇa, Mandākin̄̄, Saṃnihitā

The first group shows some overlap with the traditional group of seven rivers (saptanadī): Gañgā, Yamunā, Sindhu, Narmadā, Godāvarī, Kụ̣̣nā and Kāverī. ${ }^{86}$ The presence of the Varuṇā, a minor river flowing past Vārānasī, in this group is striking. Noteworthy about the last group is the mention of Nairañjanā, a name otherwise attested only in Buddhist literature, and Samnihitā, a tìrtha (or river?) in Kurukșetra. ${ }^{87}$

Next are addressed the eight Yakṣa lords: Mahāvaiśravaṇa, Maṇibhadra, Suviroma, Pāñcika, Vibhāṇḍaka, Dhṛtarāṣțra, Pūrṇabhadra and Virūpākșa (193-208). These are beings that are naturally associated with the earth as well. This group of eight Yakșas is not known as a distinct set from other sources. Their invocations are rich in iconographical details. Other Yakșas, on earth, on mountains, in caves and in forts, in the sky and in the nether region, as well as the Yakșas' wives, sons and daughters, complete the list (209-213).

An invocation of the earth's mountains and its oceans follows (214215). No individual mountains or oceans are mentioned. ${ }^{88}$

\footnotetext{
${ }^{86}$ Cf. Eck 2012: 168.

${ }^{87}$ See notes ad loc. There is some variation in the individual river names among the different manuscripts, while $\mathrm{N}_{58}^{K}, S_{67}^{S}$ and $\mathrm{P}_{32}^{T}$ add some more rivers. Moreover, $\mathrm{N}_{58}^{K}, S_{67}^{S}$, $\mathrm{P}_{3^{2}}^{T}$ and $\mathrm{P}_{7^{2}}^{T}$ also invoke tïrthas and lakes.

${ }^{88}$ Several manuscripts, however, identify the seven mountains of the earth, add a list of the eight islands and give the names of the seven oceans. See n. 124 on p. 183 .
} 
The final group of invocations again introduces a new topic, creatures of terrifying character and appearance: Rākșasas, Yoginīs, Bhūtas, Pretas, Piśācas and Grahas (216-234). Each group is depicted as the entourage of Śiva in his Bhairava aspect. This section stands out from the rest of the text in terms of its formulaic structure.

All these and other deities following the command of Śiva are once again requested to bestow peace to Śiva's devotees (235). A set of exclamations of victory to Siva $(236-242)$ follows. The chapter concludes with statements about the benefits of reciting and hearing the mantra (243256).

All in all, the mantra is organised around Maheśvara, who is accompanied by his wife Umā and members of his inner circle, from which it expands to include an elaborate pantheon of gods and powers. One invocation naturally leads to another, as with the collective Mothers followed by the collective Ganas, or the constellation of the Seven Sages at the end of the astronomical section followed by other groups of sages. A general organising principle running throughout the mantra relates to divisions of space and time. The latter aspect is particularly noteworthy. The mantra integrates the astronomical and astrological lore of the period and puts it to use in a Śaiva religious setting. Additions made throughout the transmission of the text show that later copyists were concerned with adding powers they thought were missing, thus displaying a tendency towards completeness. These additions attest to the mantra's active use over time.

\section{Iconographical Aspects}

Given the importance of naming in ancient India (Gonda 1970), it is striking that the mantra does not engage with the different names of the gods. As a rule only one name is singled out as the main subject of address. By contrast, the invocations are remarkably rich in matters relating to iconography. The author of the mantra has endeavoured to call to mind and visually represent the various deities invoked. While it is not an iconographical text per se, there is much that should be of interest to students of Indian art history. ${ }^{89}$ In several cases, the mantra provides us

\footnotetext{
${ }^{89}$ Available early textual sources on iconography are limited and much of the scholarship is based on post-twelfth century texts from South India (e.g. the highly influential
} 
with the earliest known detailed description of the visual appearance of a deity. A few noteworthy features of the iconographies of the gods may be mentioned here. Further details are discussed in the notes accompanying the translation.

First of all, a clear distinction can be made between deities belonging to the inner circle and those who do not belong to Siva's immediate entourage. As mentioned above, several members of the inner circle are qualified as being three-eyed (trinayana, trinetra). As close relatives of Siva they share in the god's iconography. Thus, for example, Umā is strikingly said to bear the sickle of the moon (7b). These Śaiva marks do not apply to any of the other deities, with the unsurprising exception of the Mātṛ Rudrāṇi (36b) and the Dikpāla İsāna (10oc). All other deites are mainly characterised by an attitude of total devotion to Siva.

The mantra pays particular attention to the skin and clothes of each deity, yielding important information on their colourful appearance, which is often lost in the surviving sculptures of the period. Their skin colours are treated in a systematic manner. For example, the appearance of the Dikpālas is presented as follows (99-101): Indra (gold), Agni (blazing charcoal), Yama (sapphire), Nirṛti (dark), Varuṇa (pearl), Vāyu (copper), Kubera (splendorous), Ísaāna (pearl). A similar colour scheme, with some variations, is also applied to the corresponding Ganas of the different directions (56-75). The iconography of the Ganas of the directions, which is encountered hardly anywhere else, follows that of the guardians of the directions. Thus, the ones in Indra's direction hold thunderbolts in their hands, those in Agni's ladles, those in Yama's sticks, etc.

The mantra contains one of the earliest known textual descriptions of the Mātṛs (33-49). The formulations in this section suggest that the description of their bodily appearance may not have been based on existing sculptures, even though they were around at the time, but are rather modelled on the iconography of the corresponding male deities men-

work of Rao 1914-16). An important early source is Brhatsamhita 58 , on the installation of images. Frequently quoted in secondary literature are Matsyapurāna 93 and 249-261, Viṣnudharmottarapurāna Khạ̣da 3 and Agnipurāna 2.167, but the date of these passages is by no means certain and in any case later than the Śivadharmaśāstra. A recent addition to the corpus of literature from North India are two chapters on iconography from the Śaiva Devyāmata, edited with notes by Ślączka (2016). 
tioned earlier in the text. The wording in their invocations is clearly inspired by those of their male counterparts. It serves as a reminder that a textual description need not necessarily be related to a material form. Five of the Mātṛs have a male counterpart in the text (Brahmāṇī, Rudrāṇī, Kaumārī, Vaiṣnavī and Aindrī), but the other three (Vārāhī, Cāmuṇ̣ā and Lamboșțī) do not. So it stands to reason that their portrayals are more independent. Two features are noteworthy: the reversal of the usual order of Aindrī and Vārāhī, and the unique mention of an eighth goddess, Lamboșțīi. In appearance she resembles the South Indian goddess Jyeșțhā. ${ }^{\circ}$

While the invocations of the deities are generally accompanied by iconographic descriptions, the text's treatment of cosmic powers is considerably less detailed. A distinction can be drawn between personified and non-personified powers. The astronomical part of the mantra is revealing in this respect. It begins with the Navagrahas. These include celestial bodies, but they form a well-established set of deities as well. As a consequence the mantra gives quite a bit of information on their bodily appearance and their attributes. The same is not the case for the astronomical powers that follow, including the Karaṇa Viști, the Tithis, Yogas, Nakṣatramātṛs, Rāśis and Ursa Major (saptarși). On the other hand, even here the text displays a tendency towards deification, as is illustrated by the unique naming of the Nakṣatras as Nakṣatramātṛs 'Lunar Mansion Mothers'.

Why is it that the mantra pays so much attention to the bodily appearance of the gods? It is quite conceivable that these descriptions were included to vocalise and call to manifestation the deities who were not present in the śânti ritual in a material form. The mantra may then have served as an aid for visualisation and manifestation..$^{91}$

\section{Universal Śaivism}

After the invocations of the members of Siva's inner circle there is a significant change in the form of address of the deities. From Brahmā onwards, each deity is invoked with an accompanying qualification

\footnotetext{
${ }^{90}$ For more on the text's treatment of the Mātṛs, see n. 34 on p. 154 .

${ }^{91}$ Compare in this connection the elaborate visualisations of the daily prayer to the enthroned Śiva in Aghoraśiva's twelfth-century Pañcāvaranastava (Goodall et al. 2005).
} 
(viśeșaṇa) that draws attention to the deity's devotion to Śiva. As the following inventory of such adjectives and other references to devotional attitudes illustrates, this is a key feature of the composition of the mantra:

- Śiva: śivabhakta (104c, 107c, 114c, 184c, 204a, 211a, 214c), śive bhakta (106c, 108c, 118c), śivabhaktipara (67c, 136a), śivabhaktisamanvita (8ob), śivabhaktisamutsuka (89d), śivārcanarata (40a, 117c), śivārcanapara (14a, 147c, 156c), śivapüjāpara (119c, 198c), śivapūjāparāyaṇa (34b, 148d, 204b), śivapūjāsamudyukta (69c, 71c, 75c, 86c), śivapūjāsamutsuka (211b), śivapūjārcane rata (111d, 211d), śivapūjājapodyukta (83c), śivadhyānaparāyaṇa (31b), śivadhyānaikatatpara (14b), śivadhyānaikaparama (29a), śivadhyānaikamānasa (147d, 19od), śivadhyānaikasampanna (80a), śivadhyānārcanodyukta (155c), śivadhyātr (204a), śivapādārcanarata (150c), śivapādārcane rata (167b, 19ob), śivapādābjapūjaka (179b), śivaikāhitamānasa (40b, 92b), śivapraṇāmaparama (8oc), śivasmaraṇabhāvita (83d), śivasadbhāvabhāvita (29b, 162b, 187d), śivaprasādasampanna (31a, 152c, 204c), śivadharmaparāyaṇa (86b), śivatejaḥsamāyukta (126e), śivājñānuvidhāyin (132f, 136b, 151f, 235b), śivacodita (142d), śivayogapara (148f).

- Rudra: rudrabhakta (38a, 52a), rudrabhaktiyuta (206d), rudrārcanapara (105c, 192c), rudrārcanarata (38b, 57a, 89b), rudrārcanasamāyukta (206e), rudrārcāhitamānasa (52b), rudrārcanaparodyukta (196c), rudrapūjāpara (169b, 208c, 215c), rudrapūjārcane rata (220b), rudrapraṇāmamanas (59c), rudrapraṇāmanirata (220a), rudrapraṇāmaparama (206c), rudraparāyaṇa (217d), rudraikāhitacetaska (61c, 220c), rudraikāhitamānasa (25b), rudrapradhyānanirata (63c), rudrapādārcane sakta (183b).

- Other names: haraparāyaṇa (181b), harapādārcaka (202c), harapādārcane rata $(98 \mathrm{~b}, 175 \mathrm{~b}, 194 \mathrm{~b})$, harapādanatottama (194d), haradhyānaikaparama (194c), harārcanapara (200c), mahādevārcane sakta (136a), mahādevānubhāvita (136b), maheśvarapara (158a), maheśapādapūjaka (158b), maheśārcana- 
bhāvita (122b), parameśārcanarata (65c), parameśvarabhāvita (95b), iśānārcanatatpara (115d), paśupater nata (177b).

- Special constructions: arcayantī sadā śivam (44b), śivam sampūjjya yatnena (67c), arcayanti sadākālam devaṃ tribhuvaneśvaram (141cd), issāanam pūjayanty etāḥ sarvakālam subhāvitāh (145ab), pūjayanti sadā śivam (149d), pūjayanti sadākālam rudram bhuvananāyakam (151cd), hāțakeśvaradevasya nityam pūjāparāyaṇah (16ocd), bhāvena parameṇāśu yajante sarvadā śivam (164ab), sarvabhūtapatim devam parameśam maheśvaram püjayanti sadā nadyạ̣ (187ac).

The invocations display a model of Universal Śaivism, in which the power of all and everything ultimately rests upon Siva, to whom all gods and powers are devoted as a consequence. This dependence of all cosmic power on Śiva can be considered the overall message of the mantra. The universe is, as it were, Śaivised. The hierarchical model expressed here perfecty mirrors that of early medieval Indian kingship, which involves many types of sāmantas all empowered by their loyalty and devotion to the supreme ruler. Sanderson (2009) has argued that this shared model may well have been one of the keys to the success of Śaivism and its popularity among early medieval rulers. It comes as no surprise to encounter it here in the context of a śänti invocation that would have played an important role in ritual kingship. On the other hand, the model was certainly not restricted to Śaivism alone, for other religious traditions of the time likewise sought to integrate all cosmic order under the rule of one god or divine principle. ${ }^{92}$

\footnotetext{
${ }^{22}$ Probably the most famous example is the Bhagavadgit $\bar{a}$, whose theology of Kṛ̣na, integrating a polytheistic understanding of the world in a monotheistic model, has been designated by Malinar as a form of 'cosmological monotheism' (Malinar 2007: 237241). As Schreiner has argued, the Viṣnupurāṇa may likewise be read as a theological document that brings all divine and cosmic order under the rule of Viṣnu-Nārāyaṇa (Schreiner 2013: 621-650). For further examples, see Bisschop forthc. a. The same attitude is also clearly manifested in the Bhavisyapurāna's adaptation of the mantra, in which all references to Śiva bhakti have been systematically replaced by references to Sūrya bhakti.
} 


\section{Use of the Mantra}

The Śāntyadhyāya belongs to an extensive body of liturgical literature dedicated to the appeasement or pacification (śānti) of all cosmic power to secure the welfare of the kingdom. Within the Vedic tradition, śânti rites were particularly associated with the Atharvaveda, which is where we find some of the earliest elaborate śanti mantras..$^{93}$ Another tradition that has played a key role in the development of the Brahmanical model of śānti is the astronomical-astrological lore of Jyotihśāstra. The inclusion of the Śantyadhyāya in the Śivadharma is indicative of the Śaivas' engagement with the roles of the Atharvavedic purohita and the court astrologer. ${ }^{94}$

In style and composition the Śantyadhyāya invites comparison to two Brahmanical texts: Bṛhatsaṃhitā 47 and Viṣnudharmottarapurāṇa 2.22. ${ }^{95}$ The first concerns a mantra that forms part of the so-called 'bath of prosperity' (puşyasnāna), a rite of kingship, while the second is to be recited at the 'royal consecration' (rājyābhișeka). ${ }^{96}$ The Viṣnudharmottara prescribes a lengthy mantra of 185 verses for the last consecratory act of the rājyābhișeka, requesting all deities and powers to consecrate the king. Starting with Brahmā, Viṣnu and Śiva, the mantra addresses a host of powers, ranging from the four Pāñcarātra vyūhas (Vāsudeva,

${ }^{93}$ Cf., e.g., Atharvaveda Śaunakasaṃhitā 19.9. For studies on the historical formation and development of śānti, see Hoens 1951; Kane 1930-62, V.2: 719-814; Geslani 2012. See also the Śāntikāṇ̣̂a of Lakșmīdhara's voluminous Kṛtyakalpataru.

${ }^{94}$ On this subject, see Sanderson 2004.

${ }^{95} \mathrm{ViDhP} 2.22$ has a parallel in AgnP 2.167.

${ }^{96}$ Both mantras have been the subject of a recent study by Geslani (2012) on the incorporation of śānti rites in the formation of the Purānic abhișeka. Geslani argues that the form of the royal consecration (räjyābhișeka) found in the Viṣnudharmottarapurāna originated from Atharvavedic ritual manuals such as the Śantikalpa and the Atharvavedapariśista. This concerns in particular the introduction of a śânti rite at the start of the ritual, the so-called aindrí śānti. The aindrì mahāśānti described in the Śāntikalpa forms one of 30 variants of maháśänti rites meant to appease potential powers and ward of dangers in various situations. It was particularly suited to the concerns of kingship. Ritually prepared waters (śāntyudaka), already mentioned in earlier Atharvan texts such as the Kauśikasūtra, empowered by specific groups of mantras (mantragana), play a central role in this appeasement rite. The five sets of mantras prescribed (śarmavarman, aparājita, àyușya, abhaya, svastyayana: 5.3 .5 ) are the very same sets of mantras employed by VDhP 2.19.4-5 during what VDhP 2.19.1 calls the paurandari śânti (Geslani 2012: 337). 
Samkarșaṇa, Pradyumna and Aniruddha) to various sets of deities and sages, as well as astrological conjunctions, abstract principles, texts, places of pilgrimage and rivers. Brhatsamnhitā $47.54 \mathrm{~cd}-6$ oab includes a much smaller mantra to be recited at the king's pusyasnāna. The invocation is uttered by the king's purohita and also commences with Brahmā, Viṣnu and Śiva, before moving on to the Sādhyas, Maruts and other deities, and includes astronomical principles such as the Nakșatras and the Muhūrtas, as well as other units of time. The two mantras are clearly related and provide a powerful conclusion to the ritual. By comparison, the Viṣnudharmottarapurāna's mantra appears to be an extended version of the mantra taught by Varāhamihira in the Brrhatsamnhitā. Noteworthy is the inclusion of the four Pāñcarātra vyūhas, following the invocation of the Hindu trinity of Brahmā, Viṣnu and Śiva. This is in line with the Pāñcarātra background of the Viṣnudharmottarapurāna. ${ }^{97}$

In addition to these two Brahmanical sources, the composition of the mantra also shares many features with the strings of spells and incantations that one may find in the Buddhist Dhāraṇi literature..$^{8}$ A particularly striking case is offered by the Mahāmāyūrīvidyārājñ̄i. According to the introductory narrative of this 'Great Peahen' spell, the Mahāmāyūrì was given by the Buddha to Ananda to provide help for a monk suffering from a snakebite. The spell was used for a range of protective purposes and was highly popular across Asia for many centuries. Six early Chinese translations of the text exist, ranging from the fourth to the eighth century. ${ }^{99}$ The spell includes long strings of invocations to a host of classes of deities and powers, in which each deity is individually mentioned by name. Some of these lists, such as the well-known catalogue of Yakșas, are very extensive. ${ }^{100}$ Certain sections of the spell display remarkable correspondences with parts of the Sántyadhyāya. Particularly striking are the invocations to the Nakșatras, which, as in the Śāntyadhyāya, are listed in four groups of

\footnotetext{
${ }^{97}$ On the VDhP's Pāñcarātra ideology, see Inden 2000.

${ }^{8}$ See Hidas 2015, for an introductory survey of Dhāraṇī Sūtras.

${ }^{99}$ See DesJardins 2002, for an integrated study of the Mahāmāyūrī. On the changes and expansion of the text reflected in the different Chinese translations, see Sørensen 2006 and Overbey 2016. I refer here only to the Sanskrit text.

${ }^{100}$ For the catalogue of Yakșas, see Lévi 1915 and Sircar 1971/72.
} 
seven in accordance with the four directions where they are located. ${ }^{101}$ A comprehensive study of these correspondences falls outside the scope of the present study, but they do show that we are dealing with a shared phenomenon that extends across religious boundaries.

While the Bṛhatsamnitā and the Viṣnudharmottara both include the śänti mantra in royal consecration rites, the Śantyadhyāya's scope and appeal is much broader and in this sense somewhat more akin to that of the Dhāranī literature. As stated in the concluding section of the text, the mantra is meant to ward off dangers in general and bring about welfare to the land and the people. Whereas the Viṣnudharmottara and the Brhatsam hitā repeatedly refer to the abhișeka of the king with the refrain ete tvām abhiṣiñcantu 'may these consecrate you', the Śäntyadhyāya's refrain has a first-person subject: śāntim kurvantu me sadā 'may they always bestow peace on me.102 Furthermore, the text makes no reference to an abhișeka nor hints at the involvement of a priest. The concluding verses of the Sántyadhyāya rather state the fruits of recitation of the mantra in very general terms:

One aiming for a girl acquires a girl, one aiming for victory acquires victory, one aiming for wealth obtains wealth, one aiming for sons obtains many sons, one aiming for knowledge acquires knowledge, one aiming for union obtains union. (244)

Results relating to the success of the kingdom are, however, also mentioned:

A man who should enter battle, having heard this auspicious chapter, will conquer his enemies in battle and be honoured with fortunes. (246)

He enjoys [his kingdom] for an undecaying time, with his orders not rejected. He is not overpowered by diseases and thrives with sons and grandsons. (247)

${ }^{101}$ To give one example, the invocation of the seven Nakșatras in the east runs as follows: krttikā rohin̄i caiva mrgaśirārdrā punarvasuh | puṣyo mañgalasampanno 'śleșā bavati saptamī || ity ete saptanakșatrāḥ pūrvadvārikāsthitāh ye pūrvām diśam rakșanti paripālayanti, te 'py anayā mahāmāyūryā vidyārājñyā svāter bhikșor mama sarvasatvānām ca rakșām kurvantu, guptị̣ paritrānam parigraham paripālanam śāntiṃ svastyayanam daṇdaparihāram śastraparihāraṃ viṣadūṣaṇam viṣanāśanam sìmābandham dharaṇibandhaṃ ca kurvantu, jīvatu varșaśatam paśyatu śaradāśatam (Mahāmāyūrīvidyārājñ̄i, pp. 50-51). This listing of the Nakṣatras invites comparison to that of the Nakṣatramātṛs in the east in ŚiDhŚ 6.137-139.

${ }^{102}$ There are several variations on this phrase throughout the text. 
While these verses indicate that the mantra was not meant for royal purposes alone, chapter 2 of the Sivadharmottara provides important evidence that it did acquire a prominent place in the Śivadharma's conception of royal ritual. This chapter describes an elaborate ritual called 'the gift of knowledge' (vidyādāna). ${ }^{103}$ The 'gift of knowledge' first of all concerns the teacher's instruction of the Śaiva religion to lay devotees of Śiva, but most importantly it refers to the ritual donation of a manuscript to a Śaiva guru along with the support for its recitation. The chapter gives a fascinating account of how to copy a manuscript, a sort of Sanskrit philology avant la lettre, in which one is allowed to make corrections on the spot if one sees fit to do so, and describes a lengthy ritual in which the donor is a king who has the copied manuscript paraded through the royal capital before finally handing it over to the Śaiva guru at the temple of Śiva (ŚiDhU 2.59-6o). The scene that follows is relevant to putting the Śāntyadhyāya's mantra into context:

To secure pacification (śäntyartham) for the cows, brahmins, the kings and the towns in the kingdom, the most excellent reciter should recite one chapter [from the book].

The best reciter of books is proficient with the characteristics of metre, a true poet, has a sweet voice, is a knower of the art of the Gandharvas and clever.

Rising up the guru should then sprinkle the king a little with appeasement water (śantitoya) on the head and then also the people present there. ${ }^{104}$

There can be little doubt that the manuscript from which a chapter should be read is in fact a copy of the Sivadharma and that it is the Śantyadhyāya that should be recited on this occasion. ${ }^{105}$ The ritual

${ }^{103}$ This chapter of the Śivadharmottara has been the subject of an extensive study, accompanied by a critical edition and translation, by Florinda De Simini in the context of her PhD dissertation on the subject of vidyādāna (De Simini 2013). Citations of the text are from her edition. See also De Simini 2016b.

104 ŚiDhU 2.61-63: śāntyartham ekam adhyāyam gobrāhmaṇamahībhṛtām | rāsțtrīyanagarānāạ ca vācayed vācakottamah $\|$ chandolakṣaṇatattvajñah satkavir madhurasvarah $\mid$ gāndharvavid vidagdhaś ca śreșthah pustakavācakaḥ || śāntitoyena rājānam samutthāya gurus tatah | śirasy abhyukșayed ișat tatrastham ca janam tatah $\|$.

${ }^{105}$ The Śivadharma here may have included both the Śivadharmaśāstra and the Sivadharmottara. See also De Simini (2016b: 204-205), who, however, keeps open 'the possibility that the text of the donated manuscript and the one recited during the procedures for appeasement may or may not be the same'. 
described does not appear to be a rājäbhișeka as such, but is a more general pacification rite meant for the entire population of the kingdom headed by the king. Central to the ritual is a book, a copy of the Sivadharma, handed over to the Śaiva guru. In this way, the welfare and success of the kingdom came to be grounded in the king's donation of a copy of a Śaiva scripture to the Śaiva guru.

Aside from specifications in the text itself, we may also acquire information about the mantra's actual use from epigraphic records. Most important is the previously mentioned Kannada inscription from Tălagunda, which provides detailed procedures for the recitation of the Śāntyadhyāya:

From month to month, on the eighth and fourteenth lunar days, on the new- and full-moon days and on the festive days (parvan) that are called the Vyatipātayoga and the Sankramana [conjunctions], after performing the constant and occasional worships, the teachers of the śāstras, having recited the Śāntyadhyāya, the sixth [chapter] of the Śivadharma, in due succession, having made mandalas on the eight sides of the god, by means of the mantra from this [text] placing the offering of food, will be blessing the king who rules the country, [addressing a benediction] to the cows, the Brahmins, and to the sponsor of the rite. And during the year [in] the mahaparvans, which are the two equinox days, the two solstices, on the day of the eclipses of moon or sun, on the full-moon days of Āṣạ̣̄ha, Kārttika, Māgha, and Vaiśākha; thanks to the merits of worship on each of these days, the fruit of six months will be bestowed. In these parvans, having erected thousands of platforms to the god, having performed the proclamation of a meritorious day, the inhabitants of the schools (śästrakhandikas), having, by means of colours, drawn three mandalas called the vidyämandala, the gurumandala and the śivamandala, having revered them, they will be reciting the Śantyadhyāya. ${ }^{106}$

${ }^{106}$ Translation De Simini 2016b: 196. EC 7, Skt 185, 1l. 69-74: timga! dimgalo! 2 daștami 2 caturddaśiyamāvāsye pārnnamāsye vyatīpātasaṃkramaṇav emba parbbamgaḷalu nityanaimittikapūjeyam māḍi śāstradupādhyāyaru śivavarmmadāreneya śāmtyādhyāyamm japaṃgeydalli hēlda kramadim dēvarențudikkino! maṇdalavam māḍiy alliya mantradị̣ baliyan ikki déśava alvva kșatriyanumạ̣ gōbrāhmaṇarumam yajamānanumam harasuvaru mattam varșadolage baha mahāparvvamga! 2 ḍayana 2 viśu sōmasūryyagrahaṇav āśādakārttikamāghavaiśākhada puṃnnamigalal oṃdomdudivasada püjey ārārutimgaḷ phalaman ìguv āparbbamgaḷ! dèvargge sahasragaḍ̂geyaṃ prārambhasi puṃnyāhavācaneyam mādi śāstrakhaṃdikadavaru ramgado! vidyāmaṃdalagurumaṃ̣alaśivamaṃdalav eṃba maṃdalatrayaman uddha- 
De Simini (2016b: 196-197) has shown that the inscription includes many references to technical terminology relating to the vidyādanna as described in the Sivadharmottara. It attests to that text's spread in medieval Karnataka. The inscription indeed takes up several aspects of this chapter, but it is also worth noting that recitation of the mantra is not restricted to one particular moment in time, viz. the moment of donation of the manuscript, but that it was to be recited on a range of powerful days on the ritual calendar. The inscription thus attests to the use of the mantra in a variety of ritual settings across history. Indeed,

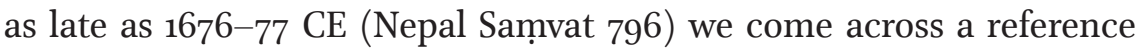
to the public recitation of the Śantyadhyāya in a document from Nepal recording the events of the year (Regmi 1966: 322).

The use of the text for recitational purposes is also evinced by the existence of various manuscripts of the Śantyadhyāya as single texts or in bundles with other texts (see Appendix I). One of the manuscripts (NGMPP, A 1174/14), a bundle of Stotras and Stavas, reports for example its recitation together with the Devimāhātmya. Another manuscript (NGMPP, A $1158 / 8=\mathrm{N}_{58}^{K}$ ) transmits the Śāntyadhyāya along with the Sāttvatabädhapraśamana, a prayer from the Viṣnudharma against afflictions, while another (NGMPP, I 54/4) starts with a nyāsa and introduces the text with the words 'now the mantra to say next'.

Finally, the Śivadharmavivarana (see Appendix II) provides a few small hints as well. The start of the commentary states that the Śantyadhyāya is meant to ward off obstacles (antarāya) that may present themselves to the worshipper. The concluding statement of the commentary indicates that it formed part of the 'daily worship' (nityapūjā) of the Siva devotee. This attests to its broader use on a daily basis.

\section{Introduction to the Edition}

The manuscript situation of the Śivadharma is complex and editing the text presents a major challenge. At the moment of writing, a total of more than 80 manuscripts of texts belonging to the Sivadharma have been identified, but this number may easily be expanded as more work

risiy arccisi śāmtyādhyāyamam japisuvaru vēdakhaṃdikada nālvarum 4 praśasta kalaśaṃa. 
is being done on the dissemination of the text. ${ }^{107}$

To avoid confusion among different scholars working on individual parts of the corpus and in order to allow for the addition of more manuscripts in future editions of texts belonging to the Sivadharma corpus, a standardised way of referencing the manuscripts is required. In the present edition I have therefore adopted a set of principles for forming the sigla, which may be followed by current and future editors of the corpus: ${ }^{108}$

1. The first element is a capital letter, which stands for the script used, e.g. $\mathrm{B}=$ Bengali, $\mathrm{D}=$ Devanāgarī, $\mathrm{G}=$ Grantha, $\mathrm{M}=$ Malayalam, N = Newari, Ś = Sāradā, T = Telugu. The only exception concerns the paper transcripts from Pondicherry. In order not to confuse these with the Devanāgarī manuscripts proper, capital $\mathrm{P}=$ Pondicherry is used as first element. To refer to an earlier edition of the text we use capital $\mathrm{E}=$ Edition.

2. The second element is a superscripted capital letter in italics, referring to the place where the manuscript is currently located. Thus: $\mathrm{C}=$ Cambridge, $\mathrm{K}=$ Kathmandu, $\mathrm{L}=$ Leiden, $\mathrm{O}=$ Oxford, $\mathrm{S}$ = Srinagar, $\mathrm{V}=$ Varanasi . To avoid possible confusion with other places that begin with the same letter, two places are referred to by two letters: $\mathrm{Ch}=$ Chennai, Ko $=$ Kolkata. The Pondicherry transcripts again form an exception: $\mathrm{T}=$ transcript. For the edition of Naraharinātha we use $\mathrm{N}$ = Naraharinātha.

3. The third element is a subscripted two-digit number (except in the case of the Pondicherry transcripts, where we allow for three digits). This refers to the last two digits of the manuscript's reference number in the library collection where it is located, e.g. 67 = Srinagar 1467, 99 = Cambridge Add. 1599. Because the NGMPP

${ }^{107}$ For a detailed survey of the transmission of Śivadharma corpus manuscripts in Nepal, see De Simini 2016a. A separate survey of Śivadharmaśāstra and Śivadharmottara manuscripts in South India is under preparation by Marco Franceschini. A first survey was presented by him at the Sivadharma workshop held at Leiden University, 26-3o September 2016: 'The transmission of the Śivadharma in Tamil Nadu'.

${ }^{108}$ This set of principles is the outcome of a meeting between Florinda De Simini, Dominic Goodall, Nirajan Kafle, Nina Mirnig and myself at the Śivadharma Workshop held at Leiden University, 26-3o September 2016. 
reel numbers will often be more familiar to scholars working with these manuscripts, we rather refer to the last two digits of the first part of the reel number in the case of these manuscripts, e.g. $82=$ A1082/3.

The objectives of the present edition of the Śantyadhyāya are modest. It aims to give the reader an insight into the transmission of the Śantyadhyāya across space and time, without, however, striving for completeness or claiming to reconstruct an underlying original text. Our present understanding of the composition and transmission of the Sivadharmaśāstra is still in its infancy and the lines of transmission are exceptionally complex. ${ }^{109}$ In the notes accompanying the translation, attention is drawn to potentially significant variants, omissions, revisions and additions. General principles of selection are discussed in the sections on the transmission in Nepal, Bengal, Kashmir and South India.

The selection of sources has been guided by a combination of practical and scientific concerns. Given the large number of early palm-leaf manuscripts surviving from Nepal, each one of them hundreds of years older than any of those from the rest of the subcontinent, preference has been given to these manuscripts, both in terms of the selection of manuscripts and in the constitution of the text. From among the available Nepalese palm-leaf manuscripts, I have selected five manuscripts that seemed to offer the most potential. ${ }^{110}$ These comprise the oldest surviving manuscript to include the Śantyadhyāya, three eleventh- to twelfth-century Śivadharma corpus manuscripts and a Malla-period manuscript in which the Śāntyadhyāya has been included in a bundle with portions from other texts. From the rest of the subcontinent, I have included the readings of a seventeenth-century paper manuscript in old Bengali script, a paper manuscript in Śāradā script and two paper transcripts from Pondicherry. Finally, I have also reported the readings of the editio princeps by Naraharinātha. ${ }^{111}$ A list of sources and the sigla

\footnotetext{
${ }^{109}$ See De Simini 2017, for an attempt to classify the manuscripts into groups and families, primarily on the basis of the transmission of Sivadharmaśāstra 12; she highlights the presence of horizontal contamination in the transmission of different parts of the text, making the reconstruction of a stemma inherently problematic.

${ }^{110}$ De Simini 2016a provides a detailed survey of the Śivadharma manuscripts from Nepal.

${ }^{11}$ There exists another, more recent edition of the Śivadharmaśāstra, published
} 
used in the edition is given below.

\section{Sources for the Edition}

$\mathrm{N}_{77}^{K o}$ Asiatic Society, Kolkata, G 4077. Palm leaf, Newari script, dated [Nepāla] Samvvat 156 (1035-36 CE).

Contains: Śivadharmaśāstra (47 fols.); Śivadharmottara (65 fols.); Śivadharmasaṃgraha (58 fols.); Umāmaheśvarasaṃvāda (35 fols.); Sivopaniṣad (22 fols.); Umottara or Uttarottaratantra (fols. 23-42, 46-49); Vṛsasārasamgraha (52 fols.); Lalitavistara (3o fols., fol. $28-38) .^{112}$

Śāntyadhyāya covers fols. 12b-22b in Roman numbering (images $17-25) .{ }^{113}$

$\mathrm{N}_{45}^{c}$ University Library of Cambridge, Add. 1645, 247 folios. Palm leaf, Newari script, dated [Nepāla] Saṃvat 259 (1139-40 CE). ${ }^{114}$

Contains: Śivadharmaśāstra (fols. 1r-38r); Śivadharmottara (fols. 38r-87r); Śivadharmasamgraha (fols. 87r-132r); Śivopaniṣad (fols. 132r-15ov); Umāmaheśvarasamvvāda (fols. 150v-18ov); Uttarottaramahāsaṃvāda (fols. 18ov-201v); Vrșasārasaṃgraha (fols. 201v-238v); Dharmaputrikā (fols. 238v-247r).

Śāntyadhyāya covers fols. 12r-19r (images 25-39).

$\mathrm{N}_{82}^{K}$ National Archives, Kathmandu, 3/393, 274 folios. Microfilmed by

under the name of Śivadharmapurāna in the Chowkhamba Sanskrit Series: Jugnu \& Sharma 2014. The readings of this publication show remarkable similarity to the readings of $\mathrm{P}_{32}^{T}$. The preface of the edition mentions, however, that it was based on a manuscript from Adyar (Jugnu \& Sharma 2014, ix), while $\mathrm{P}_{3^{2}}^{T}$ was copied from a manuscript in Kilvelur. I have not reported its readings.

${ }^{112}$ Following the description in Shastri 1928: 718-723.

${ }^{113}$ The first folio is displaced in the images. The first three syllables, nandike-, are on image $18 \mathrm{a}$, from which the text continues on image $17 \mathrm{~b}$ (with the first syllables covered by another folio). From there the text continues to $18 \mathrm{~b}$. One folio is lost in between folios $16 \mathrm{~b}$ and $17 \mathrm{a}$ (image 19).

${ }^{114}$ Available online: http://cudl.lib.cam.ac.uk/view/MS-ADD-o1645/1. For a tran-

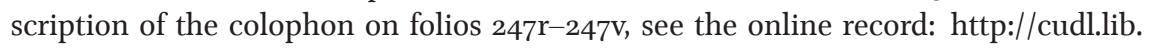
cam.ac.uk/view/MS-ADD-o1645/496 
the NGMPP, A 1082/3. ${ }^{115}$ Palm leaf, Newari script, dated [Nepāla] Samvat 189 (1069 CE). ${ }^{116}$

Contains: Śivadharmaśāstra (fols. 1v-41r); Śivadharmottara (fols. $1 \mathrm{~V}-52 \mathrm{~V}$ ); Śivadharmasamgraha (fols. 1v-57v); Umāmaheśvarasamvvāda (fols. 1v-32v); Śivopanișad (fols. 1v-19r); Vrșasārasamgraha (fols. 1v-46r); Dharmaputrikā (fols. 1v-12r); Uttarottaramahāsaṃvāda (fols. 1v-24r).

Śāntyadhyāya covers fols. 23v-27v (images 14-29).

$\mathrm{N}_{12}^{K}$ National Archives, Kathmandu, 5/841, 142 folios. Microfilmed by the NGMPP, B12/4. Palm leaf, Newari script, apograph(?) dated by another hand: [Nepāla] Samvvat 315 (1194-95 CE). ${ }^{117}$

Contains: Śivadharmaśāstra (fols. 1v-47r); Śivadharmottara (fols. 48v-109v); Śivadharmasamgraha (fols. 110r-150v*). Incomplete.

Śāntyadhyāya covers fols. 14v-24r (images 2867-2875). One folio is missing, covering the text from munih in 154 b to nadyah in $187 \mathrm{c}$.

$\mathrm{N}_{58}^{K} \quad$ National Archives, Kathmandu, 1/1376, 28 folios. Microfilmed by the NGMPP, A 1158/8. Palm leaf, Newari script, dated Bhādrapada [Nepāla] Samvvat 522 (August-September, 1402 CE).

Contains: Śāntyadhyāya (fols. 1v-2ov); Sattvabādhāpraśamana (fols. $1 \mathrm{r}-5 \mathrm{v}$ in letter numerals).

For more details, see p. 191 below.

${ }^{115}$ Thanks to Florinda De Simini, I have been able to work on the basis of high quality colour photographs of this manuscript.

${ }^{116}$ The date is recorded on the final colophon of the Dharmaputrika (12r):

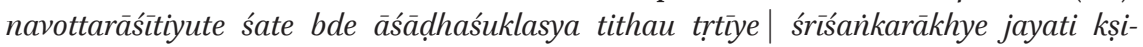
tendre śrīyangalasyottarațollake yạ̣| dharmāśayaḥ satkulalabdhajanmā guṇapriyo rāghavasinhanāmā| jñānaprakāśam śivadharmaśāstram subhapratișțam kṛtavān samagram $\mid$ karttur etena puṇyena bhūyāl lakșmīr anuttarā $\mid$ trailokyapüjitam śaivam vākyam jayati sarvada $\mid$. The first verse is an upajāti, the second anuștubh. See also Pettech 1984: 46: 'Written at Kathmandu, of which Yangala was the southern section, corresponding to the Dakṣinakoligrāma of Licchavi times. The date corresponds to May 24th, 1069.'

${ }^{117}$ Cf. De Simini 2016a: 230, n. 57, with reference to the date given on folio 47v: samvat 315 anyādrștapustake samvatsarapramāṇam drșțvā likhitam \|; 'year 315. Having seen [this] date (lit. 'year measurement') in another examined manuscript, [it] was copied [here]'. 
$\mathrm{B}_{99}^{c} \quad$ University Library of Cambridge, Add. 1599, 8o folios. Paper, Bengali script, dated Śāka Saṃvat 1604 (1682 CE). ${ }^{118}$

Contains: Śivadharmaśāstra (fols. 1v-37v); Śivadharmottara (fols. 1r-43v).

Śāntyadhyāya covers fols. 12v-18v (images 26-38).

$\hat{S}_{67}^{S} \quad$ Oriental Research Library, Srinagar, 1467, 38 folios. Paper, Śāradā script. Śivadharmaśāstra, but the text is named Śivadharmacarita. Śāntyadhyāya covers fols. 12r-19v (images 12-20).

$\mathrm{P}_{32}^{T}$ Institut Français de Pondichéry (IFP), T 32, 156 folios. Devanāgarī paper transcript of a manuscript belonging to Kīvalūr. Śivadharmaśāstra. ${ }^{119}$

Śāntyadhyāya covers fols. ${ }_{48-76 .}$.

$\mathrm{P}_{72}^{T} \quad$ Institut Français de Pondichéry (IFP), T 72b, 155 folios. Devanāgarī paper transcript of manuscript 75425, written in Grantha script, belonging to the Adyar Library, Chennai. Sivadharmaśāstra, although the text is ascribed to the Sivadharmottara. ${ }^{120}$

Śāntyadhyāya covers fols. $5^{\mathrm{O}-76}$.

$\mathrm{E}^{N}$ Yogī Naraharinātha, Śivadharma Paśupatimatam Śivadharmamahāśāstram Paśupatināthadarśanam, Kathmandu, Samvat 2055 (1998 $\mathrm{CE})$.

Śāntyadhyāya covers pages 27-44.

\section{The Transmission in Nepal}

$\mathrm{N}_{77}^{K o}$ is the oldest manuscript available for the Śantyadhyāya and, if only for this reason, deserves special attention. ${ }^{121}$ The manuscript belongs

\footnotetext{
${ }^{118}$ Available online: http://cudl.lib.cam.ac.uk/view/MS-ADD-o1599/1.

${ }^{119}$ Available online: http://muktalib7.org/IFP_ROOT/IFP/transcripts_data/Too32/ PDF/Too32.pdf.

${ }^{120}$ Available online: http://muktalib7.org/IFP_ROOT/IFP/transcripts_data/Too72/ PDF/Too72.pdf.

${ }^{121}$ As mentioned above, there exists an earlier Nepalese palm-leaf manuscript of the Sivadharma (NGMPP A 1028/4), which may be dated to the end of the tenth or early
} 
to the collection of the Asiatic Society of Kolkata and is dated $1035^{-} 3^{6}$ CE, in the colophon of the Lalitavistara, the last text included in this codex. $\mathrm{N}_{77}^{K o}$ has several unique features that set it apart from all the other manuscripts used. ${ }^{122}$ Most striking is the last of the eight texts in the bundle. In contrast to the rest of the Nepalese tradition, the text included here is not the Dharmaputrikā, but a longer work named Lalitavistara, not to be confused with the Buddhist text of the same name. ${ }^{123} \mathrm{Com}-$ pared to the main text constituted in the edition, it has two striking and unique omissions, both in the astronomical section: it lacks the invocation of Ketu in the Navagraha section (120-121) and it omits a lengthy section on Vișți, the Tithis and the Yogas (123-136). ${ }^{124}$ These omissions may well point to an earlier stage of transmission of the text, but it is difficult to assess in the absence of further manuscript evidence. The possible implications for the dating of the Śantyadhyāya have been discussed above (p. 17). $\mathrm{N}_{77}^{K o}$ has five additional omissions shared with some of the manuscripts: $14 \mathrm{ab}$ (with $\mathrm{N}_{45}^{c}, \hat{\mathrm{S}}_{67}^{s}, \mathrm{P}_{72}^{T}$ ), 32bc (with $\mathrm{N}_{45}^{c}, \mathrm{~N}_{12}^{K}, S_{67}^{s}, \mathrm{P}_{32}^{T}$, $\mathrm{P}_{72}^{T}$ ), 142ab (with $\mathrm{N}_{12}^{K}, \hat{\mathrm{S}}_{67}^{S}, \mathrm{P}_{32}^{T}, \mathrm{P}_{72}^{T}$ ), 210ab (with $\hat{S}_{67}^{S}, \mathrm{P}_{32}^{T}, \mathrm{P}_{72}^{T}$ ) and 242ad (with $\hat{\mathrm{S}}_{67}^{s}, \mathrm{P}_{7^{2}}^{T}$ ). Some of these may be due to eye-skip, but their wide spread suggests that they would already have taken place early in the transmission of the text because these omissions are shared by manuscripts from different parts of the Indian subcontinent. They are in remarkable agreement with $\hat{S}_{67}^{s}, \mathrm{P}_{32}^{T}$ and $\mathrm{P}_{72}^{T}$, all manuscripts that are not from Nepal. $\mathrm{N}_{77}^{K o}$ has two small additions shared with some of the other manuscripts: two pādas after 206b (absent only in $\mathrm{N}_{82}^{K}$ and $\mathrm{B}_{99}^{C}$ ) and two pādas after $217 \mathrm{~d}$ (with $\mathrm{E}^{N}, \mathrm{P}_{3^{2}}^{T}$ and $\mathrm{P}_{7^{2}}^{T}$ ). On the level of individual readings, the picture is somewhat similar. There are striking parallels with $\mathrm{P}_{32}^{T}$ and $\mathrm{P}_{72}^{T}$, and to a lesser extent with $\mathrm{N}_{45}^{c}$ and $\hat{S}_{67}^{s}$. Lastly it should be observed that $\mathrm{N}_{77}^{K o}$ has many trivial errors, such as lengthening of - $a$, dropping of anusvāra and visarga and dropping of syllables, as well as other bad readings of its own. The text is not transmitted in good shape.

$\mathrm{N}_{45}^{c}$ is one of several Śivadharma manuscripts in the collection of the University Library of Cambridge. It is a complete Nepalese palm-leaf

eleventh century, but it is incomplete and lacks most of the Śivadharmaśāstra, including the Śäntyadhyāya.

${ }^{122}$ See also De Simini 2017: 521-522.

${ }^{123}$ On the Śivadharma's Lalitavistara, see De Simini \& Mirnig 2017.

${ }^{124}$ The latter section is lacking in the Bhavisyapurāna parallel as well. 
manuscript, containing all eight texts traditionally associated with the Sivadharma in the Nepalese tradition. The manuscript is dated 1139-40 CE, in the colophon of the Dharmaputrikā. Although it may be an old manuscript, the text shows many scribal errors. Of all the manuscripts used, $\mathrm{N}_{45}^{c}$ contains the most trivial errors, as a quick glance at the apparatus reveals. ${ }^{125}$ Its position in the transmission of the text is not easy to identify. Together with the four other early Nepalese palm-leaf manuscripts used $\left(\mathrm{N}_{77}^{K o}, \mathrm{~N}_{82}^{K}, \mathrm{~N}_{12}^{K}, \mathrm{~N}_{58}^{K}\right)$ it lacks the significant addition after $27 \mathrm{~d}$ discussed below. However, along with the non-Nepalese manuscripts used $\left(\hat{\mathrm{S}}_{67}^{s}, \mathrm{P}_{3^{2}}^{T}\right.$ and $\left.\mathrm{P}_{72}^{T}\right)$ it lacks the two verses on the goddess Lamboșthī (48-49). ${ }^{126}$ The manuscript has a few accidental omissions as well: 9 b-1od; $14 \mathrm{ab}$ (with $\hat{\mathrm{S}}_{67}^{S}$ and $\mathrm{P}_{72}^{T}$ ); 86ab; 240ad; 244ef (with $\hat{S}_{67}^{S}$ ). There are also several additions. It adds a significant passage of 14 pādas after $213 \mathrm{~d}$, which is only partly shared in content, but not in wording, with $\mathrm{N}_{58}^{K}$ (18 pādas), $\mathrm{B}_{99}^{c}$ (22 pādas by a later hand), $\mathrm{E}^{N}$ (4 and 14 pādas) and $\hat{S}_{67}^{s}$ (18 pādas). After $238 \mathrm{~d}$ it adds four pādas that are uniquely shared with $\hat{S}_{67}^{s}$. It also has a number of additions of its own: two pādas after 132b; two pādas after 132d; eight pādas after 136d; two pādas after 147d; two pādas after 149b; two pādas after 151d; six pādas after 153d. The large number of unique additions indicates that, although $\mathrm{N}_{45}^{c}$ may be an early manuscript, it does not transmit a securely established early version of the text. On the level of individual readings, it is striking that a number of variants are shared with $\mathrm{P}_{32}^{T}$ and $\mathrm{P}_{72}^{T}$, but also, in other cases, with $\mathrm{N}_{77}^{K o}$ and $\hat{S}_{67}^{s}$.

$\mathrm{N}_{8_{2}}^{K}$, a manuscript from the National Archives Kathmandu dated 1069 $\mathrm{CE}$, is the second oldest manuscript used for this edition. It preserves a very good state of the text and it has been used as the principal source for the constitution of the text. Like $\mathrm{N}_{45}^{c}$ it contains all eight texts of the Sivadharma corpus. The manuscript is written in a very good hand with few scribal errors. It does not have any unique additions. The same holds true for $\mathrm{N}_{12}^{K}$, another very good early Nepalese palm-leaf manuscript from the National Archives in Kathmandu, containing

${ }^{125}$ I have tried to report the text of the manuscript as precisely as possible, but it should be noted that $\mathrm{N}_{45}^{c}$ does not always distinguish between śa, sa and șa, nor between $n a$ and $n a$.

${ }^{126}$ The special case of $\mathrm{B}_{99}^{c}$ will be discussed below. Unfortunately, because of the loss of a folio, $\mathrm{N}_{77}^{K o}$ is not available for this part of the text. 
all eight works belonging to the corpus. $\mathrm{N}_{12}^{K}$ is dated towards the end of the twelfth century (1194-95 CE). $\mathrm{N}_{82}^{K}$ and $\mathrm{N}_{12}^{K}$ have many readings in common, although both have readings of their own as well. ${ }^{127}$ In general the readings of $\mathrm{N}_{82}^{K}$ are closer to those of $\mathrm{E}^{N}$, which appears to be a transcription of another Nepalese manuscript. The text of $\mathrm{N}_{82}^{K}$ is remarkably close to $\mathrm{B}_{99}^{c}$. A passage for which the manuscript support is limited is $32 \mathrm{bc}$, two pādas that are only found in $\mathrm{B}_{99}^{c}, \mathrm{~N}_{82}^{K}, \mathrm{~N}_{58}^{K}$ and $\mathrm{E}^{N}$. It is quite possible that these two pādas were dropped at an early stage of the transmission of the text due to eye-skip. Another crucial passage is 120-121, the invocation to Ketu: these eight pādas are found in $\mathrm{N}_{82}^{K}$ and supported by $\mathrm{N}_{45}^{c}, \mathrm{~N}_{58}^{K}, \mathrm{~B}_{99}^{c}$ and $\mathrm{E}^{N}$. Instead of this, $\mathrm{N}_{12}^{K}$ has four different pādas that are partly shared with $\hat{S}_{67}^{S}, \mathrm{P}_{32}^{T}$ and $\mathrm{P}_{72}^{T}$. Significantly, these are precisely the two pādas missing in $\mathrm{N}_{77}^{K o}$, as discussed above. Could this indicate the introduction of Ketu at the end of the Navagraha list at two independent moments in time? The omission of $142 \mathrm{ab}$ in $\mathrm{N}_{12}^{K}$, shared with $\mathrm{N}_{77}^{K o}, \hat{\mathrm{S}}_{67}^{S}, \mathrm{P}_{3^{2}}^{T}$ and $\mathrm{P}_{72}^{T}$, suggests some shared stage of transmission of the text of these manuscripts. $\mathrm{N}_{12}^{K}$ has one unique omission: $209 \mathrm{~d}-210 a$. A significant passage for assessing the position of $\mathrm{N}_{82}^{K}$ is the addition of two pādas after 206b in all manuscripts except for $\mathrm{B}_{99}^{c}$ and $\mathrm{N}_{82}^{K}$. It provides important evidence for their close relation and their unique status vis-à-vis the other manuscripts.

$\mathrm{N}_{58}^{K}$, a manuscript from the National Archives Kathmandu dated 1402 $\mathrm{CE}$, takes up a special place in the edition, for it is the only one that contains the text of the Śantyadhyāya isolated from the rest of the Sivadharma. As such it provides important evidence for the independent transmission of the Śāntyadhyāya. Being dated more than 200 years after the other Nepalese palm-leaf manuscripts used, it also attests to a more developed form of the text. It has only two omissions within the main text: 103ad (with $\hat{S}_{67}^{s}$ ) and 125. The latter omission is preceded by a six-pāda verse (124) that forms a complete rewriting of the four-pāda verse found in the rest of the manuscripts. The manuscript contains the following passages for which it has already been shown that support of the manuscripts is comparatively limited: $14 \mathrm{ab}$ (with $\mathrm{N}_{82}^{K}, \mathrm{~N}_{12}^{K}, \mathrm{E}^{N}, \mathrm{~B}_{99}^{c}$ );

${ }^{127}$ De Simini 2017 reaches a very different conclusion regarding the position of $\mathrm{N}_{12}^{K}$ in the transmission of ŚiDhŚ 12 . In her analysis, 'this manuscript stands out as an exception among the Nepalese tradition' (p. 516). This is not corroborated by the evidence of ŚiDhŚ 6 . 
$32 \mathrm{bc}$ (with $\mathrm{N}_{82}^{K}, \mathrm{~B}_{99}^{c}, \mathrm{E}^{N}$ ); 48-49 (with $\mathrm{N}_{82}^{K}, \mathrm{~N}_{12}^{K}, \mathrm{E}^{N}, \mathrm{~B}_{99}^{c}$ ); 142ab (with $\mathrm{N}_{45}^{c}$, $\mathrm{N}_{82}^{K}, \mathrm{E}^{N}, \mathrm{~B}_{99}^{c}$ ); 242ad (with $\mathrm{N}_{45}^{c}, \mathrm{~N}_{82}^{K}, \mathrm{~N}_{12}^{K}, \mathrm{~B}_{99}^{c}, \mathrm{E}^{N}$ ). The manuscript has a large number of additions, including a number of unique ones: two pādas after 13 b; 34 pādas after $32 \mathrm{~d}$ (partly shared with $\mathrm{E}^{N}$ and $\hat{S}_{67}^{s}$ ); two pādas after 47a (partly shared with $\mathrm{P}_{32}^{T}, \mathrm{P}_{7^{2}}^{T}$ ); two pādas after $76 \mathrm{~d}$; two pādas after 101b; two pādas after 102d; 20 pādas after 104d (with $\hat{S}_{67}^{s}$ ); two pādas after 11od; two pādas after 113d ( with $\mathrm{P}_{32}^{T}, \mathrm{P}_{72}^{T}$ ); eight pādas after 122d; two pādas after $149 \mathrm{~b}$ (different from addition in $\mathrm{N}_{45}^{C}, \mathrm{P}_{32}^{T}, \mathrm{P}_{72}^{T}$ ); six pādas after 151f; four pādas after 191d (partly shared with Ś); eight pādas after 192d (last four pādas shared with $\hat{S}_{67}^{s}, \mathrm{P}_{32}^{T}, \mathrm{P}_{7^{2}}^{T}$ ); two pādas after 194d; two pādas after $206 \mathrm{~b}$ (shared with all except $\mathrm{N}_{82}^{K}, \mathrm{~B}_{99}^{c}$ ); 18 pādas after 213d (parts are shared with $\mathrm{N}_{45}^{c}, \mathrm{~B}_{99}^{c_{p c}}, \mathrm{E}^{N}, \hat{\mathrm{S}}_{67}^{S}$ ), and four pādas after 229d. Like $\mathrm{P}_{3^{2}}^{T}$ and $\mathrm{P}_{72}^{T}$ discussed below, it places $41 \mathrm{a}-42 \mathrm{~d}$ after $44 \mathrm{~d}$. In general the manuscript preserves many good readings and it provides us with a glimpse of the open and fluid transmission of the text in Nepal.

$\mathrm{E}^{N}$ stands for the printed text of the Sivadharma by Yogī Naraharinātha. Published in Kathmandu under the title of Paśupatimata, this is a somewhat curious publication, partly typed, partly handwritten and provided with a partial commentary. No mention is made of the manuscript used and no variants are reported. According to the information kindly provided by Diwakar Acharya (personal communication), it may have been based on a Devanāgarī manuscript from the time of Raṇa Bahādur Shah (1775-1806).Its readings are often in remarkable agreement with those of $\mathrm{B}_{99}^{c}$ and $\mathrm{N}_{82}^{K}$, to a lesser extent with $\mathrm{N}_{12}^{K}$. There are a number of cases where $\mathrm{B}_{99}^{c}, \mathrm{~N}_{82}^{K}$ and $\mathrm{E}^{N}$, sometimes backed up by $\mathrm{N}_{58}^{K}$, are in agreement against all the other manuscripts consulted. Attention has already been drawn above to $32 \mathrm{bc}$, two pādas uniquely found in $\mathrm{B}_{99}^{c}, \mathrm{~N}_{82}^{K}, \mathrm{~N}_{5^{8}}^{K}$ and $\mathrm{E}^{N}$. Furthermore, the significant verses $48-49$ are uniquely shared by $\mathrm{B}_{99}^{c}$, $\mathrm{N}_{82}^{K}, \mathrm{~N}_{12}^{K}, \mathrm{~N}_{58}^{K}$ and $\mathrm{E}^{N}$, while 12O-121 are found only in $\mathrm{B}_{99}^{c}, \mathrm{~N}_{82}^{K}, \mathrm{~N}_{5^{8}}^{K}$ and $\mathrm{E}^{N}$. The above cases indicate that the manuscript used by $\mathrm{E}^{N}$ transmits a version of the text closely related to that of $\mathrm{B}_{99}^{c}$ and $\mathrm{N}_{82}^{K}$. This conclusion is also borne out by the number of unique readings shared by $\mathrm{B}_{99}^{c}, \mathrm{~N}_{82}^{K}$ and $\mathrm{E}^{N}$. A telling example is 109c, where only $\mathrm{B}_{99}^{C}, \mathrm{~N}_{82}^{K}$ and $\mathrm{E}^{N}$ have the reading rudrasutāparājita, while $\mathrm{N}_{77}^{K o}, \mathrm{~N}_{45}^{C}, \mathrm{~N}_{12}^{K}, \mathrm{~N}_{58}^{K}$ and $\hat{S}_{67}^{s}$ share the hypermetrical variant aparājitā rudraratā and $\mathrm{P}_{3^{2}}^{T}$ and $\mathrm{P}_{7^{2}}^{T}$ have (a)parājitā śive bhaktā. While this might suggest that the text in $\mathrm{E}^{N}$ represents an old form of the text, such a conclusion is invalidated by several additions found in $\mathrm{E}^{N}$, 
some of which are shared by other manuscripts and some of which are unique. It is also conceivable that Naraharinātha was in fact using more than one manuscript and conflated the text. The additions found in $\mathrm{E}^{N}$ are as follows: 12 pādas after $27 \mathrm{~d}$ (partly shared with $\hat{S}_{67}^{s}, \mathrm{P}_{3^{2}}^{T}$ and $\mathrm{P}_{7^{2}}^{T}$ ); 30 pādas after $32 \mathrm{~d}$ (partly shared with $\mathrm{N}_{58}^{K}$ and $\hat{S}_{67}^{s}$ ); two pādas after 91b; four pādas after 105d; four pādas after 121d; four pādas after 213 d and fourteen pādas after 214d (partly parallel with $\mathrm{N}_{45}^{C}, \mathrm{~N}_{58}^{K}, \mathrm{~B}_{99}^{C_{\mathrm{pc}}}$ and $\hat{S}_{67}^{s}$, which add, respectively, 14, 18, 22 and 18 pādas after 213d); two pādas after 217d (with $\left.\mathrm{N}_{77}^{K o}, \mathrm{P}_{32}^{T}, \mathrm{P}_{72}^{T}\right)$. The fact that some of these additions are shared with other manuscripts, in particular $\hat{S}_{67}^{s}$, indicates that Nariharinātha did not compose them himself, but must have found them in his manuscript(s). $\mathrm{E}^{N}$ omits the following pādas: 5ocd; 58a-59d; 94cd; 174cd; 199a-20od; 212ab. These are all unique and most probably accidental omissions.

\section{The Transmission in Bengal}

The transmission in Bengal is represented here by a single manuscript in the collection of the University Library of Cambridge $\left(\mathrm{B}_{99}^{c}\right){ }^{128}$ The manuscript is written in Bengali script on yellow dyed paper and is dated Śāka Samvat 1604 (1682 CE). It is complete and contains the text of the first two works of the corpus, the Śivadharmaśāstra and the Śivadharmottara. Its readings are very good and, as already indicated above, overall very close to those of $\mathrm{N}_{8_{2}}^{K}$. In this connection it is relevant to note that the manuscript was in fact bought in Nepal (by D. Wright in 1873-76). ${ }^{129}$

For a start, all the pādas present in $\mathrm{N}_{82}^{K}$ are shared with $\mathrm{B}_{99}^{\mathrm{C}}$. The only exception is 227a-229d, but these pādas have been added later in the margin. For the rest not a single pāda is missing. The same observation holds true for those pādas, such as 32bc, 48-49 and 120a-121d noted

${ }^{128}$ The only other known manuscript in Bengali script is in the collection of the Asiatic Society of Bengal: G 9967, described in Shāstrī 1928, 714-715. It is a manuscript of the Sāntyadhyāya alone and is dated Saaka 1563 (1641/42 CE). I do not have access to it, but from Shāstrī's brief transcription of the beginning and end of the manuscript it appears that its readings are overall quite close to $\mathrm{B}_{99}^{C}$. According to the line following the colophon it was written in the town of the Kāștamaṇdapa, i.e. Kathmandu, in Nepal: śāke ${ }_{15} 63$ śrāvaṇakrṣnadvādāśyām śanau nepāle kāștamaṇdapanagare svārtham alekhi śriraghudevaśarmmanetidik. This points to a link with the Nepalese transmission, as in the case of $B_{99}^{c}$.

${ }^{129}$ http://cudl.lib.cam.ac.uk/view/MS-ADD-01599/1 
above, for which support from other manuscripts is limited. Moreover, the omission of two additional pādas found in all other manuscripts used after 206b is shared only with $\mathrm{N}_{82}^{K}$. This points to a very close relation.

As for additional passages, $\mathrm{B}_{99}^{c}$ has only one additional passage compared to $\mathrm{N}_{82}^{K}$ - after 213d, partly shared with $\mathrm{N}_{45}^{c}, \mathrm{~N}_{58}^{K}, \mathrm{E}^{N}$ and $\hat{S}_{67}^{S}$ - but this is one that has been added by a later hand. For the rest $\mathrm{B}_{99}^{c}$ follows the text of $\mathrm{N}_{82}^{K}$ throughout.

On the level of individual readings the correspondences are also remarkable. Aside from the many readings where $\mathrm{B}_{99}^{C}$ and $\mathrm{N}_{82}^{K}$ are in agreement and supported by one or more other manuscripts, and in particular $\mathrm{E}^{N}$, there are several instances where both manuscripts uniquely share or point to the same reading. Some examples of uniquely shared readings are the following: 40a, 45a, 104b, 113c, 186c (not adopted), 193d (not adopted), 20oa (not adopted), 2oob (not adopted) and 242d. On the other hand, $\mathrm{B}_{99}^{c}$ also has a few readings of its own, some of which are shared with other manuscripts: $108 \mathrm{~b}$ (with $\mathrm{N}_{58}^{K}, \mathrm{E}^{N}$ ), 112ab (with $\mathrm{N}_{45}^{c}, \mathrm{~N}_{12}^{K}$, $\mathrm{E}^{N}$ ), 121ab (eye-skip), 123b (with $\mathrm{N}_{12}^{K}, \hat{\mathrm{S}}_{67}^{S}, \mathrm{P}_{32}^{T}$ ), 124d (with $\mathrm{E}^{N}$ ), 144a (with $\mathrm{N}_{12}^{K}, \mathrm{P}_{32}^{T}, \mathrm{P}_{72}^{T}$ ), 164a, $185 \mathrm{c}$ (with $\mathrm{N}_{77}^{K o}, \mathrm{~N}_{58}^{K}, \mathrm{E}^{N}, \mathrm{~S}_{67}^{s}, \mathrm{P}_{32}^{T}, \mathrm{P}_{72}^{T}$ ), 21od. While it is conceivable that these have resulted from carelessness, the fact that some of these readings are shared with other manuscripts indicates that a different manuscript than $\mathrm{N}_{82}^{K}$ itself must have been its exemplar. That said, the text transmitted in $\mathrm{B}_{99}^{c}$ is remarkably close and must have been based on a Nepalese manuscript very similar to $\mathrm{N}_{82}^{K}$. Overall we can conclude that there is as yet no evidence for an independent Bengali recension of the text. ${ }^{130}$

\section{The Transmission in Kashmir}

$\hat{S}_{67}^{S}$, a manuscript in the collection of the Oriental Research Library in Srinagar, is one of three known Śāradā manuscripts of the Śivadharmaśâstra. ${ }^{131}$ It has many unique variants. Although it comes from the other

${ }^{130}$ On the other hand, the extensive use that the Devipurāna, by all accounts composed in Bengal, has made of the Śivadharmottara would suggest its spread to Bengal as well. See De Simini 2016b: 73-8o.

${ }^{131}$ The two other Śārada manuscripts known to me are: 1. ORL 913, Śivadharmacarita, in the Oriental Research Library of Srinagar; and 2. BHU 7/ 3986, Nandikeśvarasam hitā Śivadharmaśāstra, in the Benares Hindu University. 
end of the subcontinent, it has a remarkably large number of shared readings with the Southern manuscripts $\mathrm{P}_{32}^{T}$ and $\mathrm{P}_{72}^{T}$.

The additions in $\hat{S}_{67}^{s}$ are as follows: two pādas after $6 \mathrm{~d}$; two pādas after 18d; 16 pādas after $27 \mathrm{~d}$ (partly shared with $\mathrm{E}^{N}, \mathrm{P}_{3^{2}}^{T}$ and $\mathrm{P}_{7^{2}}^{T}$ ); 30 pādas after $32 \mathrm{~d}$ (partly shared with $\mathrm{N}_{58}^{K}$ and $\mathrm{E}^{N}$ ); two pādas after $36 \mathrm{~b}$; two pādas after $76 \mathrm{~d}$; 20 pādas after 104d; six pādas after 108d; 16 pādas after 151f; two pādas after 171b; four pādas after 191d (partly shared with $\mathrm{N}_{5^{8}}^{K}$ ); four pādas after 192d (with $\mathrm{P}_{3^{2}}^{T}$ and $\mathrm{P}_{72}^{T}$ ); 18 pādas after 213d (only partly parallel in content with $\mathrm{N}_{45}^{c}, \mathrm{~B}_{99}^{c}$ and $\mathrm{E}^{N}$ ); six pādas after $235 \mathrm{~d}$; four pādas after $238 \mathrm{~d}$ (with $\mathrm{N}_{45}^{c}$ ); four pādas after $241 \mathrm{~d}$ ( shared with $\mathrm{N}_{58}^{K}, \mathrm{P}_{32}^{T}$ and $\mathrm{P}_{72}^{T}$ ); two pādas after 256d (partly shared with $\mathrm{P}_{32}^{T}$ and $\mathrm{P}_{72}^{T}$ ).

As can be observed from this list, $\hat{S}_{67}^{s}$ has a large number of unique additions, indicating a separate line of transmission. Some additions are shared with other manuscripts, in particular $\mathrm{P}_{3^{2}}^{T}$ and $\mathrm{P}_{72}^{T}$. A few of them are, however, also shared with $\mathrm{N}_{45}^{c}, \mathrm{~N}_{58}^{K}$ and $\mathrm{E}^{N}$. Its omissions display a somewhat similar pattern: $14 \mathrm{ab}$ (with $\mathrm{N}_{77}^{K o}, \mathrm{~N}_{45}^{c}$ and $\mathrm{P}_{72}^{T}$ ); $25 \mathrm{~cd} ; 32 \mathrm{bc}$ (with $\mathrm{N}_{77}^{K o}, \mathrm{~N}_{45}^{C}, \mathrm{~N}_{12}^{K}, \mathrm{P}_{32}^{T}, \mathrm{P}_{72}^{T}$ ); 48a-49d (with $\mathrm{N}_{45}^{C}, \mathrm{P}_{32}^{T}, \mathrm{P}_{72}^{T}$ ); 5oc-52b; 57ab; 71a-72d; 8oab; 103ad (with $\mathrm{N}_{58}^{K}$ ); 142ab (with $\mathrm{N}_{77}^{K o}, \mathrm{~N}_{12}^{K}, \hat{S}_{67}^{S}, \mathrm{P}_{32}^{T}, \mathrm{P}_{72}^{T}$ ); 145cd; 154ad; 156ad (with $\mathrm{P}_{7^{2}}^{T}$ ); 158a-159b; 166cd; 172cd; 197ad (with $\mathrm{P}_{7^{2}}^{T}$ ); $210 a b$ (with $\mathrm{N}_{77}^{K o}, \mathrm{P}_{3^{2}}^{T}, \mathrm{P}_{7^{2}}^{T}$ ); 220cd; 242ad (with $\mathrm{N}_{77}^{K o}, \mathrm{P}_{72}^{T}$ ); 244ef (with $\mathrm{N}_{45}^{C}$ ). In three cases $\hat{S}_{67}^{s}$ reverses the order of the verses: $8 \mathrm{~cd}$ after $6 \mathrm{~d}$; $35 \mathrm{ab}$ after $35 \mathrm{~d}$; $240 \mathrm{Od}$ after $241 \mathrm{~d}$. The overall pattern suggests a contaminated transmission. ${ }^{12}$

\section{The Transmission in Southern India}

For the transmission of the text in the South, I have restricted myself to two paper transcripts from Pondicherry: $\mathrm{P}_{32}^{T}$ and $\mathrm{P}_{72}^{T}{ }^{1}{ }^{133}$ The text in both manuscripts is in relatively good shape. The two manuscripts clearly point to a Southern recension of the text. This is easily shown by comparison of shared additions, omissions and revisions. The same is also con-

\footnotetext{
${ }^{132}$ See also De Simini 2017: 525-527.

${ }^{133}$ The study of the Southern manuscripts of the Sivadharma is still in its infancy and the present observations must therefore be considered preliminary. Marco Franceschini (University of Bologna) is currently engaged in a study of the transmission of the manuscripts of the Śivadharmaśāstra and Śivadharmottara in the South. In his presentation, 'The transmission of the Sivadharma in Tamil Nadu', at the Sivadharma workshop at Leiden University (26-30 September 2016) he presented an overview of ca. 20 manuscripts (palm-leaf and paper) from South India. See also De Simini 2017: 516-520.
} 
firmed by the readings of the Malayalam manuscript of the Sivadharmavivarana, which shares several common features with $\mathrm{P}_{3^{2}}^{T}$ and $\mathrm{P}_{7^{2}}^{T}$ (see Appendix II).

The following shared additions are found in $\mathrm{P}_{3^{2}}^{T}$ and $\mathrm{P}_{7_{2}}^{T}$ : two pādas after $2 \mathrm{~d} ; 12$ respectively 14 pādas after $27 \mathrm{~d}$ (partly shared with $\mathrm{E}^{N}$ and $\hat{S}_{67}^{s}$ ); two pādas after 47e; two pādas after 47f; two pādas after 8ob; two, respectively four pādas after $84 \mathrm{~b} ; 12$ pādas in $\mathrm{P}_{3^{2}}^{T}$ after 102d, respectively 14 pādas in $\mathrm{P}_{72}^{T}$ after 103d; two pādas after 123d; two pādas after 127d; two pādas after 149b; four pādas after 185d; four pādas after 192d (with $\mathrm{N}_{58}^{K}, \hat{S}_{67}^{s}$ ); two pādas after $217 \mathrm{~d}$ ( with $\mathrm{N}_{77}^{K o}, \mathrm{E}^{N}$ ); four pādas after $235 \mathrm{~d}$; four pādas after 241d (partly parallel with $S_{67}^{S}$ ); four pādas after $256 \mathrm{~d}$ (partly parallel with $\hat{S}_{67}^{s}$ ). The readings in these additional passages show some variations, but overall they clearly reflect the same recension of the text.

The shared omissions are as follows: $32 \mathrm{bc}$ (with $\mathrm{N}_{77}^{K o}, \mathrm{~N}_{45}^{C}, \mathrm{~N}_{12}^{K}$ and $\hat{S}_{67}^{s}$ ); 48a-49d (with $\mathrm{N}_{45}^{c}$ and $\hat{S}_{67}^{s}$ ); 112ad; 129ab; 142ab (with $\mathrm{N}_{77}^{K o}, \mathrm{~N}_{12}^{K}$ and $\hat{S}_{67}^{s}$ ); 156ad; $210 \mathrm{ab}$ (with $\mathrm{N}_{77}^{K o}, \hat{S}_{67}^{s}$ ). The following textual revisions are found in both manuscripts: $41 \mathrm{a}-42 \mathrm{~d}$ is placed after $44 \mathrm{~d}$ (with $\mathrm{N}_{58}^{K}$ ); 120a-121d is replaced by four pādas (compare $\mathrm{N}_{12}^{K}$ and $\hat{S}_{67}^{s}$, but with different readings); $131 \mathrm{c}-13^{2} \mathrm{~d}$ is replaced by two pādas.

There can be little doubt about their common origin, but it is striking to note that several of these additions and omissions are shared with the Śāradā manuscript $\left(\hat{S}_{67}^{S}\right)$, and sometimes supported by $\mathrm{N}_{77}^{K o}$.

Aside from shared readings, both manuscripts also have additions and omissions of their own. In $\mathrm{P}_{3^{2}}^{T}$ the following additions occur: two pādas after 85d; two pādas after 96b; two pādas after 186d; two pādas after 23od; two pādas after 244f. The omissions in $\mathrm{P}_{32}^{T}$ are as follows: $15 \mathrm{ad} ; 87 \mathrm{a}-$ 89d; 108ad; 133c-134b; 165ad; 194cd; 223cd. Two passages are replaced: $159 \mathrm{c}-161 \mathrm{~b}$ follows after $162 \mathrm{~d}$ and 229a-23od is found before 223a. $\mathrm{P}_{72}^{T}$ has only one addition (six pādas after 159b) and the following omissions: $14 \mathrm{ab}$ (with $\mathrm{N}_{77}^{K o}$, $\mathrm{N}_{45}^{c}$ and $\hat{S}_{67}^{s}$ ); 22ad; 53cd; 59c-65b; 104ad; 148af; 197ad (with $\left.\hat{S}_{67}^{s}\right) ; 228 \mathrm{a}-229 \mathrm{~b} ; 242 \mathrm{ad}$ (with $\hat{S}_{67}^{s}$ ). Finally, 20a-27d is placed after the additional passage following $27 \mathrm{~d}$.

These unique features show that the readings of $\mathrm{P}_{3^{2}}^{T}$ and $\mathrm{P}_{7^{2}}^{T}$ do not derive from one and the same exemplar, but already reflect a longer history of transmission. A similar conclusion is borne out by comparison of the variations of readings on an individual level. $\mathrm{P}_{3^{2}}^{T}$ in particular shows 
a number of unique additions and alterations of the text. ${ }^{134}$

Compared to the other manuscripts used, I have been less strict in reporting the variants of $\mathrm{P}_{32}^{T}$ and $\mathrm{P}_{72}^{T}$. Thus, I have refrained from reporting ante correctionem readings and also the many cases where $\mathrm{P}_{32}^{T}$ and $\mathrm{P}_{72}^{T}$ apply final sandhi to the $p \bar{a} d a$ boundaries (-ạ for -o etc.).

\section{The Critical Apparatus}

The critical apparatus of the edition of the Śāntyadhyāya consists of three layers. The bottom layer is a positive apparatus, showing first the quoted portion of the reading adopted in the main text, followed by a lemmasign and the sources of the text that support the adopted reading, separated by a comma, after which are given the variants and their attestations. The order of listing of the sources is as follows: $\mathrm{N}_{77}^{K_{0}}, \mathrm{~N}_{45}^{c}, \mathrm{~N}_{82}^{K}, \mathrm{~N}_{12}^{K}$, $\mathrm{N}_{58}^{K}, S_{67}^{S}, \mathrm{P}_{32}^{T}, \mathrm{P}_{72}^{T}, \mathrm{E}^{N}$. To save space and make the apparatus more accessible to the reader, sigma-signs have been used to indicate where different manuscripts belonging to the same group share the same reading. The layer above the bottom layer reports omissions and additions in individual sources of one or more pādas in comparison to the main text of the edition. If there are different additional pādas, these are separated by a bullet. The same layer also occasionally reports the loss of folios in individual sources. The top layer is reserved for testimonia of the text. The symbols and abbreviations used in the apparatus are given below.

\section{Symbols and Abbreviations in the Apparatus}

$\Sigma$ Reading in all sources, with only one or two variants.

$\mathbf{K}^{\Sigma}$ Reading in all Kathmandu manuscripts.

$\mathbf{N}^{\Sigma}$ Reading in all Nepalese manuscripts.

$\mathbf{P}^{\Sigma}$ Reading in both Pondicherry transcripts.

$\sqcup$ Indicates a gap left by the scribe (if the gap is large a number is used to indicate number of syllables; e.g. $\left.\sqcup\left[-4^{-}\right] \sqcup\right)$.

${ }^{134}$ As mentioned above (n. 111 on p. $5^{2}$ ), the readings of $\mathrm{P}_{3^{2}}^{T}$ are remarkably close to the text of the Śivadharmaśāstra published in Jugnu \& Sharma 2014. 
- En-dashes indicate a lost or illegible syllable in the manuscript.

() Parentheses enclose syllables that are poorly legible in the manuscript.

++ Plus-signs enclose syllables that have been added in the manuscript.

<> Angular brackets enclose syllables that have been deleted in the manuscript.

- A bullet is used to separate lemmas within the same $p \bar{a} d a$.

$\begin{array}{llll}\text { conj. } & \text { conjecture } & \text { em. } & \text { emendation } \\ \text { ac } & \text { before correction } & \text { pc } & \text { after correction } \\ \text { om. } & \text { omit(s) } & \text { i.m. } & \text { in the margin } \\ \text { unmetr. } & \text { unmetrical } & \text { hypermetr. } & \text { hypermetrical }\end{array}$

For the sigla see pp. $5^{2}$ above. 


\title{
The Śāntyadhyāya
}

\author{
[Introduction]
}

nandikeśvara uvāca|

atah param idam guhyam rudrodgītam mahodayam| mahāvighnapraśamanaṃ mahāśāntikaraṃ śubham|| 1||

akālamṛtyuśamanaṃ sarvavyādhinivāraṇam| paracakrapramathanam sadāvijayavardhanam|| 2||

1-2 Cf. BhavP 1.175.14-15 : sarvapāpaharam puṇyạ̣ mahāvighnavināśanam| mahodayaṃ śāntikaraṃ lakṣahomavidhismṛtam|| apamṛtyuharaṃ vīra sarvavyādhiharaṃ param| paracakrapramathanaṃ sadāvijayavardhanam||

2d After this $\mathrm{P}_{32}^{T}$ adds two pādas : stambhanam parasainyasya ripusādakaraṃ sadā| $\mathrm{P}_{72}^{T}$ adds : stambhanaṃ ripusainyasyāpy aprasādakaraṃ sadā|

1 nandikeśvara uvāca ] $\mathrm{N}_{82}^{K} \mathrm{~N}_{12}^{K} \mathrm{E}^{N} \mathrm{~B}_{99}^{C}$, nandike - - - - $\mathrm{N}_{77}^{K o}$, om. $\mathrm{N}_{45}^{C}$, om namah śívāya| nandikeśvara uvāca $\mathrm{N}_{58}^{K}$, śrīnandikeśvaraḥ $\mathrm{P}_{3^{2}}^{T} \mathrm{P}_{72}^{T \text { pc }}$, śrīnandikeśvara uvāca $S_{67}^{S} \mathrm{P}_{72}^{T a c}$ 1 a rudrodgìtam ] $\mathrm{N}_{77}^{K o} \mathrm{~N}_{82}^{K} \mathrm{~N}_{12}^{K} \mathrm{~B}_{99}^{C} S_{67}^{S} \mathrm{P}_{72}^{T}$, rudrodgīta ${ }^{\circ} \mathrm{N}_{45}^{C} \mathrm{~N}_{58}^{K}$, rudrādgītaḥ $\mathrm{E}^{N}$, śivodgīṇaṃ $\mathrm{P}_{32}^{T} \quad$ 1c ${ }^{\circ}$ vighna $\left.{ }^{\circ}\right] \Sigma$, ovighnah $\mathrm{N}_{77}^{K o} \bullet{ }^{\circ}$ praśamanam ] $\Sigma$, ${ }^{\circ}$ prașamanam $\mathrm{N}_{82}^{K} \quad$ dd mahāo ${ }^{\circ} \Sigma$, sarva ${ }^{\circ} \hat{S}_{67}^{S} \quad$ 2a akālamṛtyuśamanam ] $\mathrm{N}_{77}^{K o} \mathrm{~N}_{82}^{K} \mathrm{~N}_{58}^{K} \mathrm{E}^{N} \hat{S}_{67}^{S} \mathrm{P}_{72}^{T}$, ākālamityupraśamanaṃ $\mathrm{N}_{45}^{C}$ (unmetr.), akālamṛtyusamanam $\mathrm{B}_{99}^{C}$, akālamṛtyumathanaṃ $\mathrm{N}_{12}^{K} \mathrm{P}_{32}^{T}$ 2b ' ${ }^{\circ}$ nivāraṇam ] $\Sigma$, ${ }^{\circ}$ vināśanam $S_{67}^{S} \quad \mathbf{2 c}^{2}$ para $\left.{ }^{\circ}\right] \Sigma$, prara ${ }^{\circ} \mathrm{N}_{77}^{K o}$, vara ${ }^{\circ} \mathrm{B}_{99}^{C} \bullet{ }^{\circ}$ cakrapramathanam ] $\mathrm{N}_{77}^{K o} \mathrm{~K}^{\Sigma} \mathrm{B}_{99}^{C} \mathrm{P}_{32}^{T}$, ${ }^{\circ}$ cakraprathamana $\mathrm{N}_{45}^{C}$, ${ }^{\circ}$ cakraprathamanam $\mathrm{E}^{N}$, oduḥkhapraśamanam $\hat{S}_{67}^{S}$, ${ }^{\circ}$ cakrapraśamanam $\mathrm{P}_{72}^{T} \quad$ 2d sadāvijaya $\left.{ }^{\circ}\right] \mathrm{N}_{77}^{K o} \mathrm{~N}_{45}^{C} \mathrm{~N}_{12}^{K} \mathrm{~N}_{58}^{K} \hat{S}_{67}^{S} \mathbf{P}^{\Sigma}$, sarvavijaya ${ }^{\circ} \mathrm{N}_{82}^{K}$, sarvvam vijaya ${ }^{\circ} \mathrm{B}_{99}^{C}$, sarvadā jaya ${ }^{\circ} \mathrm{E}^{N}$ 
sarvadevagrahānīkam samabhīṣțaphalapradam|

sarvaśāntyadhikārākhyaṃ dharmaṃ vakṣyāmi śâśvatam|| 3||

[Maheśvara]

śaśāñārdhadharas tryakṣo nāgayajñopavītakaḥ|

caturmukhaś caturbāhụ̣ sitabhasmāvaguṇțhitạ̣|| $4 \|$

varo vareṇyo varado devadevo maheśvarah|

trailokyanamitah śrīmān śāntim āśu karotu me|| 5\|

4-5 Cf. BhavP 1.176.6-8 : śaśikundendusaṃkāśo viśrutābharanair iha| caturbhujo mahātejāḥ puṣpārdhakṛtaśekharaḥ|| caturmukho bhasmadharạ̣ smaśānanilayaḥ sadā| gotrārir viśvanilayas tathā ca kratudūṣaṇaḥ|| varo vareṇyo varado devadevo maheśvaraḥ| ādityadehasaṃbhūtaḥ sa te śāntiṃ karotu vai||

3a ${ }^{\circ}$ grahānīkam ] $\Sigma$, ${ }^{\circ}$ grahānīka ${ }^{\circ} \mathrm{P}_{72}^{T}$, 'ograhāṇāṃ ca $\hat{S}_{67}^{S} \quad \mathbf{3}^{\mathbf{b}}$ samabhīșțaphalapradạ̣ ] $\mathrm{K}^{\Sigma} \mathrm{E}^{N} \mathrm{~B}_{99}^{C}$, samabhīṣtḥamaśoṣakam $\mathrm{N}_{77}^{K o}$, satabhīṣthamasāsakam $\mathrm{N}_{45}^{C}$, samabhīṣtāvataṃsakam $S_{67}^{S}$, sarvābhīṣtāivabhāsakam $\mathrm{P}_{32}^{T}$, samabhīṣțāvabhāsakam $\mathrm{P}_{72}^{T} \quad \mathbf{3} \mathbf{C}$ sarvaśāntya $\left.^{\circ}\right] \mathrm{N}_{82}^{K p c} \mathrm{~N}_{58}^{K} \mathrm{E}^{N} \hat{S}_{67}^{S} \mathbf{P}^{\Sigma}$, sarvaśāṃtyāo $\mathrm{N}_{77}^{K o}$, sarvvaśāntyāo $\mathrm{B}_{99}^{C}$, sarvaśantya ${ }^{\circ}$ $\mathrm{N}_{82}^{K a c}$, sarvaśāntyāo $\mathrm{N}_{12}^{K}$, sarvāh śāntyā $\overline{\mathrm{N}}_{45}^{C} \quad 3^{\mathrm{d}}$ dharmạ̣ ] $\Sigma$, dharma $\mathrm{N}_{45}^{C}$, dharmma $\mathrm{B}_{99}^{C} \bullet$ śáśvatạ̣ ] $\mathrm{K}^{\Sigma} \mathrm{E}^{N} \mathrm{~B}_{99}^{C} \hat{S}_{67}^{S} \mathrm{P}_{3^{2}}^{T},---\mathrm{N}_{77}^{K o}$, tattvatah $\mathrm{N}_{45}^{C} \mathrm{P}_{72}^{T} \quad$ 4b tryakṣo ] $\Sigma$, trā<khyo $>$ kșo $\mathrm{B}_{99}^{C} \bullet{ }^{\circ}$ pavītakah ] $\mathrm{N}_{82}^{K} \mathrm{~N}_{58}^{K} \mathrm{E}^{N} \mathrm{~B}_{99}^{C}$, ${ }^{\circ}$ pavìtadhṛk $\mathrm{N}_{77}^{K o}$, ${ }^{\circ}$ pravītuka $\mathrm{N}_{45}^{C}$, ${ }^{\circ}$ pavìtabhṛt $\mathrm{N}_{12}^{K}$, ${ }^{\circ}$ pavìtadhṛt $S_{67}^{S}$, ${ }^{\circ}$ pavìtavān $\mathbf{P}^{\Sigma}{ }^{4 \mathbf{c}}$ caturmukhaś ] $\Sigma$, caturmmukha ${ }^{\circ}$ $\mathrm{N}_{77}^{K o} \mathrm{~N}_{45}^{C}$, caturmukha ${ }^{\circ} \mathrm{N}_{58}^{K}$, caturmmukhyaś $\mathrm{B}_{99}^{C a c} \bullet$ caturbāhuh ] $\Sigma$, caturbāhu $\mathrm{N}_{77}^{K o} \mathrm{E}^{N}$ 4d 'ogunțhitah ] $\Sigma$, okuṇtitah $\mathrm{P}_{72}^{T} \quad \mathbf{5}^{\mathbf{a}}$ vareṇyo ] $\Sigma$, varetyam $\mathrm{N}_{45}^{C} \quad \mathbf{5}^{\mathbf{b}}$ maheśvarah ] $\Sigma$, maheśvaram $\mathrm{N}_{45}^{C} \quad{ }_{5} \mathrm{c}$ trailokyanamitah ] $\mathrm{N}_{77}^{K o} \mathrm{~K}^{\Sigma} \mathrm{B}_{99}^{C} \mathbf{P}^{\Sigma}$, trailokanamitah $\mathrm{N}_{45}^{C}$, trailokyanāthitah $\hat{S}_{67}^{S}$, trailokye namitah $\mathrm{E}^{N}$ 
[Umā]

sarvāvayavapūrṇena gātreṇa tanumadhyamā|

pītaśyāmātisaumyena snigdhavarṇena śobhanā|| 6||

lalāțatilakopetā candrarekhārdhadhārinī|
citrāmbaradharā devī sarvābharaṇabhūṣitā|| 7||

varastrīmayarūpeṇa śobhāguṇamahāspadā|

bhāvanāmātrasaṃtuștā umā devī varapradā|| 8||

sākṣād āgatya rūpeṇa śāntenāmitatejasā|

śāntiṃ karotu me prītā bhaktānāṃ bhaktivatsalā|| 9 ||

6-9 Cf. BhavP 1.177.22-25: sarvāvayavamukhyena gātreṇa ca sumadhyamā| pītaśyāmātisaumyena snigdhavarṇena śobhanā|| lalāṭatilakopetā candrarekhārdhadhārị̣ī| citrāmbaradharā devī sarvābharaṇabhūṣitā|| varā strīmayarūpāṇāṃ śobhāguṇasusampadā| bhāvanāmātrasaṃtuștā umā devī varapradā|| sākṣād āgatya rūpeṇa śāntenāmitatejasā| śāntiṃ karotu te prītā ādityārādhane ratā||

$8 \mathbf{c d ~} \hat{S}_{67}^{S}$ has these two pādas after $6 c d \quad 9 b$-1od Instead of these seven pādas $\mathrm{N}_{45}^{c}$ has sarvaktraśikhivāhanạ̣ (sic!)

6a ${ }^{\circ}$ pūrṇena ] $\mathrm{N}_{45}^{C} \mathrm{~K}^{\Sigma} \mathrm{E}^{N} \mathrm{~B}_{99}^{c}$, ${ }^{\circ}$ mukhyeṇa $\mathrm{N}_{77}^{K o}$, ${ }^{\circ}$ mukhyena $\hat{S}_{67}^{S} \mathbf{P}^{\Sigma} \quad 6 \mathbf{b}$ tanu $\left.{ }^{\circ}\right] \Sigma$, tu $\mathrm{N}_{77}^{K o}$ (unmetr.) 6c 'óyāmātisaumyena ] $\Sigma$, 'śyāmyādisaumyena $\mathrm{B}_{99}^{C}$, 'śyāmena dehena $S_{67}^{S} \quad 6 d$ snigdhavarṇena śobhanā ] $\mathrm{N}_{45}^{C} \mathrm{~N}_{82}^{K} \mathrm{~N}_{12}^{K} \mathrm{~N}_{58}^{K p c} E^{N} \mathrm{~B}_{99}^{C}$, sni(gdha)varṇṇena śobhitā $\mathrm{N}_{77}^{K o}$, snigdhavarṇe+na+ śobhanā $\mathrm{N}_{58}^{K}$, snigdhavarṇena sarvadā $S_{67}^{S}$, śivarūpeṇa sevitah $\mathrm{P}_{3^{2}}^{T}$, śivarūpeṇa śobhitā $\mathrm{P}_{72}^{T} \quad 7$ a lalāța $\left.{ }^{\circ}\right] \Sigma,---\mathrm{N}_{77}^{K o}$, lalāțe $\mathrm{E}^{N} \quad 7$ b candrarekhāo ] $\Sigma$, citralekhāo ${ }^{\circ} \mathrm{N}_{77}^{K o} \hat{S}_{67}^{S}$, candralekhāo $\mathrm{P}_{32}^{T} \quad 7 \mathrm{c}$ citrāmbara $\left.{ }^{\circ}\right] \mathbf{K}^{\Sigma} \mathrm{B}_{99}^{C} \hat{S}_{67}^{S}$, divyā$\operatorname{mbara}^{\circ} \mathrm{N}_{77}^{K o} \mathbf{P}^{\Sigma}$, cintrambara ${ }^{\circ} \mathrm{N}_{45}^{C}$, citrambara ${ }^{\circ} \mathrm{E}^{N} \quad 8 \mathbf{a}^{\circ}$ mayarūpena ] $\mathbf{K}^{\Sigma} \mathrm{E}^{N} \mathrm{~B}_{99}^{C} \hat{S}_{67}^{S}$, sarvarūpāṇāṃ $\mathrm{N}_{77}^{K o} \mathrm{~N}_{45}^{C}$, sarvanārīṇāṃ $\mathbf{P}^{\Sigma} \quad 8$ b śobhāo ${ }^{\circ} \Sigma$, sabhau $S_{67}^{S}$, saubhā $\mathrm{N}_{45}^{C} \bullet$ ${ }^{\circ}$ mahāspadā ] $\Sigma$, ${ }^{\circ}$ mahaspadā $\mathrm{B}_{99}^{C a c},{ }^{\circ}$ mahāspadam $\mathrm{P}_{32}^{T} \quad 8 d$ umā devī ] $\Sigma$, devy umāśu $S_{67}^{S}$, umā devi $\mathrm{E}^{N} \quad$ 9a sākșād ] $\Sigma$, sākhyād $\mathrm{N}_{45}^{C} \bullet$ āgatya ] $\Sigma$, āgamya $\mathrm{P}_{32}^{T} \quad \mathbf{9 c}$ prītā ] $\mathrm{N}_{77}^{K o} \mathrm{~K}^{\Sigma} \mathrm{E}^{N} \mathrm{~B}_{99}^{C} \mathbf{P}^{\Sigma}$, bhaktyā $\left.S_{67}^{S} \quad \mathbf{g d ~ b h a k t i}^{\circ}\right] \mathrm{N}_{77}^{K o} \mathrm{~N}_{12}^{K} \mathrm{~N}_{58}^{K} \mathrm{~B}_{99}^{C}$, vakti ${ }^{\circ} \mathrm{N}_{82}^{K}$, bha$\mathrm{kta}^{\circ} \mathrm{E}^{N} \hat{\mathrm{S}}_{67}^{S} \mathbf{P}^{\Sigma}$ 


\section{[Kārttikeya]}

padmarāgadyutiḥ saumyo raktamālyānulepanaḥ| abālo bālarūpeṇa șaḍvaktraḥ śikhivāhanaḥ|| 10||

pūrṇenduvadanaḥ saumyas triśikhah śaktisaṃyutaḥ| kṛttikomāgnirudrāngasamudbhūtaḥ surārcitaḥ|| 11||

kārttikeyo mahātejā varadānaikatatparaḥ| śāntiṃ karotu me nityaṃ balaṃ saukhyaṃ ca me sadā|| 12||

\section{[Nandīśa]}

śvetavastraparīdhānas tryakṣaḥ kanakasuprabhah| śūlapāṇir mahāprājño nandīśạ̣ śivabhāvitaḥ|| 13||

10-12 Cf. BhavP 1.178.1-3ab : abalo bālarūpeṇa khațvāngaśikhivāhanaḥ| pūrveṇa vadanah śrīmāṃs triśikhaḥ śaktisaṃyutaḥ|| kṛttikāyāś ca rudrasya cāngodbhūtah surārcitaḥ| kārttikeyo mahātejā ādityavaradarpitaḥ| śāntiṃ karotu te nityaṃ balaṃ saukhyaṃ ca tejasā|| ātreyī balavān deva ārogyaṃ ca khagādhipa| $\quad$ 13-14 Cf. BhavP 1.178.3cd-5ab : śvetavastraparīdhānas tryakṣaḥ kanakasuprabhaḥ|| śūlahasto mahāprājño nandīśo ravibhāvitaḥ| śāntiṃ karotu te śānto dharme ca matim uttamām|| dharmetarāv ubhau nityam acalạ samprayacchatu|

13b After this $\mathrm{N}_{5^{8}}^{K}$ adds two pādas : rudrabhakto mahāyogī rudraikāhimānasaḥ (sic, unmetr.)

1oa ${ }^{\circ}$ rāgadyutih ] $\mathbf{K}^{\Sigma} \mathrm{E}^{N} \mathrm{~B}_{99}^{C} \hat{\mathrm{S}}_{67}^{S} \mathbf{P}^{\Sigma}$, ${ }^{\circ}$ rāga - - $\mathrm{N}_{77}^{K o} \quad$ 1ob ${ }^{\circ}$ mālyāo $] \mathrm{N}_{82}^{K} \mathrm{~N}_{12}^{K} \mathrm{E}^{N} \mathrm{~B}_{99}^{C} \dot{S}_{67}^{S} \mathbf{P}^{\Sigma}$, ${ }^{\circ}$ mālāo $\mathrm{N}_{77}^{K o} \quad$ lod șaḍvaktrah ] $\mathrm{N}_{12}^{K} \mathrm{~N}_{58}^{K} \mathrm{E}^{N} \mathrm{~B}_{99}^{C} \dot{S}_{67}^{S} \mathrm{P}_{72}^{T}$, șațaktra ${ }^{\circ} \mathrm{N}_{77}^{K o}$, șaḍvaktra $\mathrm{N}_{82}^{K}$, șaṇmukhaḥ $\mathrm{P}_{32}^{T} \bullet{ }^{\circ}$ vāhanaḥ ] $\mathbf{K}^{\Sigma} \mathrm{B}_{99}^{C} \hat{S}_{67}^{S} \mathbf{P}^{\Sigma}$, ${ }^{\circ}$ vāhana $\mathrm{E}^{N}$ na saumyas ] $\Sigma$, saumyās $\mathrm{N}_{77}^{K o}$, saumya $\mathrm{N}_{45}^{C}$, śrīmān $\mathrm{N}_{58}^{K}$, śrīmāms $\hat{S}_{67}^{S} \quad \mathbf{u b}$ triśikhah ] $\mathrm{N}_{45}^{C} \mathrm{~K}^{\Sigma} \mathrm{E}^{N} \mathrm{~B}_{99}^{C} \hat{\mathrm{S}}_{67}^{S}$, trúsikham $\mathrm{N}_{77}^{K o}$, triśikhā ${ }^{\circ} \mathrm{P}_{32}^{T}$, triśivāo ${ }^{\circ} \mathrm{P}_{72}^{T} \quad$ 11c kṛttikomāgni $\left.{ }^{\circ}\right] \Sigma$, kṛttikomagni ${ }^{\circ} \mathrm{N}_{45}^{C} \bullet{ }^{\circ}$ rudrānga $\left.{ }^{\circ}\right] \Sigma$, ${ }^{\circ}$ rudrāmśsa ${ }^{\circ} \mathrm{E}^{N}$ nd ${ }^{\circ}$ samudbhūtạ̣ ] $\mathrm{N}_{45}^{C} \mathrm{~N}_{12}^{K} \mathrm{~N}_{58}^{K} \mathrm{E}^{N} \mathrm{~B}_{99}^{C} \hat{S}_{67}^{S} \mathrm{P}_{72}^{T}$, osamudbhūta $\mathrm{N}_{77}^{K o}$, o sumudbhūtaḥ $\mathrm{N}_{82}^{K}$, ogamgodbhūtaḥ $\mathrm{P}_{32}^{T} \bullet$ surārcitaḥ ] $\Sigma$, surārccikaḥ $\mathrm{E}^{N}$, surānvitạ̣ $\mathrm{N}_{45}^{C}$ 12a ${ }^{\circ}$ tejā ] $\Sigma$, o tejo $\mathrm{N}_{45}^{C} \mathrm{E}^{N} \quad \mathbf{1 2 b}$ varadānaika $\left.{ }^{\circ}\right] \Sigma$, varadāneka ${ }^{\circ} \mathrm{N}_{82}^{K} \bullet{ }^{\circ}$ tatparaḥ ] $\Sigma$, ${ }^{\circ}$ tatparam $\mathrm{N}_{45}^{C} \quad$ 12c śāntiṃ ] $\Sigma$, śānti $\mathrm{N}_{77}^{K o} \mathrm{~N}_{45}^{C} \bullet$ me nityạ̣ ] $\mathrm{N}_{77}^{K o} \mathrm{~N}_{82}^{K} \mathrm{E}^{N} \mathrm{~B}_{99}^{C} \mathbf{P}^{\Sigma}$, me prītā $\mathrm{N}_{58}^{K}$, satatam $\mathrm{N}_{45}^{C} \mathrm{~N}_{12}^{K} \dot{S}_{67}^{S} \quad$ 12d balam ] $\Sigma$, bala ${ }^{\circ} \mathrm{N}_{77}^{K o} \mathrm{~N}_{45}^{C} \bullet$ me sadā ] $\Sigma$, sarvadā $\mathrm{P}_{32}^{T} \quad$ 13a ${ }^{\circ}$ parīdhānas ] $\Sigma$, ${ }^{\circ}$ parīdhāna $\mathrm{N}_{77}^{K o}$, ${ }^{\circ}$ parīdhānās $\mathrm{N}_{45}^{C} \quad \mathbf{1 3}$ b tryakṣah ] $\Sigma$, tyakṣaḥ $\mathrm{N}_{77}^{K o}$, tryākșyāḥ $\mathrm{N}_{45}^{C} \bullet$ kanaka $\left.{ }^{\circ}\right] \quad \Sigma$, kanakaḥ $\mathrm{N}_{12}^{K}$ (unmetr.) • ${ }^{\circ}$ suprabhaḥ $\mathrm{N}_{82}^{K} \mathrm{E}^{N} \mathrm{~B}_{99}^{C}$, ${ }^{\circ}$ saprabhaḥ $\mathrm{N}_{12}^{K} \mathrm{~N}_{58}^{K} S_{67}^{S} \mathbf{P}^{\Sigma}$, o prabhāḥ $\mathrm{N}_{77}^{K o}$ (unmetr.), ${ }^{\circ}$ saprabham $\mathrm{N}_{45}^{C} \quad$ 13c ${ }^{\circ}$ pāṇir ] $\Sigma$, ${ }^{\circ}$ pāṇi $\mathrm{N}_{77}^{K o} \mathrm{~N}_{45}^{C} \mathrm{~N}_{58}^{K} \mathrm{E}^{N} \bullet{ }^{\circ}$ prājño ] $\Sigma$, ${ }^{\circ}$ prajñā $\mathrm{N}_{45}^{C} \mathrm{~N}_{12}^{K} \quad$ 13d nandīśạ ] $\Sigma$, nandīśa $\mathrm{N}_{45}^{C}$, nadīśa $\mathrm{N}_{58}^{K} \bullet$ obhāvitạ̣ ] $\Sigma$, obhāvitāḥ $\mathrm{N}_{45}^{C}$ 
śivārcanaparo nityaṃ śivadhyānaikatatparaḥ|

śāntiṃ karotu me śānto dharme ca matim uttamām|| 14||

[Vināyaka]

mahodaro mahākāyaḥ snigdhāñjanacayacchaviḥ|

ekadaṃșțrotkațo devo gajavaktro mahābalaḥ|| 15||

nāgayajñopavītī ca nāgābharaṇabhūṣitaḥ|

sarvārthasaṃpadādhāro gạ̣ādhyakṣo varapradaḥ|| 16||

rudrasya tanayo devo nāyako 'tha vināyakaḥ|

karotu me mahāśāntiṃ karmasiddhị̣ ca me sadā|| 17||

15-17 Cf. BhavP 1.178.5cd-7 : mahodaro mahākāyaḥ snigdhāñjanasamaprabhaḥ|| ekadamșțtrotkațo devo gajavaktro mahābalạ̣| nāgayajñopavītena nānābharaṇabhūṣitạ̣|| sarvārthasampadoddhāro gaṇādhyakṣo varapradaḥ| bhīmasya tanayo devo nāyako 'tha vināyakaḥ| karotu te mahāśāntiṃ bhāskarārcanatatparạ̣||

14ab Omitted in $\mathrm{N}_{77}^{K o} \mathrm{~N}_{45}^{C} \dot{S}_{67}^{S} \mathrm{P}_{72}^{T} \quad$ 15a-d Omitted in $\mathrm{P}_{32}^{T}$

$14 \mathrm{ab}] \mathrm{N}_{82}^{K} \mathrm{~N}_{12}^{K} \mathrm{E}^{N} \mathrm{~B}_{99}^{C}$, śivadhyānaikaparamah śivabhaktiparāyaṇaḥ $\mathrm{P}_{3^{2}}^{T} \quad$ 14c śāntim ] $\Sigma$, śānti $\mathrm{N}_{77}^{K o} \mathrm{~N}_{45}^{C} \bullet$ śānto ] $\Sigma$, śānta $\mathrm{N}_{77}^{K o}$, nityạ̣ $S_{67}^{S}$ 14d uttamām ] $\mathrm{N}_{82}^{K \text { Kpc }} \mathrm{N}_{12}^{K} \mathrm{~N}_{58}^{K} \mathrm{~B}_{99}^{C} \hat{S}_{67}^{S} \mathbf{P}^{\Sigma}$, uttamam $\mathrm{N}_{77}^{K o} \mathrm{~N}_{45}^{C} \mathrm{~N}_{82}^{K a c} \mathrm{E}^{N} \quad \mathbf{1 5}^{\circ}$ o kāyah ] $\mathbf{K}^{\Sigma} \mathrm{E}^{N} \mathrm{~B}_{99}^{C} \dot{S}_{67}^{S} \mathrm{P}_{72}^{T}$, okāya $\mathrm{N}_{77}^{K o} \mathrm{~N}_{45}^{C} \quad{ }_{15} \mathbf{b}$ snigdhāñjana $\left.{ }^{\circ}\right] \mathrm{N}_{77}^{K o} \mathrm{~K}^{\Sigma} \mathrm{E}^{N} \mathrm{~B}_{99}^{C} S_{67}^{S} \mathrm{P}_{72}^{T}$, snigdhañjana ${ }^{\circ} \mathrm{N}_{45}^{C} \bullet{ }^{\circ}$ cayacchaviḥ ] $\mathrm{N}_{82}^{K} \mathrm{E}^{N} \mathrm{~B}_{99}^{C}$, ${ }^{\circ}$ samadyuti $\mathrm{N}_{77}^{K o}$, ${ }^{\circ}$ cayacchati $\mathrm{N}_{45}^{C}$, ${ }^{\circ}$ ca+ya+cchivih $\mathrm{N}_{12}^{K}$, ${ }^{\circ}$ cayadyutị̆ $\mathrm{N}_{58}^{K}$, ${ }^{\circ}$ nibhah śivah $S_{67}^{S},{ }^{\circ}$ samadyutiḥ $\mathrm{P}_{72}^{T} \quad{ }_{15} \mathbf{d}^{\circ}$ vaktro $\mathrm{N}_{45}^{C} \mathrm{~K}^{\Sigma} \mathrm{E}^{N} \mathrm{~B}_{99}^{C} \dot{S}_{67}^{S} \mathrm{P}_{72}^{T},{ }^{\circ}$ vaktrā $\mathrm{N}_{77}^{K o} \bullet$ obalạ̣ ] $\mathrm{N}_{77}^{K o} \mathrm{~K}^{\Sigma} \mathrm{E}^{N} \mathrm{~B}_{99}^{C} \dot{S}_{67}^{S} \mathrm{P}_{72}^{T}$, obalaṃ $\mathrm{N}_{45}^{C} \quad{ }^{\circ}$ 16a nāga $\left.{ }^{\circ}\right] \Sigma$, bhāga ${ }^{\circ} \mathrm{N}_{77}^{K o} \bullet{ }^{\circ}$ yajñopavītì ca ] $\mathrm{N}_{77}^{K o} \mathrm{~N}_{45}^{C} \mathrm{~N}_{82}^{K} \mathrm{~N}_{58}^{K} \mathrm{~B}_{99}^{C} \dot{S}_{67}^{S} \mathrm{P}_{32}^{T}$, ${ }^{\circ}$ yajñopavitena $\mathrm{N}_{12}^{K} \mathrm{P}_{72}^{T}$, ${ }^{\circ}$ yajñopavīti ca $\mathrm{E}^{N} \quad \mathbf{1 6 b}$ nāgābharaṇa $^{\circ}$ ] $\Sigma$, nānābharaṇa ${ }^{\circ} \mathrm{E}^{N} \bullet$ obhūṣitaḥ ] $\Sigma$, obhūsitāh $\mathrm{N}_{45}^{C} \quad \mathbf{1 6 c}^{\circ}{ }^{\circ}$ sampadāa ${ }^{\circ}$ ] $\Sigma$, ${ }^{\circ}$ sampradāo $\mathrm{N}_{45}^{C} \quad \mathbf{1 6 d}$ gaṇādhyakșo ] $\Sigma$, gaṇādākṣo $\mathrm{N}_{45}^{C} \bullet{ }^{\circ}$ pradạ̣ ] $\Sigma$, opradāḥ $\mathrm{N}_{77}^{K o}$, ${ }^{\circ}$ padāḥ $\mathrm{N}_{45}^{C} \quad 17$ b nāyako 'tha ] $\Sigma$, nāyakeśa ${ }^{\circ} \mathrm{N}_{58}^{K} \bullet$ vināyakah ] $\Sigma$, vināyakam $\mathrm{N}_{45}^{C} \quad{ }_{17}$ d karma $\left.^{\circ}\right] \Sigma$, mahāo $\mathrm{P}_{72}^{T} \bullet$ me sadā ] $\mathrm{N}_{77}^{K o} \mathrm{~N}_{82}^{K} \mathrm{~N}_{58}^{K} \mathrm{~B}_{99}^{C}$, sarvadā $\mathrm{N}_{45}^{C} \mathrm{~N}_{12}^{K} E^{N} S_{67}^{S} \mathbf{P}^{\Sigma}$ 
[Mahākāla]

indranīlanibhas tryakșo dīptaśūlāyudhodyataḥ|

raktāmbaradharaḥ śrīmān kṛ̣ṇāngo nāgabhūṣaṇaḥ|| 18||

pāpāpanodam atulam alakṣmīmalanāśanam|

karotu me mahāśāntiṃ mahākālo mahābalaḥ|| 19||

[Ambikā]

pītavastraparīdhānā kanyārūpā svalan̉kṛtā|

gaṇamātāmbikā tryakṣā puṇyā gaurī sureśvarī| 20||

18-19 Cf. BhavP1.178.8-9 : indranīlanibhas tryakṣo dīptaśūlāyudhodyataḥ| raktāmbaradharaḥ śrīmān kṛ̣̣nāngo nāgabhūṣaṇaḥ|| pāpāpanodam atulam alakṣyo malanāśanaḥ| karotu te mahāśāntiṃ prītaḥ prītena cetasā|| 20-21 Cf. BhavP 1.178.10-11 : varāmbaradharā kanyā nānālaṃkārabhūṣitā| tridaśānāṃ ca jananī puṇā lokanamaskṛtā|| sarvasiddhikarā devī prasādaparamāspadā| śāntiṃ karotu te mātā bhuvanasya khagādhipa||

18d After this $\hat{S}_{67}^{S}$ adds two pādas : mahākāyo mahātejā mahābalapararūpaḥ (sic, unmetr.) $\quad 20 a-27 \mathbf{d} \mathrm{P}_{72}^{T}$ has these verses after the additional passage quoted in the apparatus to $27 \mathrm{~d}$

18a indra $\left.^{\circ}\right] \Sigma$, mandra ${ }^{\circ} \mathrm{P}_{72}^{T} \bullet{ }^{\circ}$ nibhas ] $\Sigma$, onibhās $\mathrm{N}_{45}^{C} \bullet$ tryakṣo ] $\Sigma$, tryākṣo $\mathrm{N}_{45}^{C}$ 18b dīpta $\left.{ }^{\circ}\right] \Sigma$, dīptam $\mathrm{N}_{45}^{C} \bullet{ }^{\circ}$ śūlāyudhodyatah ] $\mathrm{N}_{82}^{K p c} \mathrm{~N}_{12}^{K} \mathrm{~N}_{58}^{K} \mathrm{E}^{N} \mathrm{~B}_{99}^{C} \dot{S}_{67}^{S} \mathbf{P}^{\Sigma}$, ósuulodyatāyudhaḥ $\mathrm{N}_{77}^{K o}$, ${ }^{\circ}$ suulāyunyadyatā $\mathrm{N}_{45}^{C}$, ${ }^{\circ}$ suulāyudhādyatạ̣ $\mathrm{N}_{82}^{K a c} \quad \mathbf{1 8 c}$ raktāmbaradharaḥ śrīmān ] $\mathrm{N}_{82}^{K} \mathrm{~N}_{12}^{K} \mathrm{E}^{N} \hat{S}_{67}^{S} \mathbf{P}^{\Sigma}$, raktāvaradharah śrīmām $\mathrm{N}_{77}^{K o}$, raktāmbaraḥ śrī $\mathrm{N}_{45}^{C}$ (unmetr.), raktāmbaradharaśrīmān $\mathrm{N}_{58}^{K}$, vākṣāṃvaradhavaḥ śrīmān $\mathrm{B}_{99}^{C} \quad \mathbf{1 8 d}$ kṛ̣̣nāñgo nāga $\left.{ }^{\circ}\right] \quad \Sigma$, kṛṣnāgoo nāga ${ }^{\circ} \mathrm{N}_{77}^{K o} \mathrm{~N}_{58}^{K}$, kṛṣnāñgoraga ${ }^{\circ} \mathrm{P}_{72}^{T} \bullet{ }^{\circ}$ bhūṣaṇaḥ ] $\Sigma$, obhūṣitāṃ $\mathrm{N}_{45}^{C}$, obhūșitaḥ $S_{67}^{S} \quad 19 a{ }^{\circ}$ nodam atulam ] $\Sigma$, ${ }^{\circ}$ nodanamalam $E^{N} \quad 19 b{ }^{\circ}$ malanāśanam ] $\mathrm{N}_{45}^{C} \mathrm{~K}^{\Sigma} \mathrm{E}^{N} \mathrm{~B}_{99}^{C} \mathrm{P}_{72}^{T},{ }^{\circ}$ malanāśakaḥ $\mathrm{N}_{77}^{K o}$, ${ }^{\circ}$ nāśanam param $\hat{S}_{67}^{S},{ }^{\circ}$ malanāśanaḥ $\mathrm{P}_{32}^{T} \quad{ }^{19 c}{ }^{\circ}$ śāntim ] $\Sigma$, ósānti $\mathrm{N}_{45}^{C}$, okālaḥ $\mathrm{P}_{32}^{T} \quad$ 19d mahākālo mahābalaḥ ] $\mathrm{N}_{77}^{K o} \mathrm{~K}^{\Sigma} \dot{\mathrm{E}}^{N} \mathrm{~B}_{99}^{C} \mathrm{P}_{72}^{T}$, pìtapītena cetasā $\mathrm{N}_{45}^{C}$, prītah prītena cetasā $S_{67}^{S}$, prītiṃ prīteṇa cetasā $\mathrm{P}_{3^{2}}^{T} \quad$ 2oa pìta $\left.{ }^{\circ}\right] \Sigma$, prīta ${ }^{\circ} \mathrm{N}_{45}^{C} \bullet{ }^{\circ}$ parīdhānā $\Sigma$, ${ }^{\circ}$ parīdhāna $\mathrm{N}_{77}^{K o} \mathrm{~N}_{82}^{K a c} \quad$ 2ob kanyāo ${ }^{\circ} \quad \Sigma$, kanya ${ }^{\circ} \mathrm{N}_{77}^{K o} \bullet$ svalaǹkṛtā ] $\mathrm{N}_{77}^{K o} \mathrm{~N}_{82}^{K} \mathrm{~N}_{12}^{K} \mathrm{E}^{N} \mathrm{~B}_{99}^{C} S_{67}^{S} \mathrm{P}_{32}^{T}$, stvalaṃkṛtā $\mathrm{N}_{45}^{C}$, tvalan̉kṛtā $\mathrm{N}_{58}^{K}$, hyalaṃkṛtā $\mathrm{P}_{72}^{T} \quad$ 2oc gaṇamātāmbikā ] $\Sigma$, gaṇamambikās $\mathrm{N}_{45}^{C}$ (unmetr.), gaṇamātāmbika $\mathrm{N}_{12}^{K} \quad$ 2od puṇyā gaurī sureśvarī ] $\mathrm{N}_{82}^{K} \mathrm{~N}_{58}^{K} \mathrm{~B}_{99}^{C} \dot{S}_{67}^{S} \mathrm{P}_{32}^{T}$, śāntigaurīśvareșvarī $\mathrm{N}_{77}^{K o}$, śāntā gaurīśvareśvarī $\mathrm{N}_{45}^{C}$, puṇyā gaurīśvareśvarī $\mathrm{N}_{12}^{K} \mathrm{E}^{N}$, puṇyā gaurī maheśvarī $\mathrm{P}_{72}^{T}$ 
sarvasiddhikarī devī prasādaparamā parā|

śāntiṃ karotu me mātā siddhiṃ cāśu prayacchatu|| 21||

[Mahāmahiṣamardan̄̄]

snigdhaśyāmena varṇena mahāmahiṣamardanī|

dhanucakrapraharaṇā khaḍgapaț̣isadhāriṇī|| 22||

ātarjanodyatakarā sarvopadravanāśanī|

sarvamaṅgalamātā me śivam ācaratu dhruvam|| 23\|

22-23 Cf. BhavP 1.178.12-13 : snigdhaśyāmena varṇena mahāmahiṣamardanī| dhanuścakrapraharaṇā khaḍgapațtiśadhāriṇi|| ātarjany āyatakarā sarvopadravanāśinī| śāntim karotu te durgā bhavānī ca śivā tathā||

22a-23d Omitted in $\mathrm{P}_{72}^{T}$

21a ${ }^{\circ}$ karī ] $\Sigma$, ${ }^{\circ}$ pradā $\mathrm{P}_{32}^{T},{ }^{\circ}$ karā $\mathrm{P}_{72}^{T} \quad 21$ b prasāda $\left.{ }^{\circ}\right] \Sigma$, prasādāo $\mathrm{N}_{45}^{C} \bullet$ parā ] $\mathrm{N}_{77}^{K o} \mathrm{~N}_{45}^{C} \mathrm{~N}_{82}^{K} \mathrm{E}^{N} \mathrm{~B}_{99}^{C} \hat{\mathrm{S}}_{67}^{S} \mathrm{P}_{72}^{T}$, padā $\mathrm{N}_{12}^{K}$, sadā $\mathrm{N}_{58}^{K}$, śivā $\mathrm{P}_{32}^{T} \quad$ 21c śāntim ] $\Sigma$, śānti $\mathrm{N}_{77}^{K o} \mathrm{~N}_{45}^{C} \bullet$ mātā ] $\Sigma$, prītā $\mathrm{N}_{58}^{K} \mathrm{P}_{32}^{T} \quad$ 21d siddhim cāśu ] $\mathrm{N}_{82}^{K} \mathrm{~N}_{12}^{K} \mathrm{~B}_{99}^{C} S_{67}^{S}$, siddhi cāśu $\mathrm{N}_{77}^{K o}$, siddhim cāstu $\mathrm{N}_{45}^{C}$, siddhiś cānu ${ }^{\circ} \mathrm{E}^{N}$, siddhim āśu $\mathrm{N}_{58}^{K} \mathbf{P}^{\Sigma}$ 22a varṇena ] $\mathrm{N}_{77}^{K o} \mathbf{K}^{\Sigma} \mathrm{E}^{N} \mathrm{~B}_{99}^{C} \dot{S}_{67}^{S} \mathrm{P}_{32}^{T}$, varṇṇenā $\mathrm{N}_{45}^{C} \quad$ 22b ${ }^{\circ}$ mardanī ] $\mathrm{N}_{77}^{K o} \mathrm{~K}^{\Sigma} \mathrm{B}_{99}^{C} \mathrm{P}_{32}^{T}$, ${ }^{\circ}$ varddhanī $\mathrm{N}_{45}^{C}$, ${ }^{\circ}$ marddanaṃ $\mathrm{E}^{N}$, ${ }^{\circ}$ mardinī $S_{67}^{S} \quad 22 c$ dhanucakra $\left.{ }^{\circ}\right] \mathrm{N}_{77}^{K o} \mathrm{~N}_{45}^{C} \mathrm{~N}_{82}^{K}$, dhanuścakra ${ }^{\circ} \mathrm{N}_{12}^{K} \mathrm{~N}_{58}^{K} \mathrm{E}^{N} \mathrm{~B}_{99}^{C} S_{67}^{S} \mathrm{P}_{3^{2}}^{T}$, ${ }^{\circ}$ praharaṇā ] $\mathrm{N}_{45}^{C} \mathrm{~N}_{82}^{K} \mathrm{~N}_{12}^{K} \mathrm{E}^{N} \mathrm{~B}_{99}^{C} \mathrm{P}_{32}^{T}$, ${ }^{\circ}$ praharaṇī $\mathrm{N}_{58}^{K} \hat{S}_{67}^{S} \quad$ 22d khaḍgapaț̣isadhāriṇī ] $\mathrm{N}_{77}^{K o} \mathrm{~N}_{82}^{K p c} \mathrm{~N}_{12}^{K} \mathrm{E}^{N} \mathrm{~B}_{99}^{C} S_{67}^{S}$, khaḍgapaț̣isadhāraṇi $\mathrm{N}_{45}^{C} \mathrm{~N}_{82}^{K \text { ac }} \mathrm{N}_{58}^{K}$, śaṃkhayaștyasidhāriṇī $\mathrm{P}_{32}^{T}$ 23a ātarjanodyata ${ }^{\circ}$ ] $\mathrm{N}_{77}^{K o} \mathrm{~N}_{45}^{C} \mathrm{~N}_{58}^{K} \mathrm{P}_{32}^{T}$, ātarjany udyata ${ }^{\circ} \mathrm{N}_{82}^{K} \mathrm{E}^{N} \mathrm{~B}_{99}^{C}$, ātarjjanyuta ${ }^{\circ} \mathrm{N}_{12}^{K}$ (unmetr.), ātarjanyudita ${ }^{\circ} S_{67}^{S}$ (unmetr.) $\quad 23 \mathbf{b}{ }^{\circ}$ nāśanī ] $\mathbf{N}^{\Sigma} \mathrm{E}^{N} \mathrm{~B}_{99}^{C}$, ${ }^{\circ}$ nāśinī $S_{67}^{S} \mathrm{P}_{32}^{T}$ 23c ${ }^{\circ}$ mātā ] $\mathrm{N}_{45}^{C} \mathrm{~N}_{82}^{K} \mathrm{~N}_{12}^{K} \mathrm{E}^{N} \mathrm{~B}_{99}^{C} \hat{S}_{67}^{S} \mathrm{P}_{32}^{T}$, ${ }^{\circ}$ matā $\mathrm{N}_{77}^{K o}$ (unmetr.) 23d ācaratu dhruvam ] $\mathrm{N}_{82}^{K} \mathrm{E}^{N} \mathrm{~B}_{99}^{C} \hat{S}_{67}^{S} \mathrm{P}_{32}^{T}$, āśu prayacchatu $\mathrm{N}_{77}^{K o} \mathrm{~N}_{45}^{C}$, āvahatu dhruvam $\mathrm{N}_{12}^{K} \mathrm{~N}_{58}^{K}$ 


\section{[Bhṛngiriți]}

nirmāṃsena śarīreṇa snāyvasthisunibandhanaḥ|

atisūkṣmo 'tivikrāntas tryakṣo bhṛngirițir mahān|| 24||

rudrātmajo mahāvīro rudraikāhitamānasaḥ|

so 'pi me śāntabhāvena śāntim āśu prayacchatu|| 25||

[Caṇ̣eśvara]

pracaṇụagaṇasainyeśo mahāṭañkākṣadhārakaḥ|

akṣamālārpitakaras tryakṣaś caṇḍeśvaro varaḥ|| 26||

24-25 Cf. BhavP 1.178.14: atisūkșmo hy atikrodhas tryakṣo bhṛngirițir mahān| sūryātmako mahāvīrạ̣ sūryaikagatamānasaḥ| sūryabhaktikaro nityạ̣ śivaṃ te samprayacchatu|| 26-27 Cf. BhavP 1.178.15-16 : pracaṃḍagaṇasainyeśo mahāghaṇtākṣadhārakaḥ| akṣamālārpitakaraś cākṣacaṇḍeśvaro varaḥ|| caṇḍapāpaharo nityạ̣ brahmahatyāvināśanaḥ| śāntiṃ karotu te nityam ādityārādhane rataḥ| karoti ca mahāyogī kalyāṇānāṃ paramparām||

25cd Omitted in $\hat{S}_{67}^{S}$

24a nirmāṃsena ] $\Sigma$, nirmāsena $\mathrm{N}_{77}^{K o} \mathrm{~N}_{58}^{K} \quad$ 24b snāyvasthisunibandhanaḥ ] $\mathrm{N}_{82}^{K} \mathrm{E}^{N} \mathrm{~B}_{99}^{C} \mathbf{P}^{\Sigma}$, snāyusthisunibandhanāḥ $\mathrm{N}_{77}^{K o}$, snāyvasthisvanibandhanạ̣ $\mathrm{N}_{45}^{C}$, snāyvasthibhṛnganidhvaniḥ $\mathrm{N}_{12}^{K}$, snāvyasthiṣunibaṃdhanạ̣ $\mathrm{N}_{58}^{K}$, snāyvaskṛsunibandhanaḥ $\hat{S}_{67}^{S} \quad 24 \mathrm{c}$ atisūkṣmo ] $\Sigma$, atisūkṣme $\mathrm{N}_{77}^{K o} \bullet$ 'tivikrāntas ] $\mathrm{N}_{45}^{C} \mathrm{~K}^{\Sigma} \mathrm{B}_{99}^{C} \mathrm{E}^{N} \mathrm{P}_{32}^{T}$, 'tivikrānta $\mathrm{N}_{77}^{K o}$, tiraktāngas $S_{67}^{S}$, tyatikrāntah $\mathrm{P}_{72}^{T} \quad$ 24d tryakșo ] $\mathrm{N}_{12}^{K} \mathrm{~N}_{58}^{K} \mathrm{E}^{N} \mathrm{~B}_{99}^{C} \hat{S}_{67}^{S} \mathrm{P}_{32}^{T}$, tryākṣo $\mathrm{N}_{45}^{C}$, tyakșo $\mathrm{N}_{77}^{K o} \mathrm{~N}_{82}^{K} \bullet$ bhṛngirițir ] $\mathrm{K}^{\Sigma} \mathrm{E}^{N} \mathrm{~B}_{99}^{C p c} \mathbf{P}^{\Sigma}$, bhṛngiriți $\mathrm{N}_{77}^{K o}$, bhingirițir $\mathrm{N}_{45}^{C}$, bhṛngi< ț̣i $>$ rițir $\mathrm{B}_{99}^{C}$, bhṛngagiți $\hat{S}_{67}^{S} \quad$ 25a rudrātmajo ] $\Sigma$, rudrātmaja $\mathrm{N}_{45}^{C} \bullet$ mahāvīro ] $\Sigma$, mahāvīryo $\mathrm{N}_{12}^{K}$, rudrabhakto $\mathrm{P}_{32}^{T} \quad 25$ b rudraikāhita $\left.{ }^{\circ}\right] \mathrm{N}_{82}^{K} \mathrm{~N}_{58}^{K} \mathrm{E}^{N} \mathrm{~B}_{99}^{C}$, rudraikagata $\mathrm{N}_{77}^{K o} \mathrm{~N}_{45}^{C} \mathrm{~N}_{12}^{K} \hat{S}_{67}^{S} \mathbf{P}^{\Sigma} \quad{ }_{25}$ c me śānta $\left.{ }^{\circ}\right] \mathbf{K}^{\Sigma} \mathrm{E}^{N} \mathrm{~B}_{99}^{C}$, me śānti ${ }^{\circ} \mathrm{N}_{77}^{K o} \mathrm{~N}_{45}^{C}$, śāntātma ${ }^{\circ} \mathbf{P}^{\Sigma} \quad{ }_{25} \mathbf{d}$ śāntim āśu ] $\mathrm{N}_{45}^{C} \mathbf{K}^{\Sigma} \mathrm{E}^{N} \mathrm{~B}_{99}^{C} \mathbf{P}^{\Sigma}$, śātiñcāśu $\mathrm{N}_{77}^{K o} \quad \mathbf{2 6 a}{ }^{\circ}$ sainyeśo ] $\Sigma$, ${ }^{\circ}$ sainyastho $\mathrm{P}_{32}^{T}$, ${ }^{\circ}$ sainyena $\mathrm{P}_{72}^{T} \quad \mathbf{2 6 b}{ }^{\circ}$ țañkākșadhārakah ] $\Sigma$, oṭaṃkṣadhārakaṃ $\mathrm{N}_{45}^{C}$ (unmetr.) $\quad \mathbf{2 6 c}$ ${ }^{\circ}$ mālārpita ${ }^{\circ}$ ] $\Sigma$, ${ }^{\circ}$ mālādhṛta ${ }^{\circ} \mathrm{P}_{72}^{T} \bullet{ }^{\circ}$ karas ] $\Sigma$, ${ }^{\circ}$ kara $\mathrm{N}_{45}^{C} \mathrm{E}^{N} \quad$ 26d tryakșaś caṇụeśvaro varah ] $\mathrm{K}^{\Sigma} \mathrm{E}^{N} \mathrm{~B}_{99}^{C}$, tyakșacạ̣dośvaro varam $\mathrm{N}_{45}^{C}$, tyakṣacaṇdeśvareśvarah $\mathrm{N}_{77}^{K o}$, trya(kṣa)ścaṇḍeśvaro gạ̣aḥ $S_{67}^{S}$, tryakṣaścaṇḍeśvarah prabhuh $\mathbf{P}^{\Sigma}$ 
caṇdapāpāpaharaṇo brahmahatyādiśodhanạ̣|
karotu me mahāyogī kalyāṇānāṃ paramparām|| 27||

[Brahmā]

padmāsanaḥ padmanibhaś caturvadanapaṅkajaḥ|

kamaṇḍaludharạ̣ śrīmān devagandharvapūjitạ̣|| 28||

28-29 Cf. BhavP 1.176.1-2 : padmāsanaḥ padmavarṇaḥ padmapatranibhekṣaṇaḥ| kamaṇḍaludharaḥ śrīmān devagandharvapūjitaḥ|| caturmukho devapatị̣ sūryārcanaparaḥ sadā| surajyeșṭho mahātejāḥ sarvalokaprajāpatị̣| brahmaśabdena divyena brahmā śāntiṃ karotu te\|

27d After this $\mathrm{E}^{N}$ adds 12 pādas : śan̉khakundendutulyābhah kaṇțe marakataprabhaḥ| akșamālī śivāgrasthạ̣ svasaṃjñena vyavasthitaḥ| caturmukhaś catuḥpādas trinetraḥ sarvadojjvalaḥ| kṣitipatir vṛṣo devo dharmo dharmatamottamaḥ| îśạ vahati pṛșṭhena tasmād dharmo jagatprabhuḥ| vṛṣo vṛṣavaraḥ śrīmān karotu mama śāntikam| • $\hat{S}_{67}^{S}$ adds 16 pādas : ghaṇțātañkārasamyukto ghaṇțānādo mahāpriyaḥ| paraśvasigadāhasta śūlāngaś ca dhanurdharạ̣| rudraśaktidharopetạ̣ sarvavyādhiharo haraḥ| ghaṇtākarṇo gaṇaḥ śrīmān karotu mama śāntikam| śan̉khakundendutulyābhaḥ kaṇṭhe raktaprabhạ̣ śubhaḥ| kṛ̣ṇạ̣ samukuṭah śrīmān svayaṃ viṣnur vyavasthitaḥ| caturmukhaś caturbāhuḥ pṛthuvakṣạ̣̄ kṣitî́śvaraḥ| śāntiṃ karotu me nityaṃ kalyāṇaṃ ca prayacchatu| - $\mathrm{P}_{3^{2}}^{T}$ adds 12 pādas : śaṃkhakundendutulyābhạ̣ kaṇṭhe marakataprabhạ̣| akṣamālāśirogras tu svayaṃ jñāne vyavasthitaḥ| caturmukhaś catuṣpādah triṇetraḥ sarvadojvalaḥ| kṣiteh patir varayo devaḥ sarvadharmottamottamaḥ| îśạ̣ vahati pṛșṭhena tasmād dharmo jagatprabhuḥ| vṛṣo vṛ̣apatih śrīmān karotu mama śāntikam $\mid \bullet \mathrm{P}_{72}^{T}$ adds 14 pādas (following from 19f!) : śankhakundenduvarābhaḥ kaṇțhe marakataprabhaḥ| akṣamālādharograś ca svayaṃ viṣnur iva sthitạ̣| caturmukhaś caturbāhụ̣ triṇetraḥ satatojvalaḥ| kṣityāḥ patir vṛșo dharmaḥ sarvadharmottamottamaḥ| íśạ̣ vahati pṛșthena tasmād dharmo jagatpatị̣| vṛṣo vṛṣadharaḥ śrīmān karotu mama śāntikam| sarvajñas sarvasadṛśaḥ sarvagaḥ śîrșajạ̣ prabhuḥ| śamanaś śatrusainyānāṃ śāstā saāntiṃ karotu me|

27a caṇḍapāpāpaharaṇo ] $\mathrm{N}_{82}^{K p c} \mathrm{~N}_{58}^{K} \mathrm{E}^{N} \mathbf{P}^{\Sigma}$, caṇḍapāpāpataraṇe $\mathrm{N}_{77}^{K o}$, caṇụapāpāpahāraṇaṃ $\mathrm{N}_{45}^{C}$, caṇḍapāpāpaharaṇe $\mathrm{N}_{82}^{K a c}$, caṇḍapāpāpraharaṇo $\mathrm{N}_{12}^{K} \mathrm{~B}_{99}^{C}$, prayacchanpāpaharaṇam $\hat{S}_{67}^{S} \quad \mathbf{2 7} \mathbf{b}$ ohatyādiśodhanah ] $\mathrm{K}^{\Sigma} \mathrm{E}^{N} \mathrm{~B}_{99}^{C} \mathrm{P}_{32}^{T}$, ohatyādiśodhakam $\mathrm{N}_{77}^{K o}$, otyādiśodhanam $\mathrm{N}_{45}^{C}$ (unmetr.), ' hatyādiśodhanam $S_{67}^{S}$, ${ }^{\circ}$ hatyādiśodhakah $\mathrm{P}_{72}^{T} \quad{ }_{27} \mathbf{c}{ }^{\circ}$ yogī ] $\Sigma$, ○śāntiṃ $S_{67}^{S} \quad$ 27d kalyāṇānāṃ paramparām ] $\mathrm{N}_{82}^{K \mathrm{pc}} \mathrm{N}_{12}^{K} \mathrm{E}^{N} \mathrm{~B}_{99}^{C} \dot{S}_{67}^{S}$, kalyāṇanparamampadam $\mathrm{N}_{45}^{C}$, kalyaṇānāṃ paraṃparāṃ $\mathrm{N}_{82}^{K a c}$, kalyānām paramạ̣ śubham $\mathrm{N}_{58}^{K}$, nityaṃ kalyāṇavardhanam $\mathrm{P}_{3^{2}}^{T}$, kalyāṇaṃ paramaṃ śubham $\mathrm{N}_{77}^{K o} \mathrm{P}_{72}^{T} \quad \mathbf{2 8 a}$ padmāsanaḥ padmanibhaś ] $\Sigma$, padmāsanapadmanibhaś $\mathrm{N}_{12}^{K a c}$ (unmetr.), padmāsano mahāpadmaś $\hat{S}_{67}^{S} \quad \mathbf{2 8 b}$ caturvadana ${ }^{\circ} \Sigma$, caturvvedena $\mathrm{B}_{99}^{C} \bullet{ }^{\circ}$ pañkajah ] $\Sigma$, ${ }^{\circ}$ pańkaja $\mathrm{N}_{45}^{C} \quad \mathbf{2 8 c}{ }^{\circ}$ dharạ̣ ] $\Sigma$, odhara $\mathrm{N}_{77}^{K o} \mathrm{~N}_{58}^{K}$, ${ }^{\circ}$ dharāṃ $\mathrm{N}_{45}^{C} \quad \mathbf{2 8 d}$ deva $^{\circ}$ ] $\Sigma$, siddha ${ }^{\circ} \mathrm{P}_{72}^{T}$ 
śivadhyānaikaparamạ̣ śivasadbhāvabhāvitaḥ|

brahmaśabdena divyena brahmā śāntiṃ karotu me|| 29\|

[Viṣṇu]

tārkṣyāsanaś caturbāhuḥ śan̉khacakragadādharaḥ|

śyāmaḥ pītāmbaradharo mahābalaparākramaḥ|| 30||

yajñadehottamo devo mādhavo madhusūdanaḥ|

śivaprasādasampannaḥ śivadhyānaparāyaṇaḥ|| 31||

30-32 Cf. BhavP 1.176.3-5 : pītāmbaradharo deva ātreyīdayitah sadā| śan̉khacakragadāpāṇiḥ śyāmavarṇaś caturbhujaḥ|| yajñadehạ̣ kramo deva ātreyīdayitaḥ sadā| śaṅkhacakragadāpāṇir mādhavo madhusūdanah || sūryabhaktānvito nityạ̣ vigatir vigatatrayaḥ| sūryadhyānaparo nityaṃ viṣṇuḥ śāntiṃ karotu te||

29a śiva $\left.{ }^{\circ}\right] \Sigma$, śive $\mathrm{E}^{N} \bullet{ }^{\circ}$ paramah ] $\mathrm{N}_{45}^{C} \mathrm{~N}_{82}^{K} \mathrm{~N}_{12}^{K} \mathrm{E}^{N} \mathrm{~B}_{99}^{C}$, ${ }^{\circ}$ manasa $\mathrm{N}_{77}^{K o}$, ${ }^{\circ}$ manasah $\mathrm{N}_{58}^{K}$, ${ }^{\circ}$ nirataḥ $\hat{S}_{67}^{S} \mathbf{P}^{\Sigma} \quad \mathbf{2 9 b}$ śivasadbhāvabhāvitah ] $\mathrm{N}_{77}^{K o} \mathbf{K}^{\Sigma} \mathrm{E}^{N} \hat{S}_{67}^{S} \mathrm{P}_{32}^{T}$, śivam sambhāvabhāvinaḥ $\mathrm{N}_{45}^{C}$, śivavadbhāvabhāvitạ̣ $\mathrm{B}_{99}^{C}$, śivasadbhāvakovidaḥ $\mathrm{P}_{72}^{T} \quad$ 29c divyena ] $\Sigma$, davyena $\mathrm{N}_{45}^{C} \quad$ 29d brahmā ] $\mathrm{N}_{82}^{K} \mathrm{~B}_{99}^{c} \hat{S}_{67}^{S} \mathbf{P}^{\Sigma}$, brahma $\mathrm{N}_{77}^{K o} \mathrm{~N}_{12}^{K} \mathrm{~N}_{58}^{K} \mathrm{E}^{N}$, brāhma ${ }^{\circ} \mathrm{N}_{45}^{C} \bullet$ śāntiṃ ] $\Sigma$, sānti $\mathrm{N}_{77}^{K o}$, śānti $\mathrm{N}_{45}^{C} \quad$ 3oa tārkṣyāsanaś ] $\mathbf{N}^{\Sigma} \mathbf{P}^{\Sigma}$, tārkṣāsanaś $\mathrm{E}^{N} \mathrm{~B}_{99}^{C}$, tārkṣyārūḍhaś $S_{67}^{S} \bullet{ }^{\circ}$ bāhuh ] $\Sigma$, obāhu $\mathrm{N}_{77}^{K o} \quad 3$ ob ${ }^{\circ}$ gadādharah ] $\Sigma$, ogajādhara $\mathrm{N}_{45}^{C} \quad$ 3oc śyāmaḥ ] $\Sigma$, śyāma ${ }^{\circ} \mathrm{N}_{77}^{K o} \bullet{ }^{\circ}$ radharo ] $\Sigma$, ${ }^{\circ}$ rādhāro $\mathrm{N}_{45}^{C} \quad$ 3od mahābalaparākramaḥ ] $\mathrm{K}^{\Sigma} \mathrm{E}^{N} \mathrm{~B}_{99}^{C} \hat{S}_{67}^{S}$, mahābalākramaḥ $\mathrm{N}_{77}^{K o}$ (unmetr.), mahābalaparākramām $\mathrm{N}_{45}^{C}$, vanamālāvibhūṣitaḥ $\mathbf{P}^{\Sigma} \quad$ 31a ${ }^{\circ}$ dehottamo ] $\mathrm{N}_{77}^{K o} \mathbf{K}^{\Sigma} \mathrm{E}^{N} \mathrm{~B}_{99}^{C} S_{67}^{S}$, ${ }^{\circ}$ devottamo $\mathrm{N}_{45}^{C} \mathbf{P}^{\Sigma}$ 31c ${ }^{\circ}$ prasādasampannah ] $\Sigma$, oprasādasampanna $\mathrm{N}_{45}^{C}$, opraṇāmaparamaḥ $\mathrm{P}_{3^{2}}^{T} \quad$ 31d ${ }^{\circ}$ dhyānaparāyaṇaḥ ] $\Sigma$, odhyānaikatatparaḥ $\mathrm{P}_{32}^{T}$ 
sarvapāpapramathanạ̣ sarvāsuranikṛntakaḥ|

sarvadā śāntabhāvena viṣnụụ śāntiṃ karotu me|| 32||

32bc Omitted in $\mathrm{N}_{77}^{K o} \mathrm{~N}_{45}^{C} \mathrm{~N}_{12}^{K} \hat{S}_{67}^{S} \mathbf{P}^{\Sigma} \quad$ 32d After this $\mathrm{N}_{5^{8}}^{K}$ adds 34 pādas : arhantah śāntarūpī ca piñcikā(janva)pāṇinaḥ| digvāsā jatruvāsāś ca saumyacittaḥ samāhitaḥ| savṛttalocanaśāntaḥ śivadhyānaikacittakaḥ| śāntiṃ karotu me nityaṃmarhantaḥ śivabhāvitaḥ| jñānacī+va+rasampannā mahāmārapramarddakaḥ| vajradehaḥ surūpī ca tuṣitāyāḥ sureśvaraḥ| jitedriyasamāvastho varadābhayapāṇikaḥ| jñānadhyānarataḥ śāntaḥ śivayogaikamānasaḥ| buddhaḥ svabhāvabuddhena śāntim āśu prayacchati| śaṃkhakuḍenduvarṇṇābhạ̣ kaṇțhe marakataprabhaḥ| tryakșaś ca makuṭi śrīmān svayạ̣ viṣnur vyavasthitaḥ| caturyugaś catuṣpādah pṛthuvatsaḥ kṣiteh patị̣| vṛṣo hi bhagavān dharmmạ̣ sarvvadharmapurasthataḥ| îsam vahati pṛsțhena tasmād dharmo jagatprabhuḥ| vṛṣo vṛ̦avara śrīmān karotu mama śāntikam| naṃdā su+bha+drā surabhī suśīlā sumanā tathā| gāva śivapure pañca sthitāḥ kurvvantu śāntikam $\mid \bullet \mathrm{E}^{N}$ adds 30 pādas : ārhantaḥ śāntacetaṣkaviśvātman viśvayātitaḥ| digvāsā malapañkaś ca saumyacittasamāhitaḥ| samvartalocanaḥ śāntaḥ śivajñānaikacintakaḥ| saanntiṃ karotu me śāntaḥ śivayogena bhāvitaḥ| jitendriyaḥ samādhisthạ̣ pātracīvarabheșitạ̣| varadābhayapāṇiś ca jñānadhyānarataḥ sadā| yogadṛșțị̣ sadāyuktah śivajñānena bhāvitaḥ| śāntiṃ karotu me deva sarvasattvahite rataḥ| pītavarṇena dehena hāreṇa suvicitriṇā| sarvāngasundarī devi vijayā jayakāriṇ̄| śivārcanaratā nityaṃ śivajāpyaparāyaṇā| dharitrī lokamātā ca nityaṃ rakṣān karotu me| kṣīrodād utthito gāvo lokānāṃ hitakāmyayā| prīṇayanti sadā devān viprāṃś caiva viśeșataḥ| nityan tu devatātmānaḥ kurvantu mama śāntikam| • $S_{67}^{S}$ adds 30 pādas : arhan devaḥ śāntarūpī piñchakañcukapāṇikaḥ| digvāsāḥ kṛttivāsāś ca saumyacittas samāhitaḥ| saṃvṛttalocanaḥ śāntaḥ śivajñānaikacintakaḥ| śāntiṃ karotu me śāntaḥ śivajñānaikatānvitaḥ| jitendriyaḥ samādhisthạ̣ pātracīvarabhūṣitaḥ| varadābhayapāṇiś ca jñānadhyānaratas sadā| yogadṛștisamāyuktạ śivajñānaparāyaṇaḥ| śāntiṃ karotu me bauddhạ̣ sarvasattvahite ratạ̣| pìtavarṇena dehena hāreṇa suvicitritā| sarvāngasundarī devī jayā vijayakāṅkṣiṇī| śivārcanaratā nityạ̣ śivapūjāparāyaṇā| dharitrī lokamātā ca nityaṃ rakṣāṃ karotu me| kṣīrodād utthitā gāvo lokānāṃ hitakāmyayā| prīṇayanti sadā devān viprāś caiva viśeșataḥ| nityaṃ ca devatātmānaḥ kurvantu mama śāntikam|

32a sarvapāpapramathanaḥ ] $\mathrm{N}_{77}^{K o} \mathrm{~N}_{58}^{K}$, sarvapāpapramāthakah $\mathrm{N}_{82}^{K}$ (unmetr.), sarvapāpapramasthāno $\mathrm{N}_{45}^{C}$, sarvapāpapramathakaḥ $\mathrm{B}_{99}^{C}$, sarvapāpapraśamakaḥ $\mathrm{E}^{N}$, sarvapāpapraśamano $\mathrm{N}_{12}^{K} S_{67}^{S}$, śivārcanaparo nityaṃ $\mathrm{P}_{3^{2}}^{T}$, śivārcanaparạ śrīmān $\mathrm{P}_{72}^{T} \quad \mathbf{3}^{2} \mathbf{b}$ okṛntakaḥ ] $\mathrm{N}_{82}^{K} \mathrm{~B}_{99}^{C} \mathrm{E}^{N}$, okṛtaktah $\mathrm{N}_{58}^{K}$ (unmetr.) $\quad \mathbf{3}^{2 d}$ viṣnụ ] $\Sigma$, viṣṇu $\mathrm{N}_{12}^{K} \mathrm{~N}_{58}^{K} \bullet$ śāntiṃ ] $\Sigma$, śānti $\mathrm{N}_{45}^{C} \mathrm{~N}_{58}^{K}$ 
[Mātṛs]

[Brahmāṇī]

padmarāgaprabhā devī caturvadanapaṅkajā|

akṣamālārpitakarā kamaṇḍaludharā śubhā|| 33\|

brahmāṇī saumyavadanā śivapūjāparāyaṇā|

śāntiṃ karotu me prītā brahmaśabdena sarvadā|| 34||

\section{[Rudrāṇī]}

himaśailanibhā devī mahāvṛ̣abhavāhanī|

triśūlahastā varadā nāgābharaṇabhūṣitā|| 35||

caturbhujā caturvaktrā trinetrā pāpahāriṇī|

ārtiṃ haratu me prītā rudrāṇī nityam ujjvalā|| 36||

33-34 Cf. BhavP 1.177.1-2 : padmarāgaprabhā devī caturvadanapañkajā| akṣamālārpitakarā kamaṇḍaludharā śubhā|| brahmāṇī saumyavadanā ādityārādhane ratā| śāntiṃ karotu suprītā âsīrvādaparā khaga|| 35-34 Cf. BhavP 1.177·3-4 : mahāśveteti vikhyātā ādityadayitā sadā| himakundendusadṛ́āa mahāvṛ̦̣abhavāhinī|| triśūlahastāvaraṇā viśrutābharaṇā satî| caturbhujā caturvaktrā trinetrā pāpanāśinī| vṛṣadhvajārcanaratā rudrāṇī śāntidā bhavet||

35a-d $\hat{S}_{67}^{S}$ reverses the order of $35 \mathrm{~cd}$ and $35^{\mathrm{ab}} \quad \mathbf{3}^{6 \mathbf{b}}$ After this $\hat{S}_{67}^{S}$ adds two pādas: rudraśaktir mahāvīrā rudrārcanaparāyaṇā|

33a ${ }^{\circ}$ rāgaprabhā ] $\mathrm{N}_{77}^{K o} \mathbf{K}^{\Sigma} \mathrm{E}^{N} \mathrm{~B}_{99}^{C} \mathrm{P}_{32}^{T}$, ${ }^{\circ}$ rāgāprabhā $\mathrm{N}_{45}^{C}$, ${ }^{\circ}$ rāganibhā ${ }_{S_{67}^{S}}^{S} \mathrm{P}_{72}^{T} \quad$ 33b devī ] $\Sigma$, daivī $\mathrm{N}_{45}^{C}$, devi $\mathrm{E}^{N} \bullet$ pańkajā ] $\Sigma$, paṃkajāḥ $\mathrm{N}_{77}^{K o} \quad \mathbf{3 4}$ b ${ }^{\circ}$ parāyaṇā ] $\Sigma$, o parāyaṇām $\mathrm{N}_{45}^{C} \quad 35^{\mathbf{a}}$ devī ] $\Sigma$, daivī $\mathrm{N}_{45}^{C}$, devi $E^{N} \quad 35^{\circ}{ }^{\circ}$ vāhanī ] $\mathrm{N}_{45}^{C} \mathbf{K}^{\Sigma}$, ${ }^{\circ}$ vāhinī $\mathrm{N}_{77}^{K o} \mathrm{P}_{72}^{T}$, ${ }^{\circ}$ vāhanā $\mathrm{E}^{N} \mathrm{~B}_{99}^{C} \hat{\mathrm{S}}_{67}^{S p c} \mathrm{P}_{32}^{T}$, ${ }^{\circ}$ nistvanā $\hat{S}_{67}^{S a c} \quad$ 35d nāgā $\left.\bar{a}^{\circ}\right] \Sigma$, nāga ${ }^{\circ} \mathrm{N}_{58}^{K}$, sarvāo $\mathrm{P}_{32}^{T} \bullet$ bhūșitā ] $\Sigma$, obhūṣitām $\mathrm{N}_{45}^{C} \mathrm{~N}_{12}^{K} \quad 36 \mathrm{~b}$ trinetrā ] $\Sigma$, tṛ̣netrā $\mathrm{N}_{77}^{K o}$, tinetrā $\mathrm{N}_{45}^{C} \quad \mathbf{3 6 c}$ ārtiṃ haratu ] $\mathrm{N}_{12}^{K} \mathrm{~B}_{99}^{C} \mathbf{P}^{\Sigma}$, arttiharā tu $\mathrm{N}_{45}^{C}$, arttiṃ haratu $\mathrm{N}_{82}^{K}$, ārtti haratu $\mathrm{N}_{58}^{K} \mathrm{E}^{N}$, śāntiṃ karotu $\mathrm{N}_{77}^{K o} \hat{S}_{67}^{S}$ 36d rudrāṇī nityam ujjvalā ] $\mathbf{N}^{\Sigma} \mathrm{E}^{N} \mathrm{~B}_{99}^{C}$, māheśī nityam ujjvalā $\hat{S}_{67}^{S} \mathrm{P}_{7^{2}}^{T}$, brahmaśabdena sarvadā $\sqcup[-8-] \sqcup$ îśvarī nityam ujvalā $\mathrm{P}_{3^{2}}^{T}$ 
[Kaumārī]

mayūravāhanī devī sindūrāruṇavigrahā|

śaktihastā mahārūpā sarvālan̉kārabhūṣitā|| 37||

rudrabhaktā mahāvīryā rudrārcanaratā sadā|

kaumārī varadā devī sāāntim āśu dadātu me|| 38||

[Vaiṣnavī]

śan̉khacakragadāhastā śyāmā pītāmbarapriyā|

caturbhujā tārkṣyayānā vaiṣṇavī surapūjitā|| 39||

37-38 Cf. BhavP 1.177·5-6 : mayūravāhanā devī sindūrāruṇavigrahā| śaktihastā mahākāyā sarvālan̉kārabhūṣitā|| sūryabhaktā mahāvīryā sūryārcanaratā sadā| kaumārī varadā devī sāntim āśu karotu te $\mid$ 39-40 Cf. BhavP 1.177.7-8: gadācakradharā śyāmā pītāmbaradharā khaga| caturbhujā hi sā devī vaiṣṇavī surapūjitā|| sūryārcanaparā nityaṃ sūryaikagatamānasā| sāntiṃ karotu te nityaṃ sarvāsuravimardinī||

$37 \mathbf{a b}{ }^{\circ}$ vāhanī devī ] $\mathrm{N}_{77}^{K o} \mathrm{~N}_{45}^{C} \mathrm{~N}_{82}^{K} \mathrm{~N}_{58}^{K}$, ${ }^{\circ}$ vāhanā devi $\mathrm{E}^{N}$, ovāhanā devī $\mathrm{N}_{12}^{K} \mathrm{~B}_{99}^{C} \dot{S}_{67}^{S} \mathbf{P}^{\Sigma} \quad 37 \mathbf{c}$ ${ }^{\circ}$ rūpā ] $\Sigma$, ${ }^{\circ}$ vīryā $\mathrm{P}_{72}^{T} \quad 37 \mathrm{~d}$ sarvālañkāra $\left.{ }^{\circ}\right] \Sigma$, sarvābharaṇa ${ }^{\circ} \mathrm{N}_{77}^{K o} \mathrm{P}_{32}^{T} \quad \mathbf{3}^{8 \mathbf{a}}{ }^{\circ}$ vīryā ] $\Sigma$, ${ }^{\circ}$ punyyā $\mathrm{P}_{32}^{T},{ }^{\circ} \operatorname{devī~} \mathrm{P}_{72}^{T} \quad 38 \mathrm{c}$ devī ] $\Sigma$, daivī $\mathrm{N}_{45}^{C}$, devi $\mathrm{E}^{N} \quad 38 \mathrm{~d}$ āśu dadātu $] \mathrm{N}_{82}^{K} \mathrm{~N}_{58}^{K} \mathrm{E}^{N}$, āśurddadātu $\mathrm{N}_{77}^{K o}$, āśu karotu $\mathrm{N}_{45}^{C} \mathrm{~N}_{12}^{K} \mathrm{E}^{N} \mathrm{~B}_{99}^{C} S_{67}^{S} \mathbf{P}^{\Sigma} \quad 39$ a ${ }^{\circ}$ gadāo $] \quad \Sigma$, ${ }^{\circ}$ gajāo $\mathrm{N}_{45}^{C} \quad 39 c$ tārkṣyayānā ] $\mathrm{N}_{12}^{K} S_{67}^{S}$, tākṣyayānā $\mathrm{N}_{77}^{K o}$, tārkșyāyanā $\mathrm{N}_{45}^{C}$ (unmetr.), tārkṣyāsanā $\mathrm{N}_{82}^{K} \mathrm{~N}_{58}^{K}$ (unmetr.), tārkșayānā $\mathrm{E}^{N} \mathrm{~B}_{99}^{C}$, tārkșyavāhā $\mathrm{P}_{32}^{T}$, tārkșyagatā $\mathrm{P}_{72}^{T} \quad 39 \mathrm{~d}$ sura $\left.{ }^{\circ}\right] \Sigma$, śubha ${ }^{\circ}$ $\mathrm{N}_{12}^{K}$ 
śivārcanaratā nityaṃ śivaikāhitamānasā|

śāntiṃ karotu me nityạ̣ sarvāsuravimardanī|| 40||

[Aindrī]

airāvatagajārūḍhā vajrahastā mahābalā|

netrāṇāṃ tu sahasreṇa bhūṣitā kanakaprabhā|| 41||

siddhagandharvanamitā sarvālaṅkārabhūṣitā|

aindrī devī sadākālaṃ śāntim āśu karotu me|| 42||

[Vārāhī ]

varāhaghoṇā vikaṭā varāhavaravāhanī|

śyāmāvadātavipulā śan̉khacakragadāyudhā|| 43||

41-42 Cf. BhavP 1.177.9-10 : airāvatagajārūḍhā vajrahastā mahābalā| sarvatralocanā devī varṇataḥ karburāruṇā|| siddhagandharvanamitā sarvālaṃkārabhūṣitā| indrāṇi te sadā devī sanantim āśu karotu vai|| 43-44 Cf. BhavP 1.177.11-12 : varāhaghoravikațā varāhavaravāhinī| śyāmāvadātā yā devī śan̉khacakragadādharā|| tejayantī tu nimiṣān pūjayantī sadā ravim| vārāhī varadā devī tava śāntiṃ karotu vai||

41a-42d $\mathrm{N}_{58}^{K}$ and $\mathbf{P}^{\Sigma}$ has these eight pādas after 44

40a ${ }^{\circ}$ ratā ] $\mathrm{N}_{77}^{K o} \mathrm{~N}_{45}^{C} \mathrm{~N}_{12}^{K} \mathrm{~N}_{58}^{K} \mathrm{E}^{N} \dot{\mathrm{S}}_{67}^{S} \mathbf{P}^{\Sigma}$, ${ }^{\circ}$ parā $\mathrm{N}_{82}^{K} \mathrm{~B}_{99}^{C} \bullet$ nityam ] $\Sigma$, bhaktā $\mathrm{P}_{32}^{T} \quad$ 4ob śivaikāhita $\left.{ }^{\circ}\right] \mathrm{N}_{82}^{K} \mathrm{~N}_{58}^{K} \mathrm{E}^{N} \mathrm{~B}_{99}^{C}$, rudraikagata ${ }^{\circ} \mathrm{N}_{77}^{K o} \mathrm{~N}_{45}^{C} \mathrm{~N}_{12}^{K} \dot{S}_{67}^{S} \mathbf{P}^{\Sigma} \bullet{ }^{\circ}$ mānasā ] $\mathrm{N}_{82}^{K} \mathrm{~N}_{58}^{K} \mathrm{E}^{N} \hat{S}_{67}^{S} \mathbf{P}^{\Sigma}$, ${ }^{\circ}$ mānasah $\mathrm{N}_{45}^{C} \quad 40 c$ sanntim ] $\Sigma$, śānti $\mathrm{E}^{N} \bullet$ nityam ] $\Sigma$, prītā $\mathrm{P}_{32}^{T} \quad$ 4od sarvāsuravimardanī ] $\mathrm{N}_{77}^{K o} \mathrm{~K}^{\Sigma} \mathrm{B}_{99}^{C}$, sarvāsuravimarddhaṇī $\mathrm{N}_{45}^{C}$, sarvāsuravimardinī $\mathrm{E}^{N} \mathbf{P}^{\Sigma}$, sarvadāsuramardinī $\hat{S}_{67}^{S} \quad$ 41a airāvata $\left.{ }^{\circ}\right] \Sigma$, airāvaṇā ${ }^{\circ} S_{67}^{S} \bullet{ }^{\circ}$ gajārūḍhā ] $\Sigma$, ogajārūḍhas $\mathrm{N}_{77}^{K o}$, ${ }^{\circ}$ gajārūụha $\mathrm{E}^{N}$, ${ }^{\circ}$ samārūụha $\mathrm{P}_{72}^{T} \quad$ 41b obalā ] $\Sigma$, ${ }^{\circ}$ prabhā $S_{67}^{S} \quad$ 41c netrāṇām ] $\Sigma$, netānan $\mathrm{N}_{45}^{C} \quad$ 41d kanaka $^{\circ}$ ] $\Sigma$, kāñcana ${ }^{\circ} \mathrm{P}_{72}^{T} \quad$ 42a siddha $^{\circ}$ ] $\Sigma$, siddhi ${ }^{\circ}$ $\mathrm{N}_{45}^{C} \quad$ 42b sarvālañkāra $\left.^{\circ}\right] \Sigma$, sarvābharaṇa ${ }^{\circ} \mathrm{P}_{3^{2}}^{T} \quad$ 42c $\left.^{\circ} \mathrm{kālaṃ}\right] \mathrm{K}^{\Sigma} \mathrm{E}^{N} \hat{\mathrm{S}}_{67}^{S} \mathbf{P}^{\Sigma}$, okāla $\mathrm{N}_{77}^{K o} \mathrm{~N}_{45}^{C} \mathrm{~B}_{99}^{C} \quad$ 42d śāntim āśu ] $\mathrm{N}_{82}^{K} \mathrm{~N}_{12}^{K p c} \mathrm{~N}_{58}^{K} \mathrm{E}^{N} \mathrm{~B}_{99}^{C} \dot{S}_{67}^{S} \mathbf{P}^{\Sigma}$, śāntimmmāśu $\mathrm{N}_{77}^{K o}$, śāntiśasu $\mathrm{N}_{45}^{C}$, sāantimaśu $\mathrm{N}_{82}^{K a c} \quad 43$ a varāhaghoṇā ] $\Sigma$, varāhaghorā $\mathrm{N}_{45}^{C}$, vārāhaghoṇa ${ }^{\circ} \mathrm{P}_{72}^{T} \quad 43 \mathrm{~b}$ varāha $^{\circ}$ ] $\Sigma$, varāhāvarāha ${ }^{\circ} \mathrm{N}_{82}^{K a c}$ (unmetr.) • ${ }^{\circ}$ vāhanī ] $\mathrm{N}_{45}^{C} \mathbf{K}^{\Sigma}$, ${ }^{\circ}$ vāhinī $\mathrm{N}_{77}^{K o} S_{67}^{S} \mathbf{P}^{\Sigma}$, ${ }^{\circ}$ vāhanā $\mathrm{E}^{N} \mathrm{~B}_{99}^{C} \quad{ }_{43}$ c śyāmāvadātavipulā ] $\mathrm{N}_{77}^{K o} \mathrm{~K}^{\Sigma} \mathrm{B}_{99}^{C}$, śyāmāvadānavipulā $\mathrm{N}_{45}^{C}$, śyāmāvadātāṃ vipulā $\mathrm{E}^{N}$, śyāmāvadātā vipulā $\hat{S}_{67}^{S} \mathrm{P}_{32}^{T}$, śyāmābhā sarvavaradā $\mathrm{P}_{72}^{T} \quad \mathbf{4 3}^{\circ}{ }^{\circ}$ gadāyudhā ] $\mathrm{K}^{\Sigma} \mathrm{E}^{N} \mathrm{~B}_{99}^{C p c} \hat{S}_{67}^{S} \mathrm{P}_{72}^{T}$, ${ }^{\circ}$ gadādharā $\mathrm{N}_{77}^{K o}$, ${ }^{\circ}$ gajādharā $\mathrm{N}_{45}^{C} \mathrm{P}_{32}^{T}$, ${ }^{\circ}$ gadā $<$ dharā $>$ yudhā $\mathrm{B}_{99}^{C}$ 
tarjayantī sadā vighnān arcayantī sadā śivam| vārāhī varadā devī kṣemārogyaṃ dadātu me|| 44||

\section{[Cāmuṇụā]}

ūrdhvakeśā koṭarākṣā nirmāṃsā snāyubandhanā| karālavadanā ghorā khaḍgakațtāāakodyatâ|| 45||

kapālamālinī kruddhā khațvāngavaradhāriṇī| āraktapinganayanā gajacarmāvaguṇțhitā|| 46||

45-47 Cf. BhavP 1.177.13-16 : ardhakośā kațīkṣāmā nirmāṃsā snāyubandhanāt| karālavadanā ghorā khaḍgaghaṇṭodgatā satî|| kapālamālinī krūrā khaṭāngavaradhāriṇī| āraktā pinganayanā gajacarmāvaguṇțhitā|| gośrutābharaṇā devī pretasthānanivāsinī| śivārūpeṇa ghoreṇa śivarūpabhayaṃkarī| cāmuṇḍā caṇḍarūpeṇa sadā śāntim karotu te|| caṇḍamuṇḍakarā devī muṇḍadehagatā satī| kapālamālinī krūrā khațvāṅgavaradhāriṇī||

46d-77c One folio is missing in $\mathrm{N}_{77}^{K o}$, covering the text from ${ }^{\circ}$ carmāvagunthitā up to vidyā $\bar{a}^{\circ}$ in $77 \mathrm{c}$

44a tarjayantī sadā vighnān arcayantī ] $\mathrm{N}_{82}^{K} \mathrm{~N}_{12}^{K} \mathrm{E}^{N} \mathrm{~B}_{99}^{C} \hat{S}_{67}^{S} \mathrm{P}_{72}^{T}$, tarjjayanti mahāvighnām arcayanti $\mathrm{N}_{77}^{K o} \mathrm{~N}_{45}^{C}$, tarjjayaṃtī mahāvighnān arccayantī $\mathrm{N}_{58}^{K}$, tarjayantī sadā vighnaṃ arvayantī $\mathrm{P}_{72}^{T} \quad 44 \mathrm{c}$ devī ] $\Sigma$, devi $\mathrm{E}^{N} \quad 44 \mathrm{~d}$ kșemāo $\left.{ }^{\circ}\right] \mathrm{N}_{77}^{K o} \mathrm{~K}^{\Sigma} \mathrm{B}_{99}^{C} S_{67}^{S} \mathrm{P}_{32}^{T}$, kșama ${ }^{\circ} \mathrm{N}_{45}^{C}$, kṣamāo $\mathrm{E}^{N} \mathrm{P}_{72}^{T} \bullet$ dadātu ] $\mathrm{N}_{45}^{C} \mathrm{~K}^{\Sigma} \mathrm{E}^{N} \mathrm{~B}_{99}^{C} \mathrm{P}_{72}^{T}$, karotu $\mathrm{N}_{77}^{K o} \hat{\mathrm{S}}_{67}^{S} \mathrm{P}_{32}^{T} \quad 45^{2}$ ūrdhvakeśā koțarākșā ] $\mathrm{N}_{82}^{K} \mathrm{~B}_{99}^{C}$, ūrdhvakeśotkațākșāṇi $\mathrm{N}_{77}^{K o}$, ūrdhvakeśotkațākșāmā $\mathrm{N}_{45}^{C} \mathrm{~N}_{12}^{K} \mathrm{~N}_{58}^{K} \mathrm{P}_{32}^{T}$, arddhakeśā koțarākșā $\mathrm{E}^{N}$, ūrdhvakeśańkoțakșāmā $S_{67}^{S}$, ūrdhvakeśotkațā śyāmā $\mathrm{P}_{72}^{T} \quad 45^{b}$ nirmāṃșā ] $\mathrm{N}_{82}^{K} \mathrm{~N}_{12}^{K} \mathrm{E}^{N} \mathrm{P}_{72}^{T}$, nirmāṃśā $\mathrm{N}_{77}^{K o}$, nirmāṃsạ̣ $\mathrm{N}_{45}^{C}$, nirmāsā $\mathrm{N}_{58}^{K}$, nirmmāṃśā $\mathrm{B}_{99}^{C}$, nirmāṃsa ${ }^{\circ} \hat{S}_{67}^{S} \mathrm{P}_{32}^{T} \quad{ }_{45} \mathrm{c}$ karāla $\left.{ }^{\circ}\right] \Sigma$, karālā $\mathrm{N}_{12}^{K} \quad{ }^{K} 5^{\mathrm{d}}$ khaḍakațtāakrakodyatā ] $\mathrm{N}_{82}^{K a c} \mathrm{~N}_{12}^{K} \mathrm{E}^{N} \mathrm{~B}_{99}^{C}$, khaḍgakațārikodyatā $\mathrm{N}_{77}^{K o}$, khaḍgakațarakodyatā $\mathrm{N}_{45}^{C}$, khaḍgakațtārikodyatā $\mathrm{N}_{82}^{K p c} \mathrm{~N}_{58}^{K}$, khațvāngāsikarodyatā $\hat{S}_{67}^{S}$, khaḍgaghaṇṭāyudhodyatā $\mathrm{P}_{72}^{T p c}$, khaḍgaghaṇṭāyudhojvalā $\mathrm{P}_{72}^{T a c}$, khaḍgakhețakadhāriṇī $\mathrm{P}_{72}^{T} \quad$ 46a kruddhā ] $\mathrm{N}_{82}^{K} \mathrm{E}^{N} \mathrm{~B}_{99}^{C}$, krūrā $\mathrm{N}_{45}^{C} \mathrm{~N}_{12}^{K} \mathrm{~N}_{58}^{K} S_{67}^{S}$, devī $\mathrm{N}_{77}^{K o} \mathbf{P}^{\Sigma} \quad 46 \mathrm{~b}$ khațvāṇgavara $\left.{ }^{\circ}\right] \mathrm{N}_{82}^{K} \mathrm{~N}_{12}^{K} \mathrm{~B}_{99}^{C}$, krūrā khațaannga ${ }^{\circ}$ $\mathrm{N}_{77}^{K o} \mathbf{P}^{\Sigma}$, khațvāngāvara ${ }^{\circ} \mathrm{N}_{45}^{C}$, khațvāngakara ${ }^{\circ} \mathrm{N}_{58}^{K}$, khațāngavara ${ }^{\circ} \mathrm{E}^{N}$, śūlapațtisa ${ }^{\circ}{\mathrm{S}_{67}^{S}}^{S}$ 46c ārakta ${ }^{\circ}$ ] $\Sigma$, āraktāo ${ }^{\circ} B_{99}^{C} \bullet{ }^{\circ}$ nayanā ] $\Sigma$, onayayanā $\mathrm{N}_{45}^{C}$ (unmetr.) $\quad$ 46d ${ }^{\circ}$ carmāvaguṇṭhitā ] $\mathrm{K}^{\Sigma} \mathrm{E}^{N} \mathrm{~B}_{99}^{C} \hat{S}_{67}^{S} \mathrm{P}_{32}^{T}$, ${ }^{\circ}$ carmavaguṇțhitā $\mathrm{N}_{45}^{C}$, ${ }^{\circ}$ carmāvakuṇțhitā $\mathrm{P}_{72}^{T}$ 
nānānāgaparītāngī pretasthānanivāsinī|

śivarūpeṇa ghoreṇa śivārāvabhayañkarī|

cāmuṇụā caṇụarūpeṇa mahārakṣāṃ karotu me|| 47||

[Lamboșṭ̣ị̄]

āpītena śarīrena nānābharaṇabhūṣitā|

ātarjayantī vighnāni jvalatkhaḍgāgradhāriṇī|| 48||

dhvāñkṣāsanā mahāvīrā sarvapāpapraṇāśanī|

lamboșṭī varadā devī saāntim āśu karotu me|| 49||

47e After this $\mathrm{N}_{58}^{K}$ adds two pādas : ditijatrāsanena tu| bhūtapretapiśācāś ca $\bullet \mathrm{P}_{3^{2}}^{T}$ adds : citrayaṃtrā sanotu me| pretapretapiśācebhyah $\bullet \mathrm{P}_{72}^{T}$ adds : citrayantrāsanena tu| bhūtapretapisaācebhyạ̣ $\quad{ }_{47} \mathrm{f} \quad$ After this $\mathrm{P}_{32}^{T}$ adds two pādas: āyuh striyaṃ balạ̣ saukhyam prītā bhūtvā dadātu me| $\bullet \mathrm{P}_{72}^{T}$ adds : āyuh śriyaṃ balaṃ saukhyaṃ yaśo vṛddhiśriyāvaham| $\mathbf{4 8 a - 4 9 d}$ Omitted in $\mathrm{N}_{45}^{C} \dot{S}_{67}^{S} \mathbf{P}^{\Sigma}$

47a ${ }^{\circ}$ nāgaparītāngī ] $\mathrm{N}_{45}^{C} \mathrm{~N}_{12}^{K} \mathbf{P}^{\Sigma}$, ${ }^{\circ}$ topavatāngī tu $\mathrm{N}_{82}^{K}$, onāgopavītāngī $\mathrm{E}^{N} \mathrm{~B}_{99}^{C} \mathrm{~N}_{58}^{K}$, ${ }^{\circ}$ rūpaparītāngī $\hat{S}_{67}^{S} \quad 47$ b preta $\left.^{\circ}\right] \mathbf{K}^{\Sigma} \mathrm{E}^{N} \mathrm{~B}_{99}^{C} \dot{S}_{67}^{S} \mathbf{P}^{\Sigma}$, pretāo $\mathrm{N}_{45}^{C} \quad 47 \mathbf{c}$ śiva $\left.{ }^{\circ}\right] \mathrm{N}_{45}^{C} \mathrm{~N}_{82}^{K} \mathrm{~B}_{99}^{C} \hat{S}_{67}^{S} \mathbf{P}^{\Sigma}$, śivā $\mathrm{N}_{12}^{K} \mathrm{~N}_{58}^{K} \mathrm{E}^{N} \quad 47 \mathrm{~d}$ śivārāva $\left.{ }^{\circ}\right] \mathrm{N}_{45}^{C} \mathrm{~K}^{\Sigma} \mathrm{E}^{N} \mathrm{~B}_{99}^{C} \dot{S}_{67}^{S} \mathrm{P}_{32}^{T}$, பghorā ca $\mathrm{P}_{72}^{T} \bullet$ obhayañkarī ] $\mathrm{N}_{45}^{C} \mathrm{~N}_{82}^{K} \mathrm{~N}_{12}^{K} \mathrm{~B}_{99}^{C} \hat{S}_{67}^{S} \mathbf{P}^{\Sigma}$, obhayakarī $\mathrm{N}_{58}^{K}$ (unmetr.), obhayañkarā $\mathrm{E}^{N} \quad 47 \mathrm{e}$ cāmuṇḍā ] $\mathbf{K}^{\Sigma} \mathrm{E}^{N} \mathrm{~B}_{99}^{C} \hat{\mathrm{S}}_{67}^{S} \mathbf{P}^{\Sigma}$, cāmuṇḍa $\mathrm{N}_{45}^{C} \quad 47 \mathrm{f}$ mahārakṣām ] $\mathrm{N}_{82}^{K} \mathrm{~N}_{12}^{K} \mathrm{E}^{N} \mathrm{~B}_{99}^{C} \dot{S}_{67}^{S}$, mahārakșa $\mathrm{N}_{45}^{C}$, mama rakșām $\mathrm{N}_{58}^{K} \mathbf{P}^{\Sigma} \bullet$ karotu me ] $\mathrm{N}_{45}^{C} \mathrm{~N}_{82}^{K} \mathrm{E}^{N} \mathrm{~B}_{99}^{C} \dot{S}_{67}^{S}$, karoti hi $\mathrm{N}_{58}^{K}$, karotviha $\mathbf{P}^{\Sigma} \quad 48 \mathbf{a}$

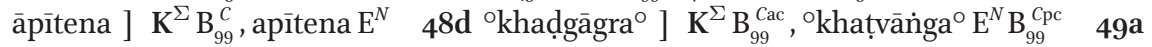
mahāvīrā ] $\mathrm{N}_{82}^{K} \mathrm{~N}_{12}^{K} \mathrm{E}^{N} \mathrm{~B}_{99}^{C}$, mahāvīryā $\mathrm{N}_{58}^{K} \quad 49 c$ lamboșţhī ] $\mathrm{N}_{82}^{K} \mathrm{~N}_{12}^{K}$, lamboșthā $\mathrm{N}_{58}^{K}$, lambaușț̣ī $\mathrm{B}_{99}^{C}$, lambaușțhi $\mathrm{E}^{N} \bullet$ varadā ] $\mathrm{N}_{82}^{K} \mathrm{~N}_{12}^{K} \mathrm{E}^{N} \mathrm{~B}_{99}^{C}$, paramā $\mathrm{N}_{5^{8}}^{K}$ 
[All Mātṛs]

ākāśamātaro divyās tathānyā lokamātaraḥ|

bhūtānāṃ mātaraḥ sarvās tathānyāsuramātaraḥ|| 50

sarvamātṛmahādevyaḥ svāyudhavyagrapāṇayaḥ|

jagad vyāpyāvatișṭhante balikāmā mahodayāḥ || 51||

rudrabhaktā mahāvīryā rudrārcāhitamānasāḥ|

śāntiṃ kurvantu me nityạ̣ mātaraḥ surapūjitāḥ̣|| $5^{2} \|$

50-52 Cf. BhavP 1.177.17-21 : ākāśamātaro devyas tathānyā lokamātaraḥ| bhūtānāṃ mātarah sarvās tathānyāḥ pitṛmātaraḥ|| vṛddhiśrāddheșu pūjyante yās tu devyo manīṣibhiḥ| mātre pramātre tanmātre iti mātṛmukhās tathâ|| pitāmahī tu tanmātā vịddhā yā ca pitāmahī| ity etās tu pitāmahyaḥ śāntim te pitṛmātaraḥ|| sarvā mātṛmahādevyaḥ svāyudhāvyagrapāṇayaḥ| jagad vyāpya pratișṭhantyo balikāmā mahodayāḥ|| śāntị̣ kurvantu te nityam ādityārādhane ratāḥ| śāntena cetasā śāntyaḥ śāntaye tava śāntidāḥ|| • BhavP 1.178.17 : ākāśamātaro divyās tathānyā devamātaraḥ| sūryāyaṇaparā devyo jagad vyāpya vyavasthitāḥ| śāntiṃ kurvantu me nityaṃ mātaraḥ surapūjitāḥ̣||

5ocd Omitted in $\mathrm{E}^{N} \quad \mathbf{5}$ oc-52b Omitted in $\hat{S}_{67}^{S}$

5oa ākāśamātaro ] $\mathrm{K}^{\Sigma} \mathrm{E}^{N} \mathrm{~B}_{99}^{C} \hat{\mathrm{S}}_{67}^{S} \mathrm{P}_{72}^{T}$, ākāśamātarā $\mathrm{N}_{45}^{C}$, ākāśe mātaro $\mathrm{P}_{32}^{T}$ • divyās ] $\mathrm{N}_{45}^{C} \mathrm{~N}_{82}^{K} \mathrm{~N}_{12}^{K} \mathrm{E}^{N} \mathrm{~B}_{99}^{C} \mathbf{P}^{\Sigma}$, devyas $\mathrm{N}_{58}^{K} \dot{S}_{67}^{S} \quad$ 5ob ${ }^{\circ}$ mātarah ] $\mathbf{K}^{\Sigma} \mathrm{E}^{N} \mathrm{~B}_{99}^{C} \dot{S}_{67}^{S} \mathbf{P}^{\Sigma}$, ${ }^{\circ}$ mātarāḥ $\mathrm{N}_{45}^{C}$ 5 oc bhūtānāṃ ] $\mathrm{N}_{45}^{C} \mathbf{K}^{\Sigma} \mathbf{P}^{\Sigma}$, bhūtānā $\mathrm{B}_{99}^{C} \bullet$ mātarạ̣ ] $\mathbf{K}^{\Sigma} \mathrm{B}_{99}^{C} \mathbf{P}^{\Sigma}$, mātarās $\mathrm{N}_{45}^{C} \quad 5^{\text {od }}$ tathānyāsuramātarah ] $\mathrm{N}_{82}^{K} \mathrm{~B}_{99}^{C}$, tathānyā devamātarāh $\mathrm{N}_{45}^{C} \mathrm{P}_{72}^{T}$, tathānyā daivamātaraḥ $\mathrm{N}_{12}^{K}$, tathānyāḥ suramātaraḥ $\mathrm{N}_{58}^{K}$, tathānyā pitṛmātarah $\mathrm{P}_{32}^{T} \quad \mathbf{5 1 a}^{\mathbf{1 a}}{ }^{\circ}$ mahādevyaḥ ] $\mathbf{K}^{\dot{\Sigma}} \mathrm{E}^{N} \mathrm{~B}_{99}^{C} \mathrm{P}_{72}^{T}$, ${ }^{\circ}$ mahādevyāh $\mathrm{N}_{45}^{C}$, ${ }^{\circ}$ gaṇā devyah $\mathrm{P}_{32}^{T} \quad \mathbf{5}^{\mathbf{1 b}}$ svāyudhavyagra $\left.{ }^{\circ}\right] \mathbf{K}^{\Sigma} \mathrm{P}_{32}^{T}$, svāyudravyāgra ${ }^{\circ} \mathrm{N}_{45}^{C}$, smāyudhạ̣vyagra ${ }^{\circ} \mathrm{B}_{99}^{C}$, ssāyudhavyagra ${ }^{\circ} \mathrm{E}^{N}$ (sic), svāyudhāsakta ${ }^{\circ}$ $\mathrm{P}_{72}^{T} \quad 5^{1 \mathrm{c}}{ }^{\circ}$ tișțhante ] $\mathrm{N}_{82}^{K} \mathrm{~N}_{58}^{K} \mathrm{E}^{N} \mathrm{~B}_{99}^{C}$, o tișththanti $\mathrm{N}_{45}^{C} \mathrm{~N}_{12}^{K}$, otișțhantyo $\mathbf{P}^{\Sigma} \quad$ 51d mahodayāh ] $\mathrm{N}_{45}^{C} \mathbf{K}^{\Sigma} \mathrm{B}_{99}^{C} \mathrm{P}_{3^{2}}^{T}$, mahodayā $\mathrm{E}^{N}$, mahodarāḥ $\mathrm{P}_{72}^{T} \quad$ 52a obhaktā ] $\mathrm{N}_{45}^{C} \mathbf{K}^{\Sigma} \mathrm{B}_{99}^{C} \mathbf{P}^{\Sigma}$,

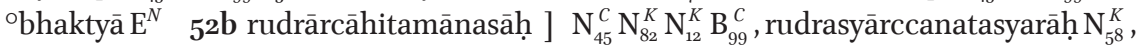
rudrārcāhitamānasā $\mathrm{E}^{N}$, rudrārcanaparāyaṇāḥ $\mathrm{P}_{3^{2}}^{T}$, raudrā rudrārcane ratāḥ $\mathrm{P}_{72}^{T} \quad \mathbf{5}^{2 \mathbf{c}}$ śāntiṃ ] $\mathbf{K}^{\Sigma} \mathrm{E}^{N} \mathrm{~B}_{99}^{C} S_{67}^{S} \mathbf{P}^{\Sigma}$, śānti $\mathrm{N}_{45}^{C} \bullet$ kurvantu ] $\mathbf{K}^{\Sigma} \mathrm{E}^{N} \hat{S}_{67}^{S} \mathbf{P}^{\Sigma}$, kurvanti $\mathrm{N}_{45}^{C}$, karotu $\mathrm{B}_{99}^{C}$ - nityam ] $\mathrm{N}_{45}^{C} \mathbf{K}^{\Sigma} \mathrm{E}^{N} \hat{S}_{67}^{S} \mathbf{P}^{\Sigma}$, nityā $\mathrm{B}_{99}^{c} \quad \mathbf{5}^{2 d}$ mātarah ] $\mathrm{N}_{45}^{C} \mathrm{~N}_{82}^{K} \mathrm{~N}_{12}^{K} \mathrm{E}^{N} \mathrm{~B}_{99}^{C} \hat{S}_{67}^{S} \mathbf{P}^{\Sigma}$, mātarāḥ $\mathrm{N}_{58}^{K} \bullet{ }^{\circ}$ pūjitāḥ ] $\mathrm{N}_{82}^{K} \mathrm{~N}_{12}^{K} E^{N} \mathrm{~B}_{99}^{C} \dot{S}_{67}^{S} \mathbf{P}^{\Sigma}$, ${ }^{\circ}$ pūjitām $\mathrm{N}_{45}^{C}$, ${ }^{\circ}$ pūjitā $\mathrm{N}_{58}^{K}$ 
[Rudras, Mātṛs and Gaṇādhipas]

ye rudrā raudrakarmāṇo rudrasthānanivāsinaḥ|

saumyāś caiva tu ye kecit sthāṇusthānanivāsinaḥ|| 53||

mātaro rudrarūpāś ca gaṇānām adhipāś ca ye|

vighnabhūtās tathā cānye digvidikṣu samāśritāḥ|| 54||

sarve suprītamanasah pratigṇhṇantu me balim|

siddhim āśu prayacchantu bhayebhyaḥ pāntu māṃ sadā|| 55||

53-55 Cf. BhavP 1.177.18-19: ye rudrā raudrakarmāṇo raudrasthānanivāsinaḥ| mātaro rudrarūpāś ca ganānām adhipāś ca ye|| vighnabhūtās tathā cānye digvidikṣu samāśritāḥ| sarve te prītamanasah pratigṇhṇantu me balim| siddhị̣ kurvantu te nityaṃ bhayebhyah pāntu sarvatah || • Mrgendra, Kriyāpāda 7·33-35 : ye rudrā raudrakarmāṇo rudrasthānanivāsinaḥ| saumyāś caiva tu ye kecit saumyasthānanivāsinah|| mātaro rudrarūpāś ca gaṇānām adhipāś ca ye| vighnabhūtās tathā cānye digvidikṣu samāśritāḥ|| sarve suprītamanasaḥ pratighṛ̣nantv imaṃ balim| siddhim juṣantu nạ̣ kṣipraṃ bhayebhyạ̣ pāntu nityaśah|| • İśgP, Sāmānyapāda 14.178-18oab = Mantrapāda 28.96cd-98 = Kriyāpāda 15.4-16ab : ye rudrā raudrakarmāṇo raudrasthānanivāsinaḥ| saumyāś caiva tu ye kecit saumyasthānanivāsinaḥ|| mātaro raudrarūpāś ca gạ̣ānām adhipāś ca ye| sarve suprītamanasạ̣ pratighṛ̣antv imaṃ balim|| siddhiṃ yacchantu me kṣipraṃ bhayebhyạ̣ pāntu māṃ sadā|

53cd Omitted in $\mathrm{P}_{72}^{T}$

53a raudrakarmāṇo ] $\mathrm{N}_{45}^{C} \mathrm{~N}_{82}^{K p c} \mathrm{~N}_{58}^{K} \mathrm{E}^{N} \mathrm{~B}_{99}^{C} \mathrm{P}_{72}^{T}$, raudrakarmāṇa $\mathrm{N}_{82}^{K a c}$, radrakarmāṇo $\mathrm{N}_{12}^{K}$, rudrakarmāṇo $S_{67}^{S} \mathrm{P}_{32}^{T} \quad \mathbf{5 3}^{\mathrm{b}}$ rudrasthāna $\left.{ }^{\circ}\right] \mathrm{N}_{45}^{C} \mathrm{~N}_{82}^{K} \mathrm{~N}_{12}^{K} \mathrm{E}^{N} \mathrm{~B}_{99}^{C} \dot{S}_{67}^{S}$, raudrasthāo $\overline{\mathrm{N}}_{58}^{K}$ (unmetr.), raudrasthāna ${ }^{\circ} \mathbf{P}^{\Sigma} \bullet{ }^{\circ}$ vāsinaḥ ] $\mathbf{K}^{\Sigma} \mathrm{E}^{N} \mathrm{~B}_{99}^{C} \hat{S}_{67}^{S} \mathbf{P}^{\Sigma}$, ${ }^{\circ}$ vāsinaṃ $\mathrm{N}_{45}^{C} \quad$ 53c saumyāś caiva tu ye kecit ] $\mathrm{N}_{45}^{C} \mathrm{~K}^{\Sigma} \mathrm{E}^{N} \mathrm{~B}_{99}^{C} \mathrm{P}_{32}^{T}$, ye rudrāh saumyavadanāḥ $S_{67}^{S} \quad \mathbf{5 3}$ sthāṇu ${ }^{\circ}$ ] $\mathrm{N}_{45}^{C} \mathrm{~K}^{\Sigma} \mathrm{E}^{N} \mathrm{~B}_{99}^{C}$, saumya ${ }^{\circ} S_{67}^{S} \mathrm{P}_{32}^{T}$ 54a rudrarūpāś ] $\mathrm{N}_{45}^{C} \mathrm{~N}_{82}^{K} \mathrm{~N}_{12}^{K} \mathrm{E}^{N} \mathrm{~B}_{99}^{C} \mathbf{P}^{\Sigma}$, raudrarūpāś $\mathrm{N}_{58}^{K}$, lokapālāśs $S_{67}^{S} \quad \mathbf{5 4 b}$ adhipāś ca ] $\mathbf{K}^{\Sigma} \mathrm{E}^{N} \mathrm{~B}_{99}^{C} \hat{S}_{67}^{S} \mathbf{P}^{\Sigma}$, adhipāra $\mathrm{N}_{45}^{C} \quad \mathbf{5 4 c}$ tathā cānye ] $\mathrm{N}_{82}^{K} \mathrm{~N}_{12}^{K} \mathrm{E}^{N} \mathrm{~B}_{99}^{C} \mathrm{P}_{72}^{T}$, tathāścānye $\mathrm{N}_{45}^{C}$, tathā cānyā $\mathrm{N}_{58}^{K}$, tathānye ca $S_{67}^{S}$, tathā ye ca $\mathrm{P}_{32}^{T} \quad 54 \mathrm{~d}$ digvidikșu ] $\mathbf{K}^{\Sigma} \mathrm{E}^{N} \mathrm{~B}_{99}^{C} \hat{S}_{67}^{S} \mathrm{P}_{32}^{T}$, daśadikșu $\mathrm{N}_{45}^{C} \mathrm{P}_{72}^{T} \quad 55^{T}$ sarve suprīta $\left.{ }^{\circ}\right] \mathrm{N}_{45}^{C} \mathbf{K}^{\Sigma} \mathrm{E}^{N} \mathbf{P}^{\Sigma}$, sarvve suprītio $\mathrm{B}_{99}^{C}$, sarve te prīta ${ }^{\circ} \hat{S}_{67}^{S} \bullet{ }^{\circ}$ manasạ ] $\mathrm{N}_{82}^{K \text { pc }} \mathrm{N}_{12}^{K} \mathrm{~N}_{58}^{K} \mathrm{E}^{N} \mathrm{~B}_{99}^{C} \dot{S}_{67}^{S} \mathbf{P}^{\Sigma}$, ${ }^{\circ}$ manasā $\mathrm{N}_{45}^{C}$, ${ }^{\circ}$ manasa $\mathrm{N}_{82}^{K a c} \quad 55^{\mathrm{b}}{ }^{\circ}$ gṛhṇantu me balim ] $\mathrm{N}_{82}^{K p c} \mathrm{~N}_{12}^{K} \mathrm{E}^{N} \mathrm{~B}_{99}^{C}$, ${ }^{\circ}$ gṛhṇantu me balih $\mathrm{N}_{45}^{C} \mathrm{~N}_{82}^{K a c}$, og̣̣hṇa me balim $\mathrm{N}_{58}^{K}$ (unmetr.), og̣̣hṇantvimaṃ balim $\hat{S}_{67}^{S} \mathrm{P}_{72}^{T}$, ${ }^{\circ}$ gṛhṇantvimāṃ balim $\mathrm{P}_{3^{2}}^{T} \quad{ }_{55} \mathrm{c}$ siddhim āśu prayacchantu ] $\mathrm{K}^{\Sigma} \mathrm{E}^{N} \mathrm{~B}_{99}^{C}$, siddhi(n̈ga)santu me nityam $\mathrm{N}_{45}^{C}$, siddhim dadantu me nityam $S_{67}^{S}$, siddhim kurvantu me nityam $\mathrm{P}_{3^{2}}^{T}$, siddhị kurvantu me kṣipram $\mathrm{P}_{72}^{T} \quad$ 55d bhayebhyah ] $\mathrm{N}_{82}^{K} \mathrm{~N}_{12}^{K} \mathrm{E}^{N} \mathrm{~B}_{99}^{C} \hat{S}_{67}^{S} \mathbf{P}^{\Sigma}$, dayebhya $\mathrm{N}_{45}^{C} \bullet$ pāntu ] $\mathrm{N}_{45}^{C} \mathrm{E}^{N} \mathrm{~B}_{99}^{C} \dot{S}_{67}^{S} \mathbf{P}^{\Sigma}$, pātu $\mathbf{K}^{\Sigma} \bullet$ māṃ ] $\mathbf{K}^{\Sigma} \mathrm{E}^{N} \mathrm{~B}_{99}^{C} \dot{S}_{67}^{S} \mathbf{P}^{\Sigma}$, me N $_{45}^{C}$ 
[Ganas : E]

aindryāṃ diśi gaṇā ye tu vajrahastā mahābalāḥ|

suśvetākṣāḥ śvetanibhās tathā vai śvetalohitāḥ|| $5^{6} \|$

divyāntarikṣabhaumāś ca pātālatalavāsinaḥ|

rudrārcanaratā hṛṣṭạ̄ śāntiṃ kurvantu me sadā|| 57||

[Gaṇas : SE]

āgneyāṃ ye gaṇāḥ sarve sruvahastā niṣangiṇaḥ|

āraktākṣā raktanibhās tathā vai raktalohitāḥ|| $5^{8}||$

56ab Cf. İ́sgP, Kriyāpāda $51.68 \mathrm{~cd}=52.42 \mathrm{~cd}=55.85 \mathrm{ab}=57.83 \mathrm{ab}=58.83 \mathrm{~cd}=60.82 \mathrm{ab}:$ ai ndryāṃ diśi gaṇā ye tu vajrahastā mahābalā $\quad$ 56-57 Cf. BhavP 1.177.20-21 : aindrādayo gaṇā ye tu vajrahastā mahābalāḥ| himakundendusadṛsāa nīlakṛṣnāngalohitāḥ|| divyāntarikṣā bhaumāś ca pātālatalavāsinaḥ| aindrāḥ śāntiṃ prakurvantu bhadrāṇi ca punah punaḥ|| • İ́sPP, Mantrapāda 30.84: tadbāhye daśadiksthebhyo gaṇebhyo vikared balim| pūrvasyāṃ diśi śivasya bhagavato ye gạ̣ā vajrapāṇayaḥ śvetāḥ śvetākṣạ̄ śvetalohitāḥ divyantarikṣabhaumāḥ pātālanivāsinaś ca tebhyas tābhyo namo namaḥ țha tha| 58-59 Cf. BhavP 1.177.22-24ab : āgneyyāṃ ye bhṛtāh sarve dhruvahatyānuṣañgiṇaḥ| sūryānuraktā raktābhā japāsumanibhās tathā|| viraktalohitā divyā āgneyyāṃ bhāskarādayaḥ| ādityārādhanaparā ādityagatamānasāḥ|| saanntiṃ kurvantu te nityaṃ prayacchantu baliṃ mama| • İ́sgP, Mantrapāda 30.84 (cont.) : āgneyyāṃ diśi śivasya bhagavato ye gaṇāḥ śaktipāṇayaḥ raktā raktākṣāḥ raktalohitā ityādi|

$57 \mathbf{a b}$ Omitted in $\hat{S}_{67}^{S} \quad \mathbf{5 8 a - 5 9 d}$ Omitted in $\mathrm{E}^{N}$

56a aindryāṃ ] $\mathrm{N}_{82}^{K} \mathrm{~N}_{12}^{K} \mathrm{E}^{N} \hat{S}_{67}^{S} \mathbf{P}^{\Sigma}$, aindrān $\mathrm{N}_{45}^{C} \mathrm{~B}_{99}^{C}$, aindryo $\mathrm{N}_{5^{K}}^{K} \quad \mathbf{5 6 b}$ obalāḥ ] $\mathrm{N}_{45}^{C} \mathrm{~N}_{82}^{K} \mathrm{~N}_{12}^{K} \mathrm{E}^{N} \mathrm{~B}_{99}^{C} \dot{S}_{67}^{S} \mathbf{P}^{\Sigma}$, obalā $\mathrm{N}_{58}^{K} \quad \mathbf{5}^{6} \mathbf{c}$ suśvetāksāạ ] $\mathrm{N}_{82}^{K} \mathrm{~N}_{58}^{K} \mathrm{E}^{N} \mathrm{~B}_{99}^{C} \dot{S}_{67}^{S} \mathbf{P}^{\Sigma}$, śvetākṣā $\mathrm{N}_{45}^{C}$ (unmetr.), śvetākṣāḥ $\mathrm{N}_{12}^{K}$ (unmetr.) $\quad \mathbf{5 6 d}$ olohitāḥ ] $\mathbf{K}^{\Sigma} \mathrm{E}^{N} \mathrm{~B}_{99}^{C} \dot{S}_{67}^{S} \mathbf{P}^{\Sigma}$, olohitāṃ $\mathrm{N}_{45}^{C} \quad 57$ a divyāntarikșa $\left.{ }^{\circ}\right] \mathrm{N}_{45}^{C} \mathrm{~N}_{82}^{K} \mathrm{~N}_{12}^{K} \mathrm{E}^{N} \mathrm{P}_{32}^{T}$, divyantarīkșa ${ }^{\circ} \mathrm{N}_{58}^{K} \mathrm{~B}_{99}^{C}$, divyantarikșā $\mathrm{P}_{72}^{T}$ - ${ }^{\circ}$ bhaumāś ] $\mathbf{K}^{\Sigma} \mathrm{E}^{N} \mathrm{~B}_{99}^{C} \mathbf{P}^{\Sigma}$, obhaumā $\mathrm{N}_{45}^{C} \quad 57$ b pātālatalavāsinaḥ ] $\mathbf{K}^{\Sigma} \mathrm{E}^{N} \mathrm{~B}_{99}^{C} \mathbf{P}^{\Sigma}$, pālatalavāsināṃ $\mathrm{N}_{45}^{C}$ (unmetr.) $\quad 57 \mathrm{c}$ rudrārcanaratā hṛștāḥ ] $\mathrm{N}_{12}^{K} \mathrm{~B}_{99}^{C} \mathrm{P}_{72}^{T}$, rudrārcanaratā hṛștāa $\mathrm{N}_{45}^{C} \mathrm{~N}_{82}^{K}$, rudrārcca+na+parā hṛștāḥ $\mathrm{N}_{58}^{K}$, rudrapraṇāmamanasaḥ $\mathrm{E}^{N}$, rudrārcanaratā hyașțaḥ $\hat{S}_{67}^{S}$, rudrārcanaratā nityaṃ $\mathrm{P}_{32}^{T} \quad 57 \mathrm{~d}$ śāntiṃ ] $\mathrm{K}^{\Sigma} \mathrm{E}^{N} \mathrm{~B}_{99}^{C} \hat{S}_{67}^{S} \mathbf{P}^{\Sigma}$, śānti $\mathrm{N}_{45}^{C}$ - sadā ] $\mathbf{K}^{\Sigma} \mathrm{E}^{N} \mathrm{~B}_{99}^{C} \hat{S}_{67}^{S} \mathbf{P}^{\Sigma}$, sadāḥ $\mathrm{N}_{45}^{C} \quad 58 \mathbf{a}$ àgneyāṃ ye gaṇāḥ sarve ] $\mathrm{N}_{82}^{K} \mathrm{~N}_{58}^{K} \mathrm{P}_{32}^{T} \mathrm{~B}_{99}^{C^{c}}$, āgneyā ye gaṇā sarvā $\mathrm{N}_{45}^{C}$, āgneyā ye gaṇāḥ sarve $\mathrm{N}_{12}^{K}$, āgneyyāṃ ye diśi gaṇāḥ $S_{67}^{S}$, ā-

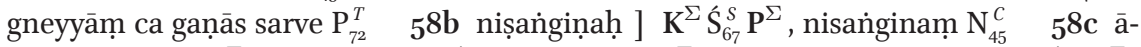
raktākṣā ] $\mathrm{N}_{45}^{C} \mathbf{K}^{\Sigma} \mathrm{B}_{99}^{C}$, āraktābhā $\hat{S}_{67}^{S}$, suraktākṣā $\mathbf{P}^{\Sigma} \quad \mathbf{5}^{8 \mathbf{d}}$ 'lohitāh ] $\mathrm{N}_{45}^{C} \mathrm{~B}_{99}^{C} \hat{S}_{67}^{S} \mathbf{K}^{\Sigma}$, olomabhiḥ $\mathrm{P}_{3^{2}}^{T}$, olocanāḥ $\mathrm{P}_{72}^{T}$ 
divyāntarikṣabhaumāś ca pātālatalavāsinaḥ| rudrapraṇāmamanasaḥ śāntiṃ kurvantu me sadā|| 59||

[Gaṇas: S]

yāmyāṃ diśi gaṇā ye tu satataṃ daṇḍapāṇayaḥ| kṛ̣̣nāḥ kṛ̣nnanibhāḥ kruddhās tathā vai kṛṣnalohitāḥ|| 6o||

divyāntarikṣabhaumāś ca pātālatalavāsinaḥ| rudraikāhitacetaskāḥ śāntiṃ kurvantu me sadā|| 61||

[Ganas: SW]

nairṛtyāṃ tu gaṇāḥ krūrā rākṣasāḥ khaḍgapāṇayaḥ| sunīlākṣā nīlanibhās tathā vai nīlalohitāḥ|| 62||

6o-61 Cf. BhavP 1.177.24cdef : bhayādityasamā ye tu satatam dạ̣dapāṇayaḥ| ādityārādhanaparāḥ kaṃ prayacchantu te sadā|| • İśgP, Mantrapāda 3o.84 (cont.) : yāmyāṃ diśi śivasya bhagavato ye gạ̣ā daṇdapāṇaya kṛṣnāḥ ityādi| 62-63 Cf. İ́śg, Mantrapāda 30.84 (cont.) : oṃ nairṛtyāṃ diśi śivasya bhagavato ye gaṇāḥ khaḍgapāṇayaḥ śyāmāḥ śyāmākṣāḥ śyāmalohitāḥ ityādi|

$59 \mathbf{c}-65 \mathrm{~b}$ Omitted in $\mathrm{P}_{72}^{T}$

59a divyāntarikṣa $\left.{ }^{\circ}\right] \mathrm{N}_{82}^{K} \mathrm{~N}_{12}^{K} \dot{S}_{67}^{S} \mathrm{P}_{32}^{T}$, divyāntirikșa ${ }^{\circ} \mathrm{N}_{45}^{C}$, divyantarīkṣa ${ }^{\circ} \mathrm{N}_{58}^{K} \mathrm{~B}_{99}^{C}$, divyantarikṣāo $\mathrm{P}_{72}^{T} \quad 59 \mathrm{c}$ rudrapraṇāmamanasạ̣ ] $\mathrm{K}^{\Sigma} \mathrm{B}_{99}^{C}$, rudrapraṇāmaparamāḥ $\mathrm{N}_{45}^{C}$, rudrapraṇāmaparamāḥ $S_{67}^{S}$, rudraikāhitacetaskāḥ $\mathrm{P}_{32}^{T} \quad 59 \mathrm{~d}$ śāntim ] $\mathrm{K}^{\Sigma} \mathrm{B}_{99}^{c} \dot{S}_{67}^{S} \mathrm{P}_{32}^{T}$, śānti $\mathrm{N}_{45}^{C} \bullet$ kurvantu me sadā ] $\mathrm{N}_{82}^{K} \mathrm{~N}_{58}^{K} \mathrm{~B}_{99}^{C} \hat{S}_{67}^{S} \mathrm{P}_{32}^{T}$, kurvanti me șadā $\mathrm{N}_{45}^{C}$, kurvantu me sadāḥ $\mathrm{N}_{12}^{K} \quad$ 6oa yāmyāṃ ] $\mathrm{N}_{82}^{K} \mathrm{~N}_{12}^{K} \mathrm{E}^{N} \mathrm{~B}_{99}^{C} \dot{S}_{67}^{S} \mathrm{P}_{32}^{T}$, yamyāṃ $\mathrm{N}_{58}^{K}$, yāmyā $\mathrm{N}_{45}^{C}$ 6oc kụṣnāḥ kṛṣnanibhāḥ kruddhās ] $\mathrm{N}_{82}^{K}$, kṛ̣ṇākṛ̣ṣnanibhāḥ krūrās $\mathrm{N}_{45}^{C} \mathrm{~N}_{58}^{K}$, kṛ̣̣nahạ kṛṣṇanibhāḥ kruddhās $\mathrm{B}_{99}^{C}$, kṛṣṇaḥ kṛṣnanibhāḥ kruddhās $\mathrm{E}^{N}$, kṛṣnāḥ kṛṣṇanibhāḥ krūrās $\mathrm{N}_{82}^{K} \hat{S}_{67}^{S}$, sukṛṣnāāṣāḥ kṛṣnanibhāḥ $\mathrm{P}_{32}^{T} \quad$ 61a divyāntarikṣa $\left.{ }^{\circ}\right] \quad \mathrm{N}_{45}^{C} \mathrm{~N}_{82}^{K} \mathrm{~N}_{12}^{K} \mathrm{E}^{N} S_{67}^{S} \mathrm{P}_{32}^{T}$, divyantarikṣa ${ }^{\circ}$ $\mathrm{N}_{58}^{K} \mathrm{~B}_{99}^{C} \quad 61 \mathrm{c}$ rudraikāhitacetaskāḥ ] $\mathrm{N}_{82}^{K} \mathrm{~N}_{12}^{K} \mathrm{~B}_{99}^{C} \mathrm{P}_{32}^{T}$, rudraikāhitacetaskā $\mathrm{N}_{77}^{C p c} \mathrm{~N}_{58}^{K} \mathrm{E}^{N}$, rudraikāhitañcataskā $\mathrm{N}_{77}^{C a c}$, parameśarcanaratāḥ $S_{67}^{S} \quad$ 61d śāntiṃ kurvantu me sadā ] $\mathrm{N}_{82}^{K} \mathrm{~N}_{58}^{K} \mathrm{E}^{N} \mathrm{~B}_{99}^{C} \mathrm{P}_{32}^{T}$, śivańkurvantu me dhruvam $\mathrm{N}_{45}^{C}$, śivam kurvantu me sadā $\mathrm{N}_{12}^{K}$, kurvantv ārogyam uttamam $S_{67}^{S} \quad$ 62a nairṛtyāṃ ] $\mathrm{N}_{82}^{K} \mathrm{~N}_{12}^{K} \mathrm{E}^{N} \mathrm{~B}_{99}^{C} \dot{S}_{67}^{S} \mathrm{P}_{3^{2}}^{T}$, nairityān $\mathrm{N}_{45}^{C}$, nairityāṃ $\mathrm{N}_{58}^{K} \bullet$ tu gaṇāḥ krūrā ] $\mathrm{N}_{45}^{C} \mathrm{~N}_{82}^{K} \mathrm{~N}_{12}^{K} \mathrm{E}^{N} \mathrm{~B}_{99}^{C} \dot{S}_{67}^{S}$, diśi gaṇāḥ krūrā $\mathrm{N}_{58}^{K}$, tu gaṇā ye tu $\mathrm{P}_{3^{2}}^{T} \quad$ 62b rākṣasāḥ khaḍgapāṇayah ] $\mathrm{N}_{58}^{K} \mathrm{~S}_{67}^{S} \mathrm{P}_{3^{2}}^{T}$ rākṣasā mṛtyurūpiṇaḥ $\mathrm{N}_{82}^{K} \mathrm{E}^{N} \mathrm{~B}_{99}^{C}$, rākșasā daṇ̣apāṇayaḥ $\mathrm{N}_{45}^{C}$, rākṣasā mṛtyupāṇayaḥ $\mathrm{N}_{12}^{K} \quad$ 62c sunīlākṣā nīlanibhās ] $\mathrm{K}^{\Sigma} \mathrm{E}^{N} \mathrm{~B}_{99}^{C} \mathrm{~S}_{67}^{S} \mathrm{P}_{3^{2}}^{T}$, supītākșā pìtākșā $\mathrm{N}_{45}^{C} \quad$ 62d tathā vai ] $\mathrm{N}_{45}^{C} \mathrm{~K}^{\Sigma} \mathrm{E}^{N} \mathrm{~B}_{99}^{C} \mathrm{P}_{3^{2}}^{T}$, tathānye $S_{67}^{S}$ - nīla $\left.{ }^{\circ}\right] \mathrm{K}^{\Sigma} \mathrm{E}^{N} \mathrm{~B}_{99}^{C} \hat{S}_{67}^{S} \mathrm{P}_{32}^{T}$, pìta ${ }^{\circ} \mathrm{N}_{45}^{C}$ 
divyāntarikṣabhaumāś ca pātālatalavāsinaḥ| rudrapradhyānaniratāḥ śāntiṃ kurvantu me sadā|| 63||

\title{
[Gaṇas:W]
}

vāruṇyāṃ vai gaṇā ye tu satataṃ pāśapāṇayaḥ| suśyāmākṣāḥ śyāmanibhās tathā vai śyāmalohitāḥ|| 64||

\author{
divyāntarikṣabhaumāś ca pātālatalavāsinaḥ| \\ parameśārcanaratāḥ kurvantu mama śāntikam|| 65||
}

[Gaṇas : NW]

vāyavyāṃ diśi gaṇā ye tu satataṃ dhvajapāṇayaḥ| supītākṣāh pịtanibhās tathā vai pītalohitāḥ|| 66||

64-65 Cf. İśgP, Mantrapāda 30.84 (cont.) : vāruṇyām diśi śivasya bhagavato ye gaṇāḥ pāśapāṇayaḥ śabalāḥ śabalākṣạ̣̄ ityādi| $\quad$ 66-67 Cf. İśgP, Mantrapāda 30.84 (cont.): vāyavyāṃ diśi śivasya bhagavato ye gaṇāḥ an̉kuśapāṇayaḥ pītāḥ ityādi|

63a divyāntarikṣabhaumāś ] $\mathrm{N}_{82}^{K} \mathrm{~N}_{12}^{K} \mathrm{E}^{N} S_{67}^{S} \mathrm{P}_{32}^{T}$, divyāntarikṣabhūmāś $\mathrm{N}_{45}^{C}$, divyāntarīkșabhaumāś $\mathrm{N}_{58}^{K} \mathrm{~B}_{99}^{C} \quad \mathbf{6 3}_{3} \mathrm{c}$ rudrapradhyānaniratāḥ ] $\mathrm{N}_{82}^{K} \mathrm{~N}_{12}^{K} \mathrm{~B}_{99}^{C}$, śivabhaktā mahātmānaḥ $\mathrm{N}_{45}^{C}$, rudraikadhyānaniratā $\mathrm{N}_{58}^{K}$, rudrasya dhyānaniratāḥ $\mathrm{E}^{N}$, rudradhyānaparā nityam $S_{67}^{S}$, rudradhyānaikaparamāḥ $\mathrm{P}_{32}^{T} \quad$ 63d śāntim kurvantu me sadā ] $\mathrm{K}^{\Sigma} \mathrm{E}^{N} \mathrm{~B}_{99}^{C} \hat{S}_{67}^{S} \mathrm{P}_{32}^{T}$, kurvantu mama śāntikam $\mathrm{N}_{45}^{C} \quad \mathbf{6 4 a}$ vāruṇyām vai gaṇā ye tu ] $\mathrm{N}_{82}^{K} \mathrm{E}^{N} \mathrm{~B}_{99}^{C}$, aparāsāngaṇā ye tu $\mathrm{N}_{45}^{C}$, aparasyāṃ gaṇā ye tu $\mathrm{N}_{12}^{K}$, paścimāyāṃ gaṇā ye tu $S_{67}^{S}$, vāruṇyāṃ ye gaṇāḥ sarve $\mathrm{N}_{5^{8}}^{K} \mathrm{P}_{3^{2}}^{T} \quad 64 \mathrm{~b}$ pāśa $\left.{ }^{\circ}\right] \mathrm{N}_{45}^{C} \mathrm{~K}^{\Sigma} \mathrm{E}^{N} \mathrm{~B}_{99}^{C} \mathrm{P}_{3^{2}}^{T}$, megha ${ }^{\circ} \hat{S}_{67}^{S}$ 64c suśyāmākșāḥ ] $\mathrm{K}^{\Sigma} \mathrm{E}^{N} \mathrm{~B}_{99}^{C} \hat{\mathrm{S}}_{67}^{S}$, suśyāmākșā $\mathrm{N}_{45}^{C}$, sumuktāksāa $\mathrm{P}_{32}^{T} \bullet$ śyāmanibhās ] $\mathrm{K}^{\Sigma} \mathrm{E}^{N} \mathrm{~B}_{99}^{C} \hat{S}_{67}^{S}$, śyāmanibhā $\mathrm{N}_{45}^{C}$, muktanibhāḥ $\mathrm{P}_{32}^{T} \quad$ 64d śyāmalohitāh ] $\mathbf{K}^{\Sigma} \mathrm{E}^{N} \mathrm{~B}_{99}^{C} \hat{S}_{67}^{S}$, śyāmalohitā $\mathrm{N}_{45}^{C}$, muktalohitāḥ $\mathrm{P}_{32}^{T} \quad \mathbf{6 5}$ a divyāntarikșa $\left.{ }^{\circ}\right] \mathrm{N}_{45}^{C} \mathrm{~N}_{82}^{K} \mathrm{~N}_{12}^{K} \mathrm{~B}_{99}^{C} \hat{S}_{67}^{S} \mathrm{P}_{32}^{T}$, divyantarīkșa ${ }^{\circ} \mathrm{N}_{58}^{K} \mathrm{E}^{N} \quad{ }_{65} \mathrm{c}$ parameśārcanaratāḥ ] $\mathrm{N}_{45}^{C} \mathrm{~K}^{\Sigma} \mathrm{B}_{99}^{C} \hat{S}_{67}^{S} \mathrm{P}_{72}^{T}$, śivabhaktiparāḥ sarve $\mathrm{E}^{N}$, śivabhaktā mahātmānaḥ $\mathrm{P}_{32}^{T} \quad$ 65d kurvantu mama śāntikạ̣ ] $\mathrm{N}_{82}^{K} \mathrm{~N}_{5^{8}}^{K} \mathrm{E}^{N} \mathrm{~B}_{99}^{C} \hat{S}_{67}^{S}$, kurvantv ārogyam uttamām $\mathrm{N}_{45}^{C}$, kurvantv ārogyam uttamaṃ $\mathrm{N}_{12}^{K} \mathrm{P}_{72}^{T}$, sāantiṃ kurvantu me sadā $\mathrm{P}_{32}^{T} \quad 66 \mathbf{a}$ vāyavyāṃ ] $\mathrm{K}^{\Sigma} \mathrm{E}^{N} \mathrm{~B}_{99}^{C} \hat{S}_{67}^{S} \mathbf{P}^{\Sigma}$, vāyavyā $\mathrm{N}_{45}^{C} \bullet$ diśi gaṇā ye tu ] $\mathrm{N}_{82}^{K} \mathrm{~N}_{58}^{K} \mathrm{~B}_{99}^{C}$, tu gaṇā ye tu $\mathrm{N}_{12}^{K} \mathrm{E}^{N} \mathrm{P}_{72}^{T}$, tu gaṇā ye vai $\mathrm{N}_{45}^{C}$, ye diśi gaṇāḥ $S_{67}^{S}$, ye gaṇāḥ sarve $\mathrm{P}_{3^{2}}^{T} \quad 66 \mathrm{c}$ supītākṣāḥ pītanibhās ] $\mathrm{K}^{\Sigma} \mathrm{E}^{N}$, supitākșā pìtanibhā $\mathrm{N}_{45}^{C} \mathrm{~B}_{99}^{C}$, āpìtākṣāḥ pītanibhās $S_{67}^{S}$, sudhūmrākșā dhūmranibhāḥ $\mathrm{P}_{3^{2}}^{T}$, supadmākṣāḥ padmanibhāḥ $\mathrm{P}_{72}^{T} \quad$ 66d tathā vai ] $\mathrm{N}_{45}^{C} \mathrm{~K}^{\Sigma} \mathrm{E}^{N} \mathrm{~B}_{99}^{C} \mathbf{P}^{\Sigma}$, tathānye $S_{67}^{S} \bullet$ pītalohitāh ] $\mathrm{N}_{82}^{K} \mathrm{~N}_{12}^{K} \mathrm{E}^{N} \mathrm{~B}_{99}^{C} \hat{S}_{67}^{S}$, pītalohitām $\mathrm{N}_{45}^{C}$, pitalohitāḥ $\mathrm{N}_{58}^{K}$, dhūmralohitāḥ $\mathrm{P}_{32}^{T}$, padmalohitāḥ $\mathrm{P}_{72}^{T}$ 
divyāntarikṣabhaumāś ca pātālatalavāsinaḥ|
śivabhaktiparāḥ sarve kṣemaṃ kurvantu me sadā|| 67||

[Ganas: N]

uttarasyāṃ gaṇā ye tu satataṃ nidhipāṇayaḥ|

śavalākṣạ̣̄ śavalanibhās tathā śavalalohitāḥ|| 68||

divyāntarikṣabhaumāś ca pātālatalavāsinaḥ|

śivapūjāsamudyuktāḥ kṣemaṃ kurvantu me sadā|| 69||

68-69 Cf. İśgP, Mantrapāda 30.84 (cont.) : om kauberyāṃ diśi śivasya bhagavato ye gaṇāḥ gadāpāṇayaḥ sitāḥ sitākṣāḥ sitalohitā ityādi|

67a divyāntarikșa $\left.{ }^{\circ}\right] \mathrm{N}_{45}^{C} \mathrm{~N}_{12}^{K} \hat{\mathrm{S}}_{67}^{S} \mathrm{P}_{32}^{T}$, divyantarikșa ${ }^{\circ} \mathrm{B}_{99}^{C}$, divyantarīkșa ${ }^{\circ} \mathrm{N}_{82}^{K} \mathrm{~N}_{58}^{K} \mathrm{E}^{N}$, divyantarikṣāo $\mathrm{P}_{72}^{T} \bullet$ obhaumāśs ] $\mathrm{K}^{\Sigma} \mathrm{E}^{N} \mathrm{~B}_{99}^{C} \hat{S}_{67}^{S} \mathbf{P}^{\Sigma}$, obhaumāṃś $\mathrm{N}_{45}^{C} \quad{ }_{67}$ b pātāla ${ }^{\circ}$ ] $\mathbf{K}^{\Sigma} \mathrm{E}^{N} \mathrm{~B}_{99}^{C} \dot{S}_{67}^{S} \mathbf{P}^{\Sigma}$, pātālā $\mathrm{N}_{45}^{C} \quad \mathbf{6 7}_{7} \mathbf{c}$ śivabhaktiparāh sarve ] $\mathrm{N}_{45}^{C} \mathrm{~N}_{82}^{K} \mathrm{~N}_{5^{8}}^{K} \mathrm{E}^{N} \mathrm{~B}_{99}^{C} \dot{S}_{67}^{S}$, śivabhaktā mahātmānaḥ $\mathrm{N}_{12}^{K}$, śivapūjāsamāyuktāḥ $\mathrm{P}_{32}^{T}$, parameśārcanaratāḥ $\mathrm{P}_{72}^{T} \quad 67 \mathrm{~d}$ kṣemaṃ kurvantu me sadā ] $\mathrm{N}_{82}^{K} \mathrm{~N}_{58}^{K} \mathrm{~B}_{99}^{C} \hat{S}_{67}^{S}$, kṣemaṃ kurvāntu me sadā $\mathrm{N}_{45}^{C}$, kurvantu mama śāntikaṃ $\mathrm{N}_{12}^{K}$, kṣamaṃ kurvantu me sadā $\mathrm{E}^{N}$, kṣemaṃ kurvantu sarvadā $\mathrm{P}_{32}^{T}$, śāntiṃ kurvantu me sadā $\mathrm{P}_{72}^{T} \quad$ 68a uttarasyāṃ gaṇā ye tu ] $\mathrm{N}_{82}^{K} \mathrm{~N}_{58}^{K} \mathrm{E}^{N} \mathrm{~B}_{99}^{C}$, uttarā dig gạ̣ā ye tu $\mathrm{N}_{45}^{C}$, uttarāyā gạ̣ā ye vai $\mathrm{N}_{12}^{K}$, bhaumyāṃ diśi gaṇā ye tu $\hat{S}_{67}^{S}$, kauberyāṃ ye gaṇāh sarve $\mathrm{P}^{\Sigma} \quad$ 68c śavalākṣāḥ śavalanibhās ] $\mathrm{K}^{\Sigma} \mathrm{E}^{N}$, supītākṣā pītanibhā $\mathrm{N}_{45}^{C}$, śavalākșāḥ śavanibhās $\mathrm{B}_{99}^{c}$, supītākșāḥ pītanibhāḥ $\mathrm{P}^{\Sigma}$, āpingākṣāḥ pinganibās $\hat{S}_{67}^{S} \quad 68 \mathrm{~d}$ tathā śavalalohitāḥ ] $\mathrm{N}_{12}^{K} \mathrm{~B}_{99}^{C}$, tathā vai pītalohitāḥ $\mathrm{N}_{45}^{C} \mathbf{P}^{\Sigma}$, tathā vai śavalohitāḥ $\mathrm{N}_{82}^{K a c}$, tathā vai śavalalohitāḥ $\mathrm{N}_{82}^{K p c} \mathrm{~N}_{58}^{K} \mathrm{E}^{N}$ (unmetr.), tathānye pingalohitāḥ $S_{67}^{S} \quad$ 69a divyāntarikșa $\left.{ }^{\circ}\right] \mathrm{N}_{45}^{C} \mathrm{~N}_{82}^{K} \mathrm{~N}_{12}^{K} \dot{S}_{67}^{S} \mathrm{P}_{32}^{T}$, divyantarīkșa ${ }^{\circ} \mathrm{N}_{58}^{K} \mathrm{E}^{N}$, divyantarikșa ${ }^{\circ} \mathrm{B}_{99}^{C} \mathrm{P}_{72}^{T} \quad 69$ b pātālatalavāsinaḥ ] $\mathrm{K}^{\Sigma} \mathrm{E}^{N} \hat{S}_{67}^{S} \mathbf{P}^{\Sigma}$, pātālātalavāsināḥ $\mathrm{N}_{45}^{C}$, pātālatalavāśinaḥ $\mathrm{B}_{99}^{C} \quad \mathbf{6 9 c}$ śivapūjāsamudyuktāḥ ] $\mathrm{K}^{\Sigma} \mathrm{E}^{N} \mathrm{~B}_{99}^{C}$, parameśārcanaratāḥ $\mathrm{N}_{45}^{C} S_{67}^{S} \mathrm{P}_{72}^{T}$, śivabhaktiparāḥ sarve $\mathrm{P}_{3^{2}}^{T} \quad$ 69d kṣemaṃ kurvantu me sadā $] \mathrm{K}^{\Sigma} \mathrm{E}^{N} \mathrm{~B}_{99}^{C}$, kurvantv ārogyam uttamam $\mathrm{N}_{45}^{C} \hat{\mathrm{S}}_{67}^{S}$, śāntiṃ kurvantu me sadā $\mathbf{P}^{\Sigma}$ 
[Gaṇas : NE]

aiśānyāṃ vai gaṇā ye tu praśāntāḥ śūlapāṇayaḥ|

sūkṣmāḥ susūkṣmasadṛ́sās tathā vai sūkṣmalohitāḥ̣|| 70||

divyāntarikṣabhaumāś ca pātālatalavāsinaḥ|

śivapūjāsamudyuktāḥ kṣemaṃ kurvantu me sadā|| 71\|

[Ganas: Below]

adhobhāge gaṇā ye tu satataṃ śūlapāṇayaḥ|

dhūmrā dhūmranibhāḥ sarve tathā vai dhūmralohitāḥ|| 72||

70-71 Cf. BhavP 1.178.25-27 : aiśānyāṃ saṃsthitā ye tu praśāntāḥ śūlapāṇayaḥ| bhasmoddhūlitadehāś ca nīlakaṇțhā vilohitāḥ|| divyāntarikṣā bhaumāś ca pātālatalavāsinaḥ| sūryapūjākarā nityaṃ pūjayitvāṃśumālinam|| tataḥ suprītamanaso lokapālaị̣ samanvitāḥ| sāantiṃ kurvantu me nityaṃ kaṃ prayacchantu pūjitāḥ|| • İ́śg, Mantrapāda 30.84 (cont.) : om aiśānyāṃ diśi śivasya bhagavato ye gaṇāḥ śūlapāṇayaḥ kapilā ityādi| 72-73 Cf. İśgP, Mantrapāda 30.84 (cont.) : om adhastād diśi śivasya bhagavato ye gaṇāḥ cakrapāṇayạ̣ dhūmrā dhūmrākṣā dhūmralohitā divyantarikṣabhaumāḥ ityādi|

71a-72d Omitted in $\hat{S}_{67}^{S}$

$70 a$ aiśānyāṃ vai gaṇā ye tu ] $\mathrm{N}_{82}^{K} \mathrm{E}^{N} \mathrm{~B}_{99}^{C} \mathrm{P}_{3^{2}}^{T}$, īsānyā diśi gạ̣ā ye tu $\mathrm{N}_{45}^{C}$ (unmetr.), aiśānyā vai gaṇā ye tu $\mathrm{N}_{12}^{K}$, aiśānyāṃ diśi gaṇā ye tu $\mathrm{N}_{5^{8}}^{K}$, aiśānyāṃ ye diśi gaṇā+ḥ+ $S_{67}^{S}$, aiśānyāṃ tu gaṇā ye tu $\mathrm{P}_{72}^{T} \quad 7 \mathrm{ob}$ praśāntāh ] $\mathrm{N}_{45}^{C} \mathrm{~N}_{82}^{K} \mathrm{~N}_{5^{8}}^{K} \mathrm{E}^{N} \mathrm{~B}_{99}^{C} \mathrm{P}^{\Sigma}$, praśāntā $\mathrm{N}_{12}^{K} \hat{S}_{67}^{S} \bullet{ }^{\circ}$ pāṇayaḥ ] $\mathrm{K}^{\Sigma} \mathrm{E}^{N} \mathrm{~B}_{99}^{C} \hat{S}_{67}^{S} \mathbf{P}^{\Sigma}$, ${ }^{\circ}$ pāṇayam $\mathrm{N}_{45}^{C} \quad 70 \mathrm{c}$ sūkṣmāḥ susūkṣmasadṛśās ] $\mathrm{N}_{82}^{K} \mathrm{E}^{N} \mathrm{~B}_{99}^{C}$, susūkṣmākṣā sūkṣmanibhā $\mathrm{N}_{45}^{C}$, susūkṣmāḥ sūkṣmasadṛsāḥ $\mathrm{N}_{12}^{K}$, susūkṣmākṣaa sūkṣmanibhās $S_{67}^{S}$, suśvetākṣāh śvetanibhāh $\mathrm{P}_{32}^{T}$, susūkṣmākṣāh sūkșmanibhās $\mathrm{N}_{5^{8}}^{K} \mathrm{P}_{72}^{T} \quad$ 7od tathā vai ] $\mathrm{N}_{45}^{C} \mathrm{~K}^{\Sigma} \mathrm{E}^{N} \mathrm{~B}_{99}^{C} \mathbf{P}^{\Sigma}$, tathānye $\grave{S}_{67}^{S} \bullet$ sūkșma $\left.{ }^{\circ}\right] \mathrm{N}_{45}^{C} \mathrm{~K}^{\Sigma} \mathrm{E}^{N} \mathrm{~B}_{99}^{C} \dot{S}_{67}^{S} \mathrm{P}_{72}^{T}$, śveta ${ }^{\circ} \mathrm{P}_{32}^{T}$ 71a divyāntarikșa ${ }^{\circ}$ ] $\mathrm{N}_{45}^{C} \mathrm{~N}_{82}^{K} \mathrm{~N}_{12}^{K} \mathrm{P}_{32}^{T}$, divyantarīkșa ${ }^{\circ} \mathrm{N}_{5^{8}}^{K} \mathrm{E}^{N}$, divyantarikṣa ${ }^{\circ} \mathrm{B}_{99}^{C} \mathrm{P}_{72}^{T} \bullet$ obhaumāś ] $\mathrm{K}^{\Sigma} \mathrm{E}^{N} \mathrm{~B}_{99}^{C} \mathbf{P}^{\Sigma}$, obhaumāṃśs $\mathrm{N}_{45}^{C} \quad 7 \mathbf{c}$ śivapūjāsamudyuktāḥ ] $\mathrm{K}^{\Sigma} \mathrm{B}_{99}^{C} \mathrm{P}_{72}^{T}$, śivapūjāsamudyuktā $\mathrm{N}_{45}^{C} \mathrm{E}^{N}$, parameśārcanaratāḥ $\mathrm{P}_{3^{2}}^{T} \quad$ 71d kṣemạ̣ kurvantu me sadā ] $\mathrm{N}_{45}^{C} \mathrm{~K}^{\Sigma} \mathrm{E}^{N} \mathrm{~B}_{99}^{C} \mathrm{P}_{72}^{T}$, śāntiṃ kurvantu me sadā $\mathrm{P}_{32}^{T} \quad$ 72a adhobhāge ] $\mathrm{K}^{\Sigma} \mathrm{E}^{N} \mathrm{~B}_{99}^{C}$, anābhāge $\mathrm{N}_{45}^{C}$, aiśānyāṃ vai $\mathrm{P}_{32}^{T}$, adhobhāga ${ }^{\circ} \mathrm{P}_{72}^{T} \quad{ }_{72} \mathbf{b}$ satatam śūlapāṇayạ̣ ] $\mathrm{N}_{82}^{K} \mathrm{~N}_{58}^{K} \mathrm{E}^{N} \mathrm{~B}_{99}^{C}$, dhūmāñkā dhūmasannibhāḥ $\mathrm{N}_{45}^{C}$, dhūmrākṣā dhūmrasannibhāḥ $\mathrm{N}_{12}^{K}$, satataṃ cakrapāṇayaḥ $\left.\mathbf{P}^{\Sigma} \quad 7^{2} \mathrm{~cd} \quad\right] \quad \mathrm{N}_{82}^{K} \mathrm{~N}_{58}^{K} \mathrm{E}^{N} \mathrm{~B}_{99}^{C}$, dhūmā sulohitā nityaṃ dhūmā yā dhūmavavarcasāḥ $\mathrm{N}_{45}^{C}$, dhūmrās sulohitā nityaṃ dhūmrābhrādhūmravarcasāḥ $\mathrm{N}_{12}^{K}$, sunīlākṣā nīlanibhāḥ tathā vai nīlalohitāḥ $\mathrm{P}_{32}^{T}$, sudhūmrākṣā dhūmranibhāḥ tathā vai dhūmralohitāḥ $\mathrm{P}_{72}^{T}$ 


\section{divyāntarikṣabhaumāś ca pātālatalavāsinaḥ| śivaṃ kurvantu me nityam alakṣmīmalanāśanam|| 73||}

[Gaṇas: Above]

ūrdhvabhāge gaṇā ye tu mahābalaparākramāḥ| susūkṣmākṣāḥ sūkṣmanibhās tathā vai sūkṣmalohitāḥ|| 74||

divyāntarikṣabhaumāś ca pātālatalavāsinaḥ| śivapūjāsamudyuktā aśubhaṃ nāśayantu me|| 75||

74-75 Cf. İśgP, Mantrapāda 30.84 (cont.) : ūrdhvāyāṃ diśi śivasya bhagavato ye gaṇāḥ padmapāṇayaḥ sūkṣmāḥ sūkṣmākṣāḥ sūkṣmalohitā ityādi|

73a divyāntarikșa $\left.{ }^{\circ}\right] \mathrm{N}_{45}^{C} \mathrm{~N}_{82}^{K} \mathrm{~N}_{12}^{K} \hat{\mathrm{S}}_{67}^{S} \mathrm{P}_{32}^{T}$, divyantarīkșa ${ }^{\circ} \mathrm{N}_{58}^{K} \mathrm{E}^{N}$, divyantarikșa ${ }^{\circ} \mathrm{B}_{99}^{C} \mathrm{P}_{72}^{T} \bullet$ obhaumāś ] $\mathbf{K}^{\Sigma} \mathrm{E}^{N} \mathrm{~B}_{99}^{C} \hat{S}_{67}^{S} \mathbf{P}^{\Sigma}$, obhūmāś $\mathrm{N}_{45}^{C} \quad 73^{\circ}{ }^{\circ}$ vāsinah ] $\mathbf{K}^{\Sigma} \mathrm{E}^{N} \mathrm{~B}_{99}^{C} \dot{\mathrm{S}}_{67}^{S} \mathbf{P}^{\Sigma}$, ${ }^{\circ}$ vāsināḥ $\mathrm{N}_{45}^{C} \quad 73 \mathrm{c}$ śivaṃ kurvantu me nityam ] $\mathrm{K}^{\Sigma} \mathrm{E}^{N} \mathrm{~B}_{99}^{C}$, śiva kurvantu me nityam $\mathrm{N}_{45}^{C}$, śivapūjāsamāyuktā $\dot{S}_{67}^{S}$, śivapūjāsamāyuktāḥ $\mathrm{P}_{32}^{T}$, śivapūjāsamudyuktāḥ $\mathrm{P}_{72}^{T} \quad 73$ d alakșmīmalanāśanam ] $\mathrm{N}_{45}^{C} \mathrm{~N}_{82}^{K} \mathrm{E}^{N} \mathrm{~B}_{99}^{C}$, alakșmīmalanāśanāḥ $\mathrm{N}_{12}^{K}$, alakṣmīmalanāśanah $\mathrm{N}_{12}^{K}$, aśubhaṃ nāśayantu me $S_{67}^{S} \mathrm{P}_{32}^{T}$, śāntiṃ kurvantu me sadā $\mathrm{P}_{72}^{T} \quad$ 74a ūrdhvabhāge ] $\mathbf{K}^{\Sigma} \mathrm{B}_{99}^{C} S_{67}^{S} \mathrm{P}_{32}^{T}$, ūdhvabhāge $\mathrm{N}_{45}^{C}$, arddhabhāge $\mathrm{E}^{N}$, ūrdhvabhāga ${ }^{\circ} \mathrm{P}_{72}^{T} \quad 74 \mathbf{b}$ mahābalaparākramāḥ ] $\mathrm{N}_{82}^{K} \mathrm{E}^{N} \mathrm{~B}_{99}^{C} \hat{S}_{67}^{S}$, svatantā dhyāyinah parāḥ $\mathrm{N}_{45}^{C}$, svatantradhyāyino parāh $\mathrm{N}_{12}^{K}$, svatantradhyāyinah parāḥ $\mathrm{N}_{58}^{K}$, satatam pāśapāṇayạ̣ $\mathrm{P}_{32}^{T}$, satatam padmapāṇayaḥ $\mathrm{P}_{72}^{T} \quad{ }_{74 \mathrm{c}}$ susūkṣmākṣāḥ sūkṣmanibhās ] $\mathrm{N}_{82}^{K} \mathrm{E}^{N} \mathrm{~B}_{99}^{C}{ }^{S}{ }_{67}^{S} \mathrm{P}_{32}^{T}$, sūkṣmāḥ sūkṣmanibhāś caiva $\mathrm{N}_{12}^{K} \mathrm{~N}_{58}^{K}$, susūkṣmā sūkṣmanibhā $\mathrm{N}_{45}^{C}$ (unmetr.), susū $\sqcup s a ̄ h ̣ ~ s u ̄ k s ̣ m a \sqcup \sqcup \sqcup \mathrm{P}_{72}^{T}$ 74d tathā vai ] $\mathrm{N}_{45}^{C} \mathrm{~N}_{82}^{K} \mathrm{~N}_{58}^{K} \mathrm{E}^{N} \mathrm{~B}_{99}^{C} \mathbf{P}^{\Sigma}$, tathā ca N $\mathrm{N}_{12}^{K}$, tathānye $\hat{S}_{67}^{S} \quad 75^{\mathbf{a}}$ divyāntarikṣa ${ }^{\circ}$ ] $\mathrm{N}_{45}^{C} \mathrm{~N}_{82}^{K} \mathrm{~N}_{12}^{K} \hat{S}_{67}^{S} \mathrm{P}_{32}^{T}$, divyantarīkșa ${ }^{\circ} \mathrm{N}_{58}^{K} \mathrm{~B}_{99}^{C}$, divyantarikșa ${ }^{\circ} \mathrm{E}^{N} \mathrm{P}_{72}^{T} \quad 7^{2}{ }^{\circ}$ samudyuktā ] $\mathrm{N}_{45}^{C} \mathrm{~K}^{\Sigma} \mathrm{B}_{99}^{C}$, ${ }^{\circ}$ samādyukta ${ }^{N}$, ${ }^{\circ}$ sadodyuktā $S_{67}^{S}$, ${ }^{\circ}$ samāyuktāḥ $\mathrm{P}_{3^{2}}^{T}$, ${ }^{\circ}$ samāsaktāḥ $\mathrm{P}_{72}^{T} \quad 75^{\mathbf{d}}$ aśubhaṃ nāśayantu me ] $\mathrm{K}^{\Sigma} \mathrm{E}^{N} \mathrm{~B}_{99}^{C} \hat{S}_{67}^{S} \mathrm{P}_{32}^{T}$, aśubhaṃ yāntu yāta me $\mathrm{N}_{45}^{C}$, śāntiṃ kurvantu me sadā $\mathrm{P}_{72}^{T}$ 


\title{
[Ganas : Conclusion]
}

ete gaṇā mahātmāno mahābalaparākramāḥ| śivaṃ sampūjya yatnena baliṃ teșām vinikṣipet| tataḥ suprītamanasạ̣ śāntiṃ kurvantu me sadā|| $76 \|$

\section{[Dikpālas]}

\section{[Indra in Amarāvatī]}

\author{
amarāvatī nāma purī pūrvabhāge vyavasthitā| \\ vidyādharasamākīrṇā siddhagandharvasevitā| \\ ratnaprākārarucirā sarvaratnopaśobhitā|| 77||
}

76 Cf. İ́sPP, Mantrapāda 3o.84 (cont.) : ebhir vikārya prīṇāti mantraị̣ pāriṣadāṃ balim|| 77-8o Cf. BhavP 1.178.28-31 : amarāvatī purī nāma pūrvabhāge vyavasthitā| vidyādharagaṇākīrṇā siddhagandharvasevitā|| ratnaprākārarucirā mahāratnopaśobhitā| tatra devapatiḥ śrīmān vajrapāṇir mahābalaḥ| gopatir gosahasreṇa śobhamānena śobhate|| airāvatagajārūḍho gaurikābho mahādyutiḥ| devendrah satatam hṛșța ādityārādhane rataḥ|| sūryajñānaikaparamaḥ sūryabhaktisamanvitaḥ| sūryapraṇāmaḥ paramāṃ śāntiṃ te 'dya prayacchatu||

76d After this $\hat{S}_{67}^{S}$ adds two pādas : te pi me prītamanasah pratig̣̣hṇantv imāṃ balim| 76f After this $\mathrm{N}_{58}^{K}$ adds two pādas: prayacchanty āśu martyānāṃ tadbhaktānāṃ viśeșatah

76a gaṇā ] $\mathrm{N}_{45}^{C} \mathrm{~K}^{\Sigma} \mathrm{B}_{99}^{c} \hat{S}_{67}^{S} \mathrm{P}^{\Sigma}$, gaṇāḥ $\mathrm{E}^{N} \quad{ }_{76}$ b mahābalaparākramāḥ ]

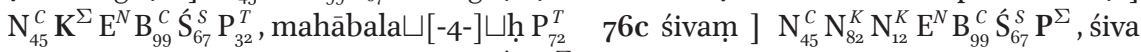
$\mathrm{N}_{58}^{K} \bullet$ sampūjya yatnena ] $\mathrm{N}_{82}^{K} \mathrm{E}^{N} \mathrm{~B}_{99}^{C} \dot{S}_{67}^{S} \mathbf{P}^{\Sigma}$, sampūjya yaṃnena $\mathrm{N}_{45}^{C}$, sampūjayantena $\mathrm{N}_{12}^{K} \quad{ }_{76} \mathbf{d}$ balim teșām ] $\mathrm{K}^{\Sigma} \mathrm{E}^{N} \mathrm{~B}_{99}^{C}$, balintathā $\mathrm{N}_{45}^{c}$, balim eșām $\hat{S}_{67}^{S} \mathbf{P}^{\Sigma} \bullet$ vinikṣipet ] $\mathbf{K}^{\Sigma} \mathrm{E}^{N} \mathrm{~B}_{99}^{C} \hat{S}_{67}^{S} \mathbf{P}^{\Sigma}$, vinikșepayet $\mathrm{N}_{45}^{C}$ (unmetr.) 76 e suprītamanasah ] $\mathrm{N}_{82}^{K} \mathrm{~N}_{12}^{K} \mathrm{E}^{N} \mathrm{~B}_{99}^{C} \mathrm{P}_{72}^{T}$, supìtamanasaḥ $\mathrm{N}_{45}^{C}$, prītā mahātmānaḥ $\mathrm{N}_{58}^{K}$, samprītamanasaḥ $\hat{S}_{67}^{S}$, suprītamanasā $\mathrm{P}_{32}^{T}$ 76f śāntim kurvantu me sadā ] $\mathrm{N}_{82}^{K} \mathrm{~N}_{12}^{K} \mathrm{E}^{N} \mathrm{~B}_{99}^{C} \hat{S}_{67}^{S} \mathrm{P}_{72}^{T \text { pc }} \mathrm{P}_{72}^{T}$, saantim kurvantu sārvatah $\mathrm{N}_{45}^{C}$, śāntim āśu balaśriyaṃ $\mathrm{N}_{58}^{K}$, saanntim kurvantu nạ̣ sadā $\mathrm{P}_{72}^{T a c} \quad 77$ a amarāvatī nāma purī ] $\mathrm{N}_{82}^{K} \mathrm{~N}_{12}^{K} \mathrm{E}^{N} \mathrm{~B}_{99}^{C} \hat{S}_{67}^{S}$, amarāvatī purī nāmā $\mathrm{N}_{45}^{C}$, amarāvatī purī nāma $\mathrm{N}_{58}^{K p c} \mathrm{P}_{32}^{T}$, amavatī purī nāma $\mathrm{N}_{58}^{K a c}$ (unmetr.), nāmnāmarāvatī puryāḥ $\mathrm{P}_{72}^{T} \quad 77 \mathbf{b}$ vyavasthitā ] $\mathrm{N}_{45}^{C} \mathrm{~N}_{12}^{K} \mathrm{E}^{N} \mathrm{~B}_{99}^{C} \hat{S}_{67}^{S} \mathbf{P}^{\Sigma}$, vyavasthitāḥ $\mathrm{N}_{82}^{K} \mathrm{~N}_{58}^{K} \quad 77 \mathrm{c} \quad{ }^{\circ}$ samākīrṇā ] $\mathrm{N}_{82}^{K} \mathrm{~N}_{58}^{K} \mathrm{E}^{N} \mathrm{~B}_{99}^{C}$, ${ }^{\circ}$ gaṇākīrṇṇ̣a ${ }^{\circ} \mathrm{N}_{77}^{K o}$, ogaṇākīrṇṇe $\mathrm{N}_{45}^{C}$, ${ }^{\circ}$ gaṇākīrṇa ${ }^{\circ} \mathrm{N}_{12}^{K} S_{67}^{S} \mathbf{P}^{\Sigma} \quad 77 \mathbf{d}{ }^{\circ}$ gandharvasevitā ] $\Sigma$, ${ }^{\circ}$ gāndharvasevitā $\mathrm{N}_{45}^{C}$, ${ }^{\circ}$ gandhasevitāḥ $\mathrm{N}_{58}^{K}$ (unmetr.) $77{ }^{\circ}{ }^{\circ}$ rucirā ] $\mathrm{N}_{82}^{K} \mathrm{~N}_{12}^{K} \mathrm{E}^{N} \mathrm{~B}_{99}^{C}$, ${ }^{\circ}$ racitā $\mathrm{N}_{77}^{K o} \mathrm{~N}_{45}^{C} \mathrm{~N}_{58}^{K} \hat{S}_{67}^{S}$, ${ }^{\circ}$ nicitāḥ $\mathrm{P}_{32}^{T}$, okhacitā $\mathrm{P}_{72}^{T} \quad{ }_{77} \mathrm{f}$ sarva $\left.{ }^{\circ}\right] \mathrm{N}_{45}^{C} \mathrm{~K}^{\Sigma} \mathrm{E}^{N} \mathrm{~B}_{99}^{C}$, mahāo $\mathrm{N}_{77}^{K o} \hat{S}_{67}^{S} \mathbf{P}^{\Sigma} \bullet{ }^{\circ}$ śobhitā ] $\Sigma$, ósotā $\mathrm{N}_{45}^{C}$ (unmetr.), ' 'sobhitāḥ $\mathrm{P}_{32}^{T}$ 
tatra devapatị̣ śrīmān vajrapāṇir mahābalaḥ|

netrānāạ tu sahasreṇa śobhanena virājate || 78||

airāvatagajārūḍho hemavarṇo mahādyutị̣|

devendraḥ satatạ̣ hụștạ parameśārcane ratạ̣|| 79||

śivadhyānaikasampannaḥ śivabhaktisamanvitaḥ|

śivapraṇāmaparamaḥ karotu mama śāntikam|| 80 ||

\section{[Agni in Tejovatī]}

āgneye digvibhāge tu purī tejovatī śubhā| nānādevasamākīrṇā ratnajvālāsamujjvalā|| 81||

81-83 Cf. BhavP 1.178.32-34: āgneyadigvibhāge tu purī tejasvatī śubhā| nānādevagaṇākīrṇā nānāratnopaśobhitā|| tatra jvālāsamākīrṇo dīptāngārasamadyutiḥ| purago dahano devo jvalanạ̣ pāpanāśanaḥ|| ādityārādhanarata ādityagatamānasạ̣| śāntiṃ karotu te devas tathā pāpaparikṣayam||

8oab Omitted in $\hat{S}_{67}^{S} \quad$ 8ob After this $\mathrm{P}_{32}^{T}$ adds two pādas : śivabhaktaih surādyaiś ca devair api sahānugaiḥ| $\mid \mathrm{P}_{72}^{T}$ adds : śivabhaktais tathānyaiś ca devair api sahānugaiḥ|

78a ${ }^{\circ}$ patih ] $\Sigma$, ${ }^{\circ}$ patim $\mathrm{N}_{45}^{C}$, ${ }^{\circ}$ pati $\mathrm{N}_{12}^{K} \quad 78 \mathrm{~b}{ }^{\circ}$ pāṇir $\mathrm{N}_{82}^{K} \mathrm{~N}_{12}^{K} \mathrm{E}^{N} \mathrm{~B}_{99}^{C} \hat{\mathrm{S}}_{67}^{S} \mathrm{P}_{32}^{T}$, ohasto $\mathrm{N}_{77}^{K o} \mathrm{P}_{72}^{T}$, ohastau $\mathrm{N}_{45}^{C}$, ${ }^{\circ}$ pāṇi $\mathrm{N}_{58}^{K} \bullet{ }^{\circ}$ balaḥ ] $\Sigma$, obalāḥ $\mathrm{N}_{45}^{C} \mathrm{~N}_{12}^{K} \quad$ 78d śobhanena virājate ] $\mathrm{N}_{77}^{K o} \mathrm{~N}_{82}^{K} \mathrm{~N}_{58}^{K} \mathrm{~B}_{99}^{C} \hat{S}_{67}^{S} \mathrm{P}_{72}^{T}$, śobhatena virājate $\mathrm{E}^{N}$, śobhamānena rājate $\mathrm{N}_{45}^{C}$, śobhanena virāte $\mathrm{N}_{12}^{K}$ (unmetr., vertical stroke after -śo-), śobhanena virājite $\mathrm{P}_{32}^{T} \quad 79$ a airāvata ${ }^{\circ}$ ] $\Sigma$, airāvaṇa ${ }^{\circ} S_{67}^{S} \bullet{ }^{\circ}$ rūḍho ] $\Sigma$, ${ }^{\circ}$ rūḍhā $\mathrm{N}_{45}^{C} \mathrm{P}_{72}^{T} \quad 79$ b ${ }^{\circ}$ varṇo $] \mathrm{N}_{45}^{C} \mathbf{K}^{\Sigma} \mathrm{B}_{99}^{C} \dot{S}_{67}^{S} \mathrm{P}_{72}^{T}$, ${ }^{\circ}$ varṇnā $\mathrm{N}_{77}^{K o},{ }^{\circ}$ varṇa ${ }^{\circ} \mathrm{E}^{N}$, ${ }^{\circ}$ vastro $\mathrm{P}_{32}^{T} \bullet{ }^{\circ}$ dyutiḥ ] $\Sigma$, ${ }^{\circ}$ dyuti $\mathrm{N}_{77}^{K o} \quad{ }_{79}$ c devendrah ] $\mathrm{N}_{82}^{K} \mathrm{~N}_{12}^{K} \mathrm{E}^{N} \dot{\mathrm{S}}_{67}^{S} \mathbf{P}^{\Sigma}$, devenda $\mathrm{N}_{77}^{K o} \mathrm{~N}_{45}^{C} \mathrm{~N}_{58}^{K} \mathrm{~B}_{99}^{C} \bullet$ satatam ] $\Sigma$, satamtam $\dot{S}_{67}^{S}$ (unmetr.) • hṛșțaḥ ] $\Sigma$, hṛșța $\mathrm{N}_{77}^{K o} \quad$ 79d parameśārcane ratạ̣ ] $\Sigma$, parameśānatatparaḥ $\mathrm{N}_{77}^{K o}$, parameśārcanatatparah $\mathrm{N}_{58}^{K}$ (unmetr.), parameśārcane rata $\mathrm{N}_{45}^{C} \quad$ 8oa ${ }^{\circ}$ dhyānaikasampannạ̣ ] $\mathrm{N}_{45}^{C} \mathrm{~N}_{12}^{K} \mathrm{~N}_{58}^{K} \mathrm{P}_{32}^{T}$, odhyānena sampannạ̣ $\mathrm{N}_{82}^{K} \mathrm{E}^{N} \mathrm{~B}_{99}^{C}$, ${ }^{\circ}$ dhyā(nai)kasampana $\mathrm{N}_{77}^{K o}$ (unmetr.), odhyānaikaparyāptaḥ $\mathrm{P}_{72}^{T} \quad$ 8ob ${ }^{\circ}$ samanvitạ̣ ] $\Sigma$, osamanvita $\mathrm{N}_{77}^{K o}$, ${ }^{\circ}$ samānvitaḥ $\mathrm{N}_{45}^{C} \quad$ 8oc ${ }^{\circ}$ praṇāmaparamaḥ ] $\Sigma$, ${ }^{\circ}$ praṇāmaparamaṃ $\mathrm{N}_{77}^{K o}$, ${ }^{\circ}$ pramāmaparamaḥ $\mathrm{N}_{45}^{C} \quad 81 a$ āgneye digvibhāge tu ] $\mathrm{N}_{82}^{K} \mathrm{E}^{N} \mathrm{~B}_{99}^{C} \hat{S}_{67}^{S}$, āgneyā diśi bhāge tu $\mathrm{N}_{77}^{K o}$, agneyāṃ digbhāge tuh $\mathrm{N}_{45}^{C}$ (unmetr.), agneyyāṃ digvibhāge tu $\mathrm{N}_{12}^{K}$, āgneyyāṃ digvibhāge tu $\mathrm{N}_{58}^{K}$, āgneyyāṃ divi bhāge tu $\mathrm{P}_{32}^{T}$, āgneye tu vibhāge tu $\mathrm{P}_{72}^{T} \quad$ 81b tejovatī ] $\Sigma$, tejavatī $\mathrm{N}_{77}^{K o} \mathrm{~N}_{58}^{K} \quad$ 81c ${ }^{\circ}$ devasamākīrṇā ] $\mathrm{N}_{82}^{K} \mathrm{~N}_{12}^{K} \mathrm{E}^{N} \mathrm{~B}_{99}^{C} \mathrm{P}_{72}^{T}$, ${ }^{\circ}$ devagaṇākīrṇṇa $\mathrm{N}_{77}^{K o}$, ${ }^{\circ}$ devagaṇākīrnāa $\mathrm{N}_{45}^{C} \mathrm{~N}_{58}^{K}$, ${ }^{\circ}$ ratnagaṇākīrṇā $S_{67}^{S}$, ${ }^{\circ}$ ratnasamākīrṇā $\mathrm{P}_{32}^{T} \quad$ 81d ${ }^{\circ}$ samujjvalā ] $\mathrm{K}^{\Sigma} \mathrm{E}^{N} \mathrm{~B}_{99}^{C} \mathrm{P}_{3^{2}}^{T}$, ${ }^{\circ}$ samākulāḥ $\mathrm{N}_{77}^{K o},{ }^{\circ}$ samajvalā $\mathrm{N}_{45}^{C}$, ${ }^{\circ}$ mahājvalā $S_{67}^{S}$, ${ }^{\circ}$ samākulā $\mathrm{P}_{72}^{T}$ 
tatra jvālāparītāngo dīptāngārasamadyutị̣|

bhūtikṛd dehināṃ devo jvalanaḥ pāpanāśanaḥ|| 82||

śivapūjājapodyuktaḥ śivasmaraṇabhāvitaḥ|

śāntiṃ karotu me devas tathā pāpaparikṣayam|| 83||

[Yama in Vaivasvatī]

vaivasvatī nāma purī dakṣiṇena vyavasthitā| surāsuraśatānīkapitṛrakșoragālayā|| 84||

tatrendranīlasaṃkāśo raktāntāyatalocanah| mahāmahiṣam ārūọhaḥ kṛṣnasragvastrabhūṣaṇaḥ|| 85||

84-86 Cf. BhavP 1.178.35-37 : vaivasvatī purī ramyā dakṣiṇena mahātmanaḥ| surāsuraśatākīrṇā nānāratnopaśobhitā|| tatra kundendusaṃkāśo haripingalalocanaḥ| mahāmahiṣam ārūụhaḥ kṛ̣ṇasragvastrabhūṣaṇaḥ|| antako 'tha mahātejāḥ sūryadharmaparāyaṇaḥ| ādityārādhanaparaḥ kṣemārogye dadātu te||

84b After this $\mathrm{P}_{3^{2}}^{T}$ adds two pādas : lohaprākārasamyuktā prāsādair gopuraih śubhaiḥ| - $\mathrm{P}_{72}^{T}$ adds four pādas : lohaprākārasaṃyuktā prāsādair upaśobhitā| tasya tasyāṃ madhye sthitah sarvakāmado harmyagas tathā| ${ }^{85} \mathbf{d}$ After this $\mathrm{P}_{3^{2}}^{T}$ adds two pādas: kālāntakamahākālacaṇụakodạ̣ḍasamyutạ̣|

82a ${ }^{\circ}$ parītāngo ] $\mathrm{N}_{82}^{K} \mathrm{~N}_{12}^{K} \mathrm{E}^{N} \mathrm{~B}_{99}^{C} \hat{S}_{67}^{S} \mathrm{P}_{72}^{T}$, ${ }^{\circ}$ parītāngā $\mathrm{N}_{77}^{K o} \mathrm{P}_{32}^{T}$, ${ }^{\circ}$ paritāṃgo $\mathrm{N}_{5^{8}}^{K}$, ${ }^{\circ}$ parītāngī $\mathrm{N}_{45}^{C} \quad 82 b \quad{ }^{\circ}$ dyutiḥ ] $\Sigma$, odyuti $\mathrm{N}_{77}^{K o} \quad 82 c$ bhūtikṛd ] $\Sigma$, bhūtakṛ $\mathrm{N}_{77}^{K o} \bullet$ dehināṃ ] $\Sigma$, dehinā $\mathrm{N}_{45}^{C} \mathrm{~N}_{5^{8}}^{K} \quad$ 82d jvalanah ] $\Sigma$, jvalanā $\mathrm{N}_{77}^{K o} \quad \mathbf{8 3 a}$ jjapodyuktah ] $\mathrm{N}_{77}^{C p c} \mathrm{~N}_{82}^{K} \mathrm{~N}_{12}^{K} \mathrm{E}^{N} \mathrm{P}_{72}^{T p c} \mathrm{P}_{72}^{T}$, ojapodyukta $\mathrm{N}_{77}^{K o}$, ojapodyuktih $\mathrm{N}_{77}^{C \text { cac }},{ }^{\circ} \mathrm{sa}----\mathrm{N}_{58}^{K}$, ojayedyujtah $\mathrm{B}_{99}^{C}$, ${ }^{\circ}$ samāyuktah $S_{67}^{S}$, ${ }^{\circ}$ japodyuktā $\mathrm{P}_{72}^{\text {Tac }} \quad 8_{3} \mathbf{b}$ śiva $\left.{ }^{\circ}\right] \Sigma$, śivāo $\mathrm{N}_{45}^{C} \bullet{ }^{\circ}$ smaraṇa ${ }^{\circ}$ ] $\Sigma$, o ${ }^{\circ}$ sabhāva ${ }^{\circ} \hat{S}_{67}^{S} \bullet$ bhāvitah ] $\mathrm{N}_{77}^{K o} \mathbf{K}^{\Sigma} \mathrm{E}^{N} \mathrm{~B}_{99}^{C} \hat{S}_{67}^{S} \mathrm{P}_{72}^{T p c}$, obhāvitāh $\mathrm{N}_{45}^{C} \mathrm{P}_{72}^{T \text { ac }}$, ${ }^{\circ}$ pāvitaḥ $\mathrm{P}_{72}^{T}$ 83c śāntiṃ karotu me devas ] $\mathrm{N}_{82}^{K} \mathrm{~N}_{12}^{K} \mathrm{E}^{N} \mathrm{~B}_{99}^{C} \mathbf{P}^{\Sigma}$, śāntinikarotu me deva $\mathrm{N}_{77}^{K o}$, śāntikarme tu me deva $\mathrm{N}_{45}^{C}$, śānti karotu me devas $\mathrm{N}_{58}^{K}$, sāantiṃ karotu me vahnis $\hat{\mathrm{S}}_{67}^{S} \quad$ 83d ${ }^{\circ}$ kṣayam ] $\Sigma$, okṣayāṃ $\mathrm{N}_{45}^{C} \quad$ 84a vaivasvatī nāma purī ] $\mathrm{N}_{77}^{C p c} \mathrm{~N}_{82}^{K} \mathrm{~N}_{12}^{K} E^{N} \mathrm{~B}_{99}^{C} \dot{S}_{67}^{S}$, vaivaṃsvatapurī nāma $\mathrm{N}_{77}^{C a c}$, vaivasvatī purī nāma $\mathrm{N}_{77}^{K o} \mathrm{~N}_{5^{K}}^{K p c} \mathrm{P}_{3^{2}}^{T}$, vaivasvatī pur̄̄ nā $\mathrm{N}_{5^{8}}^{K a c}$ (unmetr.), vaivasvatapurī nāma $\mathrm{P}_{72}^{T} \quad \mathbf{8 4} \mathrm{b}$ dakṣiṇena ] $\Sigma$, dakṣiṇe tu $\mathrm{P}_{3^{2}}^{T} \bullet$ vyavasthitā ] $\mathrm{N}_{77}^{K o} \mathrm{E}^{N} \mathrm{~B}_{99}^{C} \dot{S}_{67}^{S} \mathbf{P}^{\Sigma}$, vyavasthitāh $\mathrm{N}_{45}^{C} \mathrm{~N}_{58}^{K}$, vivasthitā $\mathrm{N}_{82}^{K p c}$, vivasvataḥ $\mathrm{N}_{12}^{K}$, vivasthatā $\mathrm{N}_{82}^{K a c} \quad 84 \mathrm{c}$ surāsura ${ }^{\circ}$ ] $\Sigma$, yamarūpa ${ }^{\circ} \mathrm{P}_{32}^{T} \bullet{ }^{\circ}$ śatānīka ${ }^{\circ}$ ] $\Sigma$, śatānīkā $\mathrm{N}_{77}^{K o},{ }^{\circ}$ samākīrṇā ŚS S $_{67}^{S} \quad{ }^{\circ}$ rakṣoragālayā ] $\mathrm{N}_{82}^{K} \mathrm{~N}_{12}^{K} \mathrm{~B}_{99}^{C} \mathrm{P}_{32}^{T}$, ${ }^{\circ}$ yakșoragālayā $\mathrm{N}_{77}^{K o} \mathrm{~N}_{45}^{C} \dot{S}_{67}^{S} \mathrm{P}_{72}^{T}$, ${ }^{\circ}$ ra-

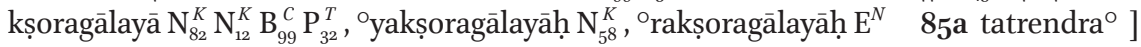
$\Sigma$, tantrendra ${ }^{\circ} \mathrm{N}_{45}^{C} \quad 85^{\mathrm{b}}$ raktāntāyata $\left.{ }^{\circ}\right] \Sigma$, raktaāyata ${ }^{\circ} \mathrm{E}^{N}$ (sic) • ${ }^{\circ}$ locanaḥ ] $\Sigma$, olocanā $\mathrm{N}_{77}^{K o}$, ${ }^{\circ}$ nocanaḥ $\mathrm{N}_{45}^{C} \quad{ }_{85} \mathrm{c}$ ārūḍhạ̣ ] $\Sigma$, ārūḍha $\mathrm{N}_{77}^{K o}$, ārūdhāḥ $\mathrm{N}_{45}^{C} \mathrm{~N}_{58}^{K}$ 
antako 'tha mahātejāḥ śivadharmaparāyaṇaḥ| śivapūjāsamudyuktaḥ kṣemārogyaṃ dadātu me|| 86||

\author{
[Nirṛti in Kṛ̣ṇā]
}

nairṛte tu diśo bhāge purī kṛ̣̣neti viśrutā| mahārakṣogaṇākīrṇā piśācapretasaṃkulā|| 87||

tatra jīmūtasaṃkāśo raktasragvastrabhūṣaṇaḥ| khaḍgapāṇir mahātejāḥ karālavadanojjvalaḥ|| 88||

rakșendro nirṛtir nityam śivārcanarataḥ sadā| karotu me mahāśāntiṃ śivabhaktisamutsukaḥ|| 89||

\begin{abstract}
87-89 Cf. BhavP 1.178.38-40 : nairṛte digvibhāge tu purī kṛṣneti viśrutā| moharakṣogaṇāśaucapiśācapretasaṃkulā|| tatra kundanibho devo raktasragvastrabhūṣaṇaḥ| khaḍgapāṇir mahātejāḥ karālavadanojjvalaḥ|| rakṣendro vasate nityam ādityārādhane rataḥ| karotu me sadā śāntiṃ dhanaṃ dhānyaṃ prayacchatu||
\end{abstract}

\title{
86ab Omitted in $\mathrm{N}_{45}^{C} \quad$ 87a-89d Omitted in $\mathrm{P}_{32}^{T}$
}

86a antako 'tha ] $\mathrm{N}_{77}^{K o} \mathbf{K}^{\Sigma} \mathrm{E}^{N} \mathrm{~B}_{99}^{c} \dot{S}_{67}^{S}$, śrīmān yamo $\mathbf{P}^{\Sigma}$ • otejāh ] $\mathbf{K}^{\Sigma} \mathrm{B}_{99}^{c} \mathbf{P}^{\Sigma}$, otejā $\mathrm{N}_{77}^{K o} \hat{S}_{67}^{S}$, ${ }^{\circ}$ tejah $\mathrm{E}^{N} \quad \mathbf{8 6 \mathbf { b }}{ }^{\circ}$ dharmaparāyaṇah ] $\mathrm{N}_{77}^{K o} \mathbf{K}^{\Sigma} \mathrm{E}^{N} \mathrm{~B}_{99}^{C} \mathbf{P}^{\Sigma}$, odhyānaikatatparah $S_{67}^{S} \quad 86 \mathrm{c}{ }^{\circ}$ samudyuktah ] $\mathrm{N}_{82}^{K} \mathrm{~N}_{58}^{K} \mathrm{E}^{N} \mathrm{~B}_{99}^{C} \mathrm{P}_{72}^{T}$, ${ }^{\circ}$ samāyuktah $\mathrm{N}_{77}^{K o} \dot{S}_{67}^{S}$, o samāyukta $\mathrm{N}_{45}^{C}$, ${ }^{\circ}$ samāsaktah $\mathrm{N}_{12}^{K}$, ojapodyuktah $\mathrm{P}_{32}^{T} \quad 86 \mathrm{~d}$ kṣemārogyam ] $\Sigma$, kṣemarogyam $\mathrm{N}_{45}^{C}$, kṣeemārogā $\mathrm{B}_{99}^{C} \bullet$ dadātu ] $\Sigma$, karotu $\hat{S}_{67}^{S} \mathrm{P}_{32}^{T} \quad$ 87a nairṛte ] $\mathrm{N}_{77}^{K o} \mathrm{~N}_{45}^{C} \mathrm{~N}_{58}^{K} \mathrm{E}^{N} \mathrm{~B}_{99}^{C} \dot{S}_{67}^{S} \mathrm{P}_{72}^{T}$, nairụtyām $\mathrm{N}_{82}^{K}$, nairṛtye ] $\mathrm{N}_{12}^{K}$ • diśo ] $\mathrm{N}_{82}^{K} \mathrm{~N}_{12}^{K} \mathrm{E}^{N} \mathrm{~B}_{99}^{C} \hat{S}_{67}^{S}$, disā $\mathrm{N}_{77}^{K o} \mathrm{~N}_{45}^{C} \mathrm{~N}_{58}^{K}$, śiśo $\mathrm{P}_{72}^{T} \quad{ }_{77} \mathbf{b}$ purī ] $\mathrm{N}_{45}^{C} \mathrm{~K}^{\Sigma} \mathrm{B}_{99}^{C} \hat{S}_{67}^{S} \mathrm{P}_{72}^{T}$, purā $\mathrm{E}^{N} \bullet$ kṛ̣ṇneti viśrutā ] $\mathrm{N}_{82}^{K} \mathrm{~N}_{12}^{K} \mathrm{~B}_{99}^{C} \hat{\mathrm{S}}_{67}^{S}$, kṛ̣ṣ̣e tu viśrutā $\mathrm{N}_{77}^{K o} \mathrm{~N}_{45}^{C}$, krụṣneti viśrutāḥ $\mathrm{E}^{N}$, kṛṣnavatī śubhā $\mathrm{N}_{58}^{K}$, kṛṣnāvatī śubhā $\mathrm{P}_{72}^{T} \quad 8_{7} \mathrm{c}{ }^{\circ}$ rakșo ${ }^{\circ}$ ] $\mathrm{N}_{77}^{K o} \mathrm{~N}_{82}^{K} \mathrm{~N}_{58}^{K} \mathrm{E}^{N} \mathrm{~B}_{99}^{C} \mathrm{P}_{72}^{T}$, ${ }^{\circ}$ yakș̣a ${ }^{\circ} \mathrm{N}_{45}^{C} \mathrm{~N}_{12}^{K} \hat{S}_{67}^{S}$ • orakṣoganākīrṇā ] $\mathrm{N}_{45}^{C} \mathrm{~N}_{82}^{K} \mathrm{~N}_{12}^{K} \mathrm{E}^{N} \mathrm{~B}_{99}^{C} S_{67}^{S} \mathrm{P}_{72}^{T}$, ${ }^{\circ}$ rakṣogaṇākīrṇa $\mathrm{N}_{77}^{K o}$, ${ }^{\circ}$ rakșoragākīrṇā $\mathrm{N}_{58}^{K} \quad{ }^{2}$ d d piśācapreta $\left.{ }^{\circ}\right] \mathrm{N}_{5^{8}}^{K} \mathrm{P}_{72}^{T}$, piśācabhūta ${ }^{\circ} \mathrm{N}_{82}^{K} \mathrm{E}^{N} \mathrm{~B}_{99}^{C} \hat{S}_{67}^{S}$ (unmetr.), piśācāpeta ${ }^{\circ} \mathrm{N}_{45}^{C}$, piśācāpreta ${ }^{\circ} \mathrm{N}_{77}^{K o} \mathrm{~N}_{12}^{K} \bullet{ }^{\circ}$ saṃkulā ] $\mathrm{N}_{45}^{C} \mathrm{~K}^{\Sigma} \mathrm{E}^{N} \hat{S}_{67}^{S} \mathrm{P}_{72}^{T}$, ${ }^{\circ}$ samı <yutā>kulā $\mathrm{B}_{99}^{C} \quad 88 \mathbf{a}$ jīmūta $\left.{ }^{\circ}\right] \mathrm{N}_{77}^{K o} \mathrm{~K}^{\Sigma} \mathrm{E}^{N} \mathrm{~B}_{99}^{C} \hat{S}_{67}^{S} \mathrm{P}_{72}^{T}$, jīmūrtta ${ }^{\circ}$ $\mathrm{N}_{45}^{C} \quad 88 \mathrm{~b}$ rakta $\left.{ }^{\circ}\right] \mathrm{N}^{\Sigma} \mathrm{E}^{N} \mathrm{~B}_{99}^{C} \hat{S}_{67}^{S}$, kṛṣna ${ }^{\circ} \mathrm{P}_{72}^{T} \bullet$ obhūṣaṇah ] $\mathrm{N}^{\Sigma} \mathrm{E}^{N} \mathrm{P}_{72}^{T}$, obhūṣaṇa $\mathrm{B}_{99}^{C}$, obhūṣitah $\hat{S}_{67}^{S} \quad 88 \mathrm{c}{ }^{\circ}$ pāṇir ] $\mathrm{N}_{45}^{C} \mathrm{~K}^{\Sigma} \mathrm{E}^{N} \mathrm{P}_{72}^{T}$, ${ }^{\circ}$ pāṇi $\mathrm{N}_{77}^{K o} \mathrm{~B}_{99}^{C} \bullet{ }^{\circ}$ tejāḥ ] $\mathrm{K}^{\Sigma} \mathrm{E}^{N} \mathrm{P}_{72}^{T}$, ${ }^{\circ}$ te $(\mathrm{jā})$ $\mathrm{N}_{77}^{K o}$, o te $\mathrm{N}_{45}^{C}$ (unmetr.), ${ }^{\circ}$ prājñah $S_{67}^{S} \quad 88 \mathrm{~d}$ ○jjvalaḥ ] $\mathrm{N}^{\Sigma} \hat{S}_{67}^{S} \mathrm{P}_{72}^{T}$, ${ }^{\circ}$ jvalāḥ $\mathrm{E}^{N} \mathrm{~B}_{99}^{C} \quad \mathbf{8 9 a}$ rakșendro ] $\mathrm{N}^{\Sigma} \mathrm{E}^{N} \mathrm{~B}_{99}^{C} S_{67}^{S}$, rakșarāț $\mathrm{P}_{7^{2}}^{T} \bullet$ nirṛtir ] $\mathrm{B}_{99}^{C}$, nairṛti $\mathrm{N}_{82}^{K}$, nirṛti $\mathrm{N}_{12}^{K} \mathrm{~N}_{58}^{K}$ nairṛtir $\mathrm{E}^{N} \dot{\mathrm{S}}_{67}^{S} \mathrm{P}_{72}^{T}$, niriti $\mathrm{N}_{77}^{K o} \mathrm{~N}_{45}^{C}$, • nityam ] $\mathrm{N}_{12}^{K} \mathrm{~N}_{58}^{K} \mathrm{E}^{N} \mathrm{~B}_{99}^{C} \dot{S}_{67}^{S} \mathrm{P}_{72}^{T}$, nitya $\mathrm{N}_{45}^{C} \mathrm{~N}_{82}^{K} \quad$ 89d ${ }^{\circ}$ samutsukah ] $\mathrm{N}_{45}^{C} \mathrm{~K}^{\Sigma} \mathrm{E}^{N} \mathrm{~B}_{99}^{C} \mathrm{P}_{72}^{T}$, ${ }^{\circ}$ samudbhavah $\mathrm{N}_{77}^{K o}$, oparāyaṇạ̣ $\hat{S}_{67}^{S}$ 
[Varuṇa in Śuddhavatī]

paścime tu diśo bhāge purī śuddhavatī śubhā|

nānāgaṇasamākīrnā nānākiṃnarasaṃkulā|| 9o||

tatra mauktikasaṃkāśạ̣ paripingalalocanaḥ|

śuklavastraparīdhānạ̣ pāśahasto mahābalaḥ|| 91||

varuṇaḥ parayā bhaktyā śivaikāhitamānasaḥ|

rogaśokārtisaṃtāpaṃ nirnāśayatu me sadā|| 92||

[Vāyu in Gandhavatī]

vāyavye digvibhāge tu purī gandhavatī śubhā|

ṛ̣isiddhaganāāhyușțā hemaprākāratoraṇā|| 93||

9o-92 Cf. BhavP 1.178.41-42 : paścime tu diśo bhāge purī śuddhavatī sadā| nānābhogisamākīrṇā nānākiṃnarasevitā|| tatra kundendusaṃkāśo haripingalalocanaḥ| śāntiṃ karotu me prītạ̣ śāntaḥ śāntena cetasā||

91b After this $\mathrm{E}^{N}$ reads $94 \mathrm{~cd}$

9oa paścime ] $\Sigma$, nairrte $\mathrm{P}_{3^{2}}^{T} \bullet$ diśo ] $\Sigma$, diśā $\mathrm{N}_{77}^{K o} \mathrm{~N}_{58}^{K} \quad$ 9ob śuddhavatī ] $\Sigma$, śuddhāvatī $\mathrm{E}^{N} \quad 9$ oc ${ }^{\circ}$ gaṇa $\left.^{\circ}\right] \quad \Sigma$, ${ }^{\circ}$ ratna $^{\circ} \mathrm{N}_{77}^{K o} \mathrm{P}_{72}^{T} \bullet{ }^{\circ}$ samākīṇā ] $\Sigma$, ${ }^{\circ}$ samākīrṇna $\mathrm{N}_{77}^{K o}$ 9od okiṃnarasaṃkulā ] $\mathrm{N}_{77}^{K o} \mathrm{~N}_{45}^{C} \mathrm{~N}_{12}^{K} \mathrm{~N}_{58}^{K} \mathbf{P}^{\Sigma}$, okiṃnaraśobhitā $\mathrm{N}_{82}^{K} \mathrm{E}^{N} \mathrm{~B}_{99}^{C}$, okiraṇasaṃkulā $\hat{S}_{67}^{S}$ 91a mauktika $\left.{ }^{\circ}\right] \Sigma$, mūktaka ${ }^{\circ} \mathrm{N}_{45}^{C} \bullet{ }^{\circ}$ saṃkāśạ ] $\Sigma$, ${ }^{\circ}$ saṃkāśa $\mathrm{N}_{77}^{K o}$, ${ }^{\circ}$ saṃkāśā $\mathrm{N}_{82}^{K a c} \quad$ 91c ${ }^{\circ}$ parīdhānaḥ ] $\mathrm{N}_{82}^{K} \mathrm{~N}_{12}^{K} \mathrm{E}^{N} \mathrm{~B}_{99}^{C} \hat{\mathrm{S}}_{67}^{S} \mathbf{P}^{\Sigma}$, ${ }^{\circ}$ parīdhāna $\mathrm{N}_{77}^{K o}$, ${ }^{\circ}$ parīdhānā $\mathrm{N}_{45}^{C}$, ${ }^{\circ}$ paridhānaḥ $\mathrm{N}_{58}^{K}$ (unmetr.), ' ${ }^{\circ}$ parīdhāneḥ $\mathrm{B}_{99}^{\text {Cac }}$ 91d 'obalạ̣ ] $\Sigma$, obalā $\mathrm{N}_{45}^{C}$, ${ }^{\circ}$ dyutiḥ $\mathrm{P}_{3^{2}}^{T} \quad$ 92a parayā ] $\Sigma$, paramā $\mathrm{N}_{45}^{C} \quad$ 92b śivaikāhitamānasaḥ ] $\Sigma$, śivaikāntitamānasām $\mathrm{N}_{45}^{C}$, śivakagatamānasaḥ $S_{67}^{S} \quad \mathbf{9 2 c}^{\circ}{ }^{\circ}$ śokārti $\left.{ }^{\circ}\right] \quad \Sigma$, 'ósokārtta ${ }^{\circ} \mathrm{N}_{77}^{K o} \bullet{ }^{\circ}$ saṃtāpaṃ ] $\mathrm{K}^{\Sigma} \mathrm{E}^{N} \mathrm{P}_{32}^{T}$, ${ }^{\circ}$ santāpa ${ }^{\circ} \mathrm{N}_{77}^{K o} \mathrm{P}_{72}^{T}{ }^{\circ}$ santāpām $\mathrm{N}_{45}^{C}$, ${ }^{\circ}$ śantāpam $\mathrm{B}_{99}^{C} \quad$ 92d nirnāśayatu me sadā ] $\mathrm{N}_{12}^{K} \hat{S}_{67}^{S}$, nirnāśayaṃtu me $\mathrm{N}_{77}^{K o}$ (unmetr), nityaṃ nirnāśayatu me $\mathrm{N}_{82}^{K}$ (unmetr.), nirṇṇāśayaṃtu sarvadā $\mathrm{N}_{45}^{C}$, nirnāśayaṃtu me sadā $\mathrm{N}_{58}^{K}$, nityaṃ nirnnāśyantu me $\mathrm{B}_{99}^{C}$, nityaṃ nirṇnāśayantu me $\mathrm{E}^{N}$, vināśayatu me sadā $\mathrm{P}_{32}^{T}$, bhayaṃ nirnāśayantu me $\mathrm{P}_{72}^{T} \quad$ 93a vāyavye ] $\mathrm{N}_{77}^{K o} \mathrm{~N}_{58}^{K} \mathrm{E}^{N} \mathrm{~B}_{99}^{C} \dot{S}_{67}^{S} \mathbf{P}^{\Sigma}$, vāyavyān $\mathrm{N}_{45}^{C} \mathrm{~N}_{12}^{K}$, vāyave $\mathrm{N}_{82}^{K} \bullet$ digvibhāge ] $\Sigma$, diśo bhāge $\mathrm{P}_{72}^{T} \quad 93 \mathrm{c}$ rșisidddha $\left.{ }^{\circ}\right] \Sigma$, rișisiddhi ${ }^{\circ} \mathrm{N}_{77}^{K o} \mathrm{~N}_{5^{8}}^{K}$, iṣisiddha ${ }^{\circ} \mathrm{N}_{45}^{C} \bullet{ }^{\circ}$ gaṇādhyușțā ]

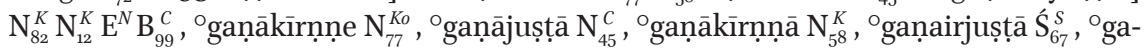
ṇākīrṇā $\mathbf{P}^{\Sigma} \quad 9 \mathbf{3}^{\circ}{ }^{\circ}$ toraṇā ] $\Sigma$, o toraṇāḥ $\mathrm{E}^{N}$ 
tatra tāmreṇa dehena kṛ̣napiningalalocanaḥ|

paṭavyāptāntarālīno dhvajayaștyāyudhodyatạ̣|| 94||

pavanaḥ paramo devaḥ parameśvarabhāvitaḥ|

kṣemārogyaṃ balaṃ śāntiṃ karotu satatam mama|| 95||

[Kubera in Mahodayā]

mahodayā nāma purī uttareṇa mahojjvalā|

anekayakṣasaṃkīṇā nānāratnopaśobhitā|| 96||

tatra devo gadāhastaś citrasragvastrabhūṣaṇaḥ|

hrasvabāhur mahātejāḥ paripingalalocanaḥ|| 97||

kubero varadaḥ śrīmān harapādārcane rataḥ|

śāntiṃ karotu me prītạ̣ śāntaḥ śāntena cetasā| | 98||

94cd $\mathrm{E}^{N}$ reads these two pādas after 91b $96 \mathrm{~b}$ After this pāda $\mathrm{P}_{3^{2}}^{T}$ adds two pādas: hemaprākārasaṃyuktā ratnaprāsādasaṃyutā|

94a tatra ] $\Sigma$, tișțhan $\mathrm{E}^{N} \bullet$ tāmreṇa ] $\Sigma$, dhūmreṇa $\mathrm{P}_{3^{2}}^{T} \bullet$ dehena ] $\Sigma$, lohena $\mathrm{P}_{72}^{T}$ 94b olocanah ] $\Sigma$, olocanāh $\mathrm{N}_{45}^{C}$, olohitah $\mathrm{P}_{72}^{T} \quad$ 94c pațavyāptāo $] \mathrm{N}_{77}^{K o} \mathrm{~K}^{\Sigma} \mathrm{E}^{N} \mathrm{~B}_{99}^{C} \dot{S}_{67}^{S}$, pațuvyāptā ${ }^{\circ} \mathrm{N}_{45}^{C}$, pațavyāptya ${ }^{\circ} \mathrm{P}_{32}^{T}$, nabhovyāptā ${ }^{\circ} \mathrm{P}_{72}^{T} \quad$ 94d ${ }^{\circ}$ yaștyaā $\left.{ }^{\circ}\right] \mathbf{K}^{\Sigma} \mathrm{E}^{N} \mathrm{~B}_{99}^{C} \hat{S}_{67}^{S} \mathbf{P}^{\Sigma}$, ${ }^{\circ}$ yașțā ${ }^{\circ} \mathrm{N}_{77}^{K o}$, ${ }^{\circ}$ cașța ${ }^{\circ} \mathrm{N}_{45}^{C}$, ${ }^{\circ}$ chatrāo $\mathrm{E}^{N} \quad$ 95a pavanah ] $\Sigma$, pavana $\mathrm{N}_{45}^{C} \bullet \operatorname{devah}$ ] $\Sigma$, devo $\mathrm{N}_{77}^{K o}$, deva $\mathrm{N}_{45}^{C} \quad$ 95 b obhāvitaḥ ] $\Sigma$, obhāvitā $\mathrm{N}_{45}^{C} \quad \mathbf{9 5}^{\mathrm{c}}$ kṣemārogyam ] $\mathrm{N}_{77}^{K o} \mathrm{~K}^{\Sigma} \mathrm{B}_{99}^{C} \grave{\mathrm{S}}_{67}^{S} \mathrm{P}_{72}^{T}$, kṣemarogam $\mathrm{N}_{45}^{C}$, kṣamārogyam $\mathrm{E}^{N}$, kṣemārogya ${ }^{\circ} \mathrm{P}_{32}^{T} \bullet$ śāntim ] $\Sigma$, śānti $\mathrm{N}_{45}^{C} \quad 95$ d satatam ] $\Sigma$, vijayam $\mathrm{P}_{32}^{T} \bullet$ mama ] $\mathbf{K}^{\Sigma} \mathrm{E}^{N} \mathrm{~B}_{99}^{C p c} \hat{\mathrm{S}}_{67}^{S} \mathbf{P}^{\Sigma}$, mamah $\mathrm{N}_{77}^{K o} \mathrm{~N}_{45}^{C}$, mamā $\mathrm{B}_{99}^{C a c} \quad$ 96a mahodayā nāma pur̄ ] $\Sigma$, mahodadhipurī nāma $\mathrm{P}_{72}^{T} \quad \mathbf{9 6 \mathbf { b }}$ uttareṇa ] $\Sigma$, uttare ca $\mathrm{P}_{72}^{T} \bullet$ mahojjvalā ] $\Sigma$, mahojvalaḥ $\mathrm{N}_{77}^{K o} \quad \mathbf{9 6 c}{ }^{\circ}$ saṃkīrṇā ] $\Sigma$, osaṃkīrṇna $\mathrm{N}_{77}^{K o} \quad$ 97a gadāhastaś ] $\Sigma$, gaḍāhastā $\mathrm{N}_{77}^{K o} \mathrm{~N}_{45}^{C} \mathrm{~N}_{58}^{K} \quad$ 97b citra $\left.{ }^{\circ}\right] \Sigma$, cintrāa $\mathrm{N}_{45}^{C}$ - obhūṣaṇaḥ ] $\mathbf{K}^{\Sigma} \mathrm{E}^{N} \mathrm{~B}_{99}^{C} \dot{S}_{67}^{S} \mathbf{P}^{\Sigma}$, obhūṣitā $\mathrm{N}_{77}^{K o}$, obhūṣaṇa $\mathrm{N}_{45}^{C}$, obhūṣitah $\hat{S}_{67}^{S} \quad$ 97c hrasvabāhur ] $\Sigma$, hasvavāhur $\mathrm{E}^{N}$, hrasvavāhu $\mathrm{N}_{45}^{C} \bullet{ }^{\circ}$ tejāh ] $\mathrm{K}^{\Sigma} \mathrm{E}^{N} \mathrm{~B}_{99}^{C} \mathbf{P}^{\Sigma}$, tejā $\mathrm{N}_{77}^{K o} \mathrm{~N}_{45}^{C} \hat{S}_{67}^{S}$ 97d olocanaḥ ] $\Sigma$, olocanā $\mathrm{N}_{77}^{K o}$, olocanāḥ $\mathrm{N}_{45}^{C} \quad \mathbf{9 8 a}$ varadah ] $\Sigma$, varada $\mathrm{N}_{12}^{K} \quad \mathbf{9 8 b}$ rataḥ ] $\Sigma$, ratā $\mathrm{N}_{77}^{K o}$, ratāḥ $\mathrm{N}_{45}^{C} \quad \mathbf{9 8 c}$ śāntiṃ ] $\Sigma$, śānti $\mathrm{N}_{45}^{C} \quad \mathbf{9 8 c d}$ prìtaḥ śāntaḥ ] $S_{67}^{S} \mathbf{P}^{\Sigma}$, śāntaḥ prītah $\mathrm{N}_{82}^{K} \mathrm{~N}_{12}^{K} \mathrm{~B}_{99}^{C}$, prītā śānta $\mathrm{N}_{77}^{K o}$, prītā śāntaḥ $\mathrm{N}_{45}^{C}$, prītāḥ śāntaḥ $\mathrm{N}_{58}^{K}$, śāntah prīta $\mathrm{E}^{N} \quad \mathbf{9 8 d}$ śāntena cetasā ] $\mathrm{N}_{77}^{K o} \mathrm{~N}_{45}^{C} \mathrm{~N}_{58}^{K} \dot{S}_{67}^{S} \mathbf{P}^{\Sigma}$, prītena tejasā $\mathrm{N}_{82}^{K} \mathrm{E}^{N} \mathrm{~B}_{99}^{C}$, prītena cetasā $\mathrm{N}_{12}^{K}$ 


\section{[İ́āna in Yaśovatī]}

yaśovatī purī ramyā aiśānīm diśam āśritā| nānāgaṇasamākīrnāā nānāsurakṛtālayā| tejaḥprākāraparyantā niraupamyā mahodayā|| 99||

tatra mauktikasaṃkāśạ śaśāñkakṛtabhūṣaṇaḥ| trinetraḥ śāntarūpātmā akṣamālādharo haraḥ|| 100||

īśānaḥ paramo devaḥ sarvadevottamottamaḥ| so 'pi sarvātmabhāvena śāntim āśu karotu me || 101\|

99-101 Cf. BhavP 1.178.43-44: yaśovatī purī ramyā aiśānīṃ diśam āśritā| nānāgaṇasamākīrṇā nānākṛtaśubhālayā| tejaḥprākāraparyantā anaupamyā sadojvalā|| tatra kundendusaṃkāśaś cāmbujākṣo vibhūṣitaḥ| trinetraḥ śāntarūpātmā akṣamālādharādharaḥ| îsānaḥ paramo devaḥ sadā śāntiṃ prayacchatu||

$101 b$ After this $\mathrm{N}_{58}^{K}$ adds two pādas : siddhānāñcaiva yogīnāṃ sarvvadā ca namaskṛtaḥ|

99a yaśovatī ] $\Sigma$, yaśovati $\mathrm{E}^{N} \bullet$ purī ramyā ] $\Sigma$, purī ramye $\mathrm{N}_{45}^{C}$, nāma purī $\hat{S}_{67}^{S} \quad$ 99b aiśānīm ] $\mathrm{N}_{82}^{K} \mathrm{~N}_{12}^{K}$, īśānyā $\mathrm{N}_{77}^{K o}$, aiśānyān $\mathrm{N}_{45}^{C} \mathrm{~N}_{58}^{K}$, aiśānan $\mathrm{B}_{99}^{C}$, îsānị̣̄ $\mathrm{E}^{N}$, aiśāna ${ }^{\circ} \mathrm{S}_{67}^{s}$, aiśānyāṃ $\mathrm{P}_{72}^{T p c} \mathrm{P}_{72}^{T}$, saumyāyāṃ $\mathrm{P}_{72}^{T \text { ac }} \bullet$ diśam āśritā ] $\mathrm{N}_{82}^{K} \mathrm{E}^{N} S_{67}^{S}$, diśim āśritā $\mathrm{N}_{77}^{K o} \mathrm{~N}_{12}^{K} \mathrm{~N}_{58}^{K}$, diśim āśritāḥ $\mathrm{N}_{45}^{C}$, diśam āśritā $\mathrm{B}_{99}^{C}$, diśi saṃsthita $\mathrm{P}^{\Sigma} \quad 99 \mathrm{c}{ }^{\circ}$ gaṇa $\left.{ }^{\circ}\right] \Sigma$, ${ }^{\circ} \operatorname{deva}{ }^{\circ} \mathrm{N}_{58}^{K} \bullet$ ${ }^{\circ}$ samākīrṇā ] $\Sigma$, o ${ }^{\circ}$ samākīrṇna $\mathrm{N}_{77}^{K o}$, ${ }^{\circ}$ samākīrṇa $\mathrm{E}^{N} \quad$ 99d ${ }^{\circ}$ surakṛtālayā ] $\mathrm{K}^{\Sigma} \mathrm{E}^{N} \mathrm{~B}_{99}^{C}$, ${ }^{\circ}$ kṛtasurālayā $\mathrm{N}_{77}^{K o} \hat{\mathrm{S}}_{67}^{S} \mathrm{P}_{3^{2}}^{T}$, ${ }^{\circ}$ kṛtasurālaya $\mathrm{N}_{45}^{C}$, ${ }^{\circ}$ ratnasurālayā $\mathrm{P}_{72}^{T} \quad$ 99e ${ }^{\circ}$ tejaḥ $\left.{ }^{\circ}\right] \Sigma$, ${ }^{\circ}$ teja ${ }^{\circ} \mathrm{N}_{77}^{K o} \mathrm{~N}_{45}^{C} \bullet{ }^{\circ}$ prākāra $\left.{ }^{\circ}\right] \mathrm{N}_{77}^{K o} \mathrm{~N}_{45}^{C} \mathrm{~N}_{12}^{K} \mathrm{~N}_{5^{K}}^{K} \mathrm{E}^{N} \mathbf{P}^{\Sigma}$, o prākīra ${ }^{\circ} \mathrm{B}_{99}^{C}$, ${ }^{\circ}$ prakāra ${ }^{\circ} \mathrm{N}_{82}^{K}$, ${ }^{\circ}$ prakāśa ${ }^{\circ} S_{67}^{S} \mathrm{P}_{32}^{T} \quad$ 99f niraupamyā mahodayā ] $\mathrm{N}_{82}^{K} \mathrm{E}^{N} \mathrm{~B}_{99}^{C}$, niraupamyā sadojvalāḥ $\mathrm{N}_{77}^{K o}$, niropamyā sadojvalā $\mathrm{N}_{45}^{C}$, niraupamyā mahojjvalā $\mathrm{N}_{12}^{K} \hat{S}_{67}^{S}$, niraupamyā guṇānvitā $\mathrm{N}_{58}^{K}$, durnnirūpaguṇojvalā $\mathrm{P}_{32}^{T}$, niraupamyā sadaujvalā $\mathrm{P}_{72}^{T} \quad$ 10oa tatra ] $\Sigma$, yatra $\mathrm{E}^{N} \bullet$ ${ }^{\circ}$ mauktika $\left.{ }^{\circ}\right] \Sigma,{ }^{\circ}$ mauktio ${ }^{\circ} \mathrm{N}_{58}^{K}$ (unmetr.) • ${ }^{\circ}$ saṃkāśah ] $\Sigma$, ${ }^{\circ}$ saṃkāśa $\mathrm{N}_{77}^{K o} \quad$ loob śaśāṅka $\left.{ }^{\circ}\right] \Sigma$, śaśāṅkaḥ $\mathrm{N}_{45}^{C} \bullet{ }^{\circ}$ bhūṣaṇah $\mathrm{N}_{45}^{C} \mathrm{~N}_{82}^{K} \mathrm{~N}_{12}^{K} \mathrm{E}^{N} \mathrm{~B}_{99}^{C} \dot{S}_{67}^{S}$, ósekharah $\mathrm{N}_{77}^{K o} \mathrm{~N}_{58}^{K} \mathbf{P}^{\Sigma} \quad$ 1ooc trinetrah ] $\Sigma$, trinetra ${ }^{\circ} \mathrm{N}_{12}^{K} \mathrm{~N}_{58}^{K} \mathrm{E}^{N} \bullet{ }^{\circ}$ rūpātmā ] $\Sigma$, ${ }^{\circ}$ rūpāngo $\mathrm{P}_{72}^{T} \quad$ lood akṣamālādharo harạ̣ ] $\mathrm{N}_{82}^{K} \mathrm{~N}_{58}^{K} \mathrm{E}^{N} \mathrm{~B}_{99}^{C} S_{67}^{S}$, akșamālādharaḥ śubhā $\mathrm{N}_{77}^{K o}$, amāladharaḥ sadā $\mathrm{N}_{45}^{C}$ (unmetr.), akṣamālādharo varaḥ $\mathrm{N}_{12}^{K}$, akșamālākarodyataḥ $\mathrm{P}_{3^{2}}^{T}$, pyakșamālādharo haraḥ $\mathrm{P}_{72}^{T} \quad 101 \mathrm{ia}$ ísānah ] $\mathrm{N}_{82}^{K} \mathrm{~N}_{12}^{K} \hat{S}_{67}^{S} \mathrm{P}^{\Sigma}$, íśāna $\mathrm{N}_{77}^{K o} \mathrm{~N}_{45}^{C} \mathrm{~N}_{58}^{K} \mathrm{E}^{N} \mathrm{~B}_{99}^{c} \bullet$ devaḥ ] $\Sigma$, deva $\mathrm{N}_{77}^{K o} \mathrm{~N}_{12}^{K}$ $101 b{ }^{\circ}$ mottamah ] $\Sigma$, ${ }^{\circ}$ mottamā $\mathrm{N}_{45}^{C}$, ${ }^{\circ}$ momah $\mathrm{N}_{5^{8}}^{K}$ (unmetr.) 1o1d karotu me ] $\mathrm{N}_{77}^{K o} \mathrm{~N}_{45}^{c} \mathrm{~N}_{58}^{K} S_{67}^{S} \mathbf{P}^{\Sigma}$, prayacchatu ] $\mathrm{N}_{82}^{K} \mathrm{~N}_{12}^{K} \mathrm{E}^{N}$, prayacchantu $\mathrm{B}_{99}^{c}$ 


\title{
[Devas in the Seven Worlds]
}

\author{
bhūrloke 'tha bhuvarloke svarloke nivasanti ye| \\ devā divyaprabhāyuktāḥ śāntiṃ kurvantu me sadā|| 102||
}

maharloke janarloke tapoloke sthitāś ca ye |

te 'pi pramuditā devāḥ śivaṃ kurvantu me sadā|| 103\|

102-103 Cf. BhavP 1.178.45-46: bhūloke tu bhuvarloke nivasanti ca ye sadā| devādevāḥ śubhāyuktāḥ śāntiṃ kurvantu te sadā|| maharloke janoloke paraloke gatāś ca ye| te sarve muditā devāḥ śāntiṃ kurvantu te sadā||

102d After this $\mathrm{N}_{58}^{K}$ adds two pādas (eye-skip 101cd) : so pi sarvvātmabhāvena śāntim āśu karotu me $\bullet \mathrm{P}_{3^{2}}^{T}$ adds (with an insertion mark ; originally after 1ogab) 12 pādas : tapoloke ca ye devāḥ śuddhasphațikasaṃnibhāḥ| rudrabhaktāḥ mahātmānaḥ śivaṃ kurvantu me sadā| janoloke ca ye devā dhautacāmīkaraprabhāḥ| iśānaṃ praṇatā nityạ̣ śāntiṃ kurvantu me sadā| maharloke ca ye devā vimānojvalasaṃsthitāḥ| śivaikāhitacetaskāḥ pāpaṃ praśamayantu me $\quad$ 103a-d Omitted in $\mathrm{N}_{58}^{K}$ and $\hat{S}_{67}^{S} \quad \mathbf{1 0 3} \mathbf{d}$ After this $\mathrm{P}_{72}^{T}$ adds 14 pādas : janaloke ca ye devāḥ dhautacāmīkaraprabhāḥ| ísānaṃ praṇatā nityaṃ śāntiṃ kurvantu me sadā| maharloke ca ye devā vimānojvalasaṃsthitāḥ| bhavapraṇāmaparamāḥ nāśayantu bhayaṃ sadā| svarlokasthāś ca ye devā dīptimanto mahājvalāḥ| śan̉karaṃ praṇatā nityaṃ kurvantu vijayaṃ mama| bhūloke caiva ye devā bhāsayanto diśo daśa| śivaikāhitacetaskāḥ pāpaṃ praśamayantu me|

102a bhūrloke'tha ] $\mathrm{N}_{82}^{K} \mathrm{~N}_{12}^{K} \mathrm{E}^{N} \mathrm{~B}_{99}^{C} \hat{\mathrm{S}}_{67}^{S}$, bhūloko tha $\mathrm{N}_{77}^{K o}$, bhūrloke rtha $\mathrm{N}_{45}^{C}$, bhūloke tha $\mathrm{N}_{5^{8}}^{K} \mathrm{P}_{32}^{T}$, bhūloke ca $\mathrm{P}_{72}^{T} \bullet$ bhuvarloke ] $\mathrm{N}_{45}^{C} \mathrm{~N}_{12}^{K} \mathrm{E}^{N} \mathbf{P}^{\Sigma}$, bhuvaloka $\mathrm{N}_{77}^{K o}$ (unmetr.), bhuvoloke $\mathrm{N}_{82}^{K} \mathrm{~N}_{5^{8}}^{K} \mathrm{~B}_{99}^{C} \hat{S}_{67}^{S} \quad$ 102b svarloke nivasanti ye ] $\mathbf{K}^{\Sigma} \mathrm{E}^{N} \mathrm{~B}_{99}^{C} \hat{S}_{67}^{S}$, svalo(ke) nivasanti ye $\mathrm{N}_{77}^{K o}$, svaraloke nivasānti ye $\mathrm{N}_{45}^{C}$ (unmetr.), svarloke 'pi ca samsthitah $\mathrm{P}_{32}^{T}$, svargaloke vasanti ye $\mathrm{P}_{72}^{T} \quad$ 102c devā divyaprabhāyuktāḥ ] $\mathrm{N}_{45}^{C} \mathrm{~N}_{12}^{K} \mathrm{P}_{72}^{T}$, devā divyaprabhāyuktā $\mathrm{N}_{77}^{K o}$, devatā divyasaṃyuktāḥ $\mathrm{N}_{82}^{K} \mathrm{~N}_{58}^{K} \mathrm{E}^{N} \mathrm{~B}_{99}^{C}$, divyā divyaprabhāyuktāḥ $\mathrm{S}_{67}^{S}$, devo divyaprabhāyuktaḥ $\mathrm{P}_{3^{2}}^{T} \quad$ 102d śāntiṃ kurvantu ] $\Sigma$, śānti kurvāntu $\mathrm{N}_{45}^{C}$, śānti kurvantu $\mathrm{N}_{58}^{K}$, śāntiṃ kurvatu $\mathrm{P}_{32}^{T} \quad$ 103a maharloke ] $\mathrm{N}_{77}^{K o} \mathrm{~N}_{82}^{K} \mathrm{E}^{N} \mathbf{P}^{\Sigma}$, mahāloker $\mathrm{N}_{45}^{C}$, mahalloke $\mathrm{N}_{12}^{K} \mathrm{~B}_{99}^{C}$ - janarloke ] $\mathrm{N}_{77}^{K o} \mathrm{~N}_{82}^{K} \mathrm{~N}_{12}^{K}$, janalloke $\mathrm{N}_{45}^{C}$, janaloke $\mathrm{B}_{99}^{C}$ (unmetr.), janoloke $\mathrm{E}^{N} \mathbf{P}^{\Sigma} \quad$ 103 $\mathbf{b}$ tapoloke sthitāś ca ye ] $\mathrm{N}_{77}^{K o} \mathrm{~N}_{82}^{K} \mathrm{~N}_{12}^{K} \mathrm{E}^{N} \mathrm{~B}_{99}^{C}$, taporloke ca ye sthitāḥ $\mathrm{N}_{45}^{C}$, tapoloke ca sạ̣sthitāḥ $\mathrm{P}_{3^{2}}^{T}$, tapoloke ca ye sthitāḥ $\mathrm{P}_{72}^{T} \quad{ }_{103 c}$ te 'pi pramuditā devāḥ ] $\mathrm{N}_{45}^{C} \mathrm{~N}_{82}^{K} \mathrm{~N}_{12}^{K} \mathrm{E}^{N} \mathrm{~B}_{99}^{C}$, te pi me muditā devā $\mathrm{N}_{77}^{K o}$, te devā nirātaṃkāḥ $\mathrm{P}_{3^{2}}^{T}$, te pi pramudyatā devāḥ $\mathrm{P}_{72}^{T} \quad$ 103d śivam kurvantu me sadā ] $\mathrm{N}_{77}^{K o} \mathrm{~N}_{82}^{K} \mathrm{~N}_{12}^{K} \mathrm{E}^{N}$, śiva kurvanttri sarvadā $\mathrm{N}_{45}^{C}$, śiva kurvvantu me sadā $\mathrm{B}_{99}^{C}$, sāntiṃ kurvantu me sadā $\mathbf{P}^{\Sigma}$ 
satyaloke ca ye devāḥ svaprabhojjvalavigrahāḥ|

śivabhaktāḥ sumanaso bhayaṃ nirnāśayantu me|| 104||

[Devas in Caves, Forests etc.]

girikandaradurgeșu vaneșu nivasanti ye|

rudrārcanaparā devā rakșāṃ kurvantu me sadā|| 105||

104a-d Omitted in $\mathrm{P}_{72}^{T} \quad$ 104d After this $\mathrm{N}_{58}^{K}$ adds 20 pādas : tapoloke ca ye devāḥ śuddhasphațikasannibhāḥ| rudrabhaktā mahātmānaḥ śivaṃ kurvvantu me sadā| janaloke ca ye devā dhautacāmīkaraprabhāḥ| îśvaram praṇatān nityạ̣ kṣemaṃ kurvvantu me sadā| mahaloke ca ye devā vimānorddhvasaṃsthitāḥ| rudrapraṇāmaparamā bhayam nirṇṇāśayantu me| svarggaloke ca ye devā dīptamantā mahojjvalāḥ| śankkarapraṇatā nityam kurvvantu vijayam mama| bhuvaloke ca ye devā bhāsamānā diśo daśa| śi-

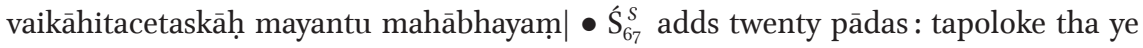
devāḥ sphațikojjvalavigrahāḥ| rudrabhaktā mahātmānaḥ śāntiṃ kurvantu me sadā| janaloke ca ye devā dhautacāmīkaraprabhāḥ| îsānapraṇatā nityạ̣ śāntiṃ kurvantu me sadā| mahalloke ca ye devās taptakāñcanasannibhāḥ| śivārcanaratā nityaṃ kṣemaṃ kurvantu me sadā| svarloke ye sthitā devā dīptavarṇā mahābalāḥ| śạ̣karapraṇatā nityaṃ kurvantu vijayaṃ mama| bhūrloke caiva ye devā bhāsayanto diśo daśa| śivaikāhi-

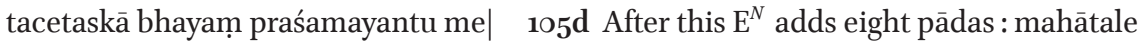
ca santi sma rasātaleṣu saṃsthitāḥ| talātale ca ye santi ye santi sutale janāḥ| nitale saṃsthitā bhūtvā vitale nivasanti ye| tale ca nilayā ye ca rakṣāṃ kurvantu sarvvadā|

104a devāh ] $\mathrm{N}_{45}^{C} \mathrm{~N}_{82}^{K} \mathrm{~N}_{12}^{K} \mathrm{E}^{N} \mathrm{~B}_{99}^{C} \dot{S}_{67}^{S}$, devā $\mathrm{N}_{77}^{K o} \mathrm{P}_{32}^{T} \quad$ 104b svaprabhojvala $\left.{ }^{\circ}\right] \mathrm{N}_{77}^{K o} \mathrm{~N}_{45}^{C} \mathrm{~N}_{12}^{K}$, prabhāvojjvala ${ }^{\circ} \mathrm{N}_{82}^{K} \mathrm{~B}_{99}^{C}$, suprabhojvala ${ }^{\circ} \mathrm{N}_{58}^{K}$, svaprabhojjvala ${ }^{\circ} \hat{S}_{67}^{S}$, prabhājojjvala ${ }^{\circ} \mathrm{E}^{N}$, bhavanty ujvala ${ }^{\circ} \mathrm{P}_{3^{2}}^{T} \bullet{ }^{\circ}$ vigrahāḥ ] $\mathrm{N}_{82}^{K} \mathrm{~N}_{12}^{K} \mathrm{E}^{N} \hat{S}_{67}^{S} \mathrm{P}_{32}^{T}$, ${ }^{\circ}$ vigrahā $\mathrm{N}_{77}^{K o} \mathrm{~N}_{45}^{C}$, ${ }^{\circ}$ sannibhā $\mathrm{N}_{58}^{K}$ 104c obhaktāḥ ] $\mathrm{N}_{82}^{K} \mathrm{~N}_{12}^{K} \mathrm{E}^{N} \mathrm{~B}_{99}^{C} \dot{S}_{67}^{S} \mathrm{P}_{32}^{T}$, obhaktā $\mathrm{N}_{77}^{K o} \mathrm{~N}_{45}^{C} \mathrm{~N}_{58}^{K} \quad$ 104d bhayam nirnāśayantu me ] $\mathrm{N}_{77}^{K o} \mathrm{~N}_{82}^{K} \mathrm{~N}_{12}^{K} \mathrm{E}^{N} \mathrm{~B}_{99}^{C} S_{67}^{S}$, bhayannināśayantu me $\mathrm{N}_{45}^{C}$, śāntiṃ kurvantu me sadā

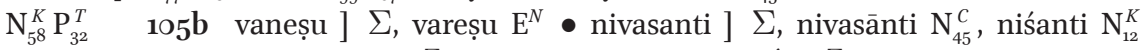
(unmetr.) 105c ${ }^{\circ}$ parā ] $\mathrm{N}_{77}^{K o} \mathrm{~K}^{\Sigma} \mathrm{E}^{N} \mathrm{~B}_{99}^{C}$, ${ }^{\circ}$ parāḥ $\mathrm{N}_{45}^{C}$, ${ }^{\circ}$ ratā $S_{67}^{S} \mathbf{P}^{\Sigma} \quad$ 105cd devā rakșām kurvantu ] $\mathrm{N}_{82}^{K} \mathrm{~N}_{12}^{K} \mathrm{E}^{N} \mathrm{~B}_{99}^{C} \hat{S}_{67}^{S}$, devā saanti kurvantu $\mathrm{N}_{77}^{K o}$, devāḥ rakṣa kurvāntu $\mathrm{N}_{45}^{C}$, devā rakṣā kurvvantu $\mathrm{N}_{58}^{K}$, devāḥ śāntiṃ kurvantu $\mathrm{P}_{3^{2}}^{T}$, nityam śāntiṃ kurvantu $\mathrm{P}_{7^{2}}^{T}$ 
[Sarasvatī]

śaraccandrāṃśugaureṇa dehenāmalatejasā|

sarasvatī śive bhaktā śāntim āśu karotu me|| 106||

[Śrī]

cārucāmīkaracchāyā sarojakarapallavā|

śivabhaktā tu śrīr devī śrīmadbhūtiṃ dadātu me || 107||

[Jayā]

cāruṇā mukhacandreṇa vicitrakanakojjvalā|

jayā devī śive bhaktā sarvakāmaṃ dadātu me|| 108||

106 Cf. BhavP 1.178.47ab : sarasvatī sūryabhaktā śāntidā vidadhātu me| 107 Cf. BhavP 1.178.47cdef : cārucāmīkarasthā yā sarojakarapallavā| sūryabhaktyāśritā devī vibhūtị̣ te prayacchatu||

108a-d Omitted in $\mathrm{P}_{32}^{T} \quad$ 108d After this $\hat{S}_{67}^{S}$ adds six pādas : vijayā ca mahābhāgā karotu mama saāntikam| triśūla vyagrahastā ca śārdūlāmbaravāsinī| jayantī ca mahābhāgā śivabhāvitacetanā|

106a śaraccandrāṃśu $\left.{ }^{\circ}\right] \mathrm{N}_{82}^{K} \mathrm{~N}_{58}^{K} \mathrm{E}^{N} \mathrm{~B}_{99}^{c} \mathrm{P}^{\Sigma}$, śaracandrāśu $\mathrm{N}_{77}^{K o}$, saracandrātio $\mathrm{N}_{45}^{C}$, śaraścandrātsu ${ }^{\circ} \mathrm{N}_{12}^{K}$, śaraccandrānka ${ }^{\circ} S_{67}^{S} \quad 106 \mathrm{~b}$ dehenāmala $\left.{ }^{\circ}\right] \mathrm{N}_{45}^{C} \mathrm{~N}_{82}^{K} \mathrm{~N}_{12}^{K} \mathrm{E}^{N} \mathrm{~B}_{99}^{C} \mathbf{P}^{\Sigma}$, dehenāmita ${ }^{\circ} \mathrm{N}_{77}^{K o} \mathrm{~N}_{58}^{K} S_{67}^{S p c}$, dehenāmṛta ${ }^{\circ} S_{67}^{S a c} \bullet{ }^{\circ}$ tejasā ] $\Sigma$, o tejasāḥ $\mathrm{N}_{45}^{C} \quad$ 106c sarasvatī ] $\Sigma$, sarāsatī $\mathrm{N}_{45}^{C}$, saraśvatī $\mathrm{N}_{58}^{K} \bullet$ śive bhaktā ] $\mathrm{N}_{45}^{C} \mathrm{~N}_{58}^{K} \mathrm{~B}_{99}^{C} \dot{S}_{67}^{S} \mathbf{P}^{\Sigma}$, śivabhaktā $\mathrm{N}_{77}^{K o} \mathrm{~N}_{82}^{K} \mathrm{~N}_{12}^{K} \mathrm{E}^{N} \quad{ }_{107}{ }^{\circ}$ cchāyā ] $\Sigma$, ohastā $\mathrm{E}^{N} \quad{ }_{107} \mathbf{b}$ sarojakarapallavā ] $\Sigma$, sarojakarapalavā $\mathrm{N}_{77}^{K o}$ (unmetr.), sarojvakarapannavā $\mathrm{N}_{45}^{C}$, sarojotpalapallavā $\mathrm{E}^{N} \quad$ 107c śivabhaktā tu śrīr devī ] $\mathrm{N}_{82}^{K} \mathrm{~N}_{58}^{K} \mathrm{~B}_{99}^{C}$, śivabhaktā tu śrī devī $\mathrm{N}_{12}^{K} \mathrm{E}^{N}$, śivabhaktā śriyā devī $\mathrm{N}_{77}^{K o} \mathrm{~N}_{45}^{C}$, śivabhaktā sadā devī $S_{67}^{S}$, śrī devī ca śive bhaktā $\mathbf{P}^{\Sigma} \quad{ }_{107}$ d śrīmadbhūtiṃ ] $\Sigma$, śrímām bhūtin $\mathrm{N}_{77}^{K o}$, śrīh sadbhūtiṃ $\hat{S}_{67}^{S} \bullet$ dadātu ] $\Sigma$, dadāntu $\mathrm{N}_{45}^{C} \quad$ 108a cāruṇā ] $\Sigma$, cārutā $\mathrm{P}_{7^{2}}^{T} \bullet$ mukha $\left.^{\circ}\right] \Sigma$, mukhya ${ }^{\circ} \mathrm{B}_{99}^{C} \quad 108 \mathbf{b}$ vicitra $\left.{ }^{\circ}\right] \mathrm{N}_{77}^{K o} \mathbf{K}^{\Sigma} \mathrm{E}^{N} S_{67}^{S} \mathrm{P}_{72}^{T}$, vicitrā $\mathrm{N}_{45}^{C} \mathrm{~B}_{99}^{C} \bullet$ ${ }^{\circ}$ kanakojjvalā ] $\mathrm{N}^{\Sigma} \hat{S}_{67}^{S} \mathrm{P}_{72}^{T}$, ${ }^{\circ}$ kuśumojjvalā $\mathrm{N}_{58}^{K}$, ${ }^{\circ}$ kusumojjvalā $\mathrm{E}^{N} \mathrm{~B}_{99}^{C} \quad$ 108c jayā devī ] $\mathrm{N}_{82}^{K} \mathrm{~N}_{58}^{K} \mathrm{E}^{N} \mathrm{~B}_{99}^{C} S_{67}^{S} \mathrm{P}_{72}^{T}$, subhagāpi $\mathrm{N}_{77}^{K o} \mathrm{~N}_{45}^{C} \mathrm{~N}_{12}^{K}$ • śive bhaktā ] $\mathrm{N}_{77}^{K o} \mathrm{~N}_{45}^{C} \mathrm{~N}_{82}^{K} \mathrm{~N}_{58}^{K} \mathrm{E}^{N} \mathrm{~B}_{99}^{C} \mathrm{P}_{72}^{T}$, mahābhāgā $S_{67}^{S} \quad$ 108d sarvakāmaṃ dadātu me ] $\mathrm{N}_{77}^{K o} \mathrm{~K}^{\Sigma} \mathrm{E}^{N} \mathrm{~B}_{99}^{C}$, sarvalokān dadātu me $\mathrm{N}_{45}^{C}$, śivabhaktā sucetanā $S_{67}^{S}$, sarvān kāmān dadātu me $\mathrm{N}_{12}^{K}$ 
[Aparājitā]

hāreṇa suvicitreṇa bhāsvatkanakamekhalā| aparājitā rudraratā karotu vijayaṃ mama|| 109||

[Navagrahas]

[Sūrya]

sindūrarāgaraktena varṇenāyatalocanaḥ|

kiraṇadvayasamyuktạ̣ saptasaptikavāhanaḥ|| 110||

gabhastimālī bhagavān śivapūjārcane rataḥ|

karotu me mahāśāntiṃ grahapị̣̄ānivāraṇīm|| 111||

109 Cf. BhavP 1.178.48: hareṇa suvicitreṇa bhāsvatkanakamekhalā| aparājitā sūryabhaktā karotu vijayaṃ tava|| 110-111 Cf. BhavP 1.175·36cd-38ab : sindūrāsanaraktābhah padmaraktābhalocanaḥ|| sahasrakiraṇo devaḥ saptāśvarathavāhanaḥ| gabhastimālī bhagavān sarvadevanamaskṛtaḥ|| karotu te mahāśāntiṃ grahapị̄ānivāriṇīm|

11od After this $\mathrm{N}_{5^{8}}^{K}$ adds two pādas : kamaladvayasamyuktaḥ surāsuranamaskṛtaḥ|

109a ${ }^{\circ}$ citreṇa ] $\Sigma$, ${ }^{\circ}$ cintreṇa $\mathrm{N}_{45}^{c},{ }^{\circ}(-\mathrm{i})--\mathrm{N}_{12}^{K} \quad$ 1ogb bhāsvatkanakhamekhalā ] $\mathrm{N}_{12}^{K} \dot{\mathrm{S}}_{67}^{S} \mathbf{P}^{\Sigma}$, bhāskarojjvalatejasā $\mathrm{N}_{82}^{K} \mathrm{~N}_{58}^{K} \mathrm{E}^{N} \mathrm{~B}_{99}^{C}$, bhāsvatakanakamekhalāḥ $\mathrm{N}_{77}^{K o}$ (unmetr.), bhānyatkanakamekhalāṃ $\mathrm{N}_{45}^{C} \quad$ 109c aparājitā rudraratā $\mathrm{N}_{77}^{K o} \mathrm{~N}_{45}^{C} \mathrm{~N}_{12}^{K} \mathrm{~N}_{58}^{K} \hat{S}_{67}^{S}$, rudrasutāparājitā $\mathrm{N}_{82}^{K} \mathrm{E}^{N} \mathrm{~B}_{99}^{C}$ (unmetr.), aparājitā śive bhaktā $\mathrm{P}_{32}^{T}$, parājitā śive bhaktā $\mathrm{P}_{72}^{T} \quad$ logd mama ] $\Sigma$, mamah $\mathrm{N}_{77}^{K o} \quad$ loa sindūrarāgaraktena ] $\mathrm{N}_{45}^{C} \mathrm{~N}_{82}^{K} \mathrm{~N}_{58}^{K} \mathrm{~B}_{99}^{C}$, sindūrarāgayuktena $\mathrm{N}_{77}^{K o}$, sindūrāruṇaraktena $\mathrm{N}_{12}^{K}$, sindūrarājaratnena $\mathrm{E}^{N}$, sindūrāruṇavaktrābjaḥ $S_{67}^{S}$, sindūrāruṇaraktāṃgaḥ $\mathbf{P}^{\Sigma} \quad 110 b$ varṇenāyata $\left.{ }^{\circ}\right] \mathrm{N}_{12}^{K} \mathrm{~N}_{58}^{K} \mathrm{~B}_{99}^{C}$, varṇṇanāyata ${ }^{\circ} \mathrm{N}_{82}^{K}$, karṇāntāyata $\left.{ }^{\circ}\right] \mathrm{N}_{77}^{K o} \mathrm{~N}_{45}^{C} \mathrm{E}^{N} \hat{S}_{67}^{S} \mathbf{P}^{\Sigma} \bullet{ }^{\circ}$ locanah ] $\mathrm{N}_{82}^{K} \mathrm{~N}_{58}^{K} \mathrm{E}^{N} \dot{\mathrm{S}}_{67}^{S} \mathbf{P}^{\Sigma}$, olocanā $\mathrm{N}_{77}^{K o}$, ○locana $\mathrm{N}_{45}^{C} \quad$ 11oc kiraṇadvayasamyuktạ ] $\mathrm{N}_{82}^{K} \mathrm{~N}_{12}^{K} \mathrm{E}^{N} \hat{S}_{67}^{S}$, sahasrakiraṇa śrīmā $\mathrm{N}_{77}^{K o}$, sahasrakiraṇah śrīmān $\mathrm{N}_{45}^{C} \mathrm{P}_{32}^{T}$, sahasrakiraṇa śrīmān $\mathrm{N}_{58}^{K}$, kiraṇādvayasamyuuktạ $\mathrm{B}_{99}^{C}$, kiranāvalisamyuktah $\mathrm{P}_{72}^{T}$ 11od saptasaptika $\left.{ }^{\circ}\right] \Sigma$, saptasaptyeka ${ }^{\circ} \hat{S}_{67}^{S}$, saptasaptaṃka ${ }^{\circ} \mathrm{N}_{82}^{K a c} \bullet{ }^{\circ}$ vāhanaḥ ] $\Sigma$, ${ }^{\circ}$ vāhanam $\mathrm{N}_{45}^{C} \quad$ 111a bhagavān ] $\Sigma$, bhagavā $\mathrm{N}_{45}^{C} \quad$ 11b śivapūjārcane rataḥ ] $\mathrm{N}_{77}^{K o} \mathrm{~K}^{\Sigma} \mathrm{E}^{N} \mathrm{~B}_{99}^{C} \hat{S}_{67}^{S}$, śivapūjārcane ratāḥ $\mathrm{N}_{45}^{C}$, śivārcanaratah sadā $\mathrm{P}_{3^{2}}^{T}$, harapādārcane ratạ̣ $\mathrm{P}_{7^{2}}^{T}$ 111 grahapīḍāo ] $\mathrm{N}_{45}^{C} \mathrm{~N}_{12}^{K} \mathrm{~N}_{5^{8}}^{K p c} \mathrm{E}^{N} \mathrm{~B}_{99}^{C} \dot{S}_{67}^{S}$, grahapīḍām $\mathrm{N}_{82}^{K} \mathbf{P}^{\Sigma}$, gra+ha+pīḍāo $\mathrm{N}_{58}^{K} \bullet{ }^{\circ}$ nivāraṇīm ] $\mathrm{N}_{82}^{K} \mathrm{~B}_{99}^{C}$, onivāriṇīṃ $\mathrm{N}_{77}^{K o}$, onivāraṇī $\mathrm{N}_{45}^{C} \mathrm{~N}_{12}^{K} \mathrm{E}^{N}$, ${ }^{\circ}$ nivāraṇaḥ $\mathrm{N}_{58}^{K}$, ${ }^{\circ}$ nivāraṇam $\hat{S}_{67}^{S}$, vyapohatu $\mathbf{P}^{\Sigma}$ 
[Soma]

jagadāpyāyanakara amṛtādhāraśītalạ̣|

somaḥ saumyena bhāvena grahapị̣̄ām vyapohatu|| 112||

\section{[Añgāraka]}

padmarāganibhāngena dehenāpingalocanaḥ| añgārakas tu me nityaṃ grahapị̣̄āṃ vyapohatu|| 113||

112 Cf. BhavP 1.175·38cd-39 : tricakraratham ārūḍha apāṃsāramayam tu yah|| daśāśvavāhano deva ātreyaś cāmṛtasravaḥ| śītāṃśur amṛtātmā ca kṣayavṛddhisamanvitaḥ| somaḥ saumyena bhāvena grahapị̣̂āṃ vyapohatu|| 113 Cf. BhavP 1.175.40: padmarāganibho bhaumo madhupingalalocanaḥ| angārako 'gnisadṛ́so grahapị̣āṃ vyapohatu||

112a-d Omitted in $\mathrm{P}^{\Sigma} \quad$ 113d After this $\mathrm{N}_{58}^{K}$ adds four pādas (hypermetr.) : rudrasambhāvasampanno rudradhyānaikamānasaḥ| grahapīdām bhayaṃ sarvva nirnnāsayantu me| $\bullet \mathbf{P}^{\Sigma}$ add : rudrasadbhāvasaṃpanno rudradhyānaikamānasaḥ| grahapīụābhayaṃ sarvaṃ vināśayatu me sadā|

112ab 'kara amṛtādhāra $\left.{ }^{\circ}\right] \mathrm{N}_{45}^{C} \mathrm{~N}_{12}^{K} \mathrm{E}^{N} \mathrm{~B}_{99}^{c}$, ${ }^{\circ}$ karo hyāmṛtādhāra ${ }^{\circ} \mathrm{N}_{77}^{K o}$, odhāro hyamṛtādhāra ${ }^{\circ} \mathrm{N}_{82}^{K}$, ${ }^{\circ}$ karo amṛtādhārah $\mathrm{N}_{58}^{K}$, ${ }^{\circ}$ karo mṛtadīpita ${ }^{\circ} \hat{S}_{67}^{S} \quad$ 112b ${ }^{\circ}$ śitalah ] $\mathrm{N}_{77}^{K o} \mathrm{~K}^{\Sigma} \mathrm{E}^{N} \mathrm{~B}_{99}^{C} \dot{S}_{67}^{S}$, ${ }^{\circ}$ sītalāḥ $\mathrm{N}_{45}^{C} \quad$ 112c somah ] $\mathbf{K}^{\Sigma} \mathrm{E}^{N} \mathrm{~B}_{99}^{C} \dot{S}_{67}^{S}$, soma $\mathrm{N}_{77}^{K o} \mathrm{~N}_{45}^{C} \bullet$ saumyena bhāvena ] $\mathrm{N}_{45}^{C} \mathrm{~N}_{82}^{K} \mathrm{~N}_{12}^{K} \mathrm{E}^{N} \mathrm{~B}_{99}^{C} \dot{S}_{67}^{S}$, syaumyena bhāvena $\mathrm{N}_{77}^{K o}$, saumye $<->$ śivabhakto $\mathrm{N}_{58}^{K}$ 112d 'pị̣̄ām ] $\mathrm{K}^{\Sigma} \mathrm{E}^{N} \mathrm{~B}_{99}^{C} \hat{S}_{67}^{S}$, ${ }^{\circ}$ pịḍa $\mathrm{N}_{77}^{K o} \mathrm{~N}_{45}^{C} \quad$ 113a ${ }^{\circ}$ nibhāngena ] $\mathrm{N}_{82}^{K} \mathrm{~N}_{12}^{K} \mathrm{E}^{N} \mathrm{~B}_{99}^{C}$, ${ }^{\circ}$ nibho bhāti $\mathrm{N}_{77}^{K o} \mathrm{~N}_{45}^{C}$, ${ }^{\circ}$ nibhā bhāti $\mathrm{N}_{58}^{K}$, ${ }^{\circ}$ nibho yasya $S_{67}^{S}$, ${ }^{\circ}$ nibhenāpi $\mathbf{P}^{\Sigma} \quad$ 113b dehenāpingalocanạ̣ ] $\mathrm{N}_{12}^{K} \mathrm{~N}_{58}^{K} \mathrm{~B}_{99}^{C} \mathrm{P}_{32}^{T}$, dehe pimgalalocanaḥ $\mathrm{N}_{77}^{K o}$, dehe pingalalocanā $\mathrm{N}_{45}^{C}$, dehenāpingalocanā $\mathrm{N}_{82}^{K \mathrm{pc}}$, dehena pingalocanā $\mathrm{N}_{82}^{K a c} \mathrm{E}^{N}$, dehah pingalalocanaḥ $\hat{S}_{67}^{S}$, dehānāpiṅgalocanaḥ $\mathrm{P}_{72}^{T} \quad \mathbf{1 1 3 c}$ añgārakas tu me nityam ] $\mathrm{N}_{82}^{K} \mathrm{~B}_{99}^{C}$, aṃgāraka śive bhaktā $\mathrm{N}_{77}^{K o}$, aṅgārakās tu satatam $\mathrm{N}_{45}^{C} \mathrm{~N}_{12}^{K}$, anggāras tu satatam $\mathrm{N}_{58}^{K}$ (unmetr.), añgārakantu me nityaṃ $\mathrm{E}^{N}$, angārakah śive bhakto $S_{67}^{S} \mathrm{P}^{\Sigma} \quad$ 113d grahapīḍām vyapohatu ] $\mathrm{N}_{45}^{C} \mathrm{~N}_{82}^{K} \mathrm{~N}_{12}^{K} \mathrm{E}^{N} \mathrm{~B}_{99}^{C} \dot{S}_{67}^{S}$, grahapīẹā vyapohatu $\mathrm{N}_{77}^{K o}$, rudrapūjārccane ratạ $\mathrm{N}_{58}^{K}$, rudrārcanaparāyaṇaḥ $\mathbf{P}^{\Sigma}$ 


\title{
[Budha]
}

kuṅkumacchavidehena cāpodyatakaraḥ sadā| śivabhakto budhaḥ śrīmān grahapīḍāṃ vyapohatu|| 114||

\author{
[Bṛhaspati]
}

dhātucāmīkaracchāyaḥ sarvajñānakṛtālayaḥ|

bṛhaspatiḥ sadākālam īśānārcanatatparaḥ|| 115||

so 'pi me śāntacittena parameṇa samāhitaḥ|

grahapị̣̂ām vinirjitya karotu vijayaṃ sadā|| 116||

114 Cf. BhavP 1.175.41: puṣparāganibheneha dehena paripingalaḥ| pītamālyāmbaradharo budhah pịḍām vyapohatu|| $\quad$ 115-116 Cf. BhavP 1.175.42-44ab : taptagaurikasaṃkāśaḥ sarvaśāstraviśāradaḥ| sarvadevagurur vipra atharvaṇavaro muniḥ|| bṛhaspatir iti khyāta arthaśāstraparaś ca yaḥ| śāntena cetasā so 'pi pareṇa susamāhitaḥ|| grahapị̣āṃ vinirjitya karotu tava śāntikam|

114a kuṅkumacchavidehena ] $\mathrm{N}_{82}^{K} \mathrm{~N}_{58}^{K} \mathrm{E}^{N} \mathrm{~B}_{99}^{C}$, kuñkumacchavinācchena $\mathrm{N}_{77}^{K o} \mathrm{~N}_{45}^{C} \mathrm{~N}_{12}^{K}$, kun̉kumāruṇagātreṇa $S_{67}^{S}$, kuñkumacchavinā śrīmad $\mathbf{P}^{\Sigma} \quad \mathbf{1 1 4}$ b cāpodyatakaraḥ sadā ] $\mathrm{N}_{82}^{K} \mathrm{~N}_{58}^{K} \mathrm{E}^{N} \mathrm{~B}_{99}^{C}$, dehena parimaṇḍalạ̣ $\mathrm{N}_{77}^{K o} \mathrm{~N}_{45}^{C} \mathrm{~N}_{12}^{K}$, dehena parimaṇditah $\dot{S}_{67}^{S} \mathbf{P}^{\Sigma} \quad$ 114c śivabhakto budhah śrīmān ] $\Sigma$, budho 'pi śivabhaktātmā $\mathrm{P}_{3^{2}}^{T} \quad$ 114d vyapohatu ] $\Sigma$, vyapotu $\mathrm{N}_{45}^{C}$ (unmetr.) 115a dhātu $\left.{ }^{\circ}\right] \Sigma$, dhauta ${ }^{\circ} \mathrm{N}_{58}^{K}, \operatorname{tapta}^{\circ} \mathbf{P}^{\Sigma} \bullet{ }^{\circ}$ cchāyah ] $\mathbf{K}^{\Sigma} \mathrm{B}_{99}^{C} \dot{S}_{67}^{S} \mathbf{P}^{\Sigma}$, ${ }^{\circ}$ cchāḥ $\mathrm{E}^{N}$ (unmetr.), ${ }^{\circ}$ cchāyā $\mathrm{N}_{77}^{K o} \mathrm{~N}_{45}^{C} \quad{ }_{115} \mathbf{c}$ bṛhaspatị̣ ] $\Sigma$, bṛhaspati $\mathrm{N}_{77}^{K o} \mathrm{~N}_{45}^{C} \mathrm{~N}_{58}^{K} \bullet$ sadākālaṃ ] $\Sigma$, mahātejāḥ $\mathrm{N}_{58}^{K}$, sadāśānta $S_{67}^{S} \quad$ 115d íśānārcanatatparaḥ ] $\Sigma$, īśānārcanatatparām $\mathrm{N}_{45}^{C}$, śicārccanavidhipriyaḥ $\mathrm{N}_{58}^{K} \quad$ 116a me śāntacittena ] $\mathrm{N}_{77}^{C p c} \mathrm{~K}^{\Sigma} \mathrm{E}^{N} \mathrm{~B}_{99}^{C} \dot{S}_{67}^{S}$, śāntena cittena $\mathrm{N}_{77}^{K o}$, me śānticittena $\mathrm{N}_{77}^{C \mathrm{Cac}}$, śāntātmabhāvena $\mathbf{P}^{\Sigma} \quad 116 b$ parameṇa ] $\Sigma$, om. $\mathrm{N}_{58}^{K}$ (unmetr.) • samāhitạ̣ ] $\mathrm{N}_{82}^{K} \mathrm{~N}_{12}^{K} \mathrm{E}^{N} \mathrm{~B}_{99}^{C} \hat{S}_{67}^{S}$, samādhinā $\mathrm{N}_{77}^{K o} \mathrm{~N}_{45}^{C} \mathrm{~N}_{58}^{K} \mathrm{P}_{72}^{T}$, subhāvitah $\mathrm{P}_{32}^{T} \quad{ }_{116 c}{ }^{\circ}$ pịdām ] $\Sigma$, ${ }^{\circ}$ pīḍā $\mathrm{N}_{77}^{K o} \mathrm{~N}_{45}^{C} \quad$ 116d sadā ] $\mathrm{N}_{82}^{K} \mathrm{~N}_{12}^{K} \mathrm{E}^{N} \mathrm{~B}_{99}^{C}$, mamah $\mathrm{N}_{77}^{K o}$, ca me $\mathrm{N}_{45}^{C}$, mama $\mathrm{N}_{58}^{K} \dot{\mathrm{S}}_{67}^{S} \mathbf{P}^{\Sigma}$ 


\section{[Śukra]}

himakundendutulyābhạ̣ suradaityendrapūjitaḥ| śukraḥ śivārcanarato grahapị̣̄ām vyapohatu|| 117||

[Śanaiścara]

bhinnāñjanacayacchāyaḥ suraktanayanadyutị̣| śanaiścarah śive bhakto grahapị̣̄ām vyapohatu|| 118||

[Rāhu]

nīlāñjananibhạ̣ śrīmān saiṃhikeyo mahābalaḥ| śivapūjāparo rāhur grahapiệām vyapohatu|| 119||

117 Cf. BhavP 1.175.44cd-46 : sūryārcanaparo nityaṃ prasādād bhāskarasya tu|| himakundenduvarṇābho daityadānavapūjitaḥ| maheśvaras tato dhīmān mahāsauro mahāmatiḥ|| sūryārcanaparo nityaṃ śukraḥ śuklanibhas tadā| nītiśāstraparo nityaṃ grahapīḍām vyapohatu||

117 ab ${ }^{\circ}$ tulyābhah sura $\left.{ }^{\circ}\right] \mathrm{K}^{\Sigma} \mathrm{E}^{N} \mathrm{~B}_{99}^{C} \mathrm{P}_{32}^{T}$, ${ }^{\circ}$ varṇṇābha sura ${ }^{\circ} \mathrm{N}_{77}^{K o}$, ${ }^{\circ}$ varṇnābhah svara ${ }^{\circ}$ $\mathrm{N}_{45}^{C}$, ${ }^{\circ}$ varṇābhah sura ${ }^{\circ} S_{67}^{S} \mathrm{P}_{72}^{T} \quad \mathbf{1 1 7}^{\circ} \mathbf{b}{ }^{\circ}$ daityendra $\left.{ }^{\circ}\right] \mathrm{N}_{77}^{K o} \mathrm{~N}_{12}^{K} \mathrm{~N}_{58}^{K} \mathrm{E}^{N} \mathrm{~B}_{99}^{C} S_{67}^{S} \mathrm{P}_{72}^{T}$, odaityaindra ${ }^{\circ} \mathrm{N}_{45}^{C}$, ${ }^{\circ}$ daisyendra ${ }^{\circ} \mathrm{N}_{82}^{K}$, ${ }^{\circ}$ daityaih $\operatorname{su}^{\circ} \mathrm{P}_{32}^{T} \bullet{ }^{\circ}$ pūjitah ] $\Sigma$, ${ }^{\circ}$ pūjakah $\mathrm{N}_{77}^{K o} \quad$ 117c śukrah ] $\Sigma$, śukra $\mathrm{N}_{77}^{K o} \mathrm{~N}_{5^{8}}^{K} \bullet$ śivārcanarato ] $\Sigma$, śivārccane nityam $\mathrm{N}_{45}^{C}$, śivārcane bhakto $\hat{S}_{67}^{S} \quad \mathbf{1 1 8 a}$ bhinnāñjanacayacchāyaḥ ] $\mathrm{N}_{45}^{C} \mathbf{K}^{\Sigma} \mathrm{E}^{N} S_{67}^{S}$, bhinnājanasamacchāyā $\mathrm{N}_{77}^{K o}$, bhinnājanacayachāyāḥ $\mathrm{B}_{99}^{C}$, bhinnāñjanasamachāyaḥ $\mathrm{P}_{32}^{T}$, nīlajīmūtasaṅkāśaḥ $\mathrm{P}_{72}^{T} \quad \mathbf{1 1 8 b}$ surakta $\left.{ }^{\circ}\right] \mathrm{N}_{45}^{C} \mathrm{~K}^{\Sigma} \mathrm{B}_{99}^{C} \grave{S}_{67}^{S} \mathrm{P}_{72}^{T}$, surakto $\mathrm{N}_{77}^{K o}$, sarakta ${ }^{\circ} \mathrm{E}^{N}$, samrakta ${ }^{\circ} \mathrm{P}_{3^{2}}^{T} \bullet{ }^{\circ}$ nayanadyutih ] $\Sigma$, onayanadyuti $\mathrm{N}_{77}^{K o}$, ${ }^{\circ}$ nayanodyutih $\mathrm{N}_{58}^{K} \quad$ 118c śanaiścarah ] $\mathrm{N}_{82}^{K} \mathrm{~B}_{99}^{C} \dot{S}_{67}^{S} \mathbf{P}^{\Sigma}$, śanaiścara $\mathrm{N}_{77}^{K o} \mathrm{~N}_{45}^{C} \mathrm{~N}_{12}^{K} \mathrm{~N}_{5^{8}}^{K} \mathrm{E}^{N} \bullet$ bhakto ] $\Sigma$, bhaktāḥ $\mathrm{N}_{45}^{C} \quad$ 118d ${ }^{\circ}$ pịdāị ] $\Sigma$, ${ }^{\circ}$ pīḍā $\mathrm{B}_{99}^{C}$ 119a nīlāñjananibhạ̣ śrīmān ] $\mathrm{N}_{77}^{K o} \mathrm{~N}_{82}^{K} \mathrm{~N}_{12}^{K} \mathrm{~B}_{99}^{C} \dot{S}_{67}^{S}$, jambuvarṇṇānibho tugrah $\mathrm{N}_{45}^{C}$, nīlāñjananibha śrīmān $\mathrm{N}_{58}^{K}$, nilāñjananibhaḥ śrīmān $\mathrm{E}^{N}$, nīlāṃjanasamah śrīmān $\mathrm{P}_{32}^{T}$, nīlameghāṃjanachāyah $\mathrm{P}_{72}^{T} \quad$ 119b saiṃhikeyo mahābalah ] $\mathrm{N}_{82}^{K} \mathrm{~N}_{12}^{K} \mathrm{E}^{N} \dot{\mathrm{S}}_{67}^{S} \mathbf{P}^{\Sigma}$, senghikeyo mahābalaḥ $\mathrm{N}_{77}^{K o}$, arddhakāyaḥ kṛtāñjaliḥ $\mathrm{N}_{45}^{C}$, saihikeyo mahābalaḥ $\mathrm{N}_{58}^{K}$, śaiṃhikeyo mahābalaḥ $\mathrm{B}_{99}^{C} \quad{ }_{119 c}{ }^{\circ}$ paro ] $\Sigma$, ${ }^{\circ}$ rato $\mathrm{P}_{32}^{T} \bullet$ rāhur ] $\Sigma$, rāhu $\mathrm{N}_{77}^{K o} \mathrm{~N}_{45}^{C} \quad$ 119d ${ }^{\circ}$ pịḍām ] $\Sigma$, ${ }^{\circ}$ pị̣̣̂ā $\mathrm{N}_{77}^{K o} \mathrm{~N}_{45}^{C} \mathrm{~N}_{12}^{K}$ 
[Ketu $]$

dhūmākāro grahaḥ ketur aiśānyāṃ diśi saṃsthitaḥ|

vartulātīvavistīrṇair locanaiś ca subhīṣaṇaḥ|| 120||

palāladhūmasaṃkāśo grahapīḍāpahārakaḥ|

ghoradaṃștrākarālī ca karotu vijayaṃ mama|| 121||

\section{[Grahas: Conclusion]}

ete grahā mahātmāno maheśārcanabhāvitāḥ|

śāntiṃ kurvantu me hṛștāḥ sadākālaṃ hitaișiṇaḥ|| 122||

120-121 Cf. BhavP 1.175·47-49: nānārūpadharo 'vyakta avijñātagatiś ca yaḥ| notpattir jāyate yasya nodayam pị̣̂itair api|| ekacūlo dvicūlaś ca triśikhaḥ pañcacūlakaḥ| sahasraśirarūpas tu candraketur iva sthitaḥ|| sūryaputro 'gniputras tu brahmaviṣnuśivātmakaḥ| anekaśikharaḥ ketuh sa te pīḍām vyapohatu|| 122 Cf. BhavP 1.175.50 : ete grahā mahātmānaḥ sūryārcanaparāḥ sadā| saantiṃ kurvantu te hṛșțạ̣̄ sadākālaṃ hitekṣaṇāḥ||

12Oa-121d Omitted in $\mathrm{N}_{77}^{K o} \bullet$ Instead of this $\mathrm{N}_{12}^{K}$ has four pādas reading : śvetapītaśikhājyotikiñcinīlāñjanaprabhaḥ| śivārcanarataḥ ketur grahapị̣āạ vyapohatu| • $S_{67}^{S}$ has : śvetapītāruṇakṛ̣ṇaḥ kvaciccāmīkaraprabhaḥ| śivārcanaratạ̣ ketur grahapīḍāṃ vyapohatu| $\bullet \mathbf{P}^{\Sigma}$ has : dhūmradehayutạ̣ krūraḥ sarvotpātasamanvitạ̣| śivārcanarataḥ ketuḥ grahapīḍām vyapohatu| 121d After this $\mathrm{E}^{N}$ adds four pādas : khaḍgasphațikahastā ca vareṇyo varadạ̣ śubhạ̣| śivabhaktaś ca janmā ca grahapị̄̂ām vyapohatu| 122d After this $\mathrm{N}_{58}^{K}$ adds eight pādas reading : (bava ca) - (bha) - ścaiva kau - (va) - (tilaṃ) garaṃ| vanigvișțisākarttaghno nāgaḥ śakunir eva ca| karaṇāni daśetāni tithibhāgānugāminaḥ| śivapūjābhiyuktāni śānti kurvvantu me sadā|

120 dhūmākāro grahạ ketur ] $\mathrm{N}_{82}^{K} \mathrm{~N}_{58}^{K} \mathrm{E}^{N} \mathrm{~B}_{99}^{C}$, ketur(nn)āma graho tyugra $\mathrm{N}_{45}^{C}$ 120 baṃsthitaḥ ] $\mathrm{N}_{82}^{K} \mathrm{~N}_{58}^{K} E^{N} \mathrm{~B}_{99}^{C}$, saṃsthitāḥ $\mathrm{N}_{45}^{C} \quad$ 120c ${ }^{\circ}$ vistīrṇair ] $\mathrm{N}_{58}^{K}$, ${ }^{\circ}$ vistīrṇe $\mathrm{N}_{82}^{K} \mathrm{E}^{N} \mathrm{~B}_{99}^{C}$, ${ }^{\circ}$ vistīrṇno $\mathrm{N}_{45}^{C} \quad \operatorname{12od}$ locanaiś ca ] $\mathrm{N}_{82}^{K} \mathrm{~N}_{58}^{K} \mathrm{~B}_{99}^{C}$, locaneśca $\mathrm{E}^{N}$, locanena $\mathrm{N}_{45}^{C} \quad 121 a b{ }^{\circ}$ saṃkāśo grahapīdāpahārakaḥ ] $\mathrm{N}_{82}^{K} \mathrm{E}^{N}$, ${ }^{\circ}$ sastārakagrahamastakaḥ $\mathrm{N}_{45}^{C}$ (unmetr.), ${ }^{\circ}$ saṃkāśo grahapiẹāạ vyapohatu $\mathrm{N}_{58}^{K}$, ${ }^{\circ}$ saṃkāśo grahapị̣̄āṃ vyapohatu <bhinnājanacayachāyo $>\mathrm{B}_{99}^{C} \quad 121 \mathrm{c}{ }^{\circ}$ daṃșțrākarālī ] $\mathrm{N}_{82}^{K} \mathrm{~B}_{99}^{C}$, ${ }^{\circ}$ daștākarālī $\mathrm{N}_{45}^{C}$, ${ }^{\circ}$ (drașț̣ā) karālī $\mathrm{N}_{58}^{K}$, odaṃșțro karālaś $\mathrm{E}^{N} \quad$ 121d mama ] $\mathrm{N}_{82}^{K} \mathrm{~N}_{58}^{K} \mathrm{E}^{N} \mathrm{~B}_{99}^{C}$, mamaḥ $\mathrm{N}_{45}^{C}$ 122b maheśārcanabhāvitāḥ ] $\mathrm{N}_{77}^{K o} \mathrm{~N}_{45}^{C} \mathrm{~N}_{82}^{K} \mathrm{~N}_{12}^{K} \mathrm{E}^{N} \mathrm{~B}_{99}^{C}$, maheśārccanabhāvitạ̣ $\mathrm{N}_{58}^{K}$, maheśārcanatatparāḥ Ś $S_{67}^{S}$, śivārcanaratāḥ sadā $\mathbf{P}^{\Sigma} \quad$ 122c kurvantu ] $\Sigma$, karotu $\mathrm{N}_{58}^{K}$ hṛșțāḥ ] $\mathrm{N}_{12}^{K} \mathrm{~N}_{58}^{K} \mathrm{E}^{N} \mathrm{P}_{32}^{T}$, hrusțāa $\mathrm{N}_{77}^{K o} \mathrm{~N}_{45}^{C} \mathrm{~N}_{82}^{K} \mathrm{~B}_{99}^{C}$, prītāḥ $S_{67}^{S}$, nityam $\mathrm{P}_{72}^{T} \quad$ 122d sadākālam ] $\Sigma$, sarvakālaṃ $\mathrm{N}_{45}^{C}$, sarvakālao $\hat{\mathrm{S}}_{67}^{S} \bullet$ hitaișiṇaḥ ] $\Sigma$, hitaiśinaḥ $\mathrm{N}_{77}^{K o} \mathrm{~N}_{45}^{C} \mathrm{~N}_{58}^{K}$ 
[Viștị]

mukhe yasya sthito mṛtyur viștiir nāma mahābalā| ṣaṇmukhā vighnakarī ca pucche ca vijayaṃkarī|| 123||

tṛtīyā saptamī caiva daśamī tu caturdaśî|

caturthī așțamī caiva ekādaśí tu pūrṇimā|| 124||

eteṣu vighnapātreṣu kṛṣne śukle samāvahā|

kurvantu me labdhavarāḥ śāntiṃ ca paramepsitām|| 125||

123a-136d Omitted in $\mathrm{N}_{77}^{K o} \quad$ 123d After this $\mathrm{P}^{\Sigma}$ add two pādas : rudrapranāamaparamā śāntim āśu karotu me| 124a-d Instead of this $\mathrm{N}_{5^{8}}^{K}$ has six pādas reading : tṛtīyāyāṃ smṛte rātrau saptamyā - - - divā| daśamyā rātribhāge ca caturdaśyāṃ divā tathā| caturthyāṃ niśibhāge tu aștamyān nise tathā| $\quad$ 125a-d Omitted in $\mathrm{N}_{58}^{K}$

123a yasya ] $\mathrm{N}_{45}^{C} \mathrm{~K}^{\Sigma} \mathrm{E}^{N} \mathrm{~B}_{99}^{C} \mathrm{P}_{32}^{T}$, yasyāḥ $\hat{S}_{67}^{S} \mathrm{P}_{72}^{T}$ • sthito ] $\mathrm{N}_{45}^{C} \mathbf{K}^{\Sigma} \mathrm{E}^{N} \mathrm{~B}_{99}^{C} \mathbf{P}^{\Sigma}$, smrto $\hat{S}_{67}^{S}$ 123b viștị ] $\mathrm{N}_{12}^{K} \mathrm{~B}_{99}^{C} S_{67}^{S} \mathrm{P}_{3^{2}}^{T}$, vișți $\mathrm{N}_{58}^{K}$, vṛștír $\mathrm{N}_{45}^{C} \mathrm{~N}_{82}^{K} \mathrm{E}^{N} \mathrm{P}_{72}^{T} \quad$ 123c șaṇmukhā ] $\mathrm{N}_{82}^{K} \mathrm{~N}_{12}^{K} \mathrm{E}^{N}$, samukhā $\mathrm{N}_{45}^{C}$, sammukhe $\mathrm{N}_{58}^{K}$, sammukhā $\hat{S}_{67}^{S}$, sumukhā $\mathbf{P}^{\Sigma} \bullet$ vighnakarī ] $\mathrm{N}_{45}^{C} \mathrm{~K}^{\Sigma} \mathrm{E}^{N} \hat{S}_{67}^{S} \mathrm{P}_{32}^{T}$, vighnakī $\mathrm{B}_{99}^{C}$ (unmetr.), vighnakartrī $\mathrm{P}_{72}^{T} \quad \mathbf{1 2 3}^{2}$ pucche ca ] $\mathrm{N}_{82}^{K} \mathrm{~N}_{12}^{K} \mathrm{E}^{N} \mathrm{~B}_{99}^{C} \hat{S}_{67}^{S} \mathrm{P}_{32}^{T}$, pumcche ca $\mathrm{N}_{45}^{C}$, pucchena $\mathrm{N}_{58}^{K}$, puștyai ca $\mathrm{P}_{72}^{T} \quad \mathbf{1 2 4 a}$ tṛtīyā saptamī ] $\mathrm{N}_{45}^{C} \mathrm{~N}_{82}^{K} \mathrm{~N}_{12}^{K} \mathrm{E}^{N} \mathrm{~B}_{99}^{C} \mathrm{P}^{\Sigma}$, tṛtīyāṃ saptamīṃ $S_{67}^{S} \quad \mathbf{1 2 4} \mathbf{b}$ daśamī tu caturdaśī ] $\mathrm{N}_{82}^{K} \mathrm{~N}_{12}^{K} \mathrm{E}^{N} \mathrm{~B}_{99}^{C}$, daśamī ca caturdaśi $\mathrm{N}_{45}^{C} \mathbf{P}^{\Sigma}$, daśamīṃ ca caturdaśīm $S_{67}^{S} \quad \mathbf{1 2 4 c}$ caturthī așțamī caiva ] $\mathrm{N}_{82}^{K} \mathrm{~N}_{12}^{K} \mathrm{~B}_{99}^{C}$, caturthī cāșțamī caiva $\mathrm{N}_{45}^{C} \mathrm{E}^{N}$, caturthīm așțamīm ekāo $\hat{S}_{67}^{S}$, caturthī tv așțamī caiva $\mathbf{P}^{\Sigma} \quad$ 124d ekādaśī tu pūrṇimā ] $\mathrm{E}^{N} \mathrm{~B}_{99}^{C}$, ekādaśyāṃ̣ayottamāṃ $\mathrm{N}_{45}^{C}$, ekādaśī tu pūrṇṇamī $\mathrm{N}_{82}^{K} \mathrm{~N}_{12}^{K}$, ${ }^{\circ}$ daśīị caiva tu pūrṇimām $S_{67}^{S}$, ekādaśī ca paurṇimā $\mathrm{P}_{3^{2}}^{T}$, ekādaśyā tu pūrṇimā $\mathrm{P}_{72}^{T} \quad$ 125a eteșu vighnapātreșu ] $\mathrm{N}_{82}^{K} \mathrm{~N}_{12}^{K} \mathrm{E}^{N} \mathrm{~B}_{99}^{C}$, etā śubhāvahā sarvā $\mathrm{N}_{45}^{C}$, vyāptāttayā ca satatam $S_{67}^{S}$, etā vighnakarā bhaktāḥ $\mathrm{P}_{32}^{T}$, etā vighnakarā bhadrāḥ $\mathrm{P}_{72}^{T} \quad$ 125ab kṛ̣ṇe śukle samāvahā $\mathrm{N}_{82}^{K} \mathrm{~N}_{12}^{K p c} \mathrm{E}^{N}$, kṛṣnā śuklā ca pakṣayoḥ $\mathrm{N}_{45}^{C}$, kṛṣne śukle samāvahāḥ $\mathrm{N}_{12}^{K a c} \mathrm{~B}_{99}^{C}$, pakșayoh śuklakṛṣnayoḥ $S_{67}^{S}$, śuklakṛṣnasamudbhavāḥ $\mathbf{P}^{\Sigma} \quad{ }_{125}$ c kurvantu me labdhavarāḥ ] $\mathrm{N}_{82}^{K} \mathrm{~N}_{12}^{K} \mathrm{E}^{N} \mathrm{~B}_{99}^{C}$, kurvantu me lavarā $\mathrm{N}_{45}^{C}$ (unmetr.), dadātu me labdhavarā $S_{67}^{S}$, bhavantu me śriyai nityaṃ $\mathrm{P}_{3^{2}}^{T}$, nāśayantu bhayaṃ nityaṃ $\mathrm{P}_{72}^{T} \quad{ }_{125} \mathrm{~d}$ śāntiṃ ca paramepsitām ] $\mathrm{N}_{12}^{K} \mathrm{~B}_{99}^{C}$, śāntiṃ ca manasepsitāṃ $\mathrm{N}_{45}^{C}$, śānti ca paramepsitām $\mathrm{N}_{82}^{K}$, śāntiś ca paramipsitam $\mathrm{E}^{N}$, śāntiṃ me manasepsitām $\hat{S}_{67}^{S}$, kurvantu vijayaṃ sadā $\mathrm{P}_{32}^{T}$, kurvantu vijayaṃ mama $\mathrm{P}_{72}^{T}$ 


\section{[Tithis]}

amāvāsī mahāpunyā piț̣devasamanvitā|

śāntiṃ ca paramāṃ puṇyāṃ śivasya paramātmanah|

śivatejaḥsamāyuktā karotu mama śāntikam|| 126||

pratipac ca mahāśāntā dvitiyā ca manoharā|

tṛtīyā ca tithị̣ śrīmān caturthī ca mahāyaśāḥ|| 127||

pañcamī nāma śāntātmā șașțhī ca tithir uttamā|

saptamī tu tithih puṇāa așțamī ca mahābalā|| 128||

127d After this $\mathrm{P}_{3^{2}}^{T}$ adds two pādas : caturthī ca mahāpuṇyā gajavaktrādhidaivatam| $\mathrm{P}_{72}^{T}$ adds : caturthī ca mahāpuṇyā gajavaktrādhidevatā|

126a amāvāsī ] $\mathrm{E}^{N}$, amāvāsyāṃ $\mathrm{N}_{45}^{C}$, amāvāsyī $\mathrm{N}_{82}^{K}$, amāvāśī $\mathrm{N}_{12}^{K} \mathrm{~B}_{99}^{C}$, ekādasyā $\mathrm{N}_{58}^{K}$ (eyeskip), amāvāsyā ] $\mathbf{P}^{\Sigma}$, amāvasyā $S_{67}^{S} \bullet$ mahāpuṇyā ] $\mathrm{N}_{82}^{K \text { pc }} \mathrm{N}_{12}^{K} \mathrm{~N}_{58}^{K} \mathrm{E}^{N} \mathrm{~B}_{99}^{C} \hat{S}_{67}^{S} \mathbf{P}^{\Sigma}$, mahāpuṇyāṃ $\mathrm{N}_{45}^{C}$, puṇyā $\mathrm{N}_{82}^{K a c}$ (unmetr.) $\quad \mathbf{1 2 6 b}{ }^{\circ}$ samanvitā ] $\mathrm{N}_{45}^{C} \mathrm{~K}^{\Sigma} \mathrm{E}^{N} \hat{S}_{67}^{S} \mathbf{P}^{\Sigma}$, ${ }^{\circ}$ sanvitā $\mathrm{B}_{99}^{C}$ (unmetr.) 126c śāntim ca paramāṃ puṇyāṃ ] $\mathrm{N}_{82}^{K} \mathrm{~N}_{12}^{K} \mathrm{~B}_{99}^{c}$, śāntā ca paramā pakvā $\mathrm{N}_{45}^{C}$, śāntiñca paramam puṇyam $\mathrm{N}_{58}^{K}$, śāntiś ca paramāṃ puṇyāṃ $\mathrm{E}^{N}$, śāntā hyeșa tu paramā $\hat{S}_{67}^{S} \mathrm{P}_{72}^{T}$, priyā hyaișā tu paramā $\mathrm{P}_{32}^{T} \quad$ 126d śivasya paramātmanạ̣ ] $\mathrm{N}_{45}^{C} \mathrm{~N}_{82}^{K p c} \mathrm{~N}_{12}^{K} \mathrm{~N}_{58}^{K} \mathrm{E}^{N} \mathrm{~B}_{99}^{C} \mathrm{P}_{32}^{T}$, śivasyāparamātmanaḥ $\mathrm{N}_{82}^{K a c}$, śivārcāyāṃ tu tatparā $S_{67}^{S}$, śivasya ca mahāpriyā $\mathrm{P}_{72}^{T} \quad \mathbf{1 2 6 e}{ }^{\circ}$ tejạ̣ $\left.{ }^{\circ}\right] \mathbf{K}^{\Sigma} \mathrm{E}^{N} \mathrm{~B}_{99}^{C} \dot{S}_{67}^{S} \mathbf{P}^{\Sigma}$, oteja ${ }^{\circ} \mathrm{N}_{45}^{C} \bullet{ }^{\circ}$ yuktā ] $\mathrm{N}_{45}^{C} \mathbf{K}^{\Sigma} \mathrm{B}_{99}^{C} \dot{S}_{67}^{S} \mathbf{P}^{\Sigma}$, oyuktāḥ $\mathrm{E}^{N} \quad 127$ a pratipac ca mahāśāntā ] $\mathrm{N}_{82}^{K} \mathrm{~N}_{12}^{K} \mathrm{E}^{N} \mathrm{~B}_{99}^{C} \dot{S}_{67}^{S}$, pratipadā ca mahāśāntī $\mathrm{N}_{45}^{C}$ (unmetr.), pratipac ca mahāsaāntiṃ $\mathrm{N}_{58}^{K}$, pratipad vahnisamyuktā $\mathbf{P}^{\Sigma} \quad{ }_{127}$ b dvitīyā ca manoharā ] $\mathrm{N}_{82}^{K} \mathrm{~N}_{12}^{K} \mathrm{~N}_{58}^{K p c} \mathrm{E}^{N} \mathrm{~B}_{99}^{C} \dot{S}_{67}^{S}$, dvitīyā ca mahoragāḥ $\mathrm{N}_{45}^{C}$, dvitīyā + ca+ mahonarā $\mathrm{N}_{58}^{K}$, dvitīyārkādhidevatā $\mathrm{P}_{32}^{T}$, dvitīyā cādhidaivatā $\mathrm{P}_{72}^{T} \quad \mathbf{1 2 7} \mathbf{c d}$ tithiḥ śrīmān caturthī ca mahāyaśāḥ ] $\mathrm{N}_{45}^{C} \mathrm{~N}_{82}^{K} \mathrm{E}^{N} \mathrm{~B}_{99}^{C}$, tithiḥ śrīmāṃ catu - - mahāyasāḥ $\mathrm{N}_{12}^{K}$, tithị śrīmāṃścaturthī +ca+ mahāyaśā $\mathrm{N}_{58}^{K}$, tithị somyā caturthī ca mahāyaśāḥ $S_{67}^{S}$, mahāpunyā dhanadena samanvitā $\mathbf{P}^{\Sigma} \quad \mathbf{1 2 8 a}$ pañcamī nāma sāāntātmā ] $\mathrm{N}_{45}^{C} \mathrm{~N}_{82}^{K} \mathrm{~N}_{5^{8}}^{K p c} \mathrm{E}^{N} \mathrm{~B}_{99}^{C}$, pañcamī nā śāntātmā $\mathrm{N}_{12}^{K}$ (unmetr.), pañcamī nāma śā+ntā+tmā $\mathrm{N}_{58}^{K}$, pañcamī nāgavịndeșțā $S_{67}^{S}$, pañcamī śrìyutā nityaṃ $\mathbf{P}^{\Sigma} \quad \mathbf{1 2 8 b}$ ca tithir uttamā ] $\mathbf{K}^{\Sigma} \mathrm{E}^{N} \mathrm{~B}_{99}^{C p c} \hat{S}_{67}^{S}$, tithir uttamā $\mathrm{N}_{45}^{C}$ (unmetr.), ca tir uttamā $\mathrm{B}_{99}^{C \text { ac }}$ (unmetr.), skandādhidaivatam $\mathrm{P}_{3^{2}}^{T}$, skandādhidevatā $\mathrm{P}_{72}^{T} \quad \mathbf{1 2 8 c}$ saptamī tu tithih puṇyā $] \mathrm{N}_{82}^{K} \mathrm{~N}_{12}^{K \text { Kpc }} \mathrm{B}_{99}^{C}$, saptī ca tathā puṇyāṃ $\mathrm{N}_{45}^{C}$ (unmetr.), saptamī tu tithi puṇyā $\mathrm{N}_{12}^{K a c} \mathrm{~N}_{58}^{K}$, saptamī ca tithiḥ puṇyā $\mathrm{E}^{N}$, saptamī ca mahābhāgā $\hat{S}_{67}^{S}$, saptamī ravisaṃyuktā $\mathbf{P}^{\Sigma} \quad \mathbf{1 2 8 d}$ așțamī ca mahābalā ] $\mathrm{K}^{\Sigma} \mathrm{E}^{N} \mathrm{~B}_{99}^{C} \hat{S}_{67}^{S}$, așțamī ca mahāphalā $\mathrm{N}_{45}^{C}$, hy așțamī rudradevatā $\mathrm{P}_{32}^{T}$, hy aștamī rudradaivatā $\mathrm{P}_{72}^{T}$ 
tithiḥ śūlabhṛtaś caiṣā pāpahā paramā smṛtā|

navamī tithir atyugrā durgāyāḥ parikīrtitā|| 129\|

daśamī śobhanā caiva tithir ekādaśî tathā|
dvādaśī caiva śāntātmā tathā tithis trayodaśîl|| 13o||

caturdaśī mahāvīryā tithị̣ śan̉karajātmajā|

pūṛnamā paripūrṇātmā tithiś ca satatojjvalā|| 131||

satataṃ tu śubhātmānas tithayaś ca krameṇa tu|

pakṣadvaye sadā hy ete candragatyānugāminaḥ|

śāntiṃ kurvantu me nityaṃ śivājñānuvidhāyinaḥ|| 132||

129ab Omitted in $\mathbf{P}^{\Sigma} \quad$ 131c-132d Instead of this $\mathbf{P}^{\Sigma}$ has two pādas : etās tu tithayah sarvā îsānārcanatatparāḥ| $\quad$ 132b After this $\mathrm{N}_{45}^{C}$ adds two pādas : nandā rudrā jayāriktā pūrṇnāyāñca pṛthak pṛthak| $\quad \mathbf{1 3 2}^{2}$ After this $\mathrm{N}_{45}^{C}$ adds two pādas : śivapūjāsamāyuktā śivadhyānaparāyaṇā|

129a tithị̣ śūlabhṛtaś caiṣā ] $\mathrm{N}_{12}^{K} \mathrm{E}^{N} \mathrm{~B}_{99}^{C}$, tithi triśūlina sāntā $\mathrm{N}_{45}^{C}$, tithi suulabhṛtaś caișā $\mathrm{N}_{82}^{K}$, tithis triśūlina+ḥ+ śāntā $\mathrm{N}_{58}^{K}$, tithis triśūlahastā yā $S_{67}^{S} \quad \mathbf{1 2 9}$ b pāpahā ] $\mathrm{N}_{45}^{C} \mathrm{~K}^{\Sigma} \mathrm{E}^{N}$, pāpāhā $\mathrm{B}_{99}^{C}$, pāpaghnī $\left.S_{67}^{S} \bullet \operatorname{smṛtā}\right] \mathrm{N}_{45}^{C} \mathrm{~N}_{82}^{K} \mathrm{~N}_{58}^{K} \mathrm{E}^{N} \mathrm{~B}_{99}^{C} S_{67}^{S}$, smṛtāḥ $\mathrm{N}_{12}^{K} \quad{ }_{129} \mathbf{c}$ tithir atyugrā ] $\mathrm{N}_{45}^{C} \mathbf{K}^{\Sigma} \mathrm{E}^{N} \mathrm{~B}_{99}^{C} \mathbf{P}^{\Sigma}$, sarvabhūtāṃ̣̣ā $S_{67}^{S} \quad \mathbf{1 2 9 d}$ durgāyāh parikīrtitā $\mathbf{K}^{\Sigma}$, durgāyā parikīrttitāḥ $\mathrm{N}_{45}^{C}$, durgāyāḥ parikīrttitāḥ $\mathrm{B}_{99}^{C}$, durgrāhyā parikīrtitāḥ $\mathrm{E}^{N}$, durjayā parikīrtitā $S_{67}^{S}$, durgā devyadhidevatā $\mathrm{P}_{32}^{T}$, durgā devyadhidaivatā $\mathrm{P}_{72}^{T} \quad \mathbf{1 3}$ oa daśamī ] $\mathbf{K}^{\Sigma} \mathrm{E}^{N} \mathrm{~B}_{99}^{C} \hat{S}_{67}^{S} \mathbf{P}^{\Sigma}$, daśamo $\mathrm{N}_{45}^{C} \bullet$ śobhanā caiva ] $\mathrm{N}_{45}^{C} \mathbf{K}^{\Sigma} \mathrm{E}^{N} \mathrm{~B}_{99}^{C}$, yamasamyuutā $\mathbf{P}^{\Sigma} \quad 13$ ob tithir ekādaśī tathā ] $\mathrm{N}_{45}^{C} \mathrm{~K}^{\Sigma} \mathrm{B}_{99}^{C}$, tithi ekādaśi tathā $\mathrm{E}^{N}$, tithir ekādaśī śubhā $S_{67}^{S}$, indreṇaikādaśī matā $\mathrm{P}_{3^{2}}^{T}$, caindreṇaikādaśī yutā $\mathrm{P}_{72}^{T} \quad \mathbf{1 3}$ oc caiva śāntātmā ] $\mathrm{N}_{45}^{C} \mathrm{~K}^{\Sigma} \mathrm{E}^{N} \mathrm{~B}_{99}^{C}$, śāntabhūpātmā $S_{67}^{S}$, viṣnusamyuktā $\mathbf{P}^{\Sigma} \quad \mathbf{1 3}$ od tathā tithis ] $\mathrm{B}_{99}^{C}$, tithi cāpi $\mathrm{N}_{45}^{C}$, tathā tithi $\mathrm{K}^{\Sigma} \mathrm{E}^{N}$, tithiś cāpi $S_{67}^{S}$, madanena $\mathbf{P}^{\Sigma} \quad \mathbf{1 3 1 a}$ mahāvīryāa ] $\mathrm{K}^{\Sigma} \mathrm{E}^{N} \mathrm{~B}_{99}^{C} \hat{S}_{67}^{S}$, mahāpunyā $\mathrm{N}_{45}^{C}$, manaḥsthena $\mathrm{P}_{32}^{T}$, maheśena $\mathrm{P}_{72}^{T} \quad \mathbf{1 3 1 b}$ tithị śańkarajātmajā ] $\mathrm{N}_{82}^{K} \mathrm{~N}_{12}^{K}$, tithi śankkarabhāvitā $\mathrm{N}_{45}^{C}$, tithị sā śakarātmajā $\mathrm{N}_{58}^{K}$, tithi śańkarajātmajā $\mathrm{E}^{N} \mathrm{~B}_{99}^{C}$, satatam śaṃkarapriyā $S_{67}^{S}$, paurṇamāsī himāṃśunā $\mathbf{P}^{\Sigma^{58}}$ 131c pūrṇamā ] $\mathrm{N}_{82}^{K} \mathrm{~B}_{99}^{C}$, pūrṇṇamī $\mathrm{N}_{45}^{C}$, pūrṇṇāmā $\mathrm{N}_{12}^{K}$, śumā (ca) $\mathrm{N}_{58}^{K}$, pūrṇamāsyā $\mathrm{E}^{N}$ (unmetr.), pūrṇimā $\hat{S}_{67}^{S} \quad$ 13ıd tithiś ca satatojjvalā ] $\mathrm{N}_{82}^{K} \mathrm{~N}_{12}^{K} \hat{S}_{67}^{S}$, tithi tvesatatojjvalā $B_{99}^{C}$, tithi tvasatatojjvalā $\mathrm{N}_{5^{8}}^{K}$, tithayạ̣ satatojjvalā $\mathrm{E}^{N}$, tithir eșām sadojvalā $\mathrm{N}_{45}^{C} \quad$ 132a satatam tu ] $\mathrm{N}_{82}^{K} \mathrm{~N}_{12}^{K} \mathrm{E}^{N} \mathrm{~B}_{99}^{C}$, satatañca $\mathrm{N}_{45}^{C} \mathrm{~N}_{58}^{K}$, ity evam ca $\hat{S}_{67}^{S} \bullet$ śubhātmanas ] $\mathrm{K}^{\Sigma} \mathrm{E}^{N} \mathrm{~B}_{99}^{C} \hat{S}_{67}^{S}$, śubhātmā $\mathrm{N}_{45}^{C}$ (unmetr.) 132b tithayaś ca ] $\mathrm{N}_{82}^{K} \mathrm{~N}_{58}^{K} \hat{S}_{67}^{S}$, tithipañca $\mathrm{N}_{45}^{C}$, tithiyaśca $\mathrm{N}_{12}^{K} \mathrm{~B}_{99}^{C}$, tithayeśca $\mathrm{E}^{N} \quad \mathbf{1 3 2 c}^{\circ}$ dvaye sadā hy ete ] $\mathrm{K}^{\Sigma} \mathrm{E}^{N} \mathrm{~B}_{99}^{C}$, odvaye mahāpuṇyā $\mathrm{N}_{45}^{C}$, odvayasamā hy etāś $\left.S_{67}^{S} \quad \mathbf{1 3 2 d}^{\text {candra }}{ }^{\circ}\right] \mathrm{K}^{\Sigma} \mathrm{E}^{N} \mathrm{~B}_{99}^{C} \hat{S}_{67}^{S}$, candrāo $\mathrm{N}_{45}^{C} \bullet{ }^{\circ}$ gatyānugāminaḥ $\mathrm{N}_{45}^{C} \mathrm{~K}^{\Sigma} \mathrm{E}^{N} \mathrm{~B}_{99}^{C}$, 'gatyāpratișțhitāḥ $S_{67}^{S} \quad \mathbf{1 3 2 f}$ śivājñānuvidhāyinaḥ ] $\mathrm{N}_{82}^{K p c} \mathrm{E}^{N} \mathrm{~B}_{99}^{C}$, śivajñānuvidhāyinaḥ $\mathrm{N}_{82}^{K a c} \mathrm{~N}_{58}^{K}$, śivadhyānāvidhāyiṇaḥ $\mathrm{N}_{45}^{C}$, śivajñānavidhāyinaḥ $\mathrm{N}_{12}^{K} \hat{\mathrm{S}}_{67}^{S}$, sadākālaṃ hitaișiṇaḥ $\mathrm{P}_{32}^{T}$, sadākālaṃ hite ratāḥ $\mathrm{P}_{72}^{T}$ 
[Yogas]

viṣkambhaḥ prītir āyuṣmān saubhāgyaṃ śobhanas tathā| atigaṇụạ sukarmā ca dhṛtị̣ śūlas tathaiva ca|| 133||

gaṇ̣o vṛddhir dhruvaś caiva vyāghāto harṣaṇas tathā| vajrasiddhir vyatīpāto variyān parighaḥ śivaḥ|| 134||

siddhị̣ sādhyaḥ śubhạ̣ śuklo brahmā aindraś ca vaidhṛtị̣| candrasya bhānoś cotpannā yogāś caite mahābalāḥ|| 135||

133c-134b Omitted in $\mathrm{P}_{32}^{T}$

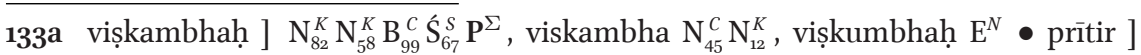
$\mathbf{K}^{\Sigma} \mathrm{E}^{N} \mathrm{~B}_{99}^{C} \hat{S}_{67}^{S} \mathbf{P}^{\Sigma}$, prīti $\mathrm{N}_{45}^{C} \quad \mathbf{1 3 3}^{\mathrm{b}}$ saubhāgyam ] $\mathbf{K}^{\Sigma}$, saubhāgya ${ }^{\circ} \mathrm{N}_{45}^{C} \mathrm{E}^{N}$, saubhāgyaḥ $\hat{S}_{67}^{S} \mathrm{P}_{32}^{T}$, saubhāgyaś $\mathrm{P}_{72}^{T} \bullet$ śobhanas ] $\mathrm{N}_{45}^{C} \mathrm{~N}_{82}^{K} \mathrm{E}^{N} \mathrm{~B}_{99}^{C} \hat{S}_{67}^{S} \mathbf{P}^{\Sigma}$, śobhanan $\mathrm{N}_{12}^{K}$, śobhanam $\mathrm{N}_{58}^{K} \quad{ }^{K} 33 c$ ogaṇụạ ] $\mathbf{K}^{\Sigma} \mathrm{E}^{N} \mathrm{~B}_{99}^{C} \hat{S}_{67}^{S} \mathrm{P}_{72}^{T}$, ogaṇạa $\mathrm{N}_{45}^{C} \bullet$ sukarmā ca ] $\mathbf{K}^{\Sigma} \mathrm{E}^{N} \mathrm{~B}_{99}^{C} \hat{S}_{67}^{S} \mathrm{P}_{72}^{T}$, sukarmāñca $\mathrm{N}_{45}^{C} \quad$ 133d dhṛtiḥ ] $\mathrm{N}_{12}^{K} \mathrm{~N}_{58}^{K} \mathrm{E}^{N} \mathrm{~B}_{99}^{C} \dot{S}_{67}^{S} \mathrm{P}_{72}^{T}$, dhṛtio $\mathrm{N}_{45}^{C} \mathrm{~N}_{82}^{K}$ • śülas ] $\mathrm{K}^{\Sigma} \mathrm{E}^{N} \mathrm{~B}_{99}^{C} \hat{S}_{67}^{S} \mathrm{P}_{72}^{T}$, śūla $\mathrm{N}_{45}^{C} \quad$ 134a vṛddhir ] $\mathrm{N}_{82}^{K} \mathrm{E}^{N} \mathrm{~B}_{99}^{C} \hat{S}_{67}^{S} \mathrm{P}_{72}^{T}$, vruddhi $\mathrm{N}_{45}^{C} \mathrm{~N}_{12}^{K} \mathrm{~N}_{58}^{K} \bullet$ caiva ] $\mathrm{N}_{45}^{C} \mathrm{~N}_{82}^{K} \mathrm{~N}_{12}^{K} \mathrm{E}^{N} \mathrm{~B}_{99}^{C} \dot{S}_{67}^{S} \mathbf{P}^{\Sigma}$, ce $\mathrm{N}_{58}^{K}$ (unmetr.) 134b harșaṇas ] $\mathbf{K}^{\Sigma} \mathrm{E}^{N} \mathrm{~B}_{99}^{C} \dot{S}_{67}^{S} \mathrm{P}_{72}^{T}$, hrasvanas $\mathrm{N}_{45}^{C} \quad{ }_{134 c}$ vajrasiddhir ] $\mathrm{N}_{82}^{K} \mathrm{~B}_{99}^{C} \hat{S}_{67}^{S}$, vajrah siddhir $\mathrm{N}_{58}^{K} \mathrm{E}^{N}$, vajrasiddhi $\mathrm{N}_{45}^{C} \mathrm{~N}_{12}^{K} \mathrm{P}_{32}^{T}$, vajras siddhir $\mathrm{P}_{72}^{T} \bullet$ vyatīpāto ] $\mathrm{N}_{45}^{C} \mathrm{~K}^{\Sigma} \mathrm{E}^{N} \mathrm{~B}_{99}^{C} S_{67}^{S} \mathrm{P}_{72}^{T}$, vyatīpātā $\mathrm{P}_{72}^{T \text { ac }}$, vyatīghātā $\mathrm{P}_{72}^{T \text { pc }}$ 134d variyān ] $\mathrm{N}_{45}^{C} \mathrm{~N}_{82}^{K} \mathrm{~N}_{12}^{K} \mathrm{E}^{N} \hat{S}_{67}^{S}$, varīyān $\mathrm{N}_{58}^{K} \mathrm{~B}_{99}^{C} \mathbf{P}^{\Sigma} \bullet$ parighạ ] $\mathrm{N}_{45}^{C} \mathrm{~N}_{12}^{K} \mathrm{~N}_{58}^{K} \mathrm{E}^{N} \mathrm{~B}_{99}^{C} \dot{\mathrm{S}}_{67}^{S} \mathbf{P}^{\Sigma}$, paridhāḥ $\mathrm{N}_{82}^{K} \quad$ 135a siddhị̣ sādhyaḥ śubhaḥ śuklo ] $\mathrm{N}_{45}^{C} \mathrm{~K}^{\Sigma} \mathrm{E}^{N} \mathrm{~B}_{99}^{C}$, siddhaḥ sādhyaḥ śuciḥ śuklo $\hat{S}_{67}^{S}$, siddhaḥ sādhyaḥ śubhạ śubhro $\mathrm{P}_{32}^{T}$, siddhaṃ sādhyam śubham śubhraṃ $\mathrm{P}_{72}^{T}$ 135b brahmā aindraś ca vaidhṛtị̣ ] $\mathrm{N}_{82}^{K} \mathrm{~B}_{99}^{C}$, brahmaindro vaidhṛtis tathā $\mathrm{N}_{45}^{C}$, brahmā aindrāś ca vaidhṛtih $\mathrm{N}_{12}^{K}$, (brahmā ai) - śca vaidhṛtis tathā $\mathrm{N}_{58}^{K}$ (unmetr.) brahma aindraś ca vaidhṛti $\mathrm{E}^{N}$, brahmendro vaidhṛtiḥ kramāt $\hat{S}_{67}^{S}$, brāhmo māhendravaidhṛtī $\mathrm{P}_{32}^{T}$, brāhmo māhendravaidhṛtị̣ $\mathrm{P}_{72}^{T} \quad \mathbf{1 3 5}^{\mathrm{c}}$ candrasya bhānoś cotpannā ] $\mathrm{N}_{82}^{K} \mathrm{~N}_{12}^{K} \mathrm{E}^{N} \mathrm{~B}_{99}^{C}$, candrāgatyā ca bhānoś ca $\mathrm{N}_{45}^{C}$, caṇḍabhānoś cotpannā $\mathrm{N}_{58}^{K}$ (unmetr.), candrasya bhānvoś cotpannā $S_{67}^{S}$, candrasya bhānor utpannā $\mathbf{P}^{\Sigma} \quad \mathbf{1 3 5}^{\mathrm{d}}$ yogāś caite ] $\mathbf{K}^{\Sigma} \mathrm{E}^{N} \mathrm{~B}_{99}^{C}$, yogā hy ete $\mathrm{N}_{45}^{C} \hat{S}_{67}^{S}$, hy ete yogā $\mathbf{P}^{\Sigma} \bullet{ }^{\circ}$ balāh ] $\mathbf{K}^{\Sigma} \mathrm{E}^{N} \mathrm{~B}_{99}^{C} \dot{S}_{67}^{S} \mathbf{P}^{\Sigma}$, obalā $\mathrm{N}_{45}^{C}$ 
śivabhaktiparāḥ sarve śivājñānuvidhāyinaḥ|
śāntiṃ kurvantu me nityaṃ tathā kilbișanāśanam|| 136||

[Nakṣatramātṛs : E]

kṛttikā paramā devī rohiṇī rucirānanā|

śrīmān mṛgaśirā bhadrā ārdrā ca paramojjvalā|| 137||

punarvasus tathā puṣyā aśleșā ca mahābalā|

nakṣatramātaro hy etāḥ prabhāmālāvibhūṣitāḥ̣|| 138||

137-139 Cf. BhavP 1.179.1-3: kṛttikā paramā devī rohị̣ī ca varānanā| śrīman mṛgaśiro bhadrā ārdrā cāpy aparojjvalā|| punarvasus tathā puṣa āśleșā ca tathādhipa| sūryārcanaratā nityạ̣ sūryabhāvānubhāvitāḥ|| arcayanti sadā devam ādityaṃ surate sadā| nakṣatramātaro hy etāḥ prabhāmālāvibhūṣitāḥ̣||

136d After this $\mathrm{N}_{45}^{C}$ adds eight pādas : saptāvimsatiyogāś ca vyāghātas tu mayāpurāḥ| tatha dīne prajāyāntu tathā kurvantu me śubham| bavabālavakaulaḥ va tautilagaravaṇijāḥ| karaṇānyamahāvīryyāḥ śānti kurvantu me sadā|

136a obhaktiparāḥ sarve ] $\mathrm{N}_{82}^{K} \mathrm{~N}_{58}^{K} \mathrm{E}^{N} \mathrm{~B}_{99}^{C}$, obhaktiratā sarve $\mathrm{N}_{45}^{C}$, obhaktiḥparāḥ sarve $\mathrm{N}_{12}^{K}$, obhaktiparā hyete $S_{67}^{S}$, obhaktiyutāḥ sarve $\mathrm{P}_{3^{2}}^{T}$, obhaktiratās sarve $\mathrm{P}_{72}^{T} \quad \mathbf{1 3} 6 \mathbf{b}$ śivājñānu $\left.{ }^{\circ}\right] \mathrm{N}_{82}^{K} \mathrm{E}^{N} \mathrm{~B}_{99}^{C}$, śivajñānāa $\mathrm{N}_{45}^{C} \mathrm{~N}_{12}^{K} \dot{S}_{67}^{S}$, śivajñānu ${ }^{\circ} \mathrm{N}_{58}^{K}$, śivabhaktio $\mathbf{P}^{\Sigma} \quad$ 136c śāntiṃ kurvantu me nityam ] $\mathrm{N}_{82}^{K} \mathrm{~N}_{12}^{K} \mathrm{E}^{N} \mathrm{~B}_{99}^{C} \hat{S}_{67}^{S} \mathrm{P}_{32}^{T}$, śānti kurvantu me nityam $\mathrm{N}_{45}^{C}$, ete śivārcanaratāḥ $\mathrm{P}_{72}^{T} \quad \mathbf{1 3 6 d}$ tathā kilbiṣanāśanam ] $\mathbf{K}^{\Sigma} \mathrm{E}^{N} \mathrm{~B}_{99}^{C} S_{67}^{S}$, sarve śāntiparāyaṇāḥ $\mathrm{N}_{45}^{C}$, śivadhyānaparāyaṇāḥ $\mathrm{P}_{32}^{T}$, kurvantu mama śāntikam $\mathrm{P}_{72}^{T} \quad$ 137a devī ] $\Sigma$, devyauh $\mathrm{N}_{45}^{C} \quad{ }_{137 \mathrm{c}}$ śrīmān mṛgaśirā bhadrā ] $\mathrm{N}_{45}^{C} \mathrm{~N}_{58}^{K} \mathrm{E}^{N}$, śrīman mrgisisirā bhadrā $\mathrm{N}_{77}^{K o}$, śrīmat mṛgaśirā bhadrā $\mathrm{N}_{82}^{K} \mathrm{~B}_{99}^{C},----_{--} \mathrm{N}_{12}^{K}$, śrīmān mṛgaśirā bhadra $S_{67}^{S}$, mṛ̣aśīrṣā śive bhaktā $\mathrm{P}_{32}^{T}$, śrīman mṛgaśiro bhadrā $\mathrm{P}_{72}^{T} \quad \mathbf{1 3 7 c}^{2}$ ārdrā ] $\Sigma$, ādrā $\mathrm{N}_{77}^{K o} \quad$ 137d paramojjvalā ] $\Sigma$, pamojjvalā $\mathrm{E}^{N}$ (unmetr.) $\mathbf{1 3 8 a}$ punarvasus ] $\Sigma$, punarvvasū $\mathrm{N}_{5^{8}}^{K} \mathrm{~B}_{99}^{C}$, punarvasu $\mathrm{N}_{82}^{K} \quad$ 138ab puṣyā aśleșā ca ] $\mathrm{N}_{82}^{K}$, puṣyā aśleșātha $\mathrm{N}_{77}^{K o} \mathrm{~N}_{45}^{C} \mathrm{E}^{N} \mathrm{~B}_{99}^{C}$, puṣya aśleșā ca $\mathrm{N}_{12}^{K}$, puṣyā a - (śā) ca $\mathrm{N}_{58}^{K}$, tiṣyaś cāśleșā ca $\hat{S}_{67}^{S}$, pușyā āśleșā ca $\mathrm{P}_{3^{2}}^{T}$, puṣyaḥ āśleșā

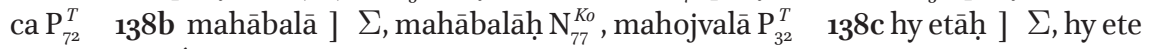
$\mathrm{N}_{77}^{K o}$, devyaḥ $S_{67}^{S} \quad \mathbf{1 3 8 d}{ }^{\circ}$ mālāvibhūṣitāh ] $\mathrm{N}_{82}^{K} \mathrm{~N}_{58}^{K} \mathrm{E}^{N} \mathrm{~B}_{99}^{C}$, ${ }^{\circ}$ mālāvabhāsitā $\mathrm{N}_{77}^{K o}$, otmālābhibhāṣitāḥ $\mathrm{N}_{45}^{C}$, ${ }^{\circ}$ mālānubhāsakāḥ $S_{67}^{S}$, ${ }^{\circ}$ mālāvabhāsitāḥ $\mathrm{N}_{12}^{K} \mathrm{P}_{3^{2}}^{T}$, ${ }^{\circ}$ mālāvabhāsakāḥ $\mathrm{P}_{72}^{T}$ 
mahādevārcane saktā mahādevānubhāvitāḥ| pūrvabhāge sthitā hy etāḥ śāntim kurvantu me sadā|| 139||

[Nakṣatramātṛs : S]

maghā sarvaguṇopetā pūrvā caiva tu phālguṇī|

uttarā phālguṇi śreșṭhā hastā citrā tathottamā|| 140||

svātī viśākhā varadā dakṣiṇasthānasaṃśritāḥ|

arcayanti sadākālaṃ devaṃ tribhuvaneśvaram|| 141||

nakṣatramātaro hy etās tejasā paribhūṣitāḥ|

mamāpi śāntikaṃ nityaṃ kurvantu śivacoditāḥ|| 142||

140-142 Cf. BhavP 1.179.4-5: maghā sarvagunopetā pūrvā caiva tu phālgunī| svātī viśākhā varadā dakṣiṇāṃ diśam āśritāḥ|| arcayanti sadā devam ādityạ̣ surapūjitam| tavāpi śāntikaṃ dyotaṃ kurvantu gaganoditāḥ̂||

142ab Omitted in $\mathrm{N}_{77}^{K o} \mathrm{~N}_{12}^{K} \hat{S}_{67}^{S} \mathbf{P}^{\Sigma}$

139a mahādevārcane saktā ] $\mathrm{N}_{82}^{K} \mathrm{E}^{N} \mathrm{~B}_{99}^{C}$, mahādevārcanaratā $\mathrm{N}_{77}^{K o} \mathrm{~N}_{58}^{K}$, mahādevārcane ratā $\mathrm{N}_{45}^{C} \mathrm{P}_{32}^{T}$ (unmetr.), mahādevārcane śaktā $\mathrm{N}_{12}^{K}$, mahārcāniratānetya $S_{67}^{S}$, mahādevārcanaratāḥ $\mathrm{P}_{72}^{T} \quad$ 139b ${ }^{\circ}$ devānubhāvitāḥ ] $\Sigma$, o devānubhāvitā $\mathrm{N}_{77}^{K o} \mathrm{~N}_{45}^{C}$, ${ }^{\circ}$ devānubhāvinah $\mathrm{N}_{58}^{K}$, ${ }^{\circ}$ devātmabhāvanāḥ $\mathrm{P}_{32}^{T} \quad$ 139c hy etāh ] $\Sigma$, hy ete $\mathrm{N}_{77}^{K o}$, ete $\mathrm{N}_{82}^{K} \quad$ 139d kurvantu ] $\Sigma$, kurvvanti $\mathrm{B}_{99}^{C} \quad$ 140a maghā ] $\Sigma$, magha $\mathrm{N}_{58}^{K}$, makhā $\mathrm{P}_{72}^{T} \quad$ 14ob pūrvā caiva tu phālguṇi ] $\mathrm{N}_{82}^{K p c} \mathrm{E}^{N} \hat{S}_{67}^{S} \mathrm{P}_{32}^{T}$, pūrvaphalguṇi uttamā $\mathrm{N}_{45}^{C}$, pūrvvā caiva tu phalgunī $\mathrm{B}_{99}^{C}$, pūrvā caiva tu phalguṇī $\mathrm{N}_{77}^{K o} \mathrm{~N}_{82}^{K a c} \mathrm{~N}_{58}^{K}$, pūrvā caiva - - - $-\mathrm{N}_{12}^{K}$, pūrvā caiva tu phālgunī $\mathrm{P}_{72}^{T} \quad$ 140c phālguṇī ] $\mathrm{E}^{N} \hat{\mathrm{S}}_{67}^{S} \mathrm{P}_{32}^{T}$, phalgun̄ī $\mathrm{N}_{77}^{K o} \mathrm{~N}_{45}^{C}$, phālgūnī $\mathrm{N}_{82}^{K p c} \mathrm{P}_{72}^{T}$, phalgunī $\mathrm{N}_{82}^{K a c} \mathrm{~B}_{99}^{C}$ - śreșțhā ] $\mathrm{N}_{45}^{C} \mathrm{~N}_{82}^{K} \mathrm{~N}_{58}^{K} \mathrm{E}^{N} \mathrm{~B}_{99}^{C} \dot{S}_{67}^{S} \mathrm{P}_{72}^{T}$, prerthā $S_{67}^{S} \quad$ 14od hastā ] $\mathrm{N}_{45}^{C} \mathrm{~N}_{82}^{K} \mathrm{~N}_{58}^{K} \mathrm{E}^{N} \mathrm{~B}_{99}^{C} \mathrm{P}_{72}^{T}$, hasta ${ }^{\circ} \mathrm{N}_{77}^{K o} \mathrm{P}_{32}^{T}$ - (-tā) $\mathrm{N}_{12}^{K}$, hasttaś $S_{67}^{S} \bullet$ tathottamā ] $\mathrm{N}_{82}^{K} \mathrm{~N}_{58}^{K} \mathrm{E}^{N} \mathrm{~B}_{99}^{C} \hat{S}_{67}^{S} \mathrm{P}_{72}^{T}$, tathaiva ca $\mathrm{N}_{77}^{K o}$, tathotamā $\mathrm{N}_{45}^{C}$, tathottarā $\mathrm{N}_{12}^{K} \mathrm{P}_{32}^{T}$ 141a svātī ] $\mathrm{N}_{45}^{C} \mathrm{~N}_{12}^{K} \mathrm{~N}_{58}^{K} \mathbf{P}^{\Sigma}$, svātmā $\mathrm{N}_{77}^{K o}$, svāti $\mathrm{N}_{82}^{K}$, svātir $\mathrm{E}^{N} \mathrm{~B}_{99}^{C} \stackrel{\mathrm{S}}{67}_{67}^{S} \bullet$ viśākhā ] $\Sigma$, viśākha $\mathrm{N}_{77}^{K o} \quad \mathbf{1 4 1 b}{ }^{\circ}$ saṃśritāh ] $\mathrm{N}_{82}^{K} \mathrm{~B}_{99}^{C}$, ${ }^{\circ}$ māśritā $\mathrm{N}_{45}^{C}$, ${ }^{\circ}$ saṃśritā $\mathrm{E}^{N}$, ${ }^{\circ}$ māsthitā $\mathrm{N}_{77}^{K o} \hat{\mathrm{S}}_{67}^{S}$, ${ }^{\circ}$ samssthitā $\mathrm{N}_{5^{8}}^{K}$, ${ }^{\circ}$ saṃsthitāḥ $\mathrm{N}_{12}^{K} \mathrm{P}^{\Sigma} \quad$ 141c arcayanti sadākālam ] $\mathbf{N}^{\Sigma} \mathrm{B}_{99}^{C}$, arcayanti mahākālam $\mathrm{E}^{N}$, ahirbudhnyam arcayanti $\hat{S}_{67}^{S}$, arcayantah sadākālaṃ $\mathrm{P}_{3^{2}}^{T}$, arcayantī sadākālaṃ $\mathrm{P}_{72}^{T} \quad$ 141d devaṃ tribhuvaneśvaram ] $\mathrm{N}_{82}^{K} \mathrm{~N}_{58}^{K} \mathrm{E}^{N} \mathrm{~B}_{99}^{C}$, devantṛnayanam param $\mathrm{N}_{77}^{K o}$, devaṃ triṇayanam param $\mathrm{N}_{45}^{C} \mathrm{P}_{32}^{T}$, devam trinayanam param $\mathrm{N}_{12}^{K}$, sadāhitam parạ̣ śivam $S_{67}^{S}$, deva $\sqcup[-4-] \sqcup$ param $\mathrm{P}_{72}^{T} \quad \mathbf{1 4 2 b}$ obhūṣitāḥ ] $\mathrm{N}_{45}^{C} \mathrm{E}^{N} \mathrm{~B}_{99}^{C}$, obhūṣitā $\mathrm{N}_{82}^{K} \mathrm{~N}_{58}^{K} \quad$ 142c nityaụ ] $\mathrm{N}_{45}^{C} \mathrm{~N}_{82}^{K} \mathrm{~N}_{5^{8}}^{K} \mathrm{E}^{N} \mathrm{~B}_{99}^{C}$, hy etā $\mathrm{N}_{77}^{K o}$, hy etāḥ $\mathrm{N}_{12}^{K} \hat{S}_{67}^{S} \mathbf{P}^{\Sigma} \quad \mathbf{1 4 2 d}{ }^{\circ}$ coditāḥ ] $\Sigma$, ${ }^{\circ}$ coditā $\mathrm{N}_{77}^{K o} \mathrm{~N}_{58}^{K}$ 
[Nakṣatramātṛs: W]

anurādhā tathā jyeșthā mūlā ṛddhibalānvitā| pūrvāṣạ̣̄hā mahāvīryā āṣāḍhā cottarā śubhā|| 143||

abhijinnāma nakṣatraṃ śravaṇā paramojjvalā| etāḥ paścimato dīptā rājante rājamūrtayaḥ|| 144||

issānaṃ pūjayanty etāḥ sarvakālaṃ subhāvitāḥ| mama śāntiṃ prakurvantu vibhūtībhị̣ samanvitāḥ|| 145||

143-145 Cf. BhavP 1.179.6-8 : anurādhā tathā jyeșțhā mūlaṃ sūryapuraḥsarā| pūrvāṣāḍhā mahāvīryā āṣāọhā cottarā tathā|| abhijinnāma nakṣatraṃ śravaṇaṃ ca bahuśrutam| etāḥ paścimato dīptā rājante cānumūrtayaḥ|| bhāskaraṃ pūjayanty etāḥ sarvakālaṃ subhāvitāḥ| sāantiṃ kurvantu te nityaṃ vibhūtiṃ ca maharddhikām||

145cd Omitted in $\hat{S}_{67}^{s}$

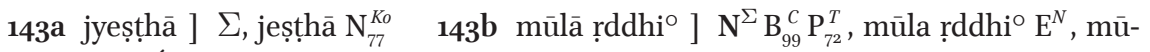
lamṛddhi ${ }^{\circ} \hat{S}_{67}^{S}$, mūlā vṛ́ścio $\mathrm{P}_{32}^{T} \quad$ 143c pūrvāșāḍhā ] $\Sigma$, pūrvāśāọhā $\mathrm{N}_{77}^{K o a c} \mathrm{~N}_{45}^{C} \quad$ 143d āṣāḍhā ] $\Sigma$, āśâḍhā $\mathrm{N}_{45}^{C}$ • śubhā ] $\Sigma$, tathā $\mathrm{N}_{12}^{K} \hat{S}_{67}^{S} \quad$ 144a abhijinnāma nakṣatraṃ ] $\mathrm{N}_{12}^{K} \mathrm{~B}_{99}^{C} \mathbf{P}^{\Sigma}$, abhiji<tā>nāma nakṣatraṃ $\mathrm{N}_{77}^{K o}$, avīcīnāma nakṣatraṃ $\mathrm{N}_{45}^{C}$, abhijirnāma nakṣatram $\mathrm{N}_{82}^{K}$, abhivirnnāma nakṣatram $\mathrm{N}_{5^{8}}^{K}$, abhijinnātmana kṣetraṃ $\mathrm{E}^{N}$, abhirnāma nakṣatraṃ $\hat{S}_{67}^{S}$ (unmetr.) $\quad \mathbf{1 4 4 b}$ śravaṇā paramojjvalā ] $\mathrm{K}^{\Sigma} \mathrm{B}_{99}^{C} \mathrm{E}^{N}$, śravaṇah paramojvalaḥ $\mathrm{N}_{77}^{K o} \hat{S}_{67}^{S} \mathrm{P}_{32}^{T}$, śravaṇo paramojvalā $\mathrm{N}_{45}^{C}$, śravaṇaṃ paramojvalā $\mathrm{P}_{72}^{T} \quad \mathbf{1 4 4 c}$ etāḥ ] $\mathrm{N}_{45}^{C} \mathrm{~N}_{82}^{K} \mathrm{~B}_{99}^{C} \mathrm{P}^{\Sigma}$, etā $\mathrm{N}_{77}^{K o} \mathrm{~N}_{12}^{K} \mathrm{~N}_{58}^{K} \mathrm{E}^{N}$, ete $S_{67}^{S} \quad$ 144d rājante $\Sigma$, rājatai $\mathrm{N}_{45}^{C}$, rājantyaḥ $\mathrm{P}_{72}^{T} \bullet$ rāja $\left.{ }^{\circ}\right] \mathrm{N}_{77}^{K o} \mathrm{~N}_{82}^{K} \mathrm{~N}_{12}^{K} \mathrm{E}^{N} \mathrm{~B}_{99}^{C}$, cāru $\mathrm{N}_{45}^{C} \mathrm{~N}_{58}^{K} \hat{S}_{67}^{S} \mathbf{P}^{\Sigma} \quad$ 145a ísāanam ] $\Sigma$, ísāāna $\mathrm{N}_{77}^{K o} \bullet$ pūjayanty etāḥ ] $\mathrm{N}_{82}^{K} \mathrm{~N}_{12}^{K} \mathrm{E}^{N} S_{67}^{S} \mathrm{P}_{72}^{T}$, pūjayaṃty etā $\mathrm{N}_{77}^{K o}$, pūjayety etāḥ $\mathrm{N}_{45}^{C}$, pūjanty etaḥ $\mathrm{N}_{58}^{K a c}$ (unmetr.), pūjanty etāḥ $\mathrm{N}_{58}^{K p c}$ (unmetr.), pūjayanty etā $\mathrm{B}_{99}^{C}$, pūjayanty etat $\mathrm{P}_{32}^{T} \quad \mathbf{1 4 5}$ b sarvakālaṃ subhāvitāḥ ] $\mathrm{N}_{45}^{C} \mathrm{~K}^{\Sigma} \mathrm{B}_{99}^{c} \mathrm{P}_{3^{2}}^{T}$, sarvakāliṣu bhāvitā $\mathrm{N}_{77}^{K o}$, sarvakālaṃ śubhāvitāḥ $\mathrm{E}^{N}$, sarvakāleșu bhāvitāḥ $\hat{S}_{67}^{S}$, sārvakālaṃ subhāvitāḥ $\mathrm{P}_{72}^{T} \quad$ 145C mama śāntiṃ prakurvantu ] $\mathrm{N}_{82}^{K} \mathrm{~N}_{58}^{K} \mathrm{E}^{N} \mathrm{~B}_{99}^{C}$, śāntikamma prakurvantu $\mathrm{N}_{77}^{K o}$, śānti kurvantu me nityaṃ $\mathrm{N}_{45}^{C}$, śāntikaṃ me prakurvanti $\mathrm{N}_{12}^{K}$, śāntiṃ kurvantu me nityaṃ $\mathrm{P}_{3_{2}}^{T}$, sāantiṃ kurvantu me prītāḥ $\mathrm{P}_{72}^{T} \quad 1_{45} \mathrm{~d}$ vibhūtībhih samanvitāh ] $\mathrm{N}_{12}^{K}$, vibhūtiñca samāhitā $\mathrm{N}_{77}^{K o}$, vibhūtiñca samāhitāḥ $\mathrm{N}_{45}^{C} \mathrm{P}_{72}^{T}$, vibhūtibhị̣ samanvitāṃ $\mathrm{B}_{99}^{C}$ (unmetr.), vibhūtibhị̣ samanvitā $\mathrm{N}_{82}^{K} \mathrm{~N}_{58}^{K} \mathrm{E}^{N}$ (unmetr.), vibhūtiṃ ca mahāhitāḥ $\mathrm{P}_{72}^{T p c}$, vibhūtiṃ ca mahābalāḥ $\mathrm{P}_{72}^{T a c}$ 
[Nakṣatramātṛs : N]

dhanișțhā śatabhiṣā ca pūrvabhādrapadā tathā| uttarābhādrarevatyau aśvinī ca maharddhikā|| 146||

bharaṇī ca mahāvīryā nityam uttarataḥ sthitāḥ|

śivārcanaparā nityaṃ śivadhyānaikamānasāḥ|

śāntiṃ kurvantu me nityaṃ sarvakālaṃ śubhodayām|| 147||

146-147 Cf. BhavP 1.179.9-11: dhanișțhā śatabhiṣā tu pūrvabhādrapadā tathā|| uttarābhādrarevatyau cāśvinī ca mahāmate| bharaṇi ca mahādevī nityam uttaratah sthitāḥ̣|| sūryārcanaratā nityam ādityagatamānasāḥ| śāntiṃ kurvantu te nityaṃ vibhūtiṃ ca maharddhikām||

147d After this $\mathrm{N}_{45}^{C}$ adds two pādas : etā pramuditā nityam dīpamānā sutejasāṃ|

146a dhanișṭhā śatabhiṣā ca ] $\mathrm{N}_{82}^{K} \mathrm{E}^{N} \mathrm{~B}_{99}^{C} \mathrm{P}_{32}^{T}$, dhanișțhā śatabhișā caiva $\mathrm{N}_{77}^{K o}$ (unmetr.), dhanișțhā satabhiṣā $\mathrm{N}_{45}^{C}$ (unmetr.), dhanișțā śatavṛsāa ca $\mathrm{N}_{12}^{K}$, dhaneșțhā śatavṛsā ca $\mathrm{N}_{58}^{K}$, dhanișțhānyā śatabhișak $S_{67}^{S}$, śravișțhayāṃ śatabhișak $\mathrm{P}_{72}^{T} \quad \mathbf{1 4 6 b}$ pūrvabhādrapadā tathā ] conj., pūrvabhadrapadas tathā $\mathrm{N}_{77}^{K o}$, pūrvabhadrapadā tathā $\mathrm{N}_{58}^{K} \mathrm{~B}_{99}^{C} \dot{S}_{67}^{S}$, pūrvā uttarabhādrapadā saha $\mathrm{N}_{45}^{C}$ (unmetr.), pūrvapadās tathā $\mathrm{N}_{82}^{\text {Kac }}$ (unmetr.), pūrvabhādrapadās tathā $\mathrm{N}_{82}^{K p c} \mathrm{E}^{N} \mathrm{P}_{32}^{T}$, pūrvā bhadrapadā tathā $\mathrm{N}_{12}^{K}$, pūrvabhādrapadā tathā $\mathrm{P}_{72}^{T} \quad \mathbf{1 4 6 c}$ uttarābhādrarevatyau ] $\mathrm{N}_{82}^{K} \mathrm{~B}_{99}^{C}$, revatī cāśvinī caiva $\mathrm{N}_{45}^{C}$, uttarābhadraraivatyo $\mathrm{N}_{12}^{K}$, uttarābhadraraivatyau $\mathrm{N}_{58}^{K}$, uttarābhadrarevatyāv $\hat{S}_{67}^{S}$, uttarābhadrarevatyā $\mathrm{N}_{77}^{K o} \mathrm{P}_{32}^{T}$, revatyuttarabhādrā ca $\mathrm{P}_{72}^{T} \quad \mathbf{1 4 6 d}$ aśvinī ca maharddhikā ] $\mathrm{N}_{82}^{K p c} \mathrm{~N}_{12}^{K} \mathrm{~N}_{58}^{K} \mathrm{~B}_{99}^{C} \hat{S}_{67}^{S} \mathrm{P}_{72}^{T}$, aśvinyaś ca maharddhikā $\mathrm{N}_{77}^{K o}$, aśvinī ca marddhikā $\mathrm{N}_{82}^{K a c}$ (unmetr.), aśvinī ca maharddhikāḥ $\mathrm{E}^{N}$, maṇḍalena vyavasthitāḥ $\mathrm{N}_{45}^{C}$, hy aśvatī sumarddhikā $\mathrm{P}_{32}^{T} \quad$ 147a bharaṇī ca ] $\Sigma$, bharaṇyaś ca $\mathrm{N}_{77}^{K o} \quad \mathbf{1 4 7} \mathbf{b}$ uttaratạ̣ sthitāḥ ] $\mathbf{K}^{\Sigma} \mathrm{B}_{99}^{C p c} S_{67}^{S}$, uttarata sthitā $\mathrm{N}_{77}^{K o}$, muttarata sthitā $\mathrm{N}_{45}^{C}$, uttarataḥ sthitaḥ $\mathrm{B}_{99}^{C \text { ac }}$, untarataḥ sthitāḥ $\mathrm{E}^{N}$, uttarasaṃsthitāḥ $\mathrm{P}^{\Sigma} \quad{ }_{147 \mathrm{c}}{ }^{\circ}$ parā nityam ] $\mathrm{K}^{\Sigma} \mathrm{E}^{N} \mathrm{~B}_{99}^{C}$, ${ }^{\circ}$ ratā nitya $\mathrm{N}_{77}^{K o}$, ${ }^{\circ}$ ratā nityaṃ $\mathrm{N}_{45}^{C} \mathrm{P}_{3^{2}}^{T}$, ${ }^{\circ}$ ratā devyaḥ $\hat{S}_{67}^{S}$, ${ }^{\circ}$ ratāḥ nityaṃ $\mathrm{P}_{72}^{T} \quad{ }^{T}{ }^{\circ} \mathrm{d}^{\circ}$ mānasāḥ ] $\Sigma$, ${ }^{\circ}$ mānasaḥ $\mathrm{N}_{77}^{K o}$, ${ }^{\circ}$ mānasā $\mathrm{N}_{58}^{K} \quad$ 147e śāntiṃ kurvantu me nityạ̣ ] $\mathrm{K}^{\Sigma} \mathrm{E}^{N} \mathrm{~B}_{99}^{C} \dot{S}_{67}^{S} \mathbf{P}^{\Sigma}$, harapādārcanaratā $\mathrm{N}_{77}^{K o}$, śānti kurvantu te nityaṃ $\mathrm{N}_{45}^{C}$, śāntim karotu me nityạ̣ $\mathrm{E}^{N} \quad{ }_{147} \mathrm{f}$ sarvakālaṃ śubhodayām ] $\mathrm{N}_{82}^{K} \mathrm{~N}_{58}^{K}$, śāntiṃ kurvantu me sadā $\mathrm{N}_{77}^{K o}$, sarvakālaṃ mahodayā $\mathrm{N}_{45}^{C}$, sarvakālaṃ subhodayam $\mathrm{N}_{12}^{K} \mathrm{~B}_{99}^{C}$, sarvakālaśubhodayām $\mathrm{E}^{N}$, sarvakālaṃ śivodayaḥ $\stackrel{S}{S}_{67}^{S}$, sarvakālaṃ śubhodayāḥ $\mathrm{P}_{32}^{T}$, balạ̣ saubhāgyam eva ca $\mathrm{P}_{72}^{T}$ 
[Rāśis : E]

meșo mṛgādhipaḥ siṃho dhanur dīptimatām varaḥ|

pūrveṇa bhāsayanty ete śivayogaparāḥ śubhāḥ|

śāntiṃ kurvantu me nityaṃ śivabhaktiparāyaṇāḥ|| 148||

\section{[Rāśis : S]}

vṛ̣aḥ kanyā ca paramā makaraś cāpi ṛddhimān| ete dakṣiṇabhāge tu pūjayanti sadā śivam| bhaktyā paramayā nityaṃ śāntiṃ kurvantu me sadā|| 149||

148 Cf. BhavP 1.179.12 : meșo mṛādhipaḥ siṃho dhanur dīptimatāṃ varaḥ| pūrveṇa bhāsayanty ete sūryayogaparāḥ śubhāḥ| śāntiṃ kurvantu te nityam bhaktyā sūryapadāṃbuje|| 149 Cf. BhavP 1.179.13 : vṛṣaḥ kanyā ca paramā makaraś cāpi buddhimān| ete dakṣiṇabhāge tu pūjayanti raviṃ sadā| bhaktyā paramayā nityaṃ śāntiṃ kurvantu te sadā||

148af Omitted in $\mathrm{P}_{72}^{T} \quad$ 149b After this $\mathrm{N}_{45}^{C}$ adds two pādas : vṛșaḥ sarvahitodyuktah sarvaṛddhisamanvitaḥ| $\mid \mathrm{N}_{58}^{K}$ adds harapādaratā nityaṃ śivabhāvena bhāvitā| $\bullet \mathrm{P}_{3^{2}}^{T}$ adds : ṛṣabhaś ca mahāvīryāḥ śivabhāvasubhāvitāḥ| $\bullet \mathrm{P}_{72}^{T}$ adds : ṛṣabhaś ca mahāvīryaḥ śivabhāvasubhāvitah|

148a meșo mṛāanhipah siṃho ] $S_{67}^{S}$, meșo vṛșādhipaḥ siṃho $\mathrm{N}_{82}^{K} \mathrm{~N}_{12}^{K} \mathrm{~B}_{99}^{c}$, meșo vṛșādhipasiṃho $\mathrm{N}_{77}^{K o}$, meṣo vṛṣādhipaḥ siṃhā $\mathrm{N}_{45}^{C}$, meșo mahāhikah siṃho $\mathrm{E}^{N}$, meșā mṛā̄dhipạ̣ siṃhā $\mathrm{N}_{58}^{K}$, meṣo mṛgādhipastham yo $\mathrm{P}_{32}^{T} \quad \mathbf{1 4 8 b}$ dhanur ] $\mathrm{N}_{58}^{K} \mathrm{E}^{N} \mathrm{~B}_{99}^{C} \hat{S}_{67}^{S} \mathrm{P}_{32}^{T}$, dhanu $\mathrm{N}^{\Sigma} \bullet$ varah ] $\mathrm{N}^{\Sigma} \mathrm{B}_{99}^{C} \hat{S}_{67}^{S} \mathrm{P}_{32}^{T}$, varāḥ $\mathrm{E}^{N} \quad{ }^{148 c}$ pūrveṇa ] $\mathrm{N}^{\Sigma} \mathrm{E}^{N} \mathrm{~B}_{99}^{C} \hat{S}_{67}^{S}$, pūrve ca $\mathrm{P}_{32}^{T} \bullet$ bhāsayanty ] $\mathrm{N}_{12}^{K} \mathrm{~N}_{58}^{K} \hat{S}_{67}^{S} \mathrm{P}_{32}^{T}$, bhāgasaty $\mathrm{N}_{77}^{K o}$, bhāșayanty $\mathrm{N}_{45}^{C} \mathrm{~N}_{82}^{K} \mathrm{E}^{N} \mathrm{~B}_{99}^{C} \quad \mathbf{1 4 8 d}$ yogaparāḥ śubhāḥ ] $S_{67}^{S}$, ${ }^{\circ}$ pūjāparāyaṇāḥ $\mathrm{N}_{82}^{K} \mathrm{E}^{N} \mathrm{~B}_{99}^{C}$, ${ }^{\circ}$ yogaparā śubhā $\mathrm{N}_{77}^{K o}$, ${ }^{\circ}$ dhyānaparāyaṇā $\mathrm{N}_{45}^{C}$, ${ }^{\circ}$ yogaparā śubhāḥ $\mathrm{N}_{12}^{K}$, ${ }^{o}$ pūjāparāyaṇā $\mathrm{N}_{58}^{K}$, ${ }^{\circ}$ pūjāparāyaṇaḥ $\mathrm{P}_{32}^{T} \quad$ 148e śāntiṃ ] $\mathrm{K}^{\Sigma} \mathrm{E}^{N} \mathrm{~B}_{99}^{C} \hat{S}_{67}^{S} \mathrm{P}_{3^{2}}^{T}$, śānti $\mathrm{N}_{77}^{K o} \mathrm{~N}_{45}^{C} \bullet$ nityam ] $\mathrm{N}_{45}^{C} \mathrm{~K}^{\Sigma} \mathrm{E}^{N} \mathrm{~B}_{99}^{C} \mathrm{P}_{32}^{T}$, bhaktā $\mathrm{N}_{77}^{K o} \mathrm{~N}_{12}^{K}$, bhaktāḥ S $S_{67}^{S} \quad 148 f$ śivabhaktiparāyanāḥ ] $\mathrm{N}_{82}^{K} \mathrm{E}^{N} \mathrm{~B}_{99}^{C}$, harapādā<bja bjapū(ja)kā N $\mathrm{N}_{77}^{K o}$, harapādābjapūjakāh $\mathrm{N}_{45}^{C} \mathrm{~N}_{12}^{K} \mathrm{P}_{32}^{T}$, śivabhaktiparāyaṇā $\mathrm{N}_{58}^{K}$, śivapādābjapūjakāḥ $S_{67}^{S} \quad$ 149a vrṣaḥ kanyā ca paramā ] $\mathrm{N}_{5^{8}}^{K}$, kanyā ca paramā devī $\mathrm{N}^{\Sigma} \mathrm{B}_{99}^{C} \mathrm{P}_{72}^{T}$, kanyā ca vṛ̣abhaḥ śrīmān $\mathrm{E}^{N}$, vṛṣā cānyā ca paramā $S_{67}^{S}$, vṛ̣saś ca varadah kanyā $\mathrm{P}_{32}^{T} \quad$ 149b cāpi ṛddhimān ] $\mathrm{N}_{45}^{C} \mathrm{~N}_{82}^{K} \mathrm{~N}_{58}^{K} E^{N} \mathrm{~B}_{99}^{C} \dot{S}_{67}^{S}$, cātibuddhimān $\mathrm{N}_{77}^{K o}$, cātidụddhimān $\mathrm{N}_{12}^{K}$, ca mahardhikāḥ $\mathrm{P}_{32}^{T}$, ca mahardhikah $\mathrm{P}_{72}^{T} \quad 149 \mathrm{c}$ ete ] $\mathrm{N}_{77}^{K o} \mathrm{~N}_{45}^{C} \mathrm{~N}_{58}^{K} \mathrm{E}^{N} \dot{S}_{67}^{S p c} \mathbf{P}^{\Sigma}$, etau $\mathrm{N}_{82}^{K} \mathrm{~B}_{99}^{C}$, etā $\mathrm{N}_{12}^{K}$, etena $\hat{S}_{67}^{S \text { ac }}$ (unmetr.) • obhāge ] $\Sigma$, odeśe $\mathrm{P}_{32}^{T} \quad$ 149d pūjayanti ] $\mathrm{N}_{77}^{K o} \mathrm{~N}_{45}^{C} \hat{S}_{67}^{S} \mathbf{P}^{\Sigma}$, pūjayatah $\mathrm{N}_{82}^{K}$, pūjayetaḥ $\mathrm{B}_{99}^{C}$, pūjayanto $\mathrm{N}_{12}^{K}$, pūjayantah $\mathrm{N}_{58}^{K} \mathrm{E}^{N} \bullet$ sadā śivam ] $\Sigma$, śidā śi(vaṃ) $\mathrm{N}_{58}^{K} \quad$ 149e bhaktyā paramayā ] $\Sigma$, bhaktyā parayā $\mathrm{N}_{77}^{K o}$ (unmetr.), bhaktyā ca parayā $\mathrm{P}_{72}^{T} \quad \mathbf{1 4 9}$ śāntiṃ ] $\Sigma$, śānti $\mathrm{N}_{45}^{C} \mathrm{~N}_{82}^{K} \bullet$ kurvantu me sadā ] $\mathrm{N}_{45}^{C} \mathrm{~N}_{12}^{K} \mathrm{~N}_{58}^{K} \mathrm{~B}_{99}^{C} \dot{S}_{67}^{S} \mathbf{P}^{\Sigma}$, me kurutāṃ sadā $\mathrm{N}_{82}^{K} \mathrm{E}^{N}$, kurva me sadā $\mathrm{N}_{77}^{K o}$ (unmetr.), kurvvantu me dā $\mathrm{N}_{58}^{K}$ 
[Rāśis :W]

mithunas tulā kumbhaś ca paścimena vyavasthitāḥ| śivapādārcanaratāḥ kṣemaṃ kurvantu me sadā|| 15ㅇ|

[Rāśis : N]

karkaṭo vṛściko mīna ete hy uttarataḥ sthitāḥ|

pūjayanti sadākālaṃ rudraṃ bhuvananāyakam|

śāntiṃ kurvantu me nityaṃ śivājñānuvidhāyinah|| 151||

15o Cf. BhavP 1.179.14ab : mithunaṃ ca tulā kuṃbhạ̣ paścime ca vyavasthitāḥ| $\quad \mathbf{1 5}^{1}$ Cf. BhavP 1.179.14cd-15 : japanty ete sadākālam ādityaṃ grahanāya kam|| śāntiṃ kurvantu te nityaṃ khakholkājñānatatparāḥ| satapodattapuṣpābhyāṃ ye smṛtāḥ satataṃ budhaih||

151d After this $\mathrm{N}_{45}^{C}$ adds two pādas : rudrabhaktā mahātmāno rudrārccaṇaparāyaṇā| 15ıf After this $\mathrm{N}_{58}^{K}$ adds six pādas (unmetr.) : janmasampadvipat kṣemah pa - - - - dhanaḥ| naișanam metro 'timetrañ ca ity etā tārakagaṇā| śivabhaktā mahātmānaḥ kurvvantu mama śāntikaṃ| $\mid \bullet S_{67}^{S}$ adds sixteen pādas : ādityavāre saṃkrāntir ghorā (śamra)tu bhītidā| śîtaraśmidine proktā dhvān̉kṣī salilavāhinī| bhaume mahodarī sā tu rājaghnī viditā matā| budhe mandākinī sā tu dviṭoghā nandakāriṇī| bṛhaspatiyute vāre nandanā vyașțikāriṇī| śukravāre miśrikarā vyāsasannasarvapradāyinī| śanaiścarī rākṣasī tu janapītākarī smṛtā| iti saṃkrāntayor nāma phalagrahayutās sadā|

15oa mithunas tulā kumbhaś ca ] $\mathrm{N}_{82}^{K} \mathrm{~B}_{99}^{C}$, tulā mithunakumbhaś ca $\mathrm{N}_{77}^{K o}$, mithunāñca tulā kumbhā $\mathrm{N}_{45}^{C}$, mithunas tulā ca kumbhaś ca $\mathrm{N}_{12}^{K}$ (unmetr.), mithunas tulā kumbhaḥ $\mathrm{N}_{58}^{K}$ (unmetr.), mithunaś ca tulā kumbhah $\mathrm{E}^{N} \hat{\mathrm{S}}_{67}^{S} \mathrm{P}_{72}^{T}$, mithunaś ca tulākuṃbhau $\mathrm{P}_{32}^{T}$ 15ob paścimena ] $\Sigma$, paścime tu $\mathrm{P}_{3^{2}}^{T} \bullet$ vyavasthitāḥ ] $\Sigma$, vyavasthitā $\mathrm{N}_{77}^{K o} \mathrm{E}^{N}$, vyavasthitaḥ $\mathrm{P}_{72}^{T} \quad{ }^{15}$ oc ${ }^{\circ}$ pādārcanaratāḥ ] $\mathrm{N}_{12}^{K} \hat{S}_{67}^{S}$, ${ }^{\circ}$ padārcanaparāḥ $\mathrm{N}_{82}^{K} \mathrm{~B}_{99}^{C}$ (unmetr.), ${ }^{\circ}$ pūjārcanaratā $\mathrm{N}_{77}^{K o}$, ${ }^{\circ}$ pādārccanaratā $\mathrm{N}_{45}^{C}$, ${ }^{\circ}$ pūjārcanaparāḥ $\mathrm{E}^{N}$, ${ }^{\circ}$ pādārcane yuktāh $\mathrm{N}_{58}^{K} \mathrm{P}_{32}^{T}$, ${ }^{\circ}$ pūjārcanaratāḥ $\mathrm{P}_{72}^{T} \quad$ 15od kṣemam ] $\mathbf{N}^{\Sigma} \mathrm{B}_{99}^{C}$, kṣamaṃ $\mathrm{E}^{N}$, śāntiṃ $\hat{S}_{67}^{S} \mathbf{P}^{\Sigma} \quad$ 151a mīna ] $\mathrm{N}_{77}^{K o} \mathrm{~N}_{45}^{C} \mathrm{~N}_{82}^{K a c} \mathrm{~N}_{12}^{K} \mathrm{~N}_{58}^{K} \mathrm{E}^{N} \mathrm{~B}_{99}^{C} \hat{S}_{67}^{S}$, mīnā $\mathrm{N}_{82}^{K \text { pc }}$, mīno $\mathrm{P}_{32}^{T}$, mīnah $\mathrm{P}_{72}^{T} \quad \mathbf{1 5 1 b}$ ete hy uttaratah sthitāḥ ] $\mathrm{N}_{82}^{K} \mathrm{E}^{N} \mathrm{~B}_{99}^{C}$, ete uttarata sthitā $\mathrm{N}_{77}^{K o}$, ete uttarata sthitāḥ $\mathrm{N}_{45}^{C}$, ete uttarataḥ sthitāḥ $\mathrm{N}_{12}^{K} \mathrm{~N}_{58}^{K}$, ete cottarataḥ sthitāḥ $S_{67}^{S}$, yenta uttaratah sthitāḥ $\mathrm{P}_{3^{2}}^{T}$, ete cottarasaṃsthitāḥ $\mathrm{P}_{72}^{T} \quad$ 151c pūjayanti ] $\mathrm{N}_{82}^{K} \mathrm{~N}_{5^{8}}^{K} \mathrm{E}^{N} \mathrm{~B}_{99}^{C}$, yajante ca $\mathrm{N}_{77}^{K o}$, yajanty ete $\mathrm{N}_{45}^{C} \mathrm{~N}_{12}^{K} \mathbf{P}^{\Sigma}$, yajanti te $S_{67}^{S}$ 151d sadākālaṃ ] $\mathrm{N}_{77}^{K o} \mathrm{~N}_{82}^{K} \mathrm{~N}_{58}^{K} \mathrm{E}^{N} \mathrm{~B}_{99}^{C} \mathbf{P}^{\Sigma}$, sadākālā $\mathrm{N}_{45}^{C}$, mahākālaṃ $\mathrm{N}_{12}^{K}$, mahādevaṃ $S_{67}^{S}$ - rudram ] $\Sigma$, rudra $\mathrm{N}_{58}^{K} \bullet$ bhuvananāyakam ] $\Sigma$, tribhuvanādhipam $\mathrm{P}_{72}^{T \mathrm{pc}}$, tribhuvaneśvaram $\mathrm{P}_{72}^{T \text { ac }} \quad$ 151e śāntiṃ kurvantu ] $\Sigma$, śānti kurvantu $\mathrm{N}_{45}^{C}$, śāntiṃ kurvanti $\mathrm{N}_{82}^{K} \bullet$ nityaṃ ] $\Sigma$, nitya $\mathrm{N}_{77}^{K o} \quad \mathbf{1 5 1 f}$ śivājñānuvidhāyinaḥ ] $\mathrm{N}_{82}^{K} \mathrm{E}^{N} \mathrm{~B}_{99}^{C} \mathrm{P}_{72}^{T a c}$, rudrajñānavidhāyinaḥ $\mathrm{N}_{77}^{K o} \mathrm{~N}_{45}^{C}$, śivajñānuvidhāyinaḥ $\mathrm{N}_{58}^{K}$, rudrājñānavidhāyakaḥ $\hat{S}_{67}^{S}$, rudrājñānuvidhāyinaḥ $\mathrm{N}_{12}^{K} \mathrm{P}_{72}^{T \text { pc }}$, rudrajñānaparāyaṇāḥ $\mathrm{P}_{72}^{T}$ 


\title{
[Saptarșis]
}

ṛṣayaḥ sapta vikhyātā dhruvāntāḥ paramojjvalāḥ|

śivaprasādasampannāḥ śāntiṃ kurvantu me sadā|| 152||

\section{[Mahāvrataṛ̦is]}

kāśyapo gālavo gārgyo viśvāmitro mahāmuniḥ| manur dakṣo vasișṭho 'tha mārkạ̣ḍaḥ pulahạ̣ kratuh|| 153\|

\author{
nārado bhṛgur ātreyo bharadvājo 'ngirā muniḥ| \\ vālmīkaḥ kauśikaḥ kaṇvaḥ śākalyo 'tha punarvasuḥ|| 154||
}

152 Cf. BhavP 1.179.16 : ṛ̣ayaḥ sapta vikhyātā dhruvāntāḥ paramojjvalāḥ| bhānuprasādāt sampannāḥ śāntiṃ kurvantu te sadā|| 153-155 Cf. BhavP 1.179.17-19: kaśyapo gālavo gārgyo viśvāmitro mahāmuniḥ| munir dakṣo vaśișțhaś ca mārkaṇḍạ̣ pulahạ̣ kratuh|| nārado bhṛgur ātreyo bhāradvājaś ca vai muniḥ| vālmīkiḥ kauśiko vātsyaḥ saākalyo 'tha punarvasuḥ|| śālaṃkāyana ity ete ṛ̣ayo 'tha mahātapāḥ| sūryadhyānaikaparamāḥ śāntim kurvantu te sadā||

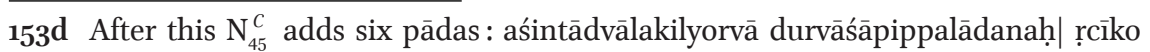
bharato 'gasti parāśaryo vibhāṇḍakaḥ| matāngo jaiminivyāśo devalo darbharomakaḥ| 154a-d Omitted in $S_{67}^{S} \quad \mathbf{1 5 4 b - 1 8 7 c}$ One folio is missing in $\mathrm{N}_{12}^{K}$, covering the text from munih up to $187 \mathrm{c}$

152a ṛșayaḥ ] $\Sigma$, ṛsaya $\mathrm{N}_{45}^{C} \mathrm{~N}_{12}^{K}$ (unmetr.) • vikhyātā ] $\Sigma$, saṃkhyātā $\mathrm{P}_{32}^{T} \quad \mathbf{1 5 2 b}$ dhruvāntāḥ ] $\Sigma$, dhruvantā $\mathrm{N}_{77}^{K o} \mathrm{~N}_{45}^{C} \mathrm{~N}_{58}^{K} \bullet$ paramojjvalāḥ ] $\Sigma$, paraparamojjvalā $\mathrm{N}_{58}^{K} \quad \mathbf{1 5 2 c}$ ${ }^{\circ}$ sampannāḥ śāntiṃ kurvantu me sadā ] $\mathrm{N}_{82}^{K} \mathrm{~N}_{12}^{K} \mathrm{E}^{N} \mathrm{~B}_{99}^{C} S_{67}^{S} \mathbf{P}^{\Sigma}$, ${ }^{\circ}$ sampaṃnā śānti kurvantu me sadā $\mathrm{N}_{77}^{K o}$, ${ }^{\circ}$ saṃpannāḥ kurvvantu mama śāntikaṃ $\mathrm{N}_{58}^{K}$, ${ }^{\circ}$ saṃpanā śānti kurvantu me sadā $\mathrm{N}_{45}^{C}$ (unmetr.) $\quad 153$ a kāśyapo ] $\Sigma$, kaśyapo $\mathrm{E}^{N} S_{67}^{S} \bullet$ gālavo gārgyo ] $\mathrm{N}_{77}^{K o} \mathrm{~N}_{82}^{K} \mathrm{~N}_{58}^{K} \mathrm{E}^{N} \mathrm{~B}_{99}^{C} S_{67}^{S} \mathrm{P}_{72}^{T p c} \mathrm{P}_{72}^{T}$, gāvo gargyo $\mathrm{N}_{45}^{C}$ (unmetr.), gālavo gārgo $\mathrm{N}_{12}^{K}$, bhārgavo gārgyo $\mathrm{P}_{72}^{T a c} \quad 1^{T} 3^{\mathrm{b}}{ }^{\circ}$ mitro ] $\Sigma$, ${ }^{\circ}$ vitro $\mathrm{N}_{58}^{K},{ }^{\circ}$ mitra $\mathrm{P}_{3^{2}}^{T} \bullet{ }^{\circ}$ munih ] $\Sigma$, ${ }^{\circ}$ muni $\mathrm{N}_{77}^{K o} \mathrm{~N}_{45}^{C} \quad{ }_{153 c}$ manur ] $\mathrm{N}_{82}^{K} \mathrm{~N}_{58}^{K} E^{N} \mathrm{~B}_{99}^{C} S_{67}^{S} \mathbf{P}^{\Sigma}$, manu $\mathrm{N}_{77}^{K o} \mathrm{~N}_{45}^{C} \mathrm{~N}_{12}^{K} \bullet$ vasisțho 'tha ] $\Sigma$, vasișţhā ca $\mathrm{N}_{77}^{K o}$, vaśișțhaś ca $\mathrm{N}_{58}^{K}$, vasișțhaś ca $\mathrm{P}_{72}^{T} \quad$ 153d mārkaṇdạ pulahạ kratuh ] $\mathrm{N}_{82}^{K} \mathrm{~N}_{12}^{K} \mathrm{E}^{N} \mathrm{~B}_{99}^{C} \mathrm{P}_{72}^{T}$, matangga pulaha kratuh $\mathrm{N}_{77}^{K o}$, mārkaṇḍa pulaha kratu $\mathrm{N}_{45}^{C}$, mārkaṇḍa pulahaḥ kratuh $\mathrm{N}_{58}^{K}$, mārkaṇḍo tha punarvasuḥ $\hat{S}_{67}^{S}$, pulastyah pulahạ kratuh $\mathrm{P}_{32}^{T} \quad{ }_{154}$ b bharadvājo ] $\mathbf{N}^{\Sigma} \mathrm{E}^{N} \mathrm{~B}_{99}^{C}$, bhāradvājo $\mathbf{P}^{\Sigma} \bullet$ 'ngirā ] $\mathrm{N}_{77}^{K o} \mathbf{K}^{\Sigma} \mathrm{E}^{N} \mathrm{~B}_{99}^{C} \mathbf{P}^{\Sigma}$, 'ńgiro $\mathrm{N}_{45}^{C} \bullet$ munih ] $\mathrm{N}_{77}^{K o} \mathrm{~N}_{82}^{K} \mathrm{~N}_{58}^{K} \mathrm{P}_{72}^{T}$, muni $\mathrm{N}_{45}^{C} \mathrm{E}^{N} \mathrm{P}_{32}^{T} \quad \mathbf{1 5 4 c}$ vālmīkah ] $\mathrm{N}_{77}^{K o} \mathrm{~N}_{45}^{C} \mathrm{~N}_{82}^{K p c} \mathrm{~N}_{58}^{K} \mathrm{~B}_{99}^{C}$, vālmikaḥ $\mathrm{N}_{82}^{\text {Kac }}$, vālmīki $\mathrm{E}^{N}$, vālmīkiḥ $\mathrm{P}_{3^{2}}^{T}$, saalmīkaḥ $\mathrm{P}_{72}^{T} \bullet$ kaṇvạ̣ ] $\mathrm{N}_{45}^{C} \mathrm{~N}_{82}^{K} \mathrm{~N}_{58}^{K} \mathrm{E}^{N} \mathrm{~B}_{99}^{C} \mathbf{P}^{\Sigma}$, kaṇțhāạ $\mathrm{N}_{77}^{K o} \quad 154 d$ śākalyo ] $\mathrm{N}_{77}^{K o} \mathrm{~N}_{45}^{C} \mathrm{~N}_{82}^{K} \mathrm{~N}_{58}^{K} \mathrm{E}^{N} \mathrm{P}_{72}^{T \text { pc }} \mathrm{P}_{72}^{T} \mathrm{~B}_{99}^{C}$, jābalyo $\mathrm{P}_{72}^{\text {Tac }} \bullet$ punarvasuh ] $\mathrm{N}_{77}^{K o} \mathrm{~N}_{58}^{K} \mathbf{P}^{\Sigma}$, punarvasu $\mathrm{N}_{45}^{C} \mathrm{~N}_{82}^{K} \mathrm{~B}_{99}^{C}$, punarvaśu $\mathrm{E}^{N}$ 
śālaṅkāyana ityādyā ṛ̣ayo 'tha mahāvratāḥ|

śivadhyānārcanodyuktāḥ śāntiṃ kurvantu me sadā|| 155||

[Ṛṣipatnīs, Ṛṣikumārikās]

ṛṣipatnyo mahāpuṇyās tathā ṛ̣ikumārikāḥ|

śivārcanaparā nityaṃ śāntiṃ kurvantu me sadā|| 156||

[Siddhas, Gandharvas, Apsarases, Vidyādharas, Garuḍas]

siddhāḥ saṃsiddhatapaso gandharvāpsarasāṃ ganāḥ|

vidyādharā mahātmāno garuḍaśs ca maharddhikāḥ̣|| 157||

156 Cf. BhavP 1.179.20 : munikanyā mahābhāgā rșikanyāḥ kumārikāḥ| sūryārcanaratā nityaṃ śāntiṃ kurvantu te sadā|| 157-158 Cf. BhavP 1.179.21-22 : siddhāḥ samṛddhatapaso ye cānye vai mahātapāḥ| vidyādharā mahātmāno garuḍaś ca tvayā saha|| ādityaparamā hy ete ādityārādhane ratāḥ| siddhị̣ te samprayacchantu āśīrvādaparāyaṇāḥ̣||

156a-d Omitted in $S_{67}^{S}$ and $\mathrm{P}_{72}^{T}$

155a śālañkāyana ] $\mathrm{N}_{77}^{K o} \mathrm{~N}_{45}^{C} \mathrm{~N}_{82}^{K} \mathrm{~N}_{58}^{K} \mathrm{~B}_{99}^{C} \dot{S}_{67}^{S} \mathbf{P}^{\Sigma}$, śākalyāyana $\mathrm{E}^{N}$ • ityādyā ] $\mathrm{N}_{77}^{K o} \mathrm{~N}_{45}^{C} \mathrm{~N}_{82}^{K} \mathrm{~B}_{99}^{C} \dot{S}_{67}^{S} \mathrm{P}_{32}^{T}$, ityādā $\mathrm{E}^{N}$, ityete $\mathrm{P}_{72}^{T} \quad \mathbf{1 5 5}^{\mathrm{b}}$ ṛșayo ] $\mathrm{N}_{77}^{K o} \mathrm{~N}_{45}^{C} \mathrm{~N}_{82}^{K} \mathrm{~N}_{58}^{K} \mathrm{E}^{N} \mathrm{~B}_{99}^{C} \dot{S}_{67}^{S} \mathrm{P}_{32}^{T}$, munayo $\mathrm{P}_{72}^{T} \bullet{ }^{\circ}$ vratāḥ ] $\mathrm{N}_{45}^{C} \mathrm{~N}_{82}^{K} \mathrm{~N}_{58}^{K} \mathrm{E}^{N} \mathrm{~B}_{99}^{C}$, o tapā $\mathrm{N}_{77}^{K o}$, obalāḥ $S_{67}^{S} \mathrm{P}_{32}^{T}$, o tapāḥ $\mathrm{P}_{72}^{T} \quad{ }_{155} \mathrm{c}$ śiva $\left.{ }^{\circ}\right] \mathrm{N}_{77}^{K o} \mathrm{~N}_{45}^{C} \mathrm{~N}_{82}^{K p c} \mathrm{~N}_{58}^{K} \mathrm{E}^{N} \mathrm{~B}_{99}^{C} \dot{S}_{67}^{S} \mathbf{P}^{\Sigma}$, śivāo $\mathrm{N}_{82}^{K a c} \bullet{ }^{\circ}$ rcanodyuktāh ] $\mathrm{N}_{45}^{C} \mathrm{~N}_{82}^{K} \mathrm{~B}_{99}^{C} \mathrm{P}_{72}^{T}$, ${ }^{\circ}$ rcanaratā $\mathrm{N}_{77}^{K o}$, ${ }^{\circ}$ rcanā ratāḥ $\mathrm{N}_{58}^{K a c}$, ${ }^{\circ}$ rcanaratāḥ $\mathrm{N}_{58}^{K p c}$, ${ }^{\circ}$ rccane dyuktāḥ $\mathrm{E}^{N}$, ${ }^{\circ}$ rcane yuktāḥ $\hat{S}_{67}^{S} \mathrm{P}_{32}^{T} \quad \mathbf{1 5 5}^{T} \mathrm{~d} \quad$ śāntim ] $\mathrm{N}_{77}^{K o} \mathrm{~N}_{82}^{K} \mathrm{E}^{N} \mathrm{~B}_{99}^{C} \dot{S}_{67}^{S} \mathbf{P}^{\Sigma}$, śānti $\mathrm{N}_{45}^{C} \mathrm{~N}_{58}^{K}$ • sadā ] $\mathrm{N}_{77}^{K o} \mathrm{~N}_{82}^{K} \mathrm{~N}_{58}^{K} \mathrm{E}^{N} \mathrm{~B}_{99}^{C} S_{67}^{S} \mathbf{P}^{\Sigma}$, sadāh $\mathrm{N}_{45}^{C} \quad \mathbf{1 5 6 a}{ }^{\circ}$ patnyo ] $\mathrm{N}_{82}^{K} \mathrm{~N}_{58}^{K} \mathrm{~B}_{99}^{C} \mathrm{P}_{32}^{T}$, ${ }^{\circ}$ panyo $\mathrm{N}_{77}^{K o}$, ${ }^{\circ}$ punyyā $\mathrm{N}_{45}^{C}$, ${ }^{\circ}$ patnyā $\mathrm{E}^{N} \quad{ }_{156 a b}{ }^{\circ}$ punyaās tathā ṛ̣ikumārikāḥ ] $\mathrm{N}_{82}^{K} \mathrm{~N}_{58}^{K} \mathrm{~B}_{99}^{C}$, ${ }^{\circ}$ puṇyā ṛ̣ikanyākumārikā $\mathrm{N}_{77}^{K o}$, obhāgāḥ ṛșikanyāḥ kumārikāḥ $\mathrm{N}_{45}^{C}$, ${ }^{\circ}$ punyā ṛ̣ikanyākumārikāḥ $\mathrm{E}^{N}$, obhāgā ṛ̣ikanyāḥ kumārikāḥ $\mathrm{P}_{3^{2}}^{T} \quad \mathbf{1 5}^{\circ}{ }^{\circ}$ parā ] $\mathrm{N}_{82}^{K} \mathrm{~N}_{58}^{K} \mathrm{~B}_{99}^{C}$, ${ }^{\circ}$ ratā $\mathrm{N}_{77}^{K o} \mathrm{~N}_{45}^{C} \mathrm{P}_{3^{2}}^{T}$, oparān $\mathrm{E}^{N} \quad{ }_{156 d}$ sāntiṃ ] $\mathrm{N}_{77}^{K o} \mathrm{~N}_{82}^{K} \mathrm{E}^{N} \mathrm{~B}_{99}^{C} \mathrm{P}_{32}^{T}$, sānti $\mathrm{N}_{45}^{C} \mathrm{~N}_{5^{8}}^{K} \bullet$ kurvantu ] $\mathrm{N}_{45}^{C} \mathrm{~N}_{82}^{K} \mathrm{~N}_{58}^{K} \mathrm{E}^{N} \mathrm{~B}_{99}^{C} \mathrm{P}_{32}^{T}$, kurvatu $\mathrm{N}_{77}^{K o} \quad$ 157a siddhāḥ saṃsiddhatapaso ] $\mathrm{N}_{82}^{K} \mathrm{~B}_{99}^{C}$, siddhāś ca siddhatapaso $\mathrm{N}_{77}^{K o} \mathrm{P}_{72}^{T}$ siddhā saṃsiddhatapaso $\mathrm{N}_{45}^{C}$, siddhāḥ saṃsiddhatapasā $\mathrm{E}^{N}$, siddhā susiddhatapaso $S_{67}^{S}$, siddhāḥ samṛddhatapaso $\mathrm{N}_{58}^{K} \mathrm{P}_{32}^{T} \quad \mathbf{1 5 7} \mathrm{b}$ gandharvāpsarasāṃ gaṇāh ] $\hat{S}_{67}^{S}$, gaṇavidyādharas tathā $\mathrm{N}_{77}^{K o}$, gandharvāpsaraso gaṇā $\mathrm{N}_{45}^{C}$, gaṇavidyādharā grahāḥ $\mathrm{N}_{82}^{K}$, sarvvāpsarasā gaṇāḥ $\mathrm{N}_{5^{8}}^{K}$ (unmetr.), gaṇo vidyādharāgrahāḥ $\mathrm{E}^{N}$, gaṇā vidyādharā grahāḥ $\mathrm{B}_{99}^{C}$, gandharvāś cāpsarogaṇāḥ $\mathrm{P}_{32}^{T}$, gandharvāpsaraso gaṇāḥ $\mathrm{P}_{72}^{T} \quad{ }_{157} \mathrm{c}$ vidyādharā mahātmāno ] $\mathrm{N}_{58}^{K} \hat{S}_{67}^{S} \mathrm{P}_{3^{2}}^{T}$, mahātmāho mahātmāno $\mathrm{N}_{77}^{K o}$, vidyādharamahātmāno $\mathrm{N}_{45}^{C}$, mahātmāno mahotsāhā $\mathrm{N}_{82}^{K} \mathrm{~B}_{99}^{C}$, mahātmāno mahotsāha $\mathrm{E}^{N}$, vidyā $\sqcup[-4-] \sqcup$ tmānaḥ $\mathrm{P}_{72}^{T} \quad{ }_{157 d}$ garuḍāś ca maharddhikāḥ ] $\mathrm{N}_{82}^{K} \mathrm{~N}_{58}^{K} \mathrm{~B}_{99}^{C} \mathrm{P}_{72}^{T}$, garuḍaś ca mahaddhikāḥ $\mathrm{N}_{77}^{K o}$, garuḍaś ca maharddhikaḥ $\mathrm{N}_{45}^{C} \mathrm{P}_{32}^{T}$, garuḍāś ca maharddhikā $\mathrm{E}^{N}$, garuḍaś ca mahābalạ̣ $\dot{S}_{67}^{S}$ 
maheśvaraparā hy ete maheśvarapadārcakāḥ| siddhim āśu prayacchantu āśirvādaparāyaṇāḥ|| 158||

\section{[Daityas : 1]}

namucir daityarājendraḥ śańkukarṇo mahābalaḥ| mahānādo 'tha vikhyāto daityaḥ paramavīryavān|| 159||

hāṭakeśvaradevasya nityam pūjāparāyaṇāḥ| balaṃ vīryaṃ ca me kṣipraṃ prayacchantu maharddhikāḥ̣|| 16o||

159-16o Cf. BhavP 1.179.23-24: namucir daityarājendrah śaṅkukarṇo mahābalah| mahānātho 'tha vikhyāto daityah paramavīryavān|| grahādhipasya devasya nityaṃ pūjāparāyaṇāḥ| balạ̣ vīryaṃ ca te ṛddhim ārogyạ̣ ca bruvantu te||

158a-159b Omitted in $\hat{S}_{67}^{S} \quad$ 159b After this $\mathrm{P}_{72}^{T}$ adds six pādas: jaṃbho nikuṃbhaś śakațaḥ śivabhaktiparāyaṇāḥ| ete daityā mahātmānạ̣ śivasadbhāvabhāvitāḥ| pușțị̣ baliṃ tathā vīryaṃ prayacchantu sukhodayam| $159 \mathrm{c}-16 \mathbf{1 b} \mathrm{P}_{3^{2}}^{T}$ has these eight pādas after $162 \mathrm{~d}$

158a ${ }^{\circ}$ parā hy ete ] $\mathrm{N}_{77}^{K o} \mathrm{~N}_{45}^{C} \mathrm{~N}_{82}^{K} \mathrm{~N}_{58}^{K} \mathrm{E}^{N} \mathrm{P}_{72}^{T}$, ${ }^{\circ}$ paro hy ete $\mathrm{B}_{99}^{C}$, o parā nityam $\mathrm{P}_{32}^{T} \quad \mathbf{1 5 8 b}$ maheśvarapadārcakāḥ ] $\mathrm{N}_{77}^{K o} \mathrm{~N}_{58}^{K} \mathrm{P}^{\Sigma}$, maheśvarasadārccakāḥ $\mathrm{N}_{45}^{C}$, maheśapādapūjakāh $\mathrm{N}_{82}^{K} \mathrm{E}^{N} \mathrm{~B}_{99}^{C}$ (unmetr.) $\quad \mathbf{1 5}^{8 \mathrm{c}}$ siddhim āśu ] $\mathrm{N}_{77}^{K o} \mathrm{~B}_{99}^{C} \mathrm{~N}_{82}^{K} \mathrm{E}^{N} \mathrm{~B}_{99}^{C} \mathbf{P}^{\Sigma}$, siddhiñcāśu $\mathrm{N}_{45}^{C} \mathrm{~N}_{58}^{K} \bullet$ prayacchantu ] $\mathrm{N}_{45}^{C} \mathrm{~N}_{82}^{K} \mathrm{~N}_{58}^{K} \mathrm{E}^{N} \mathrm{~B}_{99}^{C} \mathrm{P}^{\Sigma}$, prayacchanto $\mathrm{N}_{77}^{K o} \quad \mathbf{1 5} 8 \mathbf{d}$ ásirvāda ${ }^{\circ}$ ] $\mathrm{N}_{77}^{K o} \mathrm{~N}_{45}^{C} \mathrm{~N}_{82}^{K} \mathrm{E}^{N} \mathrm{~B}_{99}^{C}$, āśīrvāda ${ }^{\circ} \mathrm{N}_{58}^{K}$, śivadharma ${ }^{\circ} \mathbf{P}^{\Sigma} \bullet{ }^{\circ}$ parāyaṇāh ] $\mathrm{N}_{45}^{C} \mathrm{~N}_{82}^{K} \mathrm{~N}_{58}^{K} \mathrm{E}^{N} \mathrm{~B}_{99}^{C} \mathbf{P}^{\Sigma}$, ${ }^{\circ}$ parāyaṇā $\mathrm{N}_{77}^{K o} \quad 159$ a namucir ] $\mathrm{N}_{45}^{C} \mathrm{~K}^{\Sigma} \mathrm{E}^{N} \mathrm{~B}_{99}^{C} \mathbf{P}^{\Sigma}$, namuci $\mathrm{N}_{77}^{K o} \bullet$ daityarājendrah ] $\mathrm{N}_{77}^{K o} \mathrm{~N}_{45}^{C} \mathrm{~N}_{82}^{K} \mathrm{E}^{N} \mathrm{~B}_{99}^{C} \mathrm{P}_{72}^{T}$, nāma daityendraḥ $\mathrm{N}_{58}^{K} \mathrm{P}_{32}^{T} \quad \mathbf{1 5 9}^{2}$ b balah ] $\mathrm{N}_{77}^{K o} \mathrm{~N}_{45}^{C} \mathrm{~N}_{82}^{K} \mathrm{E}^{N} \mathbf{P}^{\Sigma}$, obalāh $\mathrm{N}_{58}^{K} \mathrm{~B}_{99}^{C} \quad{ }_{159}{ }^{\circ}$ nādo ] $\mathrm{N}_{77}^{K o} \mathrm{~N}_{82}^{K} \mathrm{~N}_{58}^{K} \mathrm{~N}_{45}^{C} \mathrm{~B}_{99}^{C} \mathbf{P}^{\Sigma}$, ${ }^{\circ}$ dāno $\mathrm{E}^{N}$, ${ }^{\circ}$ nātho $\hat{S}_{67}^{S}$ • vikhyāto ] $\mathrm{N}_{77}^{K o} \mathrm{~N}_{45}^{C} \mathrm{~N}_{82}^{K} \mathrm{~N}_{58}^{K} \mathrm{~B}_{99}^{C} \hat{S}_{67}^{S} \mathrm{P}_{72}^{T}$, vikhyātā $\mathrm{E}^{N}$, vitatah $\mathrm{P}_{32}^{T} \quad$ 159d daityah ] $\mathrm{N}_{82}^{K} \mathrm{~N}_{58}^{K} \mathrm{E}^{N} \mathrm{~B}_{99}^{C} \dot{S}_{67}^{S} \mathrm{P}^{\Sigma}$, daitya $\mathrm{N}_{77}^{K o} \mathrm{~N}_{45}^{C} \quad$ 16ob nityam ] $\mathrm{N}_{77}^{K o} \mathrm{~N}_{45}^{C} \mathrm{~N}_{82}^{K} \mathrm{~N}_{58}^{K} \hat{S}_{67}^{S} \mathbf{P}^{\Sigma}$, nitya ${ }^{\circ} \mathrm{E}^{N} \quad$ 16oc balam ] $\mathrm{N}_{77}^{K o} \mathrm{~N}_{45}^{C} \mathrm{~N}_{82}^{K} \mathrm{~B}_{99}^{C} S_{67}^{S} \mathbf{P}^{\Sigma}$, bala ${ }^{\circ} \mathrm{N}_{58}^{K}$, varam $\mathrm{E}^{N} \bullet$ kșipram ] $\mathrm{N}_{77}^{K o} \mathrm{~N}_{45}^{C} \mathrm{~N}_{82}^{K} \mathrm{~N}_{58}^{K} \mathrm{~B}_{99}^{C} \dot{S}_{67}^{S} \mathbf{P}^{\Sigma}$, nityam $\mathrm{E}^{N} \quad$ 16od prayacchantu ] $\mathrm{N}_{77}^{K o} \mathrm{~N}_{45}^{C} \mathrm{~N}_{82}^{K} \mathrm{E}^{N} \mathrm{~B}_{99}^{C} \dot{S}_{67}^{S}$, prayacchatu $\mathbf{P}^{\Sigma} \bullet$ maharddhikāh ] $\mathrm{N}_{77}^{K o} \mathrm{~N}_{45}^{C} \mathrm{~N}_{82}^{K} \mathrm{~N}_{58}^{K} \mathrm{E}^{N} \mathrm{~B}_{99}^{C} \mathrm{P}_{72}^{T}$, maharddhikā $S_{67}^{S}$, mahardhikaḥ $\mathrm{P}_{32}^{T}$ 
[Daityas : 2]

mahājambho hayagrīvaḥ prahlādo hy anuhlādakaḥ| tārako 'gnimukho daityaḥ kālanemir mahotkațạ̣ || 161\|

ete daityā mahātmānaḥ śivasadbhāvabhāvitāḥ|

puștịm balaṃ tathā vīryaṃ prayacchantu sukhodayam|| 162||

[Daityas : 3]

virocano hiraṇyākṣaḥ suparvaś ca sulomakaḥ| mucukundaḥ sukundaś ca daityo revatakas tathā|| 163\|

161-162 Cf. BhavP 1.179.25-26 : mahāḍhyo yo hayagrīvaḥ prahlādaḥ prabhayānvitaḥ| tānaikāgnimukho daityaḥ kālanemir mahābalaḥ|| ete daityā mahātmānaḥ sūryabhāvena bhāvitāḥ| tușțị̣ balạ̣ tathārogyam prayacchantu surārayaḥ || 163-164 Cf. BhavP 1.179.27-28 : vairocano hiranyākșas turvasuś ca sulocanah| mucakundo mukundaś ca daityo raivatakas tathā|| bhāvena parameṇemaṃ yajante satatạ̣ ravim| satatam ca śubhātmānah puṣțiṃ kurvantu te sadā||

162a-d Omitted in $\mathrm{P}_{72}^{T}$

161a hayagrīvah ] $\mathrm{N}_{82}^{K} \mathrm{~N}_{58}^{K} \mathrm{E}^{N} \hat{S}_{67}^{S} \mathrm{P}^{\Sigma}$, hayagrīva $\mathrm{N}_{77}^{K o} \mathrm{~N}_{45}^{C}$, bhayagrīvah $\mathrm{B}_{99}^{C} \quad \mathbf{1 6 1 b}$ prahlādo hy anuhlādakaḥ $\mathrm{N}_{45}^{C} \hat{S}_{67}^{S}$, prahlādaś cā(tu)śūdakaḥ $\mathrm{N}_{77}^{K o}$, prahlādo vyaktahlādakah $\mathrm{N}_{82}^{K} \mathrm{E}^{N} \mathrm{~B}_{99}^{C}$, prahlādo dhanuhlādakah $\mathrm{N}_{5^{8}}^{K}$, prahlādo daityarāṭ prabhụ̣ $\mathrm{P}_{32}^{T}$, prahlādo daityapumgavaḥ $\mathrm{P}_{72}^{T} \quad$ 161c tārako 'gnimukho daityaḥ ] $\mathrm{N}_{77}^{K o} \mathrm{~N}_{82}^{K} \mathrm{~N}_{5^{8}}^{K} \mathrm{E}^{N} \mathrm{~B}_{99}^{C} \dot{S}_{67}^{S} \mathrm{P}_{3^{2}}^{T}$, tārakāgnimukho daityaḥ $\mathrm{N}_{45}^{C}$, tārakākṣamukhā daityāḥ $\mathrm{P}_{72}^{T} \quad \mathbf{1 6 1 d}$ kālanemir ] $\mathrm{N}_{45}^{C} \mathrm{~N}_{82}^{K} \mathrm{~N}_{58}^{K} \mathrm{E}^{N} \mathrm{~B}_{99}^{C} \hat{S}_{67}^{S} \mathbf{P}^{\Sigma}$, kālanemi $\mathrm{N}_{77}^{K o} \bullet$ mahotkațah ] $\mathrm{N}_{82}^{K} \mathrm{E}^{N} \mathrm{~B}_{99}^{C}$, mahābalaḥ $\mathrm{N}_{77}^{K o} \mathrm{~N}_{58}^{K} S_{67}^{S}$, vvalottamah $\mathrm{N}_{45}^{C}$, balis tathā $\mathrm{P}_{32}^{T}$, mahātmavān $\mathrm{P}_{72}^{T}$ 162a mahātmānaḥ ] $\mathrm{N}_{45}^{C} \mathrm{~N}_{82}^{K} \mathrm{~N}_{5^{8}}^{K} \mathrm{E}^{N} \mathrm{~B}_{99}^{C} \stackrel{S}{S}_{67}^{S} \mathrm{P}_{32}^{T}$, mahātmāna $\mathrm{N}_{77}^{K o} \quad \mathbf{1 6 2 b} \quad{ }^{\circ}$ sadbhāvabhāvitāḥ ] $\mathrm{N}_{82}^{K p c} \mathrm{~N}_{58}^{K} \mathrm{E}^{N} \mathrm{~B}_{99}^{C} \dot{S}_{67}^{S} \mathrm{P}_{32}^{T}$, ${ }^{\circ}$ sadbhāvabhāvitā $\mathrm{N}_{77}^{K o}$, ${ }^{\circ}$ sambhāvabhāvitāḥ $\mathrm{N}_{45}^{C}$, ${ }^{\circ}$ sadbhāvabhāvitah $\mathrm{N}_{82}^{K a c} \quad$ 162c pușțim ] $\mathrm{N}_{77}^{K o} \mathrm{~N}_{82}^{K} \mathrm{~N}_{58}^{K} \mathrm{E}^{N} \mathrm{~B}_{99}^{C} S_{67}^{S} \mathrm{P}_{32}^{T}$, pușți ${ }^{\circ} \mathrm{N}_{45}^{C} \quad$ 162d sukhodayam ] $\mathrm{N}_{82}^{K} \mathrm{~N}_{58}^{K} \mathrm{E}^{N} \mathrm{~B}_{99}^{C} \dot{S}_{67}^{S} \mathrm{P}_{32}^{T}$, surārayah $\mathrm{N}_{77}^{K o} \mathrm{~N}_{45}^{C} \quad$ 163a virocano ] $\mathrm{N}_{82}^{K \text { pp }} \mathrm{B}_{99}^{C}$, vairocano $\mathrm{N}_{77}^{K o} \mathrm{~N}_{58}^{K} \mathrm{~N}_{45}^{C} \hat{\mathrm{S}}_{67}^{S} \mathrm{P}_{72}^{T}$, vicano $\mathrm{N}_{82}^{K a c}$ (unmetr.), vilocano $\mathrm{E}^{N}$, vairocano $\mathrm{P}_{3^{T}}^{T} \bullet$ hiraṇyākșah ] $\mathrm{N}_{77}^{K o} \mathrm{~N}_{82}^{K} \mathrm{~N}_{58}^{K} \mathrm{E}^{N} \mathrm{~B}_{99}^{C} S_{67}^{S} \mathbf{P}^{\Sigma}$, hiraṇākṣah $\mathrm{N}_{45}^{C} \quad \mathbf{1 6 3} \mathbf{b}$ suparvaś ca sulomakah ] $\mathrm{N}_{82}^{K} \mathrm{~N}_{58}^{K}$, suparṇnāa ca sulomakaṃ $\mathrm{N}_{77}^{K o}$, survvācaivavalomakaḥ $\mathrm{N}_{45}^{C}$, saparvaś ca sulomakaḥ $\mathrm{B}_{99}^{C}$, suparvāś ca sulomakah $\mathrm{E}^{N} \hat{S}_{67}^{S}$, suparvā ca sulomajạ̣ $\mathrm{P}_{32}^{T}$, suparvā ca sulomakaḥ $\mathrm{P}_{72}^{T} \quad 163 \mathrm{c}$ mucukundah ] $\mathrm{N}_{82}^{K} \mathrm{~N}_{58}^{K} \mathrm{E}^{N} \mathrm{~B}_{99}^{C} \hat{S}_{67}^{S} \mathbf{P}^{\Sigma}$, mucukunda $\mathrm{N}_{77}^{K o} \mathrm{~N}_{45}^{C} \quad$ 163d daityo revatakas ] $\mathrm{N}_{77}^{K o} \mathrm{~N}_{45}^{C} \mathrm{~N}_{82}^{K} \mathrm{~N}_{58}^{K} \mathrm{E}^{N} \mathrm{~B}_{99}^{C} \mathrm{P}_{32}^{T}$, daityāraivatakas $\hat{\mathrm{S}}_{67}^{S}$, daityo rocanakas $\mathrm{P}_{72}^{T}$ 
bhāvena parameṇāśu yajante sarvadā śivam|

satataṃ ca śubhātmānaḥ puștịm kurvantu me sadā|| 164||

[Daityapatnīs, Daityakanyakās, Daityakumāras]

daityapatnyo mahābhāgā daityānāṃ kanyakāḥ śubhāḥ|

kumārāś caiva daityānāṃ śāntiṃ kurvantu me sadā|| 165||

[Eight Nāgarājas]

[1. Ananta]

āraktena śarīreṇa raktāntāyatalocanaḥ|

mahābhogakṛtāṭopaḥ śankkhābjakṛtalāñchanaḥ|| 166||

165 Cf. BhavP 1.179.29 : daityapatayo mahābhāgā daityānāṃ kanyakāh śubhāḥ| kumārā ye ca daityānāṃ śāntiṃ kurvantu te sadā|| 166-167 Cf. BhavP 1.179·3o-31 : āraktena śarīreṇa raktāntāyatalocanāḥ| mahābhāgāḥ kṛtāṭopāḥ śan̉khādyāḥ kṛtalakṣaṇāḥ̣|| ananto nāgarājendra ādityārādhane ratạ̣| mahāpāpaviṣaṃ hatvā sāntim āśu karotu te||

165a-d Omitted in $\mathrm{P}_{3^{2}}^{T} \quad$ 166cd Omitted in $\hat{S}_{67}^{S}$

164a bhāvena parameṇāśu ] $\mathrm{N}_{77}^{K o} \mathrm{~N}_{45}^{C} \mathrm{~N}_{58}^{K} \hat{S}_{67}^{S} \mathbf{P}^{\Sigma}$, bhāvena ca pareṇāśu $\mathrm{N}_{82}^{K} \mathrm{E}^{N}$, bhāve ca na pareṇyāśu $\mathrm{B}_{99}^{C} \quad \mathbf{1 6 4}$ b yajante sarvadā ] $\mathrm{N}_{77}^{K o} \mathrm{~N}_{82}^{K} \mathrm{~N}_{58}^{K} \mathrm{E}^{N} \mathrm{~B}_{99}^{C} \dot{S}_{67}^{S} \mathrm{P}_{72}^{T}$, yamjanti parama $\mathrm{N}_{45}^{C}$, yajantaḥ sarvadā $\mathrm{P}_{32}^{T} \quad{ }_{164 c}$ satatam ca śubhātmānaḥ ] $\mathrm{N}_{77}^{K o} \mathrm{~N}_{45}^{C} \mathrm{~N}_{82}^{K} \mathrm{~N}_{58}^{K}$, satatañca subhātmānaḥ $\mathrm{B}_{99}^{C}$, satataś ca śubhātmānaḥ $\mathrm{E}^{N}$, satatam ca śubhānandāḥ $\hat{S}_{67}^{S}$, satatạ̣ ca śivātmānaṃ $\mathrm{P}_{32}^{T}$, sadāśivātmabhāvena $\mathrm{P}_{72}^{T} \quad \mathbf{1 6 5}^{\circ}{ }^{\circ}$ patnyo ] $\mathrm{N}_{77}^{K o} \mathrm{~N}_{82}^{K} \mathrm{~N}_{58}^{K} \mathrm{~B}_{99}^{C} \hat{S}_{67}^{S} \mathrm{P}_{72}^{T}$, ${ }^{\circ}$ padmo $\mathrm{N}_{45}^{C}$, ${ }^{\circ}$ patnyā $\mathrm{E}^{N} \quad \mathbf{1 6 5}$ b kanyakāh śubhāh ] $\mathrm{N}_{82}^{K} \mathrm{~N}_{58}^{K} \mathrm{E}^{N} \mathrm{~B}_{99}^{C p c} \hat{S}_{67}^{S} \mathrm{P}_{72}^{T}$, kanyakā śubhā $\mathrm{N}_{77}^{K o}$, kanyakā śubhāh $\mathrm{N}_{45}^{C} \mathrm{~B}_{99}^{C a c} \quad{ }_{165}$ c kumārāś caiva ] $\mathrm{N}_{82}^{K} \mathrm{E}^{N} \mathrm{~B}_{99}^{C}$, kumārā ye pi $\mathrm{N}_{77}^{K o}$, kumārā yaiva $\mathrm{N}_{45}^{C}$, kumārā ye ca $\mathrm{P}_{32}^{T}$, kumārakāś ca $\mathrm{P}_{72}^{T} \quad \mathbf{1 6 5}_{5} \mathrm{~d}$ śāntị̣ ] $\mathrm{N}_{77}^{K o} \mathrm{~N}_{45}^{C} \mathrm{~N}_{82}^{K} \mathrm{E}^{N} \mathrm{~B}_{99}^{C} \hat{S}_{67}^{S} \mathbf{P}^{\Sigma}$, śānti $\mathrm{N}_{58}^{K} \quad$ 166a āraktena ] $\mathrm{N}_{77}^{K o} \mathrm{~N}_{82}^{K} \mathrm{~N}_{58}^{K} \mathrm{E}^{N} \mathrm{~B}_{99}^{C} \dot{S}_{67}^{S} \mathbf{P}^{\Sigma}$, raktāyata ${ }^{\circ} \mathrm{N}_{45}^{C} \quad 166 \mathbf{b}$ olocanah ] $\mathrm{N}_{77}^{K o} \mathrm{~N}_{82}^{K} \mathrm{~N}_{58}^{K} \mathrm{E}^{N} \mathrm{~B}_{99}^{C p c} \mathbf{P}^{\Sigma}$, olocanāḥ $\mathrm{N}_{45}^{C} \mathrm{~B}_{99}^{\text {Cac }} S_{67}^{S} \quad$ 166c okṛtātopah ] $\mathrm{N}_{45}^{C} \mathrm{~N}_{82}^{K} \mathrm{~N}_{58}^{K} \mathrm{E}^{N} \mathrm{~B}_{99}^{C} \mathrm{P}^{\Sigma}$, okṛtātopa $\mathrm{N}_{77}^{K o} \quad$ 166d śańkhābjakṛtalāñchanạ ] $\mathrm{N}_{77}^{K o} \mathrm{~N}_{82}^{K} \mathrm{~N}_{58}^{K} \mathrm{E}^{N} \mathbf{P}^{\Sigma}$, śan̉khābjakṛbhūṣaṇaḥ $\mathrm{N}_{45}^{C}$ (unmetr.), śakhābjakṛtalācchanaḥ $\mathrm{B}_{99}^{C}$ 
ananto nāgarājendraḥ śivapādārcane rataḥ|

mahāpāpaviṣaṃ hatvā śāntim āśu karotu me|| 167||

\section{[2. Vāsuki]}

suśvetena tu dehena suśvetotpalaśekharah| cārubhogakṛtāțopo hāracāruvibhūṣaṇaḥ|| 168||

vāsukir nāgarājendraḥ rudrapūjāparo mahān| mahāpāpavișaṃ hatvā śāntim āśu karotu me|| 169||

\section{[3. Takșaka]}

atipìtena dehena visphuradbhogasampadā| tejasā cātidīptena kṛtasvastikalāñchanaḥ|| 170||

170-171 Cf. BhavP 1.179.32-33: atipītena dehena visphuradbhogasamppadā| tejasā cātidīptena kṛtasvastikalāñchanaḥ|| nāgarāṭt takṣakaḥ śrīmān nāmakoțyā samanvitaḥ| karotu te mahāśāntiṃ sarvadoṣaviṣāpahām||

167a ananto ] $\mathrm{N}_{77}^{K o} \mathrm{~N}_{82}^{K} \mathrm{~N}_{58}^{K} \mathrm{E}^{N} \mathrm{~B}_{99}^{C} \hat{S}_{67}^{S} \mathbf{P}^{\Sigma}$, anto $\mathrm{N}_{45}^{C}$ (unmetr.) • nāgarājendrah ] $\mathrm{N}_{82}^{K} \mathrm{~N}_{58}^{K} \mathrm{~B}_{99}^{C} S_{67}^{S} \mathrm{P}_{72}^{T}$, nāgarājendra $\mathrm{N}_{77}^{K o} \mathrm{E}^{N}$, nāgarājendrāh $\mathrm{N}_{45}^{C}$, nāma nāgendrah $\mathrm{P}_{32}^{T}$ 167b b pādārcane ratạ̣ ] $\mathrm{N}_{82}^{K} \mathrm{E}^{N} \mathrm{~B}_{99}^{C} \mathrm{P}_{72}^{T}$, ${ }^{\circ}$ pūjārcane ratā $\mathrm{N}_{77}^{K o}$, ${ }^{\circ}$ pūjāreccane ratah $\mathrm{N}_{45}^{C}$, ${ }^{\circ}$ pādarccane ratah $\mathrm{N}_{58}^{K}$, ${ }^{\circ}$ pūjāparo mahān $S_{67}^{S}$, ${ }^{\circ}$ pūjārataḥ sadā $\mathrm{P}_{32}^{T} \quad \mathbf{1 6 7 d}$ śāntim āśu ] $\mathrm{N}_{45}^{C} \mathrm{~N}_{82}^{K} \mathrm{~N}_{58}^{K} \mathrm{E}^{N} \mathrm{~B}_{99}^{C} \dot{S}_{67}^{S} \mathrm{P}_{72}^{T}$, śāntim āśụ $\mathrm{N}_{77}^{K o}$, kṣemārogyaṃ $\mathrm{P}_{32}^{T} \quad$ 168a suśvetena tu ] $\mathrm{N}_{77}^{K o} \mathrm{~N}_{82}^{K} \mathrm{E}^{N} \mathrm{~B}_{99}^{C} \dot{\mathrm{S}}_{67}^{S} \mathbf{P}^{\Sigma}$, áśvetena tu $\mathrm{N}_{45}^{C}$, suśvetena $\mathrm{N}_{58}^{K}$ (unmetr.) $\mathbf{1 6 8 b}$

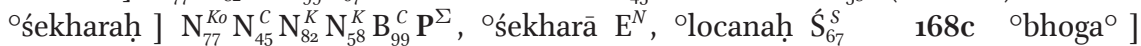
$\mathrm{N}_{77}^{K o} \mathrm{~N}_{45}^{C} \mathrm{~N}_{82}^{K} \mathrm{~N}_{58}^{K} \mathrm{~B}_{99}^{C} \stackrel{S}{S}_{67}^{S} \mathbf{P}^{\Sigma}$, obhogo $\mathrm{E}^{N}$ • otopo ] $\mathrm{N}_{82}^{K} \mathrm{~N}_{58}^{K} \mathrm{E}^{N} \mathrm{~B}_{99}^{C} \dot{S}_{67}^{S} \mathbf{P}^{\Sigma}$, otopa $\mathrm{N}_{77}^{K o} \mathrm{~N}_{45}^{C}$ 168d hāracāruvibhūṣaṇạ̣ ] $\mathrm{N}_{45}^{C} \mathrm{~N}_{82}^{K} \mathrm{~N}_{58}^{K} \mathrm{E}^{N} \mathrm{~B}_{99}^{C} \mathrm{P}_{32}^{T}$, haracāruvibhūṣaṇaḥ $\mathrm{N}_{77}^{K o}$, hārakeyūrabhūṣitaḥ $S_{67}^{S}$, haracāruvibhūṣitaḥ $\mathrm{P}_{72}^{T} \quad 169$ a vāsukir ] $\mathrm{N}_{82}^{K} \mathrm{E}^{N} \mathrm{~B}_{99}^{C} \hat{S}_{67}^{S} \mathbf{P}^{\Sigma}$, vāsuki $\mathrm{N}_{77}^{K o} \mathrm{~N}_{58}^{K}$, vāsukī $\mathrm{N}_{45}^{C} \bullet$ nāgarājendrạ̣ ] $\mathrm{N}_{77}^{K o} \mathrm{~N}_{45}^{C} \mathrm{~N}_{82}^{K} \mathrm{~B}_{99}^{C} \mathrm{P}_{72}^{T}$, nāgarājedro $\mathrm{N}_{58}^{K}$, nāma rājendrah $\mathrm{E}^{N}$, nāma nāgendro $S_{67}^{S}$, nāma nāgendraḥ $\mathrm{P}_{32}^{T} \quad \mathbf{1 6 9 \mathrm { b }}$ rudrapūjāparo mahān ] $\mathrm{N}_{45}^{C} \mathrm{~N}_{82}^{K} \mathrm{~N}_{58}^{K} \mathrm{E}^{N} \mathrm{~B}_{99}^{C}$, śivapādārcane ratah $\mathrm{N}_{77}^{K o} \mathrm{P}_{72}^{T}$, rudrapūjārate sadā $\hat{S}_{67}^{S}$, śivapūjāparo mahān $\mathrm{P}_{32}^{T} \quad$ 169d śāntim āśu karotu me ] $\mathrm{N}_{45}^{C} \mathrm{~N}_{82}^{K} \mathrm{E}^{N} \mathrm{~B}_{99}^{C}$, kșemam āśu karotu me $\mathrm{N}_{77}^{K o}$, karotu mama śāntikam $\hat{S}_{67}^{S}$, kṣemārogyam karotu me $\mathrm{P}_{32}^{T}$, kṣemārogyam dadātu me $\mathrm{N}_{58}^{K} \mathrm{P}_{72}^{T} \quad$ 17ob visphuradbhoga $\left.{ }^{\circ}\right] \mathrm{N}_{45}^{C} \mathrm{~N}_{82}^{K} \mathrm{~N}_{58}^{K} \mathrm{E}^{N} \mathrm{~B}_{99}^{C} \hat{S}_{67}^{S}$, visphuram bhoga ${ }^{\circ} \mathrm{N}_{77}^{K o}$, prasphuradbhoga ${ }^{\circ} \mathrm{P}_{72}^{T p c}$, prasphuraddeha ${ }^{\circ} \mathrm{P}_{72}^{\text {Tac }}$, prakșuradbhoga ${ }^{\circ} \mathrm{P}_{72}^{T}$ $170 c$ tejasā cātidīptena ] $\mathrm{N}_{77}^{K o} \mathrm{~N}_{82}^{K} \mathrm{~N}_{58}^{K} \mathrm{~B}_{99}^{C} S_{67}^{S} \mathrm{P}_{72}^{T}$, tejasāñcātidīpteṇa $\mathrm{N}_{45}^{C}$, tejasā cātidiptena $\mathrm{E}^{N}$, tejasā cātisaptena $\mathrm{P}_{32}^{T} \quad$ 17od $\left.{ }^{\circ} \mathrm{krrta}^{\circ}\right] \mathrm{N}_{45}^{C} \mathrm{~N}_{82}^{K} \mathrm{~N}_{58}^{K} \mathrm{E}^{N} \mathrm{~B}_{99}^{C} \stackrel{\mathrm{S}}{67}_{67}^{S} \mathrm{P}^{\Sigma}$, ${ }^{\circ} \mathrm{kṛtah}$ $\mathrm{N}_{77}^{K o} \bullet{ }^{\circ}$ Svastika $\left.{ }^{\circ}\right] \mathrm{N}_{77}^{K o} \mathrm{~N}_{82}^{K} \mathrm{~N}_{58}^{K} \mathrm{E}^{N} \mathrm{~B}_{99}^{C} \hat{S}_{67}^{S} \mathbf{P}^{\Sigma}$, ${ }^{\circ}$ svaka $^{\circ} \mathrm{N}_{45}^{C}$ (unmetr.) • olāñchanah ] $\mathrm{N}_{77}^{K o} \mathrm{~N}_{45}^{C} \mathrm{~N}_{82}^{K} \mathrm{~N}_{58}^{K} \mathrm{E}^{N} \mathrm{~B}_{99}^{C} \mathbf{P}^{\Sigma}$, olakșaṇaḥ $\hat{S}_{67}^{S}$ 
nāgarāt takṣakah śrīmān nāgakoțyā samanvitaḥ|

karotu me mahāśāntiṃ sarvadoṣaviṣāpahām|| 171||

\section{[4. Karkotaka]}

atikṛ̣nnena varṇena sphațāvikațamastakaḥ|

kaṇṭhe rekhātrayopeto ghoradaṃștrāyudhodyatạ̣|| 172||

karkoṭako mahānāgo viṣadarpabalānvitaḥ|

viṣaśastrāgnisaṃtāpaṃ hatvā śāntiṃ karotu me|| 173||

172-173 Cf. BhavP 1.179·34-35: atikṛṣnena varṇena sphuțādhikațamastakaḥ| kaṇtharekhātrayopeto ghoradaṃșțāyudhodyataḥ|| karkoțako mahānāgo viṣadarpabalānvitaḥ| viṣaśastrāgnisaṃtāpaṃ hatvā śāntiṃ karotu te||

171b After this $\hat{S}_{67}^{S}$ adds two pādas : śivarcanarato nityam śivaikagatamānasaḥ| $\mathbf{1 7 2 c d}$ Omitted in $\hat{S}_{67}^{S}$

$171 a$ nāgarāt takṣakaḥ ] $\mathrm{N}_{77}^{K o} \mathrm{~N}_{82}^{K} \mathrm{~N}_{58}^{K} \mathrm{~B}_{99}^{C} \dot{\mathrm{S}}_{67}^{S} \mathrm{P}_{32}^{T}$, nāgarāṭakṣaka $\mathrm{N}_{45}^{C}$, nāgarāḍrakṣakah $\mathrm{E}^{N}$, nāgendras takșakah. $\mathrm{P}_{72}^{T} \quad \mathbf{1 7 1 b}{ }^{\circ}$ koțyā ] $\mathrm{N}_{77}^{K o} \mathrm{~N}_{82}^{K} \mathrm{~N}_{58}^{K} \mathrm{~B}_{99}^{C} \mathrm{P}_{72}^{T}$, ${ }^{\circ}$ koțīo $\mathrm{N}_{45}^{C}$, ${ }^{\circ}$ kotyo $\mathrm{E}^{N}$, okoți ${ }^{\circ} \hat{\mathrm{S}}_{67}^{S} \mathrm{P}_{32}^{T} \bullet$ samanvitạ ] $\mathrm{N}_{77}^{K o} \mathrm{~N}_{45}^{C} \mathrm{~N}_{82}^{K} \mathrm{~N}_{58}^{K} \mathrm{~B}_{99}^{C} \dot{S}_{67}^{S} \mathrm{P}_{32}^{T}$, samanvitāḥ $\mathrm{E}^{N}$, samadyutị̣ $\mathrm{P}_{72}^{T} \quad$ 171d odoṣaviṣāpahām ] $\mathrm{N}_{82}^{K} \mathrm{~N}_{58}^{K} \mathrm{~B}_{99}^{C}$, ' doṣaviśāpaham $\mathrm{N}_{77}^{K o}$, 'opāpaviṣāpahah $\mathrm{N}_{45}^{C}$, ${ }^{\circ}$ doṣāvișāpahān $\mathrm{E}^{N}$, ${ }^{\circ}$ doșavișāpaham $\dot{\mathrm{S}}_{67}^{S}$, ${ }^{\circ}$ doṣavișāpahah $\mathrm{P}_{32}^{T}$, ${ }^{\circ}$ doṣavināśanam $\mathrm{P}_{72}^{T} \quad$ 172a varṇena ] $\mathrm{N}_{77}^{K o} \mathrm{~N}_{45}^{C} \mathrm{~N}_{82}^{K} \mathrm{~N}_{58}^{K} \mathrm{E}^{N} \mathrm{~B}_{99}^{C} \mathrm{P}_{72}^{T}$, dehena $S_{67}^{S} \mathrm{P}_{32}^{T}$ 172b sphațāvikața $\left.{ }^{\circ}\right] \mathrm{N}_{77}^{K o} \mathrm{~N}_{82}^{K} \mathrm{~B}_{99}^{C}$, sphuțāvikața ${ }^{\circ} \mathrm{N}_{45}^{C} \mathrm{~N}_{58}^{K}$, sphìtavikața ${ }^{\circ} \mathrm{E}^{N}$, sphuranmukuța ${ }^{\circ} S_{67}^{S}$, sphuradvikața ${ }^{\circ} \mathrm{P}_{32}^{T}$, kṣīradvikața ${ }^{\circ} \mathrm{P}_{72}^{T} \quad \mathbf{1 7 2 c}^{\circ}$ peto ] $\mathrm{N}_{45}^{C} \mathrm{~B}_{99}^{C} \dot{S}_{67}^{S} \mathbf{P}^{\Sigma}$, o petā $\mathrm{N}_{77}^{K o} \mathrm{~N}_{82}^{K} \mathrm{~N}_{58}^{K} \mathrm{E}^{N}$ 172d ${ }^{\circ}$ daṃșțrāyudhodyatạ ] $\mathrm{N}_{82}^{K} \mathrm{~B}_{99}^{C} \mathbf{P}^{\Sigma}$, odrașțāyudhodyatạ $\mathrm{N}_{77}^{K o}$, odrașțoyudhodyatah $\mathrm{N}_{45}^{C}$, ${ }^{\circ}$ draṃsțrāyudoyutah $\mathrm{N}_{58}^{K}$, ${ }^{\circ}$ daṃșțrāyutodyatah $\hat{S}_{67}^{S}$, ${ }^{\circ}$ daṃșțrāyudhādyataḥ $\mathrm{E}^{N} \quad$ 173a karkoțako ] $\mathrm{N}_{77}^{K o} \mathrm{~N}_{82}^{K} \mathrm{~N}_{58}^{K} \mathrm{E}^{N} \mathrm{~B}_{99}^{C} \dot{S}_{67}^{S}$, karkkauțako $\mathrm{N}_{45}^{C}$, kālakūṭo $\mathrm{P}_{32}^{T}$, kārkoțako $\mathrm{P}_{72}^{T} \bullet{ }^{\circ}$ nāgo ] $\mathrm{N}_{77}^{K o} \mathrm{~N}_{45}^{C} \mathrm{~N}_{82}^{K} \mathrm{~N}_{58}^{K} \mathrm{E}^{N} \mathrm{~B}_{99}^{C} \hat{\mathrm{S}}_{67}^{S} \mathrm{P}_{32}^{T}$, otejā $\mathrm{P}_{72}^{T} \quad \mathbf{1 7 3}^{\circ}$ obalānvitah ] $\mathrm{N}_{77}^{K o} \mathrm{~N}_{45}^{C} \mathrm{~N}_{82}^{K} \mathrm{~N}_{58}^{K} \mathrm{~B}_{99}^{C}$, obalānvitāḥ $\mathrm{E}^{N}$, o santāpam $S_{67}^{S}$, ${ }^{\circ}$ samanvitaḥ $\mathbf{P}^{\Sigma} \quad 173 \mathrm{C}$ viṣaśastrāgnisaṃtāpaṃ ] $\mathrm{N}_{77}^{K o} \mathrm{~N}_{45}^{C} \mathrm{~N}_{82}^{K} \mathrm{~N}_{5^{8}}^{K} \mathrm{E}^{N} \mathrm{~B}_{99}^{C} \hat{S}_{67}^{S} \mathrm{P}_{32}^{T}$, vișāṇy ugrāṇi sahasā $\mathrm{P}_{72}^{T} \quad$ 173d śāntim ] $\mathrm{N}_{45}^{C} \mathrm{~N}_{82}^{K} \mathrm{~N}_{58}^{K} \mathrm{E}^{N} \mathrm{~B}_{99}^{C} \dot{S}_{67}^{S} \mathbf{P}^{\Sigma}$, śānti $\mathrm{N}_{77}^{K o}$ 
[5. Padma]

padmavarṇena dehena cārupadmāyatekṣaṇaḥ| pañcabindukṛtābhāso grīvāyāṃ śubhalakṣaṇaḥ|| 174||

khyātapadmo mahānāgo harapādārcane rataḥ| karotu me mahāśāntiṃ mahāpāpaviṣakṣayam|| 175||

\section{[6. Mahāpadma]}

puṇḍarīkanibhenāpi dehenāmitatejasā|

śan̉khaśūlābjarucirair bhūṣito mūrdhni sarvadā|| 176||

mahāpadmo mahānāgo nityaṃ paśupater nataḥ|

vinikṛtya viṣaṃ ghoraṃ śāntim āśu karotu me || 177||

174-175 Cf. BhavP 1.179.36-37 : padmavarṇah padmakāntih phullapadmāyatekṣaṇaḥ| khyātaḥ padmo mahānāgo nityaṃ bhāskarapūjakaḥ|| sa te śāntiṃ śubhạ̣ śīghram acalaṃ saṃprayacchatu| śyāmena dehabhāreṇa śrīmatkamalalocanạ̣||

174cd Omitted in $\mathrm{E}^{N}$

174b cārupadmāo ] $\mathrm{N}_{77}^{K o} \mathrm{~N}_{82}^{K} \mathrm{~N}_{58}^{K} \mathrm{E}^{N} \mathrm{~B}_{99}^{c} \hat{\mathrm{S}}_{67}^{S} \mathrm{P}^{\Sigma}$, padmapatrāo $\mathrm{N}_{45}^{c}$ • otekșaṇah ] $\mathrm{N}_{45}^{C} \mathrm{~N}_{82}^{K} \mathrm{~N}_{58}^{K} \mathrm{E}^{N} \mathrm{~B}_{99}^{C} \dot{S}_{67}^{S} \mathbf{P}^{\Sigma}$, otekșaṇā $\mathrm{N}_{77}^{K o} \quad$ 174c okṛtābhāso ] $\mathrm{N}_{77}^{K o} \mathrm{~N}_{82}^{K} \mathrm{~N}_{58}^{K} \mathrm{~B}_{99}^{C} \mathrm{P}_{3^{2}}^{T}$, okrtābhogo $\mathrm{N}_{45}^{C}$, ${ }^{\circ}$ yutābhāso $\hat{S}_{67}^{S}$, ${ }^{2}$ kṣatābhāso $\mathrm{P}_{72}^{T} \quad{ }^{174 d}$ grīvāyām ] $\mathrm{N}_{77}^{K o} \mathrm{~N}_{45}^{C} \mathrm{~N}_{82}^{K} \mathrm{~N}_{58}^{K} \mathrm{~B}_{99}^{C} S_{67}^{S} \mathrm{P}_{72}^{T}$, grīvāyāḥ $\mathrm{P}_{32}^{T} \quad 1^{T} 5^{\text {a }}$ khyātapadmo ] $\mathrm{N}_{77}^{K o} \mathrm{~N}_{45}^{C} \mathrm{~N}_{82}^{K} \mathrm{~N}_{58}^{K} \mathrm{~B}_{99}^{C}$, khyātah padmo $\mathrm{E}^{N} \hat{\mathrm{S}}_{67}^{S} \mathrm{P}_{72}^{T}$, khyātah padma ${ }^{\circ} \mathrm{P}_{32}^{T}$ • ${ }^{\circ}$ nāgo ] $\mathrm{N}_{77}^{K o} \mathrm{~N}_{45}^{C} \mathrm{~N}_{82}^{K} \mathrm{~N}_{58}^{K} \mathrm{E}^{N} \mathrm{~B}_{99}^{C} \dot{S}_{67}^{S} \mathrm{P}_{32}^{T}$, obhogo $\mathrm{P}_{72}^{T} \quad{ }^{T}$ 75 karotu me ] $\mathrm{N}_{77}^{K o} \mathrm{~N}_{45}^{C} \mathrm{~N}_{82}^{K} \mathrm{~N}_{58}^{K} E^{N} \mathrm{~B}_{99}^{C} \mathrm{P}_{32}^{T}$, karotu sa $S_{67}^{S}$, karotu $\mathrm{P}_{72}^{T} \bullet{ }^{\circ}$ śāntim ] $\mathrm{N}_{77}^{K o} \mathrm{~N}_{45}^{C} \mathrm{~N}_{82}^{K} \mathrm{~N}_{58}^{K} \mathrm{~B}_{99}^{C} \mathbf{P}^{\Sigma}$, ' ${ }^{\circ}$ ánti $\mathrm{E}^{N} \quad \mathbf{1 7 5} \mathbf{d}$ mahā $\left.{ }^{\circ}\right] \mathrm{N}_{77}^{K o} \mathrm{~N}_{45}^{C} \mathrm{~N}_{82}^{K} \mathrm{~N}_{58}^{K} E^{N} \mathrm{~B}_{99}^{C} \mathbf{P}^{\Sigma}$, sarva ${ }^{\circ}$ $\hat{S}_{67}^{S}$ • ${ }^{\circ}$ vișakșayam ] $\mathrm{N}_{77}^{K o} \mathrm{~N}_{45}^{C} \mathrm{~N}_{82}^{K} \mathrm{~N}_{5^{8}}^{K} \mathrm{E}^{N} \mathrm{~B}_{99}^{C} \dot{S}_{67}^{S} \mathrm{P}_{3^{2}}^{T},{ }^{\circ}$ vi $\sqcup \sqcup$ yam $\mathrm{P}_{72}^{T} \quad \mathbf{1 7 6 a}$ puṇụarika ${ }^{\circ}$ ] $\mathrm{N}_{45}^{C} \mathrm{~N}_{82}^{K} \mathrm{~N}_{58}^{K} \mathrm{E}^{N} \mathrm{~B}_{99}^{C} \dot{S}_{67}^{S} \mathbf{P}^{\Sigma}$, pauṇdurīka ${ }^{\circ} \mathrm{N}_{77}^{K o} \bullet{ }^{\circ}$ nibhenāpi ] $\mathrm{N}_{82}^{K} \mathrm{~N}_{58}^{K} \mathrm{E}^{N} \mathrm{~B}_{99}^{C} \dot{S}_{67}^{S} \mathrm{P}_{32}^{T}$, o nibho bhāti $\mathrm{N}_{77}^{K o}$, ${ }^{\circ}$ nibhenāti $\mathrm{N}_{45}^{C}$, பபnāpi $\mathrm{P}_{72}^{T} \quad \mathbf{1 7 6 c}^{\circ}$ śūlābjarucirair ] $\mathrm{N}_{82}^{K} \mathrm{E}^{N} \mathrm{~B}_{99}^{C} \mathrm{P}_{32}^{T}$, ${ }^{\circ}$ śūlābjaruciro $\mathrm{N}_{77}^{K o} \mathrm{P}_{72}^{T}$, ${ }^{\circ}$ śūlāruciraih $\mathrm{N}_{45}^{C}$ (unmetr.), ${ }^{\circ}$ śūlabjarucirair $\mathrm{N}_{58}^{K}$, ${ }^{\circ}$ śuklābjarucirair $\hat{S}_{67}^{S} \quad$ 176d sarvadā ] $\mathrm{N}_{77}^{K o} \mathrm{~N}_{45}^{C} \mathrm{~N}_{82}^{K} \mathrm{~N}_{58}^{K} \mathrm{E}^{N} \mathrm{~B}_{99}^{C} \hat{S}_{67}^{S} \mathrm{P}_{72}^{T}$, bindubhị̣ $\mathrm{P}_{32}^{T} \quad$ 177a a nāgo ] $\mathrm{N}_{77}^{K o} \mathrm{~N}_{45}^{C} \mathrm{~N}_{82}^{K} \mathrm{~N}_{58}^{K} \mathrm{E}^{N} \mathrm{~B}_{99}^{C} S_{67}^{S} \mathrm{P}_{32}^{T}$, obhogo $\mathrm{P}_{72}^{T} \quad \mathbf{1 7 7} \mathrm{b}$ opater natah ] $\mathrm{N}_{82}^{K} \mathrm{E}^{N} \mathrm{~B}_{99}^{C}$, opate ratah $\mathrm{N}_{77}^{K o} \mathrm{~N}_{45}^{C} \mathrm{~N}_{58}^{K}$, ${ }^{\circ}$ patau ratah $S_{67}^{S} \mathbf{P}^{\Sigma} \quad{ }_{177} \mathbf{c}$ vinikrtya ] $\mathrm{N}_{82}^{K} \mathrm{~B}_{99}^{C} S_{67}^{S}$, vinihatya $\mathrm{N}_{77}^{K o} \mathrm{~N}_{58}^{K} \mathbf{P}^{\Sigma}$, vinirhatyam $\mathrm{N}_{45}^{C}$, vinișkṛtya $\mathrm{E}^{N} \quad \mathbf{1 7 7}_{\mathbf{7}} \mathbf{d}$ āśu ] $\mathrm{N}_{45}^{C} \mathrm{~N}_{82}^{K} \mathrm{~N}_{58}^{K} \mathrm{E}^{N} \mathrm{~B}_{99}^{C} \dot{S}_{67}^{S} \mathbf{P}^{\Sigma}$, āśuh $\mathrm{N}_{77}^{K o}$ 


\title{
[7. Śan̉khapāla]
}

śyāmena dehabhāreṇa śrīmatkamalalocanaḥ| viṣadarpabalonmatto grīvāyām ekarekhayā|| 178||

śan̉khapālaḥ śriyā dīptaḥ śivapādābjapūjakaḥ| mahāviṣaṃ mahāpāpaṃ hatvā śāntiṃ karotu me|| 179||

\author{
[8. Kulika]
}

atighoreṇa dehena candrārdhakṛtamastakaḥ| dipptabhogakṛtāțopaḥ śubhalakṣaṇalakșitaḥ|| 180||

178-179 Cf. BhavP 1.179.38-39ab : viṣadarpabalonmatto grīvāyāṃ rekhayānvitaḥ| śaṅkhapālaśriyā dīptaḥ sūryapādābjapūjakaḥ|| mahāviṣaṃ garaśreșțhaṃ hatvā śāntiṃ karotu te| 180-181 Cf. BhavP 1.179.39cd-40 : atigaureṇa dehena candrārdhakṛtaśekharaḥ|| dīpabhāge kṛtātopaśubhalakṣaṇalakșitạ̣| kuliko nāma nāgendro nityaṃ sūryaparāyaṇaḥ| apahṛtya viṣaṃ ghoraṃ karotu tava śāntikam||

178a śyāmena dehabhāreṇa ] $\mathrm{N}_{82}^{K} \mathrm{E}^{N} \mathrm{~B}_{99}^{C} \mathrm{P}_{32}^{T}$, śyāmena dehadehabhāreṇa $\mathrm{N}_{77}^{K o}$ (unmetr.), śyāmena vihānāpi $\mathrm{N}_{5^{8}}^{K}$ (unmetr.), atiśyāmena dehena $\hat{S}_{67}^{S}$, śyāmena dehabhāvena $\mathrm{N}_{45}^{C}$, śyāmena dehābharaṇaị $\mathrm{P}_{72}^{T} \quad \mathbf{1 7 8 b}$ śrīmat ] $\mathrm{N}_{77}^{K o} \mathrm{~N}_{45}^{C} \mathrm{~N}_{82}^{K} \mathrm{E}^{N} \mathrm{~B}_{99}^{C} \mathrm{~S}_{67}^{S} \mathrm{P}_{32}^{T}$, śrīmān $\mathrm{N}_{58}^{K} \mathrm{P}_{72}^{T} \quad 178 \mathrm{c} \quad{ }^{\circ}$ darpabalonmatto ] $\mathrm{N}_{77}^{K o} \mathrm{~N}_{82}^{K} \mathrm{~N}_{58}^{K} \mathrm{~B}_{99}^{C} \mathbf{P}^{\Sigma}$, ${ }^{\circ}$ darpabalotmatto $\mathrm{N}_{45}^{C}$, ${ }^{\circ}$ darpābalonmatto $\mathrm{E}^{N}$, odarpabalopeto $\hat{S}_{67}^{S} \quad \mathbf{1 7 8 d}$ ekarekhayā ] $\mathrm{N}_{77}^{K o} \mathrm{~N}_{82}^{K} \mathrm{~N}_{58}^{K} \mathrm{E}^{N} \mathrm{~B}_{99}^{C} \hat{S}_{67}^{S} \mathbf{P}^{\Sigma}$, ekarekhayoh $\mathrm{N}_{77}^{K o}$, ekharekhayā $\mathrm{N}_{45}^{C} \quad$ 179a $^{\circ}$ pālah śriyā ] $\mathrm{N}_{77}^{K o} \mathrm{~N}_{45}^{C} \mathrm{~N}_{82}^{K} \mathrm{~N}_{58}^{K} \mathrm{E}^{N} \mathrm{~B}_{99}^{C} \hat{S}_{67}^{S} \mathrm{P}_{32}^{T}$, ${ }^{\circ}$ vālakșiyā $\mathrm{P}_{72}^{T} \bullet$ dīptạ̣ ] $\mathrm{N}_{45}^{C} \mathrm{~N}_{82}^{K} \mathrm{~N}_{58}^{K} \mathrm{E}^{N} \mathrm{~B}_{99}^{C} \hat{S}_{67}^{S} \mathbf{P}^{\Sigma}$, dīpta $\mathrm{N}_{77}^{K o} \quad \mathbf{1 7 9}$ b ${ }^{\circ}$ pūjakaḥ ] $\mathrm{N}_{77}^{K o} \mathrm{~N}_{45}^{C} \mathrm{~N}_{82}^{K p c} \mathrm{~N}_{58}^{K} E^{N} \mathrm{~B}_{99}^{C} \hat{S}_{67}^{S} \mathbf{P}^{\Sigma}$, ${ }^{\circ}$ pūjah $\mathrm{N}_{82}^{K a c}$ (unmetr.) $\quad 179 c \quad{ }^{\circ}$ viṣam mahāpam ] $\mathrm{N}_{45}^{C} \mathrm{~N}_{82}^{K} \mathrm{~N}_{58}^{K} \mathrm{E}^{N} \mathrm{~B}_{99}^{C}$, ${ }^{\circ}$ vișam mahāghoram $\mathrm{N}_{77}^{K o} \dot{\mathrm{S}}_{67}^{S}$, oghoram viṣam hatvā $\mathbf{P}^{\Sigma}$ 179d hatvā śāntiṃ karotu me ] $\mathrm{N}_{45}^{C} \mathrm{~N}_{82}^{K} \mathrm{~N}_{58}^{K} \mathrm{E}^{N} \mathrm{~B}_{99}^{C} \hat{S}_{67}^{S}$, hutvā śāntim karotu me $\mathrm{N}_{77}^{K o}$, karotu mama śāntikam $\mathrm{P}^{\Sigma} \quad \mathbf{1 8 0 a}$ atighoreṇa ] $\mathrm{N}_{82}^{K} \mathrm{~N}_{58}^{K} \mathrm{E}^{N} \mathrm{~B}_{99}^{C} \dot{S}_{67}^{S}$, atigaureṇa $\mathrm{N}_{77}^{K o} \mathrm{~N}_{45}^{C} \mathbf{P}^{\Sigma} \bullet$ dehena ] $\mathrm{N}_{45}^{C} \mathrm{~N}_{82}^{K} \mathrm{~N}_{58}^{K} \mathrm{E}^{N} \mathrm{~B}_{99}^{C} \hat{S}_{67}^{S} \mathbf{P}^{\Sigma}$, dehe $\mathrm{N}_{77}^{K o}$ (unmetr.) 18ob candrārdhakrta $\left.{ }^{\circ}\right] \mathrm{N}_{77}^{K o} \mathrm{~N}_{82}^{K} \mathrm{~N}_{58}^{K} \mathrm{E}^{N} \mathrm{~B}_{99}^{C} \dot{S}_{67}^{S} \mathbf{P}^{\Sigma}$, candrārddhānkita ${ }^{\circ} \mathrm{N}_{45}^{C} \bullet{ }^{\circ}$ mastakah ] $\mathrm{N}_{77}^{K o} \mathrm{~N}_{45}^{C} \mathrm{~N}_{82}^{K} \mathrm{~N}_{58}^{K} E^{N} \mathrm{~B}_{99}^{C} \dot{S}_{67}^{S} \mathrm{P}_{32}^{T}$, 'óekharah $\mathrm{P}_{72}^{T} \quad$ 18oc obhoga $\left.{ }^{\circ}\right] \mathrm{N}_{45}^{C} \mathrm{~N}_{82}^{K} \mathrm{~N}_{58}^{K} \mathrm{E}^{N} \mathrm{~B}_{99}^{C} \mathrm{P}^{\Sigma}$, obhogo $S_{67}^{S} \quad$ 18ocd okrtātọopạ śubha $\left.{ }^{\circ}\right] \mathrm{N}_{45}^{C} \mathrm{~N}_{82}^{K} \mathrm{~N}_{58}^{K} \mathrm{E}^{N} \mathbf{P}^{\Sigma}$, okrtātọa śubha ${ }^{\circ} \mathrm{N}_{77}^{K o} \mathrm{~B}_{99}^{C}$, ${ }^{\circ}$ kṛtārvāpaś śaṃbhu ${ }^{\circ} \mathrm{P}_{72}^{T} \quad 18$ od olakșitah ] $\mathrm{N}_{82}^{K} \mathrm{~N}_{58}^{K} \mathrm{E}^{N} \mathrm{~B}_{99}^{C} \dot{S}_{67}^{S} \mathrm{P}^{\Sigma}$, olakṣitā $\mathrm{N}_{77}^{K o}$, lāṃcchitam $\mathrm{N}_{45}^{C}$ 
kuliko nāgarājendro nityạ̣ haraparāyaṇaḥ| apahṛtya viṣaṃ ghoraṃ karotu mama śāntikam|| 181||

[other Nāgas]

antarīkṣe ca ye nāgā ye nāgāḥ svargasaṃsthitāḥ|

girikandaradurgeșu ye nāgā bhuvi saṃsthitāḥ|| 182||

pātāle ye sthitā nāgāḥ sarve 'py atra samāhitāḥ|

rudrapādārcane saktāḥ kurvantu mama śāntikam|| 183||

[Nāginīs, Nāgakanyās, Nāgakumārikās]

nāginyo nāgakanyāś ca tathā nāgakumārikāḥ|

śivabhaktāḥ sumanasah śāntim kurvantu me sadā|| 184||

182-183 Cf. BhavP 1.179.41-42 : antarikṣe ca ye nāgā ye nāgāḥ svargasaṃsthitāḥ| girikandaradurgeșu ye nāgā bhuvi saṃsthitāḥ|| pātāle ye sthitā nāgāḥ sarve yatra samāhitāḥ| sūryapādārcanāsaktāḥ śāntiṃ kurvantu te sadā|| 184 Cf. BhavP 1.179.43: nāginyo nāgakanyāś ca tathā nāgakumārakāḥ| sūryabhaktāḥ sumanasạ̣ śāntiṃ kurvantu te sadā||

181a kuliko ] $\mathrm{N}_{77}^{K o} \mathrm{~N}_{45}^{C} \mathrm{~N}_{82}^{K} \mathrm{~N}_{58}^{K} \mathrm{~B}_{99}^{C} \dot{S}_{67}^{S}$, kulike $\mathrm{E}^{N}$, guliko $\mathbf{P}^{\Sigma}$ • nāgarājendro ] $\mathrm{N}_{77}^{K o} \mathrm{~N}_{58}^{K} \hat{S}_{67}^{S} \mathrm{P}_{32}^{T}$ nāma rājendro $\mathrm{N}_{45}^{C}$, nāgarājeśo $\mathrm{N}_{82}^{K} \mathrm{E}^{N} \mathrm{~B}_{99}^{C}$, nāma nāgendraḥ $\mathrm{P}_{72}^{T} \quad \mathbf{1 8 1 b}$ nityam haraparāyaṇaḥ ] $\mathrm{N}_{45}^{C} \mathrm{~N}_{82}^{K} \mathrm{~N}_{58}^{K} \mathrm{E}^{N} \mathrm{~B}_{99}^{C} \hat{S}_{67}^{S} \mathrm{P}^{\Sigma}$, nityaharapāyaṇa $\mathrm{N}_{77}^{K o} \quad$ 181c apahṛtya ] $\mathrm{N}_{77}^{K o} \mathrm{~N}_{82}^{K} \mathrm{~N}_{58}^{K} \mathrm{E}^{N} \mathrm{~B}_{99}^{C} \dot{S}_{67}^{S} \mathbf{P}^{\Sigma}$, ayam kṛtya $\mathrm{N}_{45}^{C}$ • vișam ] $\mathrm{N}_{45}^{C} \mathrm{~N}_{82}^{K} \mathrm{~N}_{58}^{K} \mathrm{E}^{N} \mathrm{~B}_{99}^{C} \dot{S}_{67}^{S} \mathbf{P}^{\Sigma}$, vișa $\mathrm{N}_{77}^{K o}$ (unmetr.) 182a antarìkșe ] $\mathrm{N}_{45}^{C} \mathrm{~N}_{82}^{K} \mathrm{E}^{N} \mathrm{~B}_{99}^{C} \dot{S}_{67}^{S} \mathrm{P}_{32}^{T}$, antarikṣe $\mathrm{N}_{77}^{K o} \mathrm{~N}_{58}^{K} \mathrm{P}_{72}^{T}$ 182b nāgāḥ svargasaṃsthitāḥ ] $\mathrm{N}_{45}^{C} \mathrm{~N}_{82}^{K} \mathrm{~N}_{58}^{K} \mathrm{E}^{N} \mathrm{~B}_{99}^{C} \hat{\mathrm{S}}_{67}^{S} \mathbf{P}^{\Sigma}$, nāgā svarggasamsthitā $\mathrm{N}_{77}^{K o} \quad$ 182c c ${ }^{\circ}$ kandara $\left.^{\circ}\right] \mathrm{N}_{77}^{K o} \mathrm{~N}_{45}^{C} \mathrm{~N}_{82}^{K} \mathrm{E}^{N} \mathrm{~B}_{99}^{C} \hat{S}_{67}^{S} \mathbf{P}^{\Sigma}$, ${ }^{\circ}$ gandara ${ }^{\circ} \mathrm{N}_{58}^{K}$ 182d bhuvi ] $\mathrm{N}_{77}^{K o} \mathrm{~N}_{45}^{C} \mathrm{~N}_{82}^{K} \mathrm{~N}_{58}^{K} \mathrm{E}^{N} \mathrm{~B}_{99}^{C} \hat{S}_{67}^{S}$, bhūmi ${ }^{\circ} \quad \mathbf{P}^{\Sigma} \bullet$ saṃsthitāh ] $\mathrm{N}_{45}^{C} \mathrm{~N}_{82}^{K} \mathrm{~N}_{58}^{K} \mathrm{E}^{N} \mathrm{~B}_{99}^{C} \hat{S}_{67}^{S} \mathbf{P}^{\Sigma}$, sasthitā $\mathrm{N}_{77}^{K o} \quad$ 183a ye sthitā ] $\mathrm{N}_{45}^{C} \mathrm{~N}_{82}^{K} \mathrm{~N}_{58}^{K} \mathrm{E}^{N} \mathrm{~B}_{99}^{C} \mathbf{P}^{\Sigma}$, saṃsthitā $\mathrm{N}_{77}^{K o} \hat{S}_{67}^{S} \bullet$ nāgāh ] $\mathrm{N}_{82}^{K} \mathrm{~N}_{58}^{K} \mathrm{E}^{N} \mathrm{~B}_{99}^{C} \mathbf{P}^{\Sigma}$, nāgā $\mathrm{N}_{77}^{K o} \mathrm{~N}_{45}^{C} \quad \mathbf{1 8 3} \mathbf{b}$ atra ] $\mathrm{N}_{77}^{K o} \mathrm{~N}_{82}^{K} \mathrm{~N}_{58}^{K} \mathrm{E}^{N} \mathrm{~B}_{99}^{C} \dot{S}_{67}^{S} \mathbf{P}^{\Sigma}$, atha $\mathrm{N}_{45}^{C}$ • samāhitāḥ ] $\mathrm{N}_{45}^{C} \mathrm{~N}_{82}^{K} \mathrm{~N}_{58}^{K} \mathrm{E}^{N} \mathrm{~B}_{99}^{C} S_{67}^{S} \mathbf{P}^{\Sigma}$, samāhitā $\mathrm{N}_{77}^{K o} \quad{ }^{183}$ c pādārcane ] $\mathrm{N}_{77}^{K o} \mathrm{~N}_{45}^{C} \mathrm{~N}_{82}^{K} \mathrm{E}^{N} \mathrm{~B}_{99}^{C} \mathbf{P}^{\Sigma}$, ${ }^{\circ}$ pādārcanāo $\mathrm{N}_{58}^{K} \hat{S}_{67}^{S}$ • saktāh ] $\mathrm{N}_{82}^{K} \mathrm{~B}_{99}^{C} \dot{S}_{67}^{S} \mathrm{P}_{72}^{T}$, saktā $\mathrm{N}_{77}^{K o}$, śaktāḥ $\mathrm{N}_{45}^{C} \mathrm{E}^{N}$, yuktāḥ $\mathrm{N}_{5^{8}}^{K} \mathrm{P}_{3^{2}}^{T} \quad \mathbf{1 8 3 d}$ kurvantu mama śāntikam ] $\mathrm{N}_{82}^{K} \mathrm{~N}_{58}^{K} \mathrm{E}^{N} \mathrm{~B}_{99}^{C}$, śāntiṃ kurvantu me sadā $\mathrm{N}_{77}^{K o} \hat{\mathrm{S}}_{67}^{S} \mathbf{P}^{\Sigma}$, śānti kurvantu me sadā $\mathrm{N}_{45}^{C} \quad \mathbf{1 8 4 b}{ }^{\circ}$ kumārikāḥ ] $\mathrm{N}_{45}^{C} \mathrm{~N}_{82}^{K} \mathrm{~N}_{58}^{K} \mathrm{E}^{N} \mathrm{P}_{32}^{T}$, okumārakāḥ $\mathrm{N}_{77}^{K o} \mathrm{~B}_{99}^{C} \mathrm{~S}_{67}^{S} \mathrm{P}_{72}^{T} \quad$ 184c obhaktāḥ sumanasah ] $\mathrm{N}_{82}^{K} \mathrm{~N}_{5^{8}}^{K} \mathrm{E}^{N} \mathrm{~B}_{99}^{C} \dot{S}_{67}^{S} \mathbf{P}^{\Sigma}$, obhaktā sumanasah $\mathrm{N}_{77}^{K o}$, obhaktāśumanasāḥ $\mathrm{N}_{45}^{C} \quad \mathbf{1 8 4 d}$ śāntim ] $\mathrm{N}_{77}^{K o} \mathrm{~N}_{82}^{K} \mathrm{~N}_{58}^{K} \mathrm{E}^{N} \mathrm{~B}_{99}^{C} \hat{S}_{67}^{S} \mathbf{P}^{\Sigma}$, śānti $\mathrm{N}_{45}^{C}$ 


\section{[Śrutiphala of Nāga section]}

ya idaṃ nāgasaṃsthānaṃ kīrtayec chṛnuyād api|

na tasya sarpā hiṃsanti na viṣaṃ kramate sadā|| 185||

[Rivers : 1]

gan̉gā puṇyā mahādevī yamunā narmadā nadī|

gomatī cāpi kāverī varuṇā devikā tathā|| 186||

sarvabhūtapatiṃ devaṃ parameśaṃ maheśvaram| pūjayanti sadā nadyaḥ śivasadbhāvabhāvitāḥ|| 187||

185 Cf. BhavP 1.179.44 :ya idaṃ nāmasaṃsthānaṃ kīrtayec chṛnuyāt tathā| na taṃ sarpā vihimsati na viṣaṃ kramate sadā|| 186-188 Cf. BhavP 1.18o.1-2 : gañgā puṇyā mahādevī yamunā narmadā nadī| gautamī cāpi kāverī varuṇā devikā tathâ|| sarvagrahapatị̣ devaṃ lokeśaṃ lokanāyakam| pūjayanti sadā nadyaḥ sūryasadbhāvabhāvitāḥ| śāntiṃ kurvantu te nityaṃ sūryadhyānaikamānasāḥ̂||

185d After this $\mathrm{P}_{3^{2}}^{T}$ adds four pādas : cintitam sidhyate nityam tathā pāpaparikṣayaḥ| siddhim āśu prayacchanti sarvavighnavivarjitāḥ| $\mid \mathrm{P}_{7^{2}}^{T}$ adds : cintitạ̣ labhate nityaṃ tathā pāpaparikṣayam| siddhim āśu prayacchanti sarvavighnavivarjitām| 186d After this $\mathrm{P}_{3^{2}}^{T}$ adds two pādas : kauśikā ca sarasvatī tāmraparṇī ca sarayūḥ|

185 idam ] $\mathrm{N}_{77}^{K o} \mathrm{~N}_{45}^{C} \mathrm{~N}_{82}^{K} \mathrm{E}^{N} \mathrm{~B}_{99}^{c} \mathrm{P}^{\Sigma}$, iman $\mathrm{N}_{58}^{K p c}$, imān $\mathrm{N}_{58}^{K a c}$, imaṃ $\hat{S}_{67}^{S}$ • osamsthānam ] $\mathrm{N}_{45}^{C} \mathrm{~N}_{82}^{K} \mathrm{~N}_{58}^{K} \mathrm{E}^{N} \mathrm{~B}_{99}^{C} \dot{S}_{67}^{S} \mathbf{P}^{\Sigma}$, ${ }^{\circ}$ saṃsthāna $\mathrm{N}_{77}^{K o} \quad \mathbf{1 8 5}_{5} \mathbf{b}$ kīrtayec chṛṇuyād api ] $\mathrm{N}_{82}^{K} \mathrm{~N}_{58}^{K} \mathrm{E}^{N} \mathrm{~B}_{99}^{C} \hat{S}_{67}^{S} \mathrm{P}_{32}^{T}$, kīrttaye śṛ̣uyāpi vā $\mathrm{N}_{77}^{K o}$, kīrttiye śṛ̣uyād api $\mathrm{N}_{45}^{C}$, kīrtayed vā śṛ̣noti vā $\mathrm{P}_{72}^{T} \quad{ }_{185} \mathrm{c}$ sarpā ] $\mathrm{N}_{77}^{K o} \mathrm{~N}_{82}^{K} \mathrm{~N}_{58}^{K} \mathrm{E}^{N} S_{67}^{S}$, rppā $\mathrm{N}_{45}^{C}$ (unmetr.), nāgā $\mathrm{P}_{32}^{T}$ - himssanti ] $\mathrm{N}_{77}^{K o} \mathrm{~N}_{58}^{K} \mathrm{E}^{N} \mathrm{~B}_{99}^{C} S_{67}^{S} \mathbf{P}^{\Sigma}$, hi santi $\mathrm{N}_{45}^{C} \mathrm{~N}_{82}^{K} \quad$ 185d na viṣaṃ kramate ] $\mathrm{N}_{82}^{K} \mathrm{~N}_{58}^{K} \mathrm{E}^{N} \mathrm{~B}_{99}^{C}$, vișan na kramate $\mathrm{N}_{77}^{K o}$, vișam na kramate $\mathrm{N}_{45}^{C}$, vișam nākramate $S_{67}^{S} \mathbf{P}^{\Sigma}$ • sadā ] $\mathrm{N}_{77}^{K o} \mathrm{~N}_{45}^{C} \mathrm{~N}_{82}^{K} \mathrm{~N}_{58}^{K} E^{N} \mathrm{~B}_{99}^{C} \mathrm{P}_{32}^{T}$, kvacit $S_{67}^{S}$, ca tam $\mathrm{P}_{72}^{T}$ 186a mahādevī ] $\mathrm{N}_{77}^{K o} \mathrm{~N}_{82}^{K} \mathrm{~N}_{58}^{K} \mathrm{E}^{N} \mathrm{~B}_{99}^{C} S_{67}^{S} \mathbf{P}^{\Sigma}$, madevī $\mathrm{N}_{45}^{C}$ (unmetr.) 186c cāpi kāverī ] $\mathbf{P}^{\Sigma}$, bhāśikāvīrī $\mathrm{N}_{77}^{K o}$, dāvikāverī $\mathrm{N}_{82}^{K} \mathrm{~B}_{99}^{C}$, nāma kāverī $\mathrm{N}_{45}^{C}$, devakāvairī $\mathrm{N}_{58}^{K a c}$, devikāvairī $\mathrm{N}_{58}^{K p c}$, tāpikāverī $\mathrm{E}^{N}$, cāmbikāndevī $S_{67}^{S} \quad \mathbf{1 8 6 d}$ varuṇā ] $\mathrm{N}_{77}^{K o} \mathrm{~N}_{45}^{C} \mathrm{~N}_{82}^{K} \mathrm{~N}_{58}^{K} \mathrm{E}^{N} \hat{S}_{67}^{S} \mathbf{P}^{\Sigma}$, varaṇā $\mathrm{B}_{99}^{C} \bullet$ devikā ] $\mathrm{N}_{82}^{K} \mathrm{E}^{N} \mathrm{~B}_{99}^{C} S_{67}^{S} \mathbf{P}^{\Sigma}$, devikās $\mathrm{N}_{77}^{K o} \mathrm{~N}_{58}^{K}$, devikas $\mathrm{N}_{45}^{C} \quad \mathbf{1 8 7 a}$ obhūta $\left.{ }^{\circ}\right] \mathrm{N}_{77}^{K o} \mathrm{~N}_{45}^{C} \mathrm{~N}_{82}^{K} \mathrm{E}^{N} \mathrm{~B}_{99}^{C} \mathbf{P}^{\Sigma}$, obhūtaḥ $\mathrm{N}_{58}^{K}$, ${ }^{\circ}$ deva ${ }^{\circ} \hat{S}_{67}^{S} \quad \mathbf{1 8 7}$ b parameśam ] $\mathrm{N}_{82}^{K} \mathrm{~N}_{58}^{K} E^{N} \mathrm{~B}_{99}^{C} \hat{S}_{67}^{S} \mathrm{P}^{\Sigma}$, parameśa ${ }^{\circ} \mathrm{N}_{77}^{K o}$, parameșam $\mathrm{N}_{45}^{C} \quad$ 187c pūjayanti ] $\mathrm{N}_{77}^{K o} \mathrm{~N}_{45}^{C} \mathrm{~N}_{82}^{K} \mathrm{~N}_{58}^{K} \mathrm{E}^{N} \mathrm{~B}_{99}^{C}$, pūjayantyo $\mathbf{P}^{\Sigma} \bullet$ sadā nadyaḥ ] $\mathrm{N}_{82}^{K} \mathrm{~B}_{99}^{C}$, mahānadya $\mathrm{N}_{77}^{K o}$, sadā nadyāḥ $\mathrm{N}_{45}^{C}$, mahānadyaḥ $\mathrm{N}_{58}^{K}$, mahānadyaḥ $\mathrm{E}^{N} \hat{S}_{67}^{S} \mathbf{P}^{\Sigma}$ 187d obhāvitāh ] $\mathrm{N}_{45}^{C} \mathrm{~K}^{\Sigma} \mathrm{B}_{99}^{C} \dot{S}_{67}^{S} \mathrm{P}_{72}^{T}$, obhāvitā $\mathrm{N}_{77}^{K o} \mathrm{E}^{N}$ 
śāntiṃ kurvantu me nityaṃ tathā pāpaparikṣayam|

siddhim āśu prayacchantu sarvavighnavivarjitāḥ|| 188||

[Rivers : 2]

candrabhāgā mahāpuṇā nadī godāvarī śubhā|

sarayū gaṇụakī śreșthā kauśikī ca sarasvatī|| 189||

etā nadyo mahābhāgāḥ śivapādārcane ratāḥ|

śāntiṃ kurvantu me prītāḥ śivadhyānaikamānasāḥ|| 19o||

[Rivers : 3]

nairañjanā nāma nadī śoṇaś cāpi mahānadaḥ|

mandākinī ca paramā tathā saṃnihitā śubhā|| 191||

191-192 Cf. BhavP 1.180.3-4 : nirañjanā nāma nadī śoṇaś cāpi mahānadaḥ| maṃdākinī ca paramā tathā sannihitā śubhā|| etāś cānyāś ca bahavo bhuvi divyantarikṣake| sūryārcanaratā nadyạ̣ kurvantu tava śāntikam||

191d After this $\mathrm{N}_{58}^{K}$ adds four pādas (hypermetr.): sindhu śatadrū vikhyātā lohitāś ca mahānadaḥ| mahāhradasutas tathā vaiṇyābhavā vaitaraṇi nadī| $\bullet S_{67}^{S}$ adds (hypermetr.) : śatanadrū vipāṭa sindhur lauhitaś ca mahānadaḥ| airāvatī ca veṇvādyā puṇyā vaitarạ̣ī nadī|

188a śāntiṃ ] $\Sigma$, śānti $\mathrm{N}_{45}^{C} \bullet$ me nityam ] $\Sigma$, metyam $\mathrm{N}_{5^{8}}^{K}$ (unmetr.) $\quad \mathbf{1 8 s b}^{\circ}{ }^{\circ}$ kṣayam ] $\Sigma$, okṣayaḥ $\mathrm{E}^{N} \quad{ }_{188 c}$ siddhim āśu ] $\Sigma$, siddhiñcāśuh $\mathrm{N}_{77}^{K o}$, siddhiñcāsu $\mathrm{N}_{45}^{C} \bullet$ prayacchantu ] $\Sigma$, prayacchanti $\mathrm{N}_{77}^{K o} \quad \mathbf{1 8 8 d}{ }^{\circ}$ vivarjitāh ] $\mathrm{N}_{45}^{C} \mathrm{~N}_{82}^{K} \mathrm{~N}_{58}^{K a c} \mathrm{E}^{N} \mathrm{~B}_{99}^{C} \dot{S}_{67}^{S},{ }^{\circ}$ vivarjitā $\mathrm{N}_{77}^{K o}$, ${ }^{\circ}$ vivarjitāṃ $\mathrm{N}_{12}^{K} \mathrm{~N}_{58}^{K \text { pc }}$, ${ }^{\circ}$ vināśanam $\mathbf{P}^{\Sigma} \quad \mathbf{1 8 9 b}$ godāvarī ] $\sum$, godārī $\mathrm{N}_{58}^{K}$ (unmetr.) • śubhā ] $\Sigma$, śubhāḥ $\mathrm{N}_{45}^{C} \quad{ }_{189 c}$ sarayū gaṇḍaki ] $\mathrm{N}_{77}^{K o} \mathrm{~N}_{12}^{K} \mathrm{E}^{N} \mathrm{~B}_{99}^{C} \mathrm{P}_{72}^{T}$, śarayū gaṇ̣akī $\mathrm{N}_{45}^{C} \mathrm{~N}_{58}^{K}$, sarayū gaṇ̣ikā $\mathrm{N}_{82}^{K}$, sarayū naṇụaki $S_{67}^{S}$, sarayūrgaṇ̣akī $\mathrm{P}_{32}^{T} \quad$ 189d kauśikī ] $\Sigma$, kauśakī $\mathrm{N}_{58}^{K} \quad$ 19oa nadyo ] $\Sigma$, nadyā $\mathrm{N}_{77}^{K o} \mathrm{~N}_{45}^{C} \bullet$ •bhāgāḥ ] $\mathrm{K}^{\Sigma} \mathrm{E}^{N} \mathrm{~B}_{99}^{C} \hat{S}_{67}^{S}$, ${ }^{\circ}$ bhāgā $\mathrm{N}_{77}^{K o} \mathrm{~N}_{45}^{C}$, ${ }^{\circ}$ punyyāḥ $\mathbf{P}^{\Sigma} \quad$ 19ob ${ }^{\circ}$ pādārcane ratāḥ ] $\mathrm{N}_{82}^{K} \mathrm{~N}_{58}^{K} \mathrm{E}^{N} \mathrm{~B}_{99}^{C} \mathbf{P}^{\Sigma}$, ${ }^{\circ}$ pūjāparāyaṇā $\mathrm{N}_{77}^{K o}$, ${ }^{\circ}$ pūjārcane ratā $\mathrm{N}_{45}^{C}$, ${ }^{\circ}$ pūjārrcane ratāḥ $\mathrm{N}_{12}^{K} \dot{S}_{67}^{S} \quad$ 19oc śāntiṃ ] $\mathbf{K}^{\Sigma} \mathrm{E}^{N} \mathrm{~B}_{99}^{C} \mathbf{P}^{\Sigma}$, śānti $\mathrm{N}_{77}^{K o} \mathrm{~N}_{45}^{C} \bullet$ me prītāh ] $\mathbf{N}^{\Sigma} \mathrm{B}_{99}^{C} \dot{S}_{67}^{S}$, geprītāḥ $\mathrm{E}^{N}$, me nityam $\mathrm{P}^{\Sigma} \quad$ 19od ${ }^{\circ}$ dhyānaika ${ }^{\circ}$ ] $\mathrm{N}_{77}^{K o} \mathrm{~N}_{82}^{K \mathrm{pc}} \mathrm{N}_{12}^{K} \mathrm{~N}_{58}^{K} \mathrm{E}^{N} \mathrm{~B}_{99}^{C} \dot{S}_{67}^{S} \mathbf{P}^{\Sigma}$, odhyāneka ${ }^{\circ} \mathrm{N}_{45}^{C}$, ${ }^{\circ}$ dhyānai ${ }^{\circ} \mathrm{N}_{82}^{K a c}$ (unmetr.) 191a nairañjanā ] $\mathbf{K}^{\Sigma} \mathrm{E}^{N} S_{67}^{S} \mathbf{P}^{\Sigma}$, nairājanā $\mathrm{N}_{77}^{K o}$, nairañjano $\mathrm{N}_{45}^{C}$, nirañjanā $\mathrm{B}_{99}^{C} \quad$ 191b śoṇaś cāpi ] $\Sigma$, śroṇasyāpi $\mathrm{N}_{77}^{K o}$, lonaś cāpi $\mathrm{N}_{12}^{K} \bullet$ mahānadaḥ ] $\Sigma$, mahānadī $\mathrm{N}_{77}^{K o} \mathrm{~N}_{58}^{K} \quad$ 191c mandākin̄i ] $\Sigma$, mandāginī $\mathrm{N}_{45}^{C} \bullet$ paramā ] $\Sigma$, paramāḥ $\mathrm{N}_{45}^{C}$, parama $\mathrm{B}_{99}^{C}$ 191d tathā ] $\Sigma$, tena $\hat{S}_{67}^{S} \bullet$ saṃnihitā śubhā ] $\mathrm{N}_{82}^{K} \mathrm{~N}_{12}^{K} \mathrm{E}^{N} \mathrm{~B}_{99}^{C} \hat{S}_{67}^{S} \mathrm{P}_{32}^{T}$, sahinitāśu $\mathrm{N}_{77}^{K o}$ (unmetr.), saṃnihatā śubhāḥ $\mathrm{N}_{45}^{C}$, sannihitā nadī $\mathrm{N}_{58}^{K}$, பnihitā śubhā $\mathrm{P}_{72}^{T}$ 
etāś cānyāś ca bahavo bhuvi divyāntarikṣagāḥ| rudrārcanaparā nadyaḥ kurvantu mama śāntikam|| 192||

[Yakșas]

[1. Mahāvaiśravaṇa]

mahāvaiśravaṇo devo yakșarājo maharddhikaḥ| yakṣakoțiparīvāro yakṣasaṃghena saṃyutạ̣|| 193||

193-194 Cf. BhavP 1.18o.5-7ab: mahāvaiśravaṇo devo yakṣarājo maharṣikaḥ| yakṣakoṭiparīvāro yakṣasaṃkhyeyasaṃyutaḥ|| mahāvibhavasaṃpannaḥ sūryapādārcane rataḥ| sūryadhyānaikaparamạ̣ sūryabhāvena bhāvitaḥ|| śāntiṃ karotu te prītaḥ padmapatrāyatekṣaṇaḥ|

192d After this $\mathrm{N}_{58}^{K}$ adds eight pādas : saptadvīpayutā dhātrī siddhagandharvapūjitā| śāntiṃ kurvvantu me nityaṃ sarvasiddhipradāyukā| guhyāni tīrthāṇi yāni āsamudrāt sarāṃsi ca| kurvvantu śāntikaṃ jagaṃ śrīkaṇțhādhișțhitāni tu| • ŚS ${ }_{67}^{S}$ adds four pādas : guhyāni yāni tīrthāṇi āsamudrasarāṃsi ca| kurvantu śāntikaṃ tāni śrīkaṇṭhāāhiṣṭhitāni ca| $\bullet \mathbf{P}^{\Sigma}$ adds : guhyāni yāni tīrthāni āsamudrāt sarāṃsi ca| kurvantu śāntikạ̣ tāni śrīkaṇṭhādhiṣṭhitāni tu|

192a etāś cānyāś ] $\mathrm{K}^{\Sigma} \mathrm{E}^{N} \mathrm{~B}_{99}^{C} \mathbf{P}^{\Sigma}$, etānyāś $\mathrm{N}_{77}^{K o}$ (unmetr.), etā cānyāś $\mathrm{N}_{45}^{C}$, etāścanyāś $\hat{\mathrm{S}}_{67}^{S} \bullet$ bahavo ] $\mathrm{N}^{\Sigma} \mathrm{E}^{N} \mathrm{~B}_{99}^{C} \hat{S}_{67}^{S}$, bahyopa $\mathrm{P}_{32}^{T}$, yā nadyah $\mathrm{P}_{72}^{T} \quad$ 192b bhuvi divyāntarikṣagāḥ ] $\mathrm{N}_{45}^{C} \mathrm{~N}_{82}^{K} \mathrm{~B}_{99}^{C}$, bhuvi divyāntarikṣagā $\mathrm{N}_{77}^{K o}$, bhuvi divyāntarīkṣagāḥ $\mathrm{N}_{12}^{K} \mathrm{~N}_{5^{8}}^{K}$, divi bhuvi divyāntarīkṣugāḥ $\mathrm{E}^{N}$, divi bhuvyantarigāḥ $\hat{S}_{67}^{S}$ (unmetr.), bhūmidivyāntarikṣagāḥ $\mathrm{P}_{3^{2}}^{T}$, bhuvi divyantarikṣagāḥ $\mathrm{P}_{72}^{T} \quad 192 \mathrm{c}$ nadyah ] $\Sigma$, nadyā $\mathrm{N}_{45}^{C} \quad$ 192d kurvantu mama śāntikam ] $\Sigma$, śāntiṃ kurvantu me sadā $\mathrm{P}_{72}^{T} \quad$ 193a mahāo ] $\Sigma$, mahān $\mathrm{P}_{32}^{T} \quad$ 193b $^{\circ}$ rājo ] $\Sigma$, ${ }^{\circ}$ rājā $\mathrm{N}_{77}^{K o} \mathrm{~N}_{45}^{C} \bullet$ maharddhikaḥ ] $\Sigma$, maharddhikā $\mathrm{N}_{77}^{K o} \quad$ 193c yakșakoți ${ }^{\circ}$ ] $\Sigma$, yakșakoțīo $\mathrm{N}_{45}^{C}$, yakșațīo $\mathrm{N}_{58}^{K} \quad$ 193d yakșasaṃghena ] $\mathrm{N}_{12}^{K} \mathrm{~N}_{58}^{K} E^{N}$, yakșasaṃkhyena ${ }^{\circ} \mathrm{N}_{77}^{K o}$, yakșasaṃkșaya ${ }^{\circ} \mathrm{N}_{45}^{C}$, yakșasaṃhena $\mathrm{N}_{82}^{K} \mathrm{~B}_{99}^{C}$, yakșasaṃ(gghe)na $\dot{S}_{67}^{S}$, yakṣāsaṃkhyeya ${ }^{\circ} \mathbf{P}^{\Sigma} \bullet$ saṃyutạ ] $\mathrm{N}_{77}^{K o} \mathrm{~N}_{82}^{K} \mathrm{~N}_{12}^{K} \mathrm{E}^{N} \mathbf{P}^{\Sigma}$, samyutāḥ $\mathrm{N}_{45}^{C}$, saṃvṛtah $\hat{S}_{67}^{S}$ 
mahāvibhavasampanno harapādārcane rataḥ| haradhyānaikaparamo harapādanatottamaḥ| śāntiṃ karotu me prītaḥ padmapatrāyatekșaṇaḥ|| 194||

[2. Maṇibhadra]

maṇibhadro mahāyakṣo maṇiratnavibhūṣitaḥ| manohareṇa hāreṇa kaṇṭhalagnena rājate|| 195||

195-196 Cf. BhavP 1.18o.7cd-8 : māṇibhadro mahāyakṣo maṇiratnavibhūṣitaḥ| manohareṇa hāreṇa kaṇțhalagnena rājate|| yakșiṇiyakșakanyābhị parivāritavigrahaḥ| sūryārcanasamāsaktạ̣ karotu tava śāntikam||

194cd Omitted in $\mathrm{P}_{3^{2}}^{T} \quad$ 194d After this $\mathrm{N}_{5^{8}}^{K}$ adds two pādas : yakṣinīyakṣakanyābhị̣ sevyate kāmyamokṣayoḥ|

194a ${ }^{\circ}$ sampanno ] $\Sigma$, osampanna $\mathrm{N}_{45}^{C} \quad 194 \mathrm{~b}{ }^{\circ}$ pādārcane ] $\Sigma$, ${ }^{\circ}$ padārcane $\mathrm{N}_{82}^{\text {Kac }} \bullet$ rataḥ ] $\mathrm{N}_{77}^{K o} \mathbf{K}^{\Sigma} \mathrm{B}_{99}^{C} \hat{S}_{67}^{S} \mathbf{P}^{\Sigma}$, ratāḥ $\mathrm{N}_{45}^{C}$, ratoḥ $\mathrm{E}^{N} \quad{ }_{194 c}$ haradhyānaika $\left.{ }^{\circ}\right] \mathrm{N}_{77}^{K o} \mathbf{K}^{\Sigma} \mathrm{E}^{N} \mathrm{~B}_{99}^{C} \hat{S}_{67}^{S}$, haradhyāneka ${ }^{\circ} \mathrm{N}_{45}^{C}$, śivadhyānaika ${ }^{\circ} \mathrm{P}_{72}^{T} \quad$ 194d $^{\circ}$ pādanatottamah ] $\mathrm{N}_{77}^{K o} \mathrm{~K}^{\Sigma} \mathrm{E}^{N} \mathrm{~B}_{99}^{C} \hat{S}_{67}^{S}$, ${ }^{\circ}$ pādāratottamaḥ $\mathrm{N}_{45}^{C} \mathrm{P}_{72}^{T} \quad$ 194e śāntim karotu ] $\Sigma$, śānti kurvantu $\mathrm{N}_{45}^{C}$, śānti kurvvantu $\mathrm{N}_{58}^{K} \bullet$ me prìtaḥ ] $\mathrm{N}_{82}^{K} \mathrm{~N}_{12}^{K} \mathrm{E}^{N} \mathrm{~B}_{99}^{C} \mathbf{P}^{\Sigma}$, me prīta $\mathrm{N}_{77}^{K o}$, me prītāḥ $\mathrm{N}_{45}^{C} \mathrm{~N}_{58}^{K}$, me prītyā $S_{67}^{S}$

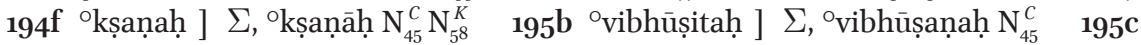
manohareṇa ] $\Sigma$, manohāreṇa $\mathrm{N}_{82}^{K a c}$, manoraheṇa $\mathrm{E}^{N} \quad$ 195d kaṇṭhalagnena ] $\Sigma$, kaṇțhalagno na $\mathrm{N}_{77}^{K o}$, kaṇțhe lagnena $\mathrm{B}_{99}^{C} \bullet$ rājate ] $\Sigma$, rājatāṃ $\mathrm{N}_{45}^{C}$ 
yakṣin̄iyakșakanyābhiḥ parivāritavigrahaḥ| rudrārcanaparodyuktah karotu mama saāntikam|| 196||

[3. Suviroma]

suviromo 'tha yakșendro maṇikuṇ̣alabhūṣitaḥ| lalāțe hemapatțena śobhanena virājate|| 197||

bahuyakṣasamākīrṇo yakșair namitavigrahaḥ| śivapūjāparo bhaktah karotu mama śāntikam|| 198||

197-198 Cf. BhavP 1.180.9-10 : suciro nāma yakṣendro maṇikuṇụalabhūṣitah| lalāțe hemapațalaprabaddhena virājate|| bahuyakṣasamākīrṇo yakṣair namitavigrahaḥ| sūryapūjāparo yuktaḥ karotu tava śāntikam||

197a-d Omitted in $\hat{S}_{67}^{S}$ and $\mathrm{P}_{72}^{T}$

196a yakṣiṇīo ] $\Sigma$, yakșaṇī̄ $\mathrm{N}_{45}^{C} \bullet{ }^{\circ}$ kanyābhiḥ ] $\Sigma$, okanyāś ca $\mathrm{N}_{77}^{K o} \quad$ 196b parivāritavigrahạ̣ ] $\mathrm{K}^{\Sigma} \mathrm{E}^{N} \mathrm{~B}_{99}^{C} \dot{S}_{67}^{S} \mathrm{P}_{3^{2}}^{T}$, parivāraparigrahạ $\mathrm{N}_{77}^{K o}$, parivārantu vigrahāḥ $\mathrm{N}_{45}^{C}$, parivāritavigrahā $\mathrm{P}_{72}^{T} \quad 196 \mathrm{c}$ rudrārcanaparodyuktạ̣ ] $\mathrm{N}_{82}^{K} \mathrm{~N}_{58}^{K}$, rudrārcanaparodyukta $\mathrm{N}_{77}^{K o}$, rudrārcanaparo bhaktāḥ $\mathrm{N}_{45}^{C}$, rudrārcanaparo yakșah $\mathrm{B}_{99}^{C}$, rudrārcanaparo bhaktah $\mathrm{N}_{12}^{K} \mathrm{P}_{32}^{T}$, rudrārcanaparo yuktaḥ $\mathrm{E}^{N}$, śivapūjāparodyuktaḥ $S_{67}^{S}$, śivapūjārcanodyuktah $\mathrm{P}_{72}^{T} \quad$ 197a suviromo 'tha ] $\mathrm{N}_{82}^{K} \mathrm{E}^{N} \mathrm{~B}_{99}^{C}$, suvīro nāma $\mathrm{N}_{77}^{K o} \mathrm{~N}_{45}^{C} \mathrm{~N}_{12}^{K} \mathrm{P}_{32}^{T}$, suviro nāma $\mathrm{N}_{58}^{K} \quad 197 \mathbf{b}$ obhūṣitah ] $\mathbf{N}^{\Sigma} \mathrm{E}^{N} \mathrm{~B}_{99}^{C}$, obhūṣaṇāḥ $\mathrm{N}_{58}^{K}$, obhūṣaṇaḥ $\mathrm{P}_{3^{2}}^{T} \quad{ }_{197 c}{ }^{\circ}$ pațțena ] $\mathrm{N}_{77}^{K o} \mathrm{~K}^{\Sigma} \mathrm{E}^{N} \mathrm{~B}_{99}^{C} \mathrm{P}_{32}^{T}$, ${ }^{\circ}$ pațena $\mathrm{N}_{45}^{C} \quad{ }_{197 d}$ śobhanena virājate ] $\mathrm{N}_{82}^{K} \mathrm{~N}_{12}^{K} \mathrm{E}^{N} \mathrm{~B}_{99}^{C}$, kāñcanena virājate $\mathrm{N}_{77}^{K o}$, kāñcanena virājatāṃ $\mathrm{N}_{45}^{C}$, śobhanena virājite $\mathrm{N}_{58}^{K}$, śobhanena virājitah $\mathrm{P}_{3^{2}}^{T}$ 198a ${ }^{\circ}$ yakșa $\left.{ }^{\circ}\right] \mathrm{N}_{77}^{K o} \mathrm{~K}^{\Sigma} \mathrm{E}^{N} \mathrm{~B}_{99}^{C} \mathrm{P}_{32}^{T}$, ${ }^{\circ}$ yakșāā $\mathrm{N}_{45}^{C} \bullet{ }^{\circ}$ samākīrṇo ] $\mathrm{N}_{45}^{C} \mathrm{~N}_{82}^{K} \mathrm{~N}_{12}^{K} \mathrm{E}^{N} \mathrm{~B}_{99}^{C} \mathrm{P}_{32}^{T}$, ${ }^{\circ}$ samākīrṇṇa $\mathrm{N}_{77}^{K o}$, ${ }^{\circ}$ samākīrṇṇā $\mathrm{N}_{58}^{K} \quad 198$ b yakșair namita $\left.{ }^{\circ}\right] \mathrm{K}^{\Sigma} \mathrm{E}^{N} \mathrm{~B}_{99}^{C} \mathrm{P}_{32}^{T}$, yakșai nāmita ${ }^{\circ}$ $\mathrm{N}_{77}^{K o}$, yakșai ṇamita ${ }^{\circ} \mathrm{N}_{45}^{C} \quad 198 \mathrm{c}$ śivapūjāparo bhaktạ ] $\mathrm{N}_{82}^{K} \mathrm{E}^{N} \mathrm{~B}_{99}^{C}$, śivapūjāparodyukta $\mathrm{N}_{77}^{K o}$, śivapūjājapodyuktah $\mathrm{N}_{45}^{C} \mathrm{~N}_{12}^{K}$, śivapūjāparodyuktah $\mathrm{N}_{58}^{K}$, śivah parāparo nityaṃ $\mathrm{P}_{32}^{T}$ 
[4. Pāñcika]

pāñciko nāma yakṣendraḥ kaṇṭhikākațakojjvalaḥ| makuțena vicitreṇa keyūrābhyāṃ virājate|| 199||

yakṣasamghaiḥ samāyukto yakṣakoțisamanvitaḥ| harārcanaparaḥ śrīmān karotu mama śāntikam|| 2oo||

\section{[5. Vibhāṇụaka]}

śrīmān vibhāṇụako yakṣo nānāratnavibhūṣitaḥ| cāruṇā kuṇḍalendreṇa karṇe nityaṃ virājate|| 201||

yakșeśvaro yakṣapatir yakṣasenāpatir dhruvaḥ| harapādārcakaḥ śrīmān karotu mama śāntikam|| 202||

199-20o Cf. BhavP 1.180.11-12 : pāñciko nāma yakṣendraḥ kaṇțhābharaṇabhūṣitaḥ| kukkuṭena vicitreṇa bahuratnānvitena tu|| yakṣavịndasamākīrṇo yakṣakoțisamanvitaḥ| sūryārcanakaraḥ śrīmān karotu tava śāntikam||

199a-2ood Omitted in $\mathrm{E}^{N}$

199a pāñciko nāma yakșendrah ] $\mathrm{N}_{82}^{K} \mathrm{~B}_{99}^{C} \mathrm{P}_{72}^{T}$, pañciko nāma yakșendro $\mathrm{N}_{77}^{K o}$, pañciko nāma yakșendroḥ $\mathrm{N}_{45}^{C}$ pañciko nāma yakșendrāḥ $\mathrm{N}_{12}^{K} \mathrm{~N}_{58}^{K a c}$, pañciko nāma yakṣendraḥ $\mathrm{N}_{58}^{K}$, yakșarājaḥ pañcaśikhaḥ $S_{67}^{S}$, vaṃciko nāma yakșendraḥ $\mathrm{P}_{3^{2}}^{T} \quad$ 199b kaṇțhikākațakojjvalah ] $\mathrm{K}^{\Sigma} \mathrm{B}_{99}^{C} \hat{S}_{67}^{S}$, kaṇthiko kaṭakojvalaḥ $\mathrm{N}_{77}^{K o}$, karṇnikākanakojvalạ̣ $\mathrm{N}_{45}^{C}$, karṇikākațakojvalah $\mathrm{P}^{\Sigma} \quad$ 199c makuṭena vicitreṇa ] $\mathrm{N}_{77}^{K o} \mathrm{~N}_{58}^{K} \mathrm{P}^{\Sigma}$, hāreṇa suvicitreṇa $\mathrm{N}_{82}^{K} \mathrm{~N}_{12}^{K} \mathrm{~B}_{99}^{C}$, makuțena vicitreṇaḥ $\mathrm{N}_{45}^{C}$, mukuțena vicitreṇa $\hat{S}_{67}^{S} \quad$ 199d virājate ] $\mathrm{N}_{77}^{K o} \mathrm{~K}^{\Sigma} \mathrm{B}_{99}^{C} \dot{S}_{67}^{S} \mathrm{P}_{72}^{T}$, virājatāṃ $\mathrm{N}_{45}^{C}$, virājitaḥ $\mathrm{P}_{32}^{T} \quad$ 20oa yakṣasaṃghaiḥ samāyukto ] $\mathrm{N}_{58}^{K}$, yakṣāsaṃkhyeyasaṃyukto $\mathrm{N}_{77}^{K o} \mathrm{P}_{32}^{T}$, yakṣasaṃhaih samāyukto $\mathrm{N}_{82}^{K} \mathrm{~B}_{99}^{C}$, yakṣasaṃghai samāyujto $\mathrm{N}_{12}^{K}$, yakṣasaṃkṣeyasaṃyukto $\mathrm{N}_{45}^{C}$, yakṣasaṃkhyeyasaṃyukto $S_{67}^{S}$, yakșāsaṃkhyeyayuktaś ca $\mathrm{P}_{72}^{T} \quad$ 2oob okoțisamanvitạ ] $\mathrm{N}_{77}^{K o} \mathrm{~N}_{12}^{K} \mathrm{~N}_{58}^{K} \dot{S}_{67}^{S} \mathbf{P}^{\Sigma}$, okoțīsamanvitaḥ $\mathrm{N}_{45}^{C}$, okoṭisamāvṛtah $\mathrm{N}_{82}^{K} \mathrm{~B}_{99}^{C} \quad$ 2ooc harārcanaparaḥ śrīmān ] $\mathrm{K}^{\Sigma} \mathrm{B}_{99}^{C}$, harārcanaparaḥ śrīn $\mathrm{N}_{77}^{K o}$ (unmetr.), harapādārccaṇe raktah $\mathrm{N}_{45}^{C}$, harārcanarato nityam $S_{67}^{S} \mathrm{P}_{3^{2}}^{T}$, harārcanaparo nityam $\mathrm{P}_{72}^{T}$ 2ood karotu ] $\mathrm{N}^{\Sigma} \mathrm{B}_{99}^{C} \mathrm{P}^{\Sigma}$, kurvantu $\hat{S}_{67}^{S} \quad$ 201a vibhāṇḍako ] $\mathrm{N}_{77}^{K o} \mathrm{~N}_{82}^{K} \mathrm{E}^{N} \hat{S}_{67}^{S}$, vibhaṇ̣̣ako $\mathrm{N}_{45}^{C} \mathrm{~N}_{12}^{K} \mathrm{~N}_{58}^{K} \mathrm{~B}_{99}^{C} \mathbf{P}^{\Sigma} \quad$ 2o1c kuṇ̣alendreṇa ] $\Sigma$, kuṇ̣alenaiva $\mathrm{P}_{72}^{T} \quad$ 2o1d nityam ] $\Sigma$, nitya $\mathrm{N}_{5^{8}}^{K} \bullet$ virājate ] $\Sigma$, virājitaḥ $\mathrm{P}_{3^{2}}^{T} \quad$ 202a yakșeśvaro yakṣapatir ] $\Sigma$, yakșeśvaro yakșapati $\mathrm{N}_{77}^{K o} \mathrm{~N}_{45}^{C}$, yakṣo yakșapatirdevo $\mathrm{P}_{32}^{T} \quad$ 202b ${ }^{\circ}$ patir dhruvah ] $\mathrm{N}_{82}^{K} \mathrm{~N}_{12}^{K} E^{N} \mathrm{~B}_{99}^{C} \dot{S}_{67}^{S}$, ${ }^{\circ}$ pati prabhuh $\mathrm{N}_{77}^{K o}$, ${ }^{\circ}$ pati dhruvam $\mathrm{N}_{45}^{C}$, ${ }^{\circ}$ patir varah $\mathrm{N}_{58}^{K}$, ${ }^{\circ}$ patir budhah $\mathbf{P}^{\Sigma} \quad$ 202c harapādārcakaḥ śrīmān ] $\mathrm{N}_{77}^{K o} \mathrm{~N}_{82}^{K} \mathrm{~N}_{12}^{K} \mathrm{E}^{N} \mathrm{~B}_{99}^{C}$, harapādārccaṇe paraḥ $\mathrm{N}_{45}^{C}$, harapādārccaka śrīmān $\mathrm{N}_{58}^{K}$, harārcanarataḥ śrīmān $\hat{S}_{67}^{S}$, harapādārcanaratạ̣ $\mathbf{P}^{\Sigma}$ 


\section{[6. Dhṛtarāșțra]}

dhṛtarāṣtro mahātejāḥ yakṣo yakṣādhipah prabhuh| divyapațtāạśukacchanno maṇikāñcanabhūṣitaḥ|| 203||

śivabhaktah śivadhyātā śivapūjāparāyaṇaḥ| śivaprasādasampannạ̣ karotu mama śāntikam|| 204||

\section{[7. Pūrṇabhadra]}

pūrṇabhadro mahāyakṣaḥ sarvālan̉kārabhūṣitaḥ| ratnapradīptapatțena hemnātīva virājate|| 205||

203-204 Cf. BhavP 1.180.13-14 : dhṛtarāṣtro mahātejā nānāyakṣādhipaḥ khaga| divyapaț̣ah śuklacchatro maṇikāñcanabhūṣitaḥ|| sūryabhaktạ̣ sūryarataḥ sūryapūjāparāyaṇaḥ| sūryaprasādasampannaḥ karotu tava śāntikam||

203d After this $\mathrm{N}_{5^{8}}^{K}$ adds four pādas : ratnasampannapațtena dehenādhivirājate| yakṣakoțisahasrais tu parivāritavigrahah|

203a ${ }^{\circ}$ rāșțro ] $\Sigma$, ${ }^{\circ}$ rāṣțo $\mathrm{N}_{77}^{K o} \mathrm{~N}_{45}^{c}$ • ${ }^{\circ}$ tejāḥ ] $\mathrm{N}_{45}^{C} \mathbf{P}^{\Sigma}$, ${ }^{\circ}$ rājo $\mathrm{N}_{77}^{K o}$, otejo $\mathrm{N}_{82}^{K}$, otejā $\mathrm{N}_{12}^{K} \mathrm{~B}_{99}^{C} \hat{S}_{67}^{S}$, ${ }^{\circ}$ rājā $\mathrm{N}_{58}^{K}$, ${ }^{\circ}$ rājo $\mathrm{E}^{N} \quad \mathbf{2 0 3 b}$ yakṣo yakșādhipah ] $\mathrm{N}_{45}^{C} \mathrm{~N}_{12}^{K} \mathrm{~N}_{58}^{K} \mathrm{E}^{N} \mathrm{~B}_{99}^{C} \dot{S}_{67}^{S} \mathbf{P}^{\Sigma}$, yakṣa yakṣādhipaḥ $\mathrm{N}_{77}^{K o}$, yakṣarājādhipa $\mathrm{N}_{58}^{K}$, yakṣo yakṣādhipa ${ }^{\circ} \mathrm{N}_{82}^{K} \quad{ }_{203}{ }^{\circ}$ pațțāmśúukachanno ] $\mathrm{N}_{77}^{K o} \mathrm{~N}_{82}^{K} \mathrm{~B}_{99}^{C} \mathcal{S}_{67}^{S} \mathbf{P}^{\Sigma}$, ${ }^{\circ}$ patțāśukacchano $\mathrm{N}_{45}^{C} \mathrm{~N}_{12}^{K}$, ${ }^{\circ}$ pațțāśuṃkacchanno $\mathrm{N}_{58}^{K}$, ${ }^{\circ}$ padmāngukachanno $\mathrm{E}^{N} \quad$ 203d ${ }^{\circ}$ kāñcana $\left.{ }^{\circ}\right] \Sigma$, ${ }^{\circ}$ kuṇḍala ${ }^{\circ} S_{67}^{S} \quad$ 204a śivabhaktah śivadhyātā ] $\mathrm{N}_{82}^{K} \mathrm{~B}_{99}^{C} \mathrm{P}_{32}^{T}$, śivabhakto śivadhyātā $\mathrm{N}_{77}^{K o}$, śivadhyātā śivabhaktāḥ $\mathrm{N}_{45}^{C}$, śivabhaktah śivadhyāna ${ }^{\circ} \mathrm{N}_{12}^{K} \mathrm{E}^{N}$, rudrapraṇāmaparamo $\mathrm{N}_{58}^{K}$, śivabhaktiśivadhyāna ${ }^{\circ} \hat{S}_{67}^{S}$, śivabhaktah śivadhyāyī $\mathrm{P}_{72}^{T} \quad 204 \mathrm{~b}$ śivapūjāparāyaṇaḥ ] $\Sigma$, rudrabhaktiparaḥ sadā $\mathrm{N}_{5^{8}}^{K} \quad 204 \mathrm{c}$ śivaprasādasampannạ̣ ] $\Sigma$, śivaprasādāsampannaḥ $\mathrm{N}_{77}^{K o}$, rudrārccanasamāyuktah 205a pūrṇabhadro ] $\Sigma$, pūrṇacandro $\mathrm{N}_{45}^{C}$, pūrvabhadro $\hat{S}_{67}^{S}$

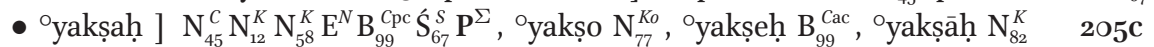
opradiptapatțena ] $\mathrm{N}_{82}^{K} \mathrm{~N}_{58}^{K} \mathrm{~B}_{99}^{C}$, ${ }^{\circ}$ pradīpapațtena $\mathrm{N}_{77}^{K o}$, opradiptavarṇneṇa $\mathrm{N}_{45}^{C}$, ${ }^{\circ}$ pradīpapațtena $\mathrm{N}_{12}^{K}$, ${ }^{\circ}$ pradīptataptena $\mathrm{E}^{N}$, o pradīpamātreṇa $\hat{S}_{67}^{S}$, ${ }^{\circ}$ dipptena pațtena $\mathbf{P}^{\Sigma} \quad{ }_{205} \mathbf{d}$ hemnātīva virājate ] $\mathrm{K}^{\Sigma} \mathrm{E}^{N} \mathrm{~B}_{99}^{C}$, hemenātivirājate $\mathrm{N}_{77}^{K o}$, deheṇāpi virājatā $\mathrm{N}_{45}^{C}$, dehenāpi virājate $\hat{S}_{67}^{S}$, haimenātīvarājite $\mathrm{P}_{3^{2}}^{T}$, haimenaiva virājate $\mathrm{P}_{72}^{T}$ 
yakșakoțisahasreṇa parivāreṇa saṃyutaḥ| rudrārcanasamāyuktaḥ karotu mama śāntikam|| 206||

\section{[8. Virūpākșa]}

virūpākṣaś ca yakṣendraḥ śvetavāso mahādyutị̣| cārukāñcanamālābhị̣ kin̉kiṇīravakānvitaiḥ|| 207||

vibhūṣitaḥ sadākālaṃ varadānaikatatparaḥ| rudrapūjāparo bhaktạ̣ karotu mama śāntikam|| 208||

207-208 Cf. BhavP 1.18o.15-16 : virūpākṣaś ca yakșendrah śvetavāsā mahādyutih| nānākāñcanamālābhir upaśobhitakandharaḥ|| sūryapūjāparo bhaktạ̣ kañjākṣaḥ kañjasaṃnibhạ̣| tejasādityasaṃkāśạ̣ karotu tava śāntikam||

2o6b After this $\mathrm{N}_{77}^{K o}$ adds two pādas : rudrapraṇāmaparamo rudrabhakityutah puraḥ| $\mathrm{N}_{45}^{C}$ adds : rudrapraṇāmaparamo rudraikagatamānasaḥ| $\bullet \mathrm{N}_{12}^{K}$ adds : rudrapraṇāmaparamo rudrabhaktiparah sadā $\bullet \mathrm{N}_{58}^{K}$ adds : rudrapraṇāmaparamo rudrabhakto tibhāvitaḥ| $\bullet \mathrm{E}^{N}$ adds : rudrapraṇāmaparamo rudrabhaktiyutah punaḥ| $\bullet \widehat{S}_{67}^{S}$ adds : rudrapraṇāmaparamo rudrabhaktipurassarah $\mid \bullet \mathrm{P}_{32}^{T}$ adds : samyato rudrapraṇato rudrabhaktah punaḥ punaḥ| $\mid \bullet \mathrm{P}_{72}^{T}$ adds : rudrapraṇāmaparamo rudrabhaktiratas sadā

206a ${ }^{\circ}$ koțisahasreṇa ] $\Sigma$, okoṭīsahasreṇah $\mathrm{N}_{45}^{C} \quad$ 2o6b parivāreṇa saṃyutah ] $\Sigma$, parivāritavigrahah $\mathbf{P}^{\Sigma} \quad$ 2o6c ${ }^{\circ}$ samāyuktah ] $\mathrm{N}_{45}^{C} \mathrm{~N}_{82}^{K} \mathrm{~N}_{58}^{K} \mathrm{E}^{N} \mathrm{~B}_{99}^{C}$, osamāyukta $\mathrm{N}_{77}^{K o}$, o samudyuktah $S_{67}^{S} \mathrm{~N}_{12}^{K} \mathbf{P}^{\Sigma} \quad$ 2o6d śāntikam ] $\Sigma$, sāntim $\mathrm{N}_{45}^{C}$ (unmetr.) 207a ca yakṣendrạ̣ ] $\mathrm{N}_{82}^{K} \mathrm{~N}_{12}^{K} \mathrm{E}^{N} \mathrm{~B}_{99}^{C} \dot{S}_{67}^{S} \mathbf{P}^{\Sigma}$, ca yakṣendro $\mathrm{N}_{77}^{K o}$, ca yakṣendrāḥ $\mathrm{N}_{45}^{C}$, mahāyakṣah $\mathrm{N}_{58}^{K} \quad 207 \mathbf{b}{ }^{\circ}$ vāso ] $\mathrm{N}_{82}^{K} \mathrm{~N}_{12}^{K} \mathrm{E}^{N} \mathrm{~B}_{99}^{C}$, ${ }^{\circ}$ vāsā $\mathrm{N}_{77}^{K o} \mathrm{~N}_{82}^{K} \dot{S}_{67}^{S} \mathbf{P}^{\Sigma}$, ovāsa $\mathrm{N}_{45}^{C} \bullet{ }^{\circ}$ dyutiḥ ] $\Sigma$, odyuti $\mathrm{N}_{45}^{C} \quad$ 207c ${ }^{\circ}$ mālābhị̣ ] $\mathrm{N}_{77}^{K o} \mathrm{~N}_{45}^{C} \mathrm{~N}_{12}^{K} \mathrm{~N}_{58}^{K} \hat{S}_{67}^{S} \mathbf{P}^{\Sigma}$, ${ }^{\circ}$ varṇābhih $\mathrm{N}_{82}^{K} \mathrm{E}^{N} \mathrm{~B}_{99}^{C} \quad$ 2O7d kinnkiṇīravakānvitaiḥ ] $\mathrm{N}_{77}^{K o} \mathrm{~N}_{12}^{K} \mathrm{~N}_{58}^{K} \mathrm{P}_{32}^{T}$, kin̉kiṇīravakānvitah $\mathrm{N}_{45}^{C} \hat{S}_{67}^{S} \mathrm{P}_{72}^{T}$ kińkiṇībhị̣ samanvitạ̣ $\mathrm{N}_{82}^{K} \mathrm{E}^{N} \mathrm{~B}_{99}^{C} \quad$ 208a vibhūṣitaḥ ] $\mathrm{N}_{12}^{K} S_{67}^{S} \mathbf{P}^{\Sigma}$, vibhūṣita $\mathrm{N}_{77}^{K o} \mathrm{~N}_{45}^{C}$, bhūṣitaś ca ] $\mathrm{N}_{82}^{K} \mathrm{E}^{N} \mathrm{~B}_{99}^{C}$, bhūṣitaḥ $\mathrm{N}_{58}^{K}$ (unmetr.) • ${ }^{\circ}$ kālaṃ ] $\mathrm{N}_{77}^{K o} \mathrm{~K}^{\Sigma} \mathrm{B}_{99}^{C} \dot{S}_{67}^{S} \mathbf{P}^{\Sigma}$, ${ }^{\circ}$ kāla ${ }^{\circ} \mathrm{N}_{77}^{C p c} \mathrm{E}^{N}$, ${ }^{\circ}$ kālā $\mathrm{N}_{77}^{C a c} \quad$ 2o8c oparo bhaktah ] $\mathrm{N}_{45}^{C} \mathrm{~N}_{82}^{K} \mathrm{~N}_{12}^{K} \mathrm{E}^{N} \mathrm{~B}_{99}^{C}$, ${ }^{\circ}$ karo nityam $\mathrm{N}_{77}^{K o}$, ${ }^{\circ}$ rato nityam $\hat{S}_{67}^{S}$, ${ }^{\circ}$ paro nityam $\mathrm{N}_{58}^{K} \mathbf{P}^{\Sigma}$ 
[other Yakșas]

antarīkṣagatā yakṣā ye yakṣāḥ svargavāsinaḥ|

girikandaradurgeșu ye yakșā bhūnivāsinaḥ|| 209||

antarīkṣe ca ye yakṣāḥ pātālatalavāsinaḥ|

nānārūpāyudhā yakṣā nānāveșadharās tathâ|| 210||

śivabhaktāḥ sumanasạ̣ śivapūjāsamutsukāḥ|

śāntiṃ kurvantu me hṛșṭạ̣ śāntāḥ śāntiparāyaṇāḥ|| 211||

209-211 Cf. BhavP 1.180.17-18 : antarikṣagatā yakṣā ye yakṣāḥ svargagāminaḥ| nānārūpadharā yakṣāh sūryabhaktā dụḍhavratāḥ|| tadbhaktās tadgamanasaḥ sūryapūjāsamutsukāḥ| śāntiṃ kurvantu te hṛ̣țāḥ śāntāḥ śāntiparāyaṇāḥ||

2ogd-210a Omitted in $\mathrm{N}_{12}^{K} \quad$ 210ab Omitted in $\mathrm{N}_{77}^{K o} \hat{\mathrm{S}}_{67}^{s} \mathbf{P}^{\Sigma} \quad$ 21ocd Omitted in $\mathrm{N}_{45}^{C}$

209a antarīkṣagatā ] $\mathrm{N}_{77}^{K o} \mathrm{~K}^{\Sigma} \mathrm{E}^{N} \mathrm{~B}_{99}^{C}$, antarīkṣe ca ye $\mathrm{N}_{45}^{C}$, antarikṣe ca ye $\hat{S}_{67}^{S} \mathrm{P}_{72}^{T}$, antarikṣagatā $\mathrm{P}_{32}^{T} \quad$ 209b ye yakṣāh ] $\Sigma$, ye yakṣā $\mathrm{N}_{77}^{K o} \mathrm{~N}_{45}^{C} \bullet{ }^{\circ}$ vāsinah ] $\mathrm{K}^{\Sigma} \mathrm{E}^{N} \mathrm{~B}_{99}^{C} \stackrel{\mathrm{S}}{67}_{67}^{S}$, ${ }^{\circ}$ samsthitā $\mathrm{N}_{77}^{K o}$, ${ }^{\circ}$ gāminaḥ $\mathrm{N}_{45}^{C}$, ${ }^{\circ}$ saṃsthitāḥ $\mathbf{P}^{\Sigma} \quad$ 2ogd ye yakșā bhūnivāsinaḥ ] $\mathrm{N}_{82}^{K} \mathrm{E}^{N} \mathrm{~B}_{99}^{C}$, ye yakșā bhuvi vāsinaḥ $\mathrm{N}_{77}^{K o} \mathrm{~N}_{58}^{K}$, pātālatalavāsinaḥ $\mathrm{N}_{45}^{C} \dot{S}_{67}^{S}$, yakșāḥ pātālavāsinaḥ $\mathrm{P}_{3^{2}}^{T}$, ye yakṣā bhūtavāsinah $\mathrm{P}_{72}^{T} \quad$ 210a antarīkṣe ] $\mathrm{N}_{82}^{K} \mathrm{~N}_{58}^{K} \mathrm{E}^{N} \mathrm{~B}_{99}^{C}$, sarvadikṣu $\mathrm{N}_{45}^{C} \quad$ 21ob pātālatalavāsinaḥ ] $\mathrm{K}^{\Sigma} \mathrm{E}^{N} \mathrm{~B}_{99}^{C}$, nānārūpāyudhopamāḥ $\mathrm{N}_{45}^{C} \quad 210 c$ nānārūpāyudhā ] $\mathrm{N}_{82}^{K} \mathrm{E}^{N} \mathrm{~B}_{99}^{C}$, pātālavāsino $\mathrm{N}_{77}^{K o} \mathrm{P}_{72}^{T}$, nānārūpadharā $\mathrm{N}_{12}^{K} \mathrm{~S}_{67}^{S}$, nānārūpāvidhā $\mathrm{N}_{58}^{K}$, nānārūpayutā $\mathrm{P}_{3^{2}}^{T}$ • yakșā ] $\mathrm{N}_{82}^{K} \mathrm{~N}_{12}^{K} \mathrm{E}^{N} \mathrm{P}_{72}^{T}$, yakșāḥ $S_{67}^{S} \mathrm{P}_{3^{2}}^{T} \quad$ 21od nānāveșadharās tathā ] conj., nānārūpāyudhādharā $\mathrm{N}_{77}^{K o}$, nānāveśadharās tathā $\mathrm{N}_{82}^{K} \mathrm{~N}_{58}^{K} \mathrm{E}^{N}$, nānārūpadharās tathā $\mathrm{B}_{99}^{C}$, anekārthavarapradāḥ $\mathrm{N}_{12}^{K}$, śivabhaktā dṛ̣havratāḥ $S_{67}^{S} \mathrm{P}_{32}^{T}$, nānārūpodyatāyudhāḥ $\mathrm{P}_{72}^{T}$ 211a śivabhaktāḥ sumanasạ̣ ] $\mathrm{N}_{12}^{K} \mathrm{~N}_{58}^{K} \mathrm{E}^{N} \mathrm{~B}_{99}^{C} \mathrm{P}_{72}^{T}$, śivabhaktā sumanasạ̣ $\mathrm{N}_{77}^{K o} \mathrm{~N}_{82}^{K}$, śivabhaktā sumanasāḥ $\mathrm{N}_{45}^{C}$, yogaiśvaryayutāḥ sarve $\mathrm{P}_{3^{2}}^{T}$, ye vai sumanasạ̣ sarve $\dot{S}_{67}^{S} \quad \mathbf{2 1 1 b}$ ${ }^{\circ}$ samutsukāḥ ] $\mathrm{N}_{12}^{K} \mathrm{~N}_{58}^{K} \mathrm{E}^{N} \hat{\mathrm{S}}_{67}^{S} \mathrm{P}^{\Sigma}$, o samutsukā $\mathrm{N}_{77}^{K o} \mathrm{~N}_{45}^{C}$, ${ }^{\circ}$ samutsukaḥ $\mathrm{N}_{82}^{K} \mathrm{~B}_{99}^{C} \quad$ 211c śāntiṃ kurvantu ] $\Sigma$, śānti karotuN $\mathrm{N}_{77}^{K o}$, śānti kurvvantu $\mathrm{N}_{58}^{K} \bullet$ hṛșțāḥ ] $\Sigma$, hṛșțā $\mathrm{N}_{77}^{K o}$, nityam $\mathrm{P}_{32}^{T} \quad$ 211d śāntāh ] $\mathrm{N}_{12}^{K} \mathrm{E}^{N} \mathrm{~B}_{99}^{C} \dot{S}_{67}^{S} \mathrm{P}^{\Sigma}$, saantā $\mathrm{N}_{77}^{K o} \mathrm{~N}_{82}^{K \text { pcc }} \mathrm{N}_{58}^{K}$, śāntio $\mathrm{N}_{45}^{C}$, śānta ${ }^{\circ} \mathrm{N}_{82}^{K a c} \bullet$ śāntiparāyaṇāḥ ] $\Sigma$, śāntiparāyaṇā $\mathrm{N}_{77}^{K o} \mathrm{~N}_{58}^{K}$, śāntena cetasā $\mathrm{P}_{32}^{T}$ 
[Yakṣiṇīs, Yakṣakumārikās, Yakṣakanyās]

yakṣiṇyo vividhākārās tathā yakṣakumārikāḥ| yakṣakanyā mahābhāgāḥ śivapūjārcane ratāḥ|| 212||

212-213 Cf. BhavP 1.18o.19-20 : yakṣiṇyo vividhākārās tathā yakṣakumārakāḥ| yakṣakanyā mahābhāgāḥ sūryārādhanatatparāḥ|| sāntiṃ svastyayanaṃ kṣemaṃ balaṃ kalyāṇam uttamam| siddhị̣ cāśu prayacchantu nityạ̣ ca susamāhitāḥ||

212ab Omitted in in $\mathrm{E}^{N}$

212a yakșinyyo ] $\mathrm{N}_{77}^{K o} \mathbf{K}^{\Sigma} \hat{S}_{67}^{S} \mathbf{P}^{\Sigma}$, yakș̣iṇyā $\mathrm{N}_{45}^{c}$, yakșiṇo $\mathrm{B}_{99}^{c} \quad$ 212ab vividhākārās tathā ] $\mathrm{N}_{45}^{C} \mathrm{~N}_{82}^{K p c} \mathrm{~N}_{12}^{K} \mathrm{~N}_{58}^{K} \mathrm{~B}_{99}^{C} \mathbf{P}^{\Sigma}$, vividhākārā tathā $\mathrm{N}_{77}^{K o}$, vivikārās tathā $\mathrm{N}_{82}^{K a c}$ (unmetr.), vividhākārāyudhā $S_{67}^{S} \quad$ 212b yakṣakumārikāḥ ] $\mathrm{N}_{12}^{K} \mathrm{~N}_{58}^{K} \mathrm{~B}_{99}^{C a c} \mathrm{P}_{32}^{T}$, yakșā kumārakā $\mathrm{N}_{77}^{K o}$, yakșakumārakāḥ $\mathrm{N}_{45}^{C} \mathrm{~N}_{82}^{K} \mathrm{~B}_{99}^{C p c} ́_{67}^{S} \mathrm{P}_{72}^{T} \quad 212 c$ mahābhāgāḥ ] $\mathrm{N}_{45}^{C} \mathrm{~K}^{\Sigma} \mathrm{E}^{N} \mathrm{~B}_{99}^{C p c} \hat{S}_{67}^{S} \mathrm{P}_{32}^{T}$, mahābhāgā $\mathrm{N}_{77}^{K o}$, mahābhāḥ $\mathrm{B}_{99}^{C a c}$ (unmetr.), mahābhogāḥ $\mathrm{P}_{72}^{T} \quad$ 212d śivapūjārcane ratāḥ ] $\mathrm{N}_{82}^{K p c} \mathrm{E}^{N} \mathrm{~B}_{99}^{C}$, śivārcanasutatparā $\mathrm{N}_{77}^{K o}$, śivaikārccaṇatatparāḥ $\mathrm{N}_{45}^{C}$, śivapūjārcane rataḥ $\mathrm{N}_{82}^{K a c}$, śivārcājapatatparāḥ $\mathrm{N}_{12}^{K}$, śivapūjārccanetāḥ $\mathrm{N}_{5^{8}}^{K}$ (unmetr.), śivārcaṇopamānasāḥ Ś $\hat{S}_{67}^{S}$, śivārcanaparāyaṇāḥ $\mathrm{P}_{3^{2}}^{T}$, śivadhyāna $\sqcup[-4-] \sqcup \cdot h \mathrm{P}_{72}^{T}$ 


\section{śāntim svastyayanaṃ kṣemaṃ balaṃ kalyāṇam uttamam| siddhiṃ cāśu prayacchantu nityam eva samāhitāḥ|| 213\|}

213d After this $\mathrm{N}_{45}^{c}$ adds 14 pādas : jambuṭipaśākadvīpạ̣ kuśadvīpạ̣ krauñcam eva ca| śalmalīkas tathā dvīpah plakṣadvīpās tathaiva caḥ| gomedhaś ca mahādvīpaḥ puṣkarantus tathaiva ca| ete dvīpā mahātmāno rudreṇa parikīrttitāḥ| śāntin̉kurvantu me hṛṣṭạ̣ tathā pāpaparikṣayạ̣| mairumandarakailāso malayo gandhamārddhaṇaḥ| śrīparvato hemakūṭāś ca mālyavantas tathaiva ca| $\bullet \mathrm{N}_{58}^{K}$ adds 18 pādas : jambūdvīpạ̣ kuśadvīpaḥ śākakrauṃco tha dvīpakaḥ| śālmalīkas tathā dvīpo $\sqcup \sqcup$ medho tha dvīpakaḥ| puskarantu mahādvīpo yathā<tmavya>sthitāḥ| rudrabhakti sadā sarvve śānti kurvvantu me sadā| kṣārakṣīrodadhiś caiva dadhno dadhṛtam eva ca| suroda garbhodaś caiva īkṣusvādras tatthaiva ca| saptasamudrānāmānaḥ kurvvantu mama śāṃtikaṃ| merumandarakailāsamalayagandhamādanāḥ| mahendraḥ śrīparvvatāś ca hemakūṭas tathaiva ca| • $\mathrm{B}_{99}^{c}$ adds 22 pādas in margin with an insertion mark (second hand, with many errors): merumandarakailāśa malayo gandhamādana| śrīparvvato mamāhendraś ca himakūṭas tathaiva ca| parvvatā sarvvadā sarvve parvvatāś ca maharddhikāḥ| śivabhaktāḥ sadākāraṃ kṣemaṃ kurvvantu me sadā| jambudvīpa plakṣadvīpạ̣ kuṣadvīpakạ̣| krauñcadvīpa saākadvīpam gomedhodvīpako mahān| puṣkarantu mahādīpamedadvīpā mahātmana| rudrabhaktiratā+ḥ+ sarvve śāntiṃ kurvvantu me sadā| kṣāroda kṣīrodaś caiva dadhno ghṛtoda eva ca| surodo da(hva)daṃbhaś ca ikṣuḥsvādras tathaiva ca| saptasamudranāmānaḥ kurvvantu mama śāntikaṃ| $\bullet \mathrm{E}^{N}$ adds four pādas : merūmandarakailāśo malayo gandhamādanaḥ| śrīparvato mahendraś ca himakūṭas tathaiva ca| $\bullet S_{67}^{S}$ adds 18 pādas : jambudvīpaś ca śākalyaḥ kuśaḥ krauñcābhidhas tathā| śalmaliś ca mahādvīpo gomedhānyas tathaiva ca| puṣkaraś ca mahādvīpa ete dvīpā mahotsukāḥ| śivabhaktiratās sarve śānti kurvantu me sadā| kṣārodadhiś ca kṣīrododadhi pūrṇo ghṛtodakaḥ| ikṣupūrṇaḥ surodaś ca svādudo garbhasaṃyutaḥ| ete samudrās sarve pi kurvantu mama śāntikam| merunmandirakailāso malayo gandhamadanaḥ| mahendraḥ śrīgiriś caiva hemakūțādayo nagāḥ|

213a śāntiṃ ] $\Sigma$, śānti $\mathrm{N}_{77}^{K o} \mathrm{~N}_{45}^{C} \quad$ 213b balaṃ ] $\Sigma$, bala ${ }^{\circ} \mathrm{N}_{77}^{K o}$, baliṃ $S_{67}^{S} \quad$ 213c siddhim cāśu ] $\mathrm{K}^{\Sigma} \mathrm{E}^{N} \mathrm{~B}_{99}^{C} \hat{S}_{67}^{S}$, siddhim cāśuh $\mathrm{N}_{77}^{K o}$, siddhiñ cāsu $\mathrm{N}_{45}^{C}$, siddhim āśu $\mathbf{P}^{\Sigma} \quad$ 213d nityam eva ] $\Sigma$, nitye sarve $\mathrm{N}_{45}^{C} \bullet$ samāhitāḥ ] $\Sigma$, samāhitā $\mathrm{N}_{77}^{K o}$ 


\section{[Mountains]}

parvatāḥ sarvadā sarve parvatāś ca maharddhikāḥ| śivabhaktāḥ sadākālaṃ kṣemaṃ kurvantu me sadā|| 214||

\section{[Oceans]}

sāgarāḥ sarvataḥ sarve sāgarāḥ sarvataḥ sthitāḥ| rudrapūjāparā nityaṃ kurvantu mama śāntikam|| 215||

\section{[Rākșasas]}

rākṣasāḥ sarvataḥ sarve rākṣasā ghorarūpiṇaḥ| rākṣasā ye mahāvīryā rākṣasāś ca mahābalāḥ|| 216||

214 Cf. BhavP 1.180.21 : parvatāḥ sarvatah sarve vṛkṣāś caiva maharddhikāḥ| sūryabhaktāḥ sadā sarve śāntiṃ kurvantu te sadā|| 215 Cf. BhavP 1.180.22 : sāgarāḥ sarvataḥ sarve gṛhāraṇyāni kṛtsnaśaḥ| sūryasyārādhanaparāḥ kurvantu tava śāntikam|| 216-218 Cf. BhavP 1.180.23-24: rākṣasāḥ sarvataḥ sarve ghorarūpā mahābalāḥ| sthalajā rākṣasā ye tu antarikṣagatāś ca ye|| pātāle rākṣasā ye tu nityaṃ sūryārcane ratāḥ| śāntiṃ kurvantu te sarve tejasā nityadīpitāḥ||

214d After this $E^{N}$ adds 14 pādas : jambudvīpah plakṣadvīpah śālmalị̣ kuśadvīpakam| krauñcadvīpạ̣ saākadvīpạ̣ gomadhye dvīpako mahān| puṣkaran tu mahādvīpam etaddvīpā mahātmanāḥ| rudrabhaktiratāḥ sarve śāntiṃ kurvvantu me sadā| kṣārodaḥ kṣ̂rodaś caiva dadhno ghṛtoda eva ca| surodaḥ svādudakaś ca ikṣusvādas tathaiva ca| saptasamudranāmānaḥ kurvantu mama śāntikam|

214a parvatāḥ ] $\Sigma$, parvatā $\mathrm{N}_{77}^{K o}$, ityādyāḥ $S_{67}^{S}$ • sarvadā ] $\mathrm{N}_{82}^{K} \mathrm{~N}_{12}^{K} \mathrm{E}^{N} \mathrm{~B}_{99}^{C p c}$, sarvata $\mathrm{N}_{77}^{K o} \mathrm{~N}_{45}^{C}$, sarvvatah $\mathrm{N}_{58}^{K}$, sarvvadāḥ $\mathrm{B}_{99}^{\text {Cac }}$, sarvataḥ $\mathbf{P}^{\Sigma}$, parvatās $\hat{S}_{67}^{S} \bullet$ sarve ] $\Sigma$, sarva ${ }^{\circ}$ $\mathrm{E}^{N} \quad 214$ b parvatāś ca ] $\Sigma$, parvatāḥ śrīo $\mathrm{N}_{45}^{C}$, parvatyaś ca $\mathrm{P}_{32}^{T} \bullet$ maharddhikāh ] $\Sigma$, maharddhikā $\mathrm{N}_{58}^{K} \quad 214 c$ śivabhaktāḥ ] $\Sigma$, śivabhaktā $\mathrm{N}_{77}^{K o} \mathrm{~N}_{45}^{C}$, śive bhaktās $\mathrm{P}_{72}^{T} \bullet$ sadākālaṃ ] $\Sigma$, sumanasaḥ $\mathrm{N}_{77}^{K o} \mathrm{P}_{72}^{T}$, sarvakālaṃ $\hat{S}_{67}^{S} \quad$ 214d kṣemạ̣ ] $\quad \Sigma$, śāntin $\mathrm{N}_{45}^{C} \quad \mathbf{2 1 5 a}$ sāgarāḥ sarvataḥ ] $\mathrm{N}_{45}^{C} \mathrm{~N}_{12}^{K} \mathrm{~N}_{58}^{K} \mathrm{E}^{N} \mathrm{~B}_{99}^{C} \mathrm{P}_{72}^{T}$, sāgarā saritā $\mathrm{N}_{77}^{K o}$, sāgarāḥ sarvata $\mathrm{N}_{82}^{K}$, samudrāḥ sarvatah $\mathrm{P}_{32}^{T}$, sāgarāḥ sarvatāḥ $\hat{S}_{67}^{S} \bullet$ sarve ] $\Sigma$, sarvā $\mathrm{N}_{77}^{K o} \quad \mathbf{2 1 5}$ b sāgarāḥ sarvatạ̣ sthitāḥ ] $\mathrm{N}_{82}^{K} \mathrm{~N}_{12}^{K} \mathrm{E}^{N} \mathrm{~B}_{99}^{C}$, sāgarā sarvata sthitā $\mathrm{N}_{77}^{K o}$, sāgarāḥ sarvata sthitāḥ $\mathrm{N}_{45}^{C}$, sāgarā sarvvatah sthitāḥ $\mathrm{N}_{5^{8}}^{K}$, ye cānye sāgarā bhuvi $\mathbf{P}^{\Sigma}$, yatra tatra ca saṃsthitāḥ $S_{67}^{S} \quad$ 215c

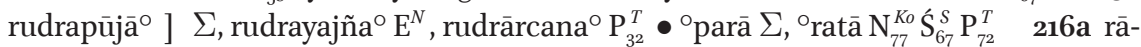
kṣasāḥ sarvataḥ ] $\Sigma$, rākṣasā sarvata $\mathrm{N}_{77}^{K o} \quad \mathbf{2 1 6 b}$ rākșasā ghora $\left.{ }^{\circ}\right] \quad \Sigma$, rākṣasāḥ para ${ }^{\circ}$ $S_{67}^{S} \bullet{ }^{\circ}$ rūpiṇaḥ ] $\Sigma$, ${ }^{\circ}$ rūpiṇa $\mathrm{N}_{77}^{K o} \quad$ 216c rākṣasā ye ] $\Sigma$, rākșasāś ca $\mathrm{N}_{45}^{C} \bullet$ mahāvīryā ] $\Sigma$, mavīrā $\mathrm{N}_{5^{8}}^{K}$ (unmetr.) 216d rākṣasāś ca ] $\Sigma$, rākṣasā ye $\hat{S}_{67}^{S} \bullet$ mahābalāh ] $\Sigma$, mahābalā $\mathrm{N}_{77}^{K o}$ 
sthalasthā rākṣasā ye tu antarīkṣe ca rākṣasāḥ| pātāle bhūtale ye ca nityaṃ rudraparāyaṇāḥ|| 217||

bhairavaṃ yasya rūpaṃ tu pretabhasmāvaguṇthitam| tejasā tasya devasya śāntiṃ kurvantu me sadā|| 218||

[Yoginīs, Ḍākinīs]

nityam ujjvalaveșeṇa yoginyo 'tha mahābalāḥ| rūpiṇyo vividhākārā ḍākinyaś ca maharddhikāḥ|| 219||

rudrapraṇāmaniratā rudrapūjārcane ratāḥ| rudraikāhitacetaskāḥ kurvantu mama śāntikam|| 220||

217ab Instead of this $\mathrm{P}_{32}^{T}$ has : svargam jalasthā nāgayakṣā ye tu yentarikṣe tu rākṣasāḥ| (unmetr.) 217d After this $\mathrm{N}_{77}^{K o} \mathrm{E}^{N} \mathbf{P}^{\Sigma}$ add two pādas : śāntiṃ kurvantu me nityam satatạ̣ śivabhāvitāḥ| 22ocd Omitted in $\hat{S}_{67}^{S}$

217a sthalasthā ] $\mathrm{N}_{77}^{K o} \mathrm{~K}^{\Sigma} \mathrm{E}^{N} \mathrm{~B}_{99}^{C} \hat{S}_{67}^{S}$, sthalastha ${ }^{\circ} \mathrm{N}_{45}^{C}$, jalasthā $\mathrm{P}_{72}^{T} \quad 217 \mathbf{b}$ antarīkṣe ] $\mathrm{N}_{77}^{K o} \mathrm{~K}^{\Sigma} \mathrm{E}^{N} \mathrm{~B}_{99}^{C}$, antarikṣe $\mathrm{N}_{45}^{C}$, ye ntarikșe $S_{67}^{S} \mathrm{P}_{72}^{T} \bullet$ ca ] $\mathbf{N}^{\Sigma} \mathrm{B}_{99}^{C}$, tu $\mathrm{E}^{N} \hat{S}_{67}^{S} \mathrm{P}_{72}^{T} \bullet$ rākșasāh ] $\Sigma$, rākṣasā $\mathrm{N}_{77}^{K o} \mathrm{~N}_{58}^{K} \quad 217 \mathrm{c}$ pātāle bhūtale ] $\mathrm{K}^{\Sigma} \mathrm{E}^{N} \mathrm{~B}_{99}^{C}$, pātāle rākṣasā $\mathrm{N}_{77}^{K o} \mathrm{~N}_{45}^{C} \hat{S}_{67}^{S} \mathrm{P}_{3^{2}}^{T}$, pātālarākșasā $\mathrm{P}_{72}^{T} \bullet$ ye ca ] $\mathrm{N}_{45}^{C} \mathbf{K}^{\Sigma} \mathrm{B}_{99}^{C}$, ye tu $\mathrm{N}_{77}^{K o} \hat{\mathrm{S}}_{67}^{S} \mathbf{P}^{\Sigma}$, ye $\mathrm{E}^{N}$ (unmetr.) 217 d nityam rudraparāyaṇāḥ ] $\mathrm{N}_{82}^{K} \mathrm{~N}_{12}^{K} \mathrm{E}^{N} \mathrm{~B}_{99}^{c}$, nitya rudrārcane ratā $\mathrm{N}_{77}^{K o} \mathrm{~N}_{58}^{K}$, nityam rudrārcane ratāh $\mathrm{N}_{45}^{C} \hat{\mathrm{S}}_{67}^{S} \mathbf{P}^{\Sigma} \quad$ 218a bhairavam ] $\Sigma$, bhairava $\mathrm{N}_{77}^{K o} \bullet$ tu ] $\Sigma$, ca $_{45}^{C} \quad \mathbf{2 1 8 b}{ }^{\circ}$ guṇṭhitam ] $\Sigma$, ${ }^{\circ}$ guṇthitah $\mathrm{N}_{77}^{K o}$, ${ }^{\circ}$ guṇțhitāh $\mathrm{N}_{45}^{C} \quad 218 \mathrm{c}$ tejasā tasya ] $\Sigma$, tejasāntasya $\mathrm{N}_{45}^{C} \bullet$ devasya ] $\Sigma$, deva $\mathrm{N}_{58}^{K}$ (unmetr.) 218d sadā ] $\Sigma$, sadāḥ $\mathrm{N}_{45}^{C} \quad$ 219a ${ }^{\circ}$ veșeṇa ] $\mathbf{P}^{\Sigma}$, ${ }^{\circ}$ veśena $\mathrm{N}_{77}^{K o} \mathbf{K}^{\Sigma} \mathrm{E}^{N} \mathrm{~B}_{99}^{C}$, ${ }^{\circ}$ veśenah $\mathrm{N}_{45}^{C}$, ${ }^{\circ}$ veśinyo $S_{67}^{S} \quad 219 \mathbf{b}$ yoginyo 'tha ] $\mathrm{N}_{77}^{K o} \mathrm{~K}^{\Sigma} \mathrm{E}^{N} \mathrm{~B}_{99}^{C} \mathrm{P}_{72}^{T p c} \mathrm{P}_{72}^{T}$, yoginyātha $\mathrm{N}_{45}^{C}$, yogino 'tha $\mathrm{P}_{72}^{T \text { ac }}$, yoginyaś ca $\hat{S}_{67}^{S} \bullet{ }^{\circ}$ balāḥ ] $\Sigma$, obalā $\mathrm{E}^{N} \quad$ 219c rūpinyo vividhākārā ] $\mathrm{N}_{77}^{K o} \mathrm{~K}^{\Sigma} \mathrm{B}_{99}^{C} \hat{S}_{67}^{S}$, rūpiṇyā vividhākārāḥ $\mathrm{N}_{45}^{C}$, rūpiṇyo vividhākāro $\mathrm{E}^{N}$, anekarūpadhāriṇya $\mathrm{P}_{32}^{T}$, anekarūpadhāriṇyo $\mathrm{P}_{72}^{T} \quad$ 219d ḍākiṇyaś ] $\mathrm{N}_{77}^{K o} \mathrm{~N}_{82}^{K} \mathrm{~B}_{99}^{C} \hat{S}_{67}^{S} \mathbf{P}^{\Sigma}$, ḍāgiṇyaś $\mathrm{N}_{45}^{C}$, dākiṇyāś $\mathrm{N}_{12}^{K} \mathrm{E}^{N}$, ḍakiś $\mathrm{N}_{58}^{K}$ (unmetr.) • maharddhikāḥ ] $\Sigma$, mahaddhikā $\mathrm{N}_{77}^{K o}$, marharddhikāḥ $\mathrm{N}_{58}^{K} \quad$ 22oa ${ }^{\circ}$ niratā ] $\mathrm{N}_{82}^{K} \mathrm{~N}_{12}^{K} \mathrm{E}^{N} \mathrm{~B}_{99}^{C}$, ${ }^{\circ}$ paramā $\mathrm{N}_{77}^{K o} \mathrm{~N}_{45}^{C} \mathrm{~N}_{58}^{K} \hat{S}_{67}^{S} \mathbf{P}^{\Sigma} \quad$ 22ob rudrapūjārcane ratāḥ ] $\mathrm{K}^{\Sigma} \mathrm{E}^{N} \mathrm{~B}_{99}^{C}$, pūjārcanaratā sadā $\mathrm{N}_{77}^{K o}$, rudrapādārccaṇe ratāḥ $\mathrm{N}_{45}^{C}$, rudrapūjāratāḥ sadā $\mathbf{P}^{\Sigma}$, rudrārcanaratāḥ sadā $S_{67}^{S} \quad$ 220c ${ }^{\circ}$ cetaskāḥ ] $\mathbf{K}^{\Sigma} \mathrm{E}^{N} \mathrm{~B}_{99}^{c} \mathbf{P}^{\Sigma}$, ${ }^{\circ}$ cetaskā $\mathrm{N}_{77}^{K o} \mathrm{~N}_{45}^{C} \quad$ 22od kurvantu mama śāntikam ] $\mathbf{N}^{\Sigma} \mathrm{E}^{N} \mathrm{~B}_{99}^{C}$, sāantiṃ kurvantu me sadā $\mathbf{P}^{\Sigma}$ 
antarīkṣagatā yāś ca ḍākinyaḥ svargasaṃsthitāḥ|

pātāle yās tu ḍākīnyo giridurgeṣu yāḥ sthitāḥ|| 221||

tṛtīyạ̣ locanaṃ yasya triśūlaṃ bhasma bhāsuram|

tejasā tasya devasya śāntim kurvantu me sadā|| 222||

[Bhūtas]

sarve bhūtā mahārūpāḥ sarve bhūtā mahojjvalāḥ|

sarve bhūtāḥ sthitāḥ saumyāḥ sarve bhūtā manojavāḥ|| 223||

antarīkṣe ca ye bhūtā ye bhūtā divi saṃsthitāḥ|

pātāle bhūtale ye tu bhūtā bhūtividhāyikāḥ̂|| 224||

223a Before this $\mathrm{P}_{32}^{T}$ inserts verses 229-231 223a-225d Added in the margin with an insertion mark in $\mathrm{N}_{12}^{K} \quad$ 223cd Omitted in $\mathrm{P}_{32}^{T}$

221a antarīkṣa $\left.{ }^{\circ}\right] \mathbf{N}^{\Sigma} \mathrm{E}^{N} \mathrm{~B}_{99}^{C}$, antarīkșāo $\mathrm{N}_{58}^{K}$, antarikșa ${ }^{\circ} \hat{S}_{67}^{S} \mathbf{P}^{\Sigma}$ • yāś ca ] $\Sigma$, ye ca $\mathrm{N}_{77}^{K o}$ 221b ḍākiṇyah ] $\mathrm{K}^{\Sigma} \mathrm{E}^{N} \mathrm{~B}_{99}^{C} \mathbf{P}^{\Sigma}$, ḍakiṃnya $\mathrm{N}_{77}^{K o}$, dāgiṇyah $\mathrm{N}_{45}^{C}$, saākinyah $\hat{S}_{67}^{S} \bullet$ svarga ${ }^{\circ}$ ] $\Sigma$, sarga ${ }^{\circ} \mathrm{N}_{12}^{K} \bullet{ }^{\circ}$ saṃsthitāḥ ] $\Sigma$, ${ }^{\circ}$ saṃsthitā $\mathrm{N}_{82}^{K} \quad$ 221c pātāle ] $\Sigma$, pātale $\mathrm{N}_{58}^{K} \bullet$ yās tu ḍākiṇyo ] $\mathrm{K}^{\Sigma} \mathrm{B}_{99}^{C}$, ${ }^{\circ}$ sampsthitā yāś ca $\mathrm{N}_{77}^{K o}$, bhūtale yās tu $\mathrm{N}_{45}^{C}$, yās tu ḍākiṇyā $\mathrm{E}^{N}$, bhūtale yāś ca $\mathbf{P}^{\Sigma}$, yās tu śākinyo $S_{67}^{S} \quad$ 221d ${ }^{\circ}$ durgeșu ] $\Sigma$, ${ }^{\circ}$ durggaṣu $E^{N} \bullet$ yāḥ sthitāh ] $\Sigma$, yā sthitā $\mathrm{N}_{77}^{K o}$, yā sthitāḥ $\mathrm{N}_{45}^{C} \mathrm{~N}_{58}^{K} \mathrm{~B}_{99}^{C} \quad$ 222a tṛtīyam ] $\Sigma$, tṛtīya ${ }^{\circ} \mathrm{E}^{N}$ • yasya ] $\Sigma$, yasyā $\mathrm{N}_{45}^{C} \quad$ 222b ' ${ }^{\circ}$ śūlaṃ bhasma bhāsuram ] $\mathrm{N}_{77}^{K o} \mathrm{~N}_{82}^{K} \mathrm{~N}_{12}^{K} \mathrm{E}^{N} \mathrm{~B}_{99}^{C} \dot{S}_{67}^{S} \mathrm{P}_{32}^{T}$, ósūlakarapahlave $\mathrm{N}_{45}^{C}$, ○śūla bhaśma bhāsuraṃ $\mathrm{N}_{58}^{K}$, 'śūlaṃ yasya bhāsuram $\mathrm{P}_{72}^{T} \quad \mathbf{2 2 2 c}$ tejasā tasya ] $\Sigma$, tejasāntasya $\mathrm{N}_{45}^{C} \quad$ 222d śāntim ] $\Sigma$, śānti $\mathrm{N}_{45}^{C} \bullet$ sadā ] $\Sigma$, sadāḥ $\mathrm{N}_{45}^{C} \quad$ 223a sarve ] $\Sigma$, sarvva ${ }^{\circ} \mathrm{E}^{N} \bullet{ }^{\circ}$ rūpāḥ $\Sigma$, ${ }^{\circ}$ rūpā $\mathrm{N}_{77}^{K o} \mathrm{~N}_{45}^{C},{ }^{\circ}$ vīryāḥ $\hat{S}_{67}^{S} \quad \mathbf{2 2 3}$ b sarve ] $\Sigma$, sarvva ${ }^{\circ} \mathrm{E}^{N} \bullet$ mahojjvalāḥ ] $\mathrm{N}_{82}^{K} \mathrm{E}^{N} \mathrm{~B}_{99}^{C} \mathbf{P}^{\Sigma}$, mahojvalā $\mathrm{N}_{77}^{K o}$, manojvalā $\mathrm{N}_{45}^{C}$, manojvalāḥ $\mathrm{N}_{12}^{K}$, mahojjvalā $\mathrm{N}_{58}^{K} \hat{S}_{67}^{S} \quad$ 223c sarve ] $\mathrm{N}^{\Sigma} \hat{S}_{67}^{S} \mathrm{P}_{72}^{T}$, sarvva ${ }^{\circ} \mathrm{E}^{N} \bullet$ bhūtāḥ sthitāḥ saumyāh ] $\mathrm{N}_{82}^{K} \mathrm{E}^{N} \mathrm{~B}_{99}^{C}$, bhūtaśitā saumyā $\mathrm{N}_{77}^{K o}$, bhūtā sthitā saumyāḥ $\mathrm{N}_{45}^{C}$, bhūtāḥ śitā+ḥ+ saumyāḥ $\mathrm{N}_{12}^{K}$, bhūtā +sthitā+ḥ saumyāḥ $\mathrm{N}_{58}^{K}$, bhūtā mahāsaumyāḥ $S_{67}^{S}$, bhūtāḥ sthitā ye tra $\mathrm{P}_{72}^{T} \quad$ 223d sarve bhūtā manojavāh ] $\mathrm{N}_{45}^{C} \mathrm{~K}^{\Sigma} \mathrm{B}_{99}^{C} \hat{S}_{67}^{S}$, sarve bhūtā manojavā $\mathrm{N}_{77}^{K o}$, sarve bhūtamanojvalāh $\mathrm{E}^{N}$, bhūtā ye nye paratra ca $\mathrm{P}_{72}^{T} \quad \mathbf{2 2 4 a}$ antarīkṣe ] $\Sigma$, antarikṣe $\hat{\mathrm{S}}_{67}^{S} \mathrm{P}_{72}^{T} \quad \mathbf{2 2 4} \mathbf{b}$ divi ] $\Sigma$, dikṣu $\mathrm{E}^{N} \bullet$ saṃsthitāḥ ] $\Sigma$, saṃsthitā $\mathrm{N}_{77}^{K o} \mathrm{~B}_{99}^{C} \quad$ 224c pātāle ] $\Sigma$, pātāla ${ }^{\circ} \mathrm{N}_{77}^{K o} \bullet$ ye tu ] $\Sigma$, ye ca $\mathrm{N}_{45}^{C}$, bhūtā $\mathrm{N}_{58}^{K} \quad$ 224d bhūtā $\Sigma$, bhūtābhūtā $\mathrm{N}_{45}^{C}$ (unmetr.), ye tu $\mathrm{N}_{82}^{K} \bullet$ bhūtividhāyikāḥ ] $\mathrm{N}_{82}^{K} \mathrm{~N}_{12}^{K} \mathrm{E}^{N} \mathrm{~B}_{99}^{C}$, bhūtavidhāyikā $\mathrm{N}_{77}^{K o}$, bhūtividhāyikaḥ $\mathrm{N}_{45}^{C}$, bhūtividhāyinaḥ $\mathrm{N}_{58}^{K} \hat{S}_{67}^{S} \mathbf{P}^{\Sigma}$ 
khațvāngaṃ vimalam yasya triśūlaṃ bhasma bhāsuram|

tejasā tasya devasya śāntiṃ kurvantu me sadā|| 225||

\section{[Pretas]}

pretāḥ pretagaṇāḥ sarve ye pretāḥ sarvatomukhāḥ| atidīptāś ca ye pretā ye pretā rudhirāśanāḥ|| 226||

antarīkṣe ca ye pretā ye pretāḥ svargavāsinaḥ| pātāle bhūtale pretā ye pretāḥ kāmarūpiṇaḥ|| 227||

śmaśāne nilayo yasya vṛ̣abho yasya vāhanam| tejasā tasya devasya śāntiṃ kurvantu me sadā|| 228||

226-228 Cf. BhavP 1.180.25-27 : pretāḥ pretagaṇāḥ sarve ye pretāḥ sarvatomukhāḥ| atidīptāś ca ye pretā ye pretā rudhirāśanāḥ|| antarikṣe ca ye pretās tathā ye svargavāsinaḥ| pātāle bhūtale vāpi ye pretāḥ kāmarūpiṇaḥ|| ekacakro ratho yasya yas tu devo vṛṣadhvajạ̣| tejasā tasya devasya śāntiṃ kurvantu te sadā ||

227a-229d Omitted in $\mathrm{B}_{99}^{C}$, but added in margin by a second hand $\left(=\mathrm{B}_{99}^{C p c}\right) \quad \mathbf{2 2 8} \mathbf{a}-\mathbf{2 2 9} \mathbf{b}$ Omitted in $\mathrm{P}_{72}^{T}$

225a khaṭvāngam ] $\mathrm{N}_{77}^{K o} \mathrm{~N}_{45}^{C} \mathrm{~N}_{82}^{K} \mathrm{~N}_{58}^{K} \mathrm{~B}_{99}^{C} \mathrm{P}_{32}^{T}$, khațvaṃga $\mathrm{N}_{12}^{K}$, khațvāñkāṃ $\mathrm{E}^{N}$ • vimalam yasya ] $\mathrm{N}_{82}^{K} \mathrm{~N}_{58}^{K} \mathrm{E}^{N} \mathrm{~B}_{99}^{C}$, yasya vimalam $\mathrm{N}_{77}^{K o} \mathrm{~N}_{45}^{C} \mathrm{~N}_{12}^{K} \dot{S}_{67}^{S} \mathbf{P}^{\Sigma} \quad$ 225b bhasma bhāsuram ] $\mathrm{N}_{82}^{K} \mathrm{E}^{N} \mathrm{~B}_{99}^{C}$, karapalave $\mathrm{N}_{77}^{K o}$, karapahlave $\mathrm{N}_{45}^{C}$, karapallave $\mathrm{N}_{12}^{K} \hat{S}_{67}^{S} \mathbf{P}^{\Sigma}$, bhasma bhāsvaram $\mathrm{N}_{58}^{K} \quad \mathbf{2 2 5}^{\mathrm{c}}$ tejasā tasya ] $\Sigma$, tejasāntasya $\mathrm{N}_{45}^{C} \quad \mathbf{2 2 5}^{\mathrm{d}}$ śāntim ] $\quad \Sigma$ śānti $\mathrm{B}_{99}^{C} \quad \mathbf{2 2 6 a}$ pretāḥ ] $\Sigma$, pretā $\mathrm{N}_{77}^{K o} \mathrm{~N}_{45}^{C} \bullet{ }^{\circ}$ gaṇāḥ ] $\Sigma$, ${ }^{\circ}$ gaṇā $\mathrm{N}_{77}^{K o} \mathrm{~N}_{58}^{K a c} \quad \mathbf{2 2 6 b}$ ye pretāḥ ] $\Sigma$ ye pretā $\mathrm{N}_{77}^{K o}$, +ye+ pretāh $\mathrm{N}_{5^{K}}^{K} \bullet$ sarvatomukhāḥ ] $\Sigma$, sarvatomukhā $\mathrm{N}_{77}^{K o}$, sarvadinmukhāḥ $\mathrm{E}^{N}$ 226c atidīptāś ca ye pretā ] $\mathrm{N}_{77}^{K o} \mathrm{~N}_{45}^{C} \mathrm{~N}_{82}^{K \text { pc }} \mathrm{E}^{N} \mathrm{~B}_{99}^{C} \mathbf{P}^{\Sigma}$, atidīptā ye pretā $\mathrm{N}_{82}^{K a c}$ (unmetr.), atidīptaś ca ye pretā $\mathrm{N}_{12}^{K}$, atidīptā ca ye pretā $\mathrm{N}_{58}^{K}$, atidīptasthale prītāh $\hat{S}_{67}^{S} \quad \mathbf{2 2 6 d}$ ye pretā ] $\Sigma$, om. $\mathrm{N}_{82}^{K a c}$ (unmetr.), yasyaitā $S_{67}^{S} \bullet$ rudhirāśanāḥ ] $\mathbf{K}^{\Sigma} \mathrm{E}^{N} \mathbf{P}^{\Sigma}$, rudhirāśanā $\mathrm{N}_{77}^{K o}$, rudhirāsanā $\mathrm{N}_{45}^{C}$, ruhirāśayah $\mathrm{B}_{99}^{C}$, rudhirās sadā $\hat{S}_{67}^{S} \quad$ 227a antarīkṣe ca ] $\mathbf{N}^{\Sigma} \mathrm{E}^{N} \mathrm{~B}_{99}^{C p c}$, antarīkṣe tu $\mathrm{P}_{32}^{T}$, antarikșe ca $\hat{S}_{67}^{S} \mathrm{P}_{72}^{T} \quad$ 227b pretāh ] $\Sigma$, pretā $\mathrm{N}_{77}^{K o} \mathrm{~N}_{45}^{C} \mathrm{~B}_{99}^{C p c}$ - ${ }^{\circ}$ vāsinaḥ ] $\mathrm{N}_{45}^{C} \mathrm{~N}_{82}^{K} \mathrm{~N}_{58}^{K} \mathrm{E}^{N} \mathrm{~B}_{99}^{C p c} \mathrm{P}_{32}^{T}$, ${ }^{\circ}$ saṃsthitā $\mathrm{N}_{77}^{K o}$, ${ }^{\circ}$ vāsivāsinah $\mathrm{N}_{12}^{K}$ (unmetr.), ogāminaḥ $\hat{S}_{67}^{S}$, o saṃsthitāḥ $\mathrm{P}_{72}^{T} \quad$ 227c bhūtale pretā ] $\mathbf{N}^{\Sigma} \hat{S}_{67}^{S} \mathrm{P}_{3^{2}}^{T}$, bhūtale pretāḥ $\mathrm{B}_{99}^{C p c}$, bhūtapretā ye $\mathrm{E}^{N} \quad$ 227d ye pretāh ] $\mathrm{N}_{12}^{K} \mathrm{~N}_{58}^{K} \mathrm{E}^{N} \hat{S}_{67}^{S} \mathrm{P}_{32}^{T}$, ye pretā $\mathrm{N}_{77}^{K o} \mathrm{~N}_{45}^{C} \mathrm{~N}_{82}^{K} \mathrm{~B}_{99}^{C p c}$, ye ye pretāḥ $\mathrm{P}_{72}^{T}$ (unmetr.) 228a śmaśāne ] $\mathrm{K}^{\Sigma} \mathrm{E}^{N} \mathrm{~B}_{99}^{C p c} S_{67}^{S}$, śmaśāna ${ }^{\circ} \mathrm{N}_{77}^{K o} \mathrm{P}_{3^{2}}^{T}$, śmaśānaṃ $\mathrm{N}_{45}^{C} \bullet$ nilayo ] $\mathrm{N}^{\Sigma} \mathrm{E}^{N} \mathrm{~B}_{99}^{C p c} \mathrm{P}_{32}^{T}$, nilayam $\mathrm{N}_{58}^{K} \hat{S}_{67}^{S} \quad \mathbf{2 2 8 b}$ vāhanam ] $\mathrm{N}_{82}^{K} S_{67}^{S} \mathrm{P}_{32}^{T}$, vāhanah $\mathrm{N}_{77}^{K o} \mathrm{~N}_{45}^{C} \mathrm{~N}_{12}^{K} \mathrm{~N}_{5^{K}}^{K} \mathrm{E}^{N} \mathrm{~B}_{99}^{C p c} \quad \mathbf{2 2 8 c}$ tejasā tasya ] $\mathrm{N}_{77}^{K o} \mathrm{~K}^{\Sigma} \mathrm{E}^{N} \mathrm{~B}_{99}^{C p c} \mathrm{P}_{32}^{T}$, tejasāntasya $\mathrm{N}_{45}^{C} \quad \mathbf{2 2 8 d}$ śāntiṃ kurvantu me sadā ] $\mathrm{N}^{\Sigma} \mathrm{E}^{N} \mathrm{~B}_{99}^{C_{\mathrm{pc}}} \mathrm{P}_{3^{2}}^{T}$, śāntim āśu karotu me $\mathrm{N}_{5^{K}}^{K} \hat{\mathrm{S}}_{67}^{S}$ 
[Piśācas]

ye piśācā mahāvīryā ṛddhimanto mahābalāḥ| nānārūpadharāḥ sarve sarve ca guṇavattarāḥ|| 229||

antarīkṣe piśācā ye svarge ye ca piśācakāḥ| bhūpātāle piśācāś ca bahurūpā manojavāḥ|| 23o||

candrārdhaṃ mastake yasya gan̉gā yena jațādhṛtā| tejasā tasya devasya śāntiṃ kurvantu me sadā|| 231||

229-231 Cf. BhavP 1.18o.28-30 : ye piśācā mahāvīryā vṛddhimanto mahābalāḥ| nānārūpadharāḥ sarve sarve ca guṇavattarāḥ|| antarikṣe piśācā ye svarge ye ca mahābalāḥ| pātāle bhūtale ye ca bahurūpā manojavāḥ|| yasyāhaṃ sārathir vīra yasya tvaṃ turagaḥ sadā| tejasā tasya devasya śāntiṃ kurvantu te 'ñjasā||

229a-231 $\mathrm{P}_{32}^{T}$ has this after 223 229b After this $\mathrm{N}_{5^{K}}^{K}$ adds four pādas : piśācā ye mahāvīryā piśācā ye maharddhikāḥ| piśācā ye mahātmānaḥ piśācāḥ kāmarūpiṇaḥ| ２3od After this $\mathrm{P}_{32}^{T}$ adds two pādas reading : nānārūpadharāḥ sarve sarve ca guṇavattarāḥ (cf. 229cd)

229a ${ }^{\circ}$ vīryā ] $\mathbf{N}^{\Sigma} \mathrm{E}^{N} \mathrm{~B}_{99}^{\text {Cpc }},{ }^{\circ}$ vīryāḥ $\mathrm{P}_{32}^{T},{ }^{\circ}$ vīrā $S_{67}^{S} \quad$ 229b ṛddhimanto mahābalāh ] $\mathrm{N}_{82}^{K} \mathrm{~N}_{58}^{K} \mathrm{E}^{N}$, riddhivantodyataprabhā $\mathrm{N}_{77}^{K o}$, ruddhimanto dyutiprabhāḥ $\mathrm{N}_{45}^{C} \hat{\mathrm{S}}_{67}^{S}$, r rddhivanto mahābalāḥ $\mathrm{N}_{12}^{K} \mathrm{~B}_{99}^{C p c}$, ye piścācā maharddhikāḥ $\mathrm{P}_{32}^{T} \quad$ 229c nānārūpadharāḥ sarve ] $\mathrm{N}_{45}^{C} \mathrm{~N}_{12}^{K} \mathrm{~N}_{5^{8}}^{K} \mathrm{E}^{N} \mathrm{~B}_{99}^{C p c} \mathrm{P}_{72}^{T}$, nānārūpadharā sarve $\mathrm{N}_{77}^{K o} \mathrm{~N}_{82}^{K}$, ye piśācāḥ mahātmāno $S_{67}^{S}$, ye piśācā mahātmānạ̣ $\mathrm{P}_{3^{2}}^{T} \quad$ 229d sarve ca guṇavattarāḥ ] $\mathrm{N}_{45}^{C} \mathrm{~K}^{\Sigma} \mathrm{E}^{N}$, sarve ca guṇavattarā $\mathrm{N}_{77}^{K o}$, sarvve sarvve ca guṇavattarāḥ $\mathrm{B}_{99}^{C p c}$ (unmetr.), ye pi te kāmarūpiṇaḥ $S_{67}^{S}$, piśācāḥ kāmarūpinaḥ $\mathrm{P}_{32}^{T}$, piśācā gunavattarāḥ $\mathrm{P}_{72}^{T} \quad 23$ oa antarīkṣe $] \mathrm{K}^{\Sigma} \mathrm{E}^{N} \mathrm{~B}_{99}^{C}$, antarikṣe $\mathrm{N}_{77}^{K o} \mathrm{~N}_{45}^{C} \hat{S}_{67}^{S} \mathbf{P}^{\Sigma} \bullet$ piśácā ye ] $\Sigma$, piśāca ye $\mathrm{N}_{77}^{K o} \quad$ 23ob svarge ye ca ] $\Sigma$, ye svarge pi $\hat{S}_{67}^{S}$. piśācakāḥ ] $\Sigma$, piśācakā $\mathrm{N}_{77}^{K o} \quad 230 c$ bhūpātāle piśācāś ca ] $\mathrm{K}^{\Sigma} \mathrm{E}^{N} \mathrm{~B}_{99}^{C}$, bhūtale ye piśācāś ca $\mathrm{N}_{77}^{K o} \mathbf{P}^{\Sigma}$, bhūpātāle piśācā ye $\mathrm{N}_{45}^{C} \hat{S}_{67}^{S} \quad$ 23od bahurūpā manojavāḥ ] $\Sigma$, bahurūpā manojavā $\mathrm{N}_{77}^{K o}$, pātālatalavāsinaḥ $\mathrm{P}_{32}^{T} \quad$ 231a candrārdham ] $\mathrm{N}_{12}^{K} \mathrm{E}^{N} \mathrm{~B}_{99}^{C} \dot{S}_{67}^{S} \mathbf{P}^{\Sigma}$, candrārddha ${ }^{\circ} \mathrm{N}_{77}^{K o} \mathrm{~N}_{45}^{C} \mathrm{~N}_{82}^{K} \mathrm{~N}_{58}^{K} \bullet$ mastake ] $\Sigma$, ${ }^{\circ}$ mastako $\mathrm{N}_{45}^{C}$, ${ }^{\circ}$ mastakam $\mathrm{N}_{58}^{K} \quad$ 231b gañgā yena jațādhṛtā ] $\mathrm{N}_{82}^{K} \mathrm{~N}_{5^{8}}^{K} \mathrm{E}^{N}$, yasyāciṃntyā śivā tanu $\mathrm{N}_{77}^{K o}$, yasya cinto tanuḥ śivaḥ $\mathrm{N}_{45}^{C}$, gañgā yena jațā dhṛtāḥ $\mathrm{N}_{12}^{K} \mathrm{~B}_{99}^{C}$, bhasma yasya vibhūṣaṇam $\mathbf{P}^{\Sigma}$, yasya bhasma vibhūṣaṇam $\hat{S}_{67}^{S}$ 231c tejasā tasya devasya ] $\Sigma$, tejasāntasya deva+sya+ $\mathrm{N}_{45}^{C} \quad$ 231d śāntiṃ ] $\Sigma$, śānti $\mathrm{N}_{58}^{K}$ 


\section{[Grahas]}

apasmāragrahāḥ sarve sarve cāpi jvaragrahāḥ| garbhabālagrahā ye tu nānārogagrahāś ca ye|| 232||

antarīkṣe grahā ye tu svarge ye tu grahottamāḥ| bhūpātāle grahā ye tu ye grahāḥ sarvatodiśaḥ|| 233||

kaṇṭhe yasya mahānīlaṃ bhūṣaṇaṃ yasya pannagaḥ| tejasā tasya devasya śāntiṃ kurvantu me sada|| 234||

232-234 Cf. BhavP 1.180.31-33 : apasmāragrahāḥ sarve sarve cāpi jvaragrahāḥ| ye ca svargasthitāḥ sarve bhūmigā ye grahottamāḥ| pātāle tu grahā ye ca ye grahāḥ sarvato gatāḥ| dakṣiṇe kiraṇe yasya sūryasya ca sthito hariḥ|| haro yasya sadā vāme lalāte kañjajạ̣ sthitạ̣| tejasā tasya devasya śāntiṃ kurvantu te sadā||

232a apasmāra ${ }^{\circ}$ ] $\Sigma$, apasmārā $\mathrm{P}_{32}^{T} \bullet{ }^{\circ}$ grahāḥ ] $\Sigma$, o grahā $\mathrm{N}_{77}^{K o} \mathrm{~N}_{45}^{C} \quad$ 232b jvaragrahāḥ ] $\Sigma$, jvaragrahā $\mathrm{N}_{77}^{K o}$, grahajvarāḥ $S_{67}^{S} \quad$ 232c tu ] $\mathrm{N}_{77}^{K o} \mathrm{~N}_{82}^{K} \mathrm{E}^{N} \mathrm{~B}_{99}^{C} \mathbf{P}^{\Sigma}$, ca $\mathrm{N}_{45}^{C} \mathrm{~N}_{12}^{K} \mathrm{~N}_{58}^{K} \dot{S}_{67}^{S} \quad$ 232d nānārogagrahāś ca ] $\mathrm{N}_{77}^{K o} \mathrm{~N}_{82}^{K} \mathrm{~N}_{58}^{K} \mathrm{E}^{N} \mathrm{~B}_{99}^{C} \hat{S}_{67}^{S} \mathrm{P}_{32}^{T}$, nānārogā grahāś ca $\mathrm{N}_{45}^{C}$, nānārūpadharāś ca $\mathrm{N}_{12}^{K}$, nānārogagrahās tu $\mathrm{P}_{72}^{T} \quad$ 233a antarīkṣe ] $\mathrm{K}^{\Sigma} \mathrm{E}^{N} \mathrm{~B}_{99}^{C}$, antarikșa ${ }^{\circ} \mathrm{N}_{77}^{K o}$, antarikșe $\mathrm{N}_{45}^{C} \dot{S}_{67}^{S} \mathbf{P}^{\Sigma} \bullet$ tu ] $\mathrm{N}_{77}^{K o} \mathrm{~N}_{45}^{C} \mathrm{~N}_{82}^{K} \mathrm{E}^{N} \mathrm{~B}_{99}^{C} \mathbf{P}^{\Sigma}$, ca $\mathrm{N}_{12}^{K} \mathrm{~N}_{58}^{K} \dot{S}_{67}^{S} \quad$ 233b svarge ye tu ] $\mathrm{N}_{77}^{K o} \mathrm{~N}_{82}^{K} \mathrm{E}^{N} \mathrm{~B}_{99}^{C} \mathrm{P}_{72}^{T}$, svargga ye $($ tu $) \mathrm{N}_{45}^{C}$, ye ca svarge $\mathrm{P}_{32}^{T}$, svarge ye ca $\mathrm{N}_{58}^{K} \hat{S}_{67}^{S} \bullet$ grahottamāh ] $\Sigma$, grahottamā $\mathrm{N}_{77}^{K o} \quad{ }_{233 c}$ bhūpātāle grahā ] $\Sigma$, bhūpātālagrahā $\mathrm{N}_{77}^{K o}$, pātāle bhūtale $\mathrm{P}_{72}^{T} \bullet$ ye tu ] $\mathrm{N}_{77}^{K o} \mathrm{~K}^{\Sigma} \mathrm{E}^{N} \mathrm{~B}_{99}^{C} \mathrm{P}_{72}^{T}$, sarve $\mathrm{N}_{45}^{C}$, ye ca $\hat{S}_{67}^{S} \mathrm{P}_{32}^{T} \quad$ 233d grahāḥ sarvatodiśah ] $\mathrm{N}_{82}^{K} \mathrm{~N}_{58}^{K} \mathrm{E}^{N} \mathrm{~B}_{99}^{C} \mathrm{P}_{72}^{T}$, grahā sarvatodisah $\mathrm{N}_{77}^{K o}$, grahā sarvacoditāḥ $\mathrm{N}_{45}^{C}$, grahās sarvatoditāḥ $\mathrm{N}_{12}^{K}$, grahāḥ sarvato diśi $\mathrm{P}_{32}^{T}$, grahāḥ sarvatạ sthitāḥ $\hat{S}_{67}^{S} \quad$ 234a kaṇthe ] $\mathrm{N}^{\Sigma} \mathrm{E}^{N} \mathrm{~B}_{99}^{C} \mathrm{P}_{32}^{T}$, kaṇtho $S_{67}^{S}$, kaṇtham $\mathrm{P}_{72}^{T} \bullet{ }^{\circ}$ nìlam ] $\mathrm{N}_{77}^{K o} \mathrm{~K}^{\Sigma} \mathrm{E}^{N} \mathrm{~B}_{99}^{C} \mathrm{P}_{72}^{T}$, ${ }^{\circ}$ nìyam $\mathrm{N}_{45}^{C}$, ${ }^{\circ}$ nìla ${ }^{\circ}$ $\mathrm{P}_{32}^{T}$, ${ }^{\circ}$ kălo $\stackrel{\mathrm{S}}{67}_{67}^{S} \quad$ 234b pannagah ] $\mathrm{N}_{82}^{K} \mathrm{~N}_{12}^{K} \mathrm{E}^{N} \mathrm{~B}_{99}^{C}$, pamnagam $\mathrm{N}_{77}^{K o} \mathrm{~N}_{45}^{C}$, pannañgam $\mathrm{N}_{58}^{K}$ (unmetr.), pannagāḥ $\hat{S}_{67}^{S} \mathbf{P}^{\Sigma} \quad{ }_{234 c}$ tejasā tasya ] $\Sigma$, tejasāntasya $\mathrm{N}_{45}^{C}$, tejasā tasa $\mathrm{N}_{12}^{K}$ 234d śāntiṃ ] $\Sigma$, śānti $\mathrm{N}_{77}^{K o}$, paușțim $\mathrm{N}_{58}^{K}$ 


\section{[All Deities]}

iti devādayaḥ sarve śivājñānuvidhāyinaḥ|

kurvantu jagatạ̣ śāntiṃ śivabhakteṣu sarvadā|| 235||

[Jaya]

jayātmayogasaṃsthāya jaya saṃśuddhacetase| jaya dānaikaśūrāya jayeśāya namo 'stu te|| 236||

jayottamāya devāya jaya kalyāṇakāriṇe| jaya prakațadehāya jaya japyāya te namaḥ|| 237||

235 Cf. BhavP 1.180.34 : iti devādayah sarve sūryayajñavidhāyinah| kurvantu jagatah śāntiṃ sūryabhakteșu sarvadā|| 236-242 Cf. BhavP 1.180.35-39: jayah sūryāya devāya tamohantre vivasvate| jayapradāya sūryāya bhāskarāya namo 'stu te|| grahottamāya devāya jayaḥ kalyāṇakāriṇe| jayaḥ padmavikāśāya budharūpāya te namaḥ|| jayaḥ dīptividhānāya jayaḥ śāntividhāyine| tamoghnāya jayāyaiva ajitāya namo namaḥ|| jayārka jaya dīptīśa sahasrakiraṇojjvala| jaya nirmitalokas tvam ajitāya namo namaḥ|| gāyatrīdeharūpāya sāvitrīdayitāya ca| dharādharāya sūryāya mārtạ̣ḍaya namo namaḥ||

235d After this $\hat{S}_{67}^{S}$ adds ten pādas : ittham nānāvidhair rūpaị̣ sthāvarair jañgamair api| krị̄̂ayā praș̣to nityam eka eva śivaḥ prabhuḥ| śāntiṃ karotu me nityam āyuḥ kalyāṇam eva ca| sampadam bhogamokṣau ca ya icched ātmane janaḥ| stotram mamaitat sa naraḥ śṛ̣otu ca paṭhed api| $\bullet \mathrm{P}_{32}^{T}$ adds four pādas : ity uktvā devadevasya saṃnidhau ca kṛtāṃjaliḥ| śivadhyānaparo bhūtvā bhaktyā stotram udairayat| $\bullet \mathrm{P}_{72}^{T}$ adds : ity uktvā devadevasya sannidhau ca kṛtāñjalị̣| śivadhyānaparo bhūtvā bhaktyā stotram udīrayan|

235a devādayaḥ ] $\Sigma$, devādaya $\mathrm{N}_{77}^{K o}$, devāyaḥ $\mathrm{N}_{77}^{C a c} \mathrm{~N}_{5^{8}}^{K}$ (unmetr.) $\quad$ 235b śivājñānu ${ }^{\circ}$ ] $\mathrm{N}_{82}^{K} \mathrm{E}^{N} \mathrm{~B}_{99}^{C} \hat{S}_{67}^{S} \mathrm{P}_{72}^{T}$, śivā jñāna ${ }^{\circ} \mathrm{N}_{77}^{K o}$, śivajñāna ${ }^{\circ} \mathrm{N}_{45}^{C} \mathrm{~N}_{58}^{K}$, śivajñānu ${ }^{\circ} \mathrm{N}_{12}^{K}$, śivadhyāna ${ }^{\circ} \mathrm{P}_{32}^{T}$ 235c jagatah śāntiṃ ] $\mathrm{K}^{\Sigma} \mathrm{E}^{N} \mathrm{~B}_{99}^{C}$, sānti jagatạ̣ $\mathrm{N}_{77}^{K o}$, jagata śāntiṃ $\mathrm{N}_{45}^{C}$ (unmetr.), śāntiṃ jagatah $S_{67}^{S} \mathbf{P}^{\Sigma} \quad$ 235d sarvadā ] $\Sigma$, sarvadāḥ $\mathrm{N}_{45}^{C} \quad \mathbf{2 3 6 a}$ jayātma ${ }^{\circ}$ ] $\Sigma$, jayātmāo $\mathrm{N}_{45}^{C} \mathrm{~N}_{12}^{K a c} \quad 236 b$ jaya saṃśuddhacetase ] $\mathrm{N}_{82}^{K} \mathrm{~N}_{58}^{K} \mathrm{E}^{N} \mathrm{~B}_{99}^{C} S_{67}^{S} \mathrm{P}_{32}^{T}$, saṃśuddhānantatejasā $\mathrm{N}_{77}^{K o}$, ja saṃśuddhatejase $\mathrm{N}_{45}^{C}$ (unmetr.), jaya saṃśuddhatejase $\mathrm{N}_{45}^{C} \mathrm{~N}_{12}^{K} \hat{\mathrm{S}}_{67}^{S}$, jaya saṃsiddhacetase $\mathrm{P}_{72}^{T} \quad$ 236cd ${ }^{\circ}$ śūrāya jayeśāya ] $\mathrm{N}_{77}^{K o} \mathrm{~K}^{\Sigma} \mathrm{B}_{99}^{C} \mathrm{P}_{32}^{T}$, ${ }^{\circ}$ sūrāyah īśānāya $\mathrm{N}_{45}^{C}$, ósūleśāya jaleśāya $\mathrm{E}^{N}$, ośūrāya jayeśāna $S_{67}^{S} \mathrm{P}_{72}^{T} \quad 237$ a jayottamāya $] \Sigma$, jayotamāya $\mathrm{N}_{77}^{K o}$ 237b okāriṇe ] $\mathrm{N}_{45}^{C} \mathrm{~K}^{\Sigma} \mathrm{E}^{N} \mathrm{~B}_{99}^{C} \hat{S}_{67}^{S}$, odhāriṇe $\mathrm{N}_{77}^{K o}$, odāyine $\mathrm{P}_{32}^{T}$, okīrtaye $\mathrm{P}_{72}^{T} \quad 237 \mathrm{c}{ }^{\circ}$ dehāya ] $\Sigma$, odevāya $\mathrm{P}_{32}^{T} \quad{ }^{\circ} \mathbf{2 3 7}$ d jaya japyāya te namah ] $\mathrm{K}^{\Sigma} \mathrm{E}^{N} \mathrm{~B}_{99}^{C} \mathrm{P}_{32}^{T}$, jitajapyāya te namah $\mathrm{N}_{77}^{K o} \mathrm{~N}_{45}^{C} \mathrm{P}_{72}^{T}$, viśveśāya namo namaḥ $\hat{\mathrm{S}}_{67}^{S}$ 
jaya lakṣmīnidhānāya jaya kāntividhāyine|

jaya vākyaviśuddhāya ajitāya namo namaḥ|| 238||

jaya triśūlahastāya jaya khațvāngadhāriṇe|

jaya nirjitalokāya jaya rūpāya te namaḥ|| 239||

jaya kāntārdhadehāya jaya candrārdhadhāriṇe|

jaya devātidevāya jaya rudrāya te namaḥ|| 240||

jaya tribhuvaneśāya jaya vikhyātakīrtaye|

jayādhārāya sarvāya jaya kartre namo namaḥ|| 241||

jaya mokṣapradātāya ș̣̦țisaṃhārakāriṇe|

brahmaviṣnvindravandyāya śiva śāntāya te namaḥ|| 242||

238d After this $\mathrm{N}_{45}^{C}$ adds four pādas : jaya jñānaviśuddhāya sarvadhyāȳ̄ namo stu te| jaya sarvāya śuddhāya așțamūrtti namo stu te $\mid S_{67}^{s}$ adds : yajñajñānaviśuddhāya sarvadhyāyin sadāśiva| jaya sarvātmaśuddhāya așțamūrtte namo stu te| 240a-d These four pādas are omitted in $\mathrm{N}_{45}^{C}$, while $\hat{S}_{67}^{S}$ has them after 241d 241d After this $\hat{S}_{67}^{S}$ adds four pādas : jaya niṣkāladehāya jaya sarvārthakāriṇe| jaya nirdagdha(kau)māya jaya rudra namo stu te $\mid \bullet \mathrm{P}_{3^{2}}^{T}$ adds : jaya nirmaktadehāya jaya sarvārthakāriṇe| jaya manmathanāśāya īśānāya namo namaḥ| $\bullet \mathrm{P}_{72}^{T}$ adds : jaya nirmaladehāya jaya sarvārthakāriṇe| jaya manmathanāśāya îsāānāya namo stu te| $\quad$ 242a-d Omitted in $\mathrm{N}_{77}^{K o}, \hat{\mathrm{S}}_{67}^{S}$ and $\mathrm{P}_{72}^{T} \quad \mathbf{2 4 2 a b}$ Omitted in $\mathrm{P}_{32}^{T} \quad \mathbf{2 4 2 d}$ After this $\mathrm{P}_{32}^{T}$ adds two pādas : jaya jātaviśuddhāya sarvavyāpin namo 'stu te|

238a onidhānāya ] $\mathrm{N}_{77}^{K o} \mathrm{~N}_{82}^{K} \mathrm{~N}_{12}^{K} \mathrm{~B}_{99}^{C} \hat{S}_{67}^{S}$, ovidhānāya $\mathrm{N}_{45}^{C} \mathrm{~N}_{58}^{K} \mathbf{P}^{\Sigma}$, odhanādhyakșa $E^{N}$ 238b kāntividhāyine ] $\Sigma$, kāntividhāyin̄i $\mathrm{N}_{77}^{K o}$, kīrttividhāyine $\mathrm{N}_{58}^{K} \quad$ 238c vākyaviśuddhāya ] $\Sigma$, te vākyaśuddhāya $\mathrm{N}_{77}^{K o} \quad$ 239a jaya triśūlahastāya ] $\Sigma$, jaya triśūlahastāya $\mathrm{N}_{77}^{K o}$, namah triśūlahastā $\mathrm{N}_{45}^{C}$ (unmetr.) $\quad 239 \mathrm{c}$ nirjita $\left.{ }^{\circ}\right] \quad \Sigma$, nirmita ${ }^{\circ} \mathrm{P}_{32}^{T} \quad$ 24ob odhāriṇe ] $\mathrm{N}_{77}^{K o} \mathbf{K}^{\Sigma} \mathrm{E}^{N} \mathrm{~B}_{99}^{C} \mathbf{P}^{\Sigma}$, ${ }^{\circ}$ maulaye $\hat{\mathrm{S}}_{67}^{S} \quad$ 24oc devātio ] $\mathrm{N}_{82}^{K} \mathrm{~N}_{12}^{K} \mathrm{E}^{N} \mathrm{~B}_{99}^{C} \dot{S}_{67}^{S} \mathrm{P}_{72}^{T}$, devānta ${ }^{\circ} \mathrm{N}_{77}^{K o}$, devādhi ${ }^{\circ} \mathrm{N}_{58}^{K}$, devādi ${ }^{\circ} \mathrm{P}_{32}^{T} \quad$ 24od jaya rudrāya ] $\mathrm{N}_{77}^{K o} \mathrm{~N}_{82}^{K} \mathrm{~N}_{58}^{K} \mathrm{E}^{N} \mathrm{~B}_{99}^{C} \hat{S}_{67}^{S} \mathrm{P}_{72}^{T}$, jaya sarvātmane $\mathrm{N}_{12}^{K}$, mahādevāya $\mathrm{P}_{32}^{T} \quad \mathbf{2 4 1 a}$ jaya tri $\left.{ }^{\circ}\right] \Sigma$, jayastṛ ${ }^{\circ} \mathrm{N}_{77}^{K o} \quad \mathbf{2 4 1 b}$ jaya vikhyāta ${ }^{\circ}$ ] $\Sigma$, vikhyātāya ca $\mathrm{N}_{77}^{K o}$, japrakhyāta ${ }^{\circ} \mathrm{N}_{45}^{C}$ (unmetr.) 241c jayādhārāya ] $\mathrm{N}_{82}^{K p c} \mathrm{~N}_{12}^{K} \mathrm{~N}_{58}^{K} \hat{S}_{67}^{S} \mathbf{P}^{\Sigma}$, jayādharāya $\mathrm{N}_{77}^{K o} \mathrm{~N}_{45}^{C} \mathrm{~N}_{82}^{K a c}$, jaya dhārāya $\mathrm{E}^{N} \mathrm{~B}_{99}^{C} \bullet$ sarvāya ] $\mathrm{N}^{\Sigma} \mathrm{E}^{N} \mathrm{~B}_{99}^{C}$, devāya $\mathrm{P}_{3^{2}}^{T}$, śarvāya $\hat{S}_{67}^{S} \mathrm{P}_{72}^{T} \quad$ 241d kartre namo ] $\Sigma$, kāntāya te $\mathrm{N}_{45}^{C} \bullet$ namaḥ ] $\mathrm{N}^{\Sigma} \mathrm{E}^{N} \mathrm{~B}_{99}^{C p c}$, 'stu te $\mathrm{B}_{99}^{C a c} \hat{S}_{67}^{S} \mathbf{P}^{\Sigma} \quad$ 242a ${ }^{\circ}$ pradātāya ] $\mathrm{N}_{82}^{K} \mathrm{E}^{N}$, ${ }^{\circ}$ pradātre ca $\mathrm{N}_{45}^{C}$, ${ }^{\circ}$ pradānāya $\mathrm{N}_{12}^{K} \mathrm{~N}_{58}^{K} \mathrm{~B}_{99}^{C} \quad \mathbf{2 4 2 b}{ }^{\circ}$ kāriṇe ] $\mathrm{K}^{\Sigma} \mathrm{E}^{N} \mathrm{~B}_{99}^{C}$, ${ }^{\circ}$ kāraṇe $\mathrm{N}_{45}^{C} \quad \mathbf{2 4 2 c}$ brahmaviṣṇinindra ${ }^{\circ}$ ] $\mathrm{N}_{82}^{K} \mathrm{~B}_{99}^{C} \mathrm{P}_{3^{2}}^{T}$, brahmaviș̣nundra ${ }^{\circ} \mathrm{N}_{77}^{C p c}$, brahmāviṣnuundra ${ }^{\circ} \mathrm{N}_{77}^{C a c}$, brahmāviṣṇvendra ${ }^{\circ} \mathrm{N}_{58}^{K}$, brahmaviṣnvīndra ${ }^{\circ} \mathrm{N}_{12}^{K} \mathrm{E}^{N} \bullet{ }^{\circ}$ vandvāya ] $\mathrm{N}_{45}^{C} \mathrm{~K}^{\Sigma} \mathrm{E}^{N} \mathrm{~B}_{99}^{C}$, ${ }^{\circ}$ rūpāya $\mathrm{P}_{32}^{T} \quad \mathbf{2 4 2 d}$ śiva śāntāya te namạ̣ ] $\mathrm{N}_{12}^{K} \mathrm{E}^{N}$, śiva śānta namo 'stu te ] $\mathrm{N}_{45}^{C}$, śiva śāntāya namo stu te $\mathrm{N}_{82}^{K} \mathrm{~B}_{99}^{C}$ (unmetr.), śiva śāṃtāya namaḥ $\mathrm{N}_{58}^{K}$, jaya śāntāya te namạ̣ $\mathrm{P}_{32}^{T}$ 


\author{
[Śrutiphala]
}

ity evaṃ śāntikādhyāyaṃ yaḥ paṭhec chṛnuyād api|

sa vidhūyāśu pāpāni śivaloke mahīyate|| 243\|

kanyārthī labhate kanyāṃ jayakāmo jayaṃ labhet|

arthakāmo labhed arthān putrakāmaḥ sutān bahūn|

vidyārthī labhate vidyāṃ yogārthī yogam āpnuyāt|| 244||

yān yān prārthayate kāmān mānavaḥ śravaṇād iha|

tat sarvạ̣ śīghram āpnoti devānāṃ ca priyo bhavet|| 245||

243 Cf. BhavP 1.18o.49-5oab : ity evaṃ śāntikādhyāyaṃ yạ paṭhec chṛụuād api| vidhinā sarvalokas tu dhyāyamāno divākaram|| sa vijitya rạ̣e śatruṃ mānạ̣ ca paramạ̣ labhet |

244ef Omitted in $\mathrm{N}_{77}^{K o}, \mathrm{~N}_{45}^{C}$ and $\hat{S}_{67}^{S} \quad$ 244f ] After this $\mathrm{P}_{32}^{T}$ adds two pādas : garbhiṇī labhate putrạ̣ kanyā vindati satpatim|

243a ity evam ] $\mathrm{K}^{\Sigma} \mathrm{E}^{N} \hat{S}_{67}^{S} \mathrm{P}_{72}^{T}$, ityeva $\mathrm{N}_{77}^{K o} \mathrm{~B}_{99}^{c}$, itevam $\mathrm{N}_{45}^{c}$, ityetac $\mathrm{P}_{32}^{T} \bullet$ śāntikāo ] $\Sigma$, sāntikāo $\mathrm{N}_{77}^{K o}$, śāntika ${ }^{\circ} \mathrm{N}_{12}^{K} \quad \mathbf{2 4 3} \mathbf{b}$ yaḥ paṭhec chṛnuyād api ] $\mathbf{K}^{\Sigma} \mathrm{B}_{99}^{C} \hat{S}_{67}^{S} \mathrm{P}_{32}^{T}$, ya pațhe śrṇuyād api $\mathrm{N}_{77}^{K o}$, yah paṭhe śṛ̣uyād api $\mathrm{N}_{45}^{C}$, ya pațhec chṛnuyād api $\mathrm{E}^{N}$, yah pațhec chṛnuyāt tathā $\mathrm{P}_{72}^{T} \quad$ 243c sa vidhūyāśu pāpāni ] $\mathrm{N}_{82}^{K} \mathrm{~N}_{58}^{K} \mathrm{~B}_{99}^{C}$, vidhūya sarvapāpānā $\mathrm{N}_{77}^{K o}$, vihāya sarvapāpāni $\mathrm{N}_{45}^{C} \mathrm{~N}_{12}^{K} \mathrm{P}_{72}^{T}$, suvidhūyāśu pāpāni $\mathrm{E}^{N}$, vihāya sarvalokāṃś ca $S_{67}^{S}$, vidhūya sarvapāpāni $\mathrm{P}_{32}^{T} \quad$ 244a kanyārthī ] $\Sigma$, kanyāthī $\mathrm{N}_{77}^{K o} \bullet$ kanyāṃ ] $\Sigma$, kanyam $\mathrm{N}_{77}^{K o}$, kanyā $\mathrm{N}_{45}^{C} \quad \mathbf{2 4 4 b}$ labhet ] $\Sigma$, labhe $\mathrm{N}_{77}^{K o} \quad \mathbf{2 4 4 c}$ labhed ] $\Sigma$, bhaved $\mathrm{N}_{58}^{K} \mathrm{~B}_{99}^{C} \bullet$ arthān ] $\mathrm{N}_{45}^{C} \mathrm{~N}_{82}^{K} \mathrm{~N}_{58}^{K} \mathrm{E}^{N} \mathrm{~B}_{99}^{C} \hat{S}_{67}^{S}$, artha $\mathrm{N}_{77}^{K o}$, artham $\mathrm{N}_{12}^{K} \mathbf{P}^{\Sigma} \quad$ 244d 'kāmaḥ sutān bahūn ] $\mathrm{N}_{82}^{K} \mathrm{~N}_{58}^{K p c} \mathrm{~B}_{99}^{C}$, ${ }^{\circ}$ kāmo bahusutāṃ $\mathrm{N}_{77}^{K o}$, ${ }^{\circ}$ kamo sutān bahūn $\mathrm{N}_{45}^{C}$ (unmetr.), ${ }^{\circ}$ kāmo 'tha putravān $\mathrm{N}_{12}^{K}$, ${ }^{\circ}$ kāmāh sutān bahūn $\mathrm{N}_{58}^{K a c}$, ${ }^{\circ}$ kāmah sutābahūn $\mathrm{E}^{N}$, ${ }^{\circ}$ kāmah bahūn sutān $\mathrm{P}_{32}^{T}$, ${ }^{\circ}$ āmo bahūn sutān $S_{67}^{S} \mathrm{P}_{32}^{T}$, ${ }^{\circ}$ kāmo labhet sutān $\mathrm{P}_{72}^{T} \quad$ 244e vidyām ] $\mathrm{K}^{\Sigma} \mathrm{E}^{N} \mathbf{P}^{\Sigma}$, vidyā $\mathrm{B}_{99}^{C} \quad$ 245a yān yān ] $\mathrm{K}^{\Sigma} \mathrm{B}_{99}^{C} \mathbf{P}^{\Sigma}$, yo yạ̣ $\mathrm{N}_{77}^{K o} \mathrm{~N}_{45}^{C}$, yat yat $\mathrm{E}^{N}$, yo yān $\hat{S}_{67}^{S}$ - prārthayate ] $\Sigma$, kāmayate $S_{67}^{S} \mathrm{P}_{3^{2}}^{T} \bullet$ kāmān ] $\Sigma$, kāmaṃ $\mathrm{N}_{77}^{K o} \quad \mathbf{2 4 5}$ b mānavaḥ śravaṇād iha ] $\Sigma$, mānavaśravaṇād iha $\mathrm{N}_{45}^{C}$, manasā ca yathepsitān $\hat{S}_{67}^{S} \quad \mathbf{2 4 5}$ c tat sarvaṃ ] $\mathrm{N}_{77}^{K o} \mathrm{~N}_{45}^{C} \mathrm{~N}_{12}^{K} \mathrm{~N}_{58}^{K} \mathrm{E}^{N} \mathrm{~B}_{99}^{C} \mathrm{P}_{72}^{T}$, tatsarva $\mathrm{N}_{82}^{K}$, tān sarvān $\mathrm{P}_{32}^{T}$, tān sarvāṃs $S_{67}^{S}$ 
śrutvādhyāyam idaṃ punyaṃ saṃgrāmaṃ praviśen naraḥ| sa nirjityāhave śatrūn kalyānaiḥ paripūjyate|| 246\|

\author{
akṣayaṃ modate kālam atiraskṛtaśāsanaḥ| \\ vyādhibhir nābhibhūyeta putrapautrapratișțhitaḥ|| 247||
}

pațhyamānam idạ̣ puṇyaṃ yam uddiśya ca paṭhyate| na tasya rogā bādhante vātapittādisaṃbhavāḥ̣|| 248||

247-256 Cf. BhavP 1.18o.5ocd-6oab : akṣayaṃ modate kālam atiraskṛtaśāsanaḥ|| vyādhibhir nābhibhūyeta putrapautraih pratișțitaḥ| bhaved ādityasadṛśas tejasā prabhayā tathā|| yān uddiśya pațhed vīra vācako mānavo bhuvi| na pīḍate ca tai rogair vātapittakaphātmakaị̣|| nākāle maraṇaṃ tasya na sarpaiś cāpi daśyate| na viṣaṃ kramate dehe na jaḍāndhyaṃ na mūkatā|| na cotpattibhayaṃ tasya nābhicārakajaṃ bhavet| ye rogā ye mahotpātā ye 'hayaś ca mahāviṣāḥ| te sarve praśamaṃ yānti śravaṇād asya bhārata|| yat puṇyạ̣ sarvatīrthānāṃ gañgādīnāṃ viśeṣatạ̣| tat puṇyạ̣ koṭiguṇitạ̣ prāpnoti śravaṇādibhiḥ|| daśānāṃ rājasūyānām anyeșāṃ ca viśeșataḥ| jīved varṣaśataṃ sāgraṃ sarvavyādhivivarjitah|| goghnaś caiva kṛtaghnaś ca brahmahā gurutalpagaḥ| śaraṇāgatadīnārtamitraviśraṃbhaghātakaḥ|| duṣțạ̣ pāpasamācāraḥ pitṛhā mātṛhā tathā| śravaṇād asya pāpebhyo mucyate nātra saṃśayaḥ|| itihāsam imaṃ puṇyam agnikāryam anuttamam| na dadyāt kasyacid vīra mūrkhasya kaluṣātmanaḥ|| sūryabhakte sadā deyaṃ sūryeṇa kathitạ̣ purā|

246a śrutvādhyāyam idaṃ puṇyam ] $\mathrm{N}_{77}^{K o} \mathrm{~N}_{45}^{C} \mathrm{~N}_{12}^{K} \mathrm{~N}_{58}^{K} \mathrm{P}_{32}^{T}$, śāntyadhyāyam paṭhan yas tu $\mathrm{N}_{82}^{K}$, śāntyādhyāyam paṭhed yas tu $\mathrm{B}_{99}^{C}$, śāntyādhyāyam imam puṇyam $\hat{S}_{67}^{S}$, śāntyādhyāyaṃ pațhan yas tu $\mathrm{E}^{N}$, śrutvādhyāyam imaṃ puṇyam $\mathrm{P}_{72}^{T} \quad \mathbf{2 4 6 \mathbf { b }}$ saṃgrāmaṃ ] $\Sigma$, samgrāme $\mathrm{N}_{77}^{K o} \mathrm{E}^{N} \bullet$ praviśen narah ] $\mathrm{N}_{82}^{K} \mathrm{E}^{N} \mathrm{~B}_{99}^{C} \hat{\mathrm{S}}_{67}^{S} \mathrm{P}_{72}^{T}$, praviśe nụpah $\mathrm{N}_{77}^{K o}$, praviśen nṛpaḥ $\mathrm{N}_{45}^{C} \mathrm{~N}_{12}^{K} \mathrm{~N}_{58}^{K} \mathrm{P}_{3^{2}}^{T} \quad$ 246c sa nirjityāhave śatrūn ] $\mathrm{N}_{82}^{K} \mathrm{~N}_{58}^{K} \mathrm{E}^{N} \mathrm{~B}_{99}^{C}$, sa vijityā ca tāṃ śatrūṃ $\mathrm{N}_{77}^{K o}$, sarvaśatrū vinirjityāṃ $\mathrm{N}_{45}^{C}$, sa vijityāhave śatrūn $\mathrm{N}_{12}^{K}$, vinirjityāśu tān śatrūn $\mathrm{P}_{3^{2}}^{T}$, vinirjitya svakān śatrūn $\hat{S}_{67}^{S}$, sa nirjityākhilān śatrūn $\mathrm{P}_{72}^{T} \quad \mathbf{2 4 6 d}$ kalyāṇaih ] $\Sigma$, kalyāṇai $\mathrm{N}_{45}^{C}$, kalpāśaiḥ $\mathrm{E}^{N} \bullet$ paripūjyate ] $\mathrm{N}_{45}^{C} \mathrm{~K}^{\Sigma} \mathrm{E}^{N}$, paripūryate $\mathrm{N}_{77}^{K o} \mathrm{~B}_{99}^{C} \mathrm{P}_{32}^{T}$, paripūritah

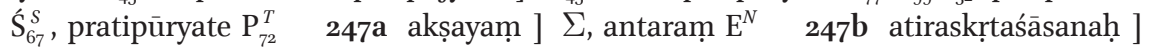
$\mathrm{N}_{82}^{K} \mathrm{~N}_{58}^{K} \mathrm{E}^{N} \mathrm{~B}_{99}^{C} \mathrm{P}_{32}^{T}$, atiraskṛtaśāsane $\mathrm{N}_{77}^{K o}$, atiraskṛtamānavaḥ $\mathrm{N}_{45}^{C}$, atiraskṛtasāśanaḥ $\mathrm{N}_{12}^{K}$, atiraskṛtamānasaḥ $S_{67}^{S}$, itarastutaśāsanaḥ $\mathrm{P}_{72}^{T} \quad \mathbf{2 4 7 \mathrm { c }}$ vyādhibhir ] $\Sigma$, vyādhibhi $\mathrm{N}_{77}^{K o} \mathrm{~N}_{58}^{K}$ 247d putrapautra $\left.{ }^{\circ}\right] \mathbf{N}^{\Sigma} \mathrm{E}^{N} \hat{S}_{67}^{S}$, putrapautraiḥ $\mathbf{P}^{\Sigma} \bullet{ }^{\circ}$ pratișțhitah ] $\Sigma$, ${ }^{\circ}$ samanvitah $\hat{S}_{67}^{S}$ 248a pațhyamānam ] $\mathrm{N}_{45}^{C} \mathrm{~K}^{\Sigma} \mathrm{B}_{99}^{C} \mathrm{P}_{32}^{T}$, pațhamānam $\mathrm{N}_{77}^{K o}$, pāṭhyamānam $\mathrm{E}^{N} \hat{S}_{67}^{S}$, śrutvādhyāyam $\mathrm{P}_{72}^{T} \bullet$ idam ] $\Sigma$, imaṃ $S_{67}^{S} \quad \mathbf{2 4 8 b}$ uddiśya ] $\Sigma$, udiśya $\mathrm{N}_{77}^{K o} \mathrm{~N}_{45}^{C} \mathrm{~N}_{12}^{K} \bullet$ ca pathyate ] $\mathrm{N}_{77}^{K o} \mathrm{~K}^{\Sigma} \mathrm{E}^{N} \mathrm{~B}_{99}^{C} \hat{S}_{67}^{S}$, paṭhe naraḥ $\mathrm{N}_{45}^{C}$, pațhennaraḥ $\mathbf{P}^{\Sigma} \quad$ 248c na tasya rogā bādhante ] $\mathrm{N}_{45}^{C} \mathrm{~N}_{82}^{K} \mathrm{~N}_{58}^{K} \mathrm{E}^{N} \hat{S}_{67}^{S}$, na tasya roga bādhyante $\mathrm{N}_{77}^{K o} \mathrm{~B}_{99}^{C}$, na tasya rogā bādhyante $\mathrm{N}_{45}^{C}$, na tạ̣ rogāḥ prabādhante $\mathrm{N}_{12}^{K}$, tasya rogā na bādhante $\mathbf{P}^{\Sigma} \quad \mathbf{2 4 8 d}{ }^{\circ}$ pittādisaṃbhavāḥ ] $\mathrm{N}_{82}^{K} \mathrm{E}^{N} \mathrm{~B}_{99}^{C} \dot{S}_{67}^{S} \mathbf{P}^{\Sigma}$, ${ }^{\circ}$ pitādisambhavā $\mathrm{N}_{77}^{K o} \mathrm{~N}_{45}^{C}$, ${ }^{\circ}$ pitādisambhavāḥ $\mathrm{N}_{12}^{K}$, ${ }^{\circ}$ pìttādisaṃbhavā $\mathrm{N}_{58}^{K}$ 
nākālamaraṇaṃ tasya na sarpaiś cāpi daṃśyate| na viṣaṃ kramate dehe na jaḍāndhatvamūkatā|| 249||

nopasargabhayam tasya notpātasya bhayam bhavet| nābhicārakṛtair doṣair lipyate sa kadācana|| 25ㅇ|

yat puṇyạ̣ sarvatīrthānāṃ gañgādīnāṃ viśeșataḥ| tat puṇyam koṭiguṇitam prāpnoti śravaṇād iha|| 251||

daśānāṃ rājasūyānām agnișțomaśatasya ca| śravaṇāt phalam āpnoti koțikoțigunottaram|| 252||

avadhyaḥ sarvadevānām anyeșāṃ ca viśeșataḥ| jīved varṣaśataṃ sāgraṃ sarvavyādhivivarjitaḥ|| 253||

goghnaś caiva kṛtaghnaś ca brahmahā gurutalpagaḥ| śaraṇāgataghātī ca mitraviśrambhaghātakaḥ|| 254||

249a nākāla $\left.{ }^{\circ}\right] \mathrm{N}_{77}^{K o} \mathrm{~K}^{\Sigma} \mathrm{E}^{N} \mathrm{~B}_{99}^{C}$, nākāle $\mathrm{N}_{45}^{C} \hat{S}_{67}^{S} \mathbf{P}^{\Sigma} \quad$ 249b sarpaiś cāpi daṃśyate ] $\mathrm{N}_{82}^{K} \mathrm{E}^{N} \mathbf{P}^{\Sigma}$, sarpaiś cāpi daśyate $\mathrm{N}_{77}^{K o} \mathrm{~N}_{12}^{K}$, sarppoś cāpi dasyate $\mathrm{N}_{45}^{C}$, sarppaiś cāpi dṛśyate $\mathrm{N}_{58}^{K}$, sarpaiś cāpi duśyate $\mathrm{B}_{99}^{C}$, sarpair nāpi daśyate $S_{67}^{S} \quad{ }_{249} \mathrm{c}$ na viṣaṃ kramate ] $\Sigma$, viṣaṃ nākramate $S_{67}^{S} \quad$ 249d jaḍāndhatvamūkatā ] $\mathrm{N}_{82}^{K} \mathrm{~N}_{12}^{K} \mathrm{E}^{N} \mathrm{~B}_{99}^{C} \mathrm{P}_{72}^{T}$, jaḍandhatvamūkatā $\mathrm{N}_{77}^{K o}$, jaḍāndhatvamūkadhā $\mathrm{N}_{45}^{C}$, jaḍāndhatvamūkatāh $\mathrm{N}_{58}^{K}$, jalāndhatvamūkataḥ $\mathrm{P}_{32}^{T}$, jaḍo ndho na mūkatā $S_{67}^{S} \quad \mathbf{2 5}$ oa nopasarga $\left.{ }^{\circ}\right] \Sigma$, na hi sarpa ${ }^{\circ} \mathrm{P}_{32}^{T} \quad \mathbf{2 5}^{\mathrm{ob}}$ notpātasya ] $\mathrm{N}_{82}^{K} \mathrm{~N}_{12}^{K} \mathrm{E}^{N} \mathrm{~B}_{99}^{C}$, notpātādio $\mathrm{N}_{77}^{K o} \mathrm{~N}_{45}^{C}$, notpādita ${ }^{\circ} \mathrm{N}_{58}^{K}$, na cotpāta ${ }^{\circ} S_{67}^{S} \mathbf{P}^{\Sigma}$ • bhavet ] $\Sigma$, tathā $\mathrm{P}_{32}^{T} \quad{ }_{25}$ oc ${ }^{\circ}$ kṛtair ] $\Sigma$, ${ }^{\circ}$ kṛte $\mathrm{N}_{77}^{K o} \quad$ 25ocd doṣair lipyate sa kadācana ] $\mathrm{N}_{12}^{K} \mathrm{E}^{N} \mathrm{~B}_{99}^{C} \hat{\mathrm{S}}_{67}^{S} \mathbf{P}^{\Sigma}$, doṣai lipyate na kadācanah $\mathrm{N}_{77}^{K o}$, dośenna lipyeta kācanaḥ $\mathrm{N}_{45}^{C}$ (unmetr.), doṣair lipyate sa kadācanạ̣ $\mathrm{N}_{82}^{K}$, doṣair lipyate na kadācana $\mathrm{N}_{58}^{K}$ 251a puṇyam ] $\Sigma$, puṇya $\mathrm{N}_{58}^{K} \bullet{ }^{\circ}$ tìrthānāṃ ] $\mathrm{N}_{77}^{K o} \mathrm{~N}_{45}^{C} \mathrm{~N}_{12}^{K} \mathrm{~N}_{58}^{K p c} \mathrm{E}^{N} \mathrm{~B}_{99}^{C} \mathbf{P}^{\Sigma}$, o tīrthānā $\mathrm{N}_{82}^{K}$, ${ }^{\circ}$ tì +rthā-nāṃ $\mathrm{N}_{58}^{K}$, ${ }^{\circ}$ tīrtheșu $S_{67}^{S} \quad$ 251c tat ] $\Sigma$, yat $\mathrm{N}_{77}^{K o} \mathrm{~N}_{58}^{K} \quad$ 251d iha ] $\Sigma$, ihah $\mathrm{N}_{77}^{K o} \mathrm{~B}_{99}^{C}$, itaḥ $S_{67}^{S} \quad$ 252a daśānāṃ ] $\Sigma$, daśānā $\mathrm{N}_{12}^{K} \mathrm{~N}_{58}^{K} \bullet{ }^{\circ}$ sūyānām ] $\Sigma$, o sūryāṇāṃm $\mathrm{N}_{45}^{C}$, ${ }^{\circ}$ sūryānām $\mathrm{N}_{12}^{K} \quad 252 b$ agnișțoma $\left.{ }^{\circ}\right] \mathrm{N}_{77}^{K o} \mathrm{~N}_{45}^{C} \mathrm{~N}_{12}^{K} \mathrm{E}^{N} \mathrm{~B}_{99}^{C} \mathbf{P}^{\Sigma}$, mașțișțoma ${ }^{\circ} \mathrm{N}_{82}^{K}$, agnișțo ${ }^{\circ} \mathrm{N}_{58}^{K}$ (unmetr.), jyotișțoma ${ }^{\circ} S_{67}^{S} \bullet{ }^{\circ}$ śatasya ] $\Sigma$, 'óatāni $S_{67}^{S} \quad \mathbf{2 5 2 c}$ āpnoti ] $\Sigma$, avāpnoti $\mathrm{N}_{45}^{C}$ 252d koțikoṭio ] $\Sigma$, koțikauți ${ }^{\circ} \mathrm{N}_{45}^{C}$, koțī koțī $\mathrm{E}^{N} \quad$ 253a avadhyah ] $\Sigma$, avadhya $\mathrm{N}_{77}^{K o} \mathrm{~N}_{12}^{K}$ - ${ }^{\circ}$ devānāṃ ] $\Sigma$, obhūtānāṃ $\mathrm{E}^{N} S_{67}^{S} \quad 253 c$ jìved varșaśatam ] $\Sigma$, jīve varșaśata ${ }^{\circ} \mathrm{N}_{45}^{C}$, jīve varșaśatam $\mathrm{N}_{12}^{K} \quad \mathbf{2 5 3}^{\circ}{ }^{\circ}$ vyādhi $\left.{ }^{\circ}\right] \Sigma$, ${ }^{\circ}$ vighna ${ }^{\circ} \mathrm{N}_{45}^{C} \quad \mathbf{2 5 4} \mathrm{b}{ }^{\circ}$ talpagah ] $\Sigma$, otalpagaḥ $\mathrm{N}_{12}^{K} \quad$ 254d ${ }^{\circ}$ viśrambhaghātakaḥ ] $\Sigma$, ${ }^{\circ}$ viśvāsaghātakā $\mathrm{N}_{45}^{C}$ 
duștapāpasamācāro mātṛhā pitṛhā tathā|
śravaṇād asya bhāvena mucyate sarvapātakaị̣|| 255||

śāntyadhyāyam idam puṇyam na deyam yasya kasyacit|

śivabhaktāya dātavyaṃ śivena kathitaṃ purā|| 256||

iti śivadharmaśāstre śāntyadhyāyaḥ ||

256d After this $\dot{S}_{67}^{S}$ adds two pādas: sarvakāmasamṛddhiś ca yah paṭhec ca dine dine $\mid \bullet \mathrm{P}_{3^{2}}^{T}$ adds four pādas : nityam khacitacittaḥ syāc chaktivyāghātavarjitaḥ| sarvakāmasamṛddhas tu yah pațheta dine dine $\mid \bullet \mathrm{P}_{7^{2}}^{T}$ adds : nityam khacitaśaktiś ca śaktivyāghātavarjitaḥ| sarvakāmasamṛddhạ̣ syāt yaḥ paṭheta dine dine|

255a dușțapāpa $\left.{ }^{\circ}\right] \mathrm{N}_{77}^{K o} \mathrm{~N}_{82}^{K} \mathrm{~N}_{58}^{K} \mathrm{E}^{N} \hat{\mathrm{S}}_{67}^{S} \mathrm{P}_{72}^{T}$, dușțapāpāpa ${ }^{\circ} \mathrm{N}_{45}^{C}$ (unmetr.), dușțah pāpa ${ }^{\circ}$ $\mathrm{N}_{12}^{K} \mathrm{P}_{32}^{T}$ • ${ }^{\circ}$ samācāro ] $\Sigma$, o ${ }^{\circ}$ samācārā $\mathrm{N}_{77}^{K o} \quad 255 \mathbf{b}$ mātṛhā pitṛhā ] $\mathbf{K}^{\Sigma} \mathrm{E}^{N} \mathrm{P}_{72}^{T}$, pitṛhā mātṛhās $\mathrm{N}_{77}^{K o}$, mātṛhā piț̣hās $\mathrm{N}_{45}^{C}$, pitṛhā mātṛhā $S_{67}^{S} \mathrm{P}_{3^{2}}^{T} \quad \mathbf{2 5 6 a}$ śāntyadhyāyam ] $\mathrm{N}_{82}^{K} \mathrm{E}^{N} \hat{S}_{67}^{S} \mathbf{P}^{\Sigma}$, śāntyādhyāyam $\mathrm{N}_{77}^{K o} \mathrm{~N}_{45}^{C} \mathrm{~N}_{12}^{K} \mathrm{~N}_{58}^{K} \mathrm{~B}_{99}^{C} \bullet$ idam ] $\Sigma$, imaṃ $\mathrm{P}_{72}^{T} \quad \mathbf{2 5 6 b}$ na deyam ] $\Sigma$, tadeya $\mathrm{N}_{77}^{K o}$, na dadyād $\mathrm{P}_{72}^{T} \quad \mathbf{2 5 6 c}$ obhaktāya dātavyam ] $\mathrm{K}^{\Sigma} \mathrm{E}^{N} \mathrm{~B}_{99}^{C} \hat{S}_{67}^{S}$, obhakte sadā deyaṃ $\mathrm{N}_{77}^{K o} \mathrm{P}_{32}^{T}$, obhaktā sadā deyaṃ $\mathrm{N}_{45}^{C}$, obhakte samādeyaḥ $\mathrm{P}_{72}^{T} \quad \mathbf{2 5 6 d}$ kathitaṃ ] $\Sigma$, kathitaḥ $\mathrm{P}_{72}^{T} \bullet$ purā ] $\Sigma$, purāḥ $\mathrm{N}_{45}^{C} \quad$ Col. iti śivadharme śāṃtyādhyāṃ samāptañ ca șașṭhamah $\mathrm{N}_{77}^{K o}$, iti śivadharmaśāstre śāṃtyādhyāya șașṭhamaḥ $\mathrm{N}_{45}^{C}$, iti śivadharmmaśāstre śāntyadhyāyah șașțḥạ $\mathrm{B}_{99}^{C}$, iti śivadharmaśāstre śāntyadhyāyah șașțhah $\mathrm{N}_{82}^{K}$, iti śivadharme nandiprokte śāntyadhyāyah ṣașțhạ $\mathrm{N}_{12}^{K}$, iti śivadharmmaśāstre naṃdikeśvaraproktaśāntyādhyāyah ṣașțha samāptaḥ $\mathrm{N}_{58}^{K}$, iti śivadharmaśāstre nandikeśvarapraṇite śāntyadhyāyo nāma șașțho 'dhyāyaḥ $\mathrm{E}^{N}$, iti nandikeśasaṃhitāyāṃ śāntyadhyāyah șașțhah $S_{67}^{S}$, iti śāntyadhyāyạ̣ șașțhah $\mathrm{P}_{3^{2}}^{T}$, iti śivadharmaśāstre śivadharmottare nandikeśvaraprokte śāntipāṭhakathanan nāma șașțho dhyāyaḥ $\mathrm{P}_{72}^{T}$ 


\title{
The Chapter on Appeasement
}

\author{
[Introduction]
}

Next I will proclaim this supreme secret, which has been extolled by Rudra, ${ }^{1}$ which confers great fortune, pacifies great obstacles, causes great appeasement, is auspicious, extinguishes untimely death, wards off all diseases, destroys the enemies' armies, always increases victory ${ }^{2}-$ an army of all deities and seizers, ${ }^{3}$ granting the desired results, the eternal law called the power of complete appeasement. (1-3)

$$
\text { [Maheśvara] }
$$

He who bears the half moon, who has three eyes, who is invested with the sacred thread of a snake, who is four-faced, ${ }^{4}$ four-armed, covered

${ }^{1}$ The compound rudrodgita is attested elsewhere in SkP Revākhanda 93.21, SkP 'Vāyu' Revākhaṇda 4.51 and SkP 'Vāyu' Revākhaṇda 60.8. In all these passages the compound is clearly a tatpuruṣa with an instrumental relation. The same meaning must underlie its use here. The Sivadharma is told to the sages by Nandikeśvara at the request of Sanatkumāra. In the opening verses of the Śivadharmaśāstra (ŚiDhŚ 1.10-11), Nandikeśvara indicates that the original teaching was given by Śiva to Pārvatī, Skanda, the Gaṇas and Nandikeśvara himself. Presumably Nandikeśvara refers to this initial moment of teaching with the reference rudrodgita. Cf. also the analysis of the Sivadharmavivarana (appendix): rudrenotkrșțatvena kathitam 'taught as being supreme by Rudra'.

${ }^{2} \mathrm{P}_{32}^{T}$, and $\mathrm{P}_{72}^{T}$ with variant readings, adds: 'paralyzing the enemy's army, always destroying the enemy'.

3 The author of the Sivadharmavivarana takes this to mean 'an army against all deities and seizers'.

4 Śiva is described here as four-faced, which is a relatively archaic feature of his iconography. For the historical development of the number of heads of Śiva, see Bakker 1997 and Törzsök 2013. Sadāśiva, the central deity of the Śaiva Siddhānta, is considered to be five-headed, but in early literature Śiva is said to be four-headed. 
with white ash, the best, the excellent, bestower of boons, ${ }^{5}$ God of gods, Maheśvara, worshipped in the three worlds, glorious - may he quickly bestow peace on me! (4-5)

\section{[Umā]}

With a body possessed of all limbs, ${ }^{6}$ and slender-waisted, with a very gentle smooth complexion that is both golden and dark, beautiful, furnished with a tilaka on her forehead, bearing the sickle of the moon, ${ }^{7}$ wearing bright clothes, the goddess, adorned with all ornaments, who has a most beautiful feminine form, great abode of beauty and of virtues, pleased simply by devotion, Umā, the goddess, bestower of boons, having arrived in person, with a peaceful form, of immeasurable splendour - may she bestow peace on me, she who is loved by her devotees and fond of devotion! ${ }^{8}(6-9)$

\section{[Kārttikeya]}

With a ruby-coloured lustre, gentle, with a red garland and red unguent, ${ }^{9}$ not a child, yet with the appearance of a child, ${ }^{10}$ six-faced, riding on a peacock, with a face like the full moon, tranquil, with three tufts of hair, furnished with a spear, sprung from the body of the Kṛttikās, Umā, Agni and

5 The sequence vara, varenya, varada is well attested in Purānic literature. It does not appear in the epics.

${ }^{6}$ The choice between sarvāvayavapūrnena and sarvāvayavamukhyena is an arbitrary one.

${ }^{7}$ Like Śiva, Umā is decorated with the sickle of the moon.

${ }^{8}$ The manuscripts are divided between bhaktivatsala and bhaktavatsalā. The phrase bhaktānām bhaktavatsala (with variant bhaktivatsala) is well attested. An early epigraphic reference may be found in the Cchoti Sadri inscription, dated [Vikrama] Samvat 597 (491 CE), which mentions Devī's bhaktavatsalatā: yā bhaktavatsalatayā prabibhartti lokān māteva [svā] kyasutapremnavivriddhasnehā 'who, out of her kindness to her devotees, sustains the worlds just like a mother full of tenderness arising from her affection to her own children' (Sircar, EI 30: 120-127, verse 2cd). BhavP 1.177.25d has given the pāda a Saura twist: ādityārādhane ratā.

${ }^{9}$ As a war god, Kārttikeya-Skanda is associated with the colour red. Mann (2012: 92), commenting on Kārttikeya's red colour, writes: 'Skanda as a red-coloured being fits his Graha-like character, but not his auspicious Senāpati character.' This argument does not seem very convincing and the present passage certainly does not contain any hints that Skanda is invoked here as a Graha.

${ }^{10}$ A similar phrase is used with reference to Kṛ̣ṇa in HV 62.10ab, *721:21, HV App. I, No. $11,{ }^{*} 8$ and ViDh 19.18a. 
Rudra, ${ }_{11}^{11}$ worshipped by the gods, Kārttikeya, of great splendour, solely intent on granting boons - may he constantly bestow peace, power and welfare on me, always! (10-12)

\section{[Nandīśa}

Wearing a garment of white cloth, three-eyed, with a beautiful golden lustre, ${ }^{12}$ a trident in his hand, ${ }^{13}$ very wise, Nandiśa, devoted to Siva, constantly intent upon worship of Śiva, solely intent upon meditation upon Siva - may he, appeased, bestow peace on me, and supreme resolve towards the [Śiva] dharma! $!^{14}\left(13^{-14}\right)$

\section{[Vināyaka]}

With a big belly, a big body, a skin like a heap of smooth collyrium, ${ }^{15}$ endowed with a single tusk ${ }^{16}$ the god who has an elephant head, very powerful, invested with the sacred thread of a snake, adorned with snakes as ornaments, the support of the accomplishment of all aims, overlord of the Ganas, granter of boons, son of Rudra, ${ }^{17}$ god, leader, Vināyaka may he bestow great peace and success in action for me always! $!^{18}\left(15^{-17}\right)$

${ }^{11}$ A reference to the complex birth of Skanda, as narrated, for example, in the Mahābhärata and the Rāmāyaña.

${ }^{12}$ After this, $\mathrm{N}_{58}^{K}$ adds: 'devoted to Rudra, great Yogin, his mind solely dedicated to Rudra' (corr. rudraikāhitamānasaḥ).

${ }^{13}$ I take śūlapānir to be short for triśūlapāṇir. Nandin's main attribute is the triśūla.

${ }^{14}$ Nandīśvara is invoked here as the promulgator of the Śivadharma.

${ }^{15}$ I have not found other textual references to Vināyaka's dark body. Could this relate to his birth from the dirt (mala) of Pārvatī's dark skin or simply to the colour of an elephant in general?

${ }^{16}$ Alternatively this may be taken as an inverted bahuvrīhi: 'with a gigantic single tusk'.

${ }^{17}$ Elsewhere I have argued that the reference to Vināyaka as 'Rudra's son' could be significant for the dating of the text (Bisschop 2010: 244). Early Purānas, such as the Vãyupurāna and the Skandapurāṇa, do not yet regard him as the son of Śiva and Pārvatī. There is, however, one exception: in SP 32.116 , in a hymn of praise, Devī is addressed as the mother of Skandacandra and Hastivaktra. On the other hand, there is no reference to this in the rest of the text, which could indicate that this notion may have started to spread around the time of composition of the Skandapurāna. On the adoption of Vināyaka/Gạ̣eśa as a member of Śiva's household, see SP IIB: 50, n. 147, and Törzsök 2004.

${ }^{18}$ The manuscript tradition is divided in $17 \mathrm{~d}$. The repetition of $m e(17 \mathrm{c})$ in $m e$ sada (17d) may have led to the smoother sarvadā in several manuscripts. 


\section{[Mahākāla]}

Resembling sapphire, three-eyed, with a blazing trident and [other] weapons raised, ${ }^{19}$ wearing a red garment, eminent, with a black body, adorned with snakes - may Mahākāla, very powerful, bestow great peace on me, ${ }^{20}$ removing evil, unequalled, destroying the taint of misfortune! ${ }^{21}(18-19)$

\section{[Ambikā]}

Wearing a garment of yellow cloth, with the appearance of a girl, well-adorned, mother of the Ganas, ${ }^{22}$ Ambikā, three-eyed, auspicious, white (Gaurī), mistress of the gods, causing all success, the goddess, intent upon favour, supreme - may the Mother bestow peace on me and quickly grant success! (2O-21)

\section{[Mahāmahiṣamardan̄̄]}

With a smooth dark colour, ${ }^{23}$ Mahāmahișamardanī (Crusher of the Great Buffalo), she who strikes with bow and discus, bearing a sword and a spear, ${ }^{24}$ with one hand raised in threat, ${ }^{25}$ destroying all misfortunes,

${ }^{19}$ For the iconography of Nandíśvara and Mahākāla, see Goodall et al. 2005: 102-108.

${ }^{20}$ The manuscripts show quite some variation, with several of them suggesting prìtena cetasā (including also the parallel in the Bhavișyapurāna), in which case, however, Mahākāla is not mentioned by name.

${ }^{21}$ The compound alakșmimalanāśana, also found in $73 \mathrm{~d}$, reappears in the Śivadharma corpus in SiUp 5.1, with reference to the bath in ashes.

${ }^{22}$ For Ambikā/Gaurī as mother of the Gaṇas, cf. SP 60.4ob (ganamātā gaṇāmbikāa). See also Coburn 1984: 98-106.

${ }^{23}$ The fact that the Warrior Goddess is described here as dark-skinned may be relevant for the time of composition of the text, for it shows that it was not yet influenced by the Devīmāhātmya. According to the Skandapurāṇa Kauśikī-Mahișāsuramardinī emerged from the dark skin left behind by Pārvatī, but in the Devīmāhātmya the colour scheme is reversed. See Yokochi 1999: 83-84.

${ }^{24}$ The bow does not occur in the earliest images of Mahișāsuramardinī, but it is found on the images executed in caves 6 and 17 at Udayagiri around the beginning of the fifth century (Harle 1971-72: 45; Yokochi 1999: 77). $\mathrm{P}_{32}^{T}$ has a significant variant: instead of the sword (khadga) and the spear (pattisa), it mentions the conch (śankha), stick (yaști) and knife (asi). The conch as one of her attributes appears to be characteristic of the Deccan (Yokochi 1999: 71). Schmid (2003: 27-29) observes that the earliest in situ images of Mahișāsuramardinī are found in a Vaiṣnava context and that it is only from the sixth century onwards that this form of the Goddess gets associated with Śaivism.

${ }^{25}$ The manuscripts are divided, with some suggesting rather a separate feminine 
mother of all happiness - may she constantly act auspicious towards me! (22-23)

$$
\text { [Bhṛngiriți] }
$$

With a body without flesh, composed of sinews and bones, completely emaciated, very mighty, three-eyed, the great Bhṛngiriți, ${ }^{26}$ son of Rudra, great hero, his mind solely directed towards Rudra - may he too, with a peaceful disposition, quickly bestow peace on me! (24-25)

\section{[Caṇ̣eśvara]}

The fierce general of the Ganas, who holds the great shaft of an axe, ${ }^{27}$ his

$\bar{a} \operatorname{tarjan} \bar{\imath}$ (also supported by the Bhavișyapurāna parallel), which should then be taken in the sense of tarjani 'threatening'.

${ }^{26}$ The reference to the emaciated Bhṛngiriți/Bhṛngin is significant. His name is not attested in literary works before the Skandapurāna (Bisschop 2010: 244-246). The emaciated figure identified as Bhṛngin begins to appear in material culture in the caves of Elephanta, Ellora, Badami and Aihole from the sixth century onwards (Bautze-Picron 2010: 104-107). The first epigraphical reference to Bhṛngin appears in the second verse of the Senakapat stone inscription of the time of Śivagupta Bālārjuna (beginning of the 7 th c.?):

nirdagdho 'py atra netrajvalanakavalanais tāvakīnair manobhūr bhüyo janmānayā te gamita iti rușā rūkșitena tvayeyam |

kiṃ bhoh śambho ranāmbhonidhim adhiśayitā tyajyate lajjyate no bhringìti proktikārī ripur aśami yayā sāvatāt pārvatī vah $\|$ (Dikshit \& Sircar, EI 31: 31-36; Shastri 1995 II, 154-159; normalized)

May Pārvatī protect you, she who subdued [her] adversary Bhṛngin, who is in the habit of speaking: 'Why, oh Śambhu, don't you abandon and shame this woman (i.e. Pārvatī), who is reposing upon the ocean of delight of love-battle, you, stiffened with anger because the Mind-born (Kāma) has been restored to life by her for you, although he had been burned in this world by gulps of fire from your [third] eye?'

The inscription actually reads śrnigiti but, as Yuko Yokochi has suggested to me (personal communication), this is no doubt a mistake for bhrngitti. Dikshit \& Sircar, followed by Shastri, conjecture janmānuyāne, but the inscription appears to read janmānayā te. An alternative conjecture janmānaye 'on the path of rebirth' may be considered.

${ }^{27}$ I have taken akṣa in the sense of 'shaft', although this meaning is not reported in the dictionaries. The rosary is already mentioned in $26 \mathrm{c}$, which precludes taking it as short for akșamālā. The manuscripts are unanimous. 
hand fixed to the rosary, three-eyed, the excellent Caṇdeśvara, ${ }^{28}$ remover of fierce crimes, who purifies from killing a brahmin and the like - may the Great Yogin prepare a succession of fortunes for me! ${ }^{29}(26-27)$

\section{[Brahmā]}

Seated on a lotus, resembling a lotus, with four lotus-faces, bearing a water-jar, eminent, worshipped by Devas and Gandharvas, solely intent upon meditation on Śiva, devoted to the true reality of Śiva - may Brahmā bestow peace on me, with the divine Vedic word (brahmaśabda)! $!^{30}(28-29)$

${ }^{28}$ Canḍeśvara has played various roles in Śaivism, as consumer of offerings, punisher of transgressions of Śaiva initiates and, in the South, as super-bhakta who cuts off his father's legs through devotion (after worshipping Śiva with milk, at which his father gets angry). In South India he has a shrine to the northeast of the central icon. Here he appears as general of the Ganas as well as chastiser of crimes. For a general study of Candeśvara, see Goodall 2009. On the broader significance of this description of Caṇdeśvara in the Śivadharmaśāstra, see Bisschop 2010: 240-241.

${ }^{29}$ After this, $\mathrm{E}^{N}, \mathrm{P}_{3^{2}}^{T}$ and $\mathrm{P}_{72}^{T}$ add 12 to 14 pādas, which run approximately (translation largely based on $\mathrm{E}^{N}$ ):

With the [white] splendour of conch-shell, jasmine and the moon, with a blazing emerald around his neck, holding a rosary, standing in front of Śiva, intent upon his own knowledge (= the Śivadharma), four-faced, four-armed, three-eyed, constantly shining, lord of the earth, the god Vṛsa, supreme among all dharmas, carries the lord on his back. Therefore dharma is the master of the world. May Vṛșa, lord of the bulls, bestow peace on me!

This is almost certainly a later addition, as can be gleaned from the different phrasing in the various manuscripts. The addition is also found in several of the single-text manuscripts of the Śantyadhyāya and it is also commented upon in the Sivadharmavivarana (see appendix). The verses may have been added to bring the present passage in line with the set of eight Gaṇeśas known from Śaiva Siddhānta literature (cf. Bisschop 2010: 243). Vrṣa, the bull of dharma, is invoked here as the embodiment of the Śivadharma. $S_{67}^{S}$ has a similar addition, but it takes the subject to be Kṛṣna rather than Vṛ̣a. Its invocation of Kụṣna is preceded by two verses dedicated to the Gaṇa Ghaṇțākarṇa. This Gaṇa is referred to in ŚiDhŚ 8.85 and 8.87. For Ghaṇtākarṇa's iconography in the Agnipurāna see de Mallmann 1963: 60-62.

${ }^{30} \mathrm{Cf}$. ŚiDhŚ 8.81: brahmaṇā brahmaśabdena stūyateyah sadā harah | sa śivaḥ śāsvato devo goṣu mārīn vyapohatu \|. 
[Viṣnu]

Seated on Garuḍa (Tārkșya), four-armed, bearing a conch, discus and mace, dark, wearing a yellow garment, ${ }^{31}$ of great power and heroism, the god who is supreme as the body of sacrifice, ${ }^{32}$ the god Mādhava, killer of Madhu, endowed with the grace of Siva, devoted to meditation on Siva, destroyer of all evils, destroyer of all demons - may Viṣnu at all times bestow peace on me, with a peaceful disposition!33 $(30-32)$

${ }^{31}$ The yellow garment is also listed as a characteristic of Viṣnu's appearance in Bṛhatsaṃhitā 24.18c and 58.32ab. Bṛhatsamnitā 58.34 further specifies that a four-armed image of Viṣnu should have the conch and discus in the left hand and the mace and śāntimudrā in the right hand. Instead of the śäntimudrā, the more common attribute from the Gupta period onwards is the lotus (Shastri 1969: 134). No fourth attribute is mentioned in the Śivadharmaśāstra. Cf. also 'Kāśyapa' quoted by M.R. Bhat (1982: 562) from Utpaladeva's commentary on the Bṛhatsaṃhitā: viṣnuś caturbhujah śārngi śankhacakragadādharaḥ | śrīvatsāinkaḥ pìtavāsā vanamālāvibhūṣitaḥ || (the last pāda is shared with the variant in $\mathrm{P}_{32}^{T}$ and $\mathrm{P}_{72}^{T}$ ).

${ }^{32}$ Alternatively, yajñadehottamo may be taken to mean 'supreme with a body consisting of sacrifice'. The close relation between Viṣnu and sacrifice is well established, as in the notions of yajñavarāha and yajñapuruṣa. Perhaps this is a reference to Viṣnu as the supreme Puruṣa, whose body constitutes the sacrifice in the Puruṣasūkta (Rgveda 10.90).

${ }^{33}$ A large number of manuscripts omit $32 b c$. The wide distribution of readings in the ending of $32 \mathrm{a}$ may be related to this omission as well.

After this verse, $E^{N}$ and $S_{67}^{S}$ add 30 pādas dedicated to Arhant, Buddha, Jayā/Vijayā, Pṛthivī (Dharitrī) and the cows of Kṣīroda. There are significant differences in phrasing. $\mathrm{N}_{58}^{K}$ has a similar addition, of 34 verses, dedicated to Arhant, Buddha, Vṛșabha, and the five cows in Śivapura (Nandā, Subhadrā, Surabhī, Suśīā, Sumanā). The following provisional translation is based largely on $\hat{S}_{67}^{S}$ :

The Arhant, the god with a peaceful form, a feather-brush and a cloth in his hands, naked, covered with dust and mire $\left(\mathrm{E}^{N}\right)$, steady-minded and concentrated, his eyes turned inwards, peaceful, contemplating only the knowledge of Śiva - may he, appeased, endowed with oneness with Śiva, bestow peace on me!

In control of his senses, absorbed in intense concentration, adorned with a vessel and a monk's robe, his fingers in the gestures of granting a boon and freedom from fear, always delighting in reflection on knowledge, furnished with yogic perception, devoted to the knowledge of Śiva - may the Buddha, delighting in the welfare of all beings, bestow peace on me! 


\section{$[\text { Mātṛs }]^{34}$}

\section{[1. Brahmāṇī]}

\section{With a ruby-coloured appearance, the goddess with four lotus-faces, her}

With a ravishing, very wonderful $\left(\mathrm{E}^{N}\right)$ yellow-coloured body, beautiful in all her limbs, the goddess, Jayā, wishing for victory, constantly delighting in the worship of Śiva, devoted to the worship of Śiva, and the Earth (Dharitrī), mother of the people - may she always protect me!

The Cows arisen from the milk ocean (Kșīroda), desiring the welfare of the people, always delighting the gods and brahmins in particular, furnished with a divine soul - may they always bestow peace on me!

On the adoption of the Arhant and the Buddha in the lists of deities in the Sivadharmaśāstra, see Bisschop forthc. a. Note that the Buddha and the god of the Arhants (arhatām devaḥ) are mentioned in Bṛhatsaṃhitā 58.44-45.

34 The iconography of the Mātrkās described in this section supports a date of the sixth century at the earliest for the composition of the text. For example, the text refers to the Mātṛās' vehicles, which start to appear in sculpture only from around this time. Among the earliest sets of Mātṛkās with vehicles are the ones from Śāmalājī dated from ca. 520 CE, and two sets from Deogarh (Schastok 1985: 70-71). See also Panikkar 1997, for a historical overview of Mātṛkā iconography. The Kāpālika iconography of the last two Mātṛs is quite striking. Also noteworthy is the addition of an eighth goddess, Lamboșthī, and the mix-up of the regular and well-established order of Vārāhī and Aindrī. Some instances of groups of Mātṛs that do have Aindrī followed by Vārāhī are known, however, e.g.:

1. a broken panel from Nagar, Bharatpur, in Rajasthan, ca. 6th/7th c. CE (AIIS o16855, now at the archaeological museum of the Amber fort outside Jaipur)

2. a set on the Paraśurāmeśvara temple at Bhuvaneśvara, ca. 7th c. CE (Pannikar 1997, plates 104-105)

3. a set on the doorway of a Sūrya temple at Umri, Tikamgarh, in Madhya Pradesh, ca. 825 CE (Meister 1986, fig. 7)

For a table recording different sets of Mātṛkās, see Meister 1997: 234-235 (Meister fails to notice the non-standard order on the Paraśurāmeśvara temple). The standard set of seven mothers derived from male counterparts is not attested in literature before the seventh century. One of the earliest sources is the Skandapurāna, which lists them along with many other Mothers in its Koțivarșa Māhātmya ( $\left.\mathrm{SP}_{\mathrm{Bh}} 171\right)$. One of the earliest iconographical descriptions, along with the present passage, may be MtP 261.24-39. The set of eight rather than seven goddesses is a distinctive Tantric feature (Hatley 2012: 107-108), but note that it also appears in Elephanta in the Mātṛkā shrine in the east wing (Schastok 1985: 62, fig. 117). 
hand fixed on a rosary, bearing a water-jar, auspicious — may Brahmānīi, with a pleasant face, devoted to worship of Śiva, pleased, always bestow peace on me with the Vedic word (brahmaśabda)! ${ }^{35}\left(33^{-34}\right)$

\section{[2. Rudrānīī]}

Resembling the mountain of snow [in whiteness], the goddess riding on a great bull, a trident in her hand, granter of boons, adorned with ornaments of snakes, four-armed, four-faced, three-eyed, remover of evils may Rudrāṇi, pleased, blazing constantly, remove pain for me! $\left(35^{-36}\right)$

\section{[3. Kaumārī]}

Riding on a peacock, the goddess with a body red like vermillion, a lance in her hand, of majestic appearance, adorned with all decorations, devoted to Rudra, of great strength, always delighting in worship of Rudra - may Kaumārī, granter of boons, the goddess, quickly bestow peace on me! $(37-38)$

\section{[4. Vaiṣnavīi}

With a conch, discus and mace in her hand, dark, fond of the yellow garment, four-armed, riding on Garuḍa (Tārkṣya), Vaiṣnavī, worshipped by the gods, always delighting in the worship of Śiva, her mind solely directed to Śiva - may she constantly bestow peace on me, destroying all demons! (39-40)

$$
[5 \cdot \text { Aindrī }]^{36}
$$

Mounted on the elephant Airāvata, a thunderbolt in her hand, very powerful, adorned with a thousand eyes, with a golden lustre, venerated by Siddhas and Gandharvas, adorned with all ornaments - may the goddess Aindrī at all times quickly bestow peace on me! (41-42)

35 The invocation is very similar in style to that of Brahmā at 28-29 above. The same is the case for the other Mātrs, with many adjectives taken over from their male counterparts. The invocations of the mothers in this section are clearly inspired by the earlier invocations of the male deities and as such do not necessarily provide independent evidence for sculptural representations.

${ }^{6}$ These two pādas are placed after Vārāhī (44) in $\mathrm{N}_{5^{8}}^{K}, \mathrm{P}_{3^{2}}^{T}$ and $\mathrm{P}_{7^{2}}^{T}$, thus making the sequence of the Mātṛkās conform to the regular standard. 


\section{[6. Vārāhī]}

With the snout of a boar, dreadful, riding a supreme boar, dark, pure and massive, with the conch, discus and mace as her weapons, ${ }^{37}$ always threatening obstacles, always worshipping Śiva — may Vārāhī, granter of boons, the goddess, grant peace and health to me! (43-44)

\section{[7. Cāmuṇụā $]$}

With upraised hair, hollow-eyed, without flesh, bound by sinews, with a gaping mouth, terrible, ready with a sword and a dagger, with a garland of skulls, angry, wearing a magnificent skull-staff, with eyes all red and yellow, covered with an elephant hide, her body encircled by various snakes, dwelling at the cremation ground, with a form of Siva that is dreadful,$^{38}$ creating fear with the cries of jackals $\left(s_{i} i \bar{a}\right)$ - may Cāmuṇ̣ā, with a terrible form (caṇdarūpa), grant great protection to me! ${ }^{39}\left(45^{-47)}\right.$

\section{[8. Lamboșțhī]}

With a fat body, adorned with various ornaments, threatening obstacles, bearing a sword with a blazing tip, seated on a crow, great heroine, de-

37 These Vaiṣnava attributes are found on several surviving Vārāhī sculptures. For Vārāhī riding a boar, see Rangarajan 2004: 97-100.

${ }^{38}$ In other words, she has a Bhairava appearance.

${ }^{39} \mathrm{~N}_{58}^{K}$ adds two more pādas on Cāmuṇḍā after $47 \mathrm{c}$, while $\mathrm{P}_{3^{2}}^{T}$ and $\mathrm{P}_{7^{2}}^{T}$ add two similar pādas after $47 \mathrm{C}$ and two more pādas after $47 \mathrm{f}$. No vehicle is mentioned for Cāmuṇ̣ā, but the reference to the cries of jackals may point to the presence of a jackal (see Schastok 1985, fig. 3 and 9). The name Cāmuṇạa appears in texts from about the sixth to the seventh century CE. The earliest textual evidence may be a passage in Bhāviveka's Tarkajvālā, which condemns the coarse ritual knowledge of Cāmuṇ̣̂ā (Kapstein 2003: 243, 249). See also Harșacarita p. 304, l. 3, referring to Cāmuṇ̣ā shrines. The name may have been standardized because of the popularity of the Devimāhätmya, in which she is the killer of Caṇda and Muṇda. The Skandapurāna does not mention the name Cāmuṇdāa, but in its Koțivarșa Māhātmya ( $\left.\mathrm{SP}_{\mathrm{Bh}}{ }^{171}\right)$, about the birth of the Mothers, it includes the standard seven Mothers, among which a goddess named Bahumāṃā takes the position of Cāmuṇ̂ā. See Bakker (2014: 255-256), who considers the passage to be 'the earliest textual reference to the standard Seven Mothers, notwithstanding the fact that images testifying to this iconography have made their appearance since the fifth century'. 
stroying all evils — may Lamboșțīi (she who has full lips), ${ }^{40}$ granter of boons, the goddess, bestow peace on me! (48-49)

\section{[All Mātṛs]}

The divine Mothers of the sky, and other Mothers of the world, all Mothers of spirits, and the other Mothers of the demons, all the Mothers who are great goddesses, their hands occupied with their own weapons, who stay around, pervading the world, eager for bali offerings, highly fortunate, devoted to Rudra, great heroines, their minds dedicated to the worship of Rudra - may the Mothers, worshipped by the gods, constantly bestow peace on me! $\left(5^{\circ}-5^{2}\right)$

\section{[Rudras, Mātṛs and Gaṇādhipas]}

The Rudras of fearsome deeds who dwell at the abodes of Rudra, and those special ones who are gentle, dwelling at the abodes of Sthānu, and the Mothers with a fearsome appearance, and they who are Lords of the Ganas, and others who may be obstacles, dwelling in the main and intermediate directions - may they all, with satisfied minds, accept my bali offering. May they quickly grant success! May they always protect me from dangers! ${ }^{41}\left(53^{-} 55\right)$

${ }^{40}$ The invocation of Lamboșthī occurs only in the Nepalese manuscripts, with the exception of $\mathrm{N}_{45}^{C}$ ( $\mathrm{N}_{77}^{K o}$ is not available because of loss of a folio), and it is also missing in the Bhavisyapurāna parallel. While this may suggest an early addition in the Nepalese transmission of the text, it is also conceivable that the two verses were omitted later, when the group of Saptamātṛka had been well established, which did not include a goddess Lambosthī. Groups of eight are a common feature of the invocations in the Śantyadhyāya. On the addition of an eighth goddess to the standard set of seven, see Hatley 2012: 107-108, but in the texts he cites, the eighth goddess is named Mahālakșmī, Bhairavī or Yogeśvarī. Lamboșṭhī's appearance is the reverse of Cāmuṇ̣̂ā. SP 68.2 associates the goddess Lambaușthī (along with Vṛ̣adaṃsāa and Kiṃnarī) with the country of Siṃhala. In form and appearance she strongly resembles the goddess Jyeșțhā, who was particularly popular in South India and who is also fat, has drooping lips (lamboșțī), and is accompanied by a crow (Leslie 1991). No images of a Jyeșțā-like goddess from the North are known.

${ }^{41}$ Verses 53-55 appear almost verbatim in Mrgendra, Kriyāpāda 7.33-35, in a section on the offering of bali. Also İśānaśivagurudevapaddhati, Sāmānyapāda 14.178-180ab = Mantrapāda 28.96cd-98 = Krìāpāda 15.4-16ab. Variants of these verses are also found in several later Tantric sources, including the works of Aghoraśiva and Bhoja (TAK 4 


\section{[Ganas: E]}

The Ganas who are in the eastern (Indra's) direction, thunderbolts in their hands, ${ }^{42}$ very powerful, very white-eyed, white in appearance, white coppery, ${ }^{43}$ in heaven, in the sky and on earth, and dwelling in the bottom of the Pātāla, thrilled, delighting in the worship of Rudra - may they always bestow peace on me! $\left(5^{6-57)}\right.$

[Gaṇas: SE]

All the Ganas in the southeastern (Agni's) direction, ladles in their hands, bearing quivers, very red-eyed, red in appearance, red coppery, in heaven, in the sky and on earth, and dwelling in the bottom of the Pātāla, their minds bent to Rudra - may they always bestow peace on me! (58-59)

\section{[Gaṇas: S]}

The Ganas in the southern (Yama's) direction, always with sticks in their hands, black, black in appearance, angry, black coppery, in heaven, in the sky and on earth, and dwelling in the bottom of the Pātāla, their minds solely devoted to Rudra - may they always bestow peace on me! (6o-61)

forthc., s.v. balimantra). The reference to bali provides a hint to the ritual activity accompanying the invocation. Early epigraphical attestations for the offering of bali are found in the Bagh copper plates of the fourth-century Valkhā king Bhuluṇạa (Ramesh \& Tiwari 1990). See Willis 2009, 102-104 (with references and an image of a fifth-century balipițha from Rāmgarh, near the Gupta site Badoh).

${ }^{42}$ The appearance of the Ganas in the different directions is modelled after the iconography of the deity who is the guardian of each direction. İsānaśiva prescribes the use of the invocation of the Ganas of the ten directions (56-75) on several occasions in the Kriyāpāda of his İśānaśivagurudevapaddhati (Siddhāntasāra), abbreviating it by citing only the first two pādas, dedicated to the Ganas in the east. He gives the full version of the mantra in Mantrapāda 30.84, but in a modified prose form. See the register of testimonia in the edition.

43 The qualification lohita is applied to each group of Ganas of the different directions but in combination with different colours. I take lohita to mean 'coppery' or 'of copper appearance' here. See also the comment in the Sivadharmavivarana: 'Even though there is a distinction of colours, extension of [the designation] "coppery" (lohita) should be understood for each of the [Ganas] addressed after the statement "in the eastern direction" (56e).' 
[Gaṇas: SW]

The Ganas in the southwestern (Nirrti's) direction, angry, demonic, with swords in their hands, ${ }^{44}$ very dark-eyed, with a dark appearance, dark coppery, in heaven, in the sky and on earth, and dwelling in the bottom of the Pātāla, delighting in meditation on Rudra — may they always bestow peace on me! (62-63)

\section{[Gaṇas: W]}

The Ganas who are in the western (Varuna's) direction, always with nooses in their hands, very dark-eyed, with a dark appearance, dark coppery, in heaven, in the sky and on earth, and dwelling in the bottom of the Pātāla, delighting in worship of the supreme lord - may they always bestow peace on me! (64-65)

\section{[Ganas: NW]}

The Gaṇas in the northwestern (Vāyu's) direction, ${ }^{45}$ always with banners in their hands, very yellow-eyed, with a yellow appearance, yellow coppery, in heaven, in the sky and on earth, and dwelling in the bottom of the Pātāla, dedicated to devotion of Śiva - may they always bestow safety upon me! (66-67)

\section{[Gaṇas: N]}

The Ganas in the northern direction, always with treasures in their hands, ${ }^{46}$ with flecked eyes, with flecked appearance, ${ }^{47}$ flecked coppery, in heaven, in the sky and on earth, and dwelling in the bottom of the Pātāla, engaged in the worship of Śiva - may they always bestow safety on me! (68-69)

${ }^{44}$ I follow the reading khadgapannayah of $\mathrm{N}_{58}^{K}, \hat{\mathrm{S}}_{67}^{S}$ and $\mathrm{P}_{32}^{T}$. The parallel expression in the İsānaśivagurudevapaddhati also supports it. As in the invocations of the other Ganas we expect a reference to the weapons they hold in their hands. Nirrti's weapon is a sword (Wessels-Mevissen 2001: 99-100).

45 The adopted reading $\left(\mathrm{N}_{82}^{K}, \mathrm{~N}_{12}^{K}, \mathrm{~B}_{99}^{C}\right)$ is hypermetrical. It is possible that vāyaryām was pronounced as vāyvyam and that the different variants have arisen as different attempts to remove it.

${ }^{46}$ The northern direction is associated with Kubera, lord of treasure.

${ }^{47}$ The support for the hypermetrical reading śavalākșạh śavalanibhās is quite strong. As in the case of 66a above, the different variants may represent different attempts to remove it. 


\section{[Gaṇas: NE]}

The Gaṇas in the northeastern (İsāna's) direction, calm, with tridents (śüla) in their hands, fine, with a very fine appearance, fine coppery, in heaven, in the sky and on earth, and dwelling in the bottom of the Pātāla, engaged in the worship of Śiva - may they always bestow safety on me! $(70-71)$

\section{[Gaṇas: Below]}

The Ganas who are in the lower part, always with tridents in their hands, smoky, with a smoke-coloured appearance, smoke-coppery, in heaven, in the sky and on earth, ${ }^{48}$ and dwelling in the bottom of the Pātāla may they constantly bestow peace on me, destroying the taint of misfortune. ${ }^{49}(72-73)$

\section{[Ganas: Above]}

The Ganas who are in the upper part, of great power and heroism, very fine-eyed, with a fine appearance, fine coppery, in heaven, in the sky and on earth, and dwelling in the bottom of the Pātāla, engaged in the worship of Śiva - may they destroy misfortune for me! (74-75)

${ }^{48}$ This stereotypical phrase, which is appropriate in the previous descriptions of Ganas in the eight different horizontal directions, does not fit well with the present description of the Ganas in the lower and upper regions of the cosmos, which already involves a vertical division. The Śivadharmavivaraṇa explains: 'Even though they are stationed in the regions of "the lower part" (72a) etc., pervasion of all worlds should be understood because of their sovereign power.'

${ }^{49}$ In contrast to the previous invocations, the descriptions of the Ganas in the lower and upper part of the cosmos show no association with a particular deity. The tridents said to be in the hands of the Ganas in the lower part may be due to the influence of the description of the Ganas the northeastern direction, mentioned just before. In later Tantric Śaiva sources Brahmā is associated with the upper part and Viṣnu with the lower part (TAK III, s.v. dikpatayah). The Southern transmission of the text appears to have updated the text accordingly, with the Ganas in the lower part bearing discuses in their hands (cakrapānayah) and the ones in the upper part bearing lotuses (padmapannayah, $\left.\mathrm{P}_{7^{2}}^{T}\right)$. The same also applies to the mantra in the Íśānaśivagurudevapaddhati. 


\section{[Ganas: Conclusion]}

Having worshipped Śiva with effort, one should scatter a bali offering for these Ganas,,$^{50}$ great souls, of great power and heroism. Thereupon, with satisfied minds, may they always bestow peace on me! $!^{5^{1}}(76)$

$[\text { Dikpālas }]^{52}$

\section{[Indra in Amarāvatī]}

The city called Amarāvatī is established in the eastern part, crowded with Vidyādharas, visited by Siddhas and Gandharvas, brilliant with a bejewelled wall, decorated with all jewels. There reigns the Lord of the gods (Indra), eminent, thunderbolt in hand, very powerful, with a thousand beautiful eyes, mounted on the elephant Airāvata, golden-coloured, ${ }^{53}$ of great splendour, chief of the gods, constantly pleased, delighting in the worship of the Supreme lord, possessed of meditation on Siva, endowed with devotion to Siva, dedicated to bowing to Śiva - may he bestow peace on me! $(77-80)$

${ }^{50}$ Note again the reference to the ritual act of scattering a bali offering.

${ }^{1} \mathrm{~N}_{5^{8}}^{K}$ adds two more pādas to create two four-pāda verses.

$5^{2}$ The following list of eight Dikpālas and their abodes corresponds to the canonical list given in Kirfel 1967: 95 (with either Virūpākṣa or Nirṛti in the southwest). For other textual descriptions of the towns, which are located on Mt Meru, see Bhuvanavinyāsa 2.43.8-17 (Kirfel 1954: 98-99). In the epics, only four Dikpālas are mentioned (Wessels-Mevissen 2001: 12-13). It is only from the time of the Purānas that we find the tradition of eight Dikpālas attested, but with quite a lot of variation (see table X [p.15] in Wessels-Mevissen 2001). The earliest dated literary evidence for the eight Dikpālas is to be found in Varāhamihira's Yogayātrā (Wessels-Mevissen 2001: 15 and table XI). Another possibly early reference is Amarakośa 1.3.178-179. The present passage, not drawn upon by Wessels-Mevissen, may be one of the earliest detailed descriptions of the eight Dikpālas. Varied sets of the eight Dikpālas start to appear for the first time in the Deccan towards the end of the sixth century, while the complete canonical set is only attested from the middle of the seventh century onwards (Wessels-Mevissen 2001: $23 \mathrm{ff}$.).

${ }^{53}$ For Indra's golden appearance, cf. VDhP 3.50.3a and 3.50.8ab. 


\section{[Agni in Tejovatī]}

In the direction of Agni (southeast) is the beautiful city Tejovatī, ${ }^{54}$ crowded with various deities, blazing with the light of jewels. There, his body surrounded by flames, with a splendour equal to blazing charcoal, causing the welfare of embodied souls, may the god who is flaming (Jvalana), destroying evils, engaged in the muttering and worship of Śiva, devoted to recollection of Śiva, bestow peace on me and destruction of evils! (81-83)

\section{[Yama in Vaivasvatī]}

The city called Vaivasvatī is established in the south, the abode of Pitrrs, Rakșas and Uragas, and an array of hundreds of Suras and Asuras. There, with the appearance of sapphire, with elongated eyes that are red at the ends, mounted on a great buffalo, adorned with a black garland and cloth, may Yama, of great splendour, devoted to the Law of Śiva, engaged in the worship of Śiva, grant me peace and health! (84-86)

\section{[Nirṛti in Kṛ̣ṇ̄ā]}

In the direction of Nirrti (southwest) is the famous town called Kṛ̣nāā, 55 crowded with great Rakṣases and Gaṇas, filled with Piśācas and Pretas. There, with the appearance of a dark cloud, adorned with a red garland and cloth, sword in hand, of great splendour, ablaze with wide open mouth, may the lord of Rakșas, Nirrti, ${ }^{6}$ always delighting in the worship of Siva, continuously bestow great peace on me, eager for devotion to Śiva! (87-89)

\section{[Varuṇa in Śuddhavatī]}

In the western direction is the splendid city Suddhavatī, crowded with various Gaṇas, filled with many Kimnaras. There, with the appearance

${ }^{54}$ Bhuvanavinyāsa 2.43.14ab has the variant Tejasvinī as the name of Agni's city: tejasvinì nāma purī āgneyyām pāvakasya tu.

${ }^{55}$ Bhuvanavinyāsa 2.43.16ab refers to this city as Śuddhavatī: nairrte krṣnavarṇā ca tathā śuddhavatì śubhā.

${ }^{56}$ The manuscripts are divided between the spelling Nirṛti and Nairṛti. 
of pearl, ${ }^{57}$ with eyes all tawny, with a bright cloth and garment, ${ }^{58}$ a noose in his hand, very powerful, may Varuna, with supreme devotion, his mind solely dedicated to Śiva, constantly destroy disease, grief, injury and pain for me! ${ }^{19}(90-92)$

\section{[Vāyu in Gandhavatī]}

In the northwestern (Vāyu's) direction is the splendid city Gandhavatī, inhabited $^{60}$ by Ṛșis, Siddhas and Gaṇas, with a golden wall and doorway. There, with a copper-coloured body ${ }^{61}$ his eyes dark and tawny, holding onto an inflated cloth, furnished with the flagstaff as his weapon, ${ }^{62}$ may the Wind (Pavana), the supreme god, devoted to Parameśvara, bestow safety, health, power [and] peace on me always! (93-95)

\section{[Kubera in Mahodayā]}

In the north is the city called Mahodayā, very luminous, crowded with many Yakșas, embellished with various jewels. There, may the god, with

\footnotetext{
57 Varuṇa should have the colour of lapislazuli (snigdhavaidūryasañkāśah) according to VDhP 3.52.1c.

${ }^{8}$ Cf. VDhP $3 \cdot 5^{2.1}$ śvetāmbaradharas tathā.

59 The adopted reading receives only limited support. Several manuscripts point to a plural nirnāśayantu, but the plural is not fitting here.

${ }^{60}$ I take adhyușta as metri causa for adhyușita. The variant readings may represent different attempts to correct it.

${ }^{61}$ According to $\operatorname{VDhP} 3.58 .1 a$, Vāyu has the colour of the sky: vāyur ambaravarnas tu.

${ }^{62}$ The interpretation of the compound pațaryāptāntarālina is uncertain, but one of Vāyu's iconographic attributes is an inflated cloth. Cf. VDhP 3.58.1c: vāyvāpūritavastra. See Wessels-Mevissen 2001: 102-103, for examples from sculpture. I take the first three members of the compound as an inverted bahuvrini: 'holding onto a cloth whose inside is pervaded [by wind]'. The Śivadharmavivarana rather takes it to mean 'dwelling in the space that is enveloped in a cloth'. Wessels-Mevissen distinguishes between a 'cloth type' of Vāyu that is North Indian and a 'flag type' that is South Indian, but she also draws attention to a sculpture on the Bāla Brahmā and Svarga Brahmā temples at Ālampur, dated to the second half of the seventh century $\mathrm{CE}$, which includes a 'rare combination of the cloth and the flag' (figures 68 and 75). The same combination is also suggested by the present invocation. The various sets of Dikpālas from Ālampur in general show great correspondences in form with the descriptions in the present invocations. For example, the text only mentions the vähanas of Indra and Yama, while the sculptures from Ālampur only depict the vāhanas of Indra, Yama and Nirrti and not those of any of the other Dikpālas (Wessels-Mevissen 20o1: 39-44). The Navabrahmā temple group as a whole shows a strong interaction of both North and South Indian artistic idioms.
} 
a mace in his hand, adorned with a colourful garland and cloth, shortarmed, of great splendour, eyes all tawny, Kubera, granter of boons, prosperous, delighting in the worship of the feet of Hara, bestow peace on me, being pleased, peaceful, with a delighted mind! (96-98)

\section{[İsāāna in Yaśovatī]}

The beautiful city Yaśovatī is established in the northeastern (İsāna's) direction, crowded with various Ganas, inhabited by many gods, surrounded by a luminous wall, unequalled, very eminent. There, may he too, with the appearance of pearl, decorated by the moon, three-eyed, with a peaceful form and self, bearing a rosary, ${ }^{63}$ Hara, İsāna, the supreme god, supreme among all gods, bestow peace on me quickly, with his entire self! (99-101)

\section{$[\text { Devas in the Seven Worlds] }]^{64}$}

The gods who dwell in the Bhūloka, the Bhuvarloka, the Svarloka, provided with divine splendour - may they always bestow peace on me! The gods who are present in the Maharloka, the Janarloka and the Tapoloka - may they too, delighted, always bestow peace on me! And the gods in the Satyaloka, their bodies self-luminous, devoted to Śiva, welldisposed - may they destroy danger for me! $!^{65}(102-104)$

\footnotetext{
${ }^{63}$ Surprisingly, no mention is made of the triśūla, İsāna's major attribute.

${ }^{64}$ On the seven worlds, see Kirfel 1967: 128.

${ }_{55}$ After this, $\mathrm{N}_{58}^{K}$ and $S_{67}^{S}$ add some more verses on the appearance of the gods in these seven worlds (in reverse order) and their devotion to Śiva. In the version of $\hat{S}_{67}^{S}$ :

The gods in Tapoloka, their bodies blazing like crystal, devoted to Rudra, great souls - may they always bestow peace on me! And the gods in Janaloka, shining like purified gold, bowing down to İsāna — may they always bestow peace on me! And the gods in Mahalloka, resembling refined gold, delighting in the worship of Śiva - may they provide security for me, always! And the gods that are in Svarloka, of shining colour, very powerful, bent towards Śaṃkara - may they provide victory for me! And the gods in Bhürloka, illuminating the ten directions, their minds dedicated only to Śiva - may they extinguish danger for me!
}

These verses are clearly secondary. Similar passages occur in $\mathrm{P}_{32}^{T}$ after $102 \mathrm{~d}$ (originally placed after 1ogb) and in $\mathrm{P}_{72}^{T}$ after 103d. 
[Devas in Caves, Forests etc.]

The gods who dwell on mountains, in caves, strongholds and in forests, devoted to the worship of Rudra - may they always grant protection to me! ${ }^{66}(105)$

[Sarasvatī]

With a body white like the rays of the moon in autumn, with spotless splendour - may Sarasvatī, devoted to Śiva, quickly bestow peace on me! (106)

$$
\text { [Śrīi }
$$

With a beautiful golden complexion and a bud in her lotus hand - may the goddess Śrī, devoted to Śiva, grant prosperous fortune to me! (107)

$$
\text { [Jayā] }
$$

With a beautiful moon-like face, blazing like brilliant gold - may the goddess Jayā, devoted to Śiva, grant all desires to me! $!^{67}$ (108)

\section{[Aparājitā]}

With a wonderful pearlnecklace, wearing a bright golden girdle ${ }^{68}$ — may Aparājitā, delighting in Rudra, grant victory to me! $!^{69}$ (109)

\footnotetext{
${ }^{66}$ After this, $\mathrm{E}^{N}$ adds four verses about the seven subterranean regions (Talas): Mahātala, Rasātala, Talātala, Sutala, Nitala, Vitala and Tala.

${ }^{6} 7$ After this, $S_{67}^{S}$ adds six more pādas, invoking Vijayā and Jayantī, thus completing the group of the four sisters of Tumburu: Jayā, Vijayā, Jayantī and Aparājitā. See Goudriaan 1973 .

68 The manuscripts are divided between bhāsvatkanakamekhalā, which receives support from the Bhavișyapurāna parallel, and bhāskaroijvalatejasā.

69 Aparājitā is an epithet of Kauśikī/Vindhyavāsinī in the Skandapurāna and Vindhyavāsinī is presented like a daughter of Śiva and Pārvatī in that text. This myth may have been transmitted in a small Śaiva circle only because, as far as we can tell, it is only told in the Skandapurāna (SP III). Note in this connection the variant-but unmetrical—reading rudrasutāparäjitāa. The reading adopted in the edition is hypermetrical, but this may be allowed if we take $a p a$ - as a single syllable, thus yielding a bha-vipulā.
} 


\section{$[\text { Navagrahas }]^{70}$}

[Sūrya: Sun]

With a complexion coloured red like vermillion, ${ }^{71}$ with elongated eyes, furnished with two rays, ${ }^{72}$ riding on seven horses - may he [Sūrya], with a garland of rays, the blessed one, delighting in the worship and praise of Śiva, bestow great peace on me, warding off injury from the planets! (110-111)

\section{[Soma: Moon]}

Bringing about the prosperity of the world, ${ }^{73}$ cooling with the vessel of

$7^{\circ}$ The invocation of the planets follows the temporal order of the seven days of the week, which was established in India by the fourth century. See Pingree 1965 and Yano 2004. Material representations of the planets as a group start to appear from the second half of the fifth century, with Ketu being absent until ca. 6oo CE. They become significantly more numerous from the second half of the seventh century (Markel 1995: 94-95, 100). The first lintel depicting all nine planets comes from Uttar Pradesh (Markel 1995: figure 21). Markel (1995: 164) argues that the astrological verses in MtP 94.1-9 (included in his appendix, pp. 189-19o) would be the first source that gives 'anthropomorphic descriptions of the planetary deities in the group known as the navagraha', dating the passage, on debatable grounds, to the sixth century. He finds the first evidence for the addition of Rāhu and Ketu to the sun, the moon and the five conventional planets, to form the navagraha, in the mid-sixth century Bṛatsaṃhitā. Varāhamihira, following Garga, is the first Jyotișa author to devote considerable portions of text to Rāhu and Ketu. See p.16. The nine planets are also mentioned several times in his earlier work, the Yogayātrā, for example in Yogayātrā 3.19-21 (on these two verses, see Pingree 1959). For a study of the navagrahaśānti, see Bühnemann 1989 .

${ }^{71} \mathrm{Cf}$. VDhP 3.67.2b sindūrāruṇasaprabhaḥ 'with a lustre red like vermillion'. Note that -aruna-is also found as a variant reading in several manuscripts.

$7^{2}$ I follow the reading of $\mathrm{N}_{82}^{K}, \mathrm{~N}_{12}^{K}, \mathrm{E}^{N}$ and $\hat{S}_{67}^{S}$. The variant reading 'thousand-rayed' (sahasrakiranah) appears to be the lectio facilior, although it receives support from the parallel in the Bhavisyapurāna. In sculptures from the Gupta period onwards, Sūrya is portrayed with two lotus buds or two full-blown lotuses in his hands. See Markel 1995: 30. This iconographic feature is also described in most of the textual sources (e.g. Brhatsam hitā 58.47ab), but VDhP 3.67.4 states that he should be depicted with the reins (raśmayah) held in both of his hands. Could it be that kiraña should be understood here to denote the reins? $\mathrm{N}_{58}^{K}$ adds two pādas after 11od, referring to the two lotuses held in Sūrya's hands.

${ }^{73}$ This is one of the rare cases where $\mathrm{N}_{82}^{K}$ is not supported by $\mathrm{B}_{99}^{c}$. The ending-dhāro (instead of -kara) may have been caused by the presence of -dhära-in pāda b. In addition the hi was probably introduced as a hiatus breaker. 
nectar ${ }^{74}$ - may Soma, with a gentle disposition, drive away injury from the planets! (112)

\section{[Anggāraka: Mars]}

With a body whose limbs are like ruby, tawny-eyed — may Angāraka always drive away injury from the planets for me! ${ }^{75}$ (113)

\section{[Budha: Mercury]}

With a saffron-skinned body, his hand raised with a bow ${ }^{76}$ - may the illustrious Budha, devoted to Śiva, always drive away injury from the planets! (114)

\section{[Bṛhaspati: Jupiter]}

With a metallic golden complexion, ${ }^{77}$ abiding in all knowledge, ${ }^{78}$ Brhhaspati, at all times devoted to the praise of İsāna - may he too, furnished with a supreme peaceful mind, having defeated injury from the planets, bestow victory always! $\left(115^{-116)}\right.$

\section{[Śukra: Venus]}

With a [white] splendour equal to snow, jasmine and the moon, worshipped by the Suras and the Daitya lords ${ }^{79}$ — may Śukra, delighting in the praise of Śiva, drive away injury from the planets. (117)

${ }^{74}$ See Markel 1995: $36-37$ on this attribute of the Moon.

${ }^{75}$ After this, $\mathrm{P}_{32}^{T}$ and $\mathrm{P}_{72}^{T}$ add a verse about Angāraka's devotion to Rudra. Some of the other invocations of the planets add an adjective referring to their devotion to Siva and it is thus easily explained why someone would have felt the need to add this verse here.

${ }^{76}$ The readings in 114 ab show much variation and the adopted reading is by no means certain. The reference to Budha's bow only occurs in $\mathrm{N}_{82}^{K}, \mathrm{~N}_{58}^{K}, \mathrm{E}^{N}$ and $\mathrm{B}_{99}^{C}$. See Markel 1995: 41-44 for the depiction of Budha as an archer in Bihari and Bengali representations, observing that 'references to the archer's iconography of Budha are absent in early Brahmanical textual descriptions, except for the Agnipurāna (51.11), which prescribes a bow and a rosary' (p. 42).

${ }^{77} \mathrm{Cf}$. VDhP 3.69.3a taptajāmbūnadākāro 'with the appearance of molten gold'.

${ }^{78}$ Brhaspati is the guru of the gods.

79 This is slightly problematic. Sukra is the guru of the demons and not of the gods. One might consider a conjecture -tulyābho 'suradaityendrapüjitah. In the Bhavisyapurāna parallel the reference to the gods has been removed: daityadānavapüjitah. 


\section{[Śanaiścara: Saturn]}

With a [dark] complexion like a heap of collyrium, with the splendour of deep-red eyes - may Śanaiścara, devoted to Śiva, drive away injury from the planets. (118)

[Rāhu: Eclipser]

Like black collyrium, eminent, son of Simhikāa, ${ }^{80}$ very powerful — may Rāhu, devoted to the worship of Śiva, drive away injury from the planets. (119)

\section{[Ketu: Dragon's Tail]}

Shaped like smoke, the planet Ketu, stationed in the northeastern direction, ${ }^{81}$ highly frightening with eyes that are round and very extensive may he, having the colour of straw smoke, removing injury from the planets, with terrible fangs and gaping mouth, bring about victory for me! $!^{82}$ $(120-121)$

\section{[Grahas: Conclusion]}

May these planets, great souls, devoted to the praise of Maheśa, bestow peace on me, full of delight, wishing [me] well at all times! $!^{83}(122)$

\footnotetext{
${ }^{80}$ Rāhu is the son of Kaśyapa and Siṃhikā. $\mathrm{N}_{45}^{c}$ changes saiṃhikeyo to ardhakāyah, a reference to Rāhu's being only a head.

${ }^{81}$ Ketu is the only one of the Navagrahas for which a location in the sky is given.

${ }^{82}$ These two verses are missing in $\mathrm{N}_{77}^{K o}$, which may have important consequences for how we understand the transmission of the text as well as its original time of composition. As discussed earlier (p. 17), it seems plausible that Ketu was added as a ninth Graha at different stages in the transmission of the text. This is suggested not only by the relatively late appearance of Ketu in textual sources in general, but also by the strong variation in readings, which have either two verses or a single verse. The Bhavisyapurāna parallel again has an entirely different reading. $\mathrm{E}^{N}$ adds one more verse after 121, invoking Janman, the name of the first lunar mansion, who is described as bearing a crystal sword (khadgasphațika) in his hand.

${ }^{83} \mathrm{~N}_{58}^{K}$ adds a list of ten Karanas after this, which provides the transition to the following invocation of the Karaṇa Viști. The start of the list is damaged, but it can be easily reconstructed. Traditionally there are held to be 11 Karanas, which each form half of a lunar day (Tithi). Since there are two times 15 Tithis in a lunar month, there are 6o
} 
[The Karana] at whose commencement (mukhe) death is present, she who is named Vișți, very powerful, [the subsequent Viști] with six forms (șanmukhā), creating obstacles, and [the one] bringing victory at the end $(\text { pucche })^{85}$ - the Third, Seventh, Tenth, Fourteenth, Fourth and Eighth, Eleventh and the Night of the Full Moon — on these [Tithis] abounding in obstacles, in the bright and dark fortnight [of the moon's cycle], she is active. May they, having obtained a blessing, bestow peace on me, much longed for! (123-125)

$$
[\text { Tithis }]^{86}
$$

May Amāvāsī (the Night of the New Moon), very auspicious, connected with Pitrrs and Devas, furnished with the splendour of Śiva, bestow peace on me, the supreme and auspicious peace of Śiva, the supreme soul! And the very calm First (Pratipad), and the beautiful Second, and the emi-

Karaṇas. They are divided into two groups: four immovable (sthira) Karaṇas (Śakuni, Catuṣpāda, Nāga and Kiṃstughna), which have a fixed position and appear only once in a cycle, and seven moveable (cara) Karaṇas (Bava, Bālava, Kaulava, Taitila, Gara, Vaṇij, Viștị), which each reappear eight times in a lunar cycle. See Bṛhatsaṃhitā 10o. Only Catuṣpāda is missing in the list of $\mathrm{N}_{58}^{K}$.

${ }^{84}$ Verses $123^{-13} 6$ are missing in $\mathrm{N}_{77}^{K o}$, which may again point to an earlier stage of composition of the text (cf. Ketu above). This is further supported by their absence in the parallel of the Bhavișyapurāna. I follow the common spelling Viști, instead of the otherwise unattested Vṛsți. Vișți is the seventh of the movable Karanas and traditionally regarded as very dangerous. Cf. Bṛhatsaṃhitā 100.4cd: na hi viștikrtạn vidadhāti śubham paraghātavișādișu siddhikaram 'Nothing done during Vișți leads to benefit, [but it] causes success in slaying enemies, poisoning, etc.' It is remarkable that only this Karana should be mentioned here and none of the other ones.

${ }^{85}$ The interpretation of the verses addressing Viști is doubtful. I am grateful to Bill Mak for his help in interpreting the text. As he pointed out to me, mukha is used in the sense of 'form' in Jyotișa literature. The verse identifies the eight manifestations of Viști in a lunar month: the first one is associated with death in our text, the following six with obstacles, and the eighth with victory. $\mathrm{P}_{3^{2}}^{T}$ adds an extra two pādas, including a request for śânti, thereby separating the invocation in this verse from the one that follows.

${ }^{86} \mathrm{P}_{3^{2}}^{T}$ and $\mathrm{P}_{7^{2}}^{T}$ have a number of significant variants, which systematically includes the name of the deity presiding over each lunar day: 
nent ${ }^{87}$ Third Tithi, and the very glorious Fourth! The peaceful Fifth and the supreme Sixth Tithi, the auspicious Seventh Tithi, and the very powerful Eighth, this Tithi of the Trident-holder (Śiva), known as the destroyer of evil, the very terrifying Ninth Tithi, which is proclaimed to belong to Durgā! And the splendid Tenth and Eleventh Tithi, and the peaceful Twelfth and the Thirteenth Tithi! The very heroic Fourteenth, the Tithi who is the daughter born from Śankara! And Pūrṇamā (the Night of the Full Moon), of complete self, the constantly blazing Tithi! May the Tithis, constantly auspicious-minded, and in due order, they who are always following the course of the moon during the two halves, constantly bestow peace on me, conforming to Siva's command! (126-132)

$$
[\text { Yogas }]^{88}
$$

Viṣkambha, Prīti, Āyuṣmant, Saubhāgya, Śobhana, Atigaṇda, Sukarman, Dhṛti and Śūla, Gạ̣ḍa, Vṛddhi, Dhruva, Vyāghāta, Harṣaṇa, Vajra, Siddhi, Vyatīpāta, Variyas, Parigha, Śiva, Siddhi, Sādhya, Śubha, Śukla, Brahman, Aindra, Vaidhrtit, these Yogas, very powerful, arisen from the moon and the sun, all devoted to Śiva, following the command of Śiva — may they

\begin{tabular}{|l|l|}
\hline Amāvāsī & Pitṛs and Devas \\
Pratipad & Vahni \\
Second & Arka $\left(\mathrm{P}_{32}^{T}\right)$ \\
Third & Dhanada \\
Fourth & Gajavaktra \\
Fifth & Śr̄̄ \\
Sixth & Skanda \\
Seventh & Ravi \\
Eighth & Rudra \\
Ninth & Durgā \\
Tenth & Yama \\
Eleventh & Indra \\
Twelfth & Viṣṇu \\
Thirteenth & Madana \\
Fourteenth & Maheśa $\left(\mathrm{P}_{72}^{T}\right)$ \\
Paurṇamāsī & Himāṃśu \\
\hline
\end{tabular}

A comparison of this table with the list provided by Einoo (2005: 105-106) shows that, despite variation with respect to individual Tithis, there was a general consensus regarding most of the deities presiding over the Tithis.

${ }^{87}$ The form śrimmān is used, where grammatically śrimmatī would be required.

${ }^{88}$ This list of 27 Yogas is standard. 
continuously bestow peace on me and the destruction of sins! ${ }^{89}\left(133^{-}\right.$ 136)

[Nakșatramātṛs: E $]^{90}$

The supreme goddess Kṛttikā, sweet-faced Rohiṇī, eminent, blessed Mṛgaśirā, and highly luminous Ārdrā, Punarvasu, Pușyā, and the very powerful Aśleșā, these Nakṣatramātṛs, adorned with a garland of light, devoted to the praise of Mahādeva, inclined to Mahādeva, they who are stationed in the eastern part - may they always bestow peace on me! (137-139)

\section{[Nakṣatramātṛs: S]}

Maghā, abiding in all qualities, and Pūrvaphālguṇī, the superior Uttaraphālguṇī, Hastā and the excellent Citrā, Svātī, Viśākhā, granter of boons, occupying the southern abode, they worship Deva, the Lord of the three worlds (Tribhuvaneśvara), all the time, these Nakṣatramātṛs, adorned with splendour - may they continuously bestow peace on me, impelled by Śiva. $(140-142)$

\section{[Nakșatramātṛs: W]}

Anurādhā, Jyeșțhā, Mūlā, endowed with wealth and power, the very heroic Pūrvāṣạ̣̄hā, and the splendid Uttarāṣāṭhā, the Nakṣatra named Abhijit, highly luminous Śravanāa, these of kingly form reign, blazing in the west, they worship İsāna all the time, well-disposed — may

\footnotetext{
${ }^{89}$ After this, $\mathrm{N}_{45}^{c}$ adds eight more pādas on the 27 Yogas and also includes a request to Bava, Bālava, Kaulava, Tautila (= Taitila), Gara and Vaṇija for peace. These are the six other moveable Karanas, thus to be added to the already mentioned Vișți (123-125).

90 The designation Nakșatramātṛs, in reference to the Nakṣatras (lunar mansions), appears not to be attested elsewhere. They are invoked as goddesses $(d e v \bar{v})$. Hazra (1954: 16) uses the order of the Nakșatras mentioned here as an argument for dating the Śivadharmaśāstra to a time before 550 CE. The arrangement from Kṛttikā to Bharaṇī represents the old order, whereas Varāhamihira used the order from Aśvinī to Revatī, which henceforth became the model. On this change of order, see also Yano 2003: 378. The lists in Kirfel 1967: 36 and 138, however, both display the order Kṛttikā to Bharaṇi. Both orders can be found in post-Varāhamihira texts and as such this order does not allow us to date the text before Varāhamihira as Hazra has done.
} 
they bestow peace on me, they who are endowed with great powers! $!^{11}$ $(143-145)$

[Nakșatramātṛs: N]

Dhanișthā, Śatabhiṣā, ${ }^{92}$ Pūrvabhādrapadā, Uttarabhadrā, Revatī, the very prosperous Aśvinī, and the very heroic Bharaṇī, they who are always stationed in the north, continously devoted to the praise of Śiva, their minds fixed on meditation on Śiva - may they constantly bestow peace on me, at all times, with an auspicious rise. (146-147)

[Rāśis: E $]^{93}$

Meșa (Aries), Siṃha (Leo), the king of animals, ${ }^{94}$ and Dhanu (Sagittarius), best of the lights, they shine in the east, intent upon worship of Śiva — may they bestow peace on me, bright, intent on union with Śiva! ${ }^{95}$ (148)

${ }^{91}$ The lengthening of the vowel in vibhütībhih $\left(\mathrm{N}_{12}^{K}\right)$ is metri causa. Pāda $145 \mathrm{~d}$ shows a lot of variation; a reading starting with vibhütim ca may also be considered.

$9^{2}$ The adopted reading is metrically bad, but it receives the best support and is even found in the parallel in the Bhavisyapurāna. The metrically correct readings of $\hat{S}_{67}^{S}$ and $\mathrm{P}_{7^{2}}^{T}$, on the other hand, are quite likely secondary.

93 This section on the zodiac signs (Rāśi) does not follow the regular order, which would rather be: 1) Aries, 2) Taurus, 3) Gemini, 4) Cancer, 5) Leo, 6) Virgo, 7) Libra, 8) Scorpio, 9) Sagittarius, 10) Capricorn, 11) Aquarius, 12) Pisces. Instead we have here the Hellenistic trigon (Sanskrit trikona) system, arranged according to four triangles, groups of three zodiac signs: East $(1,5,9)$, South $(2,6,10)$, West $(3,7,11)$, North $(4,8,12)$. This system was not recognized everywhere, which has led to a number of ambiguities in the transmission of the first two groups in the different manuscript traditions. The trigon system is found in some of the Jātakas, but it is not common elsewhere. See Pingree's note on Yavanajātaka 1.66-67 (Pingree 1978: 223-228). I am grateful to Bill Mak for pointing this out to me.

${ }^{94}$ I adopt mrgādhipah, instead of $v r s ̦ a \overline{d h i p a h, ~ t h e ~ r e a d i n g ~ o f ~ t h e ~ m a j o r i t y ~ o f ~ t h e ~}$ Nepalese manuscripts. Reading vrșādhipah, i.e. Taurus, is problematic because the inclusion of Taurus would interfere with the trigon system underlying this section. It seems likely that the source text originally read mrgädhipah, which was changed to vrșậ̣hipah early in the transmission because a scribe expected the second zodiac sign to be Taurus. Note that mrgādhipah is also the reading of the Bhavisyapurāna parallel.

95 The combined reading of $\mathrm{N}_{77}^{K o}, \mathrm{~N}_{12}^{K}, \hat{S}_{67}^{S}$ is supported by the Bhavisyapurāna parallel. 
[Rāśis: S]

Vṛṣa (Taurus), ${ }^{96}$ Kanyā (Virgo), the supreme goddess, and Makara (Capricorn), the prosperous, always worship Siva in the southern part, constantly, with highest devotion - may they always bestow peace on me! (149)

\section{[Rāśis: W]}

Mithuna (Gemini), Tulā (Libra) and Kumbha (Aquarius), established in the west - may they, delighting in honouring the feet of Siva, always bestow peace on me! (150)

\section{[Rāśis: N]}

Karkața (Cancer), Vṛścika (Scorpio) and Mīna (Pisces), they who are present in the north, at all times worship Rudra, Lord of the worlds may they, following the command of Siva, constantly bestow peace on me! ${ }^{97}(151)$

${ }^{96}$ The reading adopted in pāda a (vrșaḥ kanyā ca paramā), yielding a na-vipulā, is found only in $\mathrm{N}_{58}^{K}$, but receives some support from $S_{67}^{S}$ and $\mathrm{P}_{32}^{T}$. Moreover, it is also the reading of the parallel in the Bhavisyapurāna. It includes Taurus among the group of three Rāsis located in the south. The reading of the majority of the Nepalese manuscripts (kanyā ca paramā devī), although well supported, is problematic, because only two zodiac signs would be mentioned in the south, which would again interfere with the trigon system. The change may have occurred at the same time that Taurus was introduced in the previous verse. The amount of variation in this part of the text is quite striking. $\mathrm{N}_{45}^{C}, \mathrm{P}_{32}^{T}$ and $\mathrm{P}_{72}^{T}$ have two additional pādas after 149b, mentioning Vṛșa $\left(\mathrm{N}_{45}^{C}\right)$ and Rṣabha $\left(\mathrm{P}_{3^{2}}^{T}, \mathrm{P}_{7^{2}}^{T}\right)$, but in very different wording, suggesting that these two pādas were added independently of each other (see also the additional two pādas in $\left.\mathrm{N}_{58}^{K}\right)$.

${ }_{97}$ After this, $\mathrm{N}_{5^{8}}^{K}$ adds six pādas referring to the Tārakagaṇas, nine classes of asterisms. There are some problems in the readings of this passage, but the list corresponds largely with the classification found in Varāhamihira's Ṭikanikayātrā 1.20 (Janma, Sampat, Vipat, Kṣema, Apāya, Śubha, Kașța, Maitra, Atimaitra): tārās tu janmasampadvipatkarā kṣemāpāyaśubhakașțā $\mid$ maitrātimaitrasaṃjñāś caitāḥ saṃjñānurūpaphalāh $\| . S_{67}^{s}$ adds 16 pādas referring to the Saṃkrāntis, the sun's transitions to another house, in accordance with the seven days of the week: Ghorā (Sunday), Dhvānkṣī (Monday), Mahodarī (Tuesday), Mandākinī (Wednesday), Nandanā (Thursday), Miśrikarā (Friday), Rākșasī (Saturday). I have only found these Saṃkrāntis in the Bṛhatpārāśarahorāśāstra: sūryādau sūryasaṃkrāntir ghorā dhvāñkși mahodarī| nandā mandākin̄̄ miśrā rākșasasyeti saptadhā \| (Bṛhatpārāśarahorāśāstra 91.1). The passage may well have been added at a very late moment in the transmission of the text in Kashmir, as is also suggested by the fact that it does not occur in any of the other manuscripts. 


\section{[Seven Sages: Ursa Major]}

The renowned Seven Sages, ending in Dhruva (the polar star), highly luminous - may they, possessed with the favour of Śiva, bestow peace on me, always! (152)

\section{[Sages of Great Vows]}

Kāśyapa, Gālava, Gārgya, the great sage Viśvāmitra, Manu, Dakṣa, Vasișțha, Mārkaṇdạ, Pulaha, Kratu, ${ }^{98}$ Nārada, Bhṛgu, Ātreya, Bharadvāja, the sage Angiras, Vālmīka, Kauśika, Kaṇva, Sākalya, Punarvasu, Śālañkāyana - may these and other sages of great vows, engaged in meditation and praise of Śiva, always bestow peace on me! (153-155)

[Wives, Daughters and Sons of the Sages]

The wives of the sages, very pure, the sages' daughters and sons, ${ }^{99}$ may they, constantly intent upon praise of Śiva, bestow peace on me, always! $(156)$

[Siddhas, Gandharvas, Apsarases, Vidyādharas, Garuḍas]

The Siddhas, who have perfected their asceticism, the flocks of Gandharvas and Apsarases, the Vidyādharas, ${ }^{100}$ great souls, and the Garuḍas, very prosperous - may they, intent upon Maheśvara, worshipping the foot of Maheśvara, quickly bestow success, being intent upon benediction! $(157-158)$

${ }^{98} \mathrm{~N}_{45}^{C}$ inserts more sages, not all of whose names have been transmitted correctly.

${ }^{99}$ I take rșikumärikāh to refer to the sons as well as the daughters of the sages, in accordance with the format followed in the Daitya section below. The variant reading rșikanyākumārikāḥ may also be considered.

${ }^{100}$ The reading of $\mathrm{N}_{77}^{K o}, \mathrm{~N}_{82}^{K}$ and $\mathrm{B}_{99}^{C}$ looks defective in $157 \mathrm{bc}$ and may have arisen due to an early eye-skip. With some hesitation I follow the reading suggested by most of the other manuscripts. 
[Daityas: 1]

Namuci, the king of the Daitya lords, the very powerful Śankukarṇa, ${ }^{101}$ the Daitya called Mahānāda, of great heroism — may they, constantly intent upon the worship of god Hātakeśvara, ${ }^{102}$ very prosperous, quickly bestow power and heroism on me! (159-160)

[Daityas: 2]

Mahājambha, Hayagrīva, Prahlāda, Anuhlādaka, Tāraka, the Daitya Agnimukha, Kālanemi, Mahotkața - may these Daityas, great souls, devoted to the reality of Śiva, grant prosperity, power and heroism, resulting in joy! (161-162)

[Daityas: 3]

Virocana, Hiranyākșa, Suparva, and Sulomaka, Mucukunda, Sukunda, and the Daitya Revataka, they constantly worship Siva, instantly, with supreme intent - may they, constantly benevolent, grant prosperity to me, always! (163-164)

[Wives, Daughters and Sons of the Daityas]

The illustrious wives of the Daityas, the splendid daughters of the Daityas and the sons of the Daityas - may they always bestow peace on me! (165)

${ }^{101} \mathrm{P}_{72}^{T}$ adds three more Daityas: Jambha, Nikumbha and Śakața.

${ }^{102}$ Hāțakeśvara: Śiva as lord of Pātāla. Many of the Daityas mentioned here feature in Purāṇic descriptions of the Pātāla. See, for example, Kirfel 1967: 145, based on the descriptions in the Vāyu-and Brahmāṇdapurāna: in the first Tala are e.g. the abodes of Namuci, Mahānāda and Śankukarna, in the second those of Mahājambha, Hayagrīva and others, in the third those of Prahlāda, Anuhlāda, Tāraka and others, etc. Although not all of the Daityas mentioned in the Purānic descriptions are listed here, the order of the Daityas listed corresponds with those of the Daityas dwelling in ever lower Talas. 


\title{
[Eight Nāgarājas] ${ }^{103}$
}

\author{
[1. Ananta]
}

With a red body, elongated eyes that are red at the edges, swelling with pride with his great hood, marked by a conch and a lotus ${ }^{104}$ may Ananta, king of the Nāga lords, delighting in the praise of Siva's feet, destroy the poison of great evil and quickly bestow peace on me! $(166-167)$

\section{[2. Vāsuki]}

With a very white body, with a crown of very white lotuses, swelling with pride with a handsome hood, adorned with a charming necklace — may Vāsuki, king of the Nāga lords, the great one, intent upon the worship of Rudra, destroy the poison of great evil and quickly bestow peace on me! $(168-169)$

${ }^{103}$ On the Nāgarājas, see Vogel 1926: 189-219. As for the colours associated with the Nāgarājas, there are different traditions (Wayman 1987: 68-69). Rao 1914-16, II: 556557, referring to the Mayaśilpa, gives the following details for the seven serpent lords: Vāsuki (pearlwhite); Takșaka (glistening red, with a svastika on the hood); Karkoțaka (black, with three white stripes on the hood); Padma (rosy, with a white streak and adorned with coral ornaments); Mahāpadma (white, with the mark of the triśüla on the hood); Kulika (red, with the mark of the crescent moon on the head). Many of these features are shared in remarkable detail with the descriptions in the present passage. The Mayaśilpa passage quoted by Rao in Appendix B, p. 274, runs as follows:
śvetadehaś ca kartavyas sphuranmauktikasannibhaḥ|
raktāingas svastikopetas sutejās takșako mahān \|
krșnahạ kārkoțakaḥ kaṇțhe śuklarekhātrayānvitạ̣|
raktapadmanibhạ̣ padmạ̣ śiraś śuklas savidrumạ̣ \|
śan்khavarno mahāpadmo mastake krșnaśūladhṛk |
hemābhaś śañkhapālas syāt sitarekhādharo gale \|
kuliko raktadehas tu candrārdhakrtamastakah |
dvijihvā bāhuvat saptaphañāmaṇisamanvitāh $\|$
akșasūtradharās sarve kuṇdikāpucchasamyyutāḥ|
ekabhogās tribhogā vā hy etajjātās sutādayaḥ\|

${ }^{104}$ The attributes of conch and lotus connect Ananta with Viṣnu. 


\section{[3. Takșaka ${ }^{105}$}

With a very yellow body, rich in quivering coils, and with a very luminous splendour, marked by the Svastika — may Takșaka, the illustrious Nāga lord, accompanied by a crore of Nāgas, bestow peace on me, destroying the poison of all crimes! (170-171)

\section{[4. Karkoțaka]}

With a very black colour, an expanding hood over his head, provided with three lines on his neck, ${ }^{106}$ furnished with terrible fangs as weapons may the great Nāga Karkoțaka, possessed of poisonous pride and power, destroy the pain of poison, weapon and fire, and bestow peace on me! (172-173)

\section{[5. Padma]}

With a lotus-coloured body, his elongated eyes like handsome lotus [petals], illuminated with five spots - may the great Nāga called Padma, delighting in the praise of Hara's feet, bestow peace on me, destroying the poison of great evil! (174-175)

\section{[6. Mahāpadma]}

And with a body like a white lotus, of immeasurable splendour, always adorned on his head with [the marks of] a brilliant conch, trident and lotus - may the great Nāga Mahāpadma, constantly bowing to Paśupati, destroy the terrible poison and quickly bestow peace on me! (176-177)

$$
\text { [7. Śan̉khapāla] }
$$

With a dark body-mass, his eyes like beautiful lotuses, intoxicated with poisonous pride and power, with a single line on his neck — may Śankkhapāla, bright with lustre, worshipping the lotus-feet of Śiva, destroy great evil, the great poison, and bestow peace on me! (178-179)

\footnotetext{
${ }^{105}$ For an identified Takșaka sculpture, see Sircar (1971: 138-140), who refers to a Nāga sculpture in the State Museum Lucknow, whose pedestal is inscribed in eighth-century Siddhamātṛkā script with the words śrītakhakanāga, i.e. śrìtakṣakanāga.

${ }^{106}$ Cf. Niśvāsamukha 3.168, where Karkotaka is called Trirekhin.
} 


\section{[8. Kulika]}

With a very terrifying body, his head furnished with the sickle of the moon, swelling with pride with a shining hood, marked with an auspicious mark - may Kulika, the best of the Nāga kings, always intent upon Hara, remove the terrible poison and bestow peace on me! (180-181)

\section{[Other Nāgas]}

The Nāgas in the sky, the Nāgas abiding in heaven, the Nāgas abiding on earth, at mountains, in caves and forts, the Nāgas present in the nether region - may all of them, assembled here, dedicated to the praise of Rudra's feet, bestow peace on me! (182-183)

\section{[Wives, Daughters and Sons of Nāgas]}

The Nāgas' wives, the Nāgas' daughters and the Nāgas' sons — may they, devoted to Śiva, benevolent, always bestow peace on me! (184)

\section{[Śrutiphala of Nāga section]}

Snakes do not harm the one who will recite or hear this totality of Nāgas, nor does poison ever reach him. $(185)^{107}$

\section{[Rivers: 1]}

The holy great goddess Gangā, Yamunā, the river Narmadā, Gomatī, Kāverī, Varuṇā, ${ }^{108}$ as well as Devikā; ${ }^{109}$ [these] rivers always worship Deva, the lord of all creatures, the supreme lord, Maheśvara, devoted to the reality of Śiva - may they constantly bestow peace on me and destroy evil; let them quickly grant success, being free from all obstacles! (188)

\footnotetext{
${ }^{107}$ This is the only case in which the text inserts a statement about the śrutiphala. $\mathrm{P}_{3^{2}}^{T}$ and $\mathrm{P}_{72}^{T}$ add four more pādas about the results to be obtained from the recitation.

${ }^{108}$ The Varunā is a tributary of the Ganges, joining the great river at Vārāṇasī (SP IIA, 222).

109 Hazra (1954: 16-17) takes the mention of Devikā here and Candrabhāgā below as evidence for a northwestern origin of the text. $\mathrm{P}_{32}^{T}$ adds four more rivers after this: Kauśikā, Sarasvatī, Tāmraparṇī and Sarayū.
} 
[Rivers: 2]

The very holy Candrabhāgā, the splendid river Godāvarī, Sarayū, the superior Gaṇạakī, Kauśikī and Sarasvatī - may these highly fortunate rivers, pleased, delighting in praising Siva's feet, bestow peace on me, single-minded in their meditation on Śiva! (190)

[Rivers: 3]

The river called Nairañjanā, ${ }^{110}$ and the great river Śṇa, the supreme Mandākinī, and the splendid Samnihitā ${ }^{111}$ — may these and many other rivers, flowing on earth, in heaven and in the sky, intent upon Rudra's praise, bestow peace on me! $(192)^{112}$

[Yakșas]

[1. Mahāvaiśravaṇa $]^{113}$

The god Mahāvaiśravana, the prosperous lord of the Yakșas, with a retinue of crores of Yakșas, joined by a host of Yakșas, endowed with great majesty, delighting in the praise of Hara's feet, single-minded in meditation on Hara, best of those who bow down to Hara's feet ${ }^{14}$ - may he, delighted, bestow peace on me, his elongated eyes like lotus-petals! (193-194)

110 The presence of the Nairañjanā in this list is noteworthy. The name of this river, identified with the present-day Phalgu, flowing near Bodhgayā (Dey 1971: 135), features in Buddhist sources (e.g. Buddhacarita 12.91a), but appears not to be attested elsewhere outside of Buddhist literature.

${ }^{111}$ This may be the Saṃnihitī or Samnihityā, a tīrtha in Kurukṣetra (MBh 3.81.167169). See SP IIA: 232, and Kane IV: $801 . \mathrm{N}_{58}^{K}$ and $\hat{S}_{67}^{S}$ add a few more rivers in the northwest with some variations in naming and spelling.

${ }^{112} \mathrm{~N}_{58}^{K}$ adds eight more pādas, invoking the earth with her continents and all tìrthas and lakes on earth, inhabited by Śrīkanthha. The last four pādas of this addition are shared with $\hat{S}_{67}^{S}, \mathrm{P}_{32}^{T}$ and $\mathrm{P}_{72}^{T}$.

${ }^{113}$ Vaiśravana is another name for Kubera, who is mentioned in his function as Dikpāla above (96-98). Here he appears in a different identity, as the lord of the Yakșas.

${ }^{114} \mathrm{~N}_{58}^{K}$ adds two pādas on worship by the Yakșiṇīs and Yakșas' daughters for pleasure and liberation. 


\section{[2. Manibhadra ${ }^{115}$}

The great Yakṣa Manibhadra, decorated with gems and jewels, shines with a captivating necklace attached to his neck, his body surrounded by Yakșiṇis and Yakșa daughters — may he, fully engaged in the praise of Rudra, bestow peace on me! (195-196)

\section{[3. Suviroma $]^{116}$}

And the Yakșa lord Suviroma, decorated with gems and earrings, shines with a beautiful golden turban on his forehead, surrounded by many Yakșas, his body venerated by Yakșas - may he, devoted and intent upon worshipping Śiva, bestow peace on me! (197-198)

\section{[4. Pāñcika] $]^{117}$}

The Yakșa lord named Pāñcika, blazing with a necklace and armlets, shines with a brilliant tiara and two bracelets, joined by hosts of Yakșas, accompanied by crores of Yakșas - may the illustrious one, intent upon praising Hara, bestow peace on me! (199-200)

${ }^{115}$ Maṇibhadra is one of the better known Yakșas, second only to Kubera/Vaiśravana. See Misra 1981: 81-85. Stadtner 2002 discusses a Gupta sculpture of Manibhadra, dated to 431 CE. For an earlier inscribed Manibhadra sculpture found at Pawaya, now at the Gwalior Archaeological Museum, see Huntington: oo138o6. A recently published copperplate inscription of Vainyagupta attests to the worship of Manibhadra in eastern India in the sixth century (Furui 2016). See also Quintanilla 2007: 27-30, for epigraphic and other references to Manibhadra.

${ }^{116}$ The name Suviroma, while different in meaning, recalls the Yakșa Suciloma ('needle-haired'), who, according to Pāli sources, converted to Buddhism (Misra 1981: 117-118). Note the reference to the turban, which, like that of other Yakșas, is a prominent feature of his sculpture at Bharhut: AIIS 34309. The Bharhut sculpture bears the label suchilomo yakho (CII II/II: 79 [B9]). The variant reading suviro näma in a number of manuscripts looks secondary.

${ }^{117}$ Pāñcika is the consort of Hārītī and is well represented in Gandhāran and postGandhāran art. See Misra 1981: 73-8o. 


\section{[5. Vibhāṇḍaka $]^{118}$}

The illustrious Yakṣa Vibhāṇdaka, decorated with various jewels, constantly shines with a beautiful supreme earring on his ear, the lord of Yakșas, master of Yakșas, staunch general of the Yakșas - may the illustrious one, honouring the feet of Hara, bestow peace on me! (2O1-2O2)

\section{[6. Dhṛtarāșțra ${ }^{119}$}

The very lustrous Yakșa Dhṛtarāṣtra, powerful lord of Yakṣas, covered with a divine turban and garment, decorated with jewels and gold may he, devoted to Śiva, meditating on Śiva, intent upon worshipping Śiva, endowed with the favour of Śiva, bestow peace on me! (203-204)

\section{[7. Pūrṇabhadra $]^{120}$}

The great Yakșa Pūrṇabhadra, decorated with all kinds of adornments, shines incredibly with a golden turban bright with jewels - may he, furnished with an entourage of a thousand crores of Yakșas, ${ }^{121}$ engaged in praising Rudra, bestow peace on me! (205-206)

${ }^{118}$ Vibhānd diaka was a sage of the line of Kaśyapa. When he chanced on the Apsaras Urvaśi he shed his seed. The seed was swallowed by an antelope, who gave birth to Rṣyaśrnnga. The birth story is told in MBh 3.110. Could this be related to the Yakșa Virūḍhaka, who is the Dikpāla guarding the southern direction in Buddhist sources (see note below)?

${ }^{119}$ Dhṛtarāștra is another well-known character from the Mahābhārata. However, in Buddhist mythology Dhṛtarāșțra is one of the four great kings (caturmahārājika), guardians of the directions: Dhṛtarāșțra/Dhatarațța (E), king of the Gandharvas; Virūḍhaka/Virūḷha (S), king of the Kumbhāṇ̣as; Virūpākṣa/Virūpakkha (W), king of the Nāgas; Vaiśravaṇa/Vessavaṇa (N), king of the Yakșas. See Kirfel 1967: 195-196; Haldar 1977: 80-81; Wessels-Mevissen 2001: 18-19. For sculptures of two of them, with labels referring to them as Yakșas (kupiro yakho [B1], virudako yakho [B4]), on the pillars of the great stūpa at Bharhut, see Cunningham 1879: 19-20.

${ }^{120}$ Pūrnabhadra is a prominent Yakșa. See Misra 1981: 85-87.

${ }^{121}$ After this, all manuscripts except $\mathrm{B}_{99}^{C}$ and $\mathrm{N}_{82}^{K}$ add two more pādas on Pūrṇabhadra's devotion to Rudra. While this may suggest a case of eye-skip on the part of $\mathrm{B}_{99}^{C}$ and $\mathrm{N}_{82}^{K}$ (rudra- $\rightarrow$ rudra-), it is quite conceivable that the two pādas were added accidentally in an early archetype. This is suggested by the wide distribution of variants in the second pāda of the addition. The scenario may have been as follows: somebody accidentally started a pāda rudraprañāmaparamo (an easy slip of the pen), which was subsequently completed by different scribes with an additional pāda in different ways. The content of these two pādas is redundant. 


\section{[8. Virūpākșa ${ }^{122}$}

And the best of Yakșas, Virūpākṣa, who has a white garment, very luminous, adorned with beautiful golden garlands furnished with tinkling bells, ${ }^{123}$ at all times solely intent upon granting boons - may he, intent upon worshipping Rudra, devoted, bestow peace on me! (207-208)

\section{[Other Yakșas]}

The Yakșas in the sky, the Yakșas dwelling in heaven, the Yakșas dwelling on earth, on mountains, in caves and forts, the Yakșas in the sky, those dwelling in the bottom of the nether region, the Yakșas with various kinds of weapons, wearing various dresses, devoted to Śiva, good-minded, eager for worship of Śiva - may they, delighted, bestow peace on me, peaceful, intent upon peace! (209-211)

[Wives, Sons and Daughters of the Yakșas]

The Yakșas' wives, of various appearances, and the Yakșas' sons, the illustrious Yakșas' daughters, delighting in the worship and praise of Śiva may they quickly grant peace, blessing, safety, power, the highest happiness, constantly, all together! (212-213)

${ }^{122}$ Virūpākșa is one of the four guardians of the directions in Buddhist literature. See the note on Dhṛtarāsțra above.

${ }^{123}$ I follow the readings of the majority of the manuscripts, which is grammatically irregular but quite probably original. The variant reading in $\mathrm{N}_{82}^{K}, \mathrm{~B}_{99}^{C}$ and $\mathrm{E}^{N}$ has 'endowed and decorated with bells that have a beautiful golden colour' instead. 
$[\text { Mountains }]^{124}$

And may all mountains always, the very prosperous mountains, devoted to Siva all the time, all the time bestow peace on me! (214)

$$
[\text { Oceans }]^{125}
$$

May all the oceans everywhere, the oceans present everywhere, always intent upon worshipping Rudra, bestow peace on me! (215)

$$
\text { [Rākṣasas] }]^{126}
$$

All the Rākṣasas everywhere, the Rākșasas of terrifying form, the very heroic Rākșasas, the very powerful Rākṣasas, the Rākṣasas present on earth, the Rākșasas in the sky, those in the nether region and on the surface of the earth, constantly intent upon Rudra ${ }^{127}$ — may they always

${ }^{124}$ There are many different additions related to this section. Before this, $\mathrm{N}_{45}^{C}$ adds 14 pādas, referring to the islands Jambudvīpa, Saākadvīpa, Kuśadvīpa, Krauñca, Saālmalika, Plakșadvīpa, Gomedha and Puṣkara, and the mountains Meru, Mandara, Kailāsa, Malaya, Gandhamādana, Śrīparvata, Hemakūța and Mālyavat. A later hand in $\mathrm{B}_{99}^{C}$ has added 22 pādas with many mistakes, which are of similar content but different in wording. The passage first lists the mountains Meru, Mandara, Kailāsa, Malaya, Gandhamādana, Śrīparvata, Mahendra and Himakūța, followed by the islands Jambudvīpa, Plakṣadvīpa, Kuśadvīpa, Krauñcadvīpa, Śākadvīpa, Gomedhadvīpa and Puṣkaradvīpa, and the seven oceans Kșāroda, Kṣīroda, Dadhna, Ghṛtoda, Suroda, Dahvadambha(?) and Ikșusvāda. $\mathrm{N}_{58}^{K}$ adds 18 pādas, listing roughly the same islands, oceans and mountains, but in slightly different terms. $\mathrm{E}^{N}$ adds four pādas, which only list the mountains in the same form as we find them in the additional passage in $\mathrm{B}_{99}^{c}$, while it has the names of the continents and the seven oceans after 214. $\hat{S}_{67}^{S}$ presents yet a different case, listing the islands as Jambudvīpa, Śākalya, Kuśa, Krauñca, Śālmali, Gomedha and Puṣkara, the oceans as Kṣārodadhi, Kṣ̄rodadhi, Dadhna, Ghṛtodaka, Ikṣupūrṇa, Suroda and Svāduda, and the mountains as Meru, Mandara, Kailāsa, Malaya, Gandhamādana, Mahendra, Śrīgiri and Hemakūṭa. It looks like different transmitters were responsible for the additions of these passages, all dealing with similar content but with differences in wording and phrasing. For clarity's sake I have silently normalized the various names, which show variations in the different manuscripts.

${ }^{125}$ Before this, $\mathrm{E}^{N}$ adds four pādas, referring to the islands (see note above) and the seven oceans Kṣāroda, Kṣiroda, Dadhna, Ghṛtoda, Suroda, Svāduka and Ikṣusvāda.

${ }^{126}$ At this point in the text, the structure of the invocation changes, with invocations dedicated to deities that show a close connection to Śiva's Bhairava form. Cf. also the comment in the Śivadharmavivarana.

${ }^{127}$ After this, $\mathrm{N}_{77}^{K o}, \mathrm{E}^{N}, \mathrm{P}_{32}^{T}$ and $\mathrm{P}_{72}^{T}$ share two additional pādas: 'may they always bestow peace on me, being constantly intent upon Siva'. This addition is superfluous. 
bestow peace on me, through the splendour of that god who has a terrifying form and who is covered with the ashes of the dead! (216-218)

\section{[Yoginīs and Ḍākiṇīs]}

The very powerful Yoginīs, with a dress that is constantly blazing, beautiful, of various appearances, and the very prosperous Ḍākin̄īis ${ }^{128}$ - may they, delighting in bowing to Rudra, delighting in the worship and praise of Rudra, their minds dedicated only to Rudra, bestow peace on me! The Dâkiṇīs in the sky, those abiding in heaven, the Ḍākiṇis in the nether region, those present on mountains and forts ${ }^{129}$ - may they always bestow peace on me, through the splendour of that god who has a third eye, a trident and shining ashes! (219-222)

\section{[Bhūtas]}

All the spirits (Bhūtas) of great form, all the spirits of great splendour, all the spirits present, all the auspicious spirits, swift as thought, the spirits in the sky, the spirits abiding in heaven, the spirits in the nether region and on the surface of the earth, granting riches - may they always bestow peace on me, through the splendour of that god who has a spotless skull-staff, a trident and shining ashes! $!^{130}(223-225)$

\section{[Pretas]}

All the ghosts (Pretas), the groups of ghosts, the ghosts facing all directions, the very brilliant ghosts, the ghosts feeding on blood, the ghosts in the sky, the ghosts dwelling in heaven, the ghosts in the nether region and on the surface of the earth, the ghosts taking form at will — may they always bestow peace on me, through the splendour of that god who has his abode on the cremation ground, whose vehicle is a bull! (226-228)

${ }^{128}$ Dākinīs are attested for the first time in the Gangdhār stone inscription of Viśvavarman, dated Mālava-Vikrama era 48o (ca. 423-424 CE), referring to a temple dedicated to the Mothers (mātrnāịn veśman) that is filled with Dākiṇis (ḍākinisamprakìnam). For the text of the inscription, see Sircar 1965: 399-405. The same inscription mentions the establishment of a Viṣnu temple and a well, all by Mayūrākṣa, the minister of Viśvavarman.

${ }^{129}$ Or alternatively: 'on hillforts'.

${ }^{130}$ Instead of bhasma bhāsuram quite a few manuscripts have karapallave '[a trident] in his fingers'. 


\section{[Piśācas]}

The very heroic Piśācas, prosperous, very powerful, all bearing various forms, all most excellent, the Piśācas in the sky, the Piśācas in heaven, the Piśācas on earth and in the nether region, of many forms, swift as thought - may they always bestow peace on me, through the splendour of that god who has the digit of the moon on his head and who bears the Gañgā in his twisted hair! (229-231)

\section{[Grahas]}

All the epilepsy-seizers, and all the fever-seizers, the foetus-seizers, and those seizers of various diseases, the seizers in the sky, the best of seizers in heaven, the seizers on earth and in the nether region, the seizers in all directions - may they always bestow peace on me, through the splendour of that god who has a dark-blue neck and who has a snake for an ornament! (232-234)

\section{[All Deities]}

May all these deities and others, who are following the command of Śiva, bestow peace on earth to the devotees of Siva! ${ }^{13}$ (235)

$$
\text { [Jaya] }
$$

Victory to [You] residing in your own yoga, victory to [You] of pure consiousness, victory to the Single Hero of Boons, victory to the Lord, homage be to You!

Victory to the Supreme God, victory to the Maker of Happiness, victory to [You] with a manifest body, victory to [You] who are the object of the muttering of prayers, homage to You!

${ }^{13^{1}}$ After this, $\mathrm{P}_{3^{2}}^{T}$ and $\mathrm{P}_{72}^{T}$ add a transitional verse introducing the following Stotra. $\hat{S}_{67}^{S}$ has two different verses, the first of which starts with four pādas quoted by Jayaratha in his commentary on Tantrāloka 1.159: ittham nānāvidhair rūpaih sthāvaraih jañgamair api $\mid$ krị̂daya prasrto nityam eka eva śivah prabhụ̣ || 'Thus Śiva, the Lord, the one and only, issues forth through play, with manifold forms, both moving and unmoving. The verse is connected in $S_{67}^{S}$ with the refrain 'may he always bestow peace on me, long life and happiness!' The second verse states that one who desires success, enjoyment and release should hear and recite it. 
Victory to the Receptacle of Fortune, victory to the Bestower of Splendour, victory to [You] of pure speech, to the Unconquered, homage, homage! $!^{12}$

Victory to [You] with the trident in his hand, victory to [You] bearing the skull-staff, victory to [You] who have conquered the world, victory to Form, homage to You!

Victory to [You] half of whose body is your beloved, victory to [You] who wear the digit of the moon, victory to the Supergod of Gods, victory to Rudra, homage to You!

Victory to the Lord of the Three Worlds, victory to [You] of celebrated fame, victory to the Entire Support, victory to the Creator, homage, homage! ${ }^{133}$

Victory to the Bestower of Liberation, to the Effecter of Creation and Destruction, to [You] worshipped by Brahmā, Viṣnu and Indra!

Śiva, homage be to You, the Gentle! $(236-242)^{134}$

\section{[Śrutiphala]}

He who recites or hears this chapter on appeasement will rejoice in Sivaloka, having quickly shaken off evils.

One aiming for a girl obtains a girl, one aiming for victory obtains victory, one aiming for wealth obtains wealth, one aiming for sons obtains many sons, one aiming for knowledge obtains knowledge, one aiming for union obtains union.

Whatever desires one may aim for, a man obtains all of that here quickly by listening, and he becomes dear to the gods.

A man who should enter battle after hearing this auspicious chapter ${ }^{135}$ will conquer his enemies in battle and be honoured with fortunes.

\footnotetext{
${ }^{132} \mathrm{~N}_{45}^{C}$ and $\hat{S}_{67}^{S}$ insert four pādas, invoking Śiva as the one of pure knowledge, the one who contemplates all, the one who is completely pure and who has eight embodiments (aștamūrti).

${ }^{133} \hat{S}_{67}^{S}, \mathrm{P}_{3^{2}}^{T}$ and $\mathrm{P}_{72}^{T}$ insert four pādas (with variants) invoking Siva as the one who has a nonmaterial $\left(\hat{\mathrm{S}}_{67}^{S}\right.$; em. niṣkala-) or spotless $\left(\mathrm{P}_{7^{2}}^{T}\right)$ body, who accomplishes all goals, destroyer of Kāma.

${ }^{134} \mathrm{P}_{3^{2}}^{T}$ adds two more pādas invoking Śiva as being free from birth and all-pervading.

${ }^{135}$ Variant in $\mathrm{N}_{82}^{K}$ : 'A man who should enter battle, while reciting the chapter on appeasement ...'
} 
He will enjoy [his kingdom] for an endlessly long time, with his orders not rejected. He will not be overpowered by diseases and thrive with sons and grandsons.

Diseases arising from wind and bile do not bind the one for whose sake this auspicious recitation is recited.

He will not encounter death at an inappropriate time, and he will not be bitten by snakes. Poison will not enter his body and there will be no paralysis, blindness or dumbness [for him].

For him there will be no danger from misfortunes, there will be no fear of calamity, he will never be polluted by vices created by black magic.

The merit of all Tìrthas, in particular of such as the river Gangā, that merit, multiplied by crores, one obtains in this world by hearing it.

By hearing it, one will obtain the fruit of ten Rājasūya sacrifices and of a hundred Agnișțomas, multiplied by crores and crores.

He cannot be slain by all the gods and other beings in particular; he will live the full hundred years free from all diseases.

A cow-killer and an ungrateful person, a brahmin-slayer and one who violates his teacher's bed, one who murders someone who comes for protection and one who betrays the trust of friends, one engaging in wicked evil, a mother's killer and a father's killer - by hearing it intently, such a person will be released from all sins.

This auspicious chapter on appeasement should not be given to just anyone. It should be given to the devotee of Śiva. It was told earlier by Siva. ${ }^{136}$ $\left(243^{-256)}\right.$

Thus in the Śivadharmaśāstra, the Sixth, the Chapter on Appeasement.

${ }^{136}$ The first and the last verse of the Śantyadhyāya both specify that it was Rudra/Śiva who first taught this invocation in the past. $\mathrm{P}_{3^{2}}^{T}$ and $\mathrm{P}_{72}^{T}$ add four more pādas about the results of daily recitation, while $S_{67}^{S}$ only has the last two of them. 
Peter Bisschop - 978-90-04-38436-1 Downloaded from Brill. com $04 / 26 / 2023$ 11:40:27AM via free access 


\section{Appendix}

\section{Single-Text and Composite Manuscripts}

Aside from manuscripts belonging to the Śivadharma proper, there exist several single-text manuscripts of the Śäntyadhyāya, as well as composite manuscripts in which the Śäntyadhyāya has been included in a bundle with other texts. ${ }^{1}$ These provide further testimony for the use of the mantra. Brief descriptions of several of these manuscripts are presented here, mainly drawing on their colophons and post-colophon statements. Furthermore, for each manuscript I have added a note on three passages that are important from a text-critical point of view:

- the addition of Vṛșa after $27 \mathrm{~d}$ in some manuscripts

- the omission of $32 \mathrm{bc}$ in some manuscripts

- the addition of Arhat, Buddha, etc. after $32 \mathrm{~d}$ in some manuscripts One of the manuscripts listed here (NGMPP A 1158/8) has been used for the constitution of the text in the edition, and is referenced throughout the critical apparatus $\left(\mathrm{N}_{58}^{K}\right)$ because of its relevance for the study of the further transmission of the text in Nepal.

\section{Śāntyadhyāya Single-Text Manuscripts}

1. University of Pennsylvania Libraries, Ms. Coll. 390 item 715, 28 folios. Paper, dated Śaka 1732 (1810 CE). ${ }^{2}$

${ }^{1}$ Florinda De Simini kindly provided me with images of several of the manuscripts in Kathmandu discussed in this appendix.

${ }^{2}$ Digital images of this manuscripts are available online: http://openn.library. upenn.edu/Data/ooo2 $/ \mathrm{html} / \mathrm{mscoll} 390$ _item715.html 
Attributed to the Śivadharmaśāstra: iti śrīsivadharmmaśāstre +nandikaśvasamvvāde+ śāṃtikādhyāyah sampūrṇah śubham \| likhitam śrībhavāniśaṃkareṇa śrísāke 1732 mārgaśuklāștami śubham || viṣnudattasyedam pustakạ̣ \| (fol. 28r).

The addition after $27 \mathrm{~d}$ is included and overall corresponds to the readings of $\mathrm{E}^{N} ; 3^{2 b c}$ is included; the addition after $32 \mathrm{~d}$ is included and overall corresponds to the readings of $S_{67}^{s}$.

2. National Archives, Kathmandu, 6/2301, 24 folios. Microfilmed by the NGMPP, A 1120/12. Paper.

Attributed to the Nandikeśvarasamhitā of the Sivadharma of the Skandapurāna: iti skandapurāne śivadharmme nandikeśvaraproktāyām saṃhitāyāịn śāntyadhyāya samāptạ̣ (fol. 24v).

The manuscript has many small corrections. A second hand has added a final line on the last folio: samvat $1902 .^{3}$

The addition after $27 \mathrm{~d}$ is included and overall corresponds to the readings of $E^{N} ; 32 b c$ is included; the addition after $32 \mathrm{~d}$ is lacking.

3. Private collection, Rājopadhyāya, 13 folios (incomplete). Microfilmed by the NGMPP, G 19/16. Paper, no colophon.

The addition after $27 \mathrm{~d}$ is included and overall corresponds to the readings of $E^{N} ; 32 b c$ is included; the addition after $32 \mathrm{~d}$ is lacking.

4. the Asha Archives, Kathmandu, 4390, 16 folios (incomplete; first folio missing). Paper, dated [Nepāla] Saṃvat 975 (1854/55 CE). ${ }^{4}$ Attributed to the Śivadharmaśāstra: iti śivadharmme śāstre nandikeśvarapran̄ite șașțho dhyāya samāptam \| saṃvat 975 adhika ākhāḍa krșṇadvādasi budhavāra siddha yāinā julo śubham astu sarvvakālam \|

The addition after $27 \mathrm{~d}$ is included and overall corresponds to the readings of $\mathrm{E}^{N} ; 3^{2 \mathrm{bc}}$ is included; the addition after $3^{2 \mathrm{~d}}$ is included and overall corresponds to the readings of $\mathrm{E}^{N}$.

3 ' 2 ' corrected to ' 3 '? Taking this to be the Vikrama era, this date would correspond to $1959 / 6$ o CE.

${ }^{4}$ I thank Timothy Lubin for bringing this manuscript to my attention. 


\section{Śāntyadhyāya Composite Manuscripts}

1. Private collection, Achutananda Rājopadhyāya, 63 folios. Microfilmed by the NGMPP, E 321/26. Palmleaf, dated [Nepāla] Samvvat 316 (1196 CE).

Contains: Triśaktimāhātmya of the Varāhapurāna (folios 1v-5v; images 3-7), Śāntyadhyāya of the Śivadharmaśāstra (folios 1r-12v; images 7-25), Devimāhātmya of the Mārkaṇdeyapurāna (folios 1v4ov; images 26-64).

The colophon of the Sāntyadhyāya runs: iti śivadharmaśāstre śāntyadhyāyah șașthah samāptah $\|$ * $\|$ samvat ${ }_{31} 6$ māgha[śu]klatrayodaśyām somavāsare \| mahārājādhirājaśrīijijayakāmadevasya vijayarājye śrïtrikamanaṃdanajīvasya pustakam li[khi]tam iti \|cha\| lekhaka kestavedunāmahn mamgalam mahāśrì || śrīcampāgulmamadhyamațolake \|cha \|cha (12v).

The colophon of the Devimāhàtmya runs: iti mārkandeyapurāne sāvarṇnike manvantare devīmāhātmyam samāptam $\|$ * $\|$ samvat ${ }_{316}$ māghakrș̣nanavamyām bṛhaspatidine śrivijayakāmadevasya vijayarājye śrīcampagulpamadhyamațole śrītrikanaṃdanajīvasya pustakam likhitam iti $\left\|^{*}\right\|$ śrīvedupaṃdita likhita svahastenam iti $\|$. A later hand has added after this: nepāli 1047 sālam ${ }^{5}$ jīrnoddhārayānā śṛ̂̀ amarakāṃța upādhyām āṣā ḍ̣aśu trayodaśi 6 o deco nīhmaị (40v).

The addition after $27 \mathrm{~d}$ is lacking; $32 \mathrm{bc}$ is lacking; the addition after $3^{2 \mathrm{~d}}$ is lacking.

2. National Archives, Kathmandu, 1/1376, 28 folios. Microfilmed by the NGMPP, A 1158/8. Palmleaf, dated Bhādrapada [Nepāla] Saṃvat 522 (August-September, $1402 \mathrm{CE}$ ). This is 'the latest palm-leaf manuscript transmitting a portion of the texts of the collection' (De Simini 2016a: 214, n. 20). This manuscript has been used in the present edition and is referred to by the siglum $\mathrm{N}_{58}^{K}$.

Contains: Śāntyadhyāya (fols. 1v-2ov); Sattvabādhāpraśamana (fols. $1 \mathrm{r}-5 \mathrm{v}$ in letter numerals). The Sanntyadhyāya and the Sattvabādhāpraśamana are preceded in the microfilm by another

${ }^{5}$ Nepālī year 1047 equals 1927 CE. 
untitled text on Chandas in a different hand on four unnumbered folios.

The colophon of the Śāntyadhyāya runs: iti sivadharmmaśāstre nandikeśvaraproktaśāntyādhyāyah șaștha ${ }^{6} \quad$ samāptah \|\|$^{*} \|$ samvat 522 bhāddrapadaśukladviyāyām ${ }^{7} \|$ śubhah $\|$ dvijarāja ivābhāta divarāja dvijottamaḥ| siddharājasuta ${ }^{9}$ śrìmān gunasamśaktacetanah $h^{10} \|$ brahmopendrasundra vahnivaruno mārttaṇdajevātrka, ${ }^{12}$ sādhyākāśavasumndharānilayamah śailendrapithotpalah $\left.\right|^{13}$ sarvva[m] vyāptam anekarūpabhagavān kașțā $\overline{1}^{14}$ kalā nemișaḥ ${ }^{15}$, sarvvā yaḥ praṇato hi me [']pi śirasā sarvvātma gaurīdharaḥ $\|{ }^{16}$ śubham astu sarvvajagatām $\|$ (fol. 2ov).

The colophon of the Sattvabādhāpraśamana runs: iti viṣnudharmmeșu sattvabādhāpraśamanah samāptah| nāke khe bhuvi nāgarājabhuvane viśvarājya śiva, ${ }^{17}$ kālāle ${ }^{18}$ ca śilocaye ca mudire abje patanainale ${ }^{19} \mid$ janyusvānta ${ }^{20}$ supuṇdarīkasadane pușpe [']nile pallave, sarvvaryāpaka bhaktivatsalahare tubhyam namā

${ }^{6}$ Read śāntyadhyāyaḥ șașthaḥ.

${ }^{7}$ Read ${ }^{\circ}$ dvitīyāyām.

${ }^{8}$ Read ivābhāti.

${ }^{9}$ Read ${ }^{\circ}$ sutah.

${ }^{10}$ Read ${ }^{\circ}$ samsakta . Metre: Śloka. 'The venerable son of Siddharāja, whose mind is attached to good qualities, the best of the twice-born, shines like the lord of the birds (Garuḍa), [like] the lord of heaven (Sun).'

${ }^{11}$ Read ${ }^{\circ}$ surendra ${ }^{\circ}$.

${ }^{12}$ Read jaivātrkah.

${ }^{13}$ Read ${ }^{\circ}$ pițhotpalah.

${ }^{14}$ Read kāṣthā.

${ }^{15}$ Metri causa for nimișah.

${ }^{16}$ Metre: Saārdūlavikrīḍita. 'He who is Brahmā, Viṣnu, Indra, Agni and Varuṇa, who is Sun and Moon, who is Sādhya, ether, earth, wind and death, whose lotus seat is the lord of mountains (Kailāsa), the blessed one with many forms [by whom] everything is pervaded — the kāșțhās, the kalās and the nimișas — I bow to Him with [my] head, the Soul of All, Husband of Gaurī'

${ }^{17}$ The end of pāda a is hypometrical. It may be corrected to viśve+ndra+rājye śive or something similar.

18 'Relating to the embryo (kalala)', or short for kālālaye?

19 These are names of hells. Read patac cānale 'when falling in fire' for patanainale (unmetr.)?

${ }^{20}$ Read ${ }^{\circ}$ svānta ${ }^{\circ}$. 
sāpratam $\|^{21}$ śubham astu sarvvajagatām $\|$ (fol. 5v).

The text of the Sattvabādhäpraśamana corresponds to chapter 28 of the Viṣnudharma edited by Grünendahl (1983). It is a prayer against afflictions and thus ties in well with the subject matter of the Śantyadhyāya. Note that both colophons end with the same line śubham astu sarvajagatām. The text of the Śantyadhyāya in this manuscript is in a good state and written by a careful hand.

The addition after $27 \mathrm{~d}$ is lacking; $32 \mathrm{bc}$ is included; the addition after $32 \mathrm{~d}$ is included, but with different readings containing parts of the addition about Vṛșa found after $27 \mathrm{~d}$ in other manuscripts.

3. National Archives, Kathmandu, 5/7344, 39 folios (no foliation). Microfilmed by the NGMPP, A 1174/14. Paper thyāsapu, dated [Nepāla] Saṃvat 799 (1678-79 CE).

The bundle contains: Vārānasyaștaka (images 6972-6973); Mahādevadvādaśanāmastotra (6973-6974); Hariharastava (6974-6975); Durgāstotra (6975, 7976); Sāntyadhyāya of the Śivadharmaśāstra (6977-6985); unidentified (6986); Durgāstotra (6987-6991); unidentified (6992); Devyaștaka (6993-6994); unidentified (6995); unidentified (6996-6997); Śāntyadhyāya of the Sivadharmaśāstra (6997-7011); unidentified (7012); unidentified (7013).

The photographs are in disarray. Images $6977-6985$ cover the text of $170 \mathrm{~b}$ to the end. Images $6997-7011$ cover the text of $1 \mathrm{a}-158 \mathrm{c}$.

Image 6985 has the following post-colophon statement: śubha ${ }^{22} \|$ samvat 799 āṣādhakrṣnaśaptamyām tithau śaniñcalavāsare rikhīta sampūrṇna śubha ${ }^{23} \|$ jadī śurddham ${ }^{24}$ aśuddhạ̣ vā mama dvakhva na vijate śvadhaniyam ${ }^{25} \mid$. This is contin-

${ }^{21}$ Read namaḥ sāmpratam. Metre: Śārdūlavikrīẹita. 'In the firmament, in the sky, on the earth, in the world of the Nāga kings (Pātala) ... and in the abode (älaya?) of death, on the mountain, and in the cloud, in the lotus ... in the beautiful lotus seat in whose heart is Brahmā(?), in the flower, in the wind, in the bud, homage to you properly, who pervade all, Hari, fond of devotion.'

${ }^{22}$ Read śubham.

${ }^{23}$ Read śanaiścaravāsare likhitam sampuūrṇaṃ śubham.

${ }^{24}$ Read yadi śuddham.

${ }^{25}$ Read mama doṣo na dhìyate śodhanīyam. 
ued in what appears to be the same hand, but in thinner characters: yad atra pāthe jagadambike mayā visarggabindūkșarahīnam ${ }^{26}$ iritam $\|$ tad astu sampūrṇnatamam prasādatạ̣ saṃkalpasiddhiś ca sadaiva jāyatāṃn ${ }^{27} \|$ māhātmyam tvadvicitram danujavinihatam saptaśatyās tavedam, ${ }^{28}$ bhaktyāham pațhyamāne yajanabalijapamūdrāvihīnam \| vaisarggobindumātrākșarahīnavākyaśuddhādidoșaṃ, trailokyatrānassīle paramakarunayā devi māta $+\underline{h}+k s ̦ a m a s v a \|^{29}$ svast $^{\vec{t}^{\circ}} \|$ (image 6985).

The addition after $27 \mathrm{~d}$ is included and overall corresponds to the readings of $\mathrm{E}^{N}$, but it adds an additional four pādas (nandā sukhanasā sūraji suśl̄à sumanā tathā $\mid$ gāvaḥ śivapara paṃñca sthitāh kurvvantu śäntikam |); 32bc is included; the addition after $3^{2 d}$ is lacking.

4. Private collection, Acyuta Adhikārī, 19 folios (folios 4 and 9 missing). Microfilmed by the NGMPP, I 54/4. Paper, dated 1697.

Starts with a nyāsa on the first folio, attesting to the ritual use of the text. The Śantyadhyaya itself is introduced with the words atha pāțāntaramamtrah 'now the mantra to say next'.

The text is attributed to the Śivadharmottara: kathitā purā $3386^{31} i t i$ śivadharmmotare namidike [...] kādhyāyasamāptam sampūrnam subham [...] rikte bhrguvāara+śukle likhet sāmn+ti+kādhyāya (sidhyurmamāse) harirāmātmajạ̣ śrīpradyumnena likhyaṃ 1697 sāla ${ }^{32}$ (21r).

The addition after $27 \mathrm{~d}$ is included and overall corresponds to the readings of $\mathrm{E}^{N}$; nothing can be said about the presence of $32 \mathrm{bc}$ or

${ }^{26}$ Read ${ }^{\circ}$ bindvakșara ${ }^{\circ}$.

${ }^{27}$ Metre: Vamśastha.

${ }^{28}$ The reference is to the recitation of the 70o-verse Devimāhātmya, also known as Saptaśatì. The first post-colophon statement refers to the copying (likhitam) of the Śantyadhyāya, the second to the recitation (pāṭe ... ìritam) of the Śäntyadhyāya combined with the recitation of the Devimāhātmya?

${ }^{29}$ Metre: defective Sragdharā.

$3^{\circ}$ Read svasti.

${ }^{31}$ This probably refers to the number of syllables copied.

$3^{2}$ Śaka or Vikrama Samvat? 
the addition after $32 \mathrm{~d}$ because of the loss of a folio.

5. National Archives, Kathmandu, 1/1108, 109 folios. Microfilmed by the NGMPP, A 1299/9. Paper, no date.

According to the online NGMCP catalogue, the bundle contains: Jvaropakaraṇa, Budhaśāntistotra of the Viṣnudharma, Bhìmasenapūjāvidhi, Śukrastuti, Sarvakāmārthadabhìmasenastotra, Sāntyadhyāya.

The Śantyadhyāya covers folios 37r-69r (images 65-97). Colophon: iti śivadharmmaśāstre śāntyadhyāyah samāptaṃ ${ }^{33} \|$ (69r).

The addition after $27 \mathrm{~d}$ is lacking; $32 \mathrm{bc}$ is included; the addition after $32 \mathrm{~d}$ is lacking.

6. Institut Français de Pondichéry, RE25374. Palmleaf, Grantha script, dated 1821 CE. ${ }^{34}$

Contains: a Tamil commentary on the Sivadharmottara (208 folios); Śivadharmottara (11 folios, incomplete); Śāntyadhyāya (2 folios, no foliation, incomplete).

The surviving fragment of the Sanntyadhyāya only covers the first 30 verses of the text. The addition after $27 \mathrm{~d}$ is found not after $27 \mathrm{~d}$ but after 19d. The surviving text shows most correspondences with the readings of $\mathrm{P}_{32}^{T}$ and $\mathrm{P}_{72}^{T}$.

\section{A Commentary on the Śāntyadhyāya}

The Oriental Research Institute and Manuscripts Library in Trivandrum holds a palm-leaf manuscript of the Sivadharma (No. 12763), written in Malayalam script, consisting of two parts. ${ }^{35}$ The first part contains the text of the Śivadharmaśästra, the second part a pratīka-style

\footnotetext{
${ }^{33}$ Read samāptah.

34 The description and transcription of this manuscript has been kindly provided to me by Marco Franceschini.

${ }^{35}$ I am grateful to S.A.S. Sarma (Pondicherry) for providing me with photographs of the three folios of the manuscript that contain the Śantyadhyāya.
} 
commentary on the Sivadharmaśāstra and the Sivadharmottara. In this appendix I give a transcription of the commentary on the Śivadharmaśāstra's Śāntyadhyāya, with the pratīkas printed in italics. The English translation that follows includes references to the relevant verse numbers of the müla text. In translating the commentary I have followed the commentator's interpretation, which means that quotes from the müla text may at times differ from the text of the main translation. The text of the pratikas largely conforms to the readings of the Southern transmission of the text.

\section{Transcription}

oṃ atha pūjakasyāntarāyaśaṅka[h] sarvvaśāntyadhikārākhyan dharmmam āha atah param ityādi rudrodgìtam rudreṇotkṛșțatvena kathitạ̣ mahodayam mahojvalam rājñah paracakrapramathanam sad̄̄ vijayavarddhanam sarvadevagrahānīkam sarvvadevagrahāñ jetum pūjakasya balabhūtam evambhūtan dharmmam vakṣāmi śāsvatam īśvaroktim anatikramyety arthạ śaśāṃkārddhadharaḥ ityādi śāntim karotu ma iti brūteti śivapūjakānāṃ bhāṣaṇaprakāram upadiśati sarvvāvayavamukhyena gātreṇopalakṣitā krșnāmgngḥ ata evendranīanibhaḥ gaṇamātā nāma kācī devī prasādaparamā prasādabahulā nirmmāṃsena śarīrenopalakșitah akșamālāśirogrạ akṣamālālan̉kṛtaśirogrạ̣ kṣiteḥ patị̣ kṣitinivāsagatyoḥ kṣiteh prāṇinivāsasya lokasamūhasya pātā dharmmeṇa hi lokā rakṣyante dharmmottamottamaḥ dharmmeṇottamā devāḥ śivaddhyānaikaparamaḥ śivaddhyānenaikotkṛ̦țạ brahmaśabdena vedaśabdenopalakṣitaḥ avadātā śuddhā kṣemārogyam kṣemārogyañ cetyartthah śivarūpena śiva - - ṇa rūpeṇābhāvabhayañkarīti sambandhaḥ citrayantrāsanena vicitrayantrāsanavatā caṇ ḍaūpeṇopalakṣitā rakșām karotīti sambandhạ̣ vakṣyamāṇābhyo devatābhyo bhūmau balin datvā japyam āha ye rudrā ityādi etac ca pūjābhājanayor mmadhye pyayasva japyate liṃam aindryāṃ diśi vacanoktānāṃ pratyekaṃ rūpabhede [']pi lohitasya vyāpitvam mantavyam adhobhāga ityādiṣu pradeśasthānām api vibhūtitas sarvvalokavyāptir mmantavyā pațavyāptāntarālīnah pațasamvṛte [']vakāśa āsīna ityartthah bhürlloka ityādinā bhūlokam ārabhyorddhveșțalokeșu varttamānānāṃ vibhūtaya ucyante satyaloka ityādinā tv āvṛtteșu varttamānānāṃ vibhūtayah mahābhogakrtātopaḥ mahāśarīrakṛtodyogạ̣ bhairavam yasya rū- 
pan tv ityādinā rākṣasādīnām ārāddhyam śivarūpam abhidhīyate upasaṃharati iti devādaya iti ātmayogasamsthāya yogaśabdaś śaktivacanah svarūpabhūtaśaktisaṃsthāya te tubhyan nama iti saṃbandhạ te iti ca padam prathamāntatvena vipariṇamyatvañ jayeti sarvvatra sambandhanīyam naikaśūrāya naikasmin kuśalāya prakațadehāya prakațaśabdo jñānavacanạ jñānam eva deho yasya tasmai japyāya japenārāddhyāya vākyaviśuddhāya vākyaviṣaye viśuddhāya vaktradoṣo hi śabde doṣam āvahati vaktraśuddhau vākyam śuddham evety ataś śuddhavākyāyety arthah jayarūpāya jayaśabdena sarvvottaratvaṃ lakṣyate sarvvottīrṇasvarūpāya ādhārāya sarvvadhārakāya pațhyamānam ityādi yam rogiṇam uddiśyedam pațhyate tasya rogās tan na bādhante nopasargganimittabhayam na bhavet śāntyaddhyāyam idaṃ punyam iti prayogāt sādhutvam mantavyam katicaśaktị̣ baddhaśaktir ity artthạ̣| oṃ iti șașṭho [']ddhyāyaḥ | oṃ samāptā nityapūjā |

\section{Translation}

Om.

Now, apprehensive of obstacles to the worshipper, he discloses 'the law called the mastery of the appeasement of all' (3cd), beginning with the words 'after this' (1a).

'Extolled by Rudra' ( $1 \mathrm{~b})$, taught as being supreme by Rudra; 'of great fortune' ( $1 \mathrm{~b})$, very glorious; 'destroying the enemies' armies, always increasing victory' (2cd) for the king; 'an army against all deities and seizers' (3b), forming a force of the worshipper to conquer all gods and seizers; it being thus, 'I shall explain the eternal law' (3d), that is to say, without transgressing the word of the Lord.

Starting with 'he who bears the half moon' (4a) and instructing one to say 'may he bestow peace on me' $(5 \mathrm{~d})$, he teaches the manner of address of the worshippers of Siva.

[Umā] is distinguished 'with a body that is supreme in all limbs' (6a). ${ }^{36}$ [Mahākāla] has a 'black body' (18d), therefore he 'resembles sapphire' (18a).

She who is named 'Mother of the Ganas' (20c),37 the special 'goddess,

${ }^{36}$ The commentary reads the variant reading -mukhyena instead of -pürnena. This is also the reading of $\mathrm{N}_{77}^{K o}, \hat{\mathrm{S}}_{67}^{S}$ and $\mathbf{P}^{\Sigma}$.

37 The commentary does not take Ambikā as the name of the goddesss. 
intent upon favour' (21ab), full of favour.

[Bhṛngiriți] is distinguished 'with a body without flesh' (24a).

[Vrșa] has 'a rosary on top of his head', the top of his head is decorated with a rosary; 'lord of the earth', protector of the earth, which is an abode and a destination, [viz. protector] of the earth, which is the abode of living beings [as well as] the collection of worlds, ${ }^{38}$ for the worlds are protected by dharma; 'the supreme of those who are supreme through dharma', [for] those who are supreme through dharma are gods. ${ }^{39}$ [Brahmā] is 'only the highest by meditation on Śiva' (29a), only superior by meditation on Śiva; ${ }^{40}$ 'with the word Brahman' (29c), viz. distinguished with the word Veda. ${ }^{41}$

[Vārāhī] is 'pure' (43c), clean; 'peace, health' (44d), that is to say, peace and health.

The auspicious [Cāmuṇ̣̂ā] 'inspires fear' (47d) in Death 'with an auspicious form' (47c), with a [...] form ${ }^{42}$ - this is the syntactic structure; distinguished with 'a terrible form' (47e), 'having the seat of a bright diagram, ${ }^{43}$ which has a seat in the form of a brilliant diagram, 'she grants protection' (47f) - this is the syntactic structure.

After offering bali on the ground to the deities who will be proclaimed, he states the prayer to be muttered: 'the Rudras' (53a) etc.

And this is muttered to the linga, after having [placed it?] in between the two vessels for worship. ${ }^{44}$

${ }^{38}$ The commentary appears to want to extend the meaning of $k s i t i$ (earth) to include the entire universe. In the sense of dwelling (nivāsa) it is connected to the earth and in the sense of destination (gati) to the universe.

39 This passage on Vṛsa comments on several verses missing in the main text, but found in an additional passage after $27 \mathrm{~d}$ in $\mathrm{E}^{N}$ and $\mathbf{P}^{\Sigma}$.

${ }^{40}$ The analysis of śivadhyānaikaparamah seems forced. Its more obvious meaning is 'solely intent upon meditation on Śiva'.

${ }^{41}$ Instead of taking brahmaśabdena with śāntim karotu me ('may he bestow peace on me, with the Vedic word'), the commentary identifies Brahmā as the one who is distinguished by the word Brahman which means Veda.

${ }^{42}$ The manuscript reports a gap in between śiva and ṇa. Perhaps the original read śiva [ghore]ṇa. Furthermore, the commentary appears to comment upon a reading śivābhàvabhayaìkari in the mūla.

${ }^{43} \mathrm{P}_{72}^{T}$ has the reading citrayantrāsanena in an added passage after $47 \mathrm{e}$. The commentary takes citrayantrāsanena as a qualification of caṇdarūpeṇa.

${ }^{44}$ The reading pyayasva does not make much sense. Perhaps it hides an original absolutive 'dhyasya? 
Even though there is a distinction of colours, extension of [the designation] 'coppery' (lohita) should be understood for each of the [Ganas] addressed after the statement 'in the eastern direction' (56e).

Even though they are stationed in the regions of 'the lower part' (72a) etc., pervasion of all worlds should be understood because of their sovereign power.

[Vāyu is] 'dwelling in the space that is enveloped in a cloth' (94c), inhabiting the space that is enclosed in a cloth - this is the meaning.

With 'in the Bhūrloka' (102a) etc., the sovereign powers of those living in the worlds from Bhūloka on in ascending order are mentioned, ${ }^{45}$ but with 'in the Satyaloka' (104a) etc. the sovereign powers of those living [in the worlds are mentioned] in reverse order. ${ }^{46}$

Ananta is 'swelling with pride with his great hood' (166c), exerting himself with his great body.

With 'of that god who has a terrifying form' (218a) etc., the form of Siva that is the object of propitiation of the Rākșasas etc. is addressed.

He sums up: 'deities and others' (235a).

'To [You] residing in your own yoga' (236a): the word 'yoga' designates power, [i.e.] to [You] standing in the power that is your very own nature, 'to You' (236d), to You 'homage' - this is the connection. And in as much as it is first and last, the word 'to You' [indicates] variability [of his many forms].

'Victory' (236a) should be connected to all [the following invocations]. 'To the Multiple Hero' (236c), ${ }^{47}$ to [You] who are multiply skilful.

'To [You] whose body is the manifest' (237c): the word 'manifest' designates knowledge, [i.e.] to [You] whose body is nothing but knowledge. 'To [You] who are the object of muttering of prayers' (237d), to [You] who are to be propitiated by muttering prayers.

'To [You] of pure speech' (238c), to [You] who are pure with respect to speech; for a fault in the mouth leads towards a fault in the word, [but] when the mouth is pure speech is pure, hence 'To [You] of pure speech'

\footnotetext{
${ }^{45}$ Correct ārabhyorddhveșțalokeșu to ārabhyorddhveșu lokeșu.

${ }_{46}$ The commentary here refers to an additional passage found after 104 in $\mathrm{N}_{58}^{K}$ and $\hat{S}_{67}^{S}$, listing the appearance of the gods in the seven worlds in reverse order (i.e. from Satyaloka down). Cf. also Chāndogya Upanișad 2.2.2-2.2.3.

47 Instead of dānaikaśūrāya 'Single Hero of Boons', the commentary analyzes naikaśūrāya separately.
} 
— this is the meaning.

'To [You] whose form is victory' (239d): with the word 'victory', the supremacy over all is designated; ${ }^{48}$ to Him whose own nature transcends all.

'To the Support' (241c), to the Supporter of all.

'Recited' etc.: 'diseases do not bind' the sick person 'to whom this recitation is recited' (248ac).

'No danger' caused by 'misfortunes' (250a); 'it will not be' (25ob).

Because of the stipulation 'this auspicious chapter on appeasement' (256a), [its] goodness should be understood.

'His power fastened', ${ }^{49}$ his power tied — this is the meaning.

Om. Thus [reads] the sixth chapter.

Om. The daily worship is completed.

${ }^{48}$ The commentary seems to take jayarūpāya as a compound.

49 The form katicaśaktih is metathesis for khacitaśaktih, the reading of $\mathrm{P}_{72}^{T}$ after $256 \mathrm{~d}$. 


\section{Bibliography}

\section{Abbreviations}

$\begin{array}{ll}\text { AgnP } & \text { Agnipurāṇa } \\ \text { AIIS } & \text { American Institute of Indian Studies, Varanasi } \\ \text { BhavP } & \text { Bhavișyapurāna } \\ \text { GarP } & \text { Garuḍapurạna } \\ \text { EC } & \text { Epigraphia Carnatica } \\ \text { EI } & \text { Epigraphia Indica } \\ \text { HV } & \text { Harivaṃśa } \\ \text { İ́sP } & \text { Íśānaśivagurudevapaddhati } \\ \text { MBh } & \text { Mahābhārata } \\ \text { MtP } & \text { Matsyapurāṇa } \\ \text { ŚiDhŚ } & \text { Śivadharmaśāstra, see Naraharinath 1998; Jugnu \& Sharma 2014 } \\ \text { ŚiDhU } & \text { Śivadharmottara, see Naraharinath 1998; De Simini 2013 } \\ \text { SkP } & \text { Skandapurāṇa Revākhaṇda } \\ \text { SkP 'Vāyu' } & \text { Skandapurāṇa 'Vāyu' Revākhaṇda } \\ \text { SP } & \text { Skandapurāṇa } \\ \text { TAK } & \text { Tāntrikābhidhānakośa } \\ \text { ViDhP } & \text { Viṣnudharmottarapurāṇa } \\ \text { ViP } & \text { Viṣnupurāṇa }\end{array}$

\section{References}

Acharya, Anil Kumar

2009 Śivadharmasañgrahasya ādyādhyāyatrayasya

samīkșātmakapāțasampādanam adhyayanañca (A Critical Edition

and sSudy of First Three Chapters of the Śivadharmasangraha). PhD thesis, Pondicherry University. 
Agnipurāṇa

(AgnP) ŚrīmaharșiKrș̣naDvaipāyanaVyāsapraṇitam Agnipurāṇam, hindībhūmikā vișayānukramaṇi saṃskrta țippaṇịh sampāditam, sampādakaḥ: Āchārya Baladevopadhyāya. Varanasi: Chowkhamba Sanskrit Sansthan, 1988.

Amarakośa

Amarakośa with the unpublished South Indian Commentaries

Amarapadavivrti of Lingayasūrin and the Amarapadapārijātā of

Mallinātha. Critical Edition with Introduction by A. A. Ramanathan.

Vol. 1. Madras: Adyar Library, 1971.

Aștakaprakaraña

Āchārya Haribhadra's Aștaka Prakaraṇa, with Hindi Translation, Annotations, and Introduction. Edited by K. K. Dīkșita. Ahmedabad:

L.D. Institute of Indology, 1999.

Bakker, Hans

2001 'Sources for Reconstructing Ancient Forms of Śiva Worship', in:

François Grimal (ed.), Les sources et le temps / Sources and Time. A colloquium, Pondicherry, 11-13 January 1997, Pondichéry: IFP EFEO, 397-412.

2014 The World of the Skandapurāna. Northern India in the Sixth and Seventh Centuries. Leiden / Boston: Brill.

Bautze-Picron, Claudine

2010 'The Buddha and His Emaciated Demons', Berliner Indologische

Studien 19: 87-122.

Bhattacharya, Gouriswar

1977 'Nandin and Vụșabha', ZDMG Supplement III/2 (XIX. Deutscher

Orientalistentag vom 28. September bis 4. Oktober 1975 in Freiburg im Breisgau): $1545^{-15} 67$.

Bhuvanavinyāsa

See Kirfel 1954 .

Bisschop, Peter

2006 Early Śaivism and the Skandapurāṇa: Sects and Centres. Groningen: Egbert Forsten.

2010 'Once Again on the Identity of Candeśvara in Early Śaivism: A Rare Canḍeśvara in the British Museum?', Indo-Iranian Journal 53: 233-249.

2014 'Invoking the Powers that Be: The Śivadharma's Mahāśānti Mantra', South Asian Studies 30.2: 133-141.

forthc. a 'Inclusivism Revisited: The Worship of Other Gods in the Śivadharmaśāstra, the Skandapurāṇa and the Niśvāsamukha', in: Vincent Eltschinger, Nina Mirnig, Marion Rastelli (eds.), Tantric Communities in Context.

forthc. b 'From Mantramārga Back to Atimārga: Atimārga as a Self-Referential Term', in: Shaman Hatley et al., Śaivism and the Tantric Traditions: Essays in Honour of Alexis Sanderson. 
Bonazolli, Giorgio

1993 'Introducing "Sivadharma" and "Sivadharmottara", Altorientalistische Forschungen 20.2: 343-349.

Brhatpārāśarahorāśāstra

Bṛatpārāśara-horāśāstra of Parāśara Muni. Edited with a Critical Study, 'Sudhā' Hindī Commentary and Notes by Deva Chandra Jhā. Varanasi: Chowkhamba Sanskrit Series Office, 1973

Brhatsaṃhitā

Varāhamihira's Bṛhatsaṃhitā with English Translation, Exhaustive Notes and Literary Comments. [Edit. by] M. Ramakrishna Bhat. 2 parts. Delhi: Motilal Banarsidass, 1981-82.

Buddhacarita

Āśvaghoșa's Buddhacarita or Acts of the Buddha. Sanskrit Text with English Translation (by) E.H. Johnson. Lahore 1936 [Reprint, New

Delhi: Motilal Banarsidass, 1995].

Bühnemann, Gudrun

1989 'The Heavenly Bodies (navagraha) in Hindu Ritual', Sambhāṣā 11: 1-9. Coburn, Thomas

1984 Devī-Māhātmya. The Crystallization of the Goddess Tradition. Delhi: Motilal Banarsidass.

Cunningham, Alexander

1879 The Stûpa of Bharhut: A Buddhist Monument Ornamented with

Numerous Sculptures Illustrative of Buddhist Legend and History in the Third Century B.C.. London: W.H. Allen and Co.

Davis, Richard

1991 Ritual in an Oscillating Universe: Worshipping Śiva in Medieval India. Princeton: Princeton University Press.

De Simini, Florinda

2013 Ritual Aspects of Manuscript Transmission in Premodern India: A Historical Study of Vidyādāna Through Textual Sources. With a First Critical Edition and English Translation of Śivadharmottara's Chapter Two "On the Gift of Knowledge". PhD thesis, Università degli Studi di Torino.

2016a 'Sivadharma Manuscripts from Nepal and the Making of a Śaiva Corpus', in: Michael Friedrich, Cosima Schwarke (eds.), One-Volume Libraries: Composite and Multiple-Text Manuscripts, Berlin: De Gruyter, 207-26o.

2016b OfGods and Books. Ritual and Knowledge Transmission in the Manuscript Cultures of Premodern India. Berlin/Boston: De Gruyter.

2017 'When Lachmann's Method Meets the Dharma of Śiva. Common Errors, Scribal Interventions, and the Transmission of the Sivadharma Corpus', in: V. Vergiani, D. Cuneo, C.A. Formigatti (eds.), Indic Manuscript Cultures through the Ages, Berlin: De Gruyter, 505-547. 
De Simini, Florinda, \& Nina Mirnig

2017 'Umā and Śiva's Playful Talks in Detail (Lalitavistara): On the

Production of Śaiva Works and their Manuscripts in Medieval Nepal.

Studies on the Śivadharma and the Mahäbhārata 1', in: V. Vergiani, D.

Cuneo, C.A. Formigatti (eds.), Indic Manuscript Cultures through the

Ages, Berlin: De Gruyter, 587-653.

DesJardins, J.F. Marc

2002 Mahāmāyūrì: explorations sur la création d'une écriture prototantrique.

PhD Thesis, McGill University, Montreal.

Dey, Nundo Lal

1971 The Geographical Dictionary of Ancient and Mediceval India. Second Edition. Delhi: Cosmo Publications.

Eck, Diana

2012 India. A Sacred Geography. New York: Three Rivers Press.

Einoo, Shingo

2005 'Ritual Calendar: Change in the Conceptions of Time and Space', Journal Asiatique 293.1: 99-124.

Eltschinger, Vincent

2014 Buddhist Epistemology as Apologetics. Studies on the History,

Self-understanding and Dogmatic Foundations of Late Indian Buddhist

Philosophy. Wien: Verlag der Österreichischen Akademie der

Wissenschaften.

Furui, Ryosuke

2016 'Ājīvikas, Manibhadra and Early History of Eastern Bengal: A New

Copperplate Inscription of Vainyagupta and its Implications', Journal

of the Royal Asiatic Society 26.4: 657-681.

Ganesan, T.

2009 Two Śaiva Teachers of the Sixteenth Century. Nigamajñāna I and his

disciple Nigamajñāna II. Pondichéry: IFP.

Ganesan, T., \& R. Sathyanarayanan

2010-11 'Bhakti as a Fundamental Element in Śaivism', Bulletin d'Études

Indiennes 28-29: 51-62.

Garuḍapurāna

(GarP) The Garudamahāpurānam. [Edited with an Introduction] by

Rājendranātha Śarman, [with a] Ślokānukramaṇī. Delhi: Nag

Publishers, 1984. [= Venkatesvara Press edition 1906.]

Geslani, Marko

2011 The Ritual Culture of Appeasement: Śānti Rites in Post-Vedic Sources. $\mathrm{PhD}$ thesis, Yale University.

2012 'Śānti Rites in the Development of the Purānic Rājyābhiṣeka', Indo-Iranian Journal 55: 321-377.

Geslani, Marko, Bill Mak, et al.

2017 'Garga and Early Astral Science in India', History of Science in South Asia 5.1: 151-191. 
Gonda, Jan

1970 Notes on Names and the Name of God in Ancient India. Amsterdam /

London: North-Holland Publishing Company.

Goodall, Dominic

1998 Bhațta Rämakantha's Commentary on the Kiranatantra. Volume One:

Chapters 1-6, Critical Edition and Annotated Translation. Pondichéry:

IFP/EFEO.

2009 'Who is Caṇdeśa?', in: Shingo Einoo (ed.), The Genesis and Development of Tantra, Tokyo: Institute of Oriental Culture, 351-423 +44 plates.

2011 'The Throne of Worship: An "Archaeological Tell” of Religious

Rivalries', Studies in History 27.2: 221-250.

Goodall, Dominic, et al.

2005 The Pañcāvaraṇastava of Aghoraśiva: A Twelfth-Century South Indian

Prescription for the Visualisation of Sadāsiva and his Retinue.

Pondicherry: IFP/EFEO.

2015 The Niśvāsatattvasaṃhitā. The Earliest Surviving Śaiva Tantra. Vol. 1. A

Critical Edition \& Annotated Translation of the Mülasūtra, Uttarasūtra

\& Nayasūtra. Pondicherry, Paris: IFP/EFEO.

Goodall, Dominic, \& Harunaga Isaacson

2011 'Tantric Traditions', in: Jessica Frazier (ed.), The Continuum Companion to Hindu Studies, London/New York: Continuum, 122-137, 189-191 (notes).

Goudriaan, Teun

1973 'Tumburu and his Sisters', in: Wiener Zeitschrift für die Kunde

Südasiens und Archiv für indische Philosophie 17: 49-95.

Griffiths, A., A. Lepoutre, W.A. Southworth, \& T. Phán (eds.)

2012 The Inscriptions of Campā at the Museum of Cham Sculpture in Đà

Nẵng. Hồ Chí Minh: VNUHCM Publishing House.

Griffiths, Arlo, \& William A. Southworth

2007 'La stèle d'installation de Śrī Satyadeveśvara: une nouvelle inscription

Sanskrite du Campā trouvée à Phu'ó'c Thiện', Journal Asiatique 295.2: 349-381.

Haldar, Jnanranjan

1977 Early Buddhist Mythology. New Delhi: Manohar Book Service.

Harivamśa

(HV) The Harivaṃśa: Being the Khila or Supplement to the Mahābhärata. For the first time critically edited by P.L. Vaidya. Poona: Bhandarkar Oriental Research Institute, 1969-1971.

Harle, J.C.

1971-72 'On the Mahiṣāsuramardinī Images of the Udayagiri Hill (Vidiśā)

Caves', in: U.P. Shah (ed.), Dr. V. S. Agrawala Commemoration Volume (= Journal of the Indian Society of Oriental Art, New Series 4): 44-48. 
Harșacarita

The Harshacarita of Bānabhatța. Text of Ucchvāsas I-VIII. Edited with an Introduction and Notes by P.V. Kane. Delhi: Motilal Banarsidass, 1956. [2nd ed., 1st ed. 1918.]

Hatley, Shaman

2010 'Tantric Śaivism in Early Medieval India: Recent Research and Future Directions', Religion Compass 4/10: 615-628.

2012 'From Mātr to Yoginī. Continuity and Transformation in the South Asian Cults of the Mother Goddesses', in: István Keul (ed.), Transformations and Transfer of Tantra in Asia and Beyond, Berlin / Boston: De Gruyter, 99-130.

Hazra, R.C.

1940 Studies in the Purānic Records on Hindu Rites and Customs. Calcutta: University of Dacca.

1954 'The Siva-dharma', Journal of the Ganganatha Jha Research Institute X (1952-53, issued in 1954): 1-20.

1956 'The Śiva-dharmottara', Journal of the Ganganatha Jha Research Institute XIII: 19-50.

Hidas, Gergely

2015 'Dhāraṇī Sūtras', in: Jonathan Silk (ed.), Brill's Encyclopedia of Buddhism Vol. I, Leiden / Boston: Brill, 129-137.

Hoens, Dirk Jan

1951 Sānti: A Contribution to Ancient Indian Terminology. Vol. 1: Sānti in the Saṃhitās, the Brāhmaṇas and the Śrautasūtras. 's-Gravenhage: H.L. Smits.

Inden, Ronald

2000 'Imperial Purānas. Kashmir as Vaiṣnava Center of the World', in: Ronald Inden, Jonathan Walters, Daud Ali, Querying the Medieval. Texts and the History of Practices in South Asia, Oxford / New York: Oxford University Press, 29-98.

İśānaśivagurudevapaddhati

İsānaśivagurudevapaddhatiḥ śrīmadīśānaśivagurudevamiśraviracitā. Edited by Gaṇapati Śāstrī. Trivandrum: Government Press.

Jugnu, Shrikrishna, \& Bhanvar Sharma

2014 Śrīmad Śivadharmapurāṇam. Śaivadharmasammata upapurāṇa.

Mūlapātha aur Mohanabodhinī Hindī țīā sahita. Varanasi:

Chowkhamba Sanskrit Series.

Kafle, Nirajan

2013 'The Lingodbhava Myth in Early Śaiva Sources', in: Nina Mirnig, Péter-Dániel Szántó, Michael Williams (eds.), Puṣpikā. Tracing Ancient India Through Texts and Traditions. Contributions to Current Research in Indology. Vol. I, Oxford: Oxbow Books, 214-263. 
2015 The Niśvāsamukha, the Introductory Book of the Niśvāsatattvasaṃhitā. Critical Edition, with an Introduction and Annotated Translation.

Appended by Śivadharmasañgraha 5-9. PhD thesis, Leiden University.

Kapstein, Matthew T.

2001 Reason's Traces. Identity and Interpretation in Indian and Tibetan

Buddhist Thought. Boston: Wisdom Publications.

Kane, P. V.

1930-62 History of Dharmaśāstra (Ancient and Mediaeval Religious and Civil

Law in India). Poona: Bhandarkar Oriental Research Institute. 5 vols.

Government Oriental Series, Class B, No. 6. [= Kane I to V.]

Kirfel, Willibald

1954 Das Purāṇa vom Weltgebäude (Bhuvanavinyāsa). Die

kosmographischen Traktate der Purāṇa's. Versuch einer Textgeschichte.

Bonn: Selbstverlag des Orientalischen Seminars der Universität Bonn.

1967 Die Kosmographie der Inder: nach Quellen dargestellt. Hildesheim:

Georg Olms Verlagsbuchhandlung.

Kochhar, Rajesh

2010 'Rāhu and Ketu in Mythological and Astronomological Contexts',

Indian Journal of History of Science 45.2: 287-297.

Krrtyakalpataru Śāntikāṇda

2008 Krrtyakalpataru of Lakșmìdhara. Śāntikāṇda (Volume XIII). Edited by

Mukund Lalji Wadekar. Vadodara: Oriental Institute.

Kunhan Raja, Chittenjoor

1933 Unpublished Upanishads. Edited by the Pandits of Adyar Library under the Supervision of Dr. C. Kunhan Raja. Adyar Library.

László, Franz

1971 Die Parallelversion der Manusmrti im Bhavișyapurāna. Abhandlungen

für die Kunde des Morgenlandes. Bd. 40, 2. Wiesbaden: Steiner Verlag.

Leslie, Julia

1991 'Śrī and Jyeșțhā', in: Roles and Rituals for Hindu Women, Madison:

University of Michigan, 107-128.

Lévi, M. Sylvain

1915 'Le catalogue géographique des Yakṣa dans la Mahāmāyūrī',Journal Asiatique 1: 19-138.

Magnone, Paolo

2005 'Śivadharmottarapurāna: A Survey', in: Petteri Koskikallio (ed.), Epics,

Khilas, and Purānas: Continuities and Ruptures. Proceedings of the

Third Dubrovnik International Conference on the Sanskrit Epics and

Purānas, September 2002, Zagreb: Croatian Academy of Arts and

Sciences, 575-596.

Mahābhārata

(MBh) The Mahābhärata. For the First Time Critically Edited by V. S.

Sukthankar and Others. Poona 1927-59. 19 vols. 


\section{Mahāmāyūrīvidyārājñ̄i}

Ārya-Mahā-Māyūrū Vidyā-Rājñ̄i. Edit. by Shūyo Takubo. Tokyo: Tokyo Sankibo, 1972.

Malinar, Angelika

2007 The Bhagavadgīta.. Doctrines and Contexts. Cambridge: Cambridge University Press.

de Mallmann, Marie-Thérèse

1963 Les enseignments iconographiques de l'Agnipurāṇa. Paris: Annales du Musée Guimet.

Markel, Stephen

1995 Origins of the Indian Planetary Deities. Lewiston, NY: Edwin Mellen Press.

Matsyapurāṇa

(MtP) Śrīmad-Dvaipāyanamuni-praṇitam Matsyapurānam, etad pustakam

Ānandāśramapaṇditaih samśodhitam. Poona: Ananda Ashrama, 1981.

Meister, Michael

[reprint of the edition by H. N. Apte, 1907.]

1986 'Regional Variations in Mātṛkā Conventions', Artibus Asiae 47.3/4:

$233-267$.

Mette, Adelheid

1997 Die Gilgitfragmente des Kāraṇdaryūha. Swisttal-Odendorf: Indica et Tibetica Verlag.

Misra, O.P., \& R.K. Sharma

2003 Archaeological Excavations in Central India (Madhya Pradesh and Chhattisgarh). New Delhi: Mittal Publications.

Misra, Ram Nath

$1981 \quad Y a k s h a$ Cult and Iconography. New Delhi: Munshiram Manoharlal.

Mookerjee, Monotosh

1946 'A Painted Book Cover from Nepal', Journal of the Indian Society of Oriental Art 14: 95-101.

Mrgendra Kriyāpāda

Mrgendrāgama Kriyāpāda et Caryāpāda avec le commentaire de

Bhațta-Nārāyanakaṇtha, ed. N. R. Bhatt. Pondicherry: IFI, 1962.

Naraharinath, Yogin

1998 Śivadharma Paśupatimatam Śivadharmamahāśāstram

Paśupatināthadarśanam. Edit. by Yogin Naraharinatha. Kathmandu, samvat 2055 (1998 CE).

Oberhammer, Gerhard

1994 Offenbarungsgeschichte als Text. Religionshermeneutische

Bemerkungen zum Phänomen in hinduistischer Tradition. Wien: De

Nobili Research Library.

Overbey, Ryan Richard

2016 'Vicissitudes of Text and Rite in the Great Peahen Queen of Spells', in:

David B. Gray, Ryan R. Overbey (eds.), Tantric Traditions in 
Pañcārthabhāṣya

Transmission and Translation, New York: Oxford University Press, $257-283$.

Pāśupatasūtras, with the Pañcārthabhāsya of Kauṇdinya. Edit. by R.

Ananthakrishna Sastri. Trivandrum: Oriental Manuscripts Library, 1940 .

Panikkar, Shivaji K.

1997 Sapta Mātrkā Worship and Sculptures. An Iconographical

Interpretation of Conflicts and Resolutions in the Storied Brāhmanical Icons. New Delhi: D.K. Printworld.

Pettech, Luciano

1984 Mediaeval History of Nepal (c. 750-1482). Second, Thoroughly Revised

Edition. Roma: Istituto Italiano per il Medio ed Estremo Oriente.

Pingree, David

1959 'The Empires of Rudradāman and Yaśodharman: Evidence from Two Astrological Geographies', Journal of the American Oriental Society 79.4: 267-270.

1965 'Representations of the Planets in Indian Astrology', Indo-Iranian Journal 8: 249-267.

1978 The Yavanajātaka of Sphujidhvaja. 2 volumes. Edited, Translated, and Commented on by David Pingree. Cambridge, MS / London: Harvard University Press.

Quintanilla, Sonya Rhie

2007 History of Early Stone Sculpture at Mathura, ca. 150 BCE-100 CE. Leiden / Boston: Brill.

Ramesh, K.V., \& S.P. Tiwari

1990 A Copper-Plate Hoard of the Gupta Period from Bagh, Madhya Pradesh. New Delhi: Archaeological Survey of India.

Rangarajan, Haripriya

2004 Images of Vārāhì. An Iconographic Study. Delhi: Sharada.

Rao, T.A. Gopinatha

1914-16 Elements of Hindu Iconography. Two Volumes. Madras: The Law Printing House.

Regamey, Constantin

1971 'Motifs vichnouites et śivaïtes dans le Kāraṇḍavyūha', in: N.N., Études tibétaines dédiées à la mémoire de Marcelle Lalou, Paris: Librarie d'Amérique et d'Orient, 411-432.

Regmi, Dilli Raman

1966 Medieval Nepal, Part IV. Select Inscriptions, 1524-1768 A.D. With

Verification and Corresponding Dates in C.E. Kathmandu: Firma K.L.

Mukhopadhyay.

Sadovski, Velizar

2013 'Structure and Contents of Lists and Catalogues in Indo-Iranian

Traditions of Oral Poetry (Speech and Performance in Veda and 
Avesta, II)', in: Jared S. Klein \& Kazuhiko Yoshida (eds.), Indic across the Millennia. From the Rigveda to Modern Indo-Aryan, Bremen: Hempen Verlag, 153-192.

Sanderson, Alexis

1988 'Śaivism and the Tantric Traditions', in: S. Sutherland, et al. (eds.), The World's Religions, London: Routledge, 660-704.

2003/04 'The Śaiva Religion among the Khmers, Part I', Bulletin de l'Ecole française d'Extrême-Orient 90-91: 349-462.

2004 'Religion and the State: Śaiva Officiants in the Territory of the King's Brahmanical Chaplain', Indo-Iranian Journal 47: 229-300.

2009 'The Śaiva Age. The Rise and Dominance of Śaivism during the Early Medieval Period', in: Sh. Einoo (ed.), Genesis and Development of Tantrism, Tokyo: Institute of Oriental Culture, 41-350.

2012/13 'The Śaiva Literature', Journal of Indological Studies 24 \& 25: 1-113.

forthc. Śaivism and Brahmanism. Gonda Lecture 2006 (revised for publication, 2011).

Schmid, Charlotte

2003 'À propos des premières images de la Tueuse de buffle: déesses et krishnaïsme ancien', Bulletin de l'Ecole française d'Extrême-Orient 9o.1: 7-67.

Schreiner, Peter

2013 Viṣnupurāṇa. Althergebrachte Kunde über Viṣnu. Aus dem Sanskrit übersetzt und herausgegeben. Berlin: Verlag der Weltreligionen.

Schwartz, Jason

2012 'Caught in the Net of Śāstra: Devotion and Its Limits in an Evolving Śaiva Corpus', Journal of Hindu Studies 5.2: 210-231.

Shastri, Ajay Mitra

$1969 \quad$ India as Seen in the Bṛhatsamnitā of Varāhamihira. Delhi: Motilal Banarsidass.

1995 Inscriptions of the Śarabhapurīyas, Pāṇuvamśins and Somavaṃśins. Part I: Introduction. Part II: Inscriptions. Delhi: Motilal Banarsidass.

Shāstrī, Haraprasāda

1928 A Descriptive Catalogue of Sanskrit Manuscripts in the Government Collection under the Care of the Asiatic Society of Bengal. Volume V. Purāna Manuscripts. Calcutta: Asiatic Society of Bengal.

Sircar, D.C.

1971 Studies in the Religious Life of Ancient and Medieval India. Delhi etc.: Motilal Banarsidass.

1971/72 'Mahāmāyūrī. List of Yakṣas', Journal of Ancient Indian History 5.1-2: $1-91$. 
Skandapurāna

(SP I) The Skandapurāna. Volume I. Adhyāyas 1-25. Critically Edited with Prolegomena and English Synopsis by R. Adriaensen, H.T. Bakker \& H. Isaacson. Groningen: Egbert Forsten, 1998.

(SP IIA) The Skandapurānana. Volume IIA. Adhyāyas 26-31.14. The Vārānasī Cycle. Critical Edition with an Introduction, English Synopsis \& Philological and Historical Commentary by Hans T. Bakker and Harunaga Isaacson. Groningen: Egbert Forsten, 2004.

(SP IIB) The Skandapurāṇa. Volume IIB. Adhyāyas 31-52. The Vāhana and Naraka Cycles. Critical Edition with an Introduction \& Annotated English Synopsis by Hans T. Bakker, Peter C. Bisschop and Yuko Yokochi, in Cooperation with Nina Mirnig and Judit Törzsök. Leiden/Boston: Brill, 2014.

(SP III) The Skandapurāna. Volume III. Adhyāyas 34.1-61, 53-69. The Vindhyavāsin̄ Cycle. Critical Edition with an Introduction \& Annotated English Synopsis by Yuko Yokochi. Leiden/Groningen: Brill / Egbert Forsten 2013.

$\left(\mathrm{SP}_{\mathrm{Bh}}\right) \quad$ Skandapurānasya Ambikākhaṇdaḥ, sampādakaḥ Kṛṣnaprasāda Bhațarāī. Kathmandu: Mahendragranthamāla 2, 1988.

Skandapurāṇa Revākhaṇda

(SkP) Bṛhat śri-Narmadāpurāṇam Revākhaṇdam (parivardhita tritīya saṃskaraṇa). Edit. by Giri On̉kārānanda. Hoshangabad: Jñānasatra Prakāśana Nyāsa, 1994. [E-text by Jürgen Neuss]

(SkP 'Vāyu') śri-Skandamahāpurāṇam, pañcama bhāga: śrí Āvantyakhaṇdam. Edit. by Kṣemarāja Śrīkṛṣnadāsa. Bombay: Ven̉kateśvara Steam Press, 1910. [E-text by Jürgen Neuss]

Ślączka, Anna

2016 'The Two Iconographic Chapters from the Devyāmata and the Art of Bengal', in: Dominic Goodall, Harunaga Isaacson (eds.), Tantric Studies: Fruits of a Franco-German Collaboration on Early Tantra, Pondicherry: IFP / EFEO, 181-246.

Sørensen, Hendrik H.

2006 'The Spell of the Great, Golden Peacock Queen: The Origin, Practices, and Lore of an Early Esoteric Buddhist Tradition in China', Pacific World Third Series 8: 89-123.

Stadtner, Donald

2002 'An Important Dated Yaksha from Mathura: Bhatishri's Manibhadra', Orientations 33.6: $26-3$.

Sternbach, Ludwik

1974 The Mānava dharmaśāstra, I-III and the Bhavisya Purāna. Varanasi: All India Kashi Raj Trust. 
Stietencron, Heinrich von

1966 Indische Sonnenpriester: Sāmba und die Śākadvipūya-Brāhmaṇa. Eine textkritische und religionsgeschichtliche Studie zum indischen Sonnenkult. Wiesbaden: Harrassowitz.

Studholme, Alexander

2002 The Origins of Om Manipadme Hūm. A Study of the

Kāraṇdaryūhasūtra. Albany, NY: State University of New York Press.

Tantrāloka

The Tantrāloka of Abhinavagupta. With Commentary by Rājānaka Jayaratha. Vol. I. Edit. with Notes by Mukund Rām Shāstrī. Srinagar: Kashmir Series of Texts and Studies, 1918.

Tāntrikābhidhānakośa

(TAK II) Tāntrikābhidhānakośa II. Dictionnaire des termes techniques de la littérature hindoue tantrique. A Dictionary of Technical Terms from Hindu Tantric Literature. Wörterbuch zur Terminologie hinduistischer Tantren. Sous la direction de H. Brunner, G. Oberhammer et A. Padoux. Wien: Verlag der Österreichischen Akademie der Wissenschaften, 2004.

(TAK III) Tāntrikābhidhānakośa III. Ț-PH. Dictionnaire des termes techniques de la littérature hindoue tantrique. A Dictionary of Technical Terms from Hindu Tantric Literature. Wörterbuch zur Terminologie hinduistischer Tantren. Fondé sous la direction de Hélène Brunner, Gerhard Oberhammer et André Padoux. Direction éditoriale du troisième volume: Dominic Goodall et Marion Rastelli. Wien: Verlag der Österreichischen Akademie der Wissenschaften, 2013.

Ṭikanikayātrā

Törzsök, Judit

Tikanikayātrā of Varāhamihira. Edit. by Vasant Kumar R. Pandit, in: Journal of the University of Bombay 26 (1957), 40-63.

2004 'Three Chapters of Śaiva Material Added to the Earliest Known Recension of the Skandapurāna', in: Hans T. Bakker (ed.), Origin and Growth of the Purānic Text Corpus with Special Reference to the Skandapurāna, Delhi: Motilal Banarsidass, 17-39.

2013 'The Heads of the Godhead. The Number of Heads/Faces of Yoginīs and Bhairavas in Early Śaiva Tantras', Indo-Iranian Journal 56: 133-155.

Viṣnudharmottarapurāṇa

(VDhP) Viṣnudharmottarapurāṇa. Edit. by Kṣemarāja Śrīkṛ̣̣̣nadāsa. Bombay: Ven̉kateśvara Press, 1912.

Viṣnupurāṇa

(ViP) The Critical Edition of the Viṣnupurānam. Edit. by M. M. Pathak. Pāda-Index prepared by Peter Schreiner. Baroda: Oriental Institute, 1997, 1999 [2 volumes]. 
Vogel, J. Ph.

1926 Indian Serpent Lore or The Nāgas in Hindu Legend and Art. London:

Probsthain.

Wayman, Alex

1987 'Researches on Poison, Garuda-birds and Nāga-serpents based on the

Sgrub thabs kun btus', in: Christopher L. Beckwith (ed.), Silver on Lapis:

Tibetan Literary History, Bloomington, IN: The Tibet Society, 63-76.

Wessels-Mevissen, Corinna

2001 The Gods of the Directions in Ancient India. Origin and Early

Development in Art and Literature (until c. 1000 A.D.). Berlin: Reimer.

Willis, Michael

2009 The Archaeology of Hindu Ritual. Temples and the Establishment of the

Gods. Cambridge: Cambridge University Press.

Williams, R.

1965 'Haribhadra', Bulletin of the School of Oriental and African Studies 28:

101-111.

Yano, Michio

2003 'Calendar, Astrology, and Astronomy', in: Gavin Flood (ed.), The

Blackwell Compendium to Hinduism, Malden, MA / Oxford: Blackwell, 376-392.

2004 'Planet Worship in Ancient India', in: Charles Burnett, et al. (ed.),

Studies in the History of the Exact Sciences in Honour of David Pingree, Leiden/Boston: Brill, 331-348.

Yokochi, Yuko

1999 'The Warrior Goddess in the Devimāhātmya', Senri Ethnological Studies 50: 71-113. 
Peter Bisschop - 978-90-04-38436-1 Downloaded from Brill. com $04 / 26 / 2023$ 11:40:27AM via free access 


\section{Index}

Abhijit, 31, 171

Agni, 17, 29, 35, 40, 148, 158, 162

Agnimukha, 31, 37, 175

Agnipurāṇa, 152, 167

Aindra, 30, 170

Aindrī, 28, 34, 41, 154, 155

Airāvata, 155, 161

Ālampur, 163

Amarakośa, 161

Amarāvatī, 17, 29, 35, 161

Amāvāsī, 3०, 36, 169

Ambikā, 28, 33, 34, 150

Ananta, 32, 37, 176, 199

Añgāraka, 3o, 36, 167

Angiras, 31, 37, 174

Aniruddha, 45

Anuhlāda, 175

Anuhlādaka, 31, 175

Anurādhā, 31, 171

Aparājitā, 30, 35, 165

Apāya, 173

Apsaras, 31, 37, 174

Ârdrā, 31, 171

Arhant, 34, 153, 154

Arka, 170

Aruṇa, 22-24

Aśleșā, 31, 171

aștamūrti, 186

Asuras, 162

Aśvinī, 10, 31, 171, 172

Atharvaveda, 44

Atharvavedapariśișța, 44

Atigaṇụa, 30, 170
Atimaitra, 173

Atimārga, 11, 17

Ātreya, 31, 37, 174

Avalokiteśvara, 14

avatāra, 34

Āyuṣmant, 30, 170

Bahumāṃsā, $15^{6}$

Bālava, 169

bali, 21, 34, 35, 157, 158, 161

Bāṇa, 11

Bava, 169

Bhairava, 33, 39, 156

Bhānu, 22

Bharadvāja, 31, 37, 174

Bharan̄ī, 10, 17, 31, 36, 171, 172

Bhaviṣyapurāṇa, 21, 23, 24, 36, 43, 55, 15०, 151, 157, 165-169, 172, 173

Bhṛgu, 31, 37, 174

Bhṛngin, 15, 151

Bhṛngiriți, 28, 33, 34, 151, 198

Bhūloka, 35, 164, 199

Bhūrloka, 164

Bhūtas, 24, 33, 39, 184

Bhuvanavinyāsa, 161, 162

Bhuvarloka, 35, 164

Brahmā, 6, 9, 28, 34, 41, 44, 45, 152, 155, 16o, 186,198

Brahman, 30, 170

Brahmāṇī, 28, 34, 41, 154, 155

Bṛhaspati, 30, 36, 167

Bṛatpārāśarahorāśāstra, 173 
Bṛhatsaṃhitā , 10, 11, 21, 44-46, 153, 154,

$$
166,169
$$

Buddha, 34, 153, 154

Buddhacarita, 179

Budha, 30, 36, 167

Cāmuṇ̣̣ā, 29, 34, 41, 156, 157, 198

Caṇụeśvara, 28, 33, 34, 151, 152

Caṇdeśvara, 33

Candrabhāgā, 26, 32, 38, 178, 179

Catuṣpāda, 169

Citrā, 31, 171

Dadhna, 183

Dahvadambha, 183

Daitya, 167, 174, 175

Daityas, 31, 37, 38, 175

Dākiṇīs, 24, 184

Dakṣa, 31, 37, 174

Deva, 171,178

Devas, 152, 164, 165, 170

Devī, 6, 7, 148, 149

Devikā, 26, 32, 38, 178

Devīmāhātmya, 49, 150, 156

Dhanada, 170

Dhanișțhā, 31, 172

Dhanu, 31, 172

Dhāraṇī, 45, 46

Dharitrī, 153, 154

dharma, 152, 198

Dharmaputrikā, 52, 53, 55, 56

Dhṛtarāṣțra, 32, 38, 181

Dhṛtarāṣțra, 181

Dhṛti, 30, 170

Dhruva, 30, 37, 170, 174

Dhvāṅkṣī, 173

Dikpāla, 40, 179, 181

Dikpālas, 17, 24, 29, 34, 35, 40, 161, 163

Durgā, 170

Gajavaktra, 170

Gālava, 31, 37, 174

Gaṇa, 6, 33, 152

Gaṇādhipas, 21, 29, 34, 157

Gaṇapati, 33
Gaṇas, 21, 24, 29, 34, 35, 40, 147, 149-152, 157-164, 197, 199

Gaṇda, 30, 170

Gaṇḍakī, 32, 38, 179

Gandhamādana, 183

Gandharvas, 31, 37, 152, 155, 161, 174

Gandhavatī, 17, 29, 35, 163

Gaṇeśa, 15, 149

Gaṅgā, 32, 38, 178, 185, 187

Gara, 169

Garga, 16, 166

Gārgīyajyotișa, 16

Gārgya, 31, 37, 174

Garuḍa, 22-24, 153, 155

Garuḍapurāṇa, 10

Garuḍas, 31, 37, 174

Gaurī, 150

Ghaṇṭākarṇa, 33, $15^{2}$

Ghorā, 173

Ghṛtoda, 183

Ghṛtodaka, 183

Godāvarī, 32, 38, 179

Gomatī, 32, 38, 178

Gomedha, 183

Gomedhadvīpa, 183

Grahas, 33, 39, 168, 185

Hara, 164, 177-181

Haribhadra, 13

Harșacarita, 11, 156

Harṣaṇa, 30, 170

Hastā, 31, 171

Hāṭakeśvara, 175

Hayagrīva, 31, 37, 175

Hemakūța, 183

Himakūṭa, 183

Himāṃśu, 170

Hiraṇyākṣa, 31, 37, 175

Ikṣupūrṇa, 183

Ikṣusvāda, 183

Indra, 17, 29, 35, 40, 158, 161, 163, 170, 186

İsāna, 17, 29, 35, 40, 16o, 164, 167, 171

İsānaśiva, 21, 158 
İsānaśivagurudevapaddhati, 21, 157-16o

Jambudvīpa, 183

Janaloka, 164

Janarloka, 35, 164

Janma, 173

Janman, 168

Jayā, 3o, 34, 35, 153, 154, 165

Jayantī, 35, 165

Jayaratha, 185

Jvalana, 162

Jyeșțhā, 31, 41, 171

Kailāsa, 183

Kālanemi, 31, 37, 175

Kāma, 151, 186

Kaṇva, 31, 37, 174

Kanyā, 31, 173

Kāpālika, 154

Karaṇa, 36, 41, 168, 169

Karanas, 36, 168, 169

Kāraṇdavyūhasūtra, 14, 15, 17, 20

Karkața, 31, 173

Karkoțaka, 32, 37, 177

Kārttikeya, 28, 33, 148, 149

Kașța, 173

Kaśyapa, 168, 181

Kāśyapa, 31, 37, 174

Kaulava, 169

Kaumārī, 28, 34, 41, 155

Kauśika, 31, 37, 174

Kauśikā, 178

Kauśikī, 32, 38, 150, 165, 179

Kāverī, 26, 32, 38, 178

Ketu, 16, 3о, 36, 55, 166, 168, 169

Kimnaras, 162

Kimstughna, 169

Kratu, 31, 37, 174

Krauñca, 183

Krauñcadvīpa, 183

Kṛṣna, 33, 148, 152

Kṛ̣ṇā, 17, 29, 35, 38, 162

Kṛttikā, 10, 11, 17, 31, 36, 171

Kṛttikās, 148
Kṣāroda, 183

Kṣārodadhi, 183

Kṣema, 173

Kṣiroda, 153, 154, 183

Kșīrodadhi, 183

Kubera, 17, 24, 29, 35, 40, 163, 164, 179, 180

Kulika, 32, 37, 176, 178

Kumbha, 31, 173

Kuśa, 183

Kuśadvīpa, 183

Lakulīśvara, 17

Lalitavistara, 52, 55

Lamboșțhī, 29, 34, 41, 154, 156, 157

linga 1, 2, 9, 15, 22, 34, 198

Madana, 170

Mādhava, 153

Madhu, 153

Maghā, 31, 171

Mahābhārata, 6, 149, 181

Mahādeva, 171

Mahājambha, 31, 37, 175

Mahākāla, 28, 33, 34, 150, 197

Mahalloka, 164

Mahāmahiṣamardanī, 28, 33, 15 O

Mahāmāyūrīvidyārājñ̄i, 45

Mahānāda, 31, 37, 175

Mahāpadma, 32, 37, 177

Maharloka, 35, 164

Mahārudra, 18

Mahātala, 165

Mahāvaiśravaṇa, 32, 38, 179

Mahendra, 183

Maheśa, 168

Maheśvara, 14, 28, 33, 39, 147, 174, 178

Mahișāsuramardinī, 150

Mahodarī, 173

Mahodayā, 17, 29, 35, 163

Mahotkața, 31, 37, 175

Maitra, 173

Makara, 31, 173

Malaya, 183

Mālyavat, 183 
Mandākinī, 32, 38, 173, 179

Mandara, 183

Manibhadra, 32, 38, 180

Manu, 31, 37, 174

Manusmrti, 21

Mārkaṇḍa, 31, 37, 174

Maruts, 45

Mātṛ, 40

Mātṛkā, 154, 155

Mātṛs, 21, 28, 29, 34, 40, 41, 154, 155, 157

Matsyapurāṇa, 11

Mayaśilpa, 176

Meru, 161, 183

Meșa, 31, 172

Mīna, 31, 173

Miśrikarā, 173

Mithuna, 31, 173

Mṛgaśirā, 31, 171

Mrgendra, 21, 157

Mucukunda, 31, 37, 175

Muhūrtas, 45

Mūlā, 31, 171

Nāga, 37, 38, 169, 176-178

Nāgarāja, 37

Nāgarājas, 32, 34, 176

Nāgarājas, 176

Nāgas, 24, 32, 177, 178

Nairañjanā, 26, 32, 38, 179

Nairañjanā, 179

Nairṛti, 162

Nakșatrakalpa, 10

Nakṣatramātṛs, 30, 36, 41, 46, 171, 172

Nakṣatras, 10, 17, 36, 45, 46, 171

Nakṣatra, 36, 171

Namuci, 31, 37, 175

Nandā, 153

Nandanā, 173

Nandikeśvara, 6, 7, 147

Nandin, 6, 13, 149

Nandinagara, 12

Nandināgara, 11-13

Nandīśa, 28, 33, 149

Nandīśvara, 13

Nandīśvara, 149
Nārada, 31, 37, 174

Narmadā, 32, 38, 178

Navagrahas, 16, 17, 24, 30, 35, 36, 41, 166,168

Nirṛti, 17, 29, 35, 40, 159, 161-163

Niśvāsamukha, 6, 7

Niśvāsatattvasaṃhitā , 6, 7

Nitala, 165

Padma, 32, 37, 176, 177

Pañcārthabhāṣya, 20

Pāñcika, 32, 38, 18o

Parameśvara, 163

Parigha, 30, 170

Pārvatī, 7, 147, 149-151, 165

Pāśupatasūtra, 20

Paśupati, 177

Pātāla, 37, 158-16o, 175

Pavana, 163

Piśācas, 33, 39, 162, 185

Pitṛs, 162, 170

Plakșadvīpa, 183

Pradyumna, 45

Prahlāda, 31, 37, 175

Pratipad, 169, 170

Pretas, 33, 39, 162, 184

Prīti, 30, 170

Pṛthivī, 34, 153

Pulaha, 31, 37, 174

Punarvasu, 31, 37, 171, 174

Pūrṇabhadra, 24, 32, 38, 181

Pūrṇamā, 30, 36, 170

Puruṣa, 153

Pūrvabhādrapadā, 31, 172

Pūrvaphālguṇī, 31, 171

Pūrvāṣāọhā, 31, 171

Puṣkara, 183

Puṣkaradvīpa, 183

Puṣyā, 31, 171

Rāhu, 16, 24, 30, 36, 166, 168

Rakṣas, 162

Rākṣasas, 33, 39, 183, 199

Rākṣas̄̄, 173

Rāmāyaṇa, 149 
Rasātala, 165

Rāśi, 31, 37, 172

Rāśis, 36, 41, 172, 173

Ravi, 170

Revākhaṇda, 147

Revataka, 31, 37, 175

Revatī, 10, 11, 31, 171, 172

Rohiṇ̄i, 31, 171

R̦ṣabha, 173

R̦̣is, 37, 163

Ṛṣyaśrnnga, 181

Rudra, 7, 8, 15, 22, 42, 147, 149, 151, 155,

157-159, 164, 165, 170, 173, 176, 178-184, 186, 187, 197

Rudraloka, 8, 22

Rudrāṇī, 28, 34, 40, 41, 155

Rudras, 7, 8, 21, 22, 29, 34, 157, 198

Sadāśiva, 147

Sādhya, 30, 45, 170

Śākadvīpa, 183

Śākalya, 31, 37, 174, 183

Śakuni, 169

Śālañkāyana, 31, 37, 174

Śālmali, 183

Śālmalika, 183

Śambhu, 151

Śamkara, 164

Saṃkarṣana, 45

Saṃkrānti, 36, 173

Saṃnihitā, 32, 38, 179

Sampat, 173

Śanaiścara, 24, 30, 36, 168

Sanatkumāra, 6, 147

Śankkara, 170

Śankkhapāla, 32, 37, 177

Śan̉kukarṇa, 31, 37, 175

Șaṇmukha, 7

śānti, 1, 23, 27, 41, 43, 44, 46

Śāntikalpa, 44

Śāntyadhyāya, 1-4, 10, 15, 16, 21-27, 33,

$34,36,44-49,51-55,57,59$,

$63,152,157,189,192,195$

Sarasvatīi, 3o, 32, 35, 38, 165, 178, 179

Sarayū, 32, 38, 178, 179
Śatabhiṣā, 31, 172

Śatānīka, 22

Satyaloka, 35, 164, 199

Satyaloka, 199

Saubhāgya, 30, 170

Śeșa, 35

Siddhāntasāra, 21, $15^{8}$

Siddhas, 31, 37, 155, 161, 163, 174

Siddhi, 30, 170

Siṃha, 31, 172

Siṃhikā, 168

Sindhu, 38

Śiva, $1,2,6,7,14,15,17,18,22,24,27,3$,

$33,34,39-45,147-149$,

152-156, 159-183, 185-187,

197-199

Śivadharmasaṃgraha, 6, 52, 53

Śivadharmaśāstra, 1-12, 14, 15, 17-22, $25^{-27}, 34,40,47,5^{\circ-55}$, 59-61, 63, 147, 152, 154, 171, 195,196

Śivadharmavivaraṇa, 4, 49, 62, 147, 152, $158,16 \circ, 163,183$

Śivadharmottara, 1, 4-6, 10-14, 17-20, $25,26,47,49,5^{\circ}, 5^{2-54}$,

Śivaloka, 186 59-61, 195, 196

Śivapura, 153

Śivapurāṇa, 10

Śivopanișad, 6, 52, 53

Skanda, 7, 147, 149, 170

Skandapurāṇa, 2, 6, 14, 15, 25, 149-151, $154,156,165$

Śobhana, 30, 170

Soma, 30, 36, 166, 167

Śoṇa, 32, 38, 179

Śravaṇā, 31, 171

Śrī, 30, 35, 165, 170

Śrīgiri, 183

Śrīkaṇṭha, 179

Śrīparvata, 183

Sthāṇu, 157

Śubha, 30, 170, 173

Subhadrā, 153

Suciloma, 18 o 
Śuddhavatī, 17, 29, 35, 162

Sukarman, 30, 170

Śukla, 30, 170

Śukra, 3o, 36, 167

Sukunda, 31, 37, 175

Sūla, 30, 170

Sulomaka, 31, 37, 175

Sumanā, 153

Sumantu, 22

Suparva, 31, 37, 175

Surabhī, 153

Suras, 162,167

Suroda, 183

Sūrya, 22-24, 30, 36, 43, 166

Suśīlā, 153

Sutala, 165

Suviroma, 32, 38, 180

Svāduda, 183

Svāduka, 183

Svargaloka, 22

Svarloka, 35, 164

Svātī, 31, 171

Taitila, 169

Takṣaka, 32, 37, 176, 177

Tala, 165

Talas, 175

Talātala, 165

Tāmraparṇī, 178

Tapoloka, 35, 164

Tāraka, 31, 37, 175

Tārakagaṇas, 173

Tarkajvālā, 156

Tārkṣya, 153, 155

Tejasvinī, 162

Tejovatī, 17, 29, 35, 162

Tikanikayātrā, 173

tìrtha, 38

Tithi, 25, 30, 36, 55, 168, 170

Tithis, 41, 169, 170

Tribhuvaneśvara, 171

Trirekhin, 177

Tulā, 31, 173

Tumburu, 35, 165
Umā, 28, 33, 39, 40, 148, 197

Umāmaheśvarasaṃvāda, 52, 53

Umottara, 52

Uragas, 162

Urvaśí, 181

Uttarabhadrā, 31, 172

Uttaraphālguṇī, 31, 171

Uttarāṣạ̣̄hā, 31, 171

Uttarottaramahāsaṃvāda, 52, 53

Uttarottaratantra, $5^{2}$

Vahni, 170

Vaidhṛti, 30, 170

Vaișṇavī, 28, 34, 41, 155

Vaiśravaṇa, 179, 181

Vaivasvatī, 17, 29, 35, 162

Vajra, 30, 170

Vālmīka, 31, 37, 174

Vanij, 169

Varāhamihira, 10, 16, 161, 166, 171, 173

Vārāhī, 29, 34, 41, 154-156, 198

Vārāṇasī, 38

Variyas, 30, 170

Varuna, 17, 29, 35, 40, 159, 162, 163

Varuṇā, 27, 32, 38, 178

Vasișțha, 31, 37, 174

Vāsudeva, 44

Vāsuki, 24, 32, 37, 176

Vāyavīyasaṃhitā, 10

Vāyu, 17, 24, 29, 35, 40, 159, 163, 199

Vāyupurāṇa, 149

Vibhāṇụaka, 24, 32, 38, 181

Vidyādharas, 31, 161, 174

Vijayā, 34, 35, 153, 165

Vināyaka, 15, 28, 33, 149

Vindhyavāsinī, 165

Vipat, 173

Virocana, 31, 37, 175

Virūḍhaka, 181

Virūpākṣa, 32, 38, 161, 181, 182

Viśākhā, 31, 171

Vișkambha, 30, 170

Viṣṇu, 9, 28, 34, 44, 45, 153, 170, 176, 184, 186

Viṣnudharma, 49 
Vișnudharmottarapurāṇa, 35, 44, 45

Vișți, 25, 30, 36, 41, 55, 168, 169

Viśvāmitra, 31, 37, 174

Vitala, 165

Vṛddhi, 30, 170

Vṛșa, 31, 33, 152, 173, 198

Vṛșabha, 153

Vrșasārasamgraha, 52, 53

Vṛścika, 31, 173

Vyāghāta, 30, 170

Vyāsa, 6, 23

Vyatīpāta, 30, 170
Yājñavalkyasmrti, 11

Yakșa, 38, 180, 181

Yakșas, 24, 32-34, 38, 45, 163, 179-182

Yakșiṇīs, 179, 180

Yama, 17, 29, 35, 40, 158, 162, 163, 170

Yamunā, 32, 38, 178

Yaśovatī, 17, 29, 35, 164

Yavanajātaka, 172

Yoga, 25, 36, 55

Yogas, 30, 41, 170

Yogayātrāa, 161, 166

Yogin, 149, $15^{2}$

Yoginīs, 24, 33, 39, 184 